\title{
BIOLOGIC RESPONSES OF LIGAMENT AND TENDON GRAFT-DERIVED FIBROBLASTS TO CLINICAL LEVELS OF CYCLIC STRAIN
}

A Dissertation
presented to
the Faculty of the Graduate School
at the University of Missouri-Columbia
In Partial Fulfillment
of the Requirements for the Degree
Doctor of Philosophy
SEBASTIAN CARDONA-RAMIREZ
Dr. James L. Cook, Dissertation Supervisor
MAY 2020


(C) Copyright by Sebastian Cardona-Ramirez 2020

All Rights Reserved 
The undersigned, appointed by the dean of the Graduate School, have examined the dissertation entitled

\section{BIOLOGIC RESPONSES OF LIGAMENT AND TENDON GRAFT-DERIVED FIBROBLASTS TO CLINICAL LEVELS OF CYCLIC STRAIN}

presented by Sebastian Cardona-Ramirez, a candidate for the degree of Doctor of Philosophy, and hereby certify that, in their opinion, it is worthy of acceptance.

Professor James L. Cook

Professor Aaron M. Stoker

Professor Richard Ma

Professor Cristi Cook

Professor Trent Guess 


\section{DEDICATION}

To all students from developing countries trying to pursue a career in science. Keep pushing, the journey is long, but the reward vastly outweighs the effort

"We must try to expand the boundaries of human wisdom, empathy and perception, and

there is no way of doing that except through education."

- J. William Fulbright 


\section{ACKNOWLDEGEMENTS}

The author wishes to thank all persons and institutions involved in the pursuing of this degree. I would like to thank The Fulbright program for believing in young scientists and encourage them to pursue their dreams with the motto "bright minds that will change the world". It has been an amazing journey full of interesting people that, for sure, are already changing the world. I would also like to thank the Colombian National Institute of Sciences (COLCIENCIAS) for tuition and stipend funding through these four years and for considering this young academic a potential scientist. Also, I would like to thank LASPAU, specially my placement advisor Lisa Tapiero for their hard work in accepting, reviewing and managing my application for doctoral programs and for making sure it was accepted by the best universities. Special thanks to Jillian Collins and Lauren Pate from the International Student and Scholar services at the University of Missouri for guiding me through the process of becoming an MU scholar and for helping me with all the administrative and legal processes to be able to find a home in the US.

Now, I would like to thank the people more closely related with the successful achievement of my academic doctoral program. First and foremost, I would like to thank Dr. James L. Cook for giving me an opportunity to thrive and for showing me how education can change people. He has been really inspiring throughout my career, even before I get to know him. I will always remember how I got fascinated by his ability to lead quality orthopaedic research and how his publications reached me when I was a young undergraduate Veterinary Medicine student in Colombia. Second, I want to thank my mentor, Dr. Richard Ma for his support and teaching on critical reasoning and for the clinical approach that is making our science being heard in the most important scientific 
meetings and reachable by everyone. Third, I want to thank Dr. Aaron Stoker for his endless direction and patience and his highly valuable input in all the scientific processes that led to this work. I also want to thank Dr. Cristi Cook for showing me the value of education across borders and how individuals can improve their whole community just by giving them a chance to attend to school. I also want to thank Dr. Trent Guess for his innovative way to approach orthopaedic diseases and for finding clever solutions to complex problems such as the use of digital tools for orthopaedic patients and his input in the biomechanical part of my dissertation.

Lastly, I want to thank the persons responsible to make me believe in science in the first place, my family. I want to thank my mom, for always encouraging me to wonder and be fascinated by nature and simple events like rain and rainbows. Huge thanks to my wife, for her endless support and always believing in me, even during the most difficult situations. I want to thank my children Sara and Juan Felipe for being the boost that my soul needed, for giving me their love and make me their role model. I want to thank my aunt for her relentless support and for all the effort she has put into my education.

Thank you, God, for all your blessings and for guiding me through the darkest loneliest times, I am invincible with you by my side.

Lastly, I want to thank the TLRO family, all the staff in the MOI, specially Dr. Ma's team for their help with this study. Special thanks to all my current and past friends, they have filled this journey with memories 


\section{TABLE OF CONTENTS}

ACKNOWLDEGEMENTS

LIST OF FIGURES — vi

LIST OF TABLES $\quad$ xxiii

LIST OF ABBREVIATIONS Xxiv

LIST OF PRODUCTS $\quad$ xxvi

ACADEMIC ABSTRACT $\quad$ xxvii

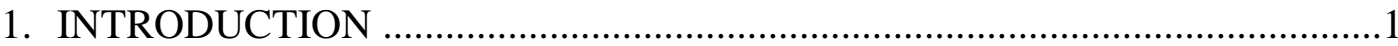

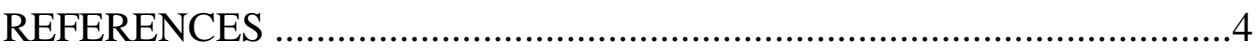

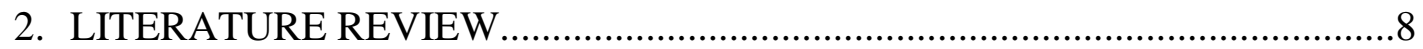

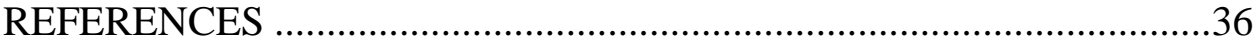

3. EFFECT OF THREE DIFFERENT CYCLIC STRAIN PROTOCOLS ON NORMAL CANINE ACL TENDON GRAFTS CELLULAR METABOLIC

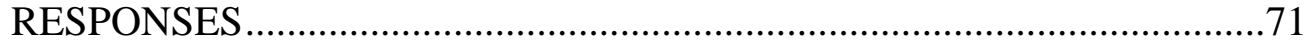

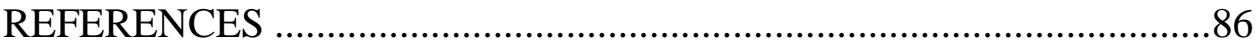

4. NORMAL CANINE ACL, PCL, SYN CELLULAR RESPONSE TO

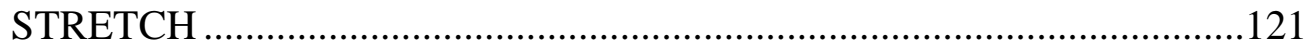

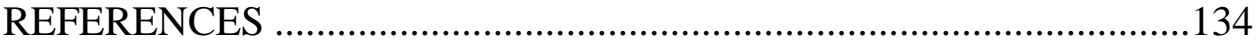

5. SEX DIFFERENCES IN ANTERIOR CRUCIATE LIGAMENT TENDON

GRAFT RESPONSE TO MECHANICAL STRAIN ..............................168 
6. NORMAL CANINE QT, PT, HT CELLS CO-CULTURED WITH SYNOVIOCYTES AND EXPOSED TO MECHANICAL STRAIN

REFERENCES

7. EFFECTS OF MECHANICAL STRAIN AND SEX ON METABOLIC RESPONSES OF HUMAN ANTERIOR CRUCIATE LIGAMENT AND SYNOVIUM .264 REFERENCES .277

8. NORMAL HUMAN TENDON DERIVED FIBROBLASTS EXPOSED TO MECHANICAL STRAIN 310

REFERENCES .323 


\section{LIST OF FIGURES}

Figure $\quad$ Page

2.1 Main events in ACL healing and repair $\quad 70$

3.1 Experimental Design-Canine Tendon Fibroblasts in monoculture 97

3.2 Metabolic activity after five days of culture-Strain differences in female 98 canine tendon fibroblasts in monoculture

3.3 Concentration of PGE-2 released to the media-Strain differences in

female canine tendon fibroblasts in monoculture

3.4 Concentration of IL-6 released to the media-Strain differences in female 100 canine tendon fibroblasts in monoculture

3.5 Concentration of IL-8 released to the media-Strain differences in female 101 canine tendon fibroblasts in monoculture

3.6 Concentration of KC released to the media-Strain differences in female 102 canine tendon fibroblasts in monoculture

3.7 Concentration of MCP-1 released to the media-Strain differences in female canine tendon fibroblasts in monoculture

3.8 Concentration of Glycosaminoglycans (GAG) released to the media-

Strain differences in female canine tendon fibroblasts in monoculture

3.9 Concentration of MMP ACTIVITY (MMPACT) released to the media-

Strain differences in female canine tendon fibroblasts in monoculture

3.10 Concentration of MMP-1 released to the media-Strain differences in female canine tendon fibroblasts in monoculture 
3.11 Concentration of MMP-2 released to the media-Strain differences in female canine tendon fibroblasts in monoculture

3.12 Concentration of MMP-3 released to the media-Strain differences in female canine tendon fibroblasts in monoculture

3.13 Metabolic activity after five days of culture-Tissue differences in female canine tendon fibroblasts in monoculture

3.14 Concentration of PGE-2 released to the media-Tissue differences in female canine tendon fibroblasts in monoculture

3.15 Concentration of IL-6 released to the media-Tissue differences in female canine tendon fibroblasts in monoculture

3.16 Concentration of IL-8 released to the media-Tissue differences in female canine tendon fibroblasts in monoculture

3.17 Concentration of KC released to the media-Tissue differences in female canine tendon fibroblasts in monoculture

3.18 Concentration of MCP-1 released to the media-Tissue differences in female canine tendon fibroblasts in monoculture

3.19 Concentration of GAG released to the media-Tissue differences in female canine tendon fibroblasts in monoculture

3.20 Concentration of MMP Activity (MMPACT) in the media-Tissue differences in female canine tendon fibroblasts in monoculture

3.21 Concentration of MMP-1 the media-Tissue differences in female canine tendon fibroblasts in monoculture 
3.22 Concentration of MMP-2 the media-Tissue differences in female canine tendon fibroblasts in monoculture

3.23 Concentration of MMP-3 the media-Tissue differences in female canine tendon fibroblasts in monoculture

3.24 Canine Quadriceps tendon (QT) fibroblasts after being stretched for 120 hours.

3.25 Canine Patellar tendon (PT) fibroblasts after being stretched for 120 hours.

3.26 Canine Hamstring tendon (HT) fibroblasts after being stretched for 120 hours.

4.1 Experimental Design-Canine Intraarticular Fibroblasts in monoculture

4.2 Metabolic Activity after five days of culture-Strain differences in female canine intraarticular fibroblasts in monoculture

4.3 Concentration of PGE2 released to the media-Strain differences in female canine intraarticular fibroblasts in monoculture

4.4 Concentration of IL-6 released to the media-Strain differences in female canine intraarticular fibroblasts in monoculture

4.5 Concentration of IL-8 released to the media-Strain differences in female canine intraarticular fibroblasts in monoculture

4.6 Concentration of $\mathrm{KC}$ released to the media-Strain differences in female canine intraarticular fibroblasts in monoculture

4.7 Concentration of MCP-1 released to the media-Strain differences in female canine intraarticular fibroblasts in monoculture 
4.8 Concentration of Glycosaminoglycans (GAG) released to the media-

Strain differences in female canine intraarticular fibroblasts in monoculture

4.9 Concentration of total MMP activity (MMPACT) released to the mediaStrain differences in female canine intraarticular fibroblasts in monoculture

4.10 Concentration of MMP-1 released to the media-Strain differences in female canine intraarticular fibroblasts in monoculture

4.11 Concentration of MMP-2 released to the media-Strain differences in female canine intraarticular fibroblasts in monoculture

4.12 Concentration of MMP-3 released to the media-Strain differences in female canine intraarticular fibroblasts in monoculture

4.13 Metabolic Activity of fibroblasts-Tissue differences in female canine intraarticular fibroblasts in monoculture

4.14 Concentration of PGE2 released to the media-Tissue differences in female canine intraarticular fibroblasts in monoculture

4.15 Concentration of IL-6 released to the media-Tissue differences in female canine intraarticular fibroblasts in monoculture

4.16 Concentration of IL-8 released to the media-Tissue differences in female canine intraarticular fibroblasts in monoculture

4.17 Concentration of KC released to the media-Tissue differences in female 160 canine intraarticular fibroblasts in monoculture 
4.18 Concentration of MCP1 released to the media-Tissue differences in female canine intraarticular fibroblasts in monoculture

4.19 Concentration of GAG released to the media-Tissue differences in female canine intraarticular fibroblasts in monoculture

4.20 Concentration of MMP Activity (MMPACT) in the media-Tissue differences in female canine intraarticular fibroblasts in monoculture

4.21 Concentration of MMP-1 released to the media-Tissue differences in female canine intraarticular fibroblasts in monoculture

4.22 Concentration of MMP-2 released to the media-Tissue differences in female canine intraarticular fibroblasts in monoculture

4.23 Concentration of MMP-3 released to the media-Tissue differences in female canine intraarticular fibroblasts in monoculture

4.24 Canine Anterior Cruciate Ligament (ACL) fibroblasts after being stretched for 120 hours

4.25 Canine Posterior Cruciate Ligament (PCL) fibroblasts after being stretched for 120 hours

5.1 Experimental Design-Canine Tendon fibroblastic differences by sex

5.2 Concentration of prostaglandin E2 (PGE2) released to the media-Canine Tendon fibroblastic differences by sex

5.3 Concentration of intereukin-8 (IL-8) released to the media-Canine Tendon fibroblastic differences by sex

5.4 Concentration of keratinocyte chemoattractant protein $(\mathrm{KC})$ released to the media-Canine Tendon fibroblastic differences by sex 
5.5 Concentration of monocyte chemoattractant protein-1 (MCP-1) released to the media-Canine Tendon fibroblastic differences by sex

5.6 Concentration of Glycosaminoglycans (GAG) released to the media-

Canine Tendon fibroblastic differences by sex

5.7 Concentration of total MMP activity (MMPACT) released to the media-

Canine Tendon fibroblastic differences by sex

5.8 Concentration of matrix metalloproteinase 2 (MMP-2) released to the

media-Canine Tendon fibroblastic differences by sex

5.9 Cellular Metabolic Activity in male fibroblasts after 120 hours-Strain differences in tendon fibroblasts from male dogs

5.10 Concentration of PGE2 released to the media in males-Strain differences in tendon fibroblasts

5.11 Concentration of IL-8 released to the media in males-Strain differences in tendon fibroblasts

5.12 Concentration of KC released to the media in males-Strain differences in tendon fibroblasts

5.13 Concentration of MCP-1 released to the media in males-Strain differences in tendon fibroblasts

5.14 Concentration of GAG released to the media in males-Strain differences in tendon fibroblasts

5.15 Concentration of MMPACT released to the media in males-Strain differences in tendon fibroblasts 
5.16 Concentration of MMP-2 released to the media in males-Strain differences in tendon fibroblasts

5.17 Cellular Metabolic Activity in males after 120 hours-Tissue differences 201 in tendon fibroblasts

5.18 Concentration of PGE2 released to the media in males-Tissue differences in tendon fibroblasts

5.19 Concentration of IL-8 released to the media in males-Tissue differences 203 in tendon fibroblasts

5.20 Concentration of $\mathrm{KC}$ released to the media in males-Tissue differences 204 in tendon fibroblasts

5.21 Concentration of MCP1 released to the media in males-Tissue differences in tendon fibroblasts

5.22 Concentration of GAG released to the media in males-Tissue differences 206 in tendon fibroblasts

5.23 Concentration of MMPACT released to the media in males-Tissue differences in tendon fibroblasts

5.24 Concentration of MMP-2 released to the media in males-Tissue differences in tendon fibroblasts

5.25 Quadriceps tendon (QT) fibroblasts from male dogs stretched after 24 and 120 hours at three different stain magnitudes.

5.26 Patellar Tendon (PT) fibroblasts from male dogs stretched after 24 and 209 120 hours at three different stain magnitudes. 
5.27 Hamstring Tendon (HT) fibroblasts from male dogs stretched after 24 and 120 hours at three different stain magnitudes.

6.1 Experimental Design-Canine Tendon Fibroblasts in Coculture with SYN 236

6.2 Metabolic Activity after 120h of culture-Monoculture vs Coculture 237

6.3 Concentration of prostaglandin E2 (PGE2) released to the media- 238

Monoculture vs Coculture

6.4 Concentration of interleukin-6 (IL-6) released to the media-Monoculture 239 vs Coculture

6.5 Concentration of intereukin-8 (IL-8), released to the media-Monoculture 240 vs Coculture

6.6 Concentration of keratinocyte chemoattractant protein $(\mathrm{KC})$ released to the media-Monoculture vs Coculture

6.7 Concentration of monocyte chemoattractant protein-1 (MCP-1) released to the media-Monoculture vs Coculture

6.8 Concentration of Glycosaminoglycans (GAG) released to the mediaMonoculture vs Coculture

6.9 Concentration of total MMP activity (MMPACT) released to the mediaMonoculture vs Coculture

6.10 Concentration of MMP-1 released to the media-Monoculture vs Coculture

6.11 Concentration of MMP-2 released to the media-Monoculture vs Coculture 
6.12 Concentration of MMP- 3 released to the media-Monoculture vs

Coculture

6.13 Metabolic Activity of graft fibroblasts-Strain differences in cocultured tendon fibroblasts

6.14 Concentration of PGE-2 released to the media when co-cultured with

SYN-Strain differences in cocultured tendon fibroblasts

6.15 Concentration of IL-8 released to the media when co-cultured with

SYN-Strain differences in cocultured tendon fibroblasts

6.16 Concentration of KC released to the media when co-cultured with SYN-

Strain differences in cocultured tendon fibroblasts

6.17 Concentration of MCP1 released to the media when co-cultured with

SYN-Strain differences in cocultured tendon fibroblasts

6.18 Concentration of GAG released to the media when co-cultured with

SYN-Strain differences in cocultured tendon fibroblasts

6.19 Concentration of MMPACT released to the media when co-cultured with SYN-Strain differences in cocultured tendon fibroblasts

6.20 Concentration of MMP-2 released to the media when co-cultured with SYN-Strain differences in cocultured tendon fibroblasts

6.21 Metabolic Activity of graft fibroblasts-Tissue differences in cocultured 256 tendon fibroblasts

6.22 Concentration of PGE2 released to the media when co-cultured with SYN-Tissue differences in cocultured tendon fibroblasts 
6.23 Concentration of IL-8 released to the media when co-cultured with

SYN-Tissue differences in cocultured tendon fibroblasts

6.24 Concentration of $\mathrm{KC}$ released to the media when co-cultured with SYN-

Tissue differences in cocultured tendon fibroblasts

6.25 Concentration of MCP1 released to the media when co-cultured with

SYN-Tissue differences in cocultured tendon fibroblasts

6.26 Concentration of GAG released to the media when co-cultured with

SYN-Tissue differences in cocultured tendon fibroblasts

6.27 Concentration of MMPACT released to the media when co-cultured

with SYN-Tissue differences in cocultured tendon fibroblasts

6.28 Concentration of MMP-2 released to the media when co-cultured with

SYN-Tissue differences in cocultured tendon fibroblasts

7.1 Experimental Design-Human Intraarticular Fibroblasts

7.2 Metabolic Activity of human intraarticular fibroblasts.

7.3 Concentration of PGE2 released to the media-Strain differences in

Human Intraarticular Fibroblasts

7.4 Concentration of interleukins 6 (IL-6) and 8 (IL-8) released to the media-Strain differences in Human Intraarticular Fibroblasts

7.5 Concentration of chemokines (KC and MCP1) released to the media-

Strain differences in Human Intraarticular Fibroblasts

7.6 Concentration of Vascular Endothelial Growth Factor (VEGF) and Macrophage Inflammatory Protein $1 \beta(\mathrm{MP} 1 \beta)$ released to the mediaStrain differences in Human Intraarticular Fibroblasts 
7.7 Concentration of GAG content released to the media-Strain differences 288 in Human Intraarticular Fibroblasts

7.8 Concentration of matrix metalloproteinases (MMP) 1,2 and 3 released to 289 the media-Strain differences in Human Intraarticular Fibroblasts

7.9 Concentration of tissue inhibitor of matrix metalloproteinases (TIMPs) 1290 and 2 released to the media-Strain differences in Human Intraarticular Fibroblasts

7.10 Metabolic Activity of fibroblasts after 120h of culture-Tissue differences in Human Intraarticular Fibroblasts

7.11 Concentration of PGE2 released to the media-Tissue differences in Human Intraarticular Fibroblasts

7.12 Concentration of IL-6 released to the media-Tissue differences in Human Intraarticular Fibroblasts

7.13 Concentration of IL- 8 released to the media-Tissue differences in Human Intraarticular Fibroblasts

7.14 Concentration of KC released to the media-Tissue differences in Human Intraarticular Fibroblasts

7.15 Concentration of MCP1 released to the media-Tissue differences in Human Intraarticular Fibroblasts

7.16 Concentration of VEGF released to the media-Tissue differences in Human Intraarticular Fibroblasts

7.17 Concentration of MP1 $\beta$ released to the media-Tissue differences in Human Intraarticular Fibroblasts 
7.18 Concentration of GAG released to the media-Tissue differences in

Human Intraarticular Fibroblasts

7.19 Concentration of MMP-1 released to the media-Tissue differences in

Human Intraarticular Fibroblasts

7.20 Concentration of MMP-2 released to the media-Tissue differences in

Human Intraarticular Fibroblasts

7.21 Concentration of MMP-3 released to the media-Tissue differences in

Human Intraarticular Fibroblasts

7.22 Concentration of TIMP-1 released to the media-Tissue differences in

Human Intraarticular Fibroblasts

7.23 Concentration of TIMP-2 released to the media-Tissue differences in

Human Intraarticular Fibroblasts

7.24 Metabolic activity of fibroblasts after 120h in culture-Sex differences in

Human Intraarticular Fibroblasts

7.25 Concentration of PGE2 released to the media-Sex differences in Human

Intraarticular Fibroblasts

7.26 Concentration of Interleukins 6 (IL-6) and (IL-8) released to the media-

Sex differences in Human Intraarticular Fibroblasts

7.27 Concentration of chemokines (KC, MCP1, MP1 $\beta)$ and Vascular

Endothelial Growth Factor (VEGF) released to the media-Sex

differences in Human Intraarticular Fibroblasts

7.28 Concentration of GAG content released to the media-Sex differences in

Human Intraarticular Fibroblasts 
7.29 Concentration of Matrix Metalloproteinases (MMP-1, 2 and 3) released

to the media-Sex differences in Human Intraarticular Fibroblasts

7.30 Concentration of Tissue Inhibitor of Matrix Metalloproteinases (TIMP- 308

1 and 2) released to the media-Sex differences in Human Intraarticular

Fibroblasts

7.31 Synovium (SYN) fibroblasts stretched after three days and six days at three different stain magnitudes.

7.32 Anterior Cruciate Ligament (ACL) fibroblasts stretched after three days and six days at three different stain magnitudes.

8.1 Experimental design-Human Tendon Fibroblasts

8.2 Metabolic activity of fibroblasts after six days of culture-Strain

differences in Human Tendon Fibroblasts

8.3 Concentration of PGE2 released to the media-Strain differences in

Human Tendon Fibroblasts

8.4 Concentration of IL-6 released to the media-Strain differences in Human

Tendon Fibroblasts

8.5 Concentration of IL-8 released to the media-Strain differences in Human

Tendon Fibroblasts

8.6 Concentration of KC released to the media-Strain differences in Human

Tendon Fibroblasts

8.7 Concentration of MCP1 released to the media-Strain differences in Human Tendon Fibroblasts 
8.8 Concentration of MP1B released to the media-Strain differences in

Human Tendon Fibroblasts

8.9 Concentration of VEGF released to the media-Strain differences in

Human Tendon Fibroblasts

8.10 Concentration of GAG released to the media-Strain differences in

Human Tendon Fibroblasts

8.11 Concentration of MMP-1 released to the media-Strain differences in

Human Tendon Fibroblasts

8.12 Concentration of MMP-2 released to the media-Strain differences in

Human Tendon Fibroblasts

8.13 Concentration of MMP-3 released to the media-Strain differences in

Human Tendon Fibroblasts

8.14 Concentration of TIMP-1 released to the media-Strain differences in

Human Tendon Fibroblasts

8.15 Concentration of TIMP-2 released to the media-Strain differences in

Human Tendon Fibroblasts

8.16 Metabolic activity of fibroblasts after six days of culture-Tissue

differences in Human Tendon Fibroblasts

8.17 Concentration of PGE2 released to the media-Tissue differences in

Human Tendon Fibroblasts

8.18 Concentration of IL-6 released to the media-Tissue differences in

Human Tendon Fibroblasts 
8.19 Concentration of IL-8 released to the media-Tissue differences in

Human Tendon Fibroblasts

8.20 Concentration of KC released to the media-Tissue differences in Human 346

Tendon Fibroblasts

8.21 Concentration of MCP1 released to the media-Tissue differences in

Human Tendon Fibroblasts

8.22 Concentration of MP1 $\beta$ released to the media-Tissue differences in

Human Tendon Fibroblasts

8.23 Concentration of VEGF released to the media-Tissue differences in

Human Tendon Fibroblasts

8.24 Concentration of GAG released to the media-Tissue differences in

Human Tendon Fibroblasts

8.25 Concentration of MMP-1 released to the media-Tissue differences in

Human Tendon Fibroblasts

8.26 Concentration of MMP-2 released to the media-Tissue differences in

Human Tendon Fibroblasts

8.27 Concentration of MMP-3 released to the media-Tissue differences in

Human Tendon Fibroblasts

8.28 Concentration of TIMP-1 released to the media-Tissue differences in

Human Tendon Fibroblasts

8.29 Concentration of TIMP-2 released to the media-Tissue differences in

Human Tendon Fibroblasts 
8.30 Metabolic Activity of fibroblasts after 120h of culture-Sex differences in 356 Human Tendon Fibroblasts

8.31 Concentration of PGE2 released to the media-Sex differences in Human 357

Tendon Fibroblasts

8.32 Concentration of IL-6 released to the media-Sex differences in Human 358

Tendon Fibroblasts

8.33 Concentration of IL-8 released to the media-Sex differences in Human 359

Tendon Fibroblasts

8.34 Concentration of KC released to the media-Sex differences in Human 360

Tendon Fibroblasts

8.35 Concentration of MCP1 released to the media-Sex differences in Human 361

Tendon Fibroblasts

8.36 Concentration of MP1 $\beta$ released to the media-Sex differences in Human 362

Tendon Fibroblasts

8.37 Concentration of VEGF released to the media-Sex differences in Human 363

Tendon Fibroblasts

8.38 Concentration of GAG released to the media-Sex differences in Human 364

Tendon Fibroblasts

8.39 Concentration of MMP-1 released to the media-Sex differences in

Human Tendon Fibroblasts

8.40 Concentration of MMP-2 released to the media-Sex differences in

Human Tendon Fibroblasts 
8.41 Concentration of MMP-3 released to the media-Sex differences in

Human Tendon Fibroblasts

8.42 Concentration of TIMP-1 released to the media-Sex differences in

Human Tendon Fibroblasts

8.43 Concentration of TIMP-2 released to the media-Sex differences in

Human Tendon Fibroblasts

8.44 Quadriceps Tendon (QT) fibroblasts stretched after three days and six

days at three different stain magnitudes.

8.45 Patellar Tendon (PT) fibroblasts stretched after three days and six days 370 at three different stain magnitudes.

8.46 Hamstring Tendon (HT) fibroblasts stretched after three days and six days at three different stain magnitudes. 


\section{LIST OF TABLES}

Table Page

7.1 Patient sex and age distribution by tissue source. Human intraarticular 285 tissues

8.1 Patient sex and age distribution by tissue source. Human tendon grafts 


\section{LIST OF ABBREVIATIONS \\ (IN ALPHABETICAL ORDER)}

2D: Two-dimensional

3D: Three-dimensional

ACL: Anterior Cruciate Ligament

ACLR: Anterior Cruciate Ligament Reconstruction

ACUC: Animal Care and Use Committee

AM: Antero-Medial

bFGF: Basic Fibroblastic Growth Factor

BPTB: Bone-Patellar Tendon-Bone

COL: Collagen

COX: Ciclo-Oxigenase

CrCL: Cranial Cruciate Ligament

DHT: Dihydrotestosterone

DMEM: Dulbecco’s Modified Eagle’s Medium

ECM: Extra Cellular Matrix

GAG: Glycosaminoglycan

HT: Hamstring Tendon

IL: Interleukin

IQR: Interquartile Range

KC: Keratinocyte Chemoattractant

MCP-1: Monocyte Chemoattractant Protein -1

MIP-1 $\beta$ : Macrophage Inflammatory Protein-1 $\beta$ 
MMP: Matrix Metalloproteinase

MMPACT: MMP Activity

MSC: Mesenchymal Stem Cell

$\mathrm{N}$ : Newton

OA: Osteoarthritis

PCL: Posterior Cruciate Ligament

PDGF: Platelet Derived Growth Factor

PG: Proteoglycan

PGE: Prostaglandin E

PICP: Procollagen-I Carboxi-terminal Propeptide

PL: Postero-lateral

PRP: Platelet Rich Plasma

PT: Patellar Tendon

PTOA: Post Traumatic Osteoarthritis

QT: Quadriceps Tendon

ROM: Range of Motion

SYN: Synovium/Synoviocytes

TGF- $\beta$-1: Transforming Growth Factor $\beta-1$

TNF: Tumor Necrosis Factor

VEGF: Vascular Endothelial Growth factor 


\section{LIST OF PRODUCTS}

(In Alphabetical order)

- 1,9-Dimethyl-Methylene Blue zinc chloride double salt: Sigma-Aldrich, St. Louis, MO, USA

- Fetal Bovine Serum: Gibco, Thermo Fisher Scientific, Waltham, MA, USA

- Insulin-Transferrin-Selenium: Gibco, Thermo Fisher Scientific, Waltham, MA, USA

- L-Ascorbic acid: Gibco, Thermo Fisher Scientific, Waltham, MA, USA

- L-Glutamine: Gibco, Thermo Fisher Scientific, Waltham, MA, USA

- Modified Eagle's medium nonessential amino acids: Gibco, Thermo Fisher Scientific, Waltham, MA, USA

- Olympus BX51 microscope: Center Valley, PA, USA

- Penicillin-streptomycin-amphotericin B: Antibiotic-Antimycotic, Gibco, Thermo Fisher Scientific, Waltham, MA, USA

- Resazurin sodium salt: Sigma-Aldrich, St. Louis, MO, USA Sodium chloride $(0.9 \% \mathrm{NaCl})$ : Hospira, Inc., Lake Forest, IL

- Sodium Pyruvate: Gibco, Thermo Fisher Scientific, Waltham, MA, USA

- Spectramax iD5 Multi-mode Microplate Reader, Molecular Devices, San Jose, CA, USA

- Trypan Blue Stain (0.4\%): Gibco, Thermo Fisher Scientific, Waltham, MA, USA

- TrypLE ${ }^{\mathrm{TM}}$ Express Enzyme: Gibco, Thermo Fisher Scientific, Waltham, MA, USA 


\title{
BIOLOGIC RESPONSES OF LIGAMENT AND TENDON GRAFT -DERIVED FIBROBLASTS TO CLINICAL LEVELS OF CYCLIC STRAIN
}

\author{
Sebastian Cardona-Ramirez \\ Dr. James L. Cook, Dissertation Supervisor \\ Dr. Richard Ma, co-advisor
}

\begin{abstract}
Anterior Cruciate Ligament (ACL) is one of the major ligaments in the knee. ACL injury is the partial or complete tear of the ACL usually as a consequence of a traumatic sportsrelated injury. ACL injury commonly affects athletes in the late adolescence and are usually more common in females than males. Factors such as metabolic response, cellular crosstalk among tissues and sex differences are not fully understood. Therefore, an increased comprehension of the biology of ACL injury and healing is required.

Management of ACL injuries is typically achieved by surgical stabilization of the knee.

For this, arthroscopic ACL reconstruction with different tendon autografts is the gold standard technique. Additionally, ligament repair by suturing the torn ends of the ACL is an alternative procedure. Although current surgical treatment, is generally successful, ACL graft and repair healing biology needs to be further explored to decrease failure rates. Moreover, whether a specific activity level would in turn affect the healing process is still not known.

Several in vitro and in vivo preclinical models have been developed to explore current ACL injury and healing biology. Among these, large animals offer the advantage to be
\end{abstract}


similar to the human. The preferred model is the spontaneous onset of ACL injury, commonly found in dogs. In both species, extracellular matrix (ECM) remodeling driven by cellular responses to load can influence disease, as well as response to treatment. However, most in vitro models do not include the multiple cell types in the joint or the mechanical environment that modulates their responses. Consequently, we aimed to evaluate the metabolic responses of fibroblasts obtained from canine intraarticular tissues potentially involved in ACL injury as well as tendon grafts commonly used for ACL reconstruction subjected to varying levels of mechanical stress in a monolayer culture. We hypothesized that fibroblasts from these tissues would produce significantly different levels of inflammatory and remodeling biomarkers in response to a spectrum of cyclic strains in vitro.

We were able to demonstrate that ACL surrounding tissues are an important source of inflammatory and degradative biomarkers, and that metabolic responses of tendon graft fibroblasts are strain and tissue dependent. Moreover, male canine tendon fibroblasts seem to increase degradative responses while females tend to increase inflammatory responses when stimulated with different strain magnitudes. Also, synoviocytes are an important source of inflammation and human intraarticular tissue fibroblasts and tendon graft-derived fibroblasts have variable responses to stress but is not differentially affected by sex. Consequently, further studies are required to elucidate complex regulation mechanisms in both canines and humans. 


\section{CHAPTER 1}

\section{INTRODUCTION}

Anterior Cruciate Ligament (ACL) injuries affect around 1.7 billion people worldwide and 1 in 3000 persons in the united states. ${ }^{1}$ Female athletes are 2-8 times more prone to ACL tears than males. ${ }^{2}$ Suturing the torn ends of the ACL was the first described technique to correct knee instability after ACL injury. ${ }^{3}$ However, an unreliable healing response has been associated with primary ACL repair. ${ }^{4}$ This has been attributed to several factors, including a hostile intra-synovial environment, specific postinflammatory responses, and intrinsic cell deficiencies. ${ }^{5,6}$ Cellular factors play an important role in ACL injury and healing. ACL rupture initiates a cascade of cytokines and catabolic enzymes. ${ }^{7 ; 8}$ Current work has shown that inflammatory and degradative molecules present after ACL injury can affect intraarticular tissues such as ligaments, tendon grafts and cartilage..$^{8-11}$ Therefore, the understanding of the early healing phase characterized by cellular release of molecules that regulate adequate balance between extracellular matrix (ECM) degradation and proliferation is crucial.

Due to the increased rate of failure with ACL repair techniques, ligament reconstruction with a tendon graft was adopted as the standard of care. ${ }^{12}$ More than 100,000 ACL reconstruction surgeries are performed each year in the US. Although generally successful, $10 \%$ of those surgeries fail for a variety of reasons including inappropriate surgical technique, inadequate graft selection, improper graft fixation and incorporation and inappropriate postoperative rehabilitation. ${ }^{13}$ There is considerable debate regarding 
the ideal autograft choice for anterior cruciate ligament (ACL) reconstruction. Common sources for ACL autografts include patellar tendon (PT), hamstring tendon (HT), and quadriceps tendon (QT). These different grafts have been associated with disparate outcomes in patients. PT, for instance, has been associated with increased prevalence of osteoarthritis (OA) after reconstruction, whereas HT has been associated with possible higher revision rates when compared to PT. ${ }^{14 ; 15}$ While material properties of various autograft tissues are known, there is little information on the biologic differences among these commonly-used ACL autografts. Additionally, the interactions between the biomechanical environment in the post-surgical healing stage with various autografts are not fully understood. Once an autograft is placed for ACL reconstruction, an inflammatory cascade ensues to begin the initial stages of graft ligamentization. The roles of the existing autograft fibroblasts in this early process are unknown.

In addition to the native ACL and the grafting tissue, it appears that surrounding tissues are also major contributors of healing molecules within the knee joint. It has been shown that synovium plays an important role in regulating the joint homeostasis by maintaining or amplifying the injury signal. ${ }^{16}$ Elevated levels of cytokines and growth factors within the knee intraarticular environment are often considered to be the contribution of synovium. ${ }^{17}$ Furthermore, the cross talk between synoviocytes and intraarticular ligaments has been shown to influence the healing response when fibroblasts are subjected to mechanical deformation. ${ }^{18}$

To evaluate the biologic events of healing in tendons and ligaments, in vitro models have been developed. However, traditional models often do not replicate the native environment within the knee joint due to the lack of mechanical strain, specifically axial 
tension for cruciate ligaments and tendon grafts that occur with knee motion, that in turn modify cellular properties. Consequently, recent 2D and 3D models have been designed to assess the effect of mechanical loading in ligament and tendon cells. ${ }^{19}$ The Flexcell@ tension is the most popular system to stress cells in monolayer culture. It has been widely used to evaluate specific cellular responses to tension loads in ligament and tendon fibroblasts. ${ }^{20-23}$ It permits to evaluate cells in monolayer or in co-culture as well as the addition of different molecular stimulus such as hormones, cytokines and growth factors. $^{24-26}$

Hence, we were interested in elucidating main responses to varying levels of clinically relevant cyclic strains of cells obtained from ligaments and tendons that are commonly involved in either ACL healing, repair or reconstruction with a tendon graft. The objective of this work was to compare the pro-inflammatory and metabolic responses of fibroblasts obtained from intraarticular tissues and tendon graft-derived fibroblasts to varying levels of mechanical strain forces. Additionally, we aimed to compare the response of male and female fibroblasts to tension load and to evaluate if tendon graft-derived fibroblasts co-cultured with synoviocytes would significantly modify the production of inflammatory and metabolism related biomarkers. Our central hypothesis was that fibroblasts from these tissues would exhibit differential responses via production of varying levels of biomarkers to a spectrum of cyclic strains in vitro. 


\section{REFERENCES}

1. Herzog MM, Marshall SW, Lund JL, et al. 2017. Cost of Outpatient Arthroscopic Anterior Cruciate Ligament Reconstruction Among Commercially Insured Patients in the United States, 2005-2013. Orthopaedic Journal of Sports Medicine 5:232596711668477.

2. Sutton KM, Bullock JM. 2013. Anterior cruciate ligament rupture: differences between males and females. The Journal of the American Academy of Orthopaedic Surgeons 21:41-50.

3. Schindler OS. 2012. Surgery for anterior cruciate ligament deficiency: a historical perspective. Knee Surgery, Sports Traumatology, Arthroscopy 20:5-47.

4. Feagin JA, Jr., Curl WW. 1976. Isolated tear of the anterior cruciate ligament: 5year follow-up study. The American journal of sports medicine 4:95-100.

5. Woo SL, Vogrin TM, Abramowitch SD. 2000. Healing and repair of ligament injuries in the knee. The Journal of the American Academy of Orthopaedic Surgeons 8:364-372.

6. Andrish J, Holmes R. 1979. Effects of synovial fluid on fibroblasts in tissue culture. Clinical orthopaedics and related research:279-283.

7. Catterall JB, Stabler TV, Flannery CR, et al. 2010. Changes in serum and synovial fluid biomarkers after acute injury (NCT00332254). Arthritis Research \& Therapy 12:R229. 
8. Lattermann C, Conley CE, Johnson DL, et al. 2018. Select Biomarkers on the Day of Anterior Cruciate Ligament Reconstruction Predict Poor Patient-Reported Outcomes at 2-Year Follow-Up: A Pilot Study. Biomed Res Int 2018:9387809.

9. Bigoni M, Sacerdote P, Turati M, et al. 2013. Acute and late changes in intraarticular cytokine levels following anterior cruciate ligament injury. Journal of orthopaedic research : official publication of the Orthopaedic Research Society $31: 315-321$.

10. Higuchi H, Shirakura K, Kimura M, et al. 2006. Changes in biochemical parameters after anterior cruciate ligament injury. International orthopaedics 30:43-47.

11. Amano K, Huebner JL, Stabler TV, et al. 2018. Synovial Fluid Profile at the Time of Anterior Cruciate Ligament Reconstruction and Its Association With Cartilage Matrix Composition 3 Years After Surgery. 46:890-899.

12. Groves EWH. 1919. The crucial ligaments of the knee-joint: Their function, rupture, and the operative treatment of the same. 7:505-515.

13. Samitier G, Marcano AI, Alentorn-Geli E, et al. 2015. Failure of Anterior Cruciate Ligament Reconstruction. The archives of bone and joint surgery 3:220240.

14. Pinczewski LA, Lyman J, Salmon LJ, et al. 2007. A 10-year comparison of anterior cruciate ligament reconstructions with hamstring tendon and patellar tendon autograft: a controlled, prospective trial. The American journal of sports medicine 35:564-574. 
15. Persson A, Fjeldsgaard K, Gjertsen JE, et al. 2014. Increased risk of revision with hamstring tendon grafts compared with patellar tendon grafts after anterior cruciate ligament reconstruction: a study of 12,643 patients from the Norwegian Cruciate Ligament Registry, 2004-2012. The American journal of sports medicine 42:285-291.

16. Iwanaga T, Shikichi M, Kitamura H, et al. 2000. Morphology and functional roles of synoviocytes in the joint. Archives of histology and cytology 63:17-31.

17. Tang Z, Yang L, Wang Y, et al. 2009. Contributions of different intraarticular tissues to the acute phase elevation of synovial fluid MMP-2 following rat ACL rupture. Journal of orthopaedic research : official publication of the Orthopaedic Research Society 27:243-248.

18. Wang C, Xie J, Jiang J, et al. 2015. Differential expressions of the lysyl oxidase family and matrix metalloproteinases-1, 2, 3 in posterior cruciate ligament fibroblasts after being co-cultured with synovial cells. Int Orthop 39:183-191.

19. Wang T, Chen P, Zheng M, et al. 2018. In vitro loading models for tendon mechanobiology. Journal of orthopaedic research : official publication of the Orthopaedic Research Society 36:566-575.

20. Breshears LA, Cook JL, Stoker AM, et al. 2010. The effect of uniaxial cyclic tensile load on gene expression in canine cranial cruciate ligamentocytes. Veterinary surgery : VS 39:433-443.

21. Hannafin JA, Attia EA, Henshaw R, et al. 2006. Effect of cyclic strain and plating matrix on cell proliferation and integrin expression by ligament fibroblasts. 
Journal of orthopaedic research : official publication of the Orthopaedic Research Society 24:149-158.

22. Henshaw DR, Attia E, Bhargava M, et al. 2006. Canine ACL fibroblast integrin expression and cell alignment in response to cyclic tensile strain in threedimensional collagen gels. Journal of orthopaedic research : official publication of the Orthopaedic Research Society 24:481-490.

23. Wang JH, Jia F, Yang G, et al. 2003. Cyclic mechanical stretching of human tendon fibroblasts increases the production of prostaglandin E2 and levels of cyclooxygenase expression: a novel in vitro model study. Connective tissue research 44:128-133.

24. Lee CY, Liu X, Smith CL, et al. 2004. The combined regulation of estrogen and cyclic tension on fibroblast biosynthesis derived from anterior cruciate ligament. Matrix biology : journal of the International Society for Matrix Biology 23:323329.

25. Qi J, Chi L, Maloney M, et al. 2006. Interleukin-1beta increases elasticity of human bioartificial tendons. Tissue engineering 12:2913-2925.

26. Rowson DT, Shelton JC, Screen HRC, et al. 2018. Mechanical loading induces primary cilia disassembly in tendon cells via TGF $\beta$ and HDAC6. Scientific reports 8:11107. 


\section{CHAPTER 2}

\section{LITERATURE REVIEW}

\section{Biology of the anterior cruciate ligament:}

The Anterior Cruciate Ligament (ACL) is an intraarticular ligament within the knee joint. Although it is intraarticular in nature, the ACL is extrasynovial, being isolated by the synovial sheet that is wrapped around the cruciate ligaments. ${ }^{1}$ The ACL follows the typical organization of connective tissues. It is covered by a vascular layer called epiligament, that merges with the periosteum at the insertion with the bone. ${ }^{2}$ The ACL possesses a characteristic hierarchical organization that comprises groups of parallel fibers' fascicles, surrounded by connective tissue. The fascicle can be in turn subdivided into subfasciculi $(100-250 \mu \mathrm{m}$ diameter) covered by the epiligament. The epiligament is rich in cellular component and nerves, both proprioceptive and sensory. ${ }^{3}$ The subfascicular units are composed of collagen fibrils $(20-155 \mathrm{~nm}) .{ }^{4}$ Furthermore, the ACL inserts into the bone through the enthesis.

The enthesis is the region where a tendon or ligament inserts to the bone in a typical transition form, via fibrocartilage or mineralized cartilage. ${ }^{5-8}$ Fibrous entheses are more common where muscles attach to diaphyseal bones and are composed of dense bands of collagen (Sharpey's fibers) that merge with the periosteum and penetrates the cortical bone. Fibrocartilaginous entheses on the other hand, contain a zone of fibrocartilage at the area of attachment of the tendon or ligament and form a typical transition zone between the collagen fibers and the bone. There is no periosteum present at the fibrocartilaginous entheses. The fibrocartilaginous zone has both mineralized and non-mineralized regions separated by a characteristic tidemark. Furthermore, fibrocartilaginous entheses are more 
common at ligament origins and insertions and at apophyseal or metaphyseal attachments of tendons. ${ }^{5}$

The specific organization of the entheses reflects the adaptation design to distribute stresses that are applied at the interface between structures with different strength and elasticity patterns. ${ }^{9}$ Moreover, fibrocartilaginous entheses must maintain integrity and function depending on directionality, tensile, compressive and shear stresses. Importantly, surgical reattachment of tendons or ligaments results in the formation of a fibrous enthesis that cannot adequately resist the complex loading patterns of fibrocartilaginous entheses. ${ }^{10}$

The cellular component of the ACL is composed by fibroblasts. They belong to the family of connective tissue cells and specialize in the production of extracellular matrix (ECM) components such as collagen and proteoglycans and provide mechanical strength to the tissue by offering a supporting framework to the ECM. Fibroblasts play a key role in the inflammatory and immune-mediated response to injury as well as homeostasis of musculoskeletal tissues. ${ }^{11}$ Additionally, the ECM -the ground substance where fibroblasts are submerged in- provides the environment required for mechanical and biological functions. ${ }^{3}$

The ACL has a typical distribution of fibroblasts. Myofibroblasts exhibit contractile properties and are important in ligament healing. ${ }^{3 ; 12 ; 13}$ The antero-medial (AM) bundle, is highly cellular, especially near the proximal end with typical round or ovoid morphology. ${ }^{13}$ The midsubstance appears to be less cellular. Cells adopt a parallel orientation with fusiform and spindle-shaped morphology. ${ }^{14}$ Distally, spheroid cells are predominant, with a lower cell density and shorter crimp formation. ${ }^{3 ;} 13$ They also have abundant cellular organelles that indicate a high cellular activity. ${ }^{3}$ Additionally, near the enthesis, 
chondrocytes can be found in the fibrocartilage and mineralized fibrocartilage zones that anchors the ligament to the bone. ${ }^{3}$

\section{Composition of Tendons and Ligaments:}

Tendons are dense bands of collagen-rich fibers that connect muscle to bone. Tendons are intended primarily to transfer forces after muscular contraction. ${ }^{15 ; 16}$ Similarly, ligaments are connective tissue bands that originate and insert on bones. They typically contribute to joint stability and to restrain excessive joint motion. Tendons and ligaments share similar cellular and extracellular biochemical composition. ${ }^{17 ;} 18$ However, these small differences in ECM structure and composition provide a wide difference in function.

Ligaments have lower collagen and increased water content compared to tendons. Importantly, tendon and ligament function and mechanical properties are highly correlated with density and morphology of collagen fibers. Moreover, collagen fiber integrity and stability are highly dependent on proper formation of covalent collagen cross-links. ${ }^{19}$ The lack of covalent cross links results in weaker mechanical and structural properties. ${ }^{20}$ Amiel et al., (1983) reported four to five times more collagen cross-links in the rabbit ACL compared to the patellar tendon. ${ }^{21}$ Additionally, $\mathrm{Ng}$ et al., (1996) demonstrated that collagen cross-links increase in patellar tendon autograft three years after ACL reconstruction in a goat model. ${ }^{22}$ Similarly, a normally higher content of elastin in ligaments (1-15\%) compared to tendons (1-5\%) is responsible for supporting up to $30 \%$ of tensile stress under uniaxial strain. Consequently, a shift in elastin content would facilitate higher loads in the reconstructed ACL graft.

Both tissues are composed of cells and fibers embedded in a three-dimensional extracellular matrix. This amorphous ground substance, commonly produced by the 
fibroblasts, is a complex mixture of type I collagen fibers with smaller quantities of types II, III, V, VI, IX and XI. ${ }^{23}$ Proteoglycans, proteins, growth factors and glycoproteins are also important components of the ligament and tendon ECM.

Within the musculoskeletal system, tissue cells are named depending on the tissue they inhabit (i.e. tenocytes within tendon, ligamentocytes within ligament), each of them having specific morphology and phenotype. Tenocytes and ligamentocytes are aligned with the collagen fibers and interconnected through long cytoplasmic extensions. Other types of cells are also seen in the connective tissue including macrophages and leukocytes. ${ }^{23}$

Different variations and adaptations in the composition and organization of the extracellular matrix provide specific mechanical properties. ${ }^{24 ; 25}$ In the case of tendons and ligaments, they are primarily intended to withstand the action of tension loads.

\section{Collagen:}

Collagen tertiary structure consists of a triple helix with three separate polypeptide molecules called alpha chain. Collagen fibrils are designed to resist tension forces. Fibrils are organized into parallel dense structures organized in a wave pattern, the crimp. ${ }^{3}$ The dominating type of collagen in ligaments and tendons is collagen type I, responsible for the tensile strength of the tissue. Type II collagen can be found in the fibrocartilaginous regions and in the insertion, sites being an indicator for applied pressure and shear stress. ${ }^{3}$ Type III collagen is found between collagen I fibers and is more abundant near the insertion sites ${ }^{26}$ It is also an indicator of remodeling after anterior cruciate ligament reconstruction. ${ }^{27}$ Type IV collagen is usually associated with the basal lamina of the intra and periligamentous vessels which can serve as a vascularization indicator. ${ }^{3 ;} 26$ Type VI collagen is found in the distal zones of the ACL and serves as a gliding component between 
functional fibrillar units and is associated with greater resistance to tension forces found in attachment sites compared to the mid-substance region. ${ }^{3}$

Mechanical tensile properties of collagen are determined by the collagen orientation, packing and fibril diameter. Additionally, hierarchical structure of collagen permits the generation of different fibrillar configurations that are specifically adapted for each specific tissue. Moreover, tendons and ligaments are subjected primarily to unidirectional tensile loads due to the parallel arrangement and dense packing of collagen fibers. ${ }^{23}$ Larger diameter fibers are present in tendons and ligaments that experience greater loads. Smaller diameter fibers produce greater surface area and control viscoelastic properties through variations in proteoglycan content. They also show a typical viscoelastic response being affected by the rate of loading. ${ }^{23}$ Typically, lower diameter collagen fibrils are common after tissue injury and repair and are associated with decreased mechanical properties of the scar tissue..$^{28}$

\section{Glycosaminoglycans:}

Glycosaminoglycans (GAGs) are molecules (carbohydrate conjugates of unbranched polysaccharides containing amino sugars) found in combination with proteoglycans (glycoproteins). GAGs possess an hexosamine unit that is highly sulfated. On the contrary, Hyaluronic acid, is a non-peptide, conjugated non-sulfated GAG consisting of Dglucuronic acid and D-glucosamine. ${ }^{29 ; 30}$

Proteoglycans (PGs) consist primarily of a core protein with covalently attached glycosaminoglycans sidechains. PGs are highly present in the interfibrillar matrix and are associated with the surfaces of collagen fibrils. Main PGs include biglycan, decorin, fibromodulin and lumican. Biosynthesis of PGs include the addition and sulfation of GAG 
sidechains within the endoplasmic reticulum and packaging within the Golgi apparatus for secretion. ${ }^{31}$ Large aggregating proteoglycans, namely aggrecan and versican, consist of a backbone of hyaluronic acid with multiple non-covalently linked proteoglycans. These aggregating PGs are highly anionic assemblies that have a high affinity for water. This leads to a high level of hydration and turgidity, important for compressive properties of the tissue. ${ }^{23 ; 29}$ PGs degradation involves the proteolytic cleavage of the core or link proteins at specific cleavage sites by specific matrix metalloproteinases (MMP) and aggrecanases. ${ }^{32}$ There are different types of degradative ECM enzymes. MMPs are zinc dependent endopeptidases that include collagenases, gelatinases and stromelysins that degrade a wide range of ECM proteins. Aggrecanases are proteolytic enzymes that cleave the core protein of aggrecan. Musculoskeletal pathology is commonly driven by an excessive degradative enzymatic activity and the inability of the tissues to assemble an effective reparative response that in turn will lead to altered composition and mechanical properties of the

\section{ECM. $^{23}$}

\section{Knee Kinematics and Biomechanics of the ACL}

The knee is a complex joint with six degrees-of-freedom, three rotational (abduction/adduction, flexion/extension and internal/external rotation) and three translational (anterior/posterior, medial/lateral, and compression/distraction). Due to the lack of boney geometric constraint, joint stability is achieved mainly by soft tissue. Here, the ACL becomes the main restrain of the knee in anterior tibial translation, causing fibers to become taut. ${ }^{33}$

According to Skelley et al,. (2015) microstructural properties in the human ACL differed between the anteromedial (AM) and posterolateral (PL) bundles. ${ }^{34}$ The AM has higher 
tissue modulus and failure stress under load compared to the PL bundle, probably due to differences in anatomical features in the femoral and tibial enthesis. ${ }^{35}$ Woo et al., (1991), showed that the ACL tensile strength is around $2160 \mathrm{~N}$ and stiffness around $242 \mathrm{~N} / \mathrm{mm} .{ }^{36}$ ACL non-linear stiffness properties are demonstrated when initial small loads are required to elongate the ligament (toe region). There is a continuous fiber recruitment which in turn increases the stiffness until fibers are fully loaded. Furthermore, a typical linear relationship between increasing loads and elongation is seen. When the yield load is reached, structural non-reversible damage occurs to the tissue becoming non-linear at maximum failure loads when complete structural integrity is altered affecting tendon and ligament cross-links. When the ligaments are kept at a constant load over a period of time, the ligaments lengthen until a steady state is obtained, called creep. ${ }^{3 ; 36}$ This is achieved by the interaction of cross-links, collagen fibers and cellular components to avoid excessive tensional stress on the ligament under repetitive or continuous exercise. ${ }^{3}$

The fibers of the ACL undergo non-isometric length changes during physiological knee motion. ${ }^{3}$ It provides varying degrees of restraint to anterior tibial translation. However, this would depend on the activation of knee-related musculature and other soft tissues. In vivo analysis has found that quadriceps activation resulted in ACL extension of around $4 \% .{ }^{37}$ Additionally, at $90^{\circ}$ flexion, the ACL did not show changes in length demonstrating the dominating function of the ACL near extension. ${ }^{38}$ Similarly, authors have shown that simultaneous activation of hamstring and quadriceps muscles, significantly decreased load on the ACL and reduced anterior tibial translation and internal rotation. ${ }^{3 ; 39-41}$ In situ forces indicate that under a $110 \mathrm{~N}$ anterior tibial load, the ACL experienced a $103 \mathrm{~N}$ at $15^{\circ}$ flexion and $59 \mathrm{~N}$ at $90^{\circ}$ flexion. The PL bundle carried the highest load at extension 
$(67 \mathrm{~N})$ while the AM bundle experienced higher forces near flexion (90N at $60^{\circ}$ flexion $)^{42}$. This situation may have important implications for reconstruction when the knee is either hyperextended or hyperflexed, leading to strains up to $8 \%$ in the reconstructed ACL graft $40 ; 43$.

\section{$\underline{\text { Anterior Cruciate Ligament Injury }}$}

Partial or complete tear or rupture of the ACL is commonly associated with traumatic sports events. ACL tears affect more than 2000,000 people in the United States each year. ${ }^{44}$ Most of ACL injuries occur under a non-contact mechanism, with no direct contact to the knee when the ACL is injured. Usually $72 \%$ of ACL injuries occurred with non-contact mechanism during sudden deceleration or landing meneuvers. ${ }^{45} 46$ Main features include rapid lower extremity deceleration resulting in forceful quadriceps contraction and change of direction or landing with a slightly hyperextended knee and typically internal rotation and flexion. It is estimated that the cost of treatment of ACL injuries is more than U\$1.7 billion annually. ${ }^{47}$

In addition to biomechanical and structural aspects, cellular factors also play an important role in ACL injury and healing. ACL rupture initiates a cascade of cytokine and catabolic enzyme activity. ${ }^{48 ;} 49$ Current work has shown that among molecules present after ACL injury, cytokines such as IL-1 could stimulate the production of matrix metalloproteinases (MMPs) which in turn could degrade collagen and reduce proteoglycan content, affecting both, ligament and cartilage. In this process downregulation of proteoglycan synthesis and upregulation of MMPs is also common. From these, it seems that IL-1 $\alpha$, IL-1 $\beta$, IL-6, IL-8, TNF $\alpha$ and MMP-13 are commonly found after ACL injury. ${ }^{49-52}$ 
Since the production of different molecules will greatly depend on the stage of the disease, individual variation of these biomarkers would produce a differential response to surgical reconstruction. ${ }^{49} ; 50$

ACL Risk factors:

Risk factors could be classified into external and internal. External factors comprise type of competition, shoe-surface interface, knee bracing and weather. ${ }^{45}$ Internal factors include femoral intercondylar notch size, lower limb alignment, posterior tibial plateau slope, intrinsic ACL material properties, patella tendon-tibia shaft angle, ACL elevation angle, hormonal variation and neuromuscular control, many of these are also specific for females. ${ }^{45 ; 53}$ Young age is also a risk factor, with adolescents from 16 to 18 years being at greater risk..$^{54}$

ACL injury tends to be two to ten times more common in female athletes than males depending on the activity. ${ }^{47}$;5; 56 Additionally, several anatomical and neuromuscular factors (mentioned earlier) are involved. ${ }^{53}$ Increased female injuries could be explained by specific motion patterns and greater forces and torques exerted in athletic activities such as smaller flexion angles, greater hip internal rotation angles, lower gluteus maximus activation and greater quadriceps-to-hamstring ratio activation resulting in increased loading at the ACL. Studies have shown that increased anterior shear forces and knee internal rotation moment could significantly increase ACL strain. ${ }^{57}$

\section{Diagnosis:}

Diagnosis of ACL tears relies on clinical history, physical exam and specific diagnostic tests. The history usually involves twisting, landing or valgus blow to the knee. ${ }^{58}$ Also, swelling could be present after a few hours. Some patients could hear a pop sound but not 
always. Pain is also variable being severe and persistent or, mild and transient. ${ }^{58} \mathrm{Chronic}$ ACL tears could involve history of instability that occur during pivoting activities.

Physical exam include different diagnostic tests. Hey Groves, identified the pivot shift phenomenon when described the antero-lateral subluxation of the tibia in the ACL deficient knee. ${ }^{59}$ Consequently, this finding led to the discovery of the "pivot-shift test", a useful diagnostic assessment tool to identify ACL deficiency. ${ }^{60}$ The pivot shift test is a specific but insensitive test for ACL tears. ${ }^{58}$ However, it is the gold standard to rule out complete ACL tear in the anesthetized patient. ${ }^{58}$ The examination under anesthesia dramatically increases pivot shift sensitivity. ${ }^{61 ; 62}$

The Lachman test is the most reliable exam for ACL tears. ${ }^{58 ; 63}$ However, it might be inconclusive in acute injury, especially when trying to differentiate between partial or complete tears. The Lachman test should be considered definitive only if its negative with a firm endpoint. The Lachman test can also lead to false positive results if there is medial collateral ligament insufficiency with consequent valgus laxity. In these cases, tibial rotation must be controlled by the examiner. Moreover, the KT-1000 is a highly accurate method for definitive ACL tear diagnosis. A $4 \mathrm{~mm}$ or higher side-to-side difference is commonly associated with definitive ACL tear. Partial tears usually have 2-3mm laxity. Furthermore, after ACL reconstruction, $4-5 \mathrm{~mm}$ laxity is commonly seen. ${ }^{64}$ Radiographic diagnosis is usually not conclusive unless there is tibial avulsion fracture in skeletally immature patients. Magnetic resonance imaging (MRI) is more commonly associated with increased sensitivity being around 80-95\%.65; 66 Additionally, high-field 1.5 tesla produces more accurate results when compared to low-field MRI. ${ }^{64}$ 


\section{Treatment:}

\section{ACL repair}

Primary ACL repair with suture was the first technique described to correct knee instability due to ACL injury. Don O’Donoghue popularized routinely ACL repair after acute injury in the 1950s and 1960s. ${ }^{67}$ Nevertheless, long term results of ACL repair showed high rates of knee instability leading to the abandonment of this technique. ${ }^{68}$

Poor healing ability of the ACL has been demonstrated. ${ }^{68 ;} 69$ Reasons for increased failure include intrinsic cellular differences. ${ }^{70 ; 71}$ In vitro studies have shown that ACL and the medial collateral ligament (MCL) have comparable rates of proliferation, similar revascularization patterns and comparable collagen production after rupture. ${ }^{72-74}$ However, the ACL lacks a proper clot formation in the wound site. ${ }^{72}$ This clot acts as a provisional scaffold rich in growth factors that are necessary for successful healing. ${ }^{75-77}$ Consequently, poor healing response in the ACL could be due to the exposure of the epiligamentous tissue to degradative enzymes present in the synovial fluid such as MMPs, leading to the removal of the provisional scaffold between the two ends of the injured ACL as well as a disorganized collagen configuration and increased type III-collagen. ${ }^{12 ;} 78$

Steadman et al., (2006) developed a technique in which micro holes were created within the femur near the ruptured ACL to produce a blood clot and hematoma formation leading to an improved healing response. ${ }^{79}$ The hematoma is a carrier of growth factors assisting in wound healing, fracture repair and in this case ligament repair. ${ }^{80 ; 81}$ This technique has been shown to be successful in patients with proximal ACL tears from a middle age cohort of patients. ${ }^{82}$ 
Based on this, Martha Murray group started to investigate ACL repair healing potential after augmentation. First, they described different stages of healing after rupture (inflammation, epiligamentous regeneration, proliferation and remodeling). ${ }^{73 ; 83}$. Inflammation is initiated by hemarthrosis. During the inflammatory phase, Murray et al., (2000) found that the ends of the ACL were swollen and edematous. There was a blood clot covering part of the ligament remnants without bridging femoral and tibial ends. Additionally, cell population consisted of fibroblasts, polymorphonuclear neutrophils, lymphocytes and macrophages seen actively phagocytizing cell and tissue debris. Also, there were dilated arterioles with proliferation of smooth muscle cells and congested capillaries and thrombus formation. ${ }^{73}$

During the epiligamentous repair phase, between three and eight weeks after rupture, the number of inflammatory cells decrease, and fibroblasts became the predominant cell type. The blood vessels show normal morphology and neo-vascularization within the ligament fascicles. Thickening of the epiligament and fibroblastic proliferation in the synovial tissue occur. The ends of the ruptured ligament are covered due to the action of myofibroblastlike cells containing $\alpha$-smooth muscle actin with contractile properties responsible for the retraction of the ligament. ${ }^{13}$

In the proliferation phase (weeks 8 to 20 ), remnant tissue is not visible and is usually adhered distally to the periligamentous tissue of the PCL. Increase in cell number and blood vessel density among collagen fascicles is observed. Fibroblasts showed a disorganized arrangement. Also, immature capillaries forming a diffuse network are present. Additionally, a continuous layer of synovial tissue is seen with abundant $\alpha$-smooth muscle actin-containing cells. ${ }^{13 ;} 73$ 
Lastly, in the remodeling and maturation phase, between one and two years after rupture, ligament ends are dense and there is no connection between remnants. There is decreased blood vessel density and a persistent synovial sheet is seen. Cell number density decreased to those seen in normal ACL. However, it seems that the ACL does not form a bridging scar after rupture probably due to proteolytic substances within the synovial fluid. ${ }^{84}$ Consequently, treatment options based on regenerative medicine have arisen, including single growth factor studies ${ }^{85 ; 86}$, platelet rich plasma (PRP) ${ }^{87 ; 88}$, mesenchymal stem cells $(\mathrm{MSC})^{89 ; 90}$ and bioscaffolds ${ }^{91 ; 92}$. Furthermore, combination of these methods has led to the introduction of augmented repair being considerably more effective than each method alone $e^{93 ; 94}$.

Currently, bioenhanced ACL repair using a collagen bioscaffold seeded with platelet concentrate has shown similar biomechanical results in a large animal model when compared to traditional ACL reconstruction techniques. ${ }^{94}$ Moreover, these promising results have led to the implementation of this technique in a human clinical trial. ${ }^{95}$ Nevertheless, although ACL repair has shown good functional outcomes after two years, long term follow up has failed to demonstrate good results, hence, emphasizing the necessity for augmentation..$^{95-97}$

\section{ACL reconstruction:}

Due to the increased rate of failure with ACL repair techniques, patients needed a different solution, leading to the idea that the ACL should be reconstructed with some kind of graft material rather than a repair. Paul Wagner in 1913 suggested the use of fascia for reconstructing complete ACL tears. The first complete ACL reconstruction was performed by Ernest William Hey Groves who utilized the entire fascia lata detached from the tibial 
insertion threaded through the femur and tibial tunnels and sown onto the periosteum while leaving the tendon attached to its origin. ${ }^{67}$ Also, Alwyn Smith modified Hey Groves technique using a strip of the fascia lata detached from the proximal muscle belly. Consequently, Groves and Smith had set the bases for ACL reconstruction.

Arthroscopic ACL reconstruction using a biologic autograft has shown significant improvement in the stability and function of the knee. Factors that affect ACLR outcomes include individual choice of the graft, bone tunnel placement, graft pre-tensioning, graft fixation technique, and initial graft fixation strength. ${ }^{98}$

\section{The Graft Choice for ACL Reconstruction}

Graft choices for ACLR typically consist of autografts or allografts. Common autografts include patellar tendon-bone graft (PT), semitendinosus/gracilis (Hamstring tendon) - HT, or the quadriceps tendon (QT) ${ }^{98}$ Often, graft choice is associated with intrinsic patient characteristics, surgeon preference and patient demographics. ${ }^{99-101}$ However, independent of the graft choices, no graft has shown ultimate failure load or stiffness comparable to the $\mathrm{ACL}^{98}$. Graft harvest, artificial fixation and biological process of healing and ligamentization will further decrease both, ultimate strength and stiffness. ${ }^{98 ; 102-105}$ Consequently, PT autografts should be used for young active athletes associated with early return to high-level activity, while HT autografts are preferred when a large skin incision or anterior knee pain is to be avoided. QT grafts are primarily used for revision surgery due to their difficulty in harvesting and compromise of knee extensor mechanism..$^{98 ; 106}$ Bone patellar tendon bone (BPTB) graft:

It was not until 1963 when Kenneth Jones suggested a new surgical technique utilizing the central third of the patellar tendon leaving the distal end attached to the bone and also 
removing the proximal part with a small block of bone and passing it through a femoral tunnel placed in the intercondylar notch. ${ }^{107}$ However, 50\% of his patients sustained a positive drawer test. Nevertheless, this technique gained popularity and patellar tendon grafting became known as Jones technique (reviewed in ${ }^{67}$ ). Bruckner suggested the use of a free central strip of bone-patellar tendon bone-graft (BPTB). This technique showed 90\% of his patients regaining normal stability. ${ }^{108}$ Kurt Frank helped popularize this technique when the first publication was available in a large patient cohort showing long-term results utilizing a free graft of the central third of the patellar tendon. This led to the adoption of the BPTB as one of the most popular graft sources. ${ }^{67}$

It is thought that BPTB grafts have a superior healing and integration capacity due to the presence of bone plugs at the end of each graft. This allows the graft to have a good fit within the bone tunnel when using either interference screw or suspensory fixation. ${ }^{109}$ Benner and Shelbourne., (2018) state that the presence of bone plugs at the end of the BPTB graft permits early biologic graft incorporation and healing at around 6 weeks after implantation. ${ }^{109}$ They also state that BPTB allows for aggressive early rehabilitation, including immediate range of motion exercises (ROM), such as full hyperextension on the day of surgery. ${ }^{110}$ According to the authors this strategy allows early full weight bearing and graft failure is rare. However, BPTB grafts do have disadvantages. The harvest technique from the extensor mechanism leaves weak points that are vulnerable for traumatic injury such as patella fracture and patellar tendon injuries. ${ }^{111}$ In addition, postoperative complications such as anterior knee pain, quadriceps weakness and difficulty with kneeling are also present. ${ }^{112-114}$ Although literature reports are sometimes conflicting ${ }^{113 ; 115}$, some references favor BPTB graft for lower revision rates and mora 
stable knee kinematics. ${ }^{116-119}$ Importantly, BPTB have been more frequently associated with postoperative OA. ${ }^{114 ; 120 ; 121}$

\section{Quadriceps tendon graft:}

since harvesting of the patellar tendon autograft was not free from harm, surgeons started to experiment with different tendon autografts including the central section of the quadriceps tendon. Blauth and Fulkerson in the 80 s tried to promote the use of the quad tendon for ACL reconstruction considering it superior. ${ }^{122}$ However, results were inferior compared to BPTB graft. Therefore, it never gained as much attention as the BPTP technique.

Nevertheless, in the 90s and 2000s, interested was revived after some authors reported comparable results to both, BPTB and hamstring autografts using a bone-QT graft. ${ }^{123-127}$ Interestingly, main finding included less surgical morbidity when using QT compared to BPTB graft. Recently, QT autograft has gained popularity being from $2.5 \%$ to $10.6 \%$ for ACL resconstruction. ${ }^{128 ;}{ }^{129}$ Additionally, QT graft has an increased collagen content and higher fibroblast density when compared to BPTB. ${ }^{130}$ Moreover, authors have also shown that the QT is significantly stronger than the PT. ${ }^{131 ; 132}$

\section{Hamstring tendon graft:}

First reports of ACLR using hamstring tendon grafts included the use of semitendinosus or gracilis either severed at its muscular junction or proximally attached through an intercondylar notch bone tunnel respectively, regaining normal knee function after two years. ${ }^{133}$ Furthermore, Marc Friedman pioneered the use a four-strand hamstring autograft with arthroscopically assistance and set the standard for ACL reconstruction with 
hamstrings. ${ }^{134}$ The four-strand HT autograft has equal or superior stability rates compared to BРTB. ${ }^{135-138}$ However, reports require thorough analysis. ${ }^{139 ;} 140$

HT graft has similar or superior strength compared to BPTB graft. ${ }^{141 ; 142}$ In fact, it is the strongest autograft among all. ${ }^{143}$ Although HT graft has a higher incidence for deep infection after harvest when compared to BPTB grafts ${ }^{144}$, donor site morbidity and anterior knee pain are lower when compared to BPTB and QT grafts. Moreover, anterior knee pain has been reported when using either PT or QT grafts, making HT grafts attractive for patients whose daily activity involve frequent kneeling, squatting, stair climbing or jumping. ${ }^{145}$ Prodromos (2018), only reports HT weakness, that can be corrected with compensatory semimembranosus hypertrophy and semitendinosus regrowth and reattachment on the proximal tibia, usually one year after the surgical intervention. ${ }^{145}$ However, some authors have shown than in few cases, HT does not fully regrow, and a knee flexion deficit may persist. ${ }^{146}$

Intra-articular scarring and stiffness appears to be increased when using PT grafts compared to HT probably due to the intraarticular nature of the PT. ${ }^{145}$ This has been corrected with accelerated rehabilitation programs and brings the question of how mechanical deprivation or overload affects the reconstructed ACL and healing process. ${ }^{147}$ It seems that HT autografts are the preferred choice for reconstruction. ${ }^{101 ;}$ 148 However, this is highly variable if surgeon location and nationality is considered. ${ }^{99 ;} 101$ Additionally, although information is conflicting, there are reports showing that HT grafts tend to fail more frequent when compared to BPTB grafts, especially in young athletes. ${ }^{149-151}$ Allografts: 
Since autografts possess an increased risk of complications due to the nature of the tissue harvest, new alternatives are required. Tendon allografts have been widely used for ACL reconstruction and involve $42.4 \%$ of primary $\mathrm{ACL}$ reconstructions and $78 \%$ of revisions. ${ }^{152-154}$ They avoid donor site morbidity and are readily available for most tissue banks. Additionally, they include a shorter operative time, improved cosmetic appearance, multiple sizes and grafts and decreased cost. ${ }^{155-157}$ Nevertheless, they carry their own risk including disease transmission, increased risk of contamination, decreased healing and integration, and it is believed that they are not as strong as autografts. ${ }^{158-160}$ However, with the advent of new technology, these weaknesses can be avoided.

It has been shown that allografts have decreased tendon-to-bone healing and decreased osteoinductive and osteoconductive properties mostly related to the sterilization process, such as the use of gamma radiation and chemical sterilization that will result in inflammatory reaction, delayed remodeling and decreased mechanical strength. ${ }^{161-164}$ Fortunately, techniques for augmenting integration and the use specific low dose gamma irradiation (1.2Mrad) allow to obtain similar results between autografts and allografts in terms of biomechanical outcomes. ${ }^{165-169}$ Additionally, a decellularization method proposed by Dong \& colleagues (2015) to increase compatibility and reduce antigenicity might lead to better outcomes. ${ }^{170}$ Although the use of allografts has great potential more research is needed to be able to replace autograft techniques.

\section{ACL Graft Positioning and Pre-tension:}

The physiologic roll-glide mechanism of the femorotibial joint is the key to restore proper knee function and avoid anterior tibial translation and altered rotation patterns. Hence, graft positioning arises as one of the most important factors in ACLR. ${ }^{98 ; 171 ; 172}$ Similarly, authors 
have shown that bone tunnel malposition is the most common technical mistake that could lead to graft failure due to a change in the biomechanical environment of the graft as a result of increased loads and strains. ${ }^{106 ;}$ 148; 173; 174 Therefore, non-physiological strain patterns throughout the functional range will increase the likelihood of ACLR failure. ${ }^{98}$ Authors have shown that when the femoral tunnel is placed anterior to the anatomic ACL footprint, results in excessive graft tension during knee flexion and correlates with poor functional outcomes. ${ }^{175}$ Consequently, anatomic graft placement results in kinematics similar to the normal knee. ${ }^{171}$

Another factor that could influence success rates in ACLR is the tension applied to the graft. Low graft tension will lead to decreased joint stability and excessive initial graft tension will lead to a restrain in range of motion leaving the graft susceptible to failure. In vitro and in vivo animal studies have investigated this aspect and have shown that grafts tend to lose their initial tension when cyclically loaded. ${ }^{176 ;} 177$ However, precise pretension magnitude in clinical setting is lacking.

\section{ACL Graft Fixation}

It is known that the fixation site is the weakest link during the early postoperative stage. ${ }^{178}$ Most fixation devices links the graft material with the bone tunnel affecting healing and mechanical properties of the reconstructed ACL. Generally, fixation devices are anchored far away from the joint, leading to higher cyclic loading magnitudes. Consequently, there is increased longitudinal and transverse graft motion within the bone tunnel leading to delayed tendon-to-bone integration. ${ }^{179}$ Fauno et al., (2005) showed that the position of the fixation sites and the fixation device significantly affects tunnel widening after ACLR. ${ }^{180}$ Similarly, Fu et al., (1999) showed that placing the fixation device close to the tunnel 
entrance increase the stiffness. ${ }^{179}$. Additionally, authors have shown that interference screw fixation led to superior ultimate strength when using a patellar tendon-bone graft compared to sutures and staples in the tibia. However, metal or biodegradable interference screws had inferior strength when compared to washerplates or sutures in the tibial attachment for quadrupled hamstring tendons. ${ }^{181}$ Interestingly, femoral fixation devices have not shown significative differential strengths among each other. ${ }^{98 ;}$ 182; 183 Furthermore, not only fixation strength is necessary but good biologic tendon-to-bone integration properties is required for successful healing.

\section{ACL Reconstruction Healing and Tendon Graft Ligamentization}

Successful intra-articular graft remodeling and intra-tunnel graft incorporation depend on the complex interplay among different factors including individual choice of graft material, graft pretensioning, and tunnel placement. ${ }^{98}$ Various animal models have been used to strengthen our understanding of the ACL ligamentization process. Traditionally, ACL graft ligamentization has been divided into three stages. The early healing phase starts from the time of surgical intervention up to six months in animal models and up to twelve months in human studies $27 ; 184 ; 185$. This stage is characterized by an initial release of growth factors such as bFGF, TGF- $\beta 1$, PDGF and cytokines such as TNF $\alpha$, IL1 $\beta$ and IL6. ${ }^{27}$

A second stage known as the proliferation stage can last from 4 weeks up to 12 weeks postoperatively. This stage is characterized by an increased cellular activity usually surpassing that of the intact ACL. There is also a particular increase in bFGF, TGF- $\beta-1$, PDGF between the third and the sixth week postoperatively and decreases by twelve weeks after surgery. ${ }^{186}$ Revascularization of the new graft starts around the fourth week postop, beginning with the release of VEGF and lasts until the twelfth week postop, just at the end 
of the proliferation phase. An increase in vascular density is found around six months postop and might correlate with the decrease in mechanical properties of the graft in which the collagen fibril density is at its lower levels. ${ }^{184}$ This remodeling stage appears to last up to two years in human studies and appears to have increased collagen synthesis shown by increased expression of COL-III. ${ }^{27}$

The third stage is known as the ligamentization/maturation phase and involves the transformation of an ACL tendon graft into a ligament-like tissue that acquires these characteristics only until six months postop. In this stage, concentration of type III collagen decreases over time, but it is still higher compared to the intact ACL. ${ }^{27}$ Additionally, mechanical properties of the reconstructed graft seem to improve in the ligamentization phase and keeps increasing up to one year postop. Maturation of the graft can start as early as nine months and lasts up to 48 months or even more. ${ }^{187}$ Although animal models have been used for deepening the understanding of ACL reconstruction, they possess several differences when compared to human studies. First, animal studies have shown extensive graft necrosis during the graft healing process, which do not seem to reach the same extent in humans (not to exceeding 30\%) based on grafts biopsies. ${ }^{27}$ An additional feature of animal models includes a different duration of each healing stage depending on the animal model being evaluated. The effect of the particular animal species on graft healing is not known. Scheffler et al., (2008) describe the early healing and remodeling phases to last around three months in animal models. ${ }^{27}$ The proliferation phase in animals seems to drastically differ with human studies. The ligamentization phase seems to be similar in both human and animal studies. ${ }^{184 ; 188 ; 189}$ Overall healing and integration in animal models 
appear to last from 6 to 12 months, which is much shorter compared to humans which can last up to 3 years. ${ }^{187}$

Despite extensive work in animals, it is still unknown when and whether a reconstructed ACL can ever reach the same biomechanical strength and stiffness of the native uninjured ACL. No animal study has shown that the reconstructed ACL reaches similar structural properties compared to the native ACL. The 'best case scenario' only reaches $50-60 \%$ of the intact ACL. It seems that one of the main features in the remodeling phase included a lower extent of neovascularization in human studies compared to animal models. Scheffler et al (2008) describes the differences in ligamentization in animal models as a process of adaptation instead of a full restoration of native ACL properties. ${ }^{27}$ Moreover, opposite to what has been shown in humans, early healing and proliferation phase in animals exhibits a greater extent of degeneration, leading to decreased mechanical properties.

\section{Perioperative Management and Physical therapy}

The objective of rehabilitation of patients after ACLR is to decrease swelling, pain, and lack of range of motion, and to provide muscle strength and dynamic stability to the lower extremity without interfering with the healing process of the graft. ${ }^{190}$ The ACL graft and the graft fixation site are significantly weaker the first few months after surgery and can be injured with less force. The graft undergoes ligamentization and is particularly weak during this stage.

Depending on the graft choice, rehabilitation protocols should be modified. Soft tissue grafts, such as hamstring tendons, are thought to be less strong than bone-tendon-bone grafts such as the patellar tendon because they require a different graft fixation. Rodeo et al., (1993) suggested that soft tissue graft incorporation at the insertion site may take from 
8 to 12 weeks. ${ }^{191}$ On the contrary, a bone-patellar tendon-bone autograft integration requires around six weeks. Consequently, tensile load to the hamstring graft should be minimized the first weeks after surgery compared to the patellar tendon graft. Additionally, harvest sites are particularly injured early after surgery, thus, avoiding high load to the quadriceps when using a QT or PT graft or, hamstring loads when using an HT graft is advised. Therefore, early healing of the graft should start with exercises that minimize the load applied to the ACL graft while maintaining a proper homeostatic stress the induces healing and integration. Exercises with the knee flexed (from $50^{\circ}$ to $100^{\circ}$ ) are recommended using both weight bearing and non-weight bearing techniques. ${ }^{190}$ Understanding how the ACL is loaded during rehabilitation can help clinicians to adjust training and exercise regimens in a safe manner. Studies indicate that ACL loading is greater in non-weight bearing exercises compared to weight-bearing exercises. ${ }^{190}$ Regardless of the activity, the ACL is loaded to a greater extent when the knee is near to full extension $\left(10^{\circ}-50^{\circ}\right.$ flexion $)$. Additionally, loads on the ACL change depending on the exercise technique. Lower ACL forces are found in exercises such as squatting and lunging with a forward trunk tilt, seated knee extension. On the contrary, other activities such as squatting with excessive forward movement of the knees beyond the toes and with the heels off the ground produce greater forces on the ACL. ${ }^{190}$ Consequently, rehabilitation exercises during early healing following ACL reconstruction could in turn produce excessive loads to the graft and its fixation. ${ }^{3}$

In the past, rehabilitation programs after ACLR included knee immobilization for around six weeks in order to avoid motion while inflammation decreased, and tendon graft starts to heal. ${ }^{192}$ However, in vitro and animal studies have shown the adverse effects of 
immobilization on cartilage, ligaments, tendons and knee associated structures. ${ }^{193}$ Several authors have shown that immobilization would lead to decreased total weight of tendons, stiffness and tensile strength. ${ }^{194 ;} 195$ Also, complete removal of load alters the structure of the healing tendon and insertion and decreases mechanical properties of the tendon. ${ }^{196}$ However, some studies have shown that the early application of load resulted in decreased load-to-failure values as well as greater macrophages and osteoclasts accumulation along the graft-tunnel interface associated with a higher inflammatory profile and a lower bone production. ${ }^{197-199}$ Similarly, Thomopoulos et al., (2003) showed superior structural, compositional and biomechanical properties in immobilized shoulder joints compared to post-operative activity in a rat model..$^{200}$

Consequently, the adoption of postoperative programs that include brace-controlled motion or continuous passive motion have been advocated. ${ }^{201 ; 202}$ Some other studies have shown that early mobilization with full weight bearing activities is possible without increasing risk of healing failure. ${ }^{147 ; 203}$ Unfortunately, how much force to the graft is too small to provide inadequate stimulus for enhanced healing, or too high to potentially injure the graft is still not known.

\section{In vitro models of Ligament and Tendon Mechanobiology}

Due to the functional importance of ligaments and tendons, mechanical evaluation is essential to understand their role in physiological and pathological conditions. Although several research has been conducted in this area, how tendons and ligaments maintain tissue integrity while subjected to cyclic loading is still unclear. Physiological loading range for tendons and ligaments usually fall under $13 \%$ of their ultimate strength. Authors have shown that physiological load to ligament and tendons is around $2-6 \%$ strain. ${ }^{204}$ 
Similarly, supraphysiological load is usually above $8 \%$ strain. Tendon and ligament cells attached to collagen fibers can sense changes in the mechanical environment. When load is applied, cells respond through the deformation of their cytoskeleton and alteration of the cell-matrix interaction. ${ }^{205}$ It is known that mechanical stimulation plays a key role in tendon and ligament development, maturation, and degradation. ${ }^{195}$

Musculoskeletal tissue cell type is of mesodermal origin. The main cell type is the fibroblast that can be differentiated according to each specific tissue type. One of the main features of connective tissue cells is mechanosensitivity. The process by which cells elaborate a biologic response to mechanical stimuli is called mechanotransduction. And encompasses many features of growth and physiological adaptation. ${ }^{206}$ Adaptation to load refers to the ability of tissues and cells to maintain specific properties such as strength and stiffness, required for the function and integrity in response to load transfer, producing distribution of stress and strain. Depending on the nature of the forces involved and the specific anatomic conditions of the tissue, the response of the tissue to stress and strain could be either physiological or pathologic. For most tissues, physiologic conditions mechanical loads may be considered trophic for musculoskeletal tissues. Under specific mechanic conditions, the cellular response involves a change in ECM composition resulting in functional adaptations in strength and stiffness. Intracellular signal that depends upon mechanical activation is not fully understood.

Forces are usually transferred through the ECM and cellular response to such deformations is the base of tissue adaptation. In ligament and tendon tissue, cells are regularly distributed throughout the ECM and are interconnected through cytoplasmic extensions and gap junctions. ${ }^{207 ; 208}$ This particular organization is thought to be responsible for the ability of 
cells to create an adaptive tissue response to mechanical or biological stimuli. ${ }^{209}$ However, normal healing after injury in tendon and ligaments is not fully restored due to fibrosis and failed connectivity among scar fibroblasts. Therefore, leading to inferior mechanical properties and lack of adaptability of the scar compared to native tissue. ${ }^{210}$

To assess the effect of mechanical loading in ligament end tendon cells adequate In vitro models are required. Various models of ligament and tendon mechanobiology have been developed. These include ex vivo loading, monolayer-2D and 3D in vitro loading. Successful models require a physiological approach for simulating in vivo conditions. Experiments performed in cells from tissues subjected to mechanical stress have revealed that cell structure and tissue organization can respond to varying amounts of forces including tension, compression and shear stress. Common methods for membrane deformation include vacuum, suction, indentation or pulling. ${ }^{211}$ Flexcell ${ }^{\circledR}$ tension is the most popular system and include monolayer models, either uniaxial (Uniflex®), or biaxial (Bioflex $\left.{ }^{\circledR}\right)$, as well as 3D culture systems (TissueTrain $\left.{ }^{\circledR}\right)$.

Uniaxial tension transferred through collagen bundles is the predominant force in both, tendon and ligament. However, secondary transverse load and shear and compression forces are also present in vivo. Additionally, Wang et al., (2018) suggested that an appropriate load range to investigate physiological mechanobiology on monolayer in vitro systems should be between $4 \%$ and $6 \% .{ }^{212}$ Similarly, authors also suggest that $10 \%$ strain on tenocytes represents over $13 \%$ strain overall tendon tissue and might represent an adequate overuse injury model. ${ }^{212}$

Ex vivo loading models are achieved by applying mechanical stress to isolated tendons or tendon fascicles within a culture environment using a bioreactor. ${ }^{212}$ Different ex vivo 
loading models have been evaluated commonly ranging from 3-10\% uniaxial tension and $0.25-1 \mathrm{~Hz} .{ }^{213-218}$ Additionally, it has been shown that physiological loading maintains structural integrity and have a therapeutic effect compared to mechanical deprivation and overloading. ${ }^{213 ; 214 ; 219}$ Loading within physiological range can induce enhanced collagen production in ex-vivo loading models. ${ }^{214 ;} 220$ Similarly, mechanical deprivation and overstretch are found to be catabolic for tendons mediated by degradative enzymes and structural damage respectively. ${ }^{212}$

Two-dimensional in vitro loading models have been developed to stress cell cultures in monolayer. Advantages of the 2D model include homogenous strain between the culture substrate, and the cells and provides accurate and controllable loading. ${ }^{212}$ However, it does not allow for evaluation of cell-matrix interaction since the ECM is not present in 2D cultures. ${ }^{212}$ Cells can be stretched either uniaxial or biaxial. Uniaxial loading is achieved by applying single direction tension to the substrate. ${ }^{212}$ It is a more physiological model for replicating tendon stresses. Nevertheless, since cruciate ligaments are twisted around each other biaxial and shear forces are also present. Physiological stress in uniaxial models have shown an increased anabolic response through collagen I production, and a decreased degradative response through lower COX-2, PGE-2 and MMPs production. Similarly, uniaxial overloading tends to increase inflammatory and catabolic factors. ${ }^{21-224}$

Biaxial loading provides multidirectional stretch to the substrate in both, longitudinal and transverse directions. Low magnitude loading using Bioflex system has shown an increased expression of inflammatory genes as well as increased ECM degradation. ${ }^{225}$; 226 Additionally, physiological loading has shown enhanced expression of ECM synthesis 
without increasing degradation. ${ }^{227 ;} 228$ Similarly, inflammatory mediators and pain modulators in tenocytes have been produced in response to supraphysiological loads. ${ }^{229-231}$ Typically, the 3D model consists of a construct seeded with tenocytes and stretched in a bioreactor. Therefore, strains transferred throughout the construct depend on the material that is being made of. 3D hydrogels are commonly used because strain can be transferred homogenously across the structure allowing a more controlled environment. ${ }^{212}$ Interesting, Patel et al., (2017) showed that physiological strain increases degradative markers in a 3D model. Moreover, authors used a more complicated model to further replicate tendon structure including longitudinal stress as well as shear stresses. ${ }^{232}$

Lastly, although several differences are found among different in vitro loading models, challenges remain when trying to find a good model that replicates in vivo scenarios. Hence, physiological relevance is only one of the many factors that should be considered when choosing a loading model. Furthermore, ease of access and manipulation arise as complementary characteristics to consider. Nevertheless, we must be aware that no model can perfectly replicate in vivo conditions and more research is needed in order to translate in vitro findings to in vivo models. 


\section{REFERENCES}

1. Arnoczky SP, Rubin RM, Marshall JL. 1979. Microvasculature of the cruciate ligaments and its response to injury. An experimental study in dogs. The Journal of bone and joint surgery American volume 61:1221-1229.

2. Frank CB. 2004. Ligament structure, physiology and function. Journal of musculoskeletal \& neuronal interactions 4:199-201.

3. Scheffler S. 2012. The cruciate ligaments: Anatomy, biology, and biomechanics. The Knee Joint: Surgical Techniques and Strategies. Paris: Springer Paris; pp. 1121.

4. Neurath M, Stofft E. 1992. [Fascicular and sub-fascicular architecture of the cruciate ligament]. Unfallchirurgie 18:125-132.

5. Benjamin M, Toumi H, Ralphs JR, et al. 2006. Where tendons and ligaments meet bone: attachment sites ('entheses') in relation to exercise and/or mechanical load. Journal of anatomy 208:471-490.

6. Apostolakos J, Durant TJ, Dwyer CR, et al. 2014. The enthesis: a review of the tendon-to-bone insertion. Muscles, Ligaments and Tendons Journal 4:333-342.

7. Woo S. 1988. Ligament, tendon, and joint capsule insertions to bone. J Injury repair of the musculoskeletal soft tissues:133-166.

8. Kolar P, Schmidt-Bleek K, Schell H, et al. 2010. The Early Fracture Hematoma and Its Potential Role in Fracture Healing. Tissue Engineering Part B: Reviews $16: 427-434$.

9. Thomopoulos S, Williams GR, Gimbel JA, et al. 2003. Variation of biomechanical, structural, and compositional properties along the tendon to bone 
insertion site. Journal of orthopaedic research : official publication of the Orthopaedic Research Society 21:413-419.

10. Hashimoto Y, Yoshida G, Toyoda H, et al. 2007. Generation of tendon-to-bone interface "enthesis" with use of recombinant BMP-2 in a rabbit model. Journal of orthopaedic research : official publication of the Orthopaedic Research Society 25:1415-1424.

11. Gattorno M, Martini A. 2016. Chapter 3 - Inflammation and Its Mediators. In: Petty RE, Laxer RM, Lindsley CB, et al. editors. Textbook of Pediatric Rheumatology (Seventh Edition). Philadelphia: W.B. Saunders; pp. 14-32.e12.

12. Nguyen DT, Ramwadhdoebe TH, van der Hart CP, et al. 2014. Intrinsic healing response of the human anterior cruciate ligament: An histological study of reattached ACL remnants. 32:296-301.

13. Murray MM, Spector M. 1999. Fibroblast distribution in the anteromedial bundle of the human anterior cruciate ligament: The presence of ?-smooth muscle actinpositive cells. 17:18-27.

14. Duthon VB, Barea C, Abrassart S, et al. 2006. Anatomy of the anterior cruciate ligament. Knee surgery, sports traumatology, arthroscopy : official journal of the ESSKA 14:204-213.

15. Alexander RM. 2002. Tendon elasticity and muscle function. Comparative biochemistry and physiology Part A, Molecular \& integrative physiology 133:1001-1011.

16. Benjamin M, Kaiser E, Milz S. 2008. Structure-function relationships in tendons: a review. Journal of anatomy 212:211-228. 
17. Birch HL. 2007. Tendon matrix composition and turnover in relation to functional requirements. International journal of experimental pathology 88:241-248.

18. Yang G, Rothrauff BB, Tuan RS. 2013. Tendon and ligament regeneration and repair: clinical relevance and developmental paradigm. Birth defects research Part C, Embryo today : reviews 99:203-222.

19. Fessel G, Gerber C, Snedeker JG. 2012. Potential of collagen cross-linking therapies to mediate tendon mechanical properties. Journal of Shoulder and Elbow Surgery 21:209-217.

20. Uzel SGM, Buehler MJ. 2011. Molecular structure, mechanical behavior and failure mechanism of the C-terminal cross-link domain in type I collagen. 4:153161.

21. Amiel D, Frank C, Harwood F, et al. 1983. Tendons and ligaments: A morphological and biochemical comparison. Journal of Orthopaedic Research 1:257-265.

22. Ng GYF, Oakes BW, Deacon OW, et al. 1996. Long-term study of the biochemistry and biomechanics of anterior cruciate ligament-patellar tendon autografts in goats. Journal of Orthopaedic Research 14:851-856.

23. Bliss S, Rawlinson JJ, Todhunter R. 2013. Tissues of the musculoskeletal system. In: Tobias KM, Johnston SA editors. Veterinary Surgery: Small Animal-EBOOK: 2-Volume Set: Elsevier Health Sciences; pp. 553-564.

24. Henshaw DR, Attia E, Bhargava M, et al. 2006. Canine ACL fibroblast integrin expression and cell alignment in response to cyclic tensile strain in three- 
dimensional collagen gels. Journal of orthopaedic research : official publication of the Orthopaedic Research Society 24:481-490.

25. Ramage L, Nuki G, Salter DM. 2009. Signalling cascades in mechanotransduction: cell-matrix interactions and mechanical loading. Scandinavian journal of medicine \& science in sports 19:457-469.

26. Neurath MF, Stofft E. 1992. Structure and function of matrix components in the cruciate ligaments. An immunohistochemical, electron-microscopic, and immunoelectron-microscopic study. Acta anatomica 145:387-394.

27. Scheffler SU, Unterhauser FN, Weiler A. 2008. Graft remodeling and ligamentization after cruciate ligament reconstruction. Knee surgery, sports traumatology, arthroscopy : official journal of the ESSKA 16:834-842.

28. Frank C, McDonald D, Bray D, et al. 1992. Collagen fibril diameters in the healing adult rabbit medial collateral ligament. Connective tissue research 27:251263.

29. Ryan CN, Sorushanova A, Lomas AJ, et al. 2015. Glycosaminoglycans in Tendon Physiology, Pathophysiology, and Therapy. Bioconjugate chemistry 26:12371251.

30. Gandhi NS, Mancera RL. 2008. The structure of glycosaminoglycans and their interactions with proteins. Chem Biol Drug Des 72:455-482.

31. Dick G, Akslen-Hoel LK, Grøndahl F, et al. 2012. Proteoglycan synthesis and Golgi organization in polarized epithelial cells. The journal of histochemistry and cytochemistry : official journal of the Histochemistry Society 60:926-935. 
32. Vynios DH. 2014. Metabolism of Cartilage Proteoglycans in Health and Disease. BioMed Research International 2014:1-9.

33. McLean SG, Mallett KF, Arruda EM. 2015. Deconstructing the anterior cruciate ligament: what we know and do not know about function, material properties, and injury mechanics. Journal of biomechanical engineering 137:020906.

34. Skelley NW, Castile RM, York TE, et al. 2015. Differences in the microstructural properties of the anteromedial and posterolateral bundles of the anterior cruciate ligament. The American journal of sports medicine 43:928-936.

35. Takahashi M, Doi M, Abe M, et al. 2006. Anatomical study of the femoral and tibial insertions of the anteromedial and posterolateral bundles of human anterior cruciate ligament. The American journal of sports medicine 34:787-792.

36. Woo SL, Hollis JM, Adams DJ, et al. 1991. Tensile properties of the human femur-anterior cruciate ligament-tibia complex. The effects of specimen age and orientation. The American journal of sports medicine 19:217-225.

37. Beynnon BD, Fleming BC, Johnson RJ, et al. 1995. Anterior cruciate ligament strain behavior during rehabilitation exercises in vivo. The American journal of sports medicine 23:24-34.

38. Beynnon B, Howe JG, Pope MH, et al. 1992. The measurement of anterior cruciate ligament strain in vivo. Int Orthop 16:1-12.

39. Markolf KL, O'Neill G, Jackson SR, et al. 2004. Effects of applied quadriceps and hamstrings muscle loads on forces in the anterior and posterior cruciate ligaments. The American journal of sports medicine 32:1144-1149. 
40. Bach JM, Hull ML. 1998. Strain inhomogeneity in the anterior cruciate ligament under application of external and muscular loads. Journal of biomechanical engineering 120:497-503.

41. More RC, Karras BT, Neiman R, et al. 1993. Hamstrings--an anterior cruciate ligament protagonist. An in vitro study. The American journal of sports medicine 21:231-237.

42. Gabriel MT, Wong EK, Woo SL, et al. 2004. Distribution of in situ forces in the anterior cruciate ligament in response to rotatory loads. Journal of orthopaedic research : official publication of the Orthopaedic Research Society 22:85-89.

43. Markolf KL, Gorek JF, Kabo JM, et al. 1990. Direct measurement of resultant forces in the anterior cruciate ligament. An in vitro study performed with a new experimental technique. The Journal of bone and joint surgery American volume 72:557-567.

44. Musahl V, Karlsson J. 2019. Anterior Cruciate Ligament Tear. New England Journal of Medicine 380:2341-2348.

45. Dai B, Herman D, Liu H, et al. 2012. Prevention of ACL injury, part I: injury characteristics, risk factors, and loading mechanism. Research in sports medicine (Print) 20:180-197.

46. Carlson VR, Sheehan FT, Boden BP. 2016. Video Analysis of Anterior Cruciate Ligament (ACL) Injuries: A Systematic Review. JBJS reviews 4.

47. Kiapour AM, Murray MM. 2014. Basic science of anterior cruciate ligament injury and repair. Bone Joint Res 3:20-31. 
48. Catterall JB, Stabler TV, Flannery CR, et al. 2010. Changes in serum and synovial fluid biomarkers after acute injury (NCT00332254). Arthritis research \& therapy 12:R229.

49. Lattermann C, Conley CE, Johnson DL, et al. 2018. Select Biomarkers on the Day of Anterior Cruciate Ligament Reconstruction Predict Poor Patient-Reported Outcomes at 2-Year Follow-Up: A Pilot Study. Biomed Res Int 2018:9387809.

50. Bigoni M, Sacerdote P, Turati M, et al. 2013. Acute and late changes in intraarticular cytokine levels following anterior cruciate ligament injury. Journal of orthopaedic research : official publication of the Orthopaedic Research Society $31: 315-321$

51. Higuchi H, Shirakura K, Kimura M, et al. 2006. Changes in biochemical parameters after anterior cruciate ligament injury. 30:43-47.

52. Amano K, Huebner JL, Stabler TV, et al. 2018. Synovial Fluid Profile at the Time of Anterior Cruciate Ligament Reconstruction and Its Association With Cartilage Matrix Composition 3 Years After Surgery. 46:890-899.

53. Smith HC, Vacek P, Johnson RJ, et al. 2012. Risk factors for anterior cruciate ligament injury: a review of the literature - part 1: neuromuscular and anatomic risk. Sports Health 4:69-78.

54. Ladenhauf HN, Graziano J, Marx RG. 2013. Anterior cruciate ligament prevention strategies. 25:64-71.

55. Mall NA, Chalmers PN, Moric M, et al. 2014. Incidence and trends of anterior cruciate ligament reconstruction in the United States. The American journal of sports medicine 42:2363-2370. 
56. Sutton KM, Bullock JM. 2013. Anterior cruciate ligament rupture: differences between males and females. The Journal of the American Academy of Orthopaedic Surgeons 21:41-50.

57. Fleming BC, Renstrom PA, Beynnon BD, et al. 2001. The effect of weightbearing and external loading on anterior cruciate ligament strain. Journal of biomechanics 34:163-170.

58. Prodromos CC, Murphy BJ. 2018. Diagnosis of Anterior Cruciate Ligament Tear. In: Prodromos C editor. The Anterior Cruciate Ligament: Reconstruction and Basic Science E-Book: Reconstruction and Basic Science, Second ed. Philadelphia, PA: Elsevier Health Sciences; pp. 46-50.

59. Groves EWH. 1919. The crucial ligaments of the knee-joint: Their function, rupture, and the operative treatment of the same. 7:505-515.

60. Galway HR, MacIntosh DL. 1980. The lateral pivot shift: a symptom and sign of anterior cruciate ligament insufficiency. Clinical orthopaedics and related research:45-50.

61. Donaldson WF, 3rd, Warren RF, Wickiewicz T. 1985. A comparison of acute anterior cruciate ligament examinations. Initial versus examination under anesthesia. The American journal of sports medicine 13:5-10.

62. van Eck CF, van den Bekerom MP, Fu FH, et al. 2013. Methods to diagnose acute anterior cruciate ligament rupture: a meta-analysis of physical examinations with and without anaesthesia. Knee surgery, sports traumatology, arthroscopy : official journal of the ESSKA 21:1895-1903. 
63. Benjaminse A, Gokeler A, van der Schans CP. 2006. Clinical diagnosis of an anterior cruciate ligament rupture: a meta-analysis. J Orthop Sports Phys Ther $36: 267-288$.

64. Prodromos C. 2017. The Anterior Cruciate Ligament: Reconstruction and Basic Science E-Book: Reconstruction and Basic Science: Elsevier Health Sciences;

65. Prodromos CC, Han YS, Keller BL, et al. 2005. Stability results of hamstring anterior cruciate ligament reconstruction at 2- to 8-year follow-up. Arthroscopy : the journal of arthroscopic \& related surgery : official publication of the Arthroscopy Association of North America and the International Arthroscopy Association 21:138-146.

66. Craig JG, Go L, Blechinger J, et al. 2005. Three-tesla imaging of the knee: initial experience. Skeletal radiology 34:453-461.

67. Schindler OS. 2012. Surgery for anterior cruciate ligament deficiency: a historical perspective. Knee Surgery, Sports Traumatology, Arthroscopy 20:5-47.

68. Strand T, Molster A, Hordvik M, et al. 2005. Long-term follow-up after primary repair of the anterior cruciate ligament: clinical and radiological evaluation 15-23 years postoperatively. Arch Orthop Trauma Surg 125:217-221.

69. Feagin JA, Jr., Curl WW. 1976. Isolated tear of the anterior cruciate ligament: 5year follow-up study. The American journal of sports medicine 4:95-100.

70. Yoshida M, Fujii K. 1999. Differences in cellular properties and responses to growth factors between human ACL and MCL cells. Journal of Orthopaedic Science 4:293-298. 
71. Xie J, Wang C, Huang DY, et al. 2013. TGF-beta1 induces the different expressions of lysyl oxidases and matrix metalloproteinases in anterior cruciate ligament and medial collateral ligament fibroblasts after mechanical injury. Journal of biomechanics 46:890-898.

72. Murray MM, Fleming BC. 2013. Biology of anterior cruciate ligament injury and repair: Kappa delta ann doner vaughn award paper 2013. Journal of orthopaedic research : official publication of the Orthopaedic Research Society 31:1501-1506.

73. Murray MM, Martin SD, Martin TL, et al. 2000. Histological changes in the human anterior cruciate ligament after rupture. The Journal of bone and joint surgery American volume 82:1387-1397.

74. Spindler KP, Clark SW, Nanney LB, et al. 1996. Expression of collagen and matrix metalloproteinases in ruptured human anterior cruciate ligament: an in situ hybridization study. Journal of orthopaedic research : official publication of the Orthopaedic Research Society 14:857-861.

75. Laurens N, Koolwijk P, De Maat MPM. 2006. Fibrin structure and wound healing. 4:932-939.

76. Enoch S, Leaper DJ. 2008. Basic science of wound healing. Surgery (Oxford) 26:31-37.

77. Vavken P, Murray MM. 2011. The potential for primary repair of the ACL. Sports Med Arthrosc Rev 19:44-49.

78. Georgiev GP, Landzhov B, Kotov G, et al. 2018. Matrix Metalloproteinase-2 and -9 Expression in the Epiligament of the Medial Collateral and Anterior Cruciate Ligament in Human Knees: A Comparative Study. Cureus 10:e3550. 
79. Steadman JR, Cameron-Donaldson ML, Briggs KK, et al. 2006. A minimally invasive technique ("healing response") to treat proximal ACL injuries in skeletally immature athletes. The journal of knee surgery 19:8-13.

80. Bolander ME. 1992. Regulation of Fracture Repair by Growth Factors. Proceedings of the Society for Experimental Biology and Medicine 200:165-170.

81. Solheim E. 1998. Growth factors in bone. International Orthopaedics 22:410-416.

82. Steadman JR, Matheny LM, Briggs KK, et al. 2012. Outcomes following healing response in older, active patients: a primary anterior cruciate ligament repair technique. The journal of knee surgery 25:255-260.

83. Naraoka T, Ishibashi Y, Tsuda E, et al. 2012. Time-dependent gene expression and immunohistochemical analysis of the injured anterior cruciate ligament. Bone Joint Res 1:238-244.

84. Andersen RB, Gormsen J. 1987. Fibrin dissolution in synovial fluid. Scandinavian journal of rheumatology 16:319-333.

85. Marui T, Niyibizi C, Georgescu HI, et al. 1997. Effect of growth factors on matrix synthesis by ligament fibroblasts. Journal of orthopaedic research : official publication of the Orthopaedic Research Society 15:18-23.

86. Kobayashi D, Kurosaka M, Yoshiya S, et al. 1997. Effect of basic fibroblast growth factor on the healing of defects in the canine anterior cruciate ligament. Knee surgery, sports traumatology, arthroscopy : official journal of the ESSKA 5:189-194. 
87. Joshi SM, Mastrangelo AN, Magarian EM, et al. 2009. Collagen-platelet composite enhances biomechanical and histologic healing of the porcine anterior cruciate ligament. The American journal of sports medicine 37:2401-2410.

88. Murray MM, Spindler KP, Abreu E, et al. 2007. Collagen-platelet rich plasma hydrogel enhances primary repair of the porcine anterior cruciate ligament. Journal of orthopaedic research : official publication of the Orthopaedic Research Society 25:81-91.

89. Kanaya A, Deie M, Adachi N, et al. 2007. Intra-articular injection of mesenchymal stromal cells in partially torn anterior cruciate ligaments in a rat model. Arthroscopy : the journal of arthroscopic \& related surgery : official publication of the Arthroscopy Association of North America and the International Arthroscopy Association 23:610-617.

90. Oe K, Kushida T, Okamoto N, et al. 2011. New strategies for anterior cruciate ligament partial rupture using bone marrow transplantation in rats. Stem cells and development 20:671-679.

91. Berry SM, Green MH. 1997. Hyaluronan: a potential carrier for growth factors for the healing of ligamentous tissues. Wound repair and regeneration : official publication of the Wound Healing Society [and] the European Tissue Repair Society 5:33-38.

92. Fisher MB, Liang R, Jung HJ, et al. 2012. Potential of healing a transected anterior cruciate ligament with genetically modified extracellular matrix bioscaffolds in a goat model. Knee surgery, sports traumatology, arthroscopy : official journal of the ESSKA 20:1357-1365. 
93. Fleming BC, Magarian EM, Harrison SL, et al. 2010. Collagen scaffold supplementation does not improve the functional properties of the repaired anterior cruciate ligament. Journal of orthopaedic research : official publication of the Orthopaedic Research Society 28:703-709.

94. Vavken P, Fleming BC, Mastrangelo AN, et al. 2012. Biomechanical outcomes after bioenhanced anterior cruciate ligament repair and anterior cruciate ligament reconstruction are equal in a porcine model. Arthroscopy : the journal of arthroscopic \& related surgery : official publication of the Arthroscopy Association of North America and the International Arthroscopy Association 28:672-680.

95. Murray MM, Kalish LA, Fleming BC, et al. 2019. Bridge-Enhanced Anterior Cruciate Ligament Repair: Two-Year Results of a First-in-Human Study. Orthop J Sports Med 7:2325967118824356.

96. Feagin JA, Curl WW. 1976. Isolated tear of the anterior cruciate ligament: 5-year follow-up study. The American journal of sports medicine 4:95-100.

97. Engebretsen L, Benum P, Sundalsvoll S. 1989. Primary suture of the anterior cruciate ligament. A 6-year follow-up of 74 cases. Acta Orthop Scand 60:561564.

98. Dargel J, Gotter M, Mader K, et al. 2007. Biomechanics of the anterior cruciate ligament and implications for surgical reconstruction. Strategies in Trauma and Limb Reconstruction 2:1-12.

99. Houck DA, Kraeutler MJ, Vidal AF, et al. 2018. Variance in Anterior Cruciate Ligament Reconstruction Graft Selection based on Patient Demographics and 
Location within the Multicenter Orthopaedic Outcomes Network Cohort. The journal of knee surgery 31:472-478.

100. Budny J, Fox J, Rauh M, et al. 2017. Emerging Trends in Anterior Cruciate Ligament Reconstruction. The journal of knee surgery 30:63-69.

101. Grassi A, Carulli C, Innocenti M, et al. 2018. New Trends in Anterior Cruciate Ligament Reconstruction: A Systematic Review of National Surveys of the Last 5 Years. Joints 6:177-187.

102. Cooper DE, Deng XH, Burstein AL, et al. 1993. The strength of the central third patellar tendon graft. A biomechanical study. The American journal of sports medicine 21:818-823; discussion 823-814.

103. Hamner DL, Brown CH, Jr., Steiner ME, et al. 1999. Hamstring tendon grafts for reconstruction of the anterior cruciate ligament: biomechanical evaluation of the use of multiple strands and tensioning techniques. The Journal of bone and joint surgery American volume 81:549-557.

104. Staubli HU, Schatzmann L, Brunner P, et al. 1996. Quadriceps tendon and patellar ligament: cryosectional anatomy and structural properties in young adults. Knee surgery, sports traumatology, arthroscopy : official journal of the ESSKA 4:100-110.

105. Marieswaran M, Jain I, Garg B, et al. 2018. A Review on Biomechanics of Anterior Cruciate Ligament and Materials for Reconstruction. Applied Bionics and Biomechanics 2018:14. 
106. Fu FH, Bennett $\mathrm{CH}, \mathrm{Ma} \mathrm{CB}$, et al. 2000. Current trends in anterior cruciate ligament reconstruction. Part II. Operative procedures and clinical correlations. The American journal of sports medicine 28:124-130.

107. Jones KG. 1963. Reconstruction of the anterior cruciate ligament. a technique using the central one-third of the patellar ligament. The Journal of bone and joint surgery American volume 45:925-932.

108. Pietsch P, Richter E, Brückner HJMU. 1969. Ergebnisse plastischer Wiederherstellungsoperationen der Kreuz-und Seitenbänder am Kniegelenk bei 80 Patienten. 72:141-181.

109. Benner WR, Shelbourne KD. 2018. The Case for the Bone-Patellar Tendon-Bone Autograft with Anterior Cruciate Ligament Reconstruction. In: Prodromos C editor. The Anterior Cruciate Ligament: Reconstruction and Basic Science EBook: Elsevier Health Sciences.

110. Shelbourne KD, Gray T. 1997. Anterior cruciate ligament reconstruction with autogenous patellar tendon graft followed by accelerated rehabilitation. A two- to nine-year followup. The American journal of sports medicine 25:786-795.

111. Lee GH, McCulloch P, Cole BJ, et al. 2008. The incidence of acute patellar tendon harvest complications for anterior cruciate ligament reconstruction. Arthroscopy : the journal of arthroscopic \& related surgery : official publication of the Arthroscopy Association of North America and the International Arthroscopy Association 24:162-166.

112. Mastrokalos DS, Springer J, Siebold R, et al. 2005. Donor site morbidity and return to the preinjury activity level after anterior cruciate ligament reconstruction 
using ipsilateral and contralateral patellar tendon autograft: a retrospective, nonrandomized study. The American journal of sports medicine 33:85-93.

113. Magnussen RA, Carey JL, Spindler KP. 2011. Does autograft choice determine intermediate-term outcome of ACL reconstruction? Knee surgery, sports traumatology, arthroscopy : official journal of the ESSKA 19:462-472.

114. Pinczewski LA, Lyman J, Salmon LJ, et al. 2007. A 10-year comparison of anterior cruciate ligament reconstructions with hamstring tendon and patellar tendon autograft: a controlled, prospective trial. The American journal of sports medicine 35:564-574.

115. Holm I, Oiestad BE, Risberg MA, et al. 2010. No difference in knee function or prevalence of osteoarthritis after reconstruction of the anterior cruciate ligament with 4-strand hamstring autograft versus patellar tendon-bone autograft: a randomized study with 10-year follow-up. The American journal of sports medicine 38:448-454.

116. Mohtadi NG, Chan DS, Dainty KN, et al. 2011. Patellar tendon versus hamstring tendon autograft for anterior cruciate ligament rupture in adults. The Cochrane database of systematic reviews:Cd005960.

117. Rahr-Wagner L, Thillemann TM, Pedersen AB, et al. 2014. Comparison of hamstring tendon and patellar tendon grafts in anterior cruciate ligament reconstruction in a nationwide population-based cohort study: results from the danish registry of knee ligament reconstruction. The American journal of sports medicine 42:278-284. 
118. Gifstad T, Foss OA, Engebretsen L, et al. 2014. Lower risk of revision with patellar tendon autografts compared with hamstring autografts: a registry study based on 45,998 primary ACL reconstructions in Scandinavia. The American journal of sports medicine 42:2319-2328.

119. Persson A, Fjeldsgaard K, Gjertsen JE, et al. 2014. Increased risk of revision with hamstring tendon grafts compared with patellar tendon grafts after anterior cruciate ligament reconstruction: a study of 12,643 patients from the Norwegian Cruciate Ligament Registry, 2004-2012. The American journal of sports medicine 42:285-291.

120. Thompson SM, Salmon LJ, Waller A, et al. 2016. Twenty-Year Outcome of a Longitudinal Prospective Evaluation of Isolated Endoscopic Anterior Cruciate Ligament Reconstruction With Patellar Tendon or Hamstring Autograft. The American journal of sports medicine 44:3083-3094.

121. Seon JK, Song EK, Park SJ. 2006. Osteoarthritis after anterior cruciate ligament reconstruction using a patellar tendon autograft. Int Orthop 30:94-98.

122. Blauth W. 1984. Die zweizügelige Ersatzplastik des vorderen Kreuzbandes aus der Quadricepssehne. J Unfallheilkunde 87:45-51.

123. Shelton W. 2018. The Case for Quadriceps Autograft. In: Prodromos C editor. The Anterior Cruciate Ligament: Reconstruction and Basic Science E-Book: Elsevier Health Sciences; pp. 84-85.

124. Stäubli H-U. 1998. The Quadriceps Tendon-Patellar Bone Construct for ACL Reconstruction. In: Imhoff AB, Burkart A editors. Knieinstabilität und 
Knorpelschaden: Das instabile Knie und der Knorpelschaden des Sportlers. Heidelberg: Steinkopff; pp. 126-139.

125. Shelton WR, Holt S. 2004. Quadriceps tendon anterior cruciate ligament reconstruction (SS-15). Arthroscopy : the journal of arthroscopic \& related surgery : official publication of the Arthroscopy Association of North America and the International Arthroscopy Association 20:e7.

126. Kim SJ, Kumar P, Oh KS. 2009. Anterior cruciate ligament reconstruction: autogenous quadriceps tendon-bone compared with bone-patellar tendon-bone grafts at 2-year follow-up. Arthroscopy : the journal of arthroscopic \& related surgery : official publication of the Arthroscopy Association of North America and the International Arthroscopy Association 25:137-144.

127. Han HS, Seong SC, Lee S, et al. 2008. Anterior cruciate ligament reconstruction : quadriceps versus patellar autograft. Clinical orthopaedics and related research 466:198-204.

128. van Eck CF, Schreiber VM, Mejia HA, et al. 2010. "Anatomic" anterior cruciate ligament reconstruction: a systematic review of surgical techniques and reporting of surgical data. Arthroscopy : the journal of arthroscopic \& related surgery : official publication of the Arthroscopy Association of North America and the International Arthroscopy Association 26:S2-12.

129. Middleton KK, Hamilton T, Irrgang JJ, et al. 2014. Anatomic anterior cruciate ligament (ACL) reconstruction: a global perspective. Part 1. Knee surgery, sports traumatology, arthroscopy : official journal of the ESSKA 22:1467-1482. 
130. Hadjicostas PT, Soucacos PN, Koleganova N, et al. 2008. Comparative and morphological analysis of commonly used autografts for anterior cruciate ligament reconstruction with the native ACL: an electron, microscopic and morphologic study. Knee Surgery, Sports Traumatology, Arthroscopy 16:10991107.

131. Staubli HU, Schatzmann L, Brunner P, et al. 1999. Mechanical tensile properties of the quadriceps tendon and patellar ligament in young adults. The American journal of sports medicine 27:27-34.

132. Slone HS, Romine SE, Premkumar A, et al. 2015. Quadriceps tendon autograft for anterior cruciate ligament reconstruction: a comprehensive review of current literature and systematic review of clinical results. Arthroscopy : the journal of arthroscopic \& related surgery : official publication of the Arthroscopy Association of North America and the International Arthroscopy Association $31: 541-554$

133. Lindeman KJZO. 1950. Uber der plastischen Ersatz der Kreuzbander durch gestierte Schneverpflanzung. Z Orthop 79:316-334.

134. Friedman M. 1988. Arthroscopic semitendinosus (gracilis) reconstruction for anterior cruciate ligament deficiency. Techniques in orthopaedics 2:74-80.

135. Barenius B, Nordlander M, Ponzer S, et al. 2010. Quality of life and clinical outcome after anterior cruciate ligament reconstruction using patellar tendon graft or quadrupled semitendinosus graft: an 8-year follow-up of a randomized controlled trial. The American journal of sports medicine 38:1533-1541. 
136. Fu F. 2011. Anterior cruciate ligament reconstruction with semitendinosus graft provided similar stability and knee function and fewer problems with kneeling compared with the bone-patellar tendon-bone graft. The Journal of bone and joint surgery American volume 93:969.

137. Gifstad T, Sole A, Strand T, et al. 2013. Long-term follow-up of patellar tendon grafts or hamstring tendon grafts in endoscopic ACL reconstructions. Knee surgery, sports traumatology, arthroscopy : official journal of the ESSKA 21:576583.

138. Chee MY, Chen Y, Pearce CJ, et al. 2017. Outcome of Patellar Tendon Versus 4Strand Hamstring Tendon Autografts for Anterior Cruciate Ligament Reconstruction: A Systematic Review and Meta-analysis of Prospective Randomized Trials. Arthroscopy : the journal of arthroscopic \& related surgery : official publication of the Arthroscopy Association of North America and the International Arthroscopy Association 33:450-463.

139. Xie X, Liu X, Chen Z, et al. 2015. A meta-analysis of bone-patellar tendon-bone autograft versus four-strand hamstring tendon autograft for anterior cruciate ligament reconstruction. The Knee 22:100-110.

140. Goldblatt JP, Fitzsimmons SE, Balk E, et al. 2005. Reconstruction of the anterior cruciate ligament: meta-analysis of patellar tendon versus hamstring tendon autograft. Arthroscopy : the journal of arthroscopic \& related surgery : official publication of the Arthroscopy Association of North America and the International Arthroscopy Association 21:791-803. 
141. Pailhe R, Cavaignac E, Murgier J, et al. 2015. Biomechanical study of ACL reconstruction grafts. Journal of orthopaedic research : official publication of the Orthopaedic Research Society 33:1188-1196.

142. Cavaignac E, Pailhe R, Reina N, et al. 2016. Can the gracilis replace the anterior cruciate ligament in the knee? A biomechanical study. Int Orthop 40:1647-1653.

143. Prodromos C, Joyce BT. 2018. Relative Strengths of Anterior Cruciate Ligament Autografts and Allografts. In: Prodromos C editor. The Anterior Cruciate Ligament: Reconstruction and Basic Science E-Book: Elsevier Health Sciences; pp. 71-73.

144. Maletis GB, Inacio MC, Reynolds S, et al. 2013. Incidence of postoperative anterior cruciate ligament reconstruction infections: graft choice makes a difference. The American journal of sports medicine 41:1780-1785.

145. Prodromos C. 2018. The Case for Hamstring Anterior Cruciate Ligament Reconstruction. In: Prodromos C editor. The Anterior Cruciate Ligament: Reconstruction and Basic Science E-Book: Elsevier Health Sciences; pp. 81-83.

146. Ahlen M, Liden M, Bovaller A, et al. 2012. Bilateral magnetic resonance imaging and functional assessment of the semitendinosus and gracilis tendons a minimum of 6 years after ipsilateral harvest for anterior cruciate ligament reconstruction. The American journal of sports medicine 40:1735-1741.

147. Shelbourne KD, Nitz P. 1990. Accelerated rehabilitation after anterior cruciate ligament reconstruction. The American journal of sports medicine 18:292-299. 
148. Middleton KK, Hamilton T, Irrgang JJ, et al. 2014. Anatomic anterior cruciate ligament (ACL) reconstruction: a global perspective. Part 1. Knee Surgery, Sports Traumatology, Arthroscopy 22:1467-1482.

149. Samuelsen BT, Webster KE, Johnson NR, et al. 2017. Hamstring Autograft versus Patellar Tendon Autograft for ACL Reconstruction: Is There a Difference in Graft Failure Rate? A Meta-analysis of 47,613 Patients. Clinical orthopaedics and related research 475:2459-2468.

150. Barrett AM, Craft JA, Replogle WH, et al. 2011. Anterior Cruciate Ligament Graft Failure:A Comparison of Graft Type Based on Age and Tegner Activity Level. The American journal of sports medicine 39:2194-2198.

151. Reinhardt KR, Hetsroni I, Marx RG. 2010. Graft Selection for Anterior Cruciate Ligament Reconstruction: A Level I Systematic Review Comparing Failure Rates and Functional Outcomes. Orthopedic Clinics 41:249-262.

152. Maletis GB, Inacio MC, Funahashi TT. 2013. Analysis of 16,192 anterior cruciate ligament reconstructions from a community-based registry. The American journal of sports medicine 41:2090-2098.

153. Carey JL, Dunn WR, Dahm DL, et al. 2009. A systematic review of anterior cruciate ligament reconstruction with autograft compared with allograft. The Journal of Bone and Joint Surgery American volume 91:2242.

154. Greenberg DD, Robertson M, Vallurupalli S, et al. 2010. Allograft compared with autograft infection rates in primary anterior cruciate ligament reconstruction. JBJS 92:2402-2408. 
155. Bottoni CR, Smith EL, Shaha J, et al. 2015. Autograft Versus Allograft Anterior Cruciate Ligament Reconstruction: A Prospective, Randomized Clinical Study With a Minimum 10-Year Follow-up. The American journal of sports medicine 43:2501-2509.

156. Cole DW, Ginn TA, Chen GJ, et al. 2005. Cost comparison of anterior cruciate ligament reconstruction: autograft versus allograft. Arthroscopy : the journal of arthroscopic \& related surgery : official publication of the Arthroscopy Association of North America and the International Arthroscopy Association 21:786-790.

157. Sun K, Zhang J, Wang Y, et al. 2011. Arthroscopic reconstruction of the anterior cruciate ligament with hamstring tendon autograft and fresh-frozen allograft: a prospective, randomized controlled study. The American journal of sports medicine 39:1430-1438.

158. Arnoczky SP. 2006. The biology of allograft incorporation. The journal of knee surgery 19:207-214.

159. Eagan MJ, McAllister DR. 2009. Biology of allograft incorporation. Clinics in sports medicine 28:203-214.

160. Jackson DW, Corsetti J, Simon TM. 1996. Biologic incorporation of allograft anterior cruciate ligament replacements. Clinical Orthopaedics and Related Research ${ }^{\circledR} 324: 126-133$.

161. Rihn JA, Harner CD. 2003. The use of musculoskeletal allograft tissue in knee surgery. Arthroscopy : the journal of arthroscopic \& related surgery : official 
publication of the Arthroscopy Association of North America and the International Arthroscopy Association 19:51-66.

162. Jackson DW, Grood ES, Goldstein JD, et al. 1993. A comparison of patellar tendon autograft and allograft used for anterior cruciate ligament reconstruction in the goat model. The American journal of sports medicine 21:176-185.

163. Harris NL, Indelicato PA, Bloomberg MS, et al. 2002. Radiographic and histologic analysis of the tibial tunnel after allograft anterior cruciate ligament reconstruction in goats. The American journal of sports medicine 30:368-373.

164. Saccomanno M. 2017. Graft-Tunnel Healing: Elsevier Health Sciences;

165. Bhatia S, Bell R, Frank RM, et al. 2012. Bony incorporation of soft tissue anterior cruciate ligament grafts in an animal model: autograft versus allograft with lowdose gamma irradiation. The American journal of sports medicine 40:1789-1798.

166. Youn I, Jones DG, Andrews PJ, et al. 2004. Periosteal augmentation of a tendon graft improves tendon healing in the bone tunnel. Clinical Orthopaedics and Related Research® 419:223-231.

167. Kyung H-S, Kim S-Y, Oh C-W, et al. 2003. Tendon-to-bone tunnel healing in a rabbit model: the effect of periosteum augmentation at the tendon-to-bone interface. Knee Surgery, Sports Traumatology, Arthroscopy 11:9-15.

168. Lui P, Zhang P, Chan K, et al. 2010. Biology and augmentation of tendon-bone insertion repair. Journal of orthopaedic surgery and research 5:59.

169. Chen CH. 2009. Strategies to enhance tendon graft--bone healing in anterior cruciate ligament reconstruction. Chang Gung medical journal 32:483-493. 
170. Dong S, Huangfu X, Xie G, et al. 2015. Decellularized versus fresh-frozen allografts in anterior cruciate ligament reconstruction: an in vitro study in a rabbit model. The American journal of sports medicine 43:1924-1934.

171. Musahl V, Plakseychuk A, VanScyoc A, et al. 2005. Varying femoral tunnels between the anatomical footprint and isometric positions: effect on kinematics of the anterior cruciate ligament-reconstructed knee. The American journal of sports medicine 33:712-718.

172. Hefzy MS, Grood ES, Noyes FR. 1989. Factors affecting the region of most isometric femoral attachments. Part II: The anterior cruciate ligament. The American journal of sports medicine 17:208-216.

173. Rupp S, Muller B, Seil R. 2001. Knee laxity after ACL reconstruction with a BPTB graft. Knee surgery, sports traumatology, arthroscopy : official journal of the ESSKA 9:72-76.

174. Abebe ES, Kim JP, Utturkar GM, et al. 2011. The effect of femoral tunnel placement on ACL graft orientation and length during in vivo knee flexion. Journal of biomechanics 44:1914-1920.

175. Sommer C, Friederich NF, Muller W. 2000. Improperly placed anterior cruciate ligament grafts: correlation between radiological parameters and clinical results. Knee surgery, sports traumatology, arthroscopy : official journal of the ESSKA 8:207-213.

176. Arnold MP, Lie DT, Verdonschot N, et al. 2005. The remains of anterior cruciate ligament graft tension after cyclic knee motion. The American journal of sports medicine 33:536-542. 
177. Boylan D, Greis PE, West JR, et al. 2003. Effects of initial graft tension on knee stability after anterior cruciate ligament reconstruction using hamstring tendons: a cadaver study. Arthroscopy : the journal of arthroscopic \& related surgery : official publication of the Arthroscopy Association of North America and the International Arthroscopy Association 19:700-705.

178. Brand J, Jr., Weiler A, Caborn DN, et al. 2000. Graft fixation in cruciate ligament reconstruction. The American journal of sports medicine 28:761-774.

179. Fu FH, Bennett CH, Lattermann C, et al. 1999. Current trends in anterior cruciate ligament reconstruction. Part 1: Biology and biomechanics of reconstruction. The American journal of sports medicine 27:821-830.

180. Fauno P, Kaalund S. 2005. Tunnel widening after hamstring anterior cruciate ligament reconstruction is influenced by the type of graft fixation used: a prospective randomized study. Arthroscopy : the journal of arthroscopic \& related surgery : official publication of the Arthroscopy Association of North America and the International Arthroscopy Association 21:1337-1341.

181. Magen HE, Howell SM, Hull ML. 1999. Structural properties of six tibial fixation methods for anterior cruciate ligament soft tissue grafts. The American journal of sports medicine 27:35-43.

182. Ahmad CS, Gardner TR, Groh M, et al. 2004. Mechanical properties of soft tissue femoral fixation devices for anterior cruciate ligament reconstruction. The American journal of sports medicine 32:635-640.

183. Zantop T, Weimann A, Rummler M, et al. 2004. Initial fixation strength of two bioabsorbable pins for the fixation of hamstring grafts compared to interference 
screw fixation: single cycle and cyclic loading. The American journal of sports medicine 32:641-649.

184. Falconiero RP, DiStefano VJ, Cook TM. 1998. Revascularization and ligamentization of autogenous anterior cruciate ligament grafts in humans. Arthroscopy : the journal of arthroscopic \& related surgery : official publication of the Arthroscopy Association of North America and the International Arthroscopy Association 14:197-205.

185. Claes S, Verdonk P, Forsyth R, et al. 2011. The "ligamentization" process in anterior cruciate ligament reconstruction: what happens to the human graft? A systematic review of the literature. The American journal of sports medicine 39:2476-2483.

186. Kuroda R, Kurosaka M, Yoshiya S, et al. 2000. Localization of growth factors in the reconstructed anterior cruciate ligament: immunohistological study in dogs. Knee Surgery, Sports Traumatology, Arthroscopy 8:120-126.

187. Rougraff B, Shelbourne KD, Gerth PK, et al. 1993. Arthroscopic and histologic analysis of human patellar tendon autografts used for anterior cruciate ligament reconstruction. The American journal of sports medicine 21:277-284.

188. Weiler A, Peters G, Maurer J, et al. 2001. Biomechanical properties and vascularity of an anterior cruciate ligament graft can be predicted by contrastenhanced magnetic resonance imaging. A two-year study in sheep. The American journal of sports medicine 29:751-761.

189. Weiler A, Peine R, Pashmineh-Azar A, et al. 2002. Tendon healing in a bone tunnel. Part I: Biomechanical results after biodegradable interference fit fixation 
in a model of anterior cruciate ligament reconstruction in sheep. Arthroscopy : the journal of arthroscopic \& related surgery : official publication of the Arthroscopy Association of North America and the International Arthroscopy Association 18:113-123.

190. Escamilla RF, Macleod TD, Wilk KE, et al. 2012. Anterior cruciate ligament strain and tensile forces for weight-bearing and non-weight-bearing exercises: a guide to exercise selection. J Orthop Sports Phys Ther 42:208-220.

191. Rodeo SA, Arnoczky SP, Torzilli PA, et al. 1993. Tendon-healing in a bone tunnel. A biomechanical and histological study in the dog. The Journal of bone and joint surgery American volume 75:1795-1803.

192. Paulos L, Noyes FR, Grood E, et al. 1991. Knee rehabilitation after anterior cruciate ligament reconstruction and repair. J Orthop Sports Phys Ther 13:60-70.

193. Noyes FR. 1977. Functional properties of knee ligaments and alterations induced by immobilization: a correlative biomechanical and histological study in primates. Clinical orthopaedics and related research:210-242.

194. Wang JH. 2006. Mechanobiology of tendon. Journal of biomechanics 39:15631582.

195. Killian ML, Cavinatto L, Galatz LM, et al. 2012. The role of mechanobiology in tendon healing. J Shoulder Elbow Surg 21:228-237.

196. Thomopoulos S, Zampiakis E, Das R, et al. 2008. The effect of muscle loading on flexor tendon-to-bone healing in a canine model. Journal of orthopaedic research : official publication of the Orthopaedic Research Society 26:1611-1617. 
197. Ma R, Schar M, Chen T, et al. 2018. Effect of Dynamic Changes in Anterior Cruciate Ligament In Situ Graft Force on the Biological Healing Response of the Graft-Tunnel Interface. The American journal of sports medicine 46:915-923.

198. Bedi A, Kovacevic D, Fox AJ, et al. 2010. Effect of early and delayed mechanical loading on tendon-to-bone healing after anterior cruciate ligament reconstruction. The Journal of bone and joint surgery American volume 92:2387-2401.

199. Brophy RH, Kovacevic D, Imhauser CW, et al. 2011. Effect of short-duration low-magnitude cyclic loading versus immobilization on tendon-bone healing after ACL reconstruction in a rat model. The Journal of bone and joint surgery American volume 93:381-393.

200. Thomopoulos S, Williams GR, Soslowsky LJ. 2003. Tendon to bone healing: differences in biomechanical, structural, and compositional properties due to a range of activity levels. Journal of biomechanical engineering 125:106-113.

201. Haggmark T, Eriksson E. 1979. Cylinder or mobile cast brace after knee ligament surgery. A clinical analysis and morphologic and enzymatic studies of changes in the quadriceps muscle. The American journal of sports medicine 7:48-56.

202. Noyes FR, Mangine RE, Barber S. 1987. Early knee motion after open and arthroscopic anterior cruciate ligament reconstruction. The American journal of sports medicine 15:149-160.

203. Janssen RPA, Van Melick N, Van Mourik JBA, et al. 2018. ACL reconstruction with hamstring tendon autograft and accelerated brace-free rehabilitation: a systematic review of clinical outcomes. BMJ Open Sport \& Exercise Medicine 4:e000301. 
204. Screen HR, Lee DA, Bader DL, et al. 2004. An investigation into the effects of the hierarchical structure of tendon fascicles on micromechanical properties. Proceedings of the Institution of Mechanical Engineers Part H, Journal of engineering in medicine 218:109-119.

205. Sun Z, Guo SS, Fässler R. 2016. Integrin-mediated mechanotransduction. The Journal of cell biology 215:445-456.

206. Peyton SR, Ghajar CM, Khatiwala CB, et al. 2007. The emergence of ECM mechanics and cytoskeletal tension as important regulators of cell function. Cell biochemistry and biophysics 47:300-320.

207. Chi SS, Rattner JB, Sciore P, et al. 2005. Gap junctions of the medial collateral ligament: structure, distribution, associations and function. Journal of anatomy 207:145-154.

208. Wall ME, Banes AJ. 2005. Early responses to mechanical load in tendon: role for calcium signaling, gap junctions and intercellular communication. Journal of musculoskeletal \& neuronal interactions 5:70-84.

209. Ko KS, McCulloch CA. 2001. Intercellular mechanotransduction: cellular circuits that coordinate tissue responses to mechanical loading. Biochem Biophys Res Commun 285:1077-1083.

210. Lo IK, Ou Y, Rattner JP, et al. 2002. The cellular networks of normal ovine medial collateral and anterior cruciate ligaments are not accurately recapitulated in scar tissue. Journal of anatomy 200:283-296. 
211. Tondon A, Haase C, Kaunas R. 2014. Mechanical Stretch Assays in Cell Culture Systems. Approximate Analytical Methods for Solving Ordinary Differential Equations:313.

212. Wang T, Chen P, Zheng M, et al. 2018. In vitro loading models for tendon mechanobiology. Journal of orthopaedic research : official publication of the Orthopaedic Research Society 36:566-575.

213. Wang T, Lin Z, Day RE, et al. 2013. Programmable mechanical stimulation influences tendon homeostasis in a bioreactor system. Biotechnology and bioengineering 110:1495-1507.

214. Wang T, Lin Z, Ni M, et al. 2015. Cyclic mechanical stimulation rescues achilles tendon from degeneration in a bioreactor system. Journal of orthopaedic research : official publication of the Orthopaedic Research Society 33:1888-1896.

215. Steiner TH, Burki A, Ferguson SJ, et al. 2012. Stochastic amplitude-modulated stretching of rabbit flexor digitorum profundus tendons reduces stiffness compared to cyclic loading but does not affect tenocyte metabolism. BMC musculoskeletal disorders 13:222.

216. Wunderli SL, Widmer J, Amrein N, et al. 2018. Minimal mechanical load and tissue culture conditions preserve native cell phenotype and morphology in tendon-a novel ex vivo mouse explant model. Journal of orthopaedic research : official publication of the Orthopaedic Research Society 36:1383-1390.

217. Freedman BR, Rodriguez AB, Hillin CD, et al. 2018. Tendon healing affects the multiscale mechanical, structural and compositional response of tendon to quasistatic tensile loading. Journal of The Royal Society Interface 15:20170880. 
218. Freedman BR, Rodriguez AB, Leiphart RJ, et al. 2018. Dynamic Loading and Tendon Healing Affect Multiscale Tendon Properties and ECM Stress Transmission. Scientific reports 8:10854.

219. Legerlotz K, Jones GC, Screen HR, et al. 2013. Cyclic loading of tendon fascicles using a novel fatigue loading system increases interleukin-6 expression by tenocytes. Scandinavian journal of medicine \& science in sports 23:31-37.

220. Maeda E, Shelton JC, Bader DL, et al. 2007. Time dependence of cyclic tensile strain on collagen production in tendon fascicles. Biochem Biophys Res Commun 362:399-404.

221. Yang G, Im H-J, Wang JHC. 2005. Repetitive mechanical stretching modulates IL-1 $\beta$ induced COX-2, MMP-1 expression, and PGE2 production in human patellar tendon fibroblasts. Gene 363:166-172.

222. Jiang C, Shao L, Wang Q, et al. 2012. Repetitive mechanical stretching modulates transforming growth factor-beta induced collagen synthesis and apoptosis in human patellar tendon fibroblasts. Biochemistry and cell biology $=$ Biochimie et biologie cellulaire 90:667-674.

223. Yang G, Crawford RC, Wang JHC. 2004. Proliferation and collagen production of human patellar tendon fibroblasts in response to cyclic uniaxial stretching in serum-free conditions. Journal of biomechanics 37:1543-1550.

224. Wang JH, Jia F, Yang G, et al. 2003. Cyclic mechanical stretching of human tendon fibroblasts increases the production of prostaglandin E2 and levels of cyclooxygenase expression: a novel in vitro model study. Connective tissue research 44:128-133. 
225. Arnoczky SP, Tian T, Lavagnino M, et al. 2002. Activation of stress-activated protein kinases (SAPK) in tendon cells following cyclic strain: the effects of strain frequency, strain magnitude, and cytosolic calcium. Journal of orthopaedic research : official publication of the Orthopaedic Research Society 20:947-952.

226. Tsuzaki M, Bynum D, Almekinders L, et al. 2003. ATP modulates load-inducible IL-1beta, COX 2, and MMP-3 gene expression in human tendon cells. J Cell Biochem 89:556-562.

227. Archambault J, Tsuzaki M, Herzog W, et al. 2002. Stretch and interleukin-1beta induce matrix metalloproteinases in rabbit tendon cells in vitro. Journal of orthopaedic research : official publication of the Orthopaedic Research Society 20:36-39.

228. Crockett RJ, Centrella M, McCarthy TL, et al. 2010. Effects of cyclic strain on rat tail tenocytes. Molecular biology reports 37:2629-2634.

229. Spang C, Backman LJ, Le Roux S, et al. 2017. Glutamate signaling through the NMDA receptor reduces the expression of scleraxis in plantaris tendon derived cells. BMC musculoskeletal disorders 18:218.

230. Fong G, Backman LJ, Hart DA, et al. 2013. Substance P enhances collagen remodeling and MMP-3 expression by human tenocytes. Journal of orthopaedic research : official publication of the Orthopaedic Research Society 31:91-98.

231. Lavagnino M, Gardner KL, Arnoczky SP. 2015. High magnitude, in vitro, biaxial, cyclic tensile strain induces actin depolymerization in tendon cells. Muscles, Ligaments and Tendons Journal 5:124-128. 
232. Patel D, Sharma S, Bryant SJ, et al. 2017. Recapitulating the Micromechanical Behavior of Tension and Shear in a Biomimetic Hydrogel for Controlling Tenocyte Response. Advanced healthcare materials 6. 


\section{Figures}

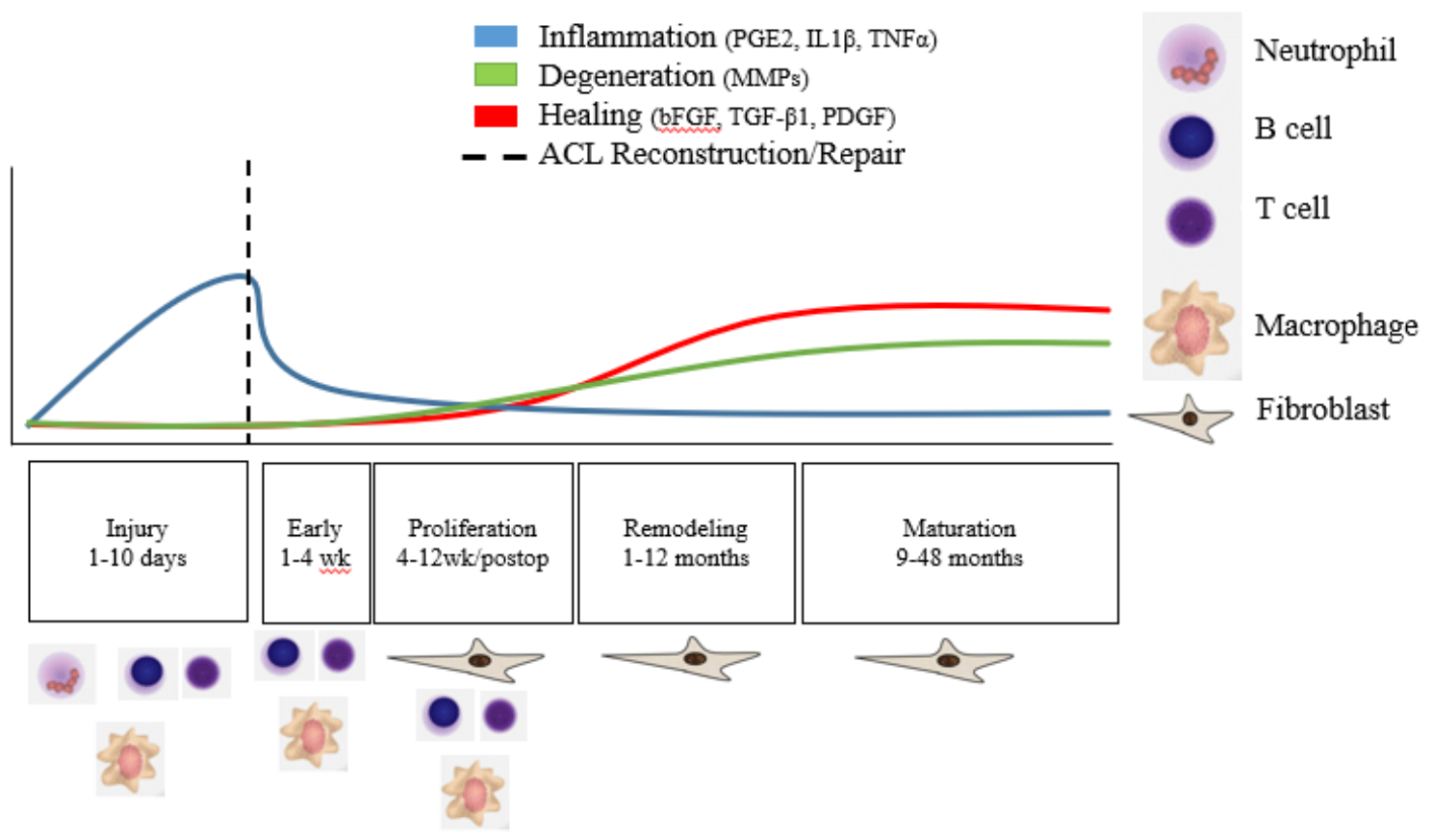

Figure 2.1. Main events in ACL healing and repair. 


\section{CHAPTER 3}

\section{EFFECT OF THREE DIFFERENT CYCLIC STRAIN PROTOCOLS ON NORMAL CANINE ACL TENDON GRAFTS CELLULAR METABOLIC RESPONSES}

\section{Introduction:}

Anterior cruciate ligament (ACL) injury is the main cause of knee instability and a leading cause of post-traumatic osteoarthritis (OA) ${ }^{1}$ Unfortunately, the ACL does not heal spontaneously after rupture, often requiring operative management in individuals who cannot function effectively with an ACL-deficient knee. ${ }^{2}$ Although generally successful, failure rates after ACL reconstruction can be as high as 20\%. According to Middleton et al.,(2014) 3 , $5.3 \%$ of ACL reconstructions fail because of poor graft healing and incorporation. ${ }^{3}$

The current standard operative technique for ACL reconstruction involves the harvest and implantation of a tendon autograft or allograft for ligament reconstruction to stabilize the injured knee. Most common autograft choices include patellar tendon (PT), semitendinosus/gracilis tendon (HT), or quadriceps tendon (QT). Although the initial strength of the graft is comparable to the native ACL, graft harvest, fixation, and the subsequent ligamentization of the tendon graft significantly decrease ultimate strength and stiffness. ${ }^{4}$ In recent studies, tendon graft choice has been associated with different outcomes including variable risk of graft failure, early onset osteoarthritis (OA), and kneeling pain. ${ }^{5}$

Additionally, success with restoration of joint stability and return to sport are associated to specific biomechanical factors within the tissue. For instance, stress deprivation or 
overuse have been associated with decreased structural properties of the grafts and increased inflammatory profiles respectively, while controlled loading has proven beneficial for postoperative healing and repair. ${ }^{6-9}$ ACL reconstruction with certain tissues, such as PT, are more related to development of lateral OA, increased production of inflammatory mediators and failure, possibly due to overloading of fibroblasts. ${ }^{6}$ While some authors have shown that PT grafts have lower revision rates, it has also been associated with increased donor site morbidity. ${ }^{10 ; 11}$ Similarly, although soft tissue grafts have yielded less stability, they have similar strength compared to PT with comparable functional scores and excellent graft ligamentization properties. ${ }^{12-15}$ Consequently, there is a lack of consensus regarding the optimal biomechanical environment for healing and function for each specific graft choice.

Successful healing of the tendon graft partly involves the understanding of the biological processes that occur during graft remodeling, maturation and incorporation. The early graft healing phase is characterized by the cellular release of molecules that regulate adequate balance between ECM degradation and synthesis. Main fibroblastic products involved in healing include cell signaling cytokines, inflammatory mediators, and degradative enzymes, as well as ECM structural components involved in maintaining the mechanical properties of the tissues. ${ }^{16}$ It is also important to recognize that these tendon graft cells are subjected to mechanical stress during rehabilitation after surgery, which can affect subsequent graft remodeling and tissue organization. Given that tendon graft fibroblasts respond to mechanical stress by altering the ECM of the tissue, it is important to understand tissue-specific differences in the biologic responses to mechanical stimulus in order to guide appropriate activity level after surgery until normal function is restored. 
The goal of this work is to evaluate the metabolic responses of common tendon graft fibroblasts used for ACL reconstruction to clinically relevant mechanical stresses in order to characterize graft types by their healing potential and their ability to respond to various biomechanical environments. We hypothesize that tendon graft fibroblasts will respond in significantly different ways with respect to inflammation, ECM degradation and metabolism in response to strain levels that simulate different physiologic conditions. These data will inform clinical practice in terms of graft choice, surgical fixation technique, and postoperative physical therapy protocols towards reducing risk for failure and improving outcomes.

\section{Materials and Methods:}

\section{Tissue processing and culture}

With ACUC approval (ACUC\#9163, 9164), tendon grafts (12 QT, 12 PT and 9 HT) were harvested from skeletally mature female dogs $(n=16)$ euthanatized for studies unrelated to the current work and free of any orthopedic disease based on orthopedic examination and radiographs. Tissues were aseptically minced into $0.5-1 \mathrm{~cm}^{2}$ pieces and digested overnight in Type 1A Clostridium histolyticum collagenase solution (Sigma-Aldrich, St. Louis, MO, USA) at a concentration of $0.5 \mathrm{mg} / \mathrm{mL}$. The aliquot was then suspended with nutritional media (Gibco DMEM, Thermo Fisher Scientific, Waltham, MA, USA) containing 10\% FBS, $0.002 \%$ Penicillin, $100 \mu \mathrm{g} / \mathrm{mL}$ Streptomycin, $25 \mu \mathrm{g} / \mathrm{mL}$ Amphotericin B, $0.002 \%$ L-Ascorbate and 0.01\% L-glutamine (Sigma Chemical Co, St. Louis, MO), cultured in T75 flasks (TPP Techno Plastic Products AG, Trasadingen, Switzerland) and incubated at $5 \% \mathrm{CO}_{2}, 37^{\circ} \mathrm{C}$, and $95 \%$ humidity.

\section{Biaxial mechanical stress}


Once confluent, passage one cells were exposed to TrypLE express (Invitrogen, Carlsbad, CA) for five minutes, then resuspended and seeded $\left(1 \times 10^{5}\right.$ cells/well $)$ in Collagen Type I-coated BioFlex® plates (Flexcell International, NC, USA). Cells were incubated for an additional $48 \mathrm{~h}$ before strain was applied. Then, nutritional culture media was replaced with $0.5 \%$ FBS media for the remaining five days of culture. Fibroblasts were subjected to continuous mechanical stimulation (2-s strain and 10 -s relaxation at a $0.5 \mathrm{~Hz}$ frequency) with a biaxial sinusoidal waveform with three different elongation strains (mechanical strain deprivation-0\%, physiologic strain-4\%, or supraphysiological strain$10 \%)^{17}$ for 5 days using the Flexcell FX-4000T strain system (Flexcell International, NC, USA) (Fig. 3-1). Media was changed every $24 \mathrm{~h}$ and stored at $-20^{\circ} \mathrm{C}$ until analysis.

\section{Biomarker assays}

Media from 24 and 120 hours of culture were assessed for various biomarkers.

Proteoglycan (GAG) was assessed using the DMMB assay as previously described. ${ }^{18}$ The concentration of prostaglandin E2 (PGE2) (Cayman Chemical, Ann Arbor, MI, USA), cytokines (IL-6, IL-8, KC and MCP-1) (Millipore, Billerica, MA, USA), total matrix metalloproteinase activity (MMPACT) (SensoLyte 520 generic MMP assay, Anaspec, Inc., Fremont, CA), and MMP production (MMP-1, MMP-2 and MMP-3) (R\&D Systems, Minneapolis, MN, USA), were all assessed using commercially available assays according to the manufacturer's protocol. At the end of the strain protocol (120h), cell viability analysis was performed using the resazurin assay (Sigma Aldrich, Saint Louis, MO). 


\section{Statistical Analysis}

For statistical analysis, normal distribution was assessed by Shapiro-Wilk test. Data were not normally distributed so non-parametric analyses were used to determine significant differences between groups. Results were reported as median \pm interquartile range (IQR). Comparisons among strains and tissue types were performed using Kruskal-Wallis test and Dunn`s test posthoc (R Core Team (2019), R version 3.6.2 Vienna, Austria), with significance set at $p<0.05$.

\section{Results:}

Mechanical strain elicited significantly different biological responses from common ACL graft fibroblasts. The metabolic activity level decreased for HT fibroblasts as strain level increased. PT demonstrated no significant changes and QT demonstrated an increase in metabolic activity at supraphysiological strain relative to mechanical strain deprivation. Moreover, the metabolic activity of the QT was significantly lower than the other graft fibroblasts with strain deprivation (Fig. 3.2). Additionally, HT and PT metabolic activity was significantly higher than QT fibroblasts when stress deprived but there was not a significant difference between cell types at physiologic and supraphysiologic strain levels (Fig. 3.13).

\section{Inflammation related responses}

There was a significantly decreased production of PGE2 when all graft fibroblasts were subjected to no strain (Fig. 3.3). Supraphysiological strain significantly overregulated the production of PGE2 across all grafts. Physiological strain, stimulated a balanced production of PGE2, being significantly different from abnormal loads, no strain and $10 \%$ strain. QT fibroblasts had a significantly lower production of PGE2 at physiological 
stress compared to HT at both time points (Fig. 3.14). Interestingly, HT significantly decreased the production of PGE2 only when subjected to supraphysiological strain. The production of IL-6 was consistently low in all graft fibroblasts regardless of the strain (Fig. 3.4). However, PT fibroblasts produced significantly more IL-6 at physiological strain relative to abnormal loads. Moreover, HT fibroblasts in the stress deprivation group produced significantly more IL-6 compared to HT fibroblasts in the physiological and supraphysiological strain groups after $120 \mathrm{~h}$ of culture. IL-6 production was also significatively higher in HT when compared to PT fibroblasts at no strain at 120h of culture (Fig. 3.15).

The production of IL-8 by QT fibroblasts was significantly lower in the stress deprivation group compared to the supraphysiological strain group at $24 \mathrm{~h}$ but was significantly higher stress deprivation group compared to the supraphysiological strain group after $120 \mathrm{~h}$ of culture (Fig. 3.5). Similarly, PT consistently showed significantly lower levels of both $\mathrm{KC}$ at $24 \mathrm{~h}$ and MCP-1 at $24 \mathrm{~h}$ and $120 \mathrm{~h}$ of culture in the strain deprivation group compared to the supraphysiological stress (Fig. 3.6 and 3.7). Whereas QT and HT fibroblasts did not show significant differences in KC production at any strain at any timepoint. Additionally, QT fibroblasts subjected to physiological strain exhibited a significantly increased production of MCP-1 compared to no strain at 24h of culture. Moreover, HT fibroblasts showed significantly increased production of MCP-1 at supraphysiological strain relative to both, no strain and $4 \%$ strain at $24 \mathrm{~h}$ of culture. When comparing differences in cytokine production between tissue types, the production of IL-8 was significantly higher in the QT and PT fibroblasts compared to the HT fibroblasts in the physiological strain group at 24h, but not at 120h of culture (Fig. 3.16). 
Moreover, there was a consistent decrease in IL-8 and KC from HT compared to QT fibroblasts after 120h of culture in both abnormal load groups, no strain and $10 \%$ strain

(Fig. 3.16 - 17). Lastly, production of MCP-1 in the supraphysiological strain group was significantly higher in the HT fibroblasts compared to the QT and PT fibroblasts at 24h of culture (Fig. 3.18).

GAG and MMP activity

Although statistical differences in GAG production were not significant, there was a trend towards higher production of GAG at the $24 \mathrm{~h}$ time point in the physiological and supraphysiological strain groups, which tended to decrease over time in the QT and HT fibroblast groups (Fig. 3.8). The production of GAG by the PT fibroblast was significantly higher than the QT and HT fibroblasts in the supraphysiological strain group at 120h of culture (Fig. 3.19).

Overall, general MMP activity was consistently increased in QT fibroblasts at physiological strain compared to both no strain and $10 \%$ strain at $24 \mathrm{~h}$ and $120 \mathrm{~h}$ of culture. Similarly, PT fibroblasts exhibited increased MMPACT at physiological strain relative to supraphysiological strain at $24 \mathrm{~h}$ and $120 \mathrm{~h}$. In addition, HT fibroblasts showed significantly higher MMPACT at $4 \%$ strain at $24 \mathrm{~h}$ but not at $120 \mathrm{~h}$ of culture (Fig. 3.9). Interestingly, MMP activity levels produced by HT fibroblasts at physiological strain was significantly higher than the QT fibroblasts at 24h but significantly lower in the HT fibroblasts compared to the QT fibroblasts at 120h (Fig. 3.20).

\section{Specific MMP Production}

The production of MMP-1 by QT fibroblasts in the strain deprivation group was significantly higher than the physiological strain group at 120h of culture (Fig. 3.10). 
Moreover, production of MMP-2 by QT fibroblasts in the strain deprivation group was significantly higher than the supraphysiological strain group at 120h of culture (Fig. 3.11). The production of MMP-3 was low by all fibroblasts at all strain levels, but production was significantly lower in HT fibroblasts compared to QT and PT fibroblasts in the strain deprived group at $24 \mathrm{~h}$ and $120 \mathrm{~h}$ of culture, respectively (Fig. 3.12).

The production of MMP-1 and MMP-3 by PT fibroblasts were significantly lower than QT fibroblasts in the physiological strain group at 24h of culture (Fig. 3.21-23). Moreover, the production of MMP-2 by HT fibroblasts was significantly higher than the QT fibroblasts in the supraphysiological strain group at 120h of culture (Fig. 3.22).

\section{Discussion}

Tendon grafts can respond to different cyclic strains due to the mechanosensitive nature of their cellular component. The results of this work support the hypothesis that inflammation, degradative enzyme metabolism, and ECM metabolism are different in QT, PT and HT fibroblasts in response to different clinically relevant tension loads. Additionally, degradative enzyme metabolism tended to be higher at supraphysiological strain, while inflammation tended to be higher when stress deprived. However, the dynamics of the cellular responses to strain were variable and dependent on tissue type. The results of the present study suggest that fibroblasts from different autograft tendons exhibit different early biological responses to mechanical stress. These biological differences may explain certain clinical phenomenon associated with specific graft types. Metabolic activity decreased and MMP activity increased in HT fibroblasts in response to increasing strain levels relative to other tendon graft fibroblasts, which may result in an overall degradative environment and initial weakening of the graft. This may provide 
some biological rationale for data that suggest HT grafts are associated with an increased risk for graft laxity relative to PT grafts..$^{9 ; 20}$

Inflammation is an adaptive response that is triggered by harmful stimuli such as tissue injury. It has the purpose of enabling effective repair responses and to re-establish tissue and organ homeostasis. However, if this balance is not reached within normal timeframes, a chronic malfunction would lead to tissue degeneration. ${ }^{21}$

The inflammatory profile of the tendon fibroblasts was evaluated by measuring PGE2, cytokines and chemokines. Mechanical strain deprivation did not induce production of PGE2 in any of the graft tenocytes. However, tendon fibroblasts increased the production of PGE2 as strain was applied to the cells in a magnitude dependent manner. Further, graft fibroblasts subjected to supraphysiological strain levels had the highest level of PGE2 production. This finding is in agreement with a previous study by Wang et al., (2003), which found that human PT fibroblasts increased production of PGE2 when stretched at either $8 \%$ or $12 \%$ compared to non-stretched controls. ${ }^{22}$

These findings suggest that abnormal mechanical loads, either stress deprivation or supraphysiological strain, might be harmful for effective healing due to a lack of inflammatory response with stress deprivation or an excessive inflammatory response with excessive strain that promotes further joint degradation and pain sensitization. ${ }^{23-25} 26$ Sauerschnig et al., (2018) found that selective COX-2 inhibitors caused impaired tendonto-bone healing and weakened the mechanical stability of the graft in a rabbit model of ACL reconstruction using a semitendinosus tendon autograft. ${ }^{27}$ In addition, Cilli et al., (2004) determined that the presence of PGE2 equal to or greater than $10 \mathrm{ng} / \mathrm{mL}$ decreased collagen production and cell proliferation compared with the control group in human PT 
fibroblasts. ${ }^{28}$ Furthermore, physiological loads seem to maintain a balanced level of PGE2 required for physiological purposes, especially in QT and PT graft fibroblasts. ${ }^{29}$ Cytokines are proteins secreted by many cell types in response to different antigenic responses and stimulate diverse responses of cells involved in immunity and inflammation. ${ }^{30}$ IL-6 is a cytokine produced by fibroblasts and numerous other cell types in response to injury or stimulation with other cytokines such as IL-1 $\beta$ and TNF- $\alpha .{ }^{30}, 31 ; 32$ IL-8 is a cytokine that induces neutrophil chemotaxis and promotes angiogenesis. ${ }^{33},{ }^{34}$ Interestingly, IL-6 and IL-8 have been identified in the synovial fluid of dogs with natural and experimental cranial cruciate ligament injury. ${ }^{32 ; 34 ; 35}$ Similar studies in humans detected increased levels of both IL-6 and IL-8 after ACL injury and one month after ACL reconstruction but lower levels just before the surgery. ${ }^{36}$ Similarly, Larsson et al (2017) found higher concentration of IL-6 and IL-8 at 4 and 8 months after ACL reconstruction immediately after ACL injury compared to delayed reconstruction possibly being the result of a second trauma induced by the reconstruction procedure itself. ${ }^{37}$

Our results did not show IL- 6 being produced by graft tenocytes at a large scale, probably due to the lack of stimulation by other cell types including macrophages and lymphocytes. ${ }^{38}$ Additionally, since IL-6 has a short life span, it would not be possible to detect it in large amounts in tenocyte cultures even at 24 hours; therefore, earlier evaluation timepoints are required. ${ }^{39 ; 40}$ On the other hand, it seems that graft tenocytes can produce IL-8 in big amounts In vitro and such concentration is affected by cell type and magnitude of mechanical stimulus. Interestingly, it seems that PT and HT had a similar initial response to increasing tensile loads by overregulating IL8 production. 
However, QT fibroblasts exhibited a more erratic response and completely shifted after 120h.

$\mathrm{KC}$ (Keratinocyte Chemoattractant) also known as GRO $\alpha$ or CXCL1 is a chemokine expressed by macrophages, neutrophils and epithelial cells characterized by neutrophil chemoattractant activity, mitogenic and angiogenic properties, and inflammatory attributes. ${ }^{30} \mathrm{KC}$ and IL-8 are members of the CXC cytokine family. They have been found in the synovial fluid of dogs with naturally occurring cranial cruciate ligament injury. ${ }^{41}$ Since in our study, lower levels of these cytokines were found in QT at physiological strain, it is possible that this graft fibroblastic type is involved in less inflammatory and degradative activation compared to PT and HT. ${ }^{42}$ Similarly, Monocyte Chemoattractant Protein (MCP-1), also known as CCL2, is a chemokine responsible for the chemotaxis of mononuclear cells that increases the expression of MMP-3 and also inhibits the synthesis of proteoglycans inducing their release from chondrocytes in vitro. ${ }^{41}$ Interestingly, some authors have shown that MCP1 stability is affected by GAG content suggesting an interaction with the ECM. ${ }^{43 ; 44}$ Our results indicate that chemokines are differentially produced in different graft fibroblasts and this production depend on the magnitude of the strain applied and the duration of the mechanical stimulus. It seems that PT fibroblasts are more reactive to changes in tension load shown by the significantly increased production of IL-8, KC and MCP1 relative to mechanical deprivation. Interestingly, $\mathrm{KC}$ production did not change significantly among tendon fibroblasts when physiologically stretched.

On the other hand, it seems that MCP1 production was significantly affected by supraphysiological strain in PT fibroblasts and was maintained after 120h. However, 
tension load did not affect QT and HT fibroblasts at 120h. This might suggest that PT fibroblasts possess a higher chemotactic ability and that inflammatory regulation in tendons depend on a more complex pathway activation process. ${ }^{45-47}$

GAGs are negatively charged molecules responsible for the structural integrity of the ECM and influence the viscoelastic properties of the tissues. ${ }^{48}$ Although GAG content is not as high in tendon as in other connective tissues, tendon GAGs are still critical to function and are important indicators of tendon injury. ${ }^{49-51}$ They are produced by fibroblasts in response to external factors such as mechanical and biological stimuli in an attempt to maintain ECM homeostasis during health, after injury, or in disease states. ${ }^{51 ; 52}$ In fact, they are being constantly degraded by common MMPs also found in the synovial fluid of the healthy knee and after ACL rupture and reconstruction. ${ }^{53-56}$ Early immobilization after ACL reconstruction has been associated with increased fibrosis and reduced structural and mechanical properties. ${ }^{57 ; 58}$ This is consistent with our results that showed increased GAG content when QT and HT graft fibroblasts were stress deprived. Interestingly, GAG production was significantly increased when PT fibroblasts were supraphysiologically stretched compared to both, QT and HT. This reaction could occur as an attempt to withstand high loads by creating more ECM components. However, since GAGs are not responsible for tensile strength, it is expected that this response is detrimental for appropriate healing of the graft. Additionally, when the femoral tunnel is placed anteriorly to the ACL footprint, the tension in the graft increases above the physiological level, resulting in higher tibio-femoral joint contact forces and altering the biomechanical environment of the joint. ${ }^{59 ; 60}$ Our findings suggest that 
abnormal stresses produce either, insufficient or excessive GAG content that might hamper an adequate healing response.

The synovial fluid within the knee joint has a high activity of MMPs under normal conditions. ${ }^{61 ; 62}$ However, such activity depends on successful proteolytic activation. ${ }^{63}$ In addition, active MMPs are being regulated by other molecules such as TIMPS. ${ }^{64}$ This might be the reason why MMP activity was mostly detected only at physiological strain in QT and HT fibroblasts at 24h, and in QT and PT at 120h. Moreover, it seems that $4 \%$ strain successfully maintains adequate degradative levels for homeostatic remodeling and avoids deposition of scar tissue, fibrosis and adherences. ${ }^{56 ; 63 ; 65-67}$ It is also possible that abnormal loads upregulate MMP inhibition with TIMPS leading to a low MMPACT. When analyzing specific MMPs production we found an initial increase in MMP1 that mitigated with time. On the contrary, MMP-2 increased after 120h, particularly when QT fibroblasts were stress deprived relative to supraphysiological stress. Interestingly, although concentration of MMP-3 concentration was consistently low, it was significantly higher when QT and PT were stress deprived at 24 and 120 hours, respectively. This might suggest that MMP-1 and 2 have specific roles in tendon fibroblastic responses to stress. Wang et al., (2017) reported a significant increase in MMP-2 gene and protein expression four and eight weeks after ACL reconstruction with a semitendinosus autograft in a rabbit model. ${ }^{68}$ Since MMP-2 can degrade denatured collagen as well as other types of collagen including I and III, it is believed that MMP-2 plays a key role after ACL reconstruction. ${ }^{56 ; 69}$ Similarly, it has been shown that MMPs 1,2 and 13 are overexpressed in healthy human hamstring tenocytes subjected to high 
frequency loading $(10 \mathrm{~Hz}) \cdot{ }^{70}$ Nevertheless, levels of MMPs after ACL reconstruction remains to be unknown.

There are several limitations to this work. First, the in vitro model utilized here is simple and does not account for interaction with other cell types. Additionally, cellular responses in monolayer cultures can greatly vary when compared with 3D cultures or whole explant models. ${ }^{17 ; 71}$ Moreover, equibiaxial load is rarely seen in vivo and other mechanical forces such as shear stress and torsion are not easily replicated in vitro. Finally, future research might include a more complete analysis on regulatory pathways including collagen metabolism, MMPs regulation through TIMPs and specific GAGs production including proteoglycans and sulfated and non-sulfated GAGs

Results shown in this work provide a framework for future investigation regarding in vitro analysis for ACL reconstruction. The exact roles for the proteins assessed in this study, presence of other molecules, and sequence and control of events in ACL graft healing are areas of ongoing work in our laboratory aimed at further characterizing the ACL ligamentization process.

There are a variety of clinical scenarios in which biomechanical environment of the tendon graft can be altered, including graft size, position in bone tunnels/sockets, graft fixation and physical rehabilitation protocol after surgery. ${ }^{3 ; 72-75}$ Similarly, mechanical deprivation has also led to undesirable results including significant muscle atrophy, arthrofibrosis and decreased mechanical properties of the graft. ${ }^{76 ; 77}$ Since the production of inflammatory mediators such as cytokines and PGE2 is key for mounting a successful healing response, the transformation of an ACL tendon graft into a ligament-like tissue will greatly depend on early postsurgical events. ${ }^{16}$ ACL graft ligamentization starts from 
the time of surgical intervention up to six months in animal models and, up to twelve months in human studies. ${ }^{16 ; 78 ; 79}$ While the remodeling stage does not initiate until the first month postoperatively, we showed that ECM composition changes as early as five days after tendon fibroblasts are subjected to loading and those responses are greatly influenced by the magnitude of the tensile load applied.

The early events in graft healing are likely modulated by cellular release of molecules that regulate the balance between the early inflammatory cascade and the subsequent ECM degradation and synthesis processes. Hence, we can conclude that the mechanical environment greatly affects the early healing response 


\section{REFERENCES}

1. Lohmander LS, Englund PM, Dahl LL, et al. 2007. The long-term consequence of anterior cruciate ligament and meniscus injuries: osteoarthritis. The American journal of sports medicine 35:1756-1769.

2. Barrack RL, Bruckner JD, Kneisl J, et al. 1990. The outcome of nonoperatively treated complete tears of the anterior cruciate ligament in active young adults. Clinical orthopaedics and related research:192-199.

3. Middleton KK, Hamilton T, Irrgang JJ, et al. 2014. Anatomic anterior cruciate ligament (ACL) reconstruction: a global perspective. Part 1. Knee Surgery, Sports Traumatology, Arthroscopy 22:1467-1482.

4. Hadjicostas PT, Soucacos PN, Koleganova N, et al. 2008. Comparative and morphological analysis of commonly used autografts for anterior cruciate ligament reconstruction with the native ACL: an electron, microscopic and morphologic study. Knee Surgery, Sports Traumatology, Arthroscopy 16:10991107.

5. Thompson SM, Salmon LJ, Waller A, et al. 2016. Twenty-Year Outcome of a Longitudinal Prospective Evaluation of Isolated Endoscopic Anterior Cruciate Ligament Reconstruction With Patellar Tendon or Hamstring Autograft. The American journal of sports medicine 44:3083-3094.

6. Killian ML, Cavinatto L, Galatz LM, et al. 2012. The role of mechanobiology in tendon healing. J Shoulder Elbow Surg 21:228-237. 
7. Yang G, Im HJ, Wang JH. 2005. Repetitive mechanical stretching modulates IL1beta induced COX-2, MMP-1 expression, and PGE2 production in human patellar tendon fibroblasts. Gene 363:166-172.

8. Ma R, Schar M, Chen T, et al. 2018. Effect of Dynamic Changes in Anterior Cruciate Ligament In Situ Graft Force on the Biological Healing Response of the Graft-Tunnel Interface. The American journal of sports medicine 46:915-923.

9. Yates CK, McCarthy MR, Hirsch HS, et al. 1992. Effects of continuous passive motion following ACL reconstruction with autogenous patellar tendon grafts. Journal of Sport Rehabilitation 1:121-131.

10. Kartus J, Movin T, Karlsson J. 2001. Donor-site morbidity and anterior knee problems after anterior cruciate ligament reconstruction using autografts. Arthroscopy : the journal of arthroscopic \& related surgery : official publication of the Arthroscopy Association of North America and the International Arthroscopy Association 17:971-980.

11. Gifstad T, Foss OA, Engebretsen L, et al. 2014. Lower Risk of Revision With Patellar Tendon Autografts Compared With Hamstring Autografts. The American journal of sports medicine 42:2319-2328.

12. Dong S, Xie G, Zhang Y, et al. 2015. Ligamentization of Autogenous Hamstring Grafts After Anterior Cruciate Ligament Reconstruction: Midterm Versus Longterm Results. 43:1908-1917.

13. Aglietti P, Giron F, Buzzi R, et al. 2004. Anterior cruciate ligament reconstruction: bone-patellar tendon-bone compared with double semitendinosus 
and gracilis tendon grafts. A prospective, randomized clinical trial. The Journal of bone and joint surgery American volume 86:2143-2155.

14. Mohtadi NG, Chan DS, Dainty KN, et al. 2011. Patellar tendon versus hamstring tendon autograft for anterior cruciate ligament rupture in adults. The Cochrane database of systematic reviews:Cd005960.

15. Mouarbes D, Menetrey J, Marot V, et al. 2019. Anterior Cruciate Ligament Reconstruction: A Systematic Review and Meta-analysis of Outcomes for Quadriceps Tendon Autograft Versus Bone-Patellar Tendon-Bone and Hamstring-Tendon Autografts. The American journal of sports medicine 47:35313540.

16. Scheffler SU, Unterhauser FN, Weiler A. 2008. Graft remodeling and ligamentization after cruciate ligament reconstruction. Knee surgery, sports traumatology, arthroscopy : official journal of the ESSKA 16:834-842.

17. Wang T, Chen P, Zheng M, et al. 2018. In vitro loading models for tendon mechanobiology. Journal of orthopaedic research : official publication of the Orthopaedic Research Society 36:566-575.

18. Farndale RW, Buttle DJ, Barrett AJ. 1986. Improved quantitation and discrimination of sulphated glycosaminoglycans by use of dimethylmethylene blue. Biochim Biophys Acta 883:173-177.

19. Cristiani R, Sarakatsianos V, Engstrom B, et al. 2018. Increased knee laxity with hamstring tendon autograft compared to patellar tendon autograft: a cohort study of 5462 patients with primary anterior cruciate ligament reconstruction. Knee surgery, sports traumatology, arthroscopy : official journal of the ESSKA. 
20. Gifstad T, Foss OA, Engebretsen L, et al. 2014. Lower risk of revision with patellar tendon autografts compared with hamstring autografts: a registry study based on 45,998 primary ACL reconstructions in Scandinavia. The American journal of sports medicine 42:2319-2328.

21. Medzhitov R. 2008. Origin and physiological roles of inflammation. Nature 454:428-435.

22. Wang JH, Jia F, Yang G, et al. 2003. Cyclic mechanical stretching of human tendon fibroblasts increases the production of prostaglandin E2 and levels of cyclooxygenase expression: a novel in vitro model study. Connective tissue research 44:128-133.

23. St-Jacques B, Ma W. 2014. Peripheral prostaglandin E2 prolongs the sensitization of nociceptive dorsal root ganglion neurons possibly by facilitating the synthesis and anterograde axonal trafficking of EP4 receptors. Exp Neurol 261:354-366.

24. Kawabata A. 2011. Prostaglandin E2 and pain--an update. Biol Pharm Bull $34: 1170-1173$.

25. Kassuya CA, Ferreira J, Claudino RF, et al. 2007. Intraplantar PGE2 causes nociceptive behaviour and mechanical allodynia: the role of prostanoid $\mathrm{E}$ receptors and protein kinases. Br J Pharmacol 150:727-737.

26. Liu B, Goode AP, Carter TE, et al. 2016. Matrix Metalloproteinase Activity and Prostaglandin E2 are Elevated in the Synovial Fluid of Meniscus Tear Patients.

27. Sauerschnig M, Stolberg-Stolberg J, Schmidt C, et al. 2018. Effect of COX-2 inhibition on tendon-to-bone healing and PGE2 concentration after anterior cruciate ligament reconstruction. European journal of medical research 23:1. 
28. Cilli F, Khan M, Fu F, et al. 2004. Prostaglandin E2 affects proliferation and collagen synthesis by human patellar tendon fibroblasts. Clin J Sport Med 14:232236.

29. Li X, Ellman M, Muddasani P, et al. 2009. Prostaglandin E2 and its cognate EP receptors control human adult articular cartilage homeostasis and are linked to the pathophysiology of osteoarthritis. Arthritis and rheumatism 60:513-523.

30. Abbas AK, Lichtman AH, Pillai S. 2014. Cellular and molecular immunology Ebook: Elsevier Health Sciences;

31. Guerne PA, Zuraw BL, Vaughan JH, et al. 1989. Synovium as a source of interleukin 6 in vitro. Contribution to local and systemic manifestations of arthritis. The Journal of clinical investigation 83:585-592.

32. Venn G, Nietfeld JJ, Duits AJ, et al. 1993. Elevated synovial fluid levels of interleukin-6 and tumor necrosis factor associated with early experimental canine osteoarthritis. Arthritis and rheumatism 36:819-826.

33. Allen PI, Conzemius MG, Evans RB, et al. 2019. Correlation between synovial fluid cytokine concentrations and limb function in normal dogs and in dogs with lameness from spontaneous osteoarthritis. Veterinary surgery : VS 48:770-779.

34. El-Hadi M, Charavaryamath C, Aebischer A, et al. 2012. Expression of interleukin-8 and intercellular cell adhesion molecule-1 in the synovial membrane and cranial cruciate ligament of dogs after rupture of the ligament. Can J Vet Res 76:8-15. 
35. de Bruin T, de Rooster H, van Bree H, et al. 2005. Interleukin-8 mRNA expression in synovial fluid of canine stifle joints with osteoarthritis. Vet Immunol Immunopathol 108:387-397.

36. Bigoni M, Turati M, Gandolla M, et al. 2016. Effects of ACL Reconstructive Surgery on Temporal Variations of Cytokine Levels in Synovial Fluid. Mediators Inflamm 2016:8243601.

37. Larsson S, Struglics A, Lohmander LS, et al. 2017. Surgical reconstruction of ruptured anterior cruciate ligament prolongs trauma-induced increase of inflammatory cytokines in synovial fluid: an exploratory analysis in the KANON trial. Osteoarthritis and Cartilage 25:1443-1451.

38. Bondeson J, Wainwright SD, Lauder S, et al. 2006. Arthritis research \& therapy 8:R187.

39. Gerhartz C, Dittrich E, Stoyan T, et al. 1994. Biosynthesis and half-life of the interleukin-6 receptor and its signal transducer gp130. 223:265-274.

40. Skutek M, Van Griensven M, Zeichen J, et al. 2001. Cyclic mechanical stretching enhances secretion of Interleukin 6 in human tendon fibroblasts. Knee Surgery, Sports Traumatology, Arthroscopy 9:322-326.

41. Garner BC, Stoker AM, Kuroki K, et al. 2011. Using animal models in osteoarthritis biomarker research. The journal of knee surgery 24:251-264.

42. Chakrabarti S. 2005. Regulation of matrix metalloproteinase-9 release from IL-8stimulated human neutrophils. Journal of Leukocyte Biology 78:279-288. 
43. Crown SE, Yu Y, Sweeney MD, et al. 2006. Heterodimerization of CCR2 chemokines and regulation by glycosaminoglycan binding. The Journal of biological chemistry 281:25438-25446.

44. Proudfoot AE, Handel TM, Johnson Z, et al. 2003. Glycosaminoglycan binding and oligomerization are essential for the in vivo activity of certain chemokines. Proceedings of the National Academy of Sciences of the United States of America 100:1885-1890.

45. Snedeker JG, Foolen J. 2017. Tendon injury and repair - A perspective on the basic mechanisms of tendon disease and future clinical therapy. Acta biomaterialia 63:18-36.

46. Abraham AC, Shah SA, Golman M, et al. 2019. Targeting the NF-kappaB signaling pathway in chronic tendon disease. Sci Transl Med 11.

47. Tian X, Jiang H, Chen Y, et al. 2018. Baicalein Accelerates Tendon-Bone Healing via Activation of Wnt/beta-Catenin Signaling Pathway in Rats. Biomed Res Int 2018:3849760.

48. Ryan CN, Sorushanova A, Lomas AJ, et al. 2015. Glycosaminoglycans in Tendon Physiology, Pathophysiology, and Therapy. Bioconjugate chemistry 26:12371251.

49. Attia M, Scott A, Duchesnay A, et al. 2012. Alterations of overused supraspinatus tendon: a possible role of glycosaminoglycans and HARP/pleiotrophin in early tendon pathology. Journal of orthopaedic research : official publication of the Orthopaedic Research Society 30:61-71. 
50. Yokota A, Gimbel JA, Williams GR, et al. 2005. Supraspinatus tendon composition remains altered long after tendon detachment. J Shoulder Elbow Surg 14:72s-78s.

51. Yoon JH, Halper J. 2005. Tendon proteoglycans: biochemistry and function. Journal of musculoskeletal \& neuronal interactions 5:22-34.

52. Sorrell JM, Caplan AI. 2009. Chapter 4 Fibroblasts-A Diverse Population at the Center of It All. International Review of Cell and Molecular Biology: Academic Press; pp. 161-214.

53. Hadler-Olsen E, Fadnes B, Sylte I, et al. 2011. Regulation of matrix metalloproteinase activity in health and disease. FEBS Journal 278:28-45.

54. Amiel D, Ishizue KK, Harwood FL, et al. 1989. Injury of the anterior cruciate ligament: the role of collagenase in ligament degeneration. Journal of orthopaedic research : official publication of the Orthopaedic Research Society 7:486-493.

55. Dahlberg L, Friden T, Roos H, et al. 1994. A longitudinal study of cartilage matrix metabolism in patients with cruciate ligament rupture--synovial fluid concentrations of aggrecan fragments, stromelysin-1 and tissue inhibitor of metalloproteinase-1. Br J Rheumatol 33:1107-1111.

56. Demirag B, Sarisozen B, Ozer O, et al. 2005. Enhancement of tendon-bone healing of anterior cruciate ligament grafts by blockage of matrix metalloproteinases. The Journal of bone and joint surgery American volume $87: 2401-2410$. 
57. Amiel D, Woo SLY, Harwood FL, et al. 1982. The Effect of Immobilization on Collagen Turnover in Connective Tissue: A Biochemical-Biomechanical Correlation. Acta Orthopaedica Scandinavica 53:325-332.

58. Galatz LM, Charlton N, Das R, et al. 2009. Complete removal of load is detrimental to rotator cuff healing. Journal of Shoulder and Elbow Surgery 18:669-675.

59. Li G, Papannagari R, E Defrate L, et al. 2006. Comparison of the ACL and ACL graft forces before and after ACL reconstruction an in-vitro robotic investigation. Acta Orthopaedica 77:267-274.

60. Abebe ES, Kim JP, Utturkar GM, et al. 2011. The effect of femoral tunnel placement on ACL graft orientation and length during in vivo knee flexion. Journal of biomechanics 44:1914-1920.

61. Xie J, Wang CL, Yang W, et al. 2018. Modulation of MMP-2 and MMP-9 through connected pathways and growth factors is critical for extracellular matrix balance of intra-articular ligaments. J Tissue Eng Regen Med 12:e550-e565.

62. Heard BJ, Martin L, Rattner JB, et al. 2012. Matrix metalloproteinase protein expression profiles cannot distinguish between normal and early osteoarthritic synovial fluid. 13:126.

63. Murphy G, Stanton H, Cowell S, et al. 1999. Mechanisms for pro matrix metalloproteinase activation. APMIS 107:38-44.

64. Brew K, Dinakarpandian D, Nagase H. 2000. Tissue inhibitors of metalloproteinases: evolution, structure and function. Biochimica et Biophysica Acta (BBA) - Protein Structure and Molecular Enzymology 1477:267-283. 
65. Chakraborti S, Mandal M, Das S, et al. 2003. Regulation of matrix metalloproteinases: an overview. Mol Cell Biochem 253:269-285.

66. Mukherjee R, Bruce JA, McClister DM, Jr., et al. 2005. Time-dependent changes in myocardial structure following discrete injury in mice deficient of matrix metalloproteinase-3. J Mol Cell Cardiol 39:259-268.

67. Visse R, Nagase H. 2003. Matrix metalloproteinases and tissue inhibitors of metalloproteinases: structure, function, and biochemistry. Circ Res 92:827-839.

68. Wang WM, Ma XJ, Huang SB, et al. 2017. A comparative study of effect of autograft compared with allograft anterior cruciate ligament reconstruction on expressions of LOXs and MMPs. Bioscience reports 37.

69. Choi H-R, Kondo S, Hirose K, et al. 2002. Expression and enzymatic activity of MMP-2 during healing process of the acute supraspinatus tendon tear in rabbits. Journal of Orthopaedic Research 20:927-933.

70. Udeze CP, Jones ER, Riley GP, et al. 2019. An in vitro investigation into the effects of $10 \mathrm{~Hz}$ cyclic loading on tenocyte metabolism. Scandinavian journal of medicine \& science in sports.

71. Wunderli SL, Widmer J, Amrein N, et al. 2018. Minimal mechanical load and tissue culture conditions preserve native cell phenotype and morphology in tendon-a novel ex vivo mouse explant model. Journal of orthopaedic research : official publication of the Orthopaedic Research Society 36:1383-1390.

72. Getelman MH, Friedman MJ. 1999. Revision anterior cruciate ligament reconstruction surgery. The Journal of the American Academy of Orthopaedic Surgeons 7:189-198. 
73. Morgan JA, Dahm D, Levy B, et al. 2012. Femoral tunnel malposition in ACL revision reconstruction. The journal of knee surgery 25:361-368.

74. Rayan F, Nanjayan SK, Quah C, et al. 2015. Review of evolution of tunnel position in anterior cruciate ligament reconstruction. World journal of orthopedics $6: 252$.

75. Wang JHC. 2006. Mechanobiology of tendon. Journal of biomechanics 39:15631582.

76. Häggmark T, Eriksson E. 1979. Cylinder or mobile cast brace after knee ligament surgery:A clinical analysis and morphologic and enzymatic studies of changes in the quadriceps muscle. The American journal of sports medicine 7:48-56.

77. Thomopoulos S, Zampiakis E, Das R, et al. 2008. The effect of muscle loading on flexor tendon-to-bone healing in a canine model. Journal of orthopaedic research : official publication of the Orthopaedic Research Society 26:1611-1617.

78. Falconiero RP, DiStefano VJ, Cook TM. 1998. Revascularization and ligamentization of autogenous anterior cruciate ligament grafts in humans. Arthroscopy : the journal of arthroscopic \& related surgery : official publication of the Arthroscopy Association of North America and the International Arthroscopy Association 14:197-205.

79. Claes S, Verdonk P, Forsyth R, et al. 2011. The "ligamentization" process in anterior cruciate ligament reconstruction: what happens to the human graft? A systematic review of the literature. The American journal of sports medicine 39:2476-2483. 
Figures

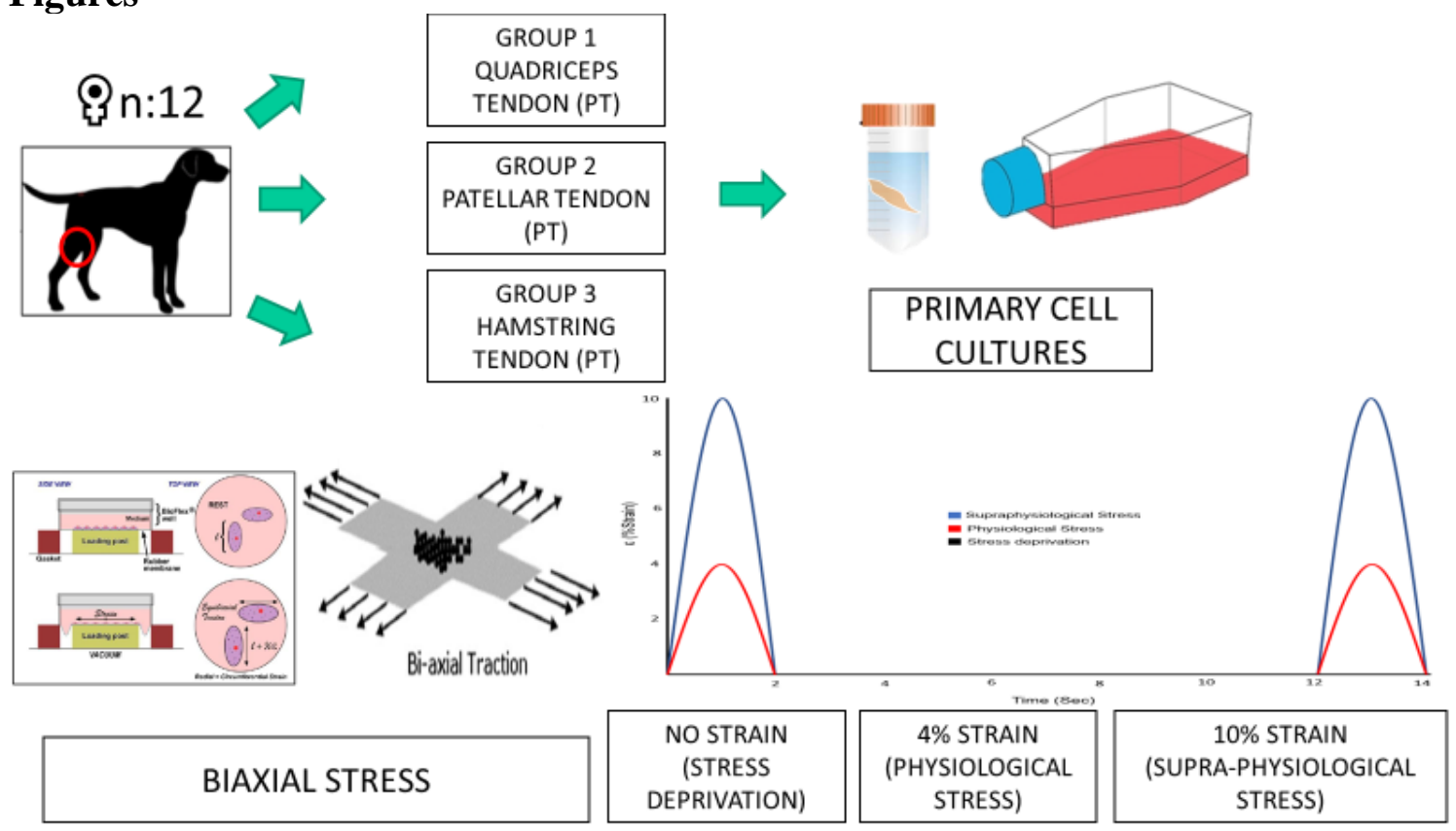

Figure 3.1. Experimental design. 

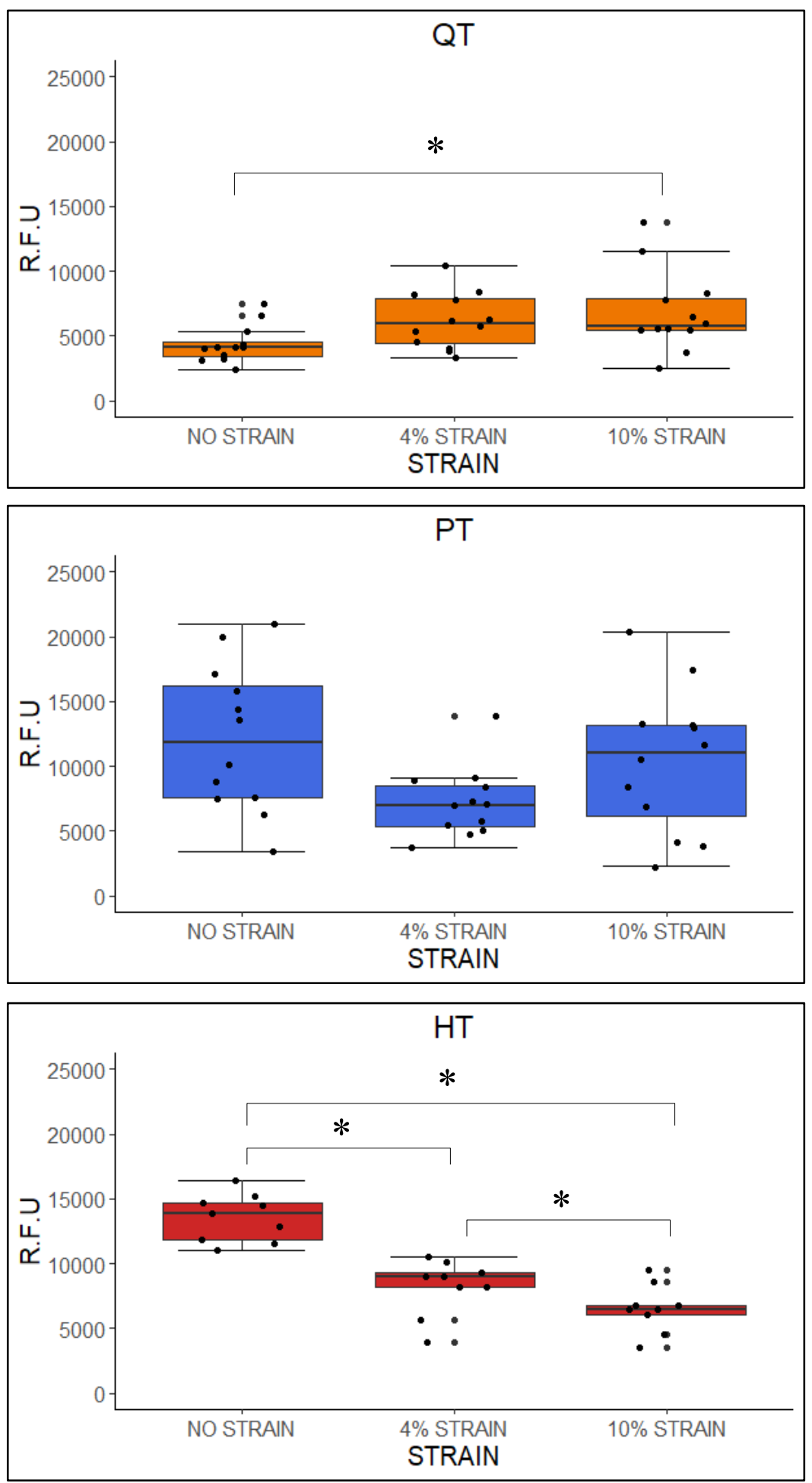

Figure 3.2. Metabolic activity after five days of culture. (*) Significant difference between strains. RFU: Resazurin Fluorescent Units QT: Quadriceps tendon; PT: Patellar tendon, HT: Hamstring tendon 

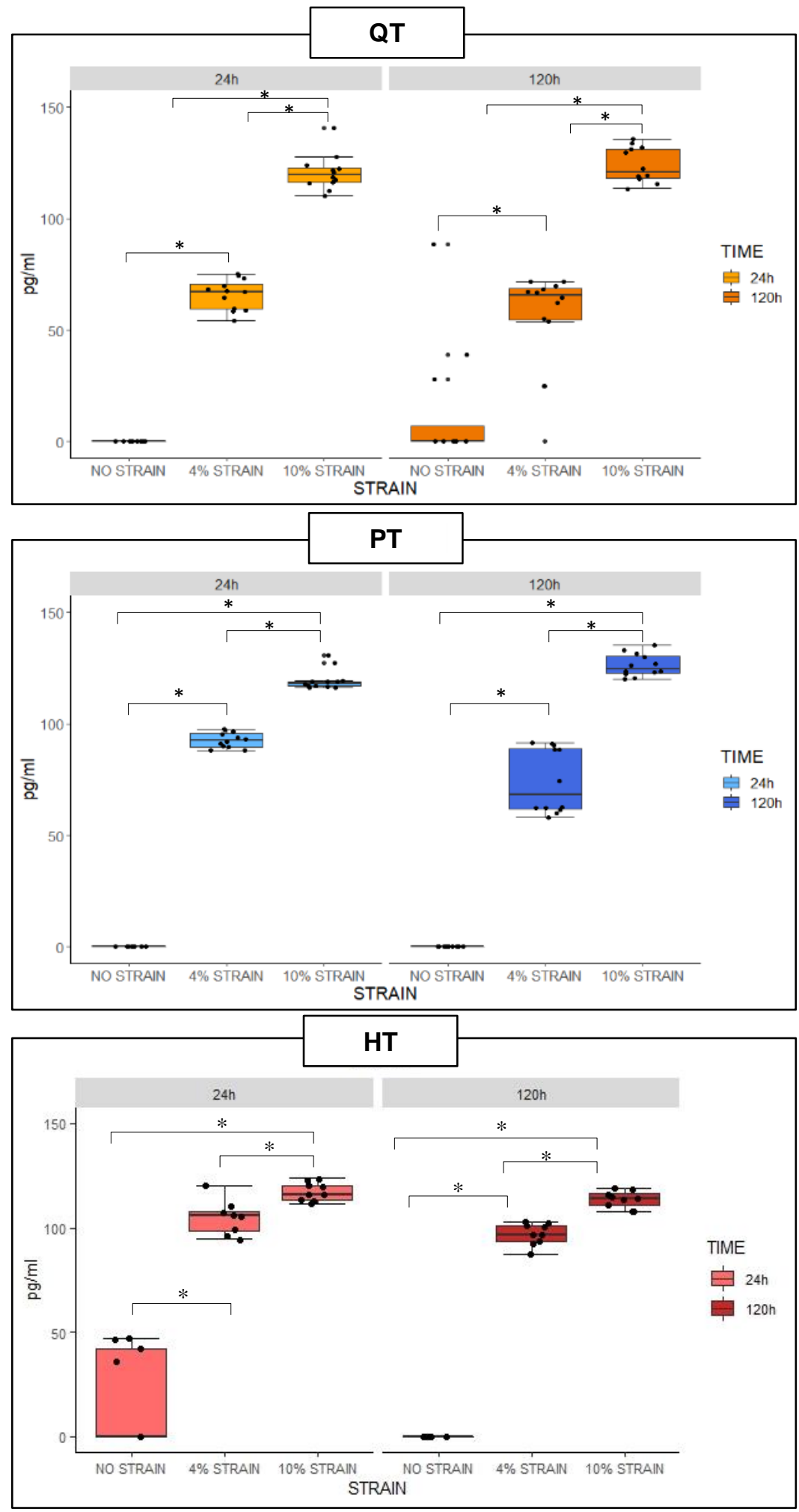

Figure 3.3. Concentration of PGE-2 released to the media. * significant difference between strains at 24 and120 hours. QT: Quadriceps tendon; PT: Patellar tendon, HT: Hamstring tendon 


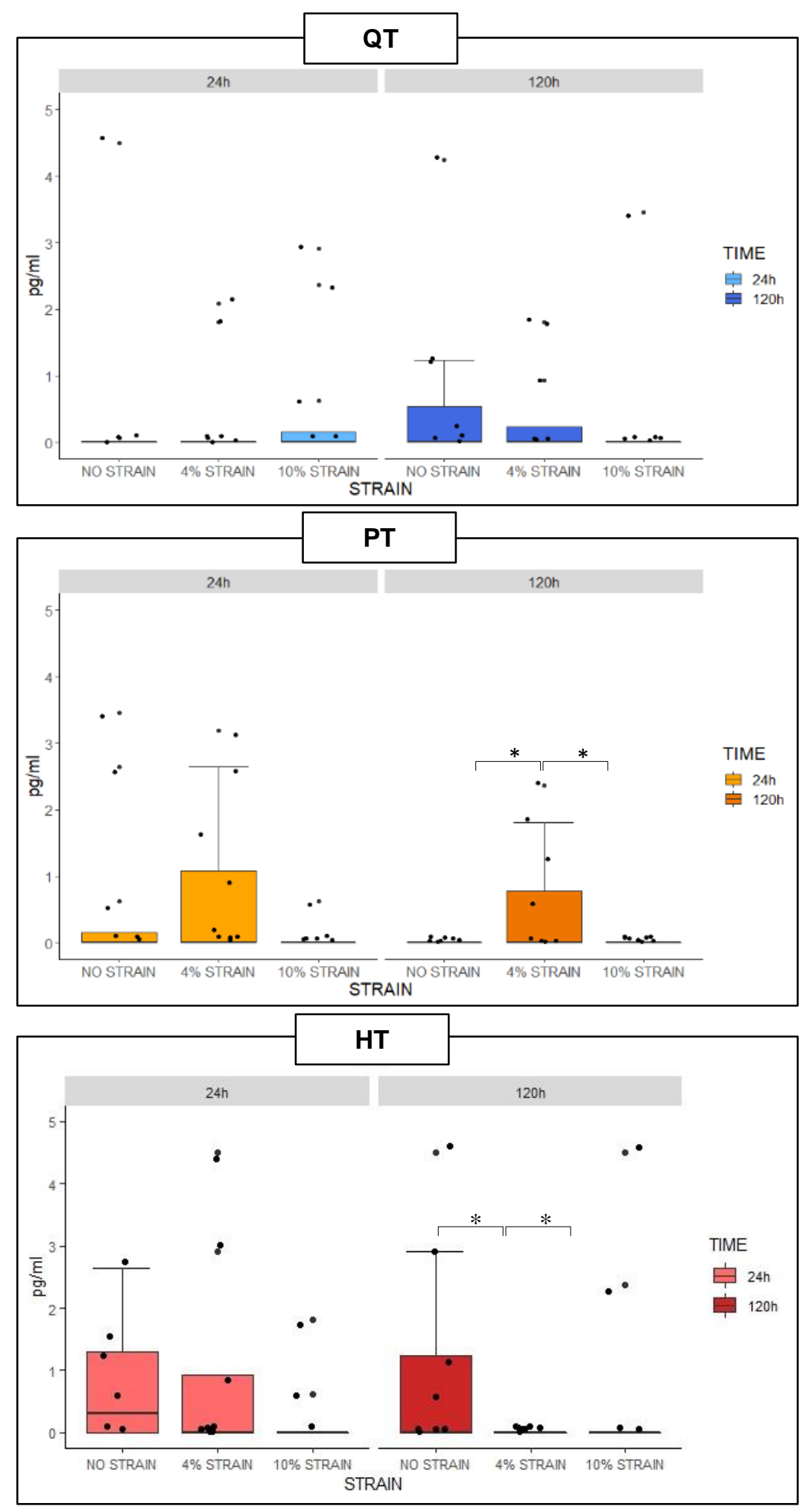

Figure 3.4. Concentration of IL-6 released to the media. * significant difference between strains at 24 and 120 hours. QT: Quadriceps tendon; PT: Patellar tendon, HT: Hamstring tendon 

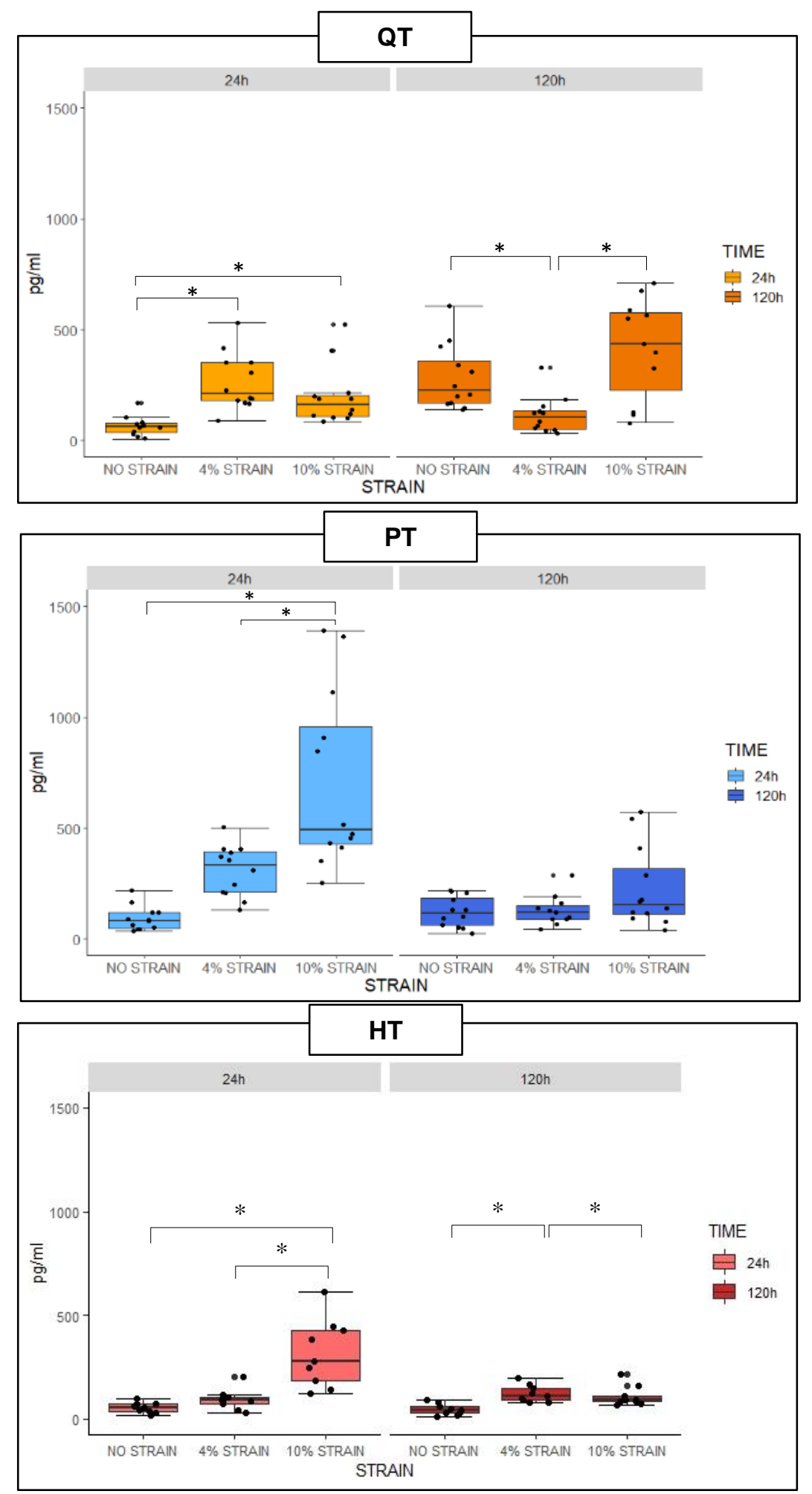

Figure 3.5. Concentration of IL-8 released to the media. * significant difference between strains at 24 and 120 hours. QT: Quadriceps tendon; PT: Patellar tendon, HT: Hamstring tendon 

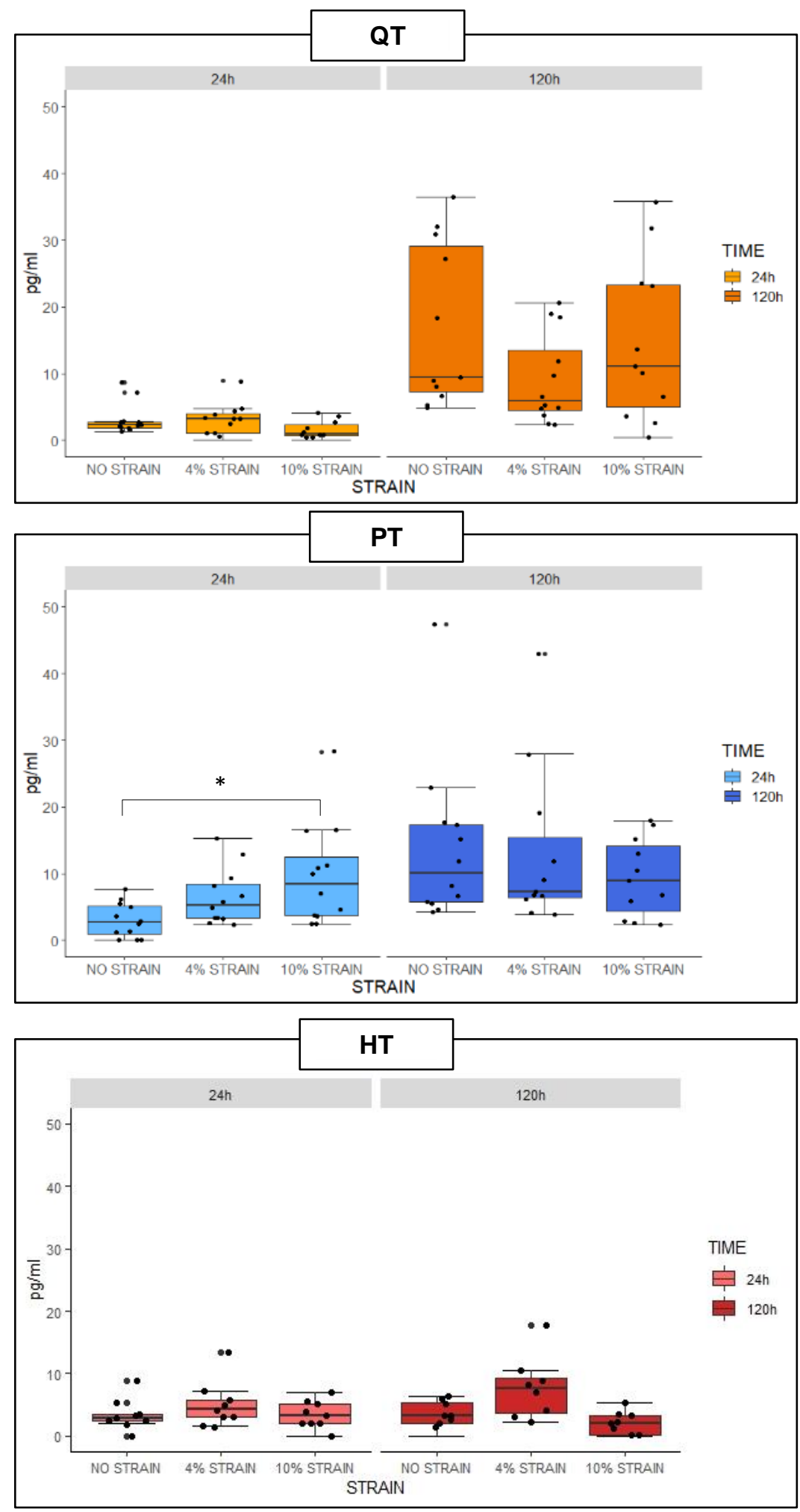

Figure 3.6. Concentration of KC released to the media. * significant difference between strains at 24 and120 hours. QT: Quadriceps tendon; PT: Patellar tendon, HT: Hamstring tendon 

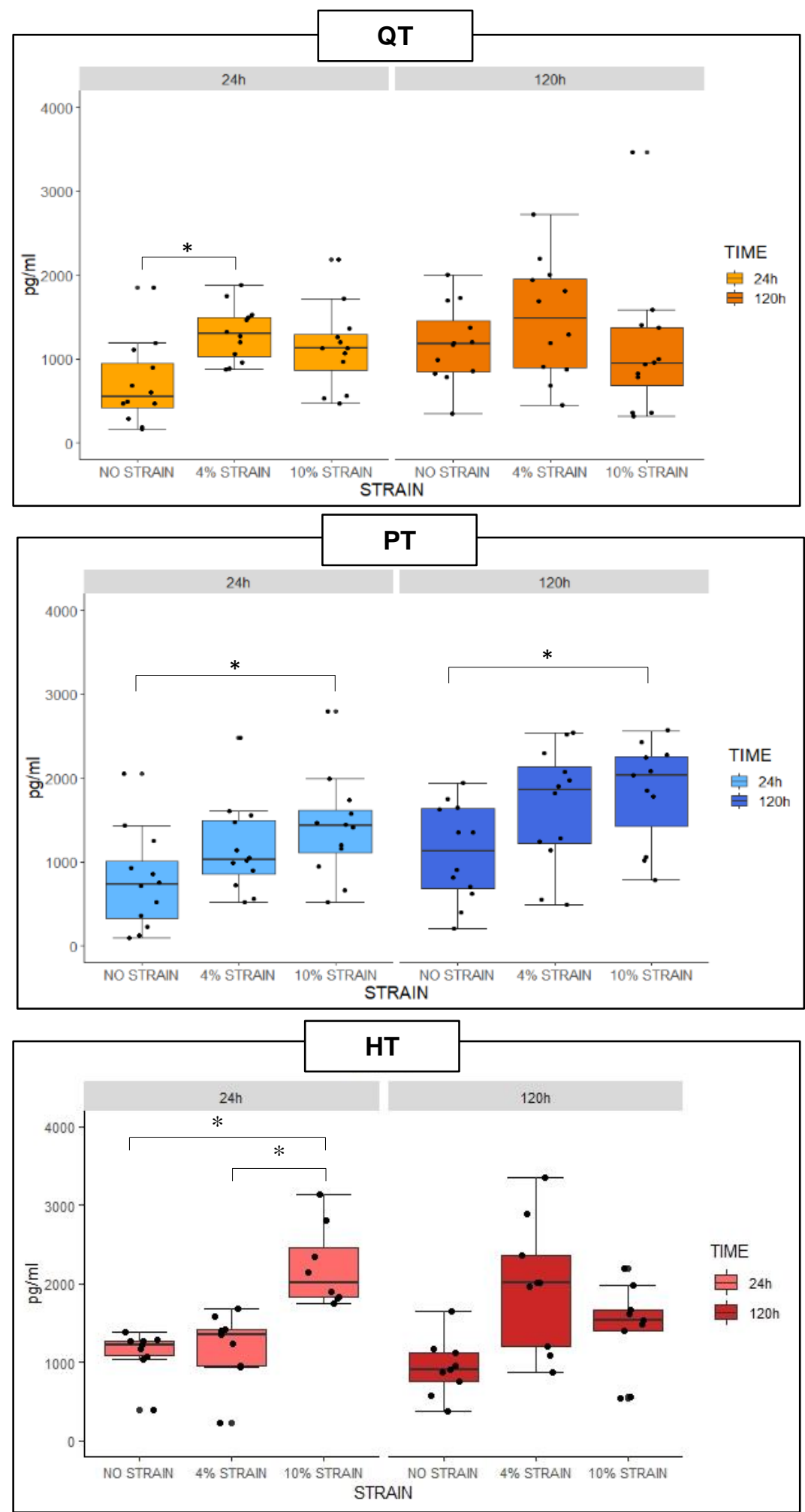

Figure 3.7. Concentration of MCP-1 released to the media. * significant difference between strains at 24 and120 hours. QT: Quadriceps tendon; PT: Patellar tendon, HT: Hamstring tendon 

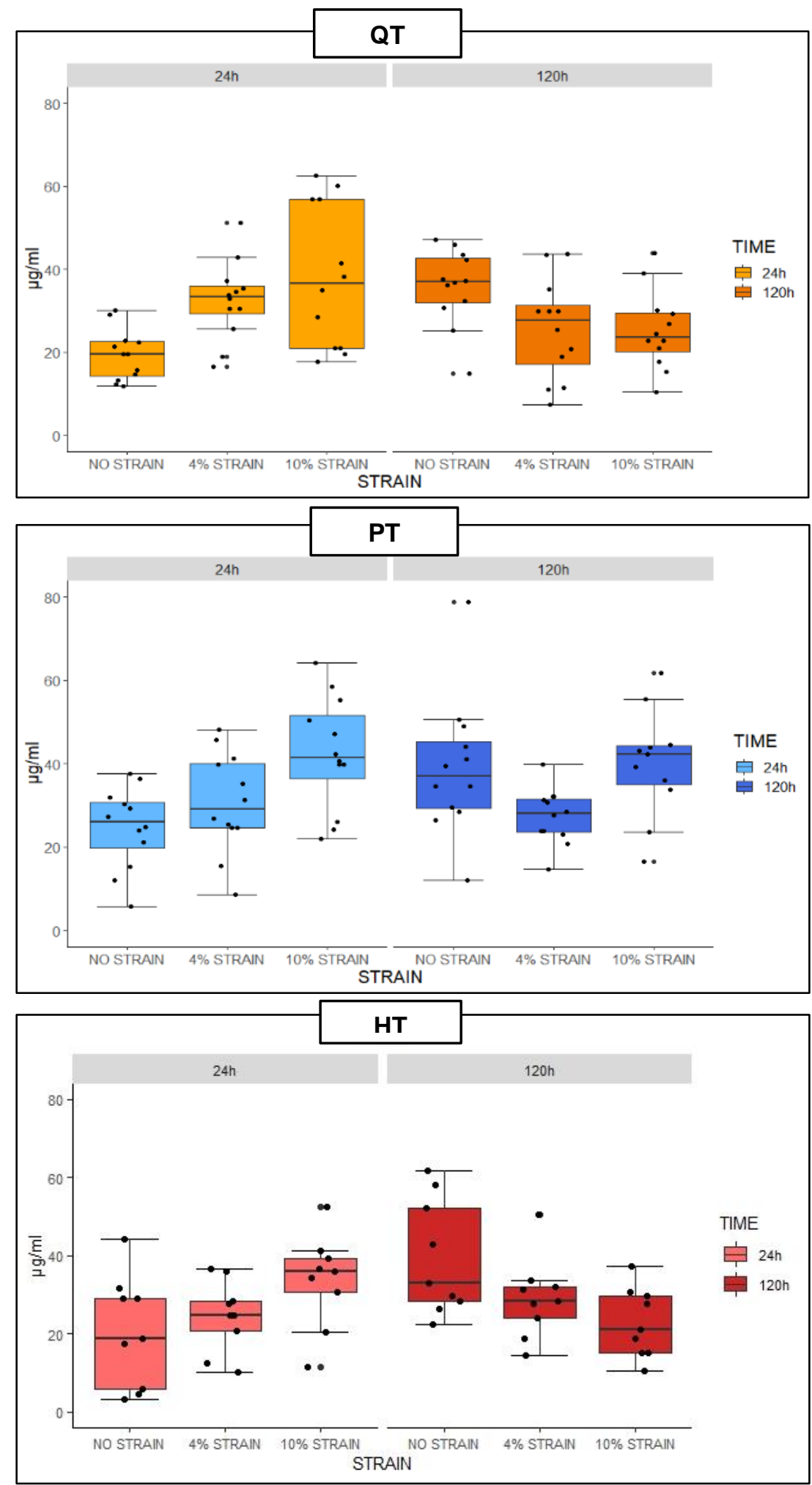

Figure 3.8. Concentration of Glycosaminoglycans (GAG) released to the media. * significant difference between strains at 24 and120 hours. QT: Quadriceps tendon; PT: Patellar tendon, HT: Hamstring tendon 

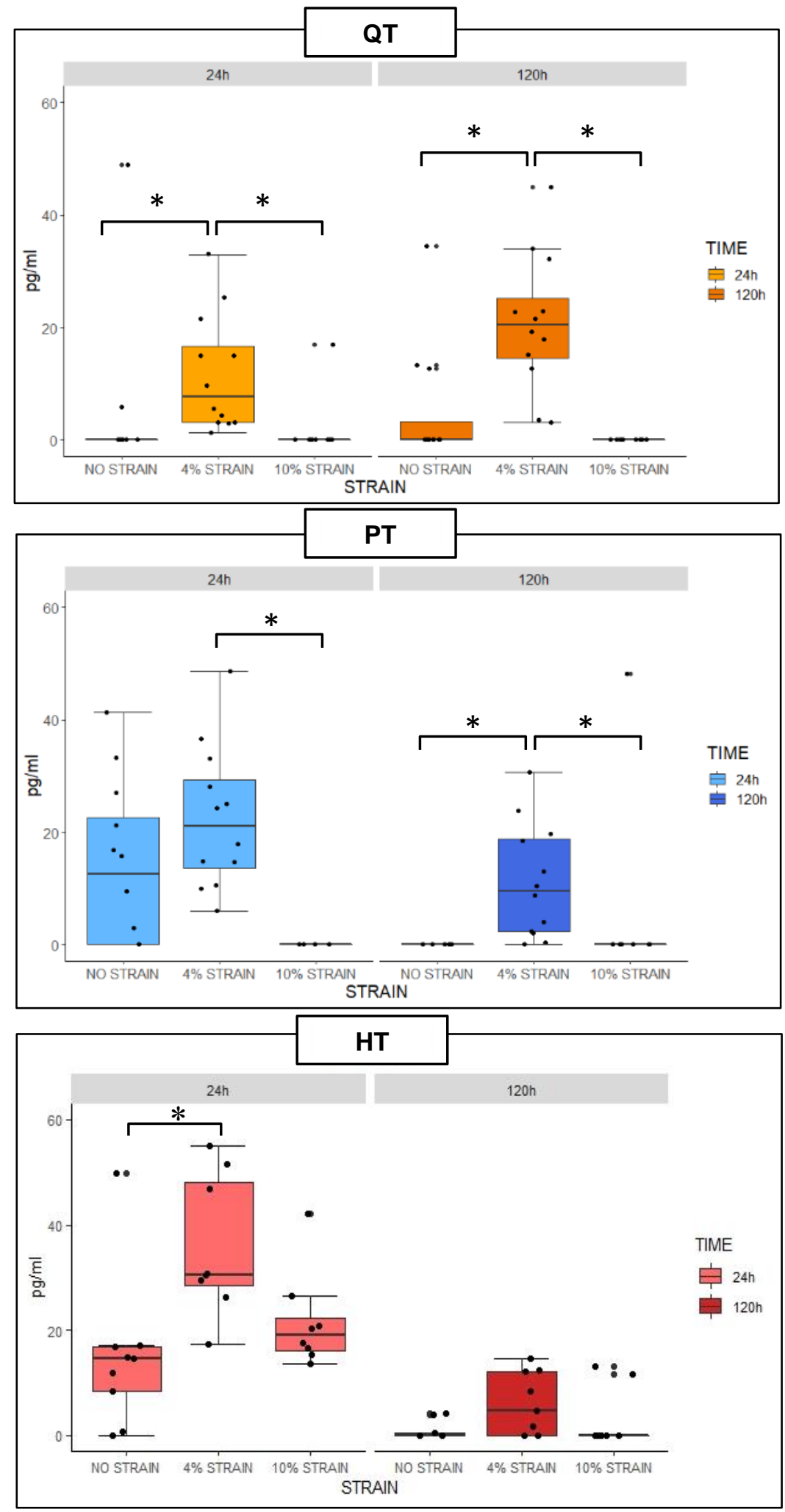

Figure 3.9. Concentration of MMP ACTIVITY (MMPACT) released to the media. * significant difference between strains at 24 and120 hours. QT: Quadriceps tendon; PT: Patellar tendon, HT: Hamstring tendon 


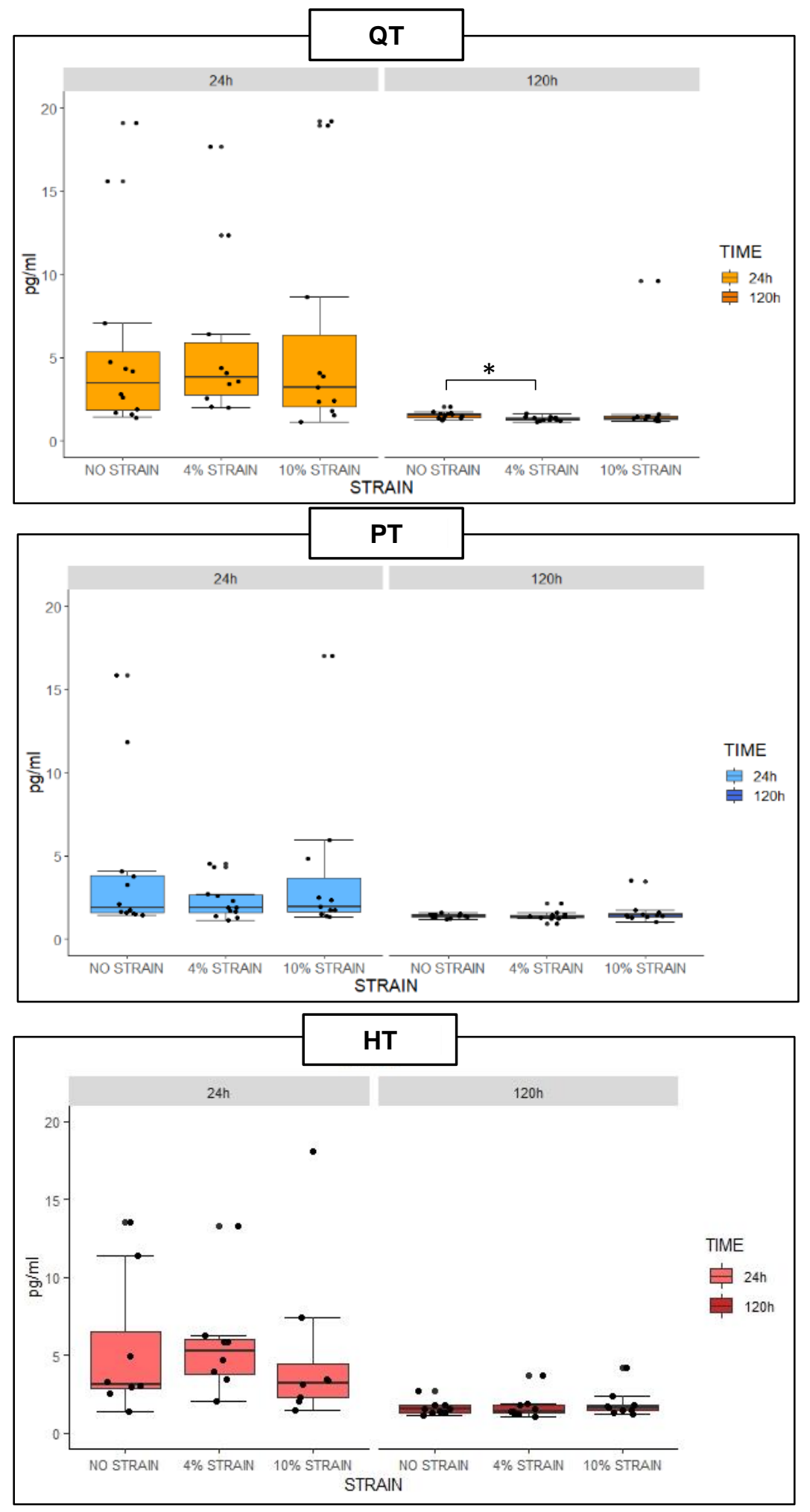

Figure 3.10. Concentration of MMP-1 released to the media. * significant difference between strains at 24 and120 hours. QT: Quadriceps tendon; PT: Patellar tendon, HT: Hamstring tendon 

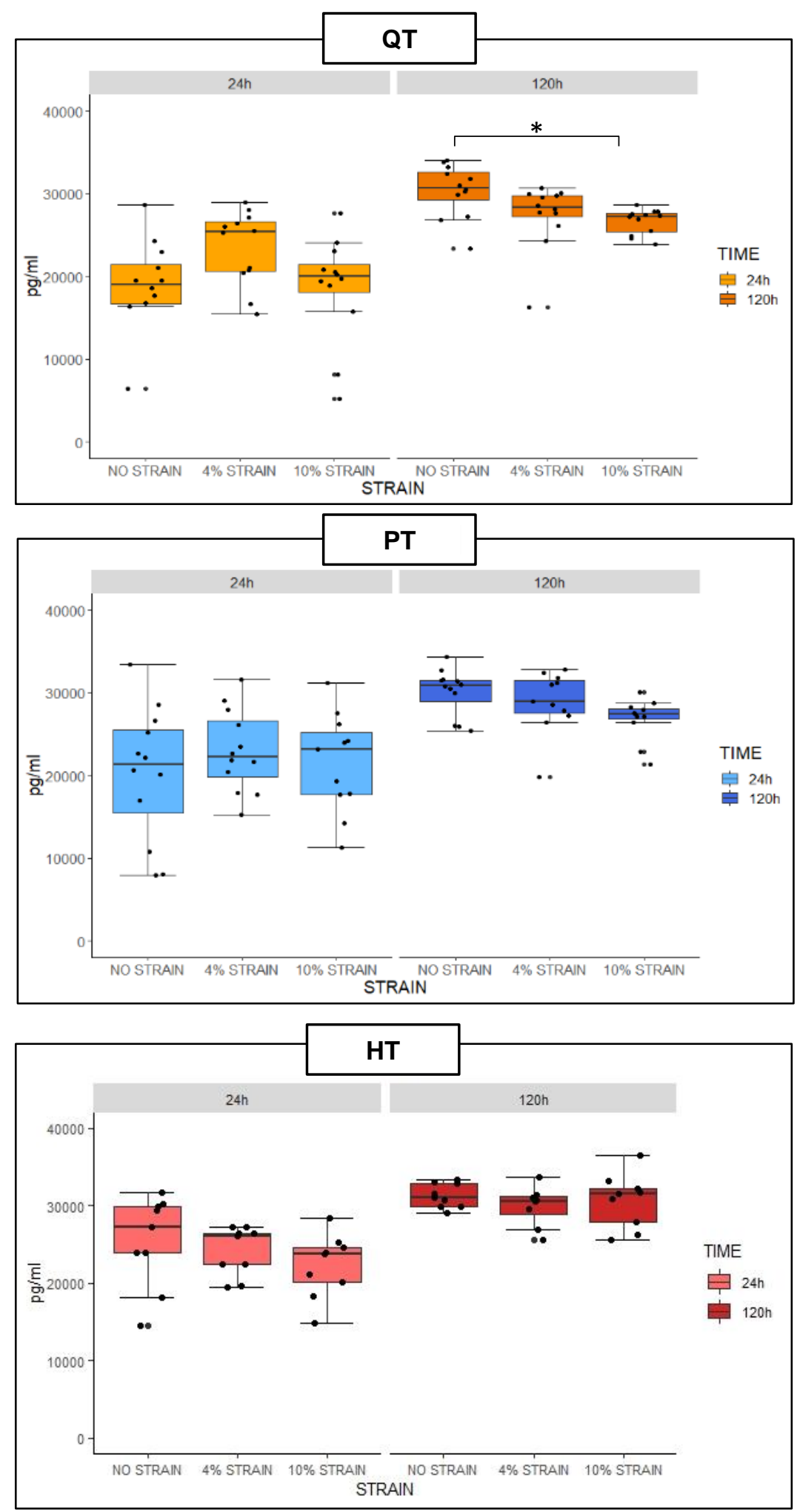

Figure 3.11. Concentration of MMP-2 released to the media. * significant difference between strains at 24 and120 hours. QT: Quadriceps tendon; PT: Patellar tendon, HT: Hamstring tendon 

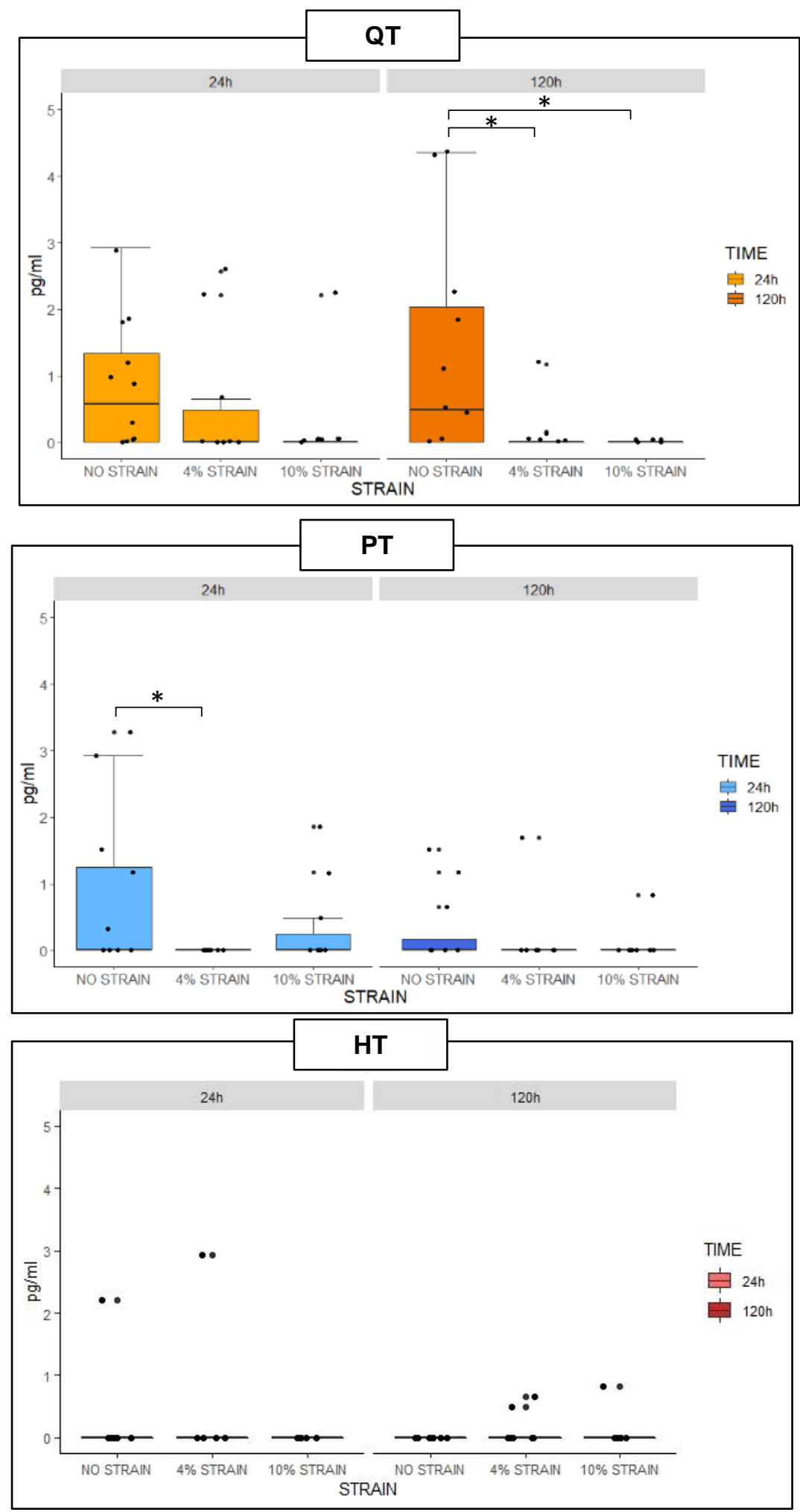

Figure 3.12. Concentration of MMP-3 released to the media. * significant difference between strains at 24 and120 hours. QT: Quadriceps tendon; PT: Patellar tendon, HT: Hamstring tendon 

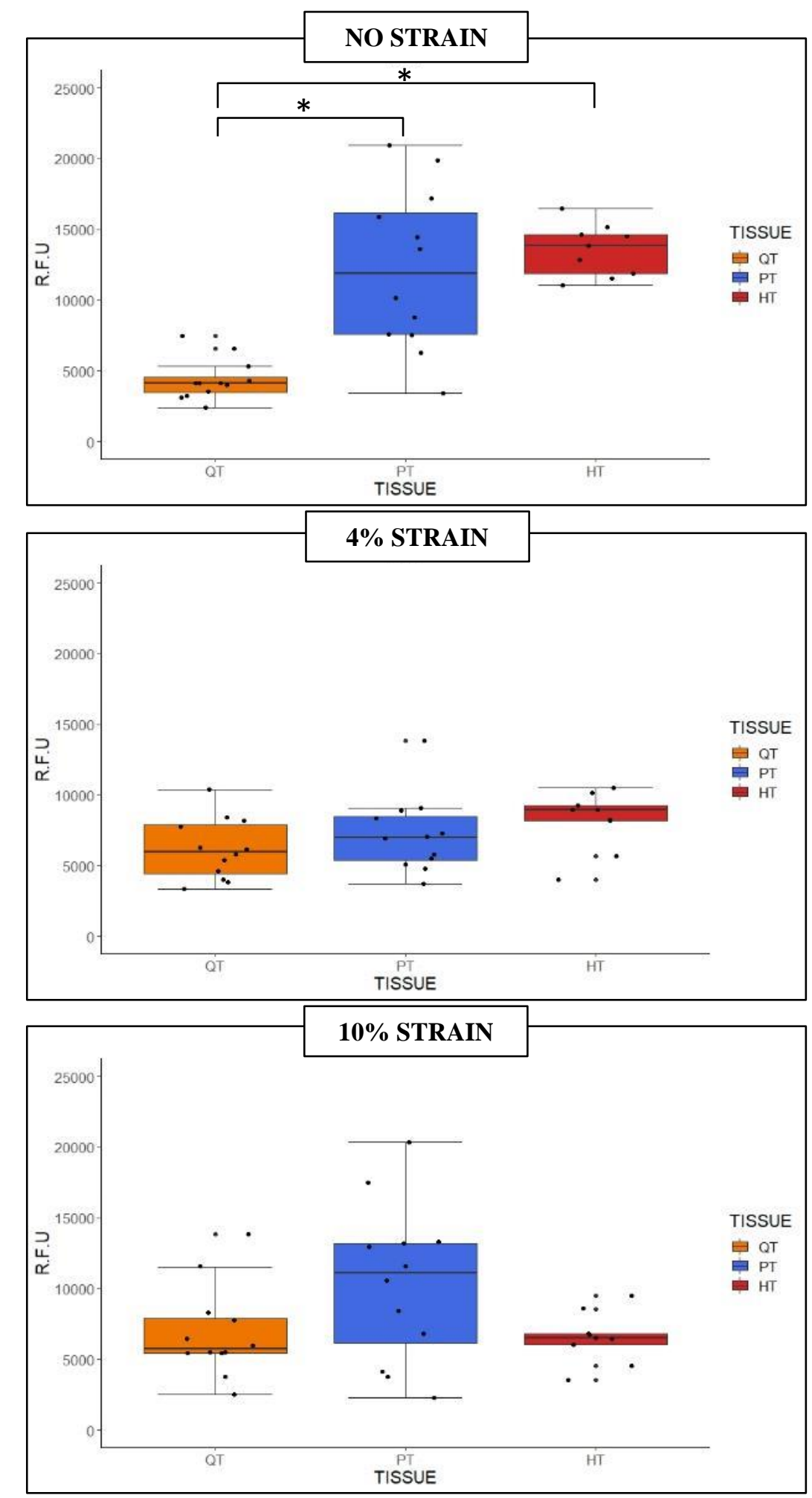

Figure 3.13. Metabolic activity after five days of culture. $\left(^{*}\right)$ significant difference between tissues. RFU: Resazurin Fluorescent Units QT: Quadriceps tendon; PT: Patellar tendon, HT: Hamstring tendon 


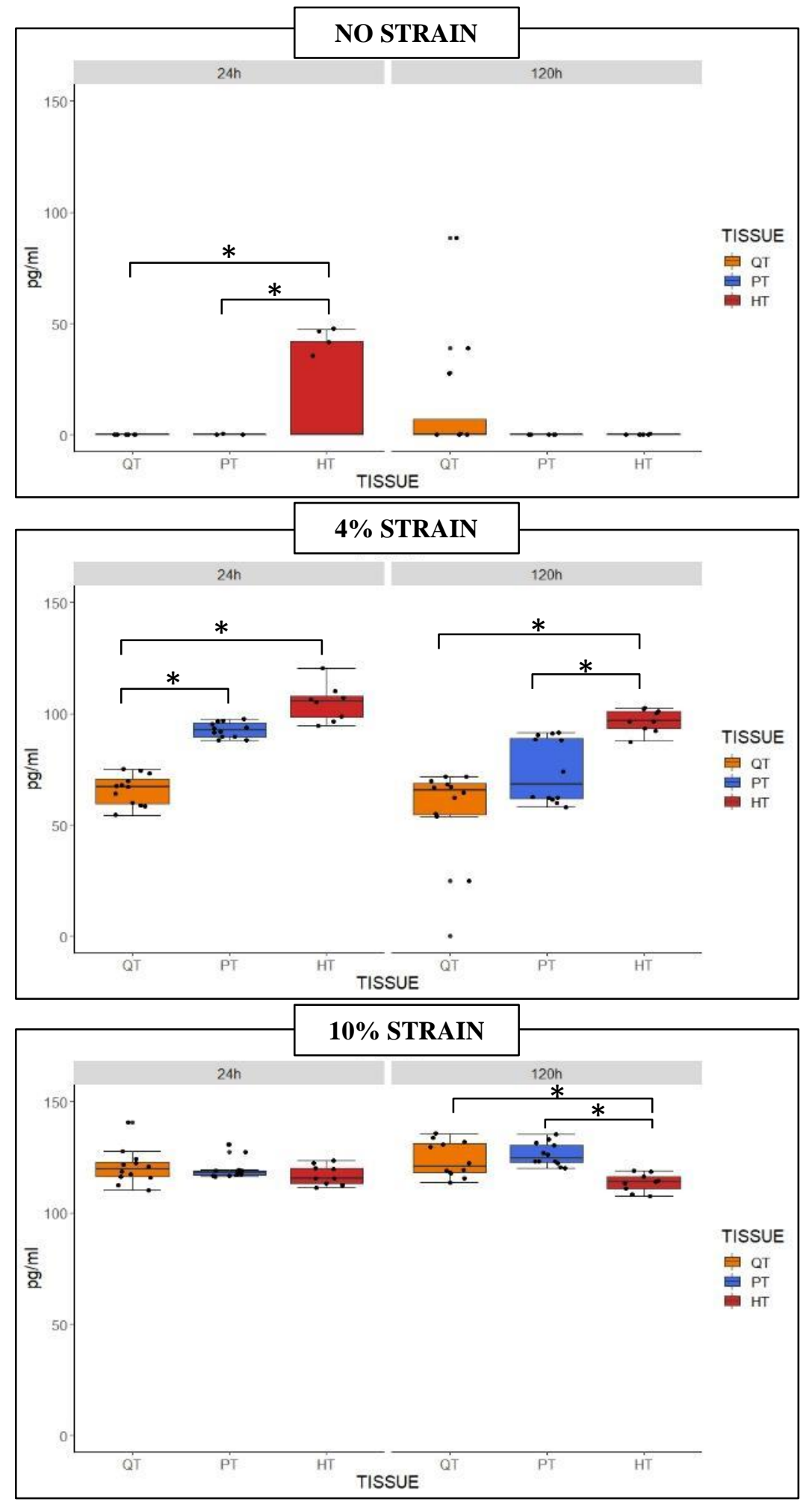

Figure 3.14. Concentration of PGE- 2 released to the media. * significant difference between tissues at 24 and 120 hours. QT: Quadriceps tendon; PT: Patellar tendon, HT: Hamstring tendon 

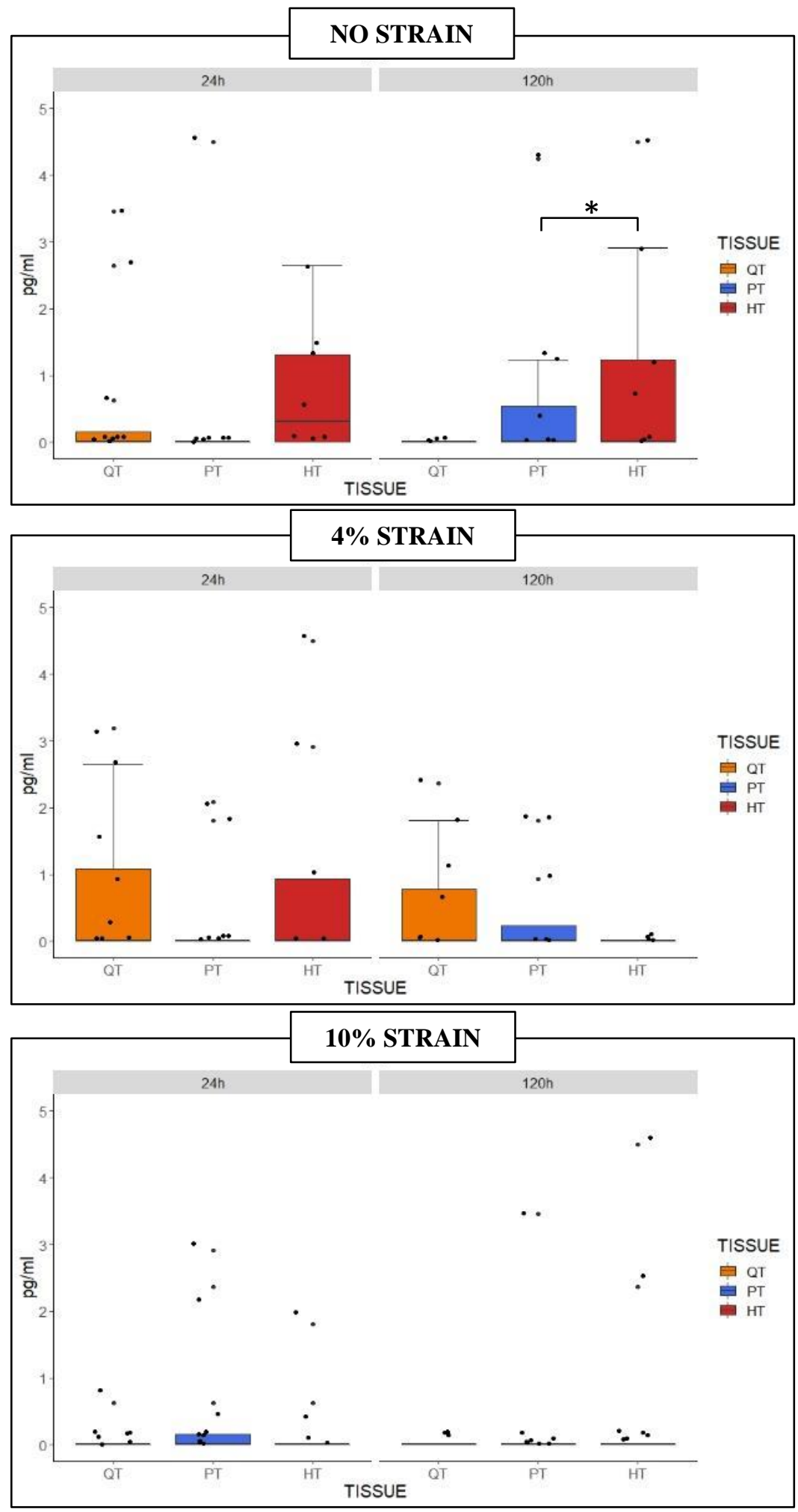

Figure 3.15. Concentration of IL-6 released to the media. * significant difference between tissues at 24 and120 hours. QT: Quadriceps tendon; PT: Patellar tendon, HT: Hamstring tendon 

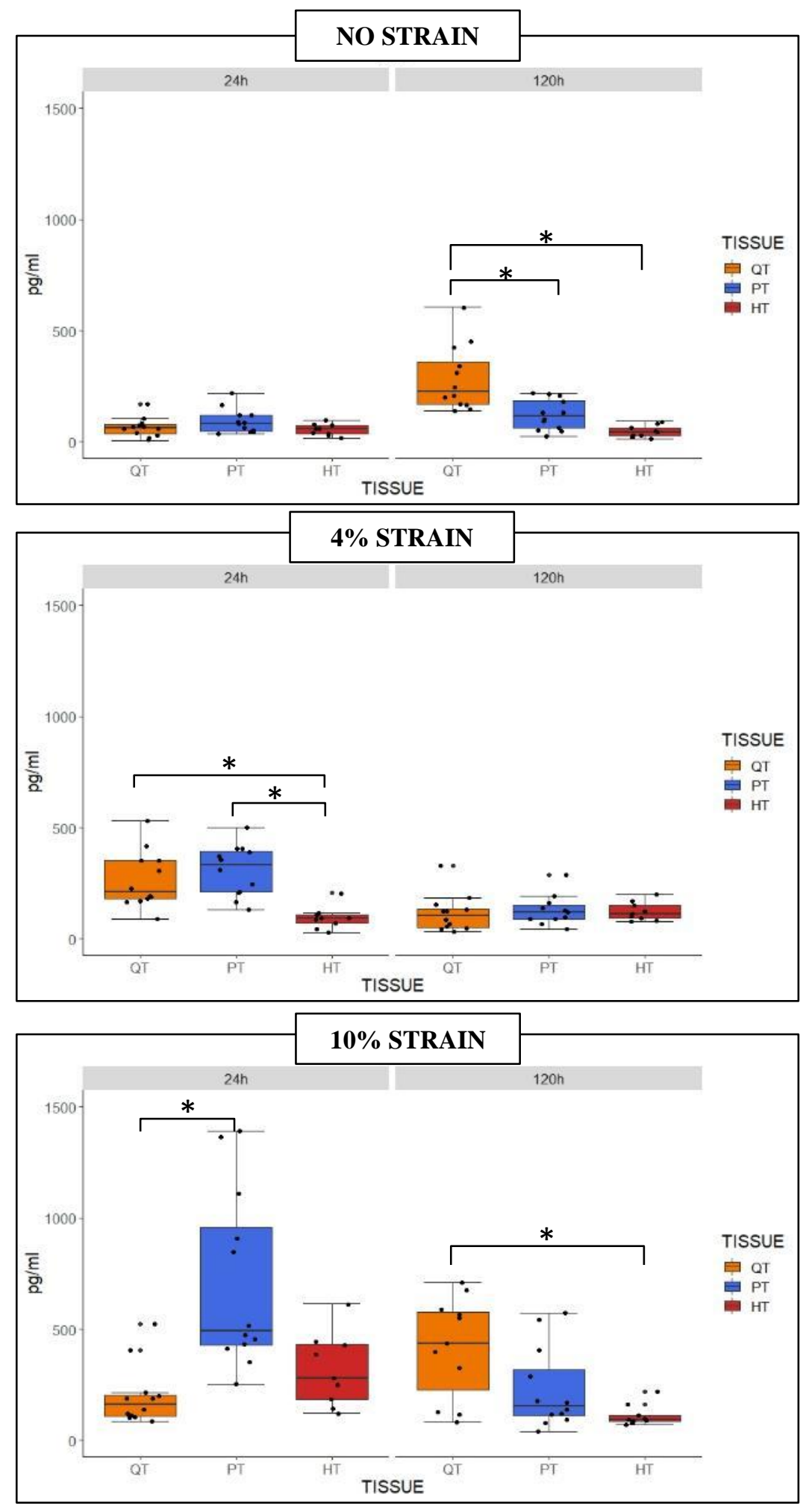

Figure 3.16. Concentration of IL-8 released to the media. * significant difference between tissues at 24 and120 hours. QT: Quadriceps tendon; PT: Patellar tendon, HT: Hamstring tendon 

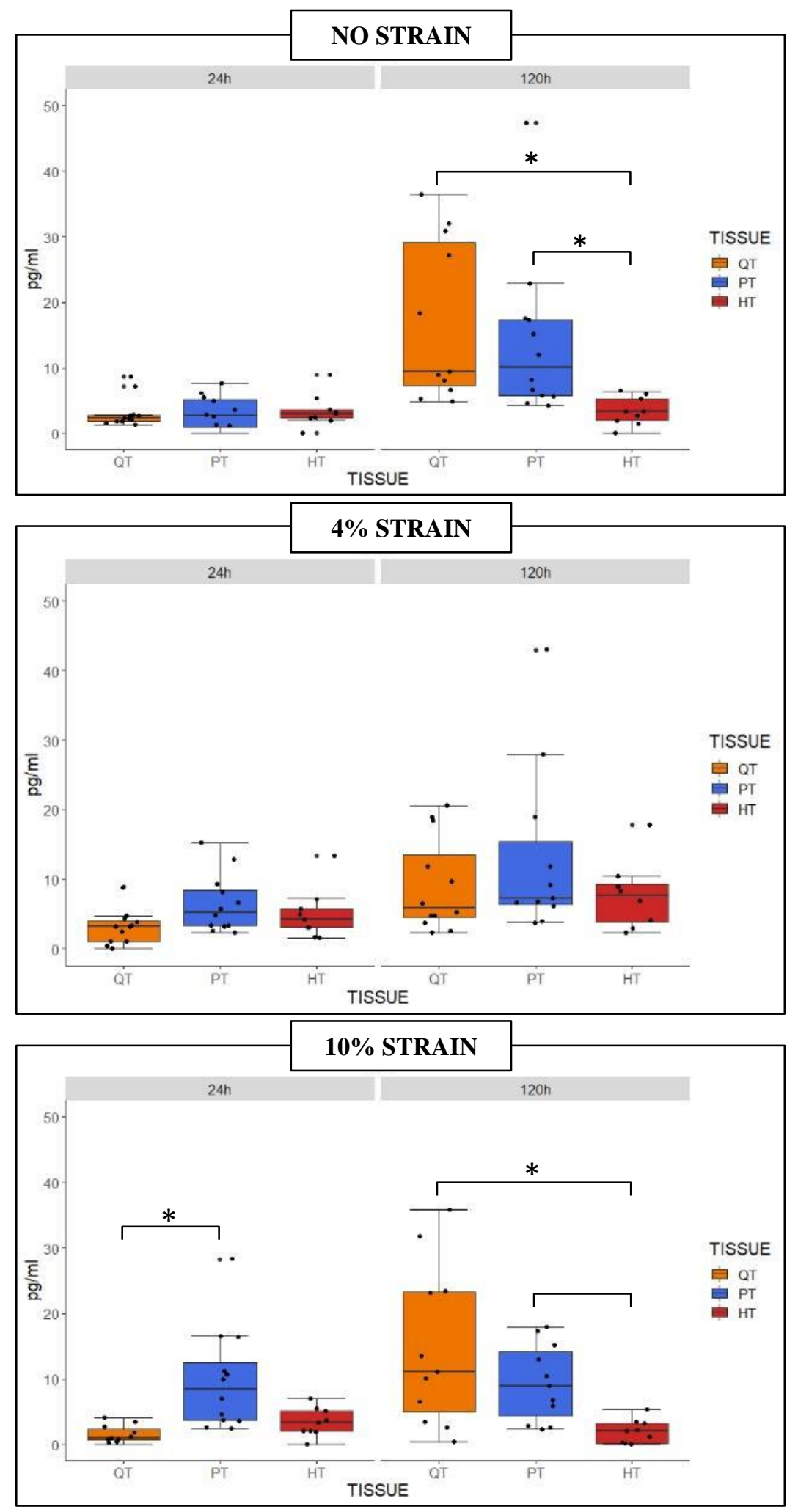

Figure 3.17. Concentration of $\mathrm{KC}$ released to the media. * significant difference between tissues at 24 and120 hours. QT: Quadriceps tendon; PT: Patellar tendon, HT: Hamstring tendon 

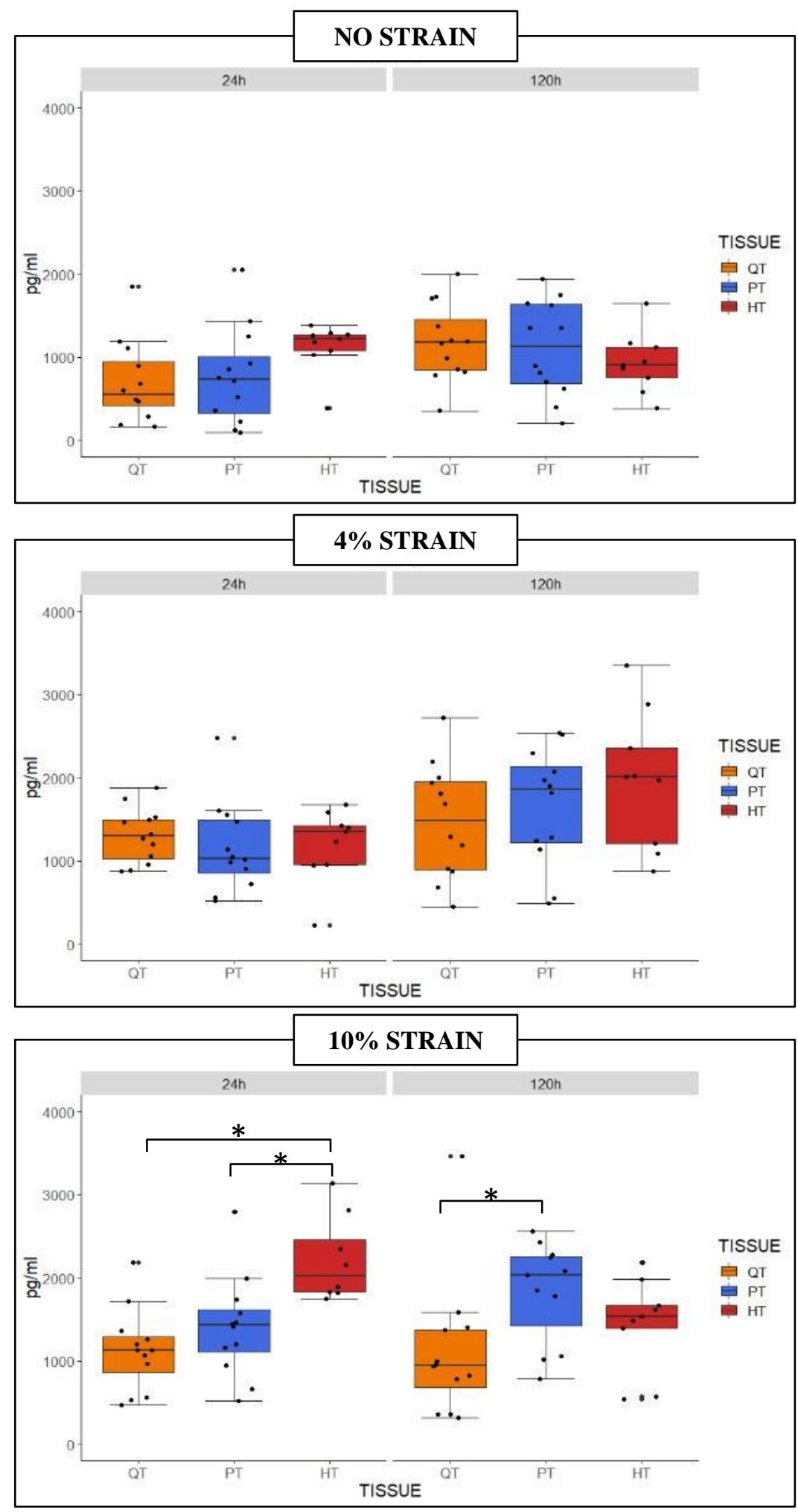

Figure 3.18. Concentration of MCP-1 released to the media. * significant difference between tissues at 24 and120 hours. QT: Quadriceps tendon; PT: Patellar tendon, HT: Hamstring tendon 

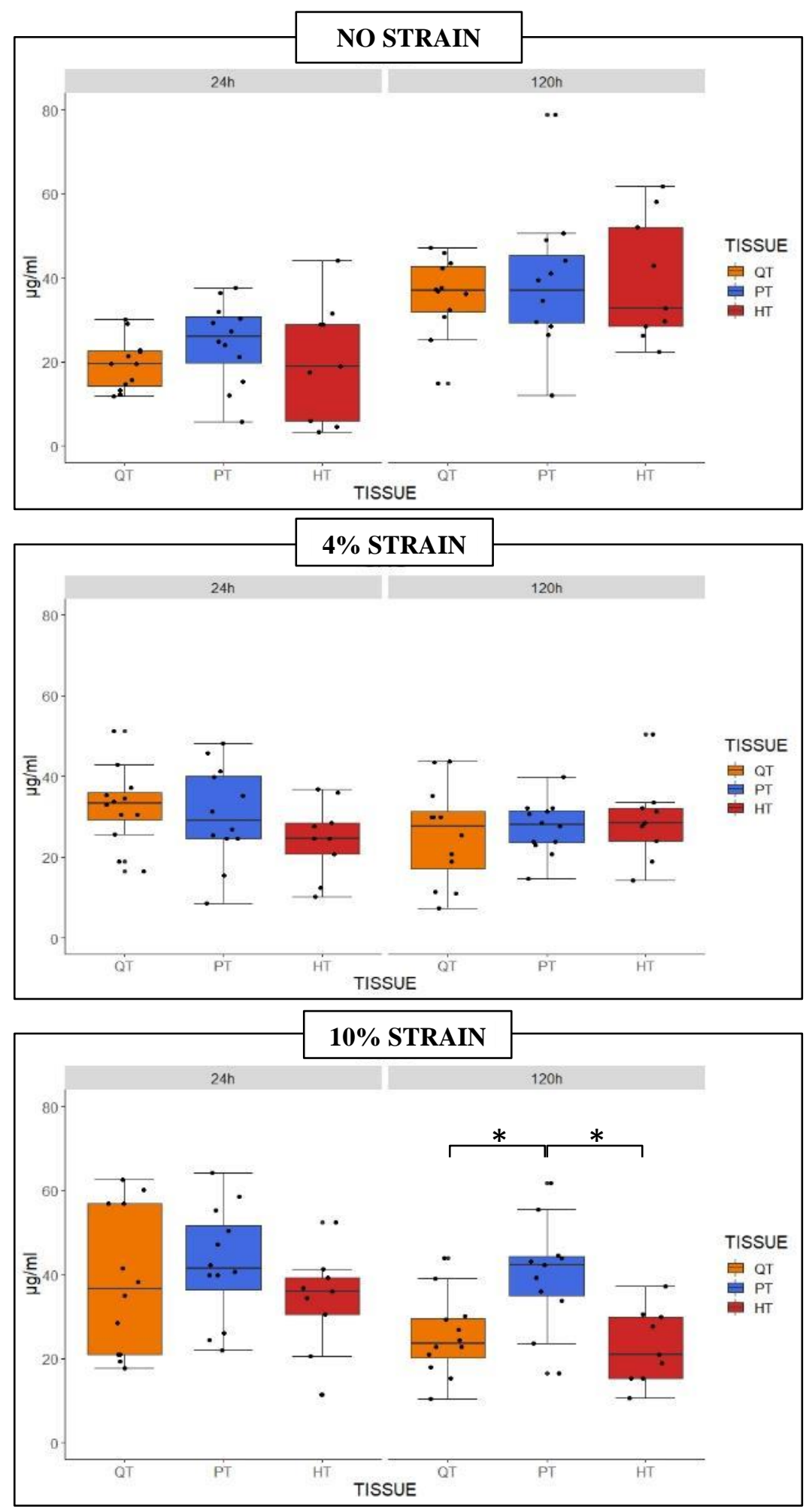

Figure 3.19. Concentration of GAG released to the media. * significant difference between tissues at 24 and120 hours. QT: Quadriceps tendon; PT: Patellar tendon, HT: Hamstring tendon 


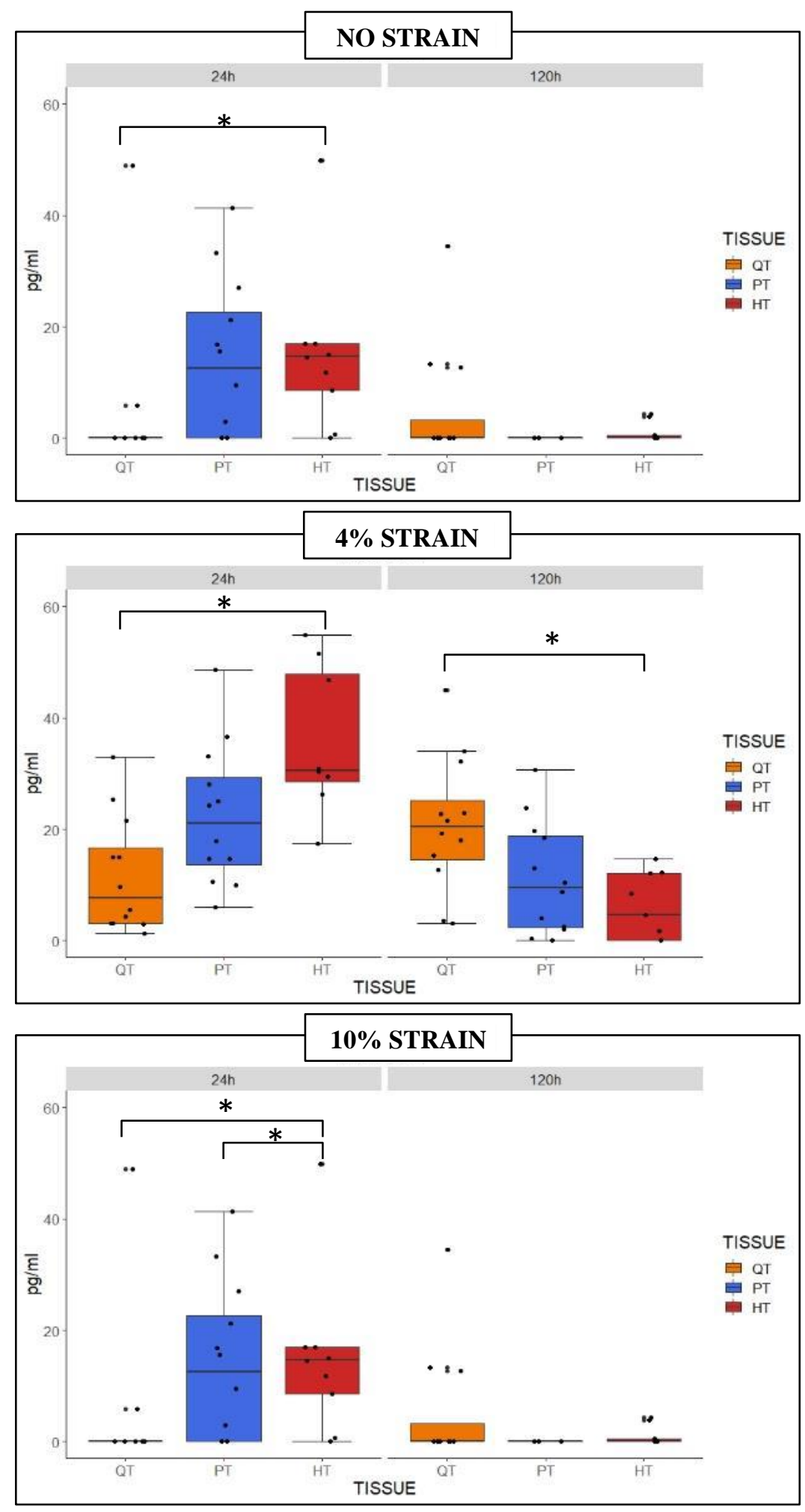

Figure 3.20. Concentration of MMP Activity (MMPACT) in the media. * significant difference between tissues at 24 and120 hours. QT: Quadriceps tendon; PT: Patellar tendon, HT: Hamstring tendon 

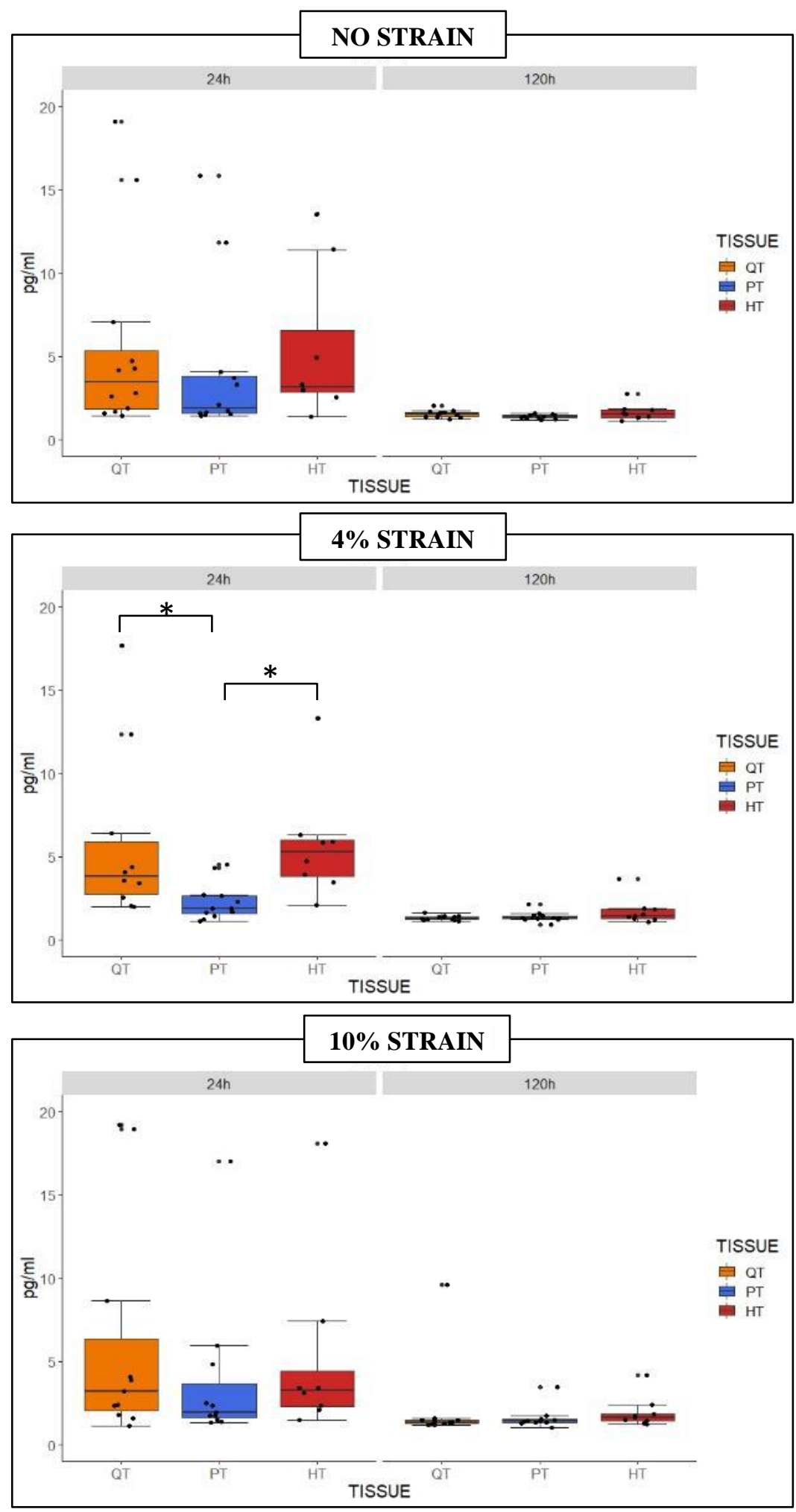

Figure 3.21. Concentration of MMP-1 in the media. * significant difference between tissues at 24 and120 hours. QT: Quadriceps tendon; PT: Patellar tendon, HT: Hamstring tendon 

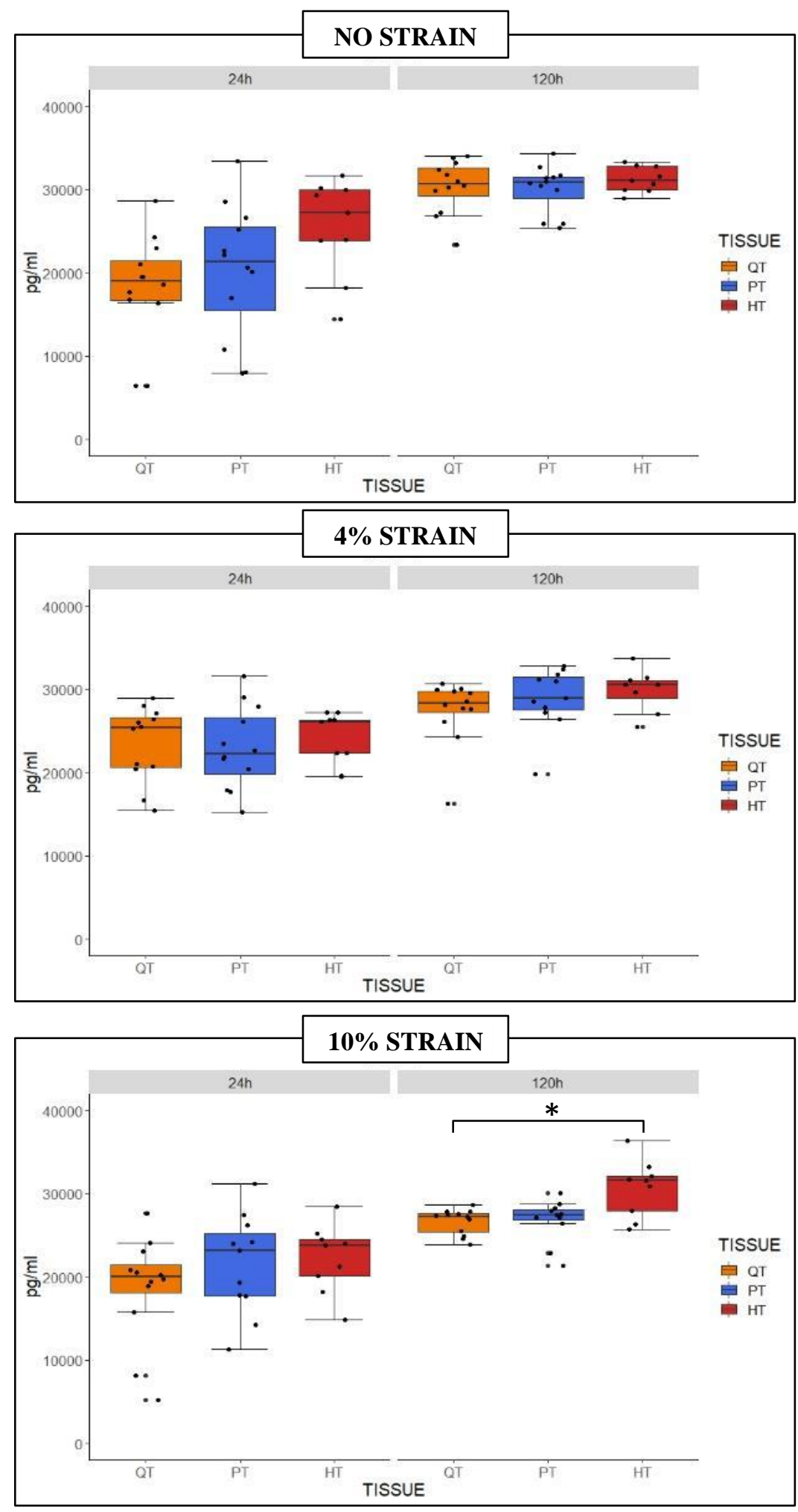

Figure 3.22. Concentration of MMP-2 in the media. * significant difference between tissues at 24 and120 hours. QT: Quadriceps tendon; PT: Patellar tendon, HT: Hamstring tendon 

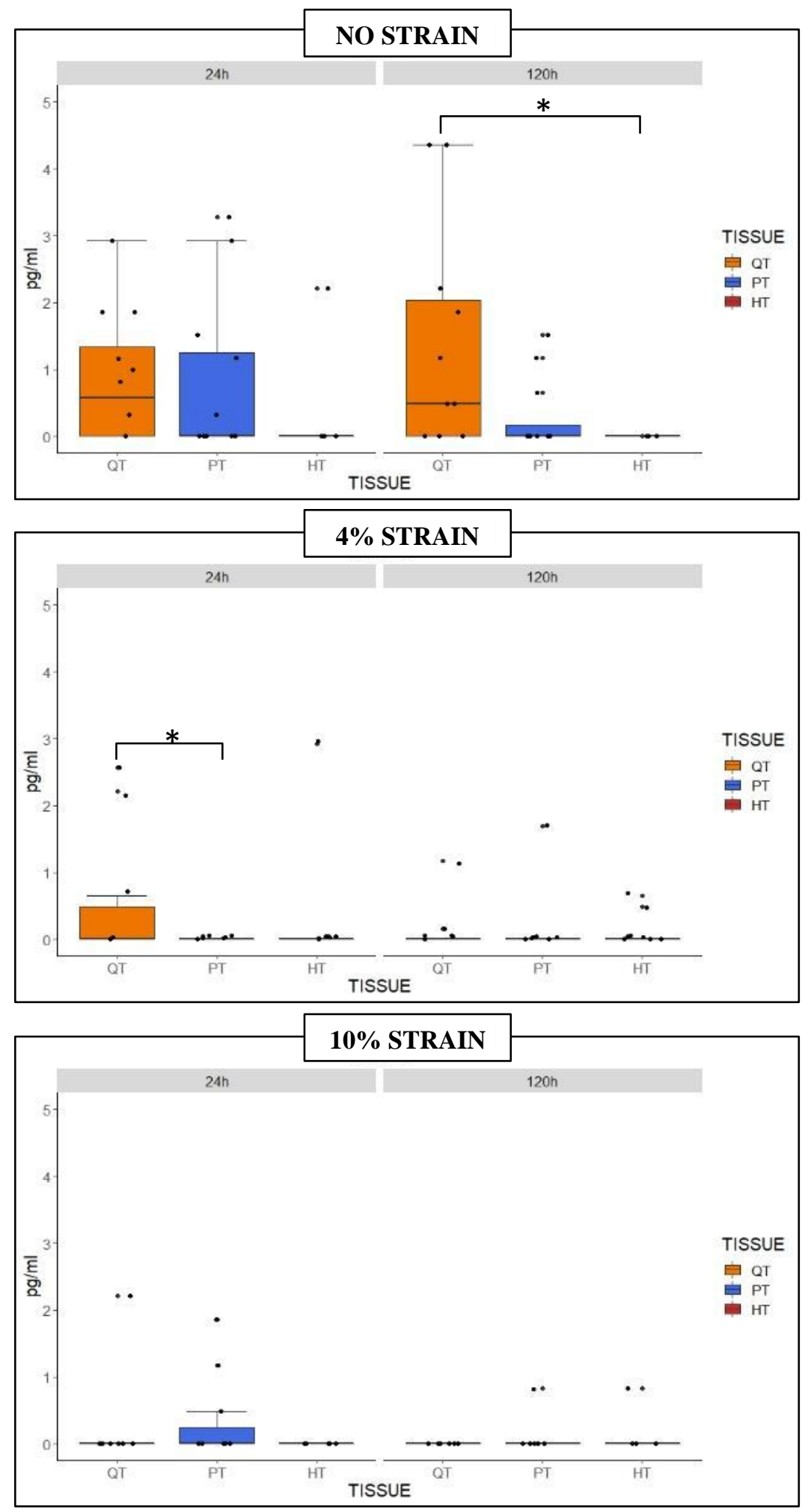

Figure 3.23. Concentration of MMP-3 in the media. * significant difference between tissues at 24 and120 hours. QT: Quadriceps tendon; PT: Patellar tendon, HT: Hamstring tendon 

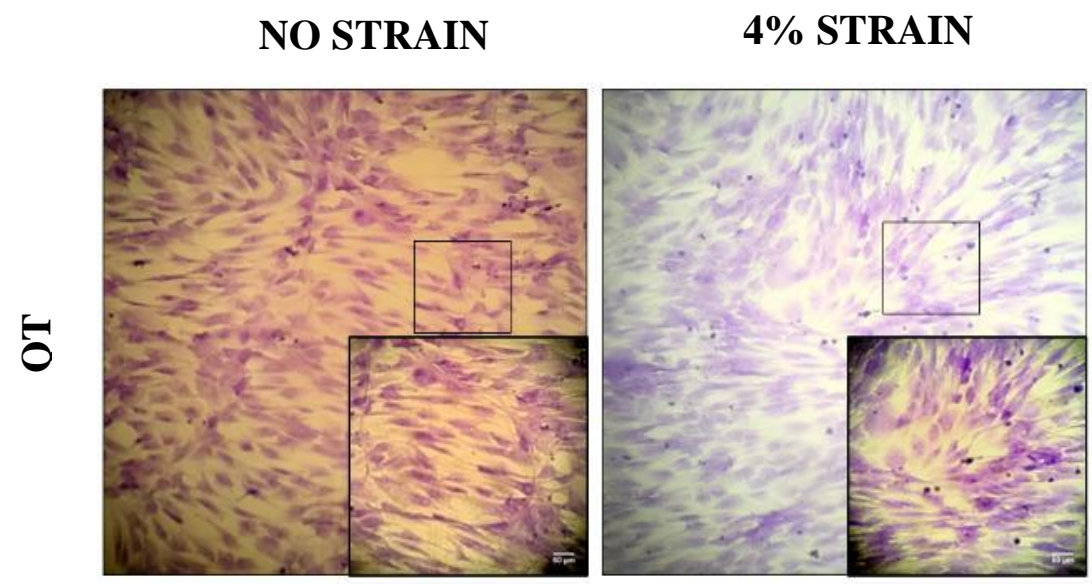

10\% STRAIN

Figure 3.24. Quadriceps tendon (QT) fibroblasts after being stretched for 120 hours.
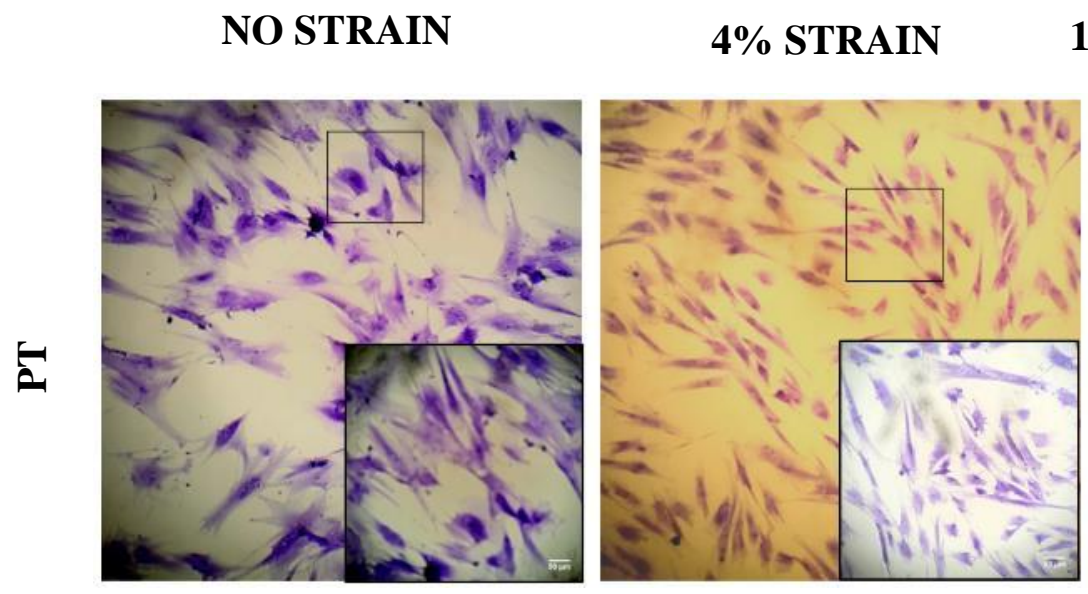

$10 \%$ STRAIN

Figure 3.25. Patellar tendon (PT) fibroblasts after being stretched for 120 hours.
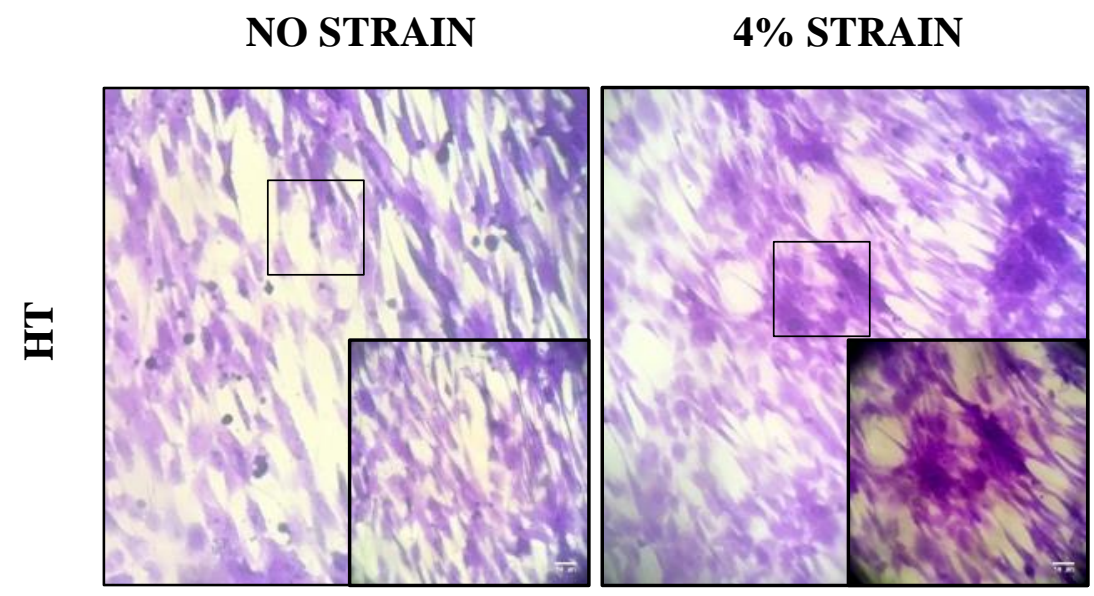

$10 \%$ STRAIN

Figure 3.26. Hamstring tendon (HT) fibroblasts after being stretched for 120 hours. 


\section{CHAPTER 4}

\section{NORMAL CANINE ACL, PCL, SYN CELLULAR RESPONSE TO STRETCH}

\section{Introduction:}

Cranial cruciate ligament (CrCL) deficiency is the most common cause of hindlimb lameness in the dog. The analogous human disorder, anterior cruciate ligament (ACL) deficiency, is the primary cause of knee instability in humans. ${ }^{1 ; 2}$ In both species, extracellular matrix (ECM) remodeling which can be modulated by cellular responses to mechanical stress can influence the overall injury and healing response. ${ }^{3 ; 4}$ Importantly, the joint is a complex organ with various intraarticular tissues playing vital roles in the joint injury response. However, the interplay between various tissue types in the knee are not fully understood.

ACL reconstruction using a tendon graft is the standard-of-care for surgical management of ACL tears in humans. However, morbidity associated with ACL reconstructive surgery include increased risk of failure in certain patients and the inability to consistently mitigate the onset of post-traumatic osteoarthritis (PTOA). ${ }^{5 ; 6}$ There is also an ongoing debate if a reconstructed ACL recapitulates all of the properties of a native ACL including its proprioceptive or reflex responses. ${ }^{7}$ Therefore, a renewed interest in primary ACL repair has developed. While attractive, an unreliable healing response has been associated with primary ACL repair. This has been attributed to several factors, including a hostile intrasynovial environment, specific post-inflammatory responses, and intrinsic cell deficiencies. ${ }^{8 ; 9}$. Therefore, understanding the early healing phase characterized by cellular release of molecules that regulate adequate balance between ECM degradation and 
proliferation may be crucial for case selection in ACL repair and to optimize the rehabilitation protocols after surgery.

Experiments performed in cells from tissues subjected to mechanical stresses have revealed that cell structure and tissue organization can respond to varying amounts of forces including tension, compression and shear stress. Common methods for membrane deformation include vacuum, suction, indentation or pulling. ${ }^{10}$ The Flexercell ${ }^{\circledR}$ System has been developed to stress cell cultures in monoculture and promote morphological and biochemical changes. Hence, this tension system allows the measurement of cellular responses to mechanical stress, including ECM interactions.

The Flexercell system has been widely used in ACL research. ${ }^{11-15}$ Due to the multiscale structure of ligaments and tendons, biomechanical research at each hierarchical scale should be conducted in order to gain a broad understanding on tendon/ligament pathology and healing. Authors have investigated this phenomenon in both tendons and ligaments. ${ }^{16-}$ ${ }^{19}$ However, few studies have included the effect of mechanical stress in ACL repair. ${ }^{12 ; 20-}$ 22

The objective of this work was to compare the pro-inflammatory and remodeling responses of fibroblasts obtained from canine intraarticular tissues of the stifle joint that may be involved in primary ACL repair (ACL, synovium (SYN), posterior cruciate ligament (PCL) when subjected to varying levels of mechanical strain forces. We hypothesized that fibroblasts from these tissues would produce significantly different levels of inflammatory and remodeling biomarkers in response to a spectrum of cyclic strains in vitro.

\section{Materials and methods:}

Tissue processing and culture 
With ACUC approval (ACUC\#9163, 9164), tissues (9 ACL, 12 PCL and 12 SYN) were harvested from skeletally mature female $\operatorname{dogs}(n=15)$ euthanatized for studies unrelated to the current work. Tissues were minced into $0.5-1 \mathrm{~cm}^{2}$ pieces and digested in $0.5 \%$ Type 1A Clostridium histolyticum collagenase solution (Sigma-Aldrich, St. Louis, MO, USA). The aliquots were then suspended with nutritional media containing $10 \%$ FBS and cultured at $5 \% \mathrm{CO}_{2}, 37^{\circ} \mathrm{C}$, and $95 \%$ humidity. Once confluent, the cells were resuspended and seeded in Collagen Type I-coated BioFlex ${ }^{\circledR}$ plates $\left(1 \times 10^{5}\right.$ cells/well $)$. Cells were incubated for an additional $48 \mathrm{~h}$ before strain was applied. Then, nutritional culture media was replaced with $0.5 \%$ FBS media for the remaining five days of culture. Fibroblasts were subjected to continuous mechanical stimulation (2-s strain and 10-s relaxation at a $0.5 \mathrm{~Hz}$ frequency) with a biaxial sinusoidal waveform with three different elongation strains (mechanical stress deprivation-0\%, physiologic strain-4\%, and high strain-10\%) ${ }^{23}$ for 5 days (120h) using the Flexcell FX-4000T strain system (Flexcell International, NC, USA) (Fig. 4.1). Media was changed every $24 \mathrm{~h}$ and stored at $-20^{\circ} \mathrm{C}$ until used for biomarker analysis.

\section{Biomarker assays}

Media was changed every $24 \mathrm{~h}$ and stored at $-20^{\circ} \mathrm{C}$ until analysis. Media from 24 and 120 hours of culture were assessed for various biomarkers. Proteoglycan (GAG) was assessed using the DMMB assay as previously described. ${ }^{24}$ The concentration of prostaglandin $\mathrm{E}_{2}$ (PGE2) (Cayman Chemical, Ann Arbor, MI, USA), cytokines (IL-6, IL-8, KC and MCP1) (Millipore, Billerica, MA, USA), total matrix metalloproteinase (MMP) activity (SensoLyte 520 generic MMP assay, Anaspec, Inc., Fremont, CA), and MMP production (MMP-1, MMP-2 and MMP-3) (R\&D Systems, Minneapolis, MN, USA), were all 
assessed using commercially available assays according to the manufacturer's protocol. At the end of the strain protocol (120h), cell viability analysis was performed using the resazurin assay (Sigma Aldrich, Saint Louis, MO).

\section{Statistical Analysis}

For statistical analysis, normal distribution was assessed by Shapiro-Wilk test. Because data were not normally distributed, non-parametric analyses were used. Significance levels were set at $\mathrm{p}<0.05$. Results were reported as median \pm interquartile range (IQR). Comparisons among strains and tissue types were performed using Kruskal-Wallis test and Dunn's test posthoc (R Core Team (2019), R version 3.6.2 Vienna, Austria).

\section{Results}

Metabolic activity was significantly affected by strain magnitude in all intraarticular fibroblasts evaluated. SYN fibroblasts exhibited a significant decrease in metabolic activity at supraphysiological strain (10\%) compared to mechanical strain deprivation (no strain). Moreover, in ACL derived fibroblasts, physiological strain (4\%) elicited an increase in metabolic activity compared to the no strain group. Interestingly, in PCL fibroblasts, no strain produced a significant increase in metabolic activity compared to $4 \%$ strain and $10 \%$ strain (Fig.4.2). Additionally, comparisons among tissue types showed that at no strain, fibroblasts derived from the PCL had a significantly higher metabolic activity compared to SYN and ACL fibroblasts. Interestingly, physiological strain (4\%) did not elicit a differential metabolic activity among the different cell types. Moreover, supraphysiological strain (10\%) produced an increased metabolic activity from PCL compared to ACL fibroblasts (Fig. 4.13). 
Inflammatory responses were also significantly affected by both strain and tissue type. At 24h, PGE2 production was significantly increased in SYN and ACL fibroblasts subjected to supraphysiological strain compared to the no strain and $4 \%$ strain groups at $24 \mathrm{~h}$. Further, the production of PGE2 by ACL fibroblasts was significantly higher in the $10 \%$ strain group compared to the no strain and 4\% strain groups at 120h. Moreover, PGE2 production was significantly increased in PCL fibroblasts at supraphysiological strain compared to 4\% strain (Fig 4.3).

Tissue type differences showed that at no strain, PCL fibroblasts tended to exhibit a significantly increased production of PGE2 compared to both SYN and ACL at 24h. Also, at $120 \mathrm{~h}$, stress deprivation elicited a significantly decreased production of PGE2 in ACL relative to SYN and PCL fibroblasts. Moreover, at $24 \mathrm{~h}$ of physiological strain, SYN fibroblasts showed a significantly decreased production of PGE2 relative to ACL and PCL but no significant differences at $120 \mathrm{~h}$. Additionally, at $24 \mathrm{~h}$ of supraphysiological strain, PCL showed a significant decrease in PGE2 production compared to SYN and ACL and no difference among tissue types at 120h (Fig. 4.14).

The production of IL-6 by all fibroblasts was significantly higher at physiological strain compared to supraphysiological strain at $24 \mathrm{~h}$ of culture. SYN fibroblasts significantly increased IL-6 production at no strain and physiological strain compared to $10 \%$ strain at 24h. Similarly, ACL production of IL-6 was also higher at $4 \%$ strain compared to $10 \%$ strain at $24 \mathrm{~h}$. Moreover, PCL production of IL-6 was significantly higher at $4 \%$ strain compared to $10 \%$ strain at $24 \mathrm{~h}$ and at no strain compared to $4 \%$ strain at $120 \mathrm{~h}$ (Fig.4.4). Additionally, PCL fibroblasts exhibited a consistent increase in IL-6 compared to ACL fibroblasts at $24 \mathrm{~h}$ and $120 \mathrm{~h}$ of mechanical strain deprivation. Furthermore, physiological 
strain was not significantly different among tissues at $24 \mathrm{~h}$ but changed after $120 \mathrm{~h}$ with ACL producing significantly lower levels of IL-6 compared to both SYN and PCL. Moreover, at 10\% strain, PCL fibroblasts showed a significant increase in IL-6 production relative to SYN and ACL fibroblasts at $24 \mathrm{~h}$ and $120 \mathrm{~h}$ (Fig.4.15).

The production of IL-8 was significantly increased in SYN fibroblasts at both physiological and supraphysiological strains compared to no strain at $24 \mathrm{~h}$ (Fig. 4.5). Moreover, ACL fibroblasts exhibited significantly higher levels of IL-8 at both, physiological and supraphysiological strain compared to no strain at 24h. PCL fibroblasts showed increased levels of IL-8 at $4 \%$ strain relative to both, no strain and $10 \%$ strain, which in turn was significantly higher than no strain at $24 \mathrm{~h}$. However, at $120 \mathrm{~h}$, mechanical strain deprivation elicited a significant increase of IL-8 from PCL fibroblasts when stress deprived compared to $4 \%$ strain.

Tissue differences showed that at $120 \mathrm{~h}$ of mechanical strain deprivation, SYN and PCL fibroblasts produced significantly more IL-8 than ACL fibroblasts. Moreover, at 4\% strain, PCL fibroblasts produced significantly more IL-8 than SYN and ACL fibroblasts at 24h. Similarly, after $120 \mathrm{~h}$, production of IL-8 from ACL fibroblasts was significantly lower than SYN and PCL fibroblasts. Also, at 10\% strain, ACL fibroblasts produced significantly more IL-8 than SYN fibroblasts at $24 \mathrm{~h}$. However, at $120 \mathrm{~h}$, SYN and PCL exhibited a significantly greater production of IL-8 compared to ACL fibroblasts (Fig. 4.16).

Production of the chemokine KC significantly increased in ACL and PCL fibroblasts at $24 \mathrm{~h}$ of physiological strain compared to no strain and supraphysiological strain. Moreover, no differences were seen in SYN at any strain or timepoint (Fig.4.6). 
Tissue differences showed that at no strain, KC production significantly increased in PCL fibroblasts compared to ACL fibroblasts at $120 \mathrm{~h}$. Moreover, at $4 \%$ strain, $\mathrm{KC}$ production was significantly higher in PCL fibroblasts relative to SYN and ACL fibroblasts at 24h. Further, at $10 \%$ strain, ACL production of KC was significantly lower than both SYN and PCL fibroblasts at 120h (Fig. 4.18).

The chemokine MCP-1 was not significantly different in SYN fibroblasts at any strain or timepoint. However, in ACL fibroblasts, MCP-1 production was significantly increased at $4 \%$ strain relative to no strain at $24 \mathrm{~h}$. Similarly, PCL fibroblasts exhibited a significant increase in MCP-1 production after 24h of physiological strain compared to no strain and supraphysiological strain (Fig. 4.7).

Tissue source showed a significant increase in MCP-1 in PCL compared to ACL fibroblasts after $120 \mathrm{~h}$ of mechanical strain deprivation. Moreover, at $4 \%$ strain, PCL fibroblasts produced significantly more MCP-1 than SYN and ACL fibroblasts at 24h. Furthermore, at $10 \%$ strain, ACL produced significantly less MCP-1 than both SYN and PCL fibroblasts at $120 \mathrm{~h}$ (Fig. 4.18).

Increased production of GAG was observed in SYN and PCL fibroblasts at 24h with escalating strain magnitudes. No strain produced significantly less GAG than $4 \%$ and $10 \%$ strain at $24 \mathrm{~h}$. However, at $120 \mathrm{~h}, 10 \%$ strain produced significantly less GAG than no strain and 4\% strain in SYN fibroblasts. ACL fibroblasts exhibited significantly more GAG at no strain compared to $4 \%$ strain and $10 \%$ strain at $120 \mathrm{~h}$. Moreover, PCL fibroblasts showed significantly less GAG at no strain compared to $4 \%$ strain and $10 \%$ strain at $24 \mathrm{~h}$ (Fig. 4.8). Tissue source comparisons showed that the production of GAG by ACL fibroblasts was significantly lower than the production by the SYN and PCL fibroblasts at no strain and 
$4 \%$ strain at $120 \mathrm{~h}$. However, at $10 \%$ strain, GAG production was significantly higher in PCL fibroblasts compared to SYN and ACL after 120h of culture (Fig. 4.19).

The level of total MMPACT was not significantly different in SYN fibroblasts at any strain or timepoint. However, ACL fibroblasts exhibited significantly higher concentration of MMPACT at physiological strain compared to both, stress deprived and supraphysiological strain groups at $24 \mathrm{~h}$ and $120 \mathrm{~h}$. Moreover, MMPACT increased in PCL fibroblasts at supraphysiological strain relative to $4 \%$ strain at $24 \mathrm{~h}$. Conversely, $4 \%$ strain showed significantly decreased levels of MMPACT after $120 \mathrm{~h}$ of supraphysiological strain compared to no strain at $120 \mathrm{~h}$ (Fig. 4.9).

Cell type comparisons indicated that at mechanical strain deprivation, PCL fibroblasts produced significantly increased levels of MMPACT compared to SYN and ACL fibroblasts at $24 \mathrm{~h}$ and $120 \mathrm{~h}$. interestingly, physiological strain did not produce considerable MMPACT at any timepoint in any of the fibroblast types. Moreover, at $10 \%$ strain, MMPACT production was significantly higher in PCL fibroblasts compared to both SYN and ACL fibroblasts at 24h and 120h (Fig. 4.20).

The production of MMP-1, MMP-2 and MMP-3 by SYN fibroblasts was significantly higher at physiological levels compared to supraphysiological strain at 24h (Fig. 4.10-4.11 and 4.12). The production of MMP-2 by ACL fibroblasts was significantly higher at physiological strain levels compared to supraphysiological strain levels at $120 \mathrm{~h}$. There was not a significant difference between strain groups for the production of MMPs by PCL fibroblasts at both timepoints. Interestingly, PCL exhibited a consistent increase in MMP1 and MMP-3 when compared to ACL fibroblasts regardless of the strain at $24 \mathrm{~h}$ and $120 \mathrm{~h}$ (Fig. 4.21 and 4.23). Moreover, mechanical strain deprivation elicited a significant increase 
in MMP-2 production compared to SYN and PCL fibroblasts at 120h. Similarly, at 4\% strain, MMP-2 production was significantly higher in ACL compared to PCL fibroblasts at $120 \mathrm{~h}$. Moreover, at $10 \%$ strain, MMP-2 production was significantly lower in SYN compared to ACL and PCL fibroblasts (Fig. 4.22).

\section{Discussion}

In this study, we demonstrated that intraarticular fibroblasts from the ACL, PCL, and synovium produce significantly different levels of inflammatory and remodeling biomarkers in response to a spectrum of cyclic strains in vitro. This work supported the hypothesis that fibroblasts from intraarticular tissues involved in ACL injury and repair produce significantly different levels of inflammatory and remodeling biomarkers in response to cyclic strains in vitro.

Fibroblasts are found across all connective tissues including skin, tendons and ligaments and are the primary source of ECM components including collagens, proteoglycans and growth factors. ${ }^{25}$ Studies have shown that fibroblasts from the anterior cruciate ligament, posterior cruciate ligament, and synovium are sensitive to mechanical strain variations. ${ }^{26-}$

${ }^{30}$ Moreover, abnormal loads applied to the tissue can contribute to the development and progression of osteoporosis, osteoarthritis and tendinopathy. ${ }^{31}$

In vivo, the PCL is a primary restraint to posterior tibial translation and internal rotation ${ }^{32}$. Some authors have reported the increase in the corresponding in situ ligament force when sectioning either the ACL or the PCL. ${ }^{33 ; 34}$ In this work we believe that either, ACL or PCL injury will lead to excessive forces in the adjacent ligament. We assume that this force would be higher than 5\% strain in cases of hyperextension, which is supraphysiological for any ligament and will lead to further cartilage degradation and a hostile environment. ${ }^{35}$ 
Additionally, the alteration of the structural properties such as ligament stiffness or initial tautness of the cruciate ligaments could modify the mechanical role of both cruciate ligaments. ${ }^{35}$ Moglo \& Shirazi-Adl (2005) showed that the mechanical contribution of a cruciate ligament was strongly dependent on the force applied in the other ligament. ${ }^{35}$ The authors suggest that alterations in ligament stiffness after ligament reconstruction surgery would alter the mechanical role not only of the treated ligament but the untreated one as well. ${ }^{35}$ Moreover, these same effects could stimulate a different metabolic response due to the variation in mechanical loads in other intraarticular structures such as the meniscus. ${ }^{36}$ In addition to the intraarticular ligaments, the synovial membrane also plays a key role in delivering nutrition and blood to both, the PCL and the ACL. The synovium also clears intra-articular debris from the joint and modulates the inflammatory response of the joint. ${ }^{37}$; ${ }^{38}$ After cruciate ligament injury there is an increase in the concentration of cytokines and growth factors in the synovial fluid, which may originate from the synovium. ${ }^{37}$ Tang et al., (2009) showed an increased production of MMP-2 in synovium explants relative to intraarticular tissues including ACL and PCL. ${ }^{37}$ Our findings demonstrate that in response to increased strain levels, the synovium produces significantly higher levels of IL-6 and IL-8 compared to the cruciate ligament fibroblasts during culture.

The poor healing ability of the ACL has been attributed to specific cellular cues and biochemical composition of the intrasynovial environment that may impair the initiation of the healing cascade. ${ }^{8 ;} 39-42$ Authors have demonstrated that a fine balance between understimulation and overloading must be met for effective ACL healing. ${ }^{43-45}$ Certain conditions must be met in order to elicit an optimal primary healing response. Close continuity of the torn ends of the ligament and a controlled functional motion may enhance 
the healing process. ${ }^{46}$ These conditions are rarely met in ACL tears to permit in situ healing without surgical intervention. Therefore, for ACL repair a reapproximation of the two ends of the ruptured ligaments has been attempted. ${ }^{47}$ However, this technique has largely failed when using suture alone. ${ }^{48}$ One possible cause for the failure of the repair is the inability of the tissue to maintain a bridging clot after repair. It is possible that the hematoma that develops is removed by enzymes present in the synovial fluid, which impairs the ability of the hematoma to deliver of growth factors that assist in wound healing and ligament repair. $49-51$

Murray et al., (2013) investigated the use of a bioactive scaffold containing a collagenplatelet composite to enhance suture repair of the torn ACL in an animal model. ${ }^{52 ;} 53$ Augmented repair showed promising results and led to similar outcomes when compared to ACL reconstruction. ${ }^{54}$ Additionally, the authors have shown that a six-week delay on ACL bioenhanced repair has a negative effect on functional outcomes on the porcine ACL. ${ }^{55}$ Similarly, authors also showed that sex plays a critical role on the biomechanical outcomes of bridge-enhanced ACL repair in this model. ${ }^{56}$ Consequently, the promising findings from the Murray et al's Bridge-Enhanced Anterior Cruciate Ligament Repair (BEAR) technique have led to the translation of this methodology into encouraging human clinical trials. ${ }^{57}$ Given the ability of this technique to salvage an individual's native ACL tissue, primary ACL repair may circumvent some of the disadvantages of ACL reconstruction including PTOA, the associated morbidity with tissue recovery from harvest when using an autograft, and delayed return to sport due to the length of time needed for the graft ligamentization after ACL reconstruction. ${ }^{8-65}$ 
Additionally, impaired healing after primary repair may be the consequence of the mechanical environment on surrounding tissues. After ACL injury, neighboring tissues must bear supraphysiological loads that act as a cue for catabolic factors such as inflammatory molecules and degradative enzymes. ${ }^{66}$ Our study shows that variable inflammatory responses from different intraarticular fibroblasts occur based on mechanical strain, which is relevant for ACL healing in the setting of injury and primary repair. Zhou et al., (2005) found a differentially increased expression of MMP-2 in overstretched ACL fibroblasts at 12 hours stretch. ${ }^{67}$ Conversely, our data shows that MMP-2 production was similar across all stretching protocols. One reason for these conflicting findings may be that authors investigated the release of MMP-2 to the culture media of these tissues after ACL injury while we investigated the fibroblastic response of normal tissues.

Unlike the PCL, the ACL fibroblasts were relatively quiescent in our experiments when subjected to different strain levels. This relative lack of biologic response has implications when considering ACL repair. Our results might explain the high rates of failure associated with ACL repair, particularly midsubstance repairs, as ACL fibroblasts appear to have dampened responses to mechanical strain for inflammatory and matrix production pathways. Bio-enhancement techniques therefore may be necessary after primary ACL repair. ${ }^{47 ; 68}$

This study has potential limitations that need to be considered for interpretation. First, samples were obtained from healthy dogs without knee conditions. This may restrict the application of some of our results for ACL repair. Consequently, our lab is also focusing on evaluating metabolic responses from fibroblasts obtained from human patients that sustained ACL injury. Second, we did not see a high production of markers that may be 
involved in ACL injury in vivo such as IL-6 and MMP-1 and 3. This could be related to a lack of cross-talking between different cell types and the absence of a proper extra cellular matrix that will expand further molecular interactions. Lastly, the system used to apply mechanical strain only involves biaxial strain, which is rarely found in vivo and other mechanical forces such as shear stress and rotation may play a role in fibroblastic responses to strain. Hence, additional investigation utilizing 3D cultures or ex vivo explant cultures with a proper bioreactor is highly recommended.

In summary, our results show that surrounding intraarticular tissues, such as the SYN and PCL, are important sources of biologic mediators depending on strain level. Moreover, findings from this work suggest that surrounding intraarticular tissues play important roles in modulating the joint biochemical environment in response to mechanical strain, which may have important implications in a post-primary ACL repair setting. Since physiological strain produced the lowest levels of inflammatory mediators by both PCL and SYN fibroblasts, early passive range of motion may be advantageous in a post-repair joint environment. These findings may have implications for joint injury and in the setting of primary intraarticular ligament repair. Future work in our laboratory is focused on elucidating the complex interplay between these tissues and their responses to mechanical stimulus using co-culture models in order to optimize treatment strategies in cruciate ligament repair. 


\section{REFERENCES}

1. Kowaleski M, Boudrieau R, Pozzi A. 2013. Stifle Joint. In: Tobias KM, Johnston SA editors. Veterinary Surgery: Small Animal-E-BOOK: 2-Volume Set: Elsevier Health Sciences; pp. 906-998.

2. Lohmander LS, Englund PM, Dahl LL, et al. 2007. The long-term consequence of anterior cruciate ligament and meniscus injuries: osteoarthritis. The American journal of sports medicine 35:1756-1769.

3. Quasnichka HL, Anderson-MacKenzie JM, Tarlton JF, et al. 2005. Cruciate ligament laxity and femoral intercondylar notch narrowing in early-stage knee osteoarthritis. Arthritis \& Rheumatism 52:3100-3109.

4. Comerford EJ, Innes JF, Tarlton JF, et al. 2004. Investigation of the composition, turnover, and thermal properties of ruptured cranial cruciate ligaments of dogs. American journal of veterinary research 65:1136-1141.

5. Barenius B, Nordlander M, Ponzer S, et al. 2010. Quality of life and clinical outcome after anterior cruciate ligament reconstruction using patellar tendon graft or quadrupled semitendinosus graft: an 8-year follow-up of a randomized controlled trial. The American journal of sports medicine 38:1533-1541.

6. Murray JR, Lindh AM, Hogan NA, et al. 2012. Does anterior cruciate ligament reconstruction lead to degenerative disease?: Thirteen-year results after bonepatellar tendon-bone autograft. The American journal of sports medicine 40:404413. 
7. Nagelli CV, Cook JL, Kuroki K, et al. 2017. Does Anterior Cruciate Ligament Innervation Matter for Joint Function and Development of Osteoarthritis? The journal of knee surgery 30:364-371.

8. Woo SL, Vogrin TM, Abramowitch SD. 2000. Healing and repair of ligament injuries in the knee. The Journal of the American Academy of Orthopaedic Surgeons 8:364-372.

9. Andrish J, Holmes R. 1979. Effects of synovial fluid on fibroblasts in tissue culture. Clinical orthopaedics and related research:279-283.

10. Tondon A, Haase C, Kaunas R. 2014. Mechanical Stretch Assays in Cell Culture Systems. In: Neu CP, Genin GM editors. Handbook of imaging in biological mechanics, 1st ed: CRC press; pp. 313-319.

11. Henshaw DR, Attia E, Bhargava M, et al. 2006. Canine ACL fibroblast integrin expression and cell alignment in response to cyclic tensile strain in threedimensional collagen gels. Journal of orthopaedic research : official publication of the Orthopaedic Research Society 24:481-490.

12. Henshaw DR, Attia E, Bhargava M, et al. 2006. Canine ACL fibroblast integrin expression and cell alignment in response to cyclic tensile strain in threedimensional collagen gels. Journal of Orthopaedic Research 24:481-490.

13. Attia E, Bohnert K, Brown H, et al. 2014. Characterization of Total and Active Matrix Metalloproteinases-1, -3, and -13 Synthesized and Secreted by Anterior Cruciate Ligament Fibroblasts in Three-Dimensional Collagen Gels. Tissue Engineering Part A 20:171-177. 
14. Lee CH, Shin HJ, Cho IH, et al. 2005. Nanofiber alignment and direction of mechanical strain affect the ECM production of human ACL fibroblast. Biomaterials 26:1261-1270.

15. Lee CY, Liu X, Smith CL, et al. 2004. The combined regulation of estrogen and cyclic tension on fibroblast biosynthesis derived from anterior cruciate ligament. Matrix biology : journal of the International Society for Matrix Biology 23:323329.

16. Fang F, Lake SP. 2017. Experimental evaluation of multiscale tendon mechanics. Journal of orthopaedic research : official publication of the Orthopaedic Research Society $35: 1353-1365$.

17. Freedman BR, Rodriguez AB, Leiphart RJ, et al. 2018. Dynamic Loading and Tendon Healing Affect Multiscale Tendon Properties and ECM Stress Transmission. Scientific reports 8:10854.

18. Hurschler C, Loitz-Ramage B, Vanderby R, Jr. 1997. A structurally based stressstretch relationship for tendon and ligament. Journal of biomechanical engineering 119:392-399.

19. Linka K, Itskov M. 2016. Mechanics of collagen fibrils: A two-scale discrete damage model. Journal of the Mechanical Behavior of Biomedical Materials 58:163-172.

20. Song F, Jiang D, Wang T, et al. 2017. Mechanical Loading Improves TendonBone Healing in a Rabbit Anterior Cruciate Ligament Reconstruction Model by Promoting Proliferation and Matrix Formation of Mesenchymal Stem Cells and Tendon Cells. Cellular Physiology and Biochemistry 41:875-889. 
21. Erik A, Krista B, Haydee B, et al. 2014. Characterization of Total and Active Matrix Metalloproteinases-1, -3, and -13 Synthesized and Secreted by Anterior Cruciate Ligament Fibroblasts in Three-Dimensional Collagen Gels. 20:171-177.

22. Kim SG, Akaike T, Sasagaw T, et al. 2002. Gene expression of type I and type III collagen by mechanical stretch in anterior cruciate ligament cells. Cell structure and function 27:139-144.

23. Wang WM, Ma XJ, Huang SB, et al. 2017. A comparative study of effect of autograft compared with allograft anterior cruciate ligament reconstruction on expressions of LOXs and MMPs. Bioscience reports 37.

24. Farndale RW, Buttle DJ, Barrett AJ. 1986. Improved quantitation and discrimination of sulphated glycosaminoglycans by use of dimethylmethylene blue. Biochim Biophys Acta 883:173-177.

25. Chiquet M, Gelman L, Lutz R, et al. 2009. From mechanotransduction to extracellular matrix gene expression in fibroblasts. Biochim Biophys Acta 1793:911-920.

26. Camelliti P, Borg TK, Kohl P. 2005. Structural and functional characterisation of cardiac fibroblasts. Cardiovasc Res 65:40-51.

27. Toyoda T, Matsumoto H, Fujikawa K, et al. 1998. Tensile load and the metabolism of anterior cruciate ligament cells. Clinical orthopaedics and related research:247-255.

28. Sambajon VV, Cillo JE, Jr., Gassner RJ, et al. 2003. The effects of mechanical strain on synovial fibroblasts. Journal of Oral and Maxillofacial Surgery 61:707712. 
29. Zhang Y, Huang W, Jiang J, et al. 2014. Influence of TNF-alpha and biomechanical stress on matrix metalloproteinases and lysyl oxidases expressions in human knee synovial fibroblasts. Knee surgery, sports traumatology, arthroscopy : official journal of the ESSKA 22:1997-2006.

30. Wang C, Xie J, Jiang J, et al. 2015. Differential expressions of the lysyl oxidase family and matrix metalloproteinases-1, 2, 3 in posterior cruciate ligament fibroblasts after being co-cultured with synovial cells. Int Orthop 39:183-191.

31. Wang JH-C, Thampatty BP. 2006. An Introductory Review of Cell Mechanobiology. Biomechanics and Modeling in Mechanobiology 5:1-16.

32. Logterman SL, Wydra FB, Frank RM. 2018. Posterior Cruciate Ligament: Anatomy and Biomechanics. Curr Rev Musculoskelet Med 11:510-514.

33. Vogrin TM, Hoher J, Aroen A, et al. 2000. Effects of sectioning the posterolateral structures on knee kinematics and in situ forces in the posterior cruciate ligament. Knee surgery, sports traumatology, arthroscopy : official journal of the ESSKA 8:93-98.

34. Dargel J, Gotter M, Mader K, et al. 2007. Biomechanics of the anterior cruciate ligament and implications for surgical reconstruction. Strategies in Trauma and Limb Reconstruction 2:1-12.

35. Moglo KE, Shirazi-Adl A. 2005. Cruciate coupling and screw-home mechanism in passive knee joint during extension--flexion. Journal of biomechanics 38:10751083.

36. Moglo KE, Shirazi-Adl A. 2003. Biomechanics of passive knee joint in drawer: load transmission in intact and ACL-deficient joints. The Knee 10:265-276. 
37. Tang Z, Yang L, Wang Y, et al. 2009. Contributions of different intraarticular tissues to the acute phase elevation of synovial fluid MMP-2 following rat ACL rupture. Journal of orthopaedic research : official publication of the Orthopaedic Research Society 27:243-248.

38. Iwanaga T, Shikichi M, Kitamura H, et al. 2000. Morphology and functional roles of synoviocytes in the joint. Archives of histology and cytology 63:17-31.

39. Sung KL, Kwan MK, Maldonado F, et al. 1994. Adhesion strength of human ligament fibroblasts. Journal of biomechanical engineering 116:237-242.

40. Kato S, Saito M, Funasaki H, et al. 2015. Distinctive collagen maturation process in fibroblasts derived from rabbit anterior cruciate ligament, medial collateral ligament, and patellar tendon in vitro. Knee Surgery, Sports Traumatology, Arthroscopy 23:1384-1392.

41. Zhang J, Pan T, Im HJ, et al. 2011. Differential properties of human ACL and MCL stem cells may be responsible for their differential healing capacity. BMC Med 9:68.

42. Nagineni CN, Amiel D, Green MH, et al. 1992. Characterization of the intrinsic properties of the anterior cruciate and medial collateral ligament cells: an in vitro cell culture study. Journal of orthopaedic research : official publication of the Orthopaedic Research Society 10:465-475.

43. Killian ML, Cavinatto L, Galatz LM, et al. 2012. The role of mechanobiology in tendon healing. J Shoulder Elbow Surg 21:228-237.

44. Brophy RH, Kovacevic D, Imhauser CW, et al. 2011. Effect of short-duration low-magnitude cyclic loading versus immobilization on tendon-bone healing after 
ACL reconstruction in a rat model. The Journal of bone and joint surgery American volume 93:381-393.

45. Bedi A, Kovacevic D, Fox AJ, et al. 2010. Effect of early and delayed mechanical loading on tendon-to-bone healing after anterior cruciate ligament reconstruction. The Journal of bone and joint surgery American volume 92:2387-2401.

46. Andriacchi T, Sabiston P, DeHaven K, et al. 1988. Ligament: injury and repair. In: Woo S, Buckwalter JA editors. Injury and Repair of the Musculoskeltal Soft Tissues: American Academy of Orthopaedic Surgeons; pp. 103-128.

47. Kiapour AM, Murray MM. 2014. Basic science of anterior cruciate ligament injury and repair. Bone Joint Res 3:20-31.

48. Strand T, Molster A, Hordvik M, et al. 2005. Long-term follow-up after primary repair of the anterior cruciate ligament: clinical and radiological evaluation 15-23 years postoperatively. Arch Orthop Trauma Surg 125:217-221.

49. Andersen RB, Gormsen J. 1987. Fibrin dissolution in synovial fluid. Scandinavian journal of rheumatology 16:319-333.

50. Bolander ME. 1992. Regulation of Fracture Repair by Growth Factors. Proceedings of the Society for Experimental Biology and Medicine 200:165-170.

51. Solheim E. 1998. Growth factors in bone. International Orthopaedics 22:410-416.

52. Murray MM, Magarian EM, Harrison SL, et al. 2010. The effect of skeletal maturity on functional healing of the anterior cruciate ligament. The Journal of bone and joint surgery American volume 92:2039-2049.

53. Murray MM, Magarian E, Zurakowski D, et al. 2010. Bone-to-bone fixation enhances functional healing of the porcine anterior cruciate ligament using a 
collagen-platelet composite. Arthroscopy : the journal of arthroscopic \& related surgery : official publication of the Arthroscopy Association of North America and the International Arthroscopy Association 26:S49-57.

54. Vavken P, Fleming BC, Mastrangelo AN, et al. 2012. Biomechanical outcomes after bioenhanced anterior cruciate ligament repair and anterior cruciate ligament reconstruction are equal in a porcine model. Arthroscopy : the journal of arthroscopic \& related surgery : official publication of the Arthroscopy Association of North America and the International Arthroscopy Association 28:672-680.

55. Magarian EM, Fleming BC, Harrison SL, et al. 2010. Delay of 2 or 6 weeks adversely affects the functional outcome of augmented primary repair of the porcine anterior cruciate ligament. The American journal of sports medicine $38: 2528-2534$.

56. Kiapour AM, Fleming BC, Murray MM. 2015. Biomechanical Outcomes of Bridge-enhanced Anterior Cruciate Ligament Repair Are Influenced by Sex in a Preclinical Model. Clinical orthopaedics and related research 473:2599-2608.

57. Murray MM, Kalish LA, Fleming BC, et al. 2019. Bridge-Enhanced Anterior Cruciate Ligament Repair: Two-Year Results of a First-in-Human Study. Orthop J Sports Med 7:2325967118824356.

58. Paschos NK. 2017. Anterior cruciate ligament reconstruction and knee osteoarthritis. World journal of orthopedics 8:212-217.

59. Lecoq FA, Parienti JJ, Murison J, et al. 2018. Graft Choice and the Incidence of Osteoarthritis After Anterior Cruciate Ligament Reconstruction: A Causal 
Analysis From a Cohort of 541 Patients. The American journal of sports medicine 46:2842-2850.

60. Simonian PT, Harrison SD, Cooley VJ, et al. 1997. Assessment of morbidity of semitendinosus and gracilis tendon harvest for ACL reconstruction. The American journal of knee surgery 10:54-59.

61. Nakamae A, Ochi M, Deie M, et al. 2012. Unsuccessful regeneration of the semitendinosus tendon harvested for anterior cruciate ligament reconstruction: Report of two cases. Orthopaedics \& Traumatology: Surgery \& Research 98:932935.

62. Nagelli CV, Hewett TE. 2017. Should Return to Sport be Delayed Until 2 Years After Anterior Cruciate Ligament Reconstruction? Biological and Functional Considerations. Sports Med 47:221-232.

63. Konrath JM, Vertullo CJ, Kennedy BA, et al. 2016. Morphologic Characteristics and Strength of the Hamstring Muscles Remain Altered at 2 Years After Use of a Hamstring Tendon Graft in Anterior Cruciate Ligament Reconstruction. The American journal of sports medicine 44:2589-2598.

64. Abourezk MN, Ithurburn MP, McNally MP, et al. 2017. Hamstring Strength Asymmetry at 3 Years After Anterior Cruciate Ligament Reconstruction Alters Knee Mechanics During Gait and Jogging. The American journal of sports medicine 45:97-105.

65. Papalia R, Franceschi F, D'Adamio S, et al. 2015. Hamstring Tendon Regeneration After Harvest for Anterior Cruciate Ligament Reconstruction: 
A Systematic Review. Arthroscopy: The Journal of Arthroscopic \& Related Surgery 31:1169-1183.

66. Yang G, Im H-J, Wang JHC. 2005. Repetitive mechanical stretching modulates IL-1 $\beta$ induced COX-2, MMP-1 expression, and PGE2 production in human patellar tendon fibroblasts. Gene 363:166-172.

67. Zhou D, Lee HS, Villarreal F, et al. 2005. Differential MMP-2 activity of ligament cells under mechanical stretch injury: An in vitro study on human ACL and MCL fibroblasts. Journal of Orthopaedic Research 23:949-957.

68. Sandberg R, Balkfors B, Nilsson B, et al. 1987. Operative versus non-operative treatment of recent injuries to the ligaments of the knee. A prospective randomized study. The Journal of bone and joint surgery American volume 69:1120-1126. 
Figures

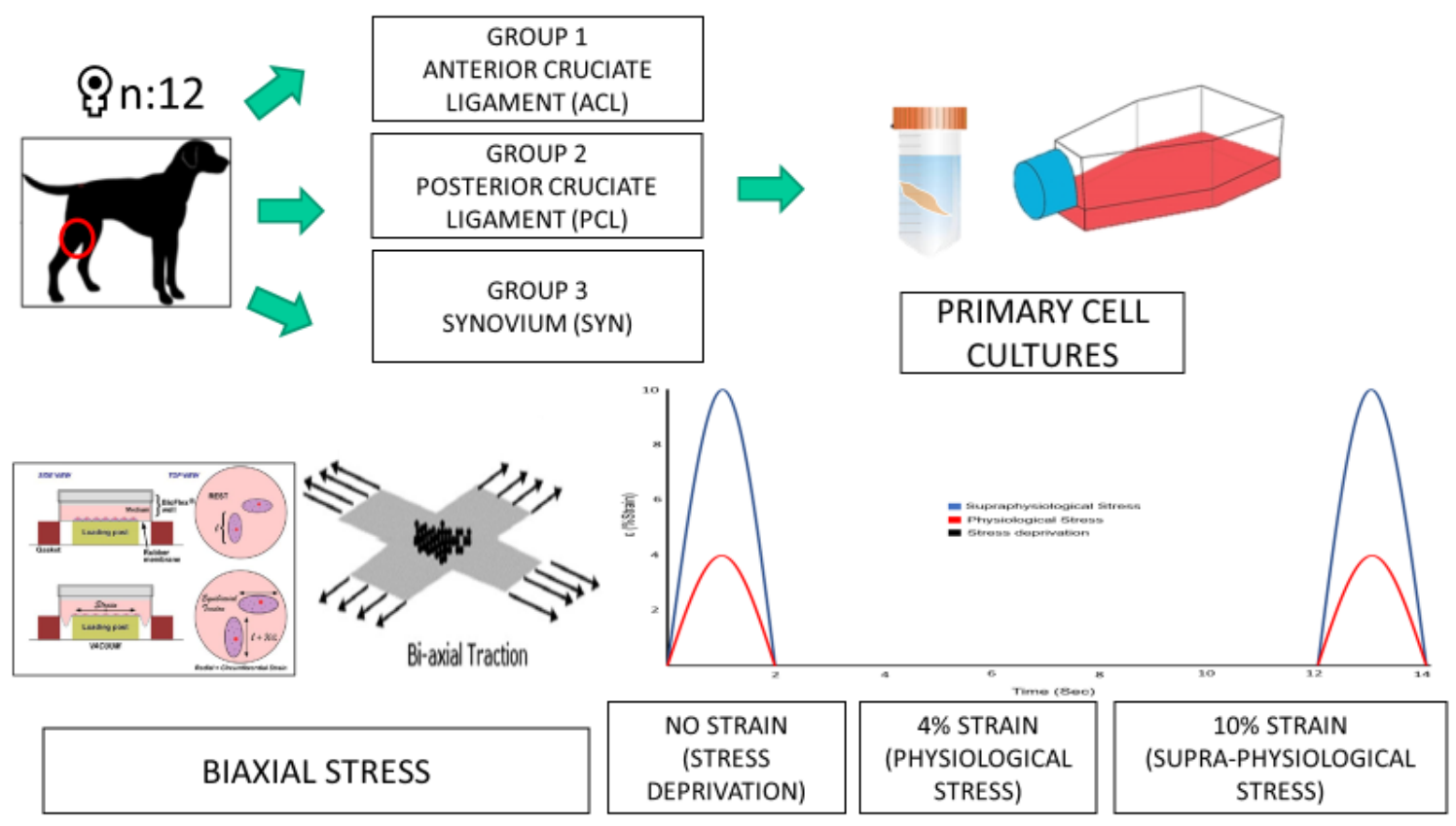

Figure 4.1. Experimental Design 

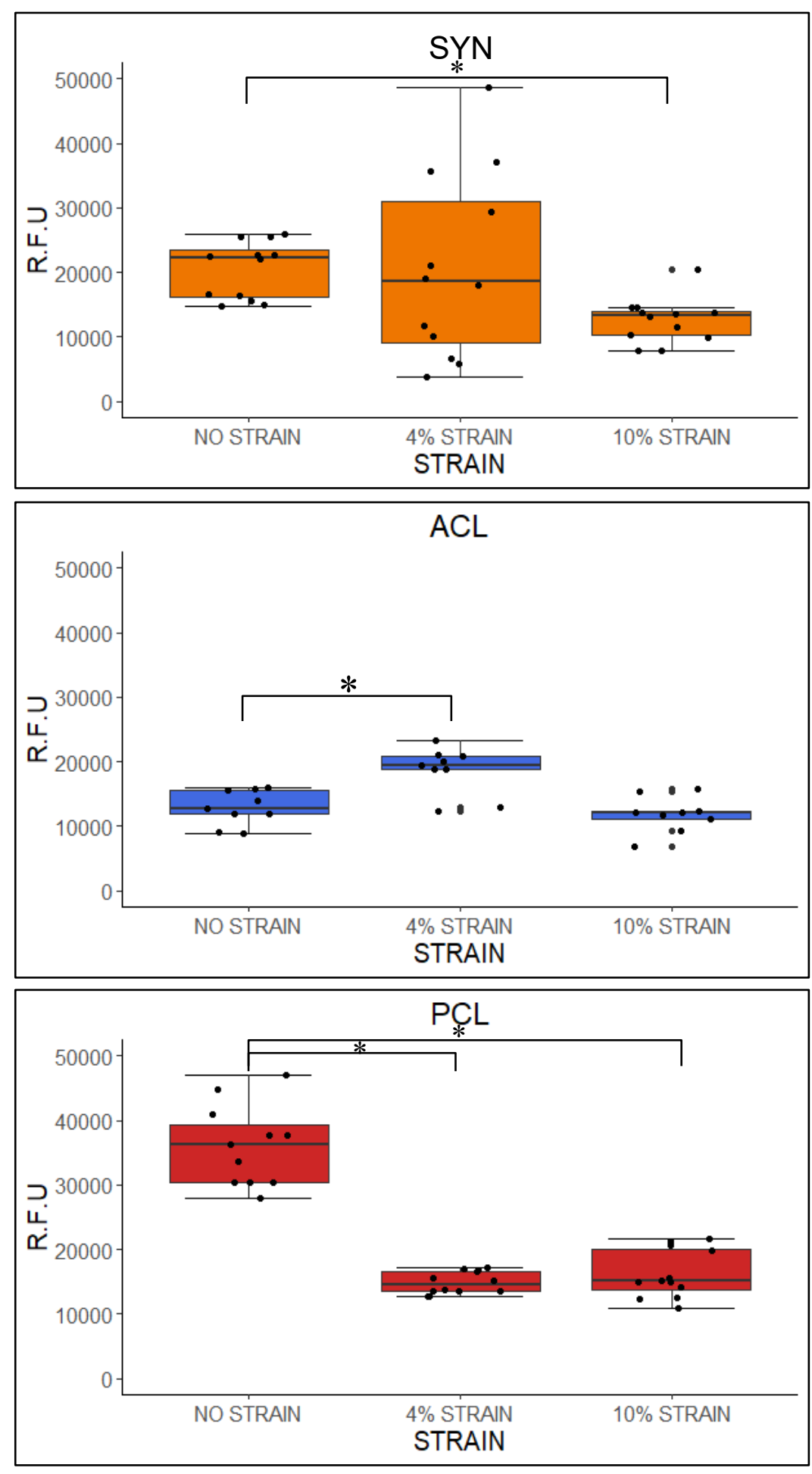

Figure 4.2. Metabolic Activity of fibroblasts. * significant difference between strains at 120 hours of culture. SYN: Synovium; ACL: Anterior cruciate ligament, PCL: Posterior cruciate ligament. R.F.U: Resazurin Fluorescent Units 

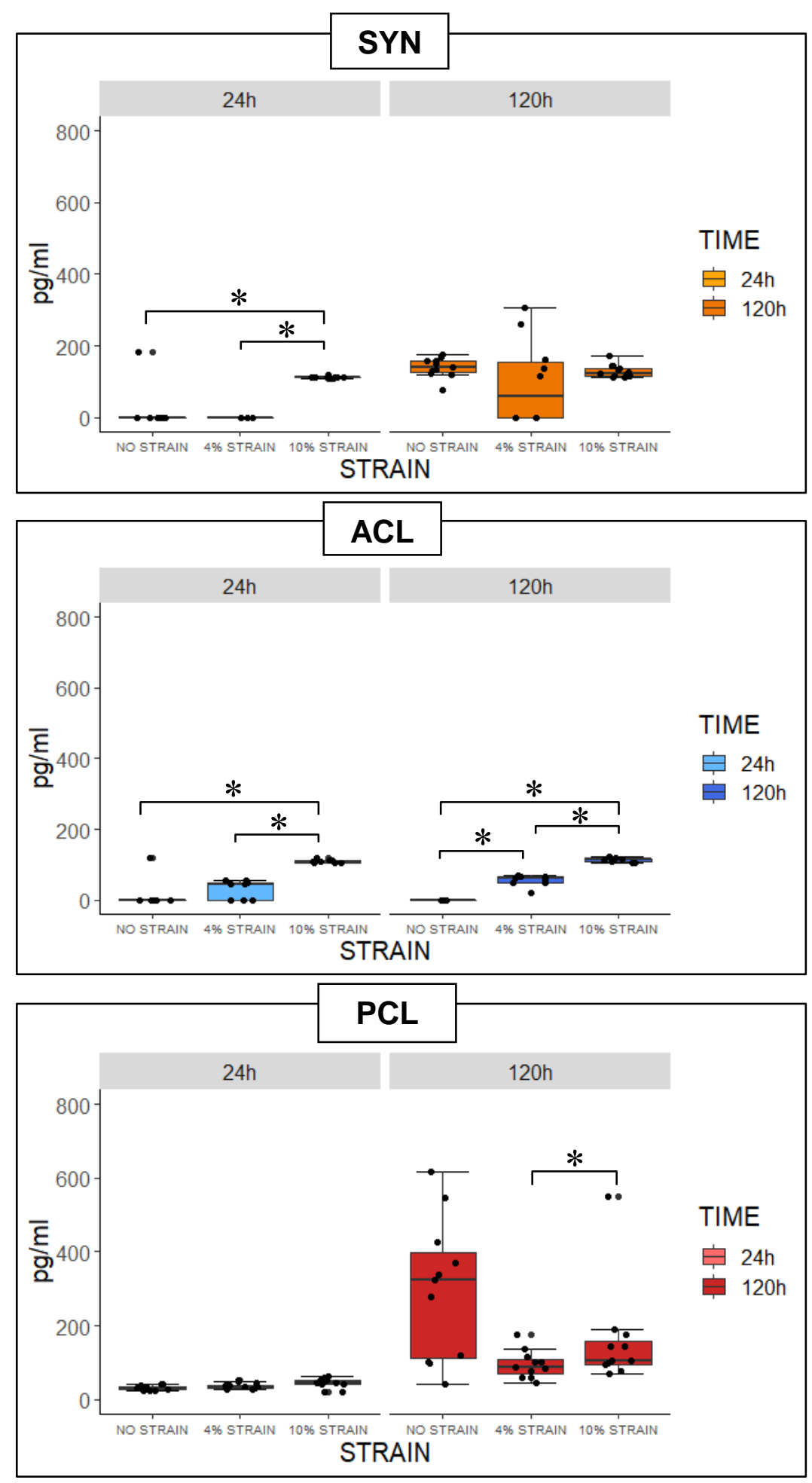

Figure 4.3. Concentration of PGE2 released to the media. * significant difference between strains at 24 and 120 hours of culture. SYN: Synovium; ACL: Anterior cruciate ligament, PCL: Posterior cruciate ligament 

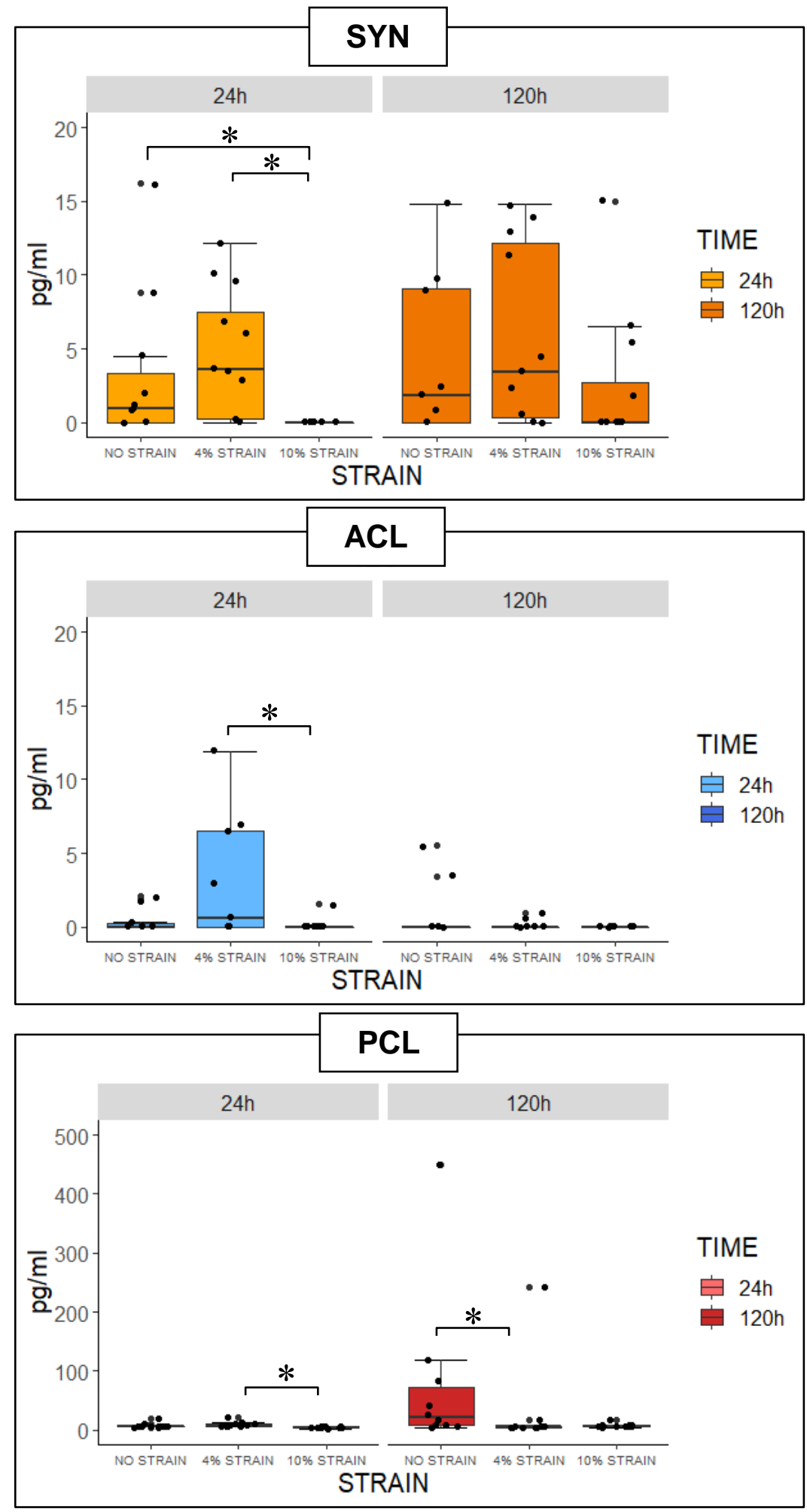

Figure 4.4. Concentration of IL-6 released to the media. * significant difference between strains at 24 and 120 hours of culture. SYN: Synovium; ACL: Anterior cruciate ligament, PCL: Posterior cruciate ligament 

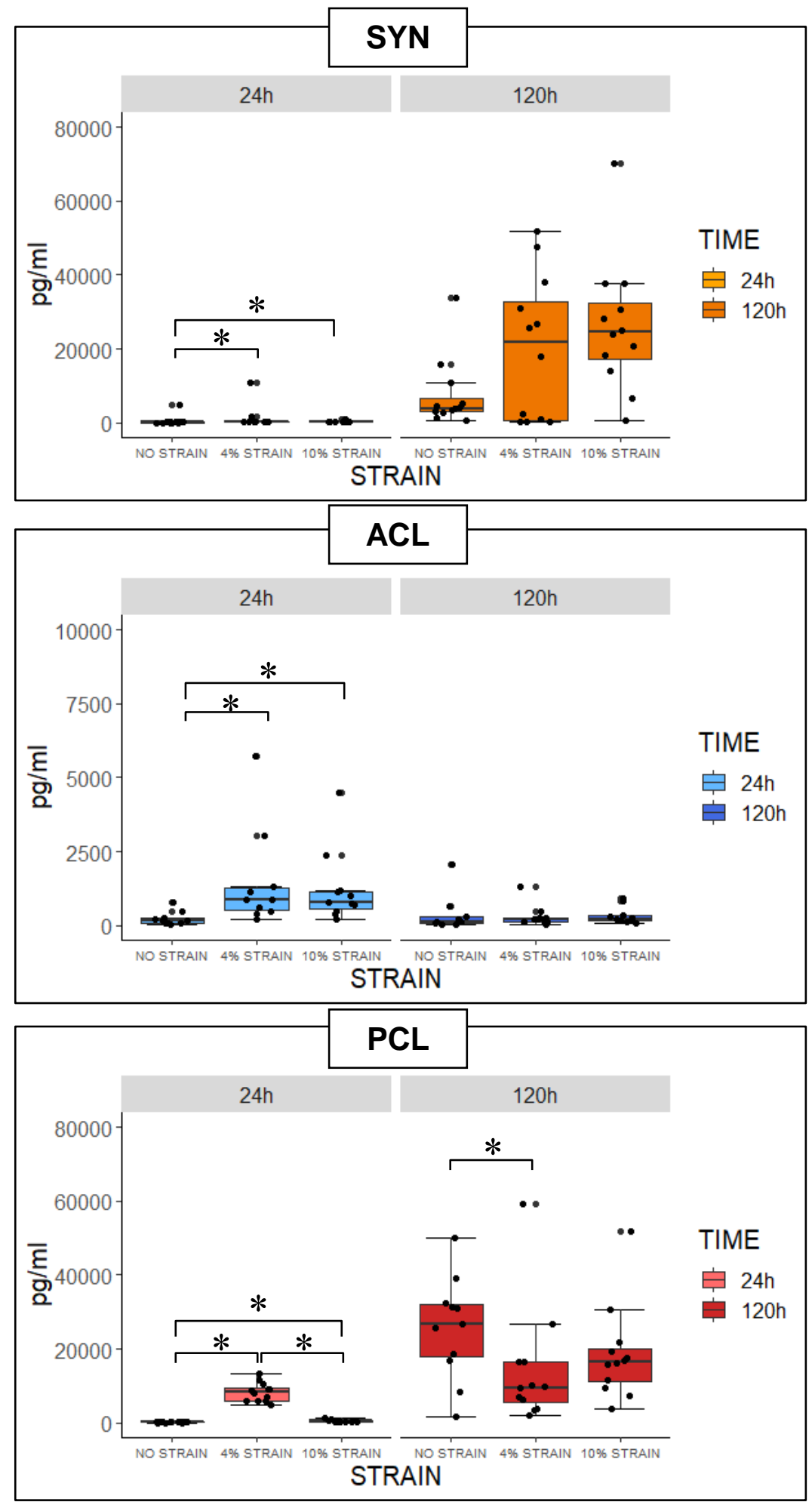

Figure 4.5. Concentration of IL-8 released to the media. * significant difference between strains at 24 and 120 hours of culture. SYN: Synovium; ACL: Anterior cruciate ligament, PCL: Posterior cruciate ligament 

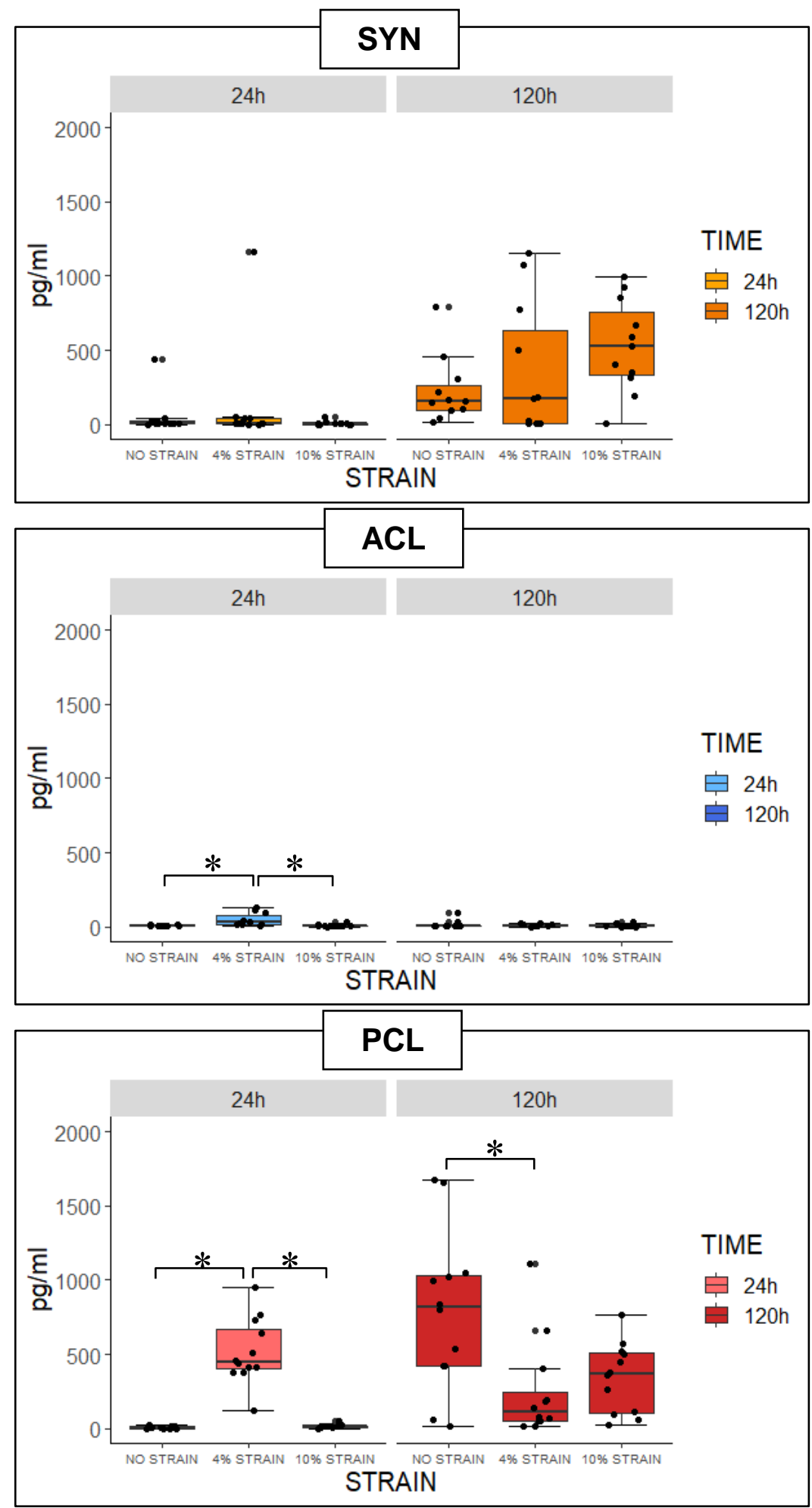

Figure 4.6. Concentration of $\mathrm{KC}$ released to the media. * significant difference between strains at 24 and 120 hours of culture. SYN: Synovium; ACL: Anterior cruciate ligament, PCL: Posterior cruciate ligament 

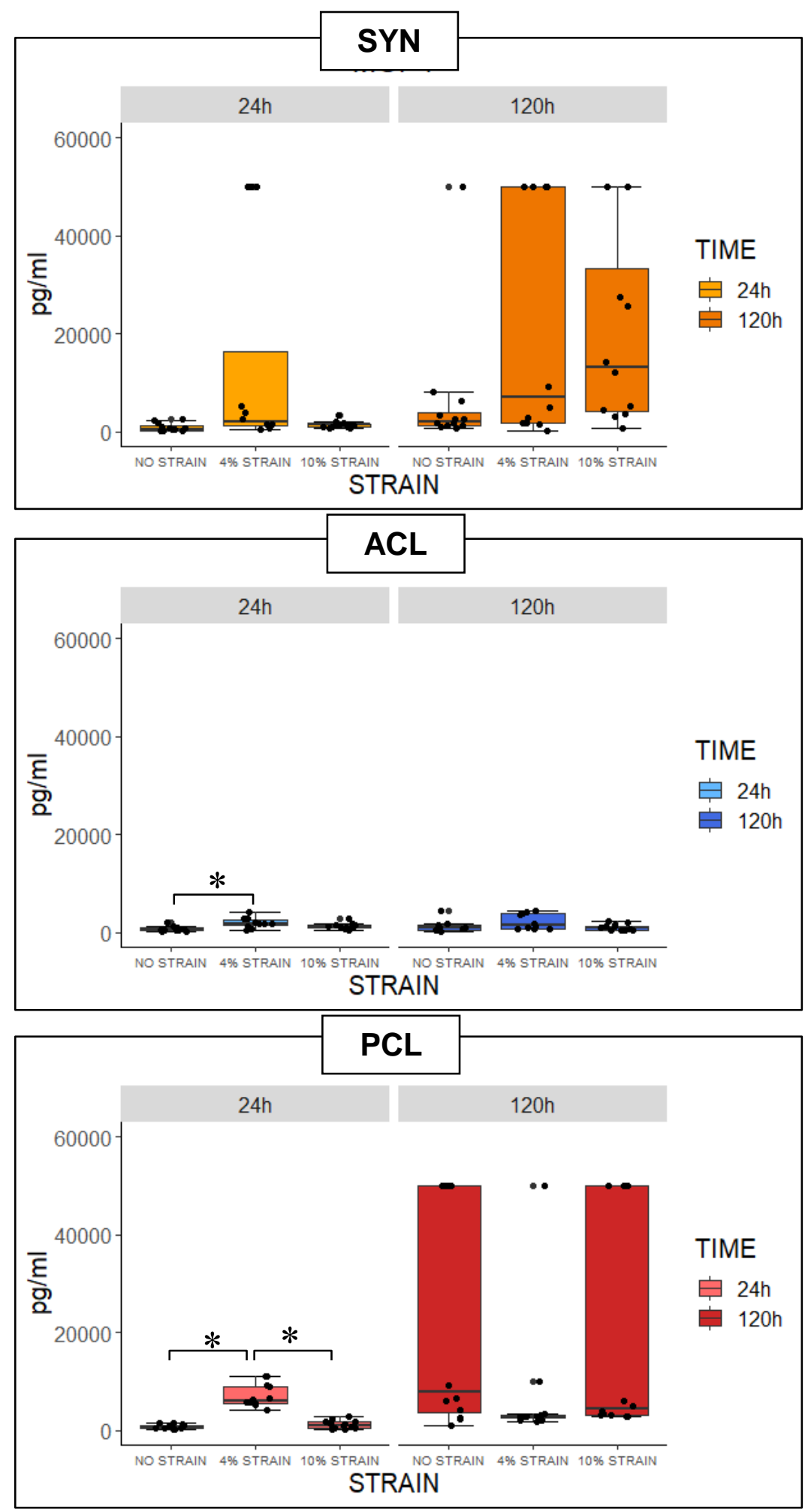

Figure 4.7. Concentration of MCP-1 released to the media. * significant difference between strains at 24 and 120 hours of culture. SYN: Synovium; ACL: Anterior cruciate ligament, PCL: Posterior cruciate ligament 

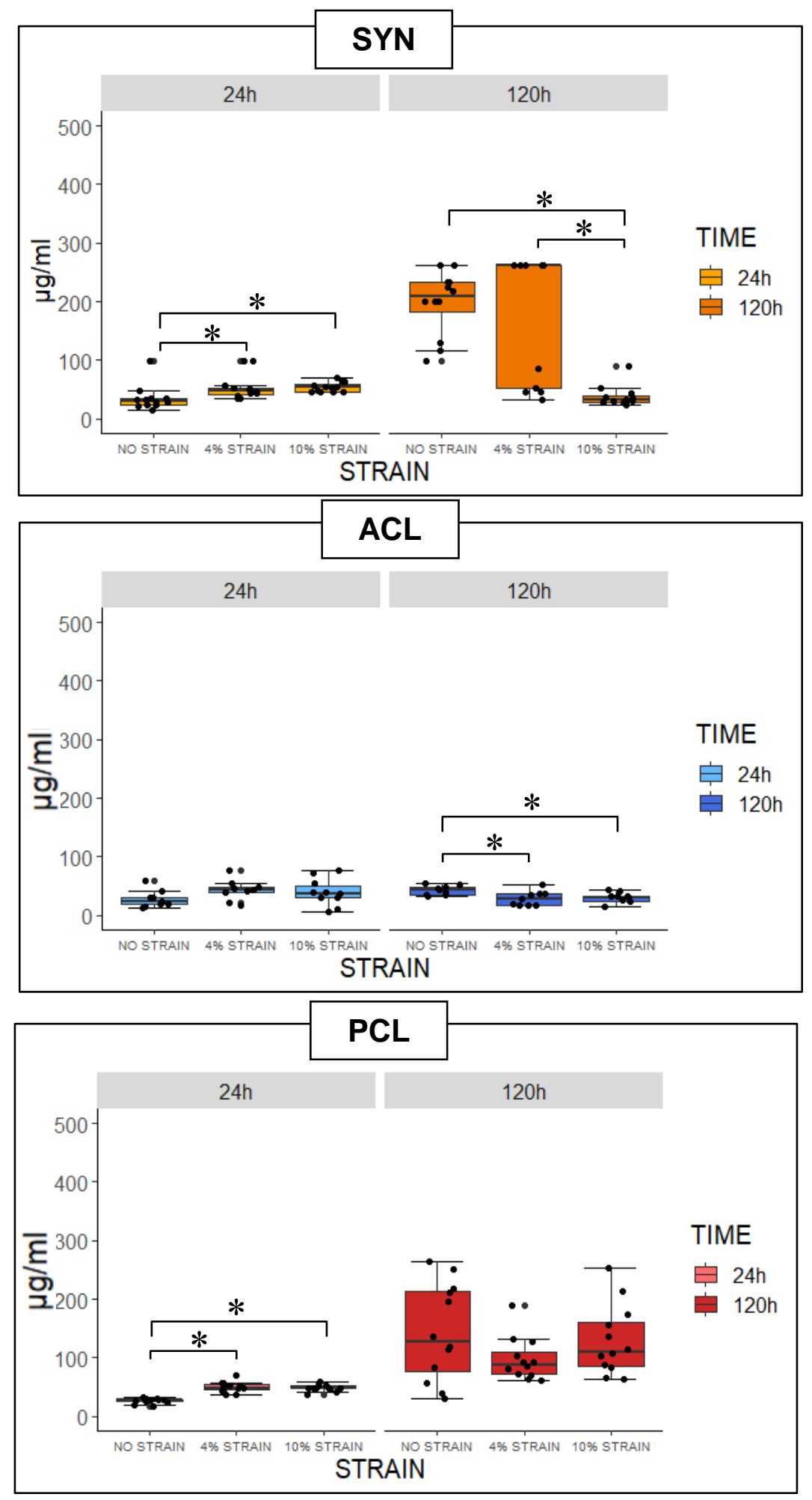

Figure 4.8. Concentration of Glycosaminoglycans (GAG) released to the media. * significant difference between strains at 24 and 120 hours of culture. SYN: Synovium; ACL: Anterior cruciate ligament, PCL: Posterior cruciate ligament 

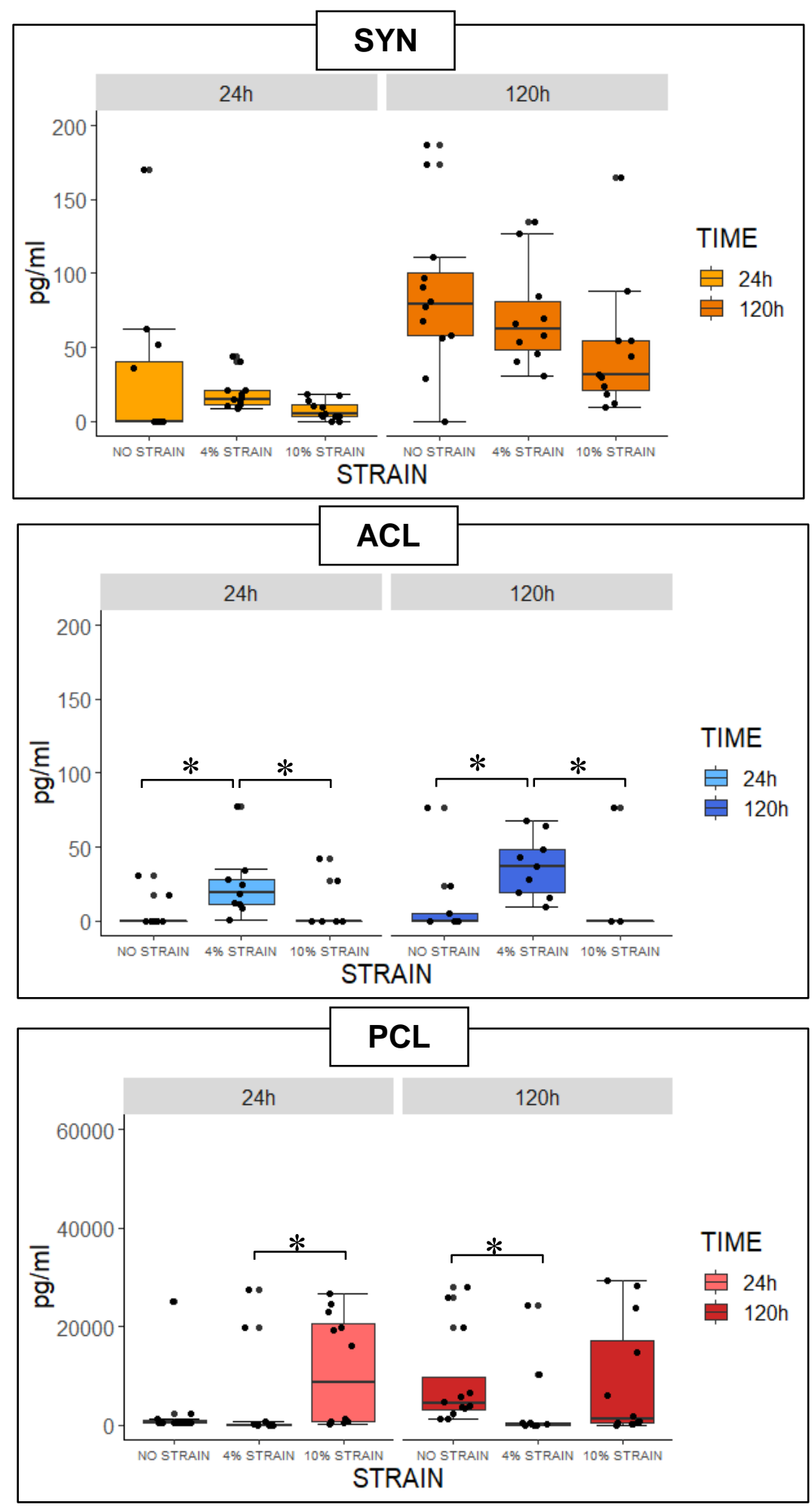

Figure 4.9. Concentration of total MMP activity (MMPACT) released to the media. * significant difference between strains at 24 and 120 hours of culture. SYN: Synovium; ACL: Anterior cruciate ligament, PCL: Posterior cruciate ligament 


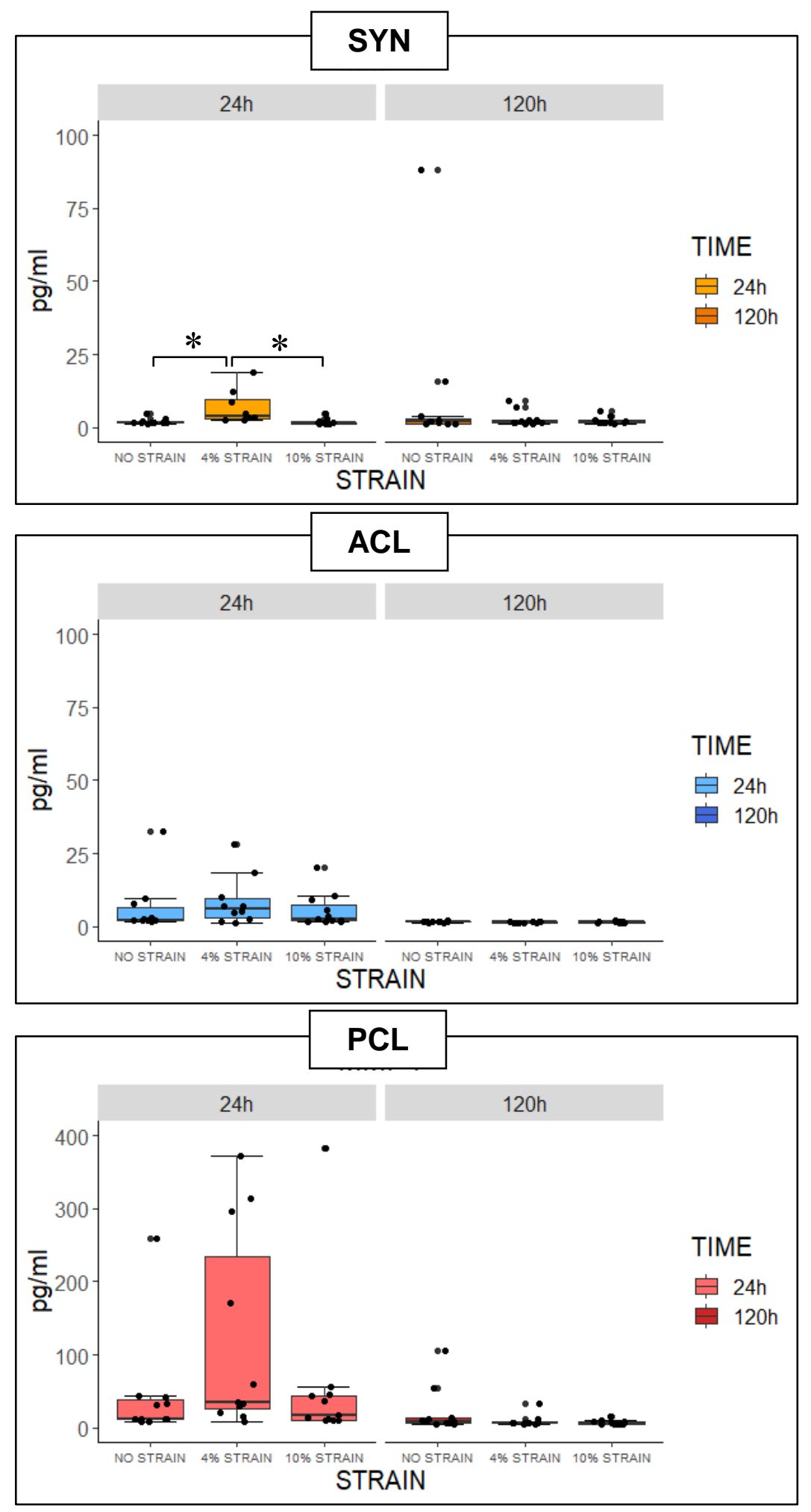

Figure 4.10. Concentration of matrix MMP-1 released to the media. * significant difference between strains at 24 and 120 hours of culture. SYN: Synovium; ACL: Anterior cruciate ligament, PCL: Posterior cruciate ligament 


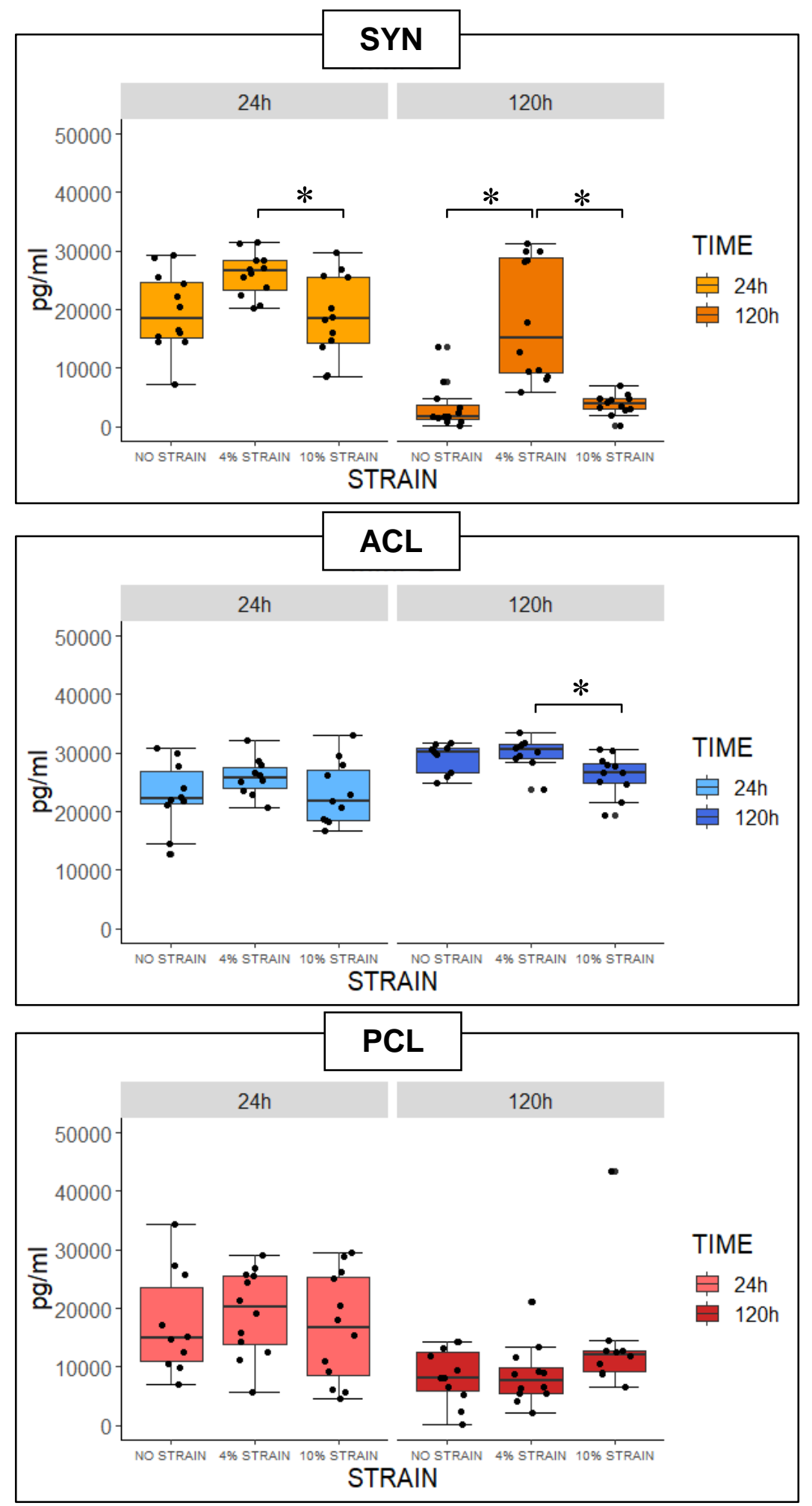

Figure 4.11. Concentration of matrix MMP-2 released to the media. * significant difference between strains at 24 and 120 hours of culture. SYN: Synovium; ACL: Anterior cruciate ligament, PCL: Posterior cruciate ligament 

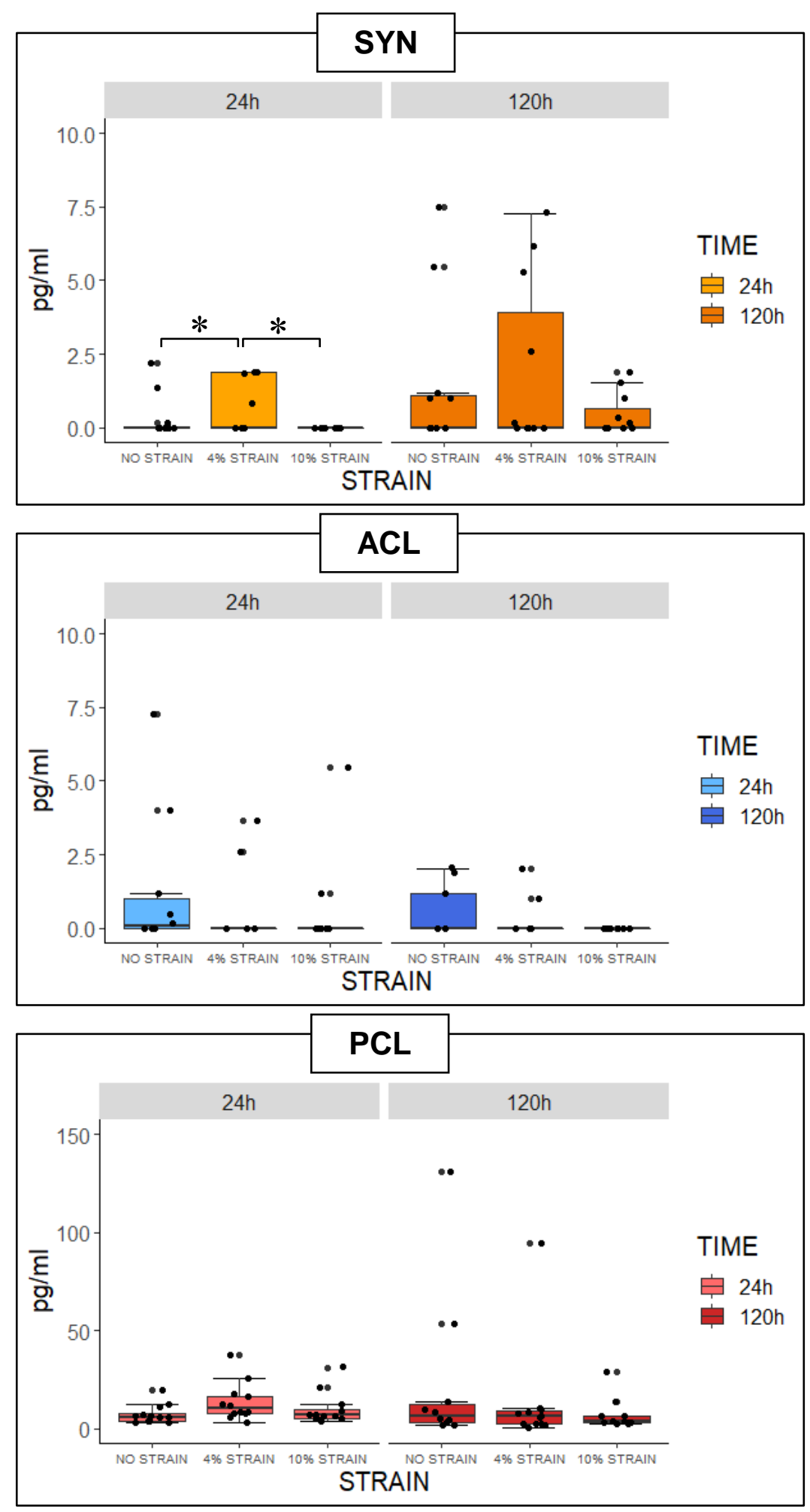

Figure 4.12. Concentration of matrix MMP-3 released to the media. * significant difference between strains at 24 and 120 hours of culture. SYN: Synovium; ACL: Anterior cruciate ligament, PCL: Posterior cruciate ligament 

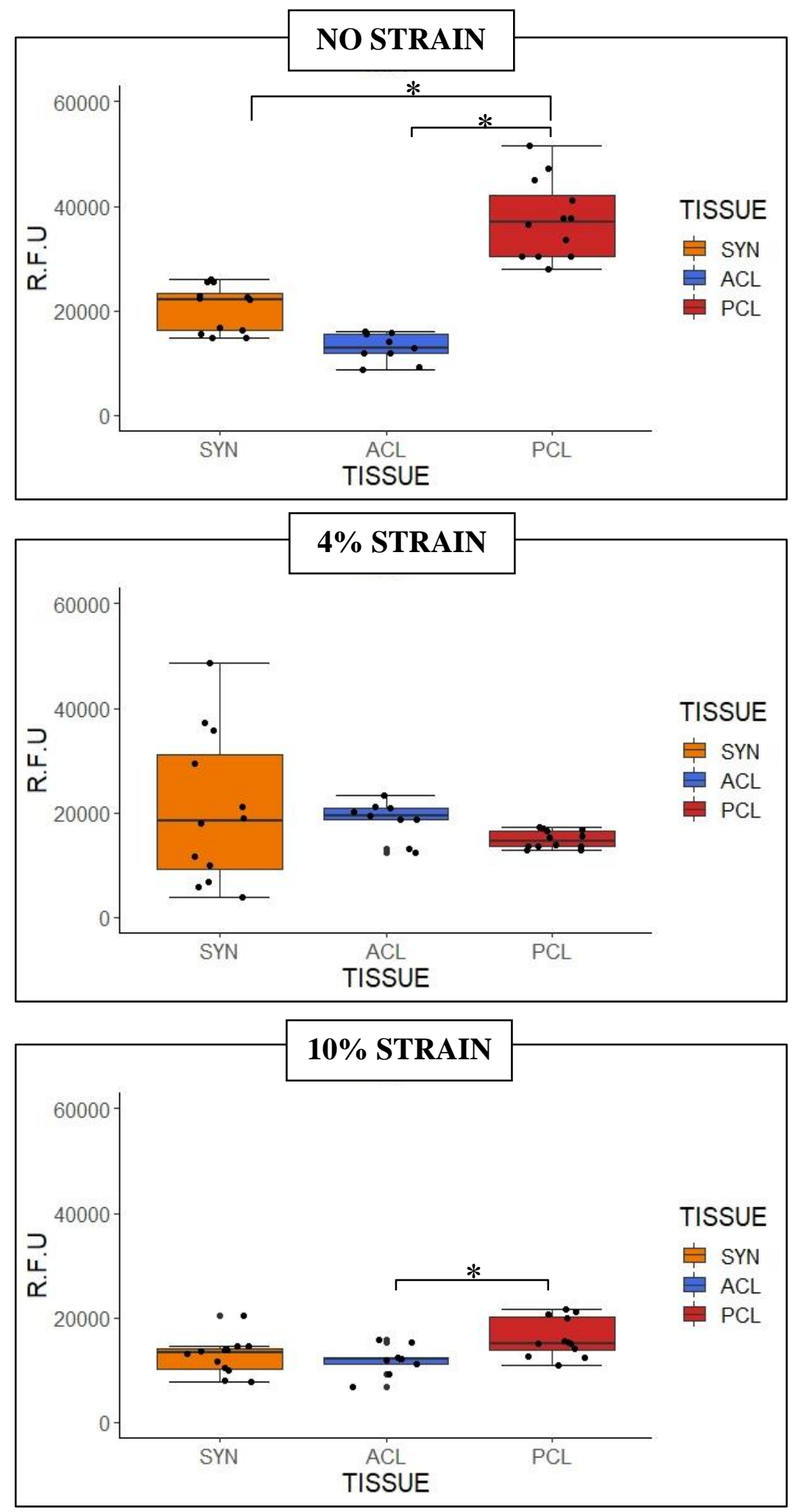

Figure 4.13. Metabolic Activity of fibroblasts. * significant difference between tissues at 120 hours of culture. SYN: Synovium; ACL: Anterior cruciate ligament, PCL: Posterior cruciate ligament. R.F.U: Resazurin Fluorescent Units 

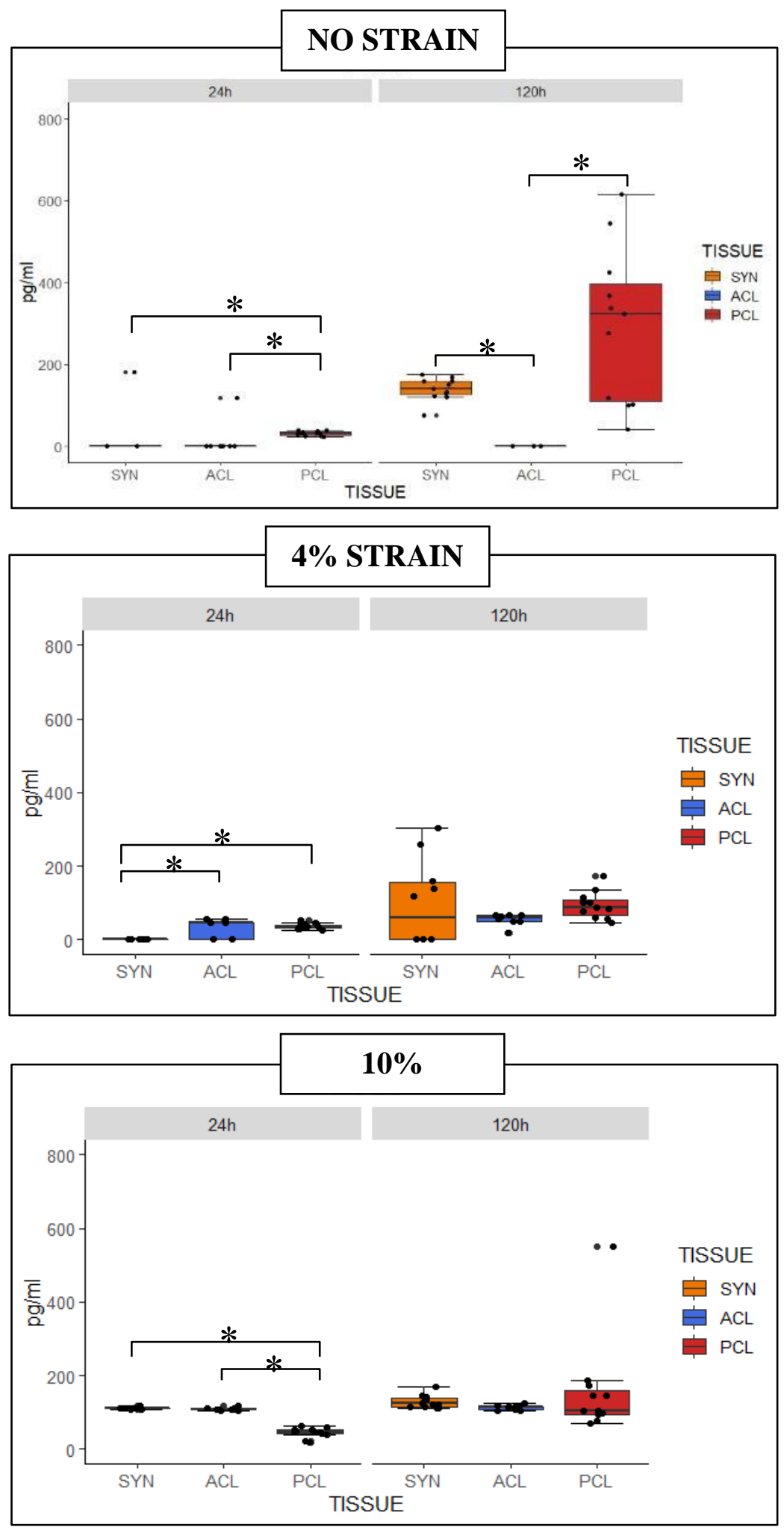

Figure 4.14. Concentration of PGE2 released to the media. * significant difference between tissues at 24 and 120 hours of culture. SYN: Synovium; ACL: Anterior cruciate ligament, PCL: Posterior cruciate ligament 


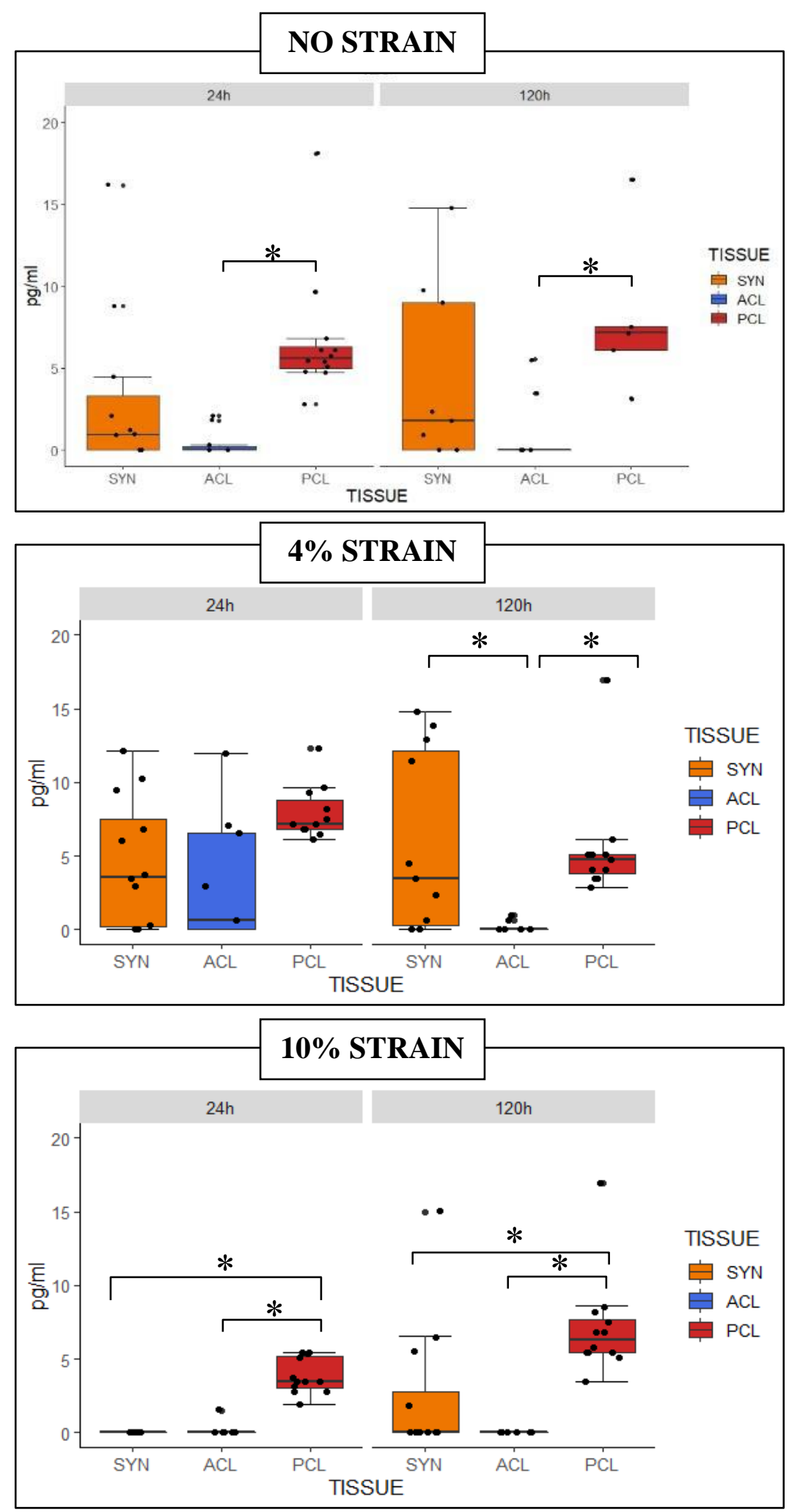

Figure 4.15. Concentration of IL-6 released to the media. * significant difference between tissues at 24 and 120 hours of culture. SYN: Synovium; ACL: Anterior cruciate ligament, PCL: Posterior cruciate ligament 

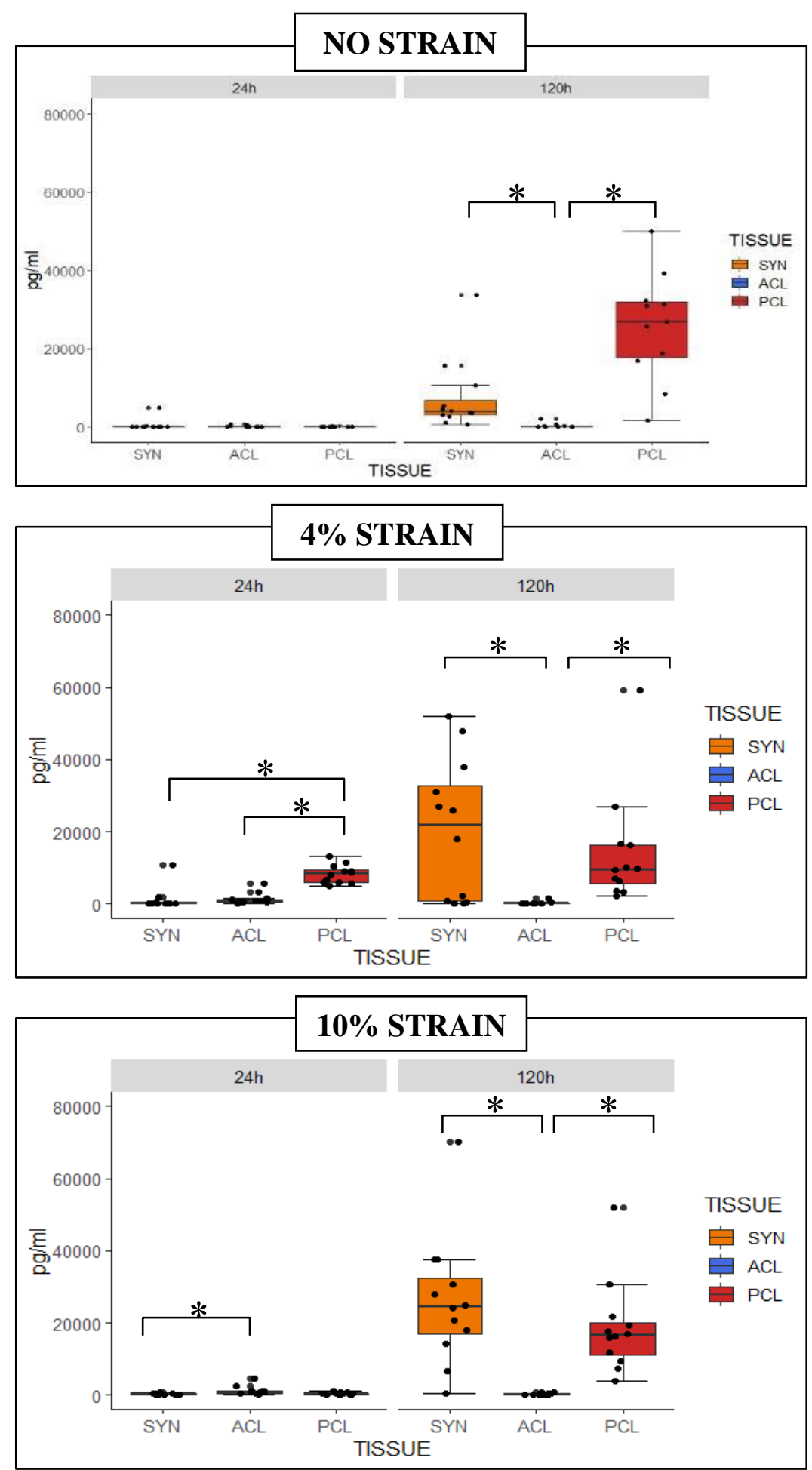

Figure 4.16. Concentration of IL-8 released to the media. * significant difference between tissues at 24 and 120 hours of culture. SYN: Synovium; ACL: Anterior cruciate ligament, PCL: Posterior cruciate ligament 

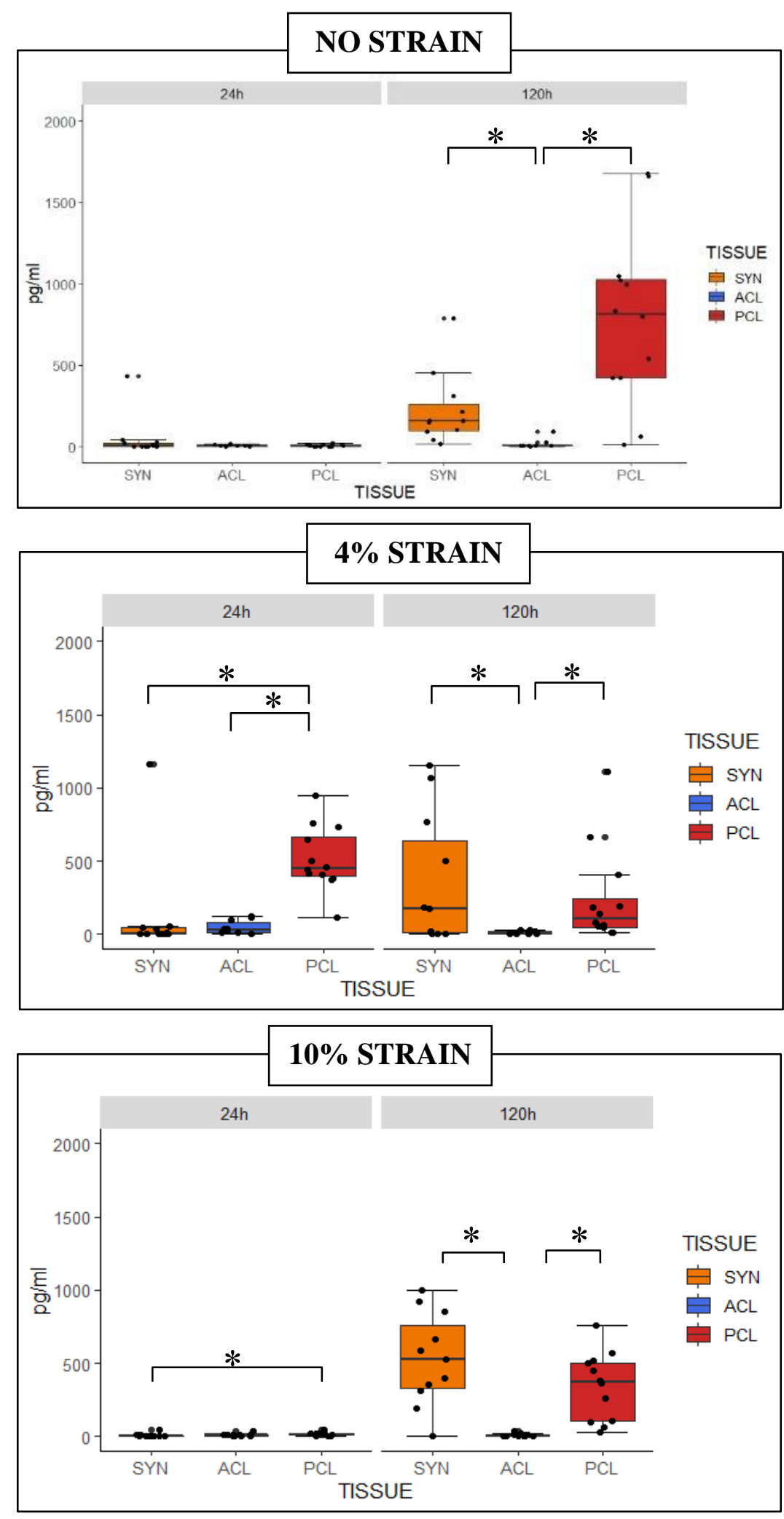

Figure 4.17. Concentration of $\mathrm{KC}$ released to the media. * significant difference between tissues at 24 and 120 hours of culture. SYN: Synovium; ACL: Anterior cruciate ligament, PCL: Posterior cruciate ligament 

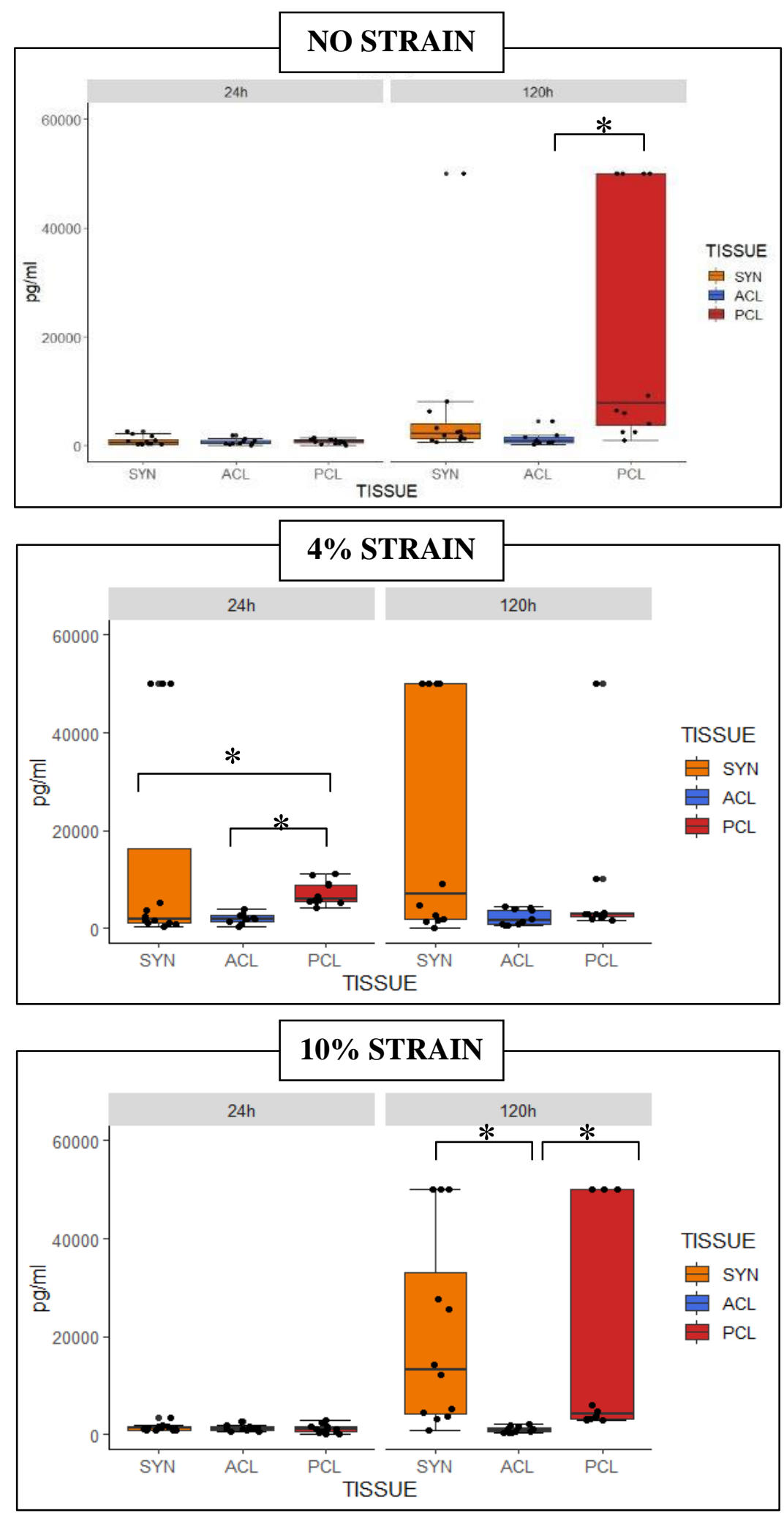

Figure 4.18. Concentration of MCP1 released to the media. * significant difference between tissues at 24 and 120 hours of culture. SYN: Synovium; ACL: Anterior cruciate ligament, PCL: Posterior cruciate ligament 

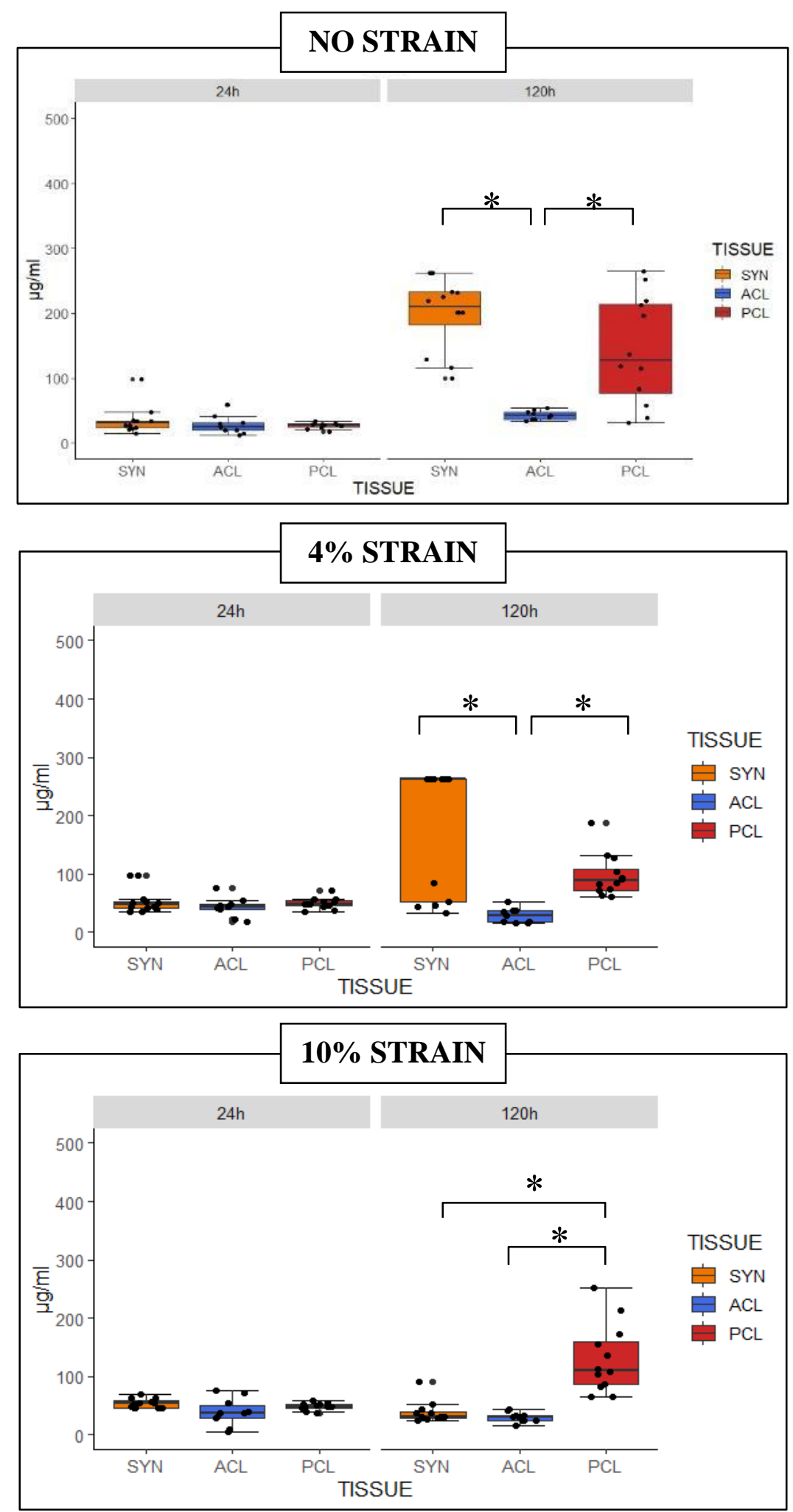

Figure 4.19. Concentration of GAG released to the media. * significant difference between tissues at 24 and 120 hours of culture. SYN: Synovium; ACL: Anterior cruciate ligament, PCL: Posterior cruciate ligament 

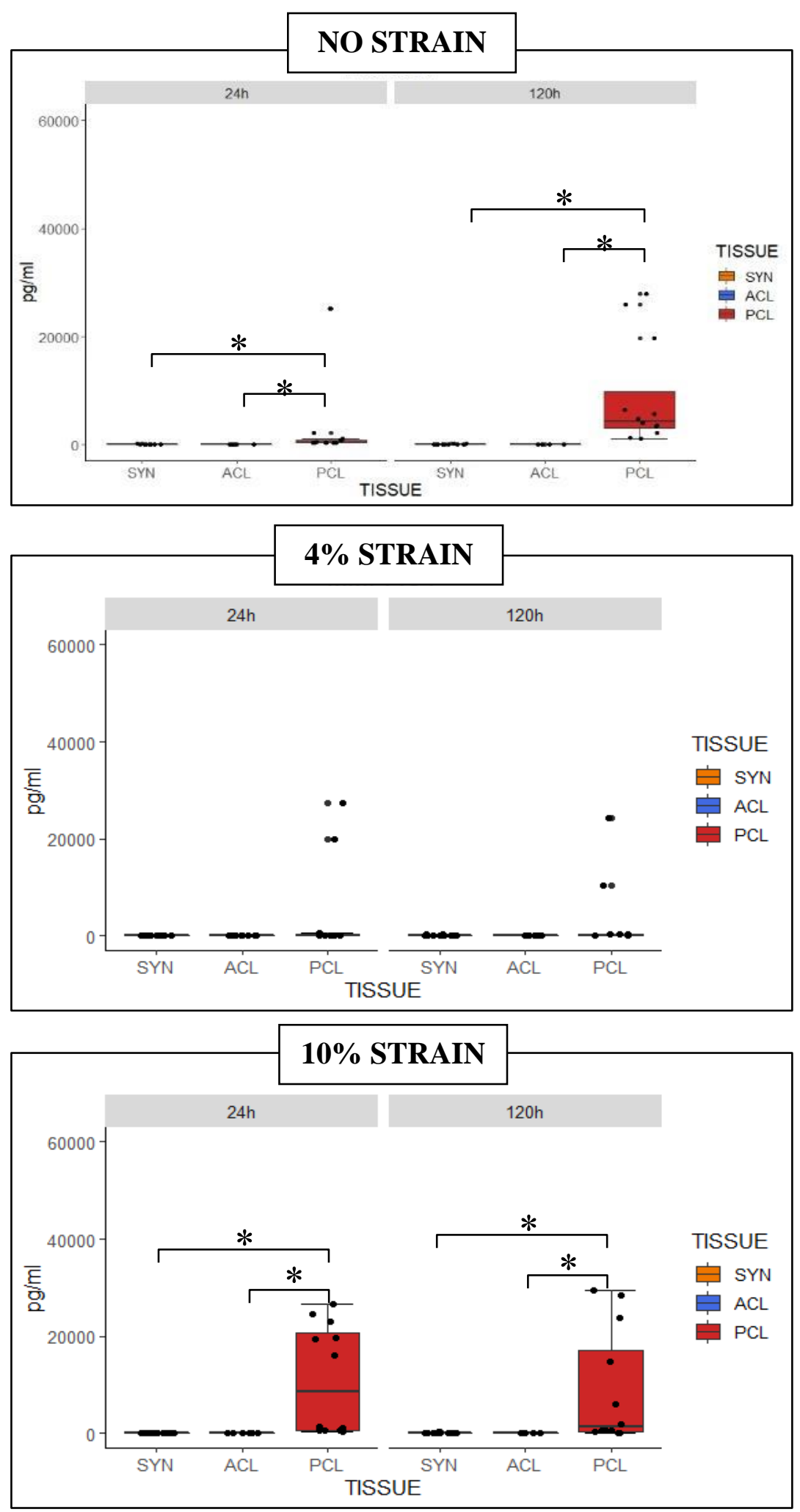

Figure 4.20. Concentration of MMP Activity (MMPACT) in the media. * significant difference between tissues at 24 and 120 hours of culture. SYN: Synovium; ACL: Anterior cruciate ligament, PCL: Posterior cruciate ligament 

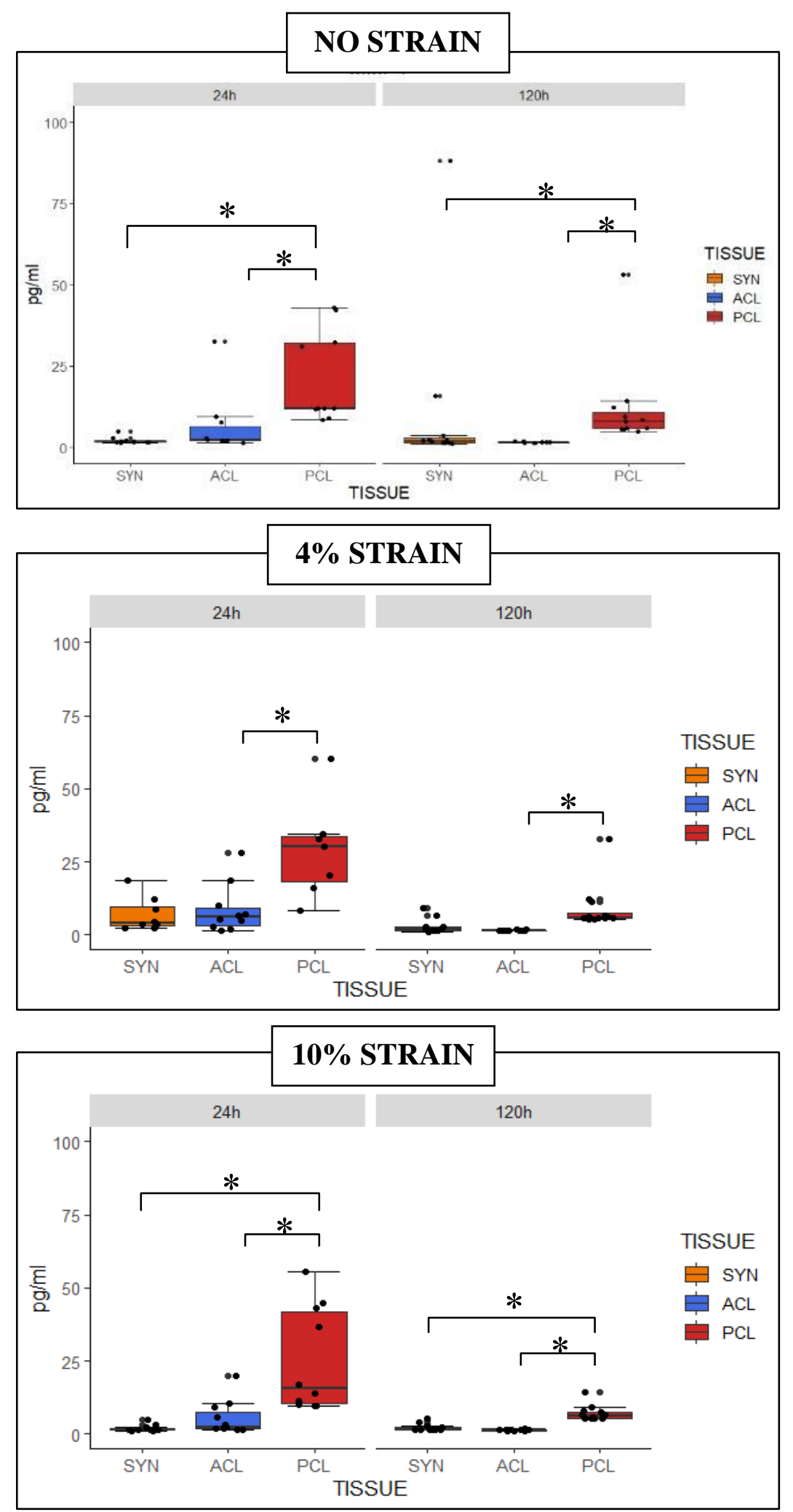

Figure 4.21. Concentration of MMP-1 released to the media. * significant difference between tissues at 24 and 120 hours of culture. SYN: Synovium; ACL: Anterior cruciate ligament, PCL: Posterior cruciate ligament 

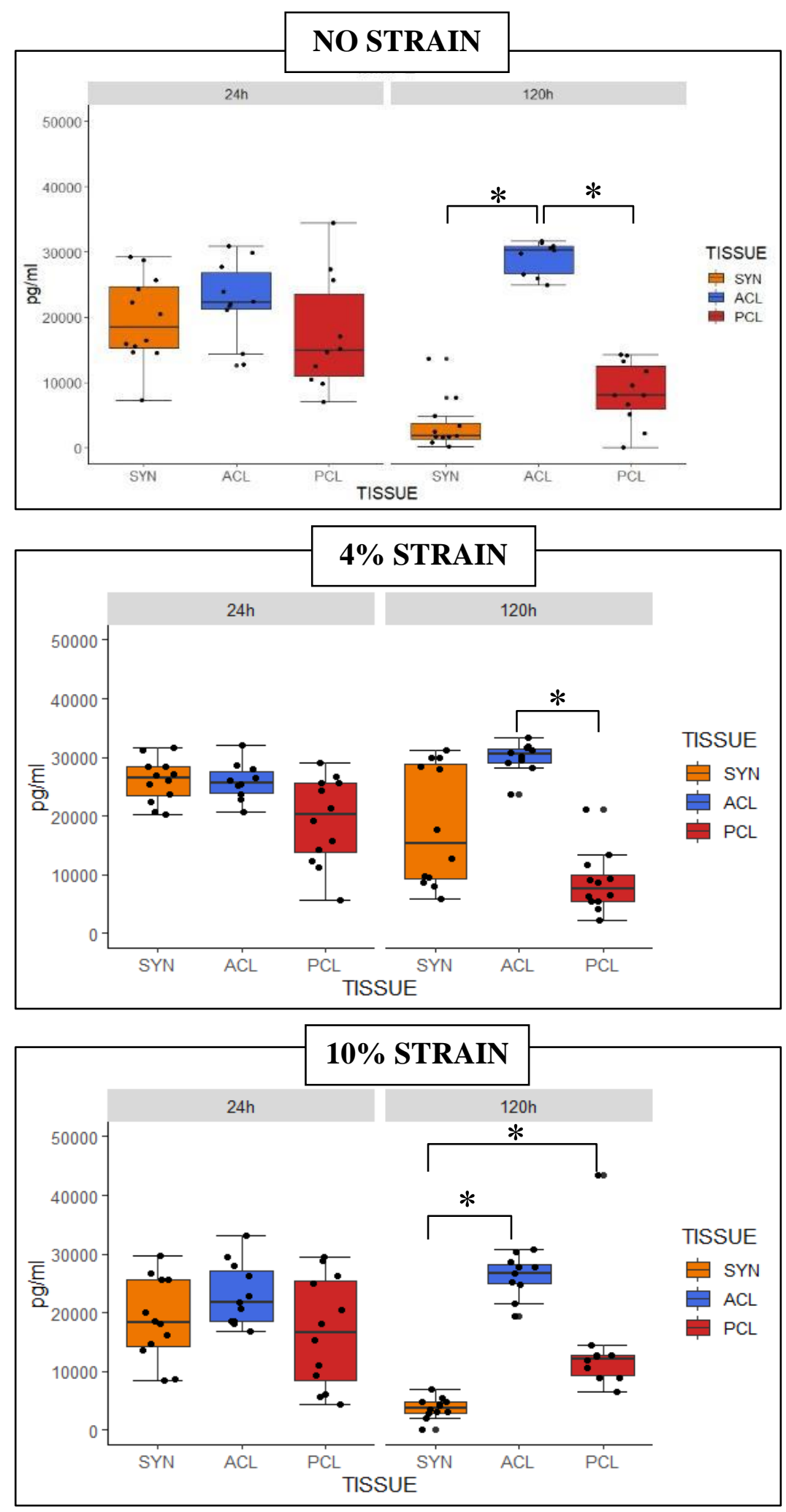

Figure 4.22. Concentration of MMP-2 released to the media. * significant difference between tissues at 24 and 120 hours of culture. SYN: Synovium; ACL: Anterior cruciate ligament, PCL: Posterior cruciate ligament 

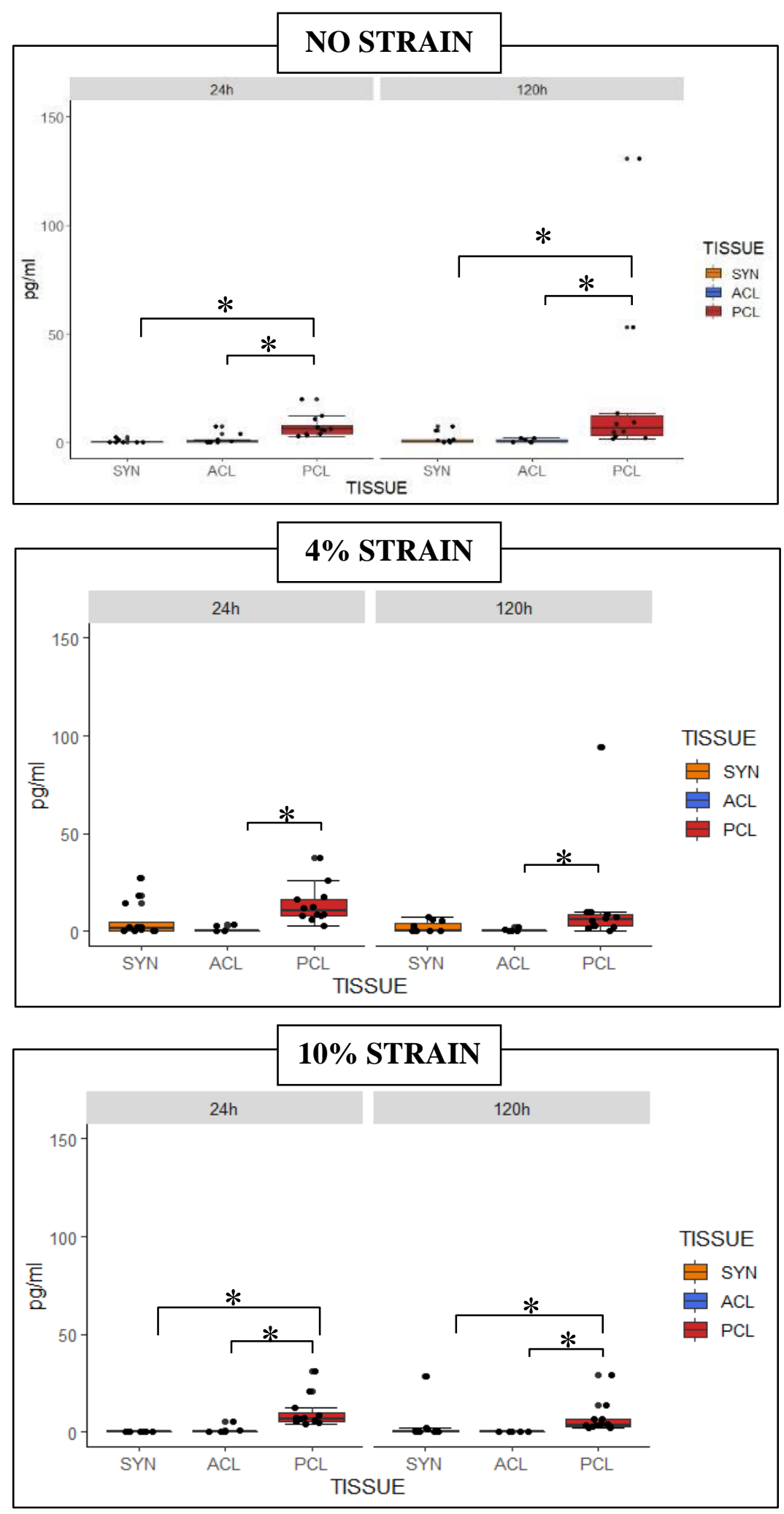

Figure 4.23. Concentration of MMP-3 released to the media. * significant difference between tissues at 24 and 120 hours of culture. SYN: Synovium; ACL: Anterior cruciate ligament, PCL: Posterior cruciate ligament 


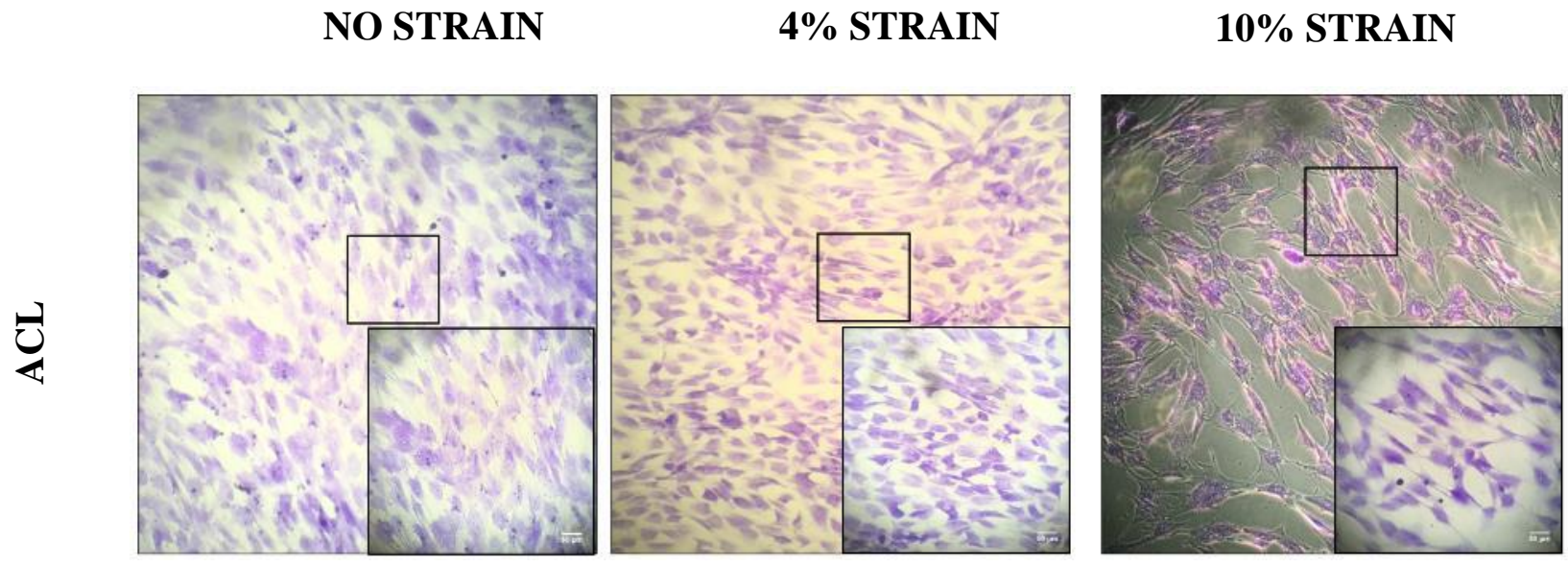

Figure 4.24. Anterior cruciate Ligament (ACL) fibroblasts after being stretched for 120 hours.

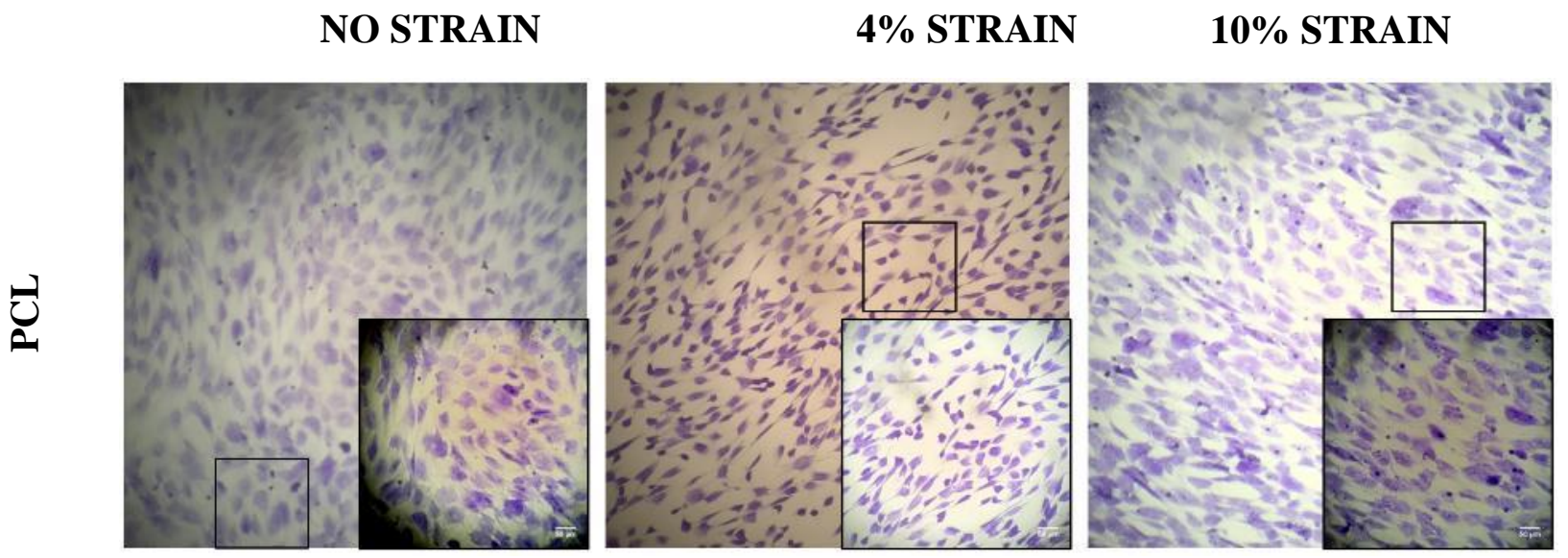

Figure 4.25. Posterior cruciate Ligament (PCL) fibroblasts after being stretched for 120 hours. 


\section{CHAPTER 5}

\section{SEX DIFFERENCES IN ANTERIOR CRUCIATE LIGAMENT TENDON GRAFT RESPONSE TO MECHANICAL STRAIN}

\section{Introduction:}

Graft choice is one of the main topics of discussion between knee surgeons in regards of anterior cruciate ligament (ACL) reconstruction. ${ }^{1}$ The ideal graft must possess good strength to allow accelerated rehabilitation, have no donor site morbidity, be readily available and heals and incorporates quickly into the bone tunnel ${ }^{2}$. Several factors have been associated with poor outcomes or re-injury after ACL reconstruction including poor surgical technique, (inappropriate position of the bone tunnel, inadequate graft pre-tension, improper graft fixation), non-adequate biomechanical factors, failure of graft incorporation, and re-injury due to trauma. ${ }^{1 ; 3-5}$ However, the effect of sex on ACL reconstruction surgery is unknown.

There are known sex disparities in ACL injury and healing after surgery. ACL injury is two to five times more common in female athletes than in their male counterparts. ${ }^{6 ; 7}$ Main reasons for increased female ACL rupture rates include increased femoral intercondylar notch height and plateau slope, decreased condylar width and notch angle, altered kinematic patterns and neuromuscular control showing lower limb alignment, smaller flexion angles, greater hip internal rotation angles, lower gluteus maximus activation and greater quadriceps-to-hamstring ratio activation in females compared to males. ${ }^{8-13}$ Similarly, females also show differences in intrinsic ACL material properties compared to males including decreased length, area and volume which in turn results in lower ultimate

stress and stiffness. ${ }^{8 ; 14}$ Additionally, the effect of female hormones and hormonal variation 
has been suggested as a possible cause for increased laxity and consequent rupture of the ACL in females. ${ }^{15-17}$ There is also ongoing debate whether sex is an adverse risk factor for patient outcomes after ACL surgery. While Thompson et al., (2016) showed increased ACL graft rupture risk associated to male sex, Tan et al., (2015) showed increased relative risk of revision surgery in females. ${ }^{18 ;} 19$ Therefore, the effect of sex on the probability of graft failure in ACL reconstruction is an active research topic.

In addition to sex, graft choice may impact success after ACL surgery. The biological differences of various tendon autografts used for ACL reconstruction are not well understood. Early ACL graft healing and remodeling require a fine balance between extracellular matrix (ECM) degradation and synthesis. A precise inflammatory response is also likely necessary to drive an effective healing response. ${ }^{20-22}$ Evidence provided by human and animal studies on ACL healing indicates that the early biological environment likely play an important role in ACL graft maturation, and that the metabolism of the graft is further modified by biomechanical cues. ${ }^{21 ; 23}$ Tensile forces can alter responses of ligament fibroblasts to various stimuli, including sex hormone regulation. ${ }^{24}$ However, it is not known if the sex of the patient is associated with significant differences in the cell's response to tensile forces. Further, the complex interplay between the mechanical environment of specific tissues used for ACL reconstruction and sex are still poorly understood. The objective of the current study is to determine if there are sex related differences in the biological responses of fibroblasts obtained from common ACL autografts to varying levels of mechanical strain. We aimed to evaluate the differences between male and female tendon graft-derived fibroblasts to different levels of cyclic strains that simulate clinical conditions. We hypothesized that male ACL graft fibroblasts 
from quadriceps tendon (QT), patellar tendon (PT), and hamstring tendon (HT) will exhibit significantly lower degradative and inflammatory levels compared to female autograft fibroblasts.

\section{Materials and methods:}

Tissue processing and culture

Male (7 QT, 9 PT, $5 \mathrm{HT}$ ) and female (12QT, 12PT, 9HT) tissues were harvested from skeletally mature dogs (9 and 12 respectively) euthanatized for studies unrelated to the current work. Tissues were aseptically minced into $0.5-1 \mathrm{~cm}^{2}$ pieces and digested overnight in Type 1A Clostridium histolyticum collagenase solution (Sigma-Aldrich, St. Louis, MO, USA) at a concentration of $0.5 \mathrm{mg} / \mathrm{mL}$. The aliquot was then suspended with nutritional media (Gibco DMEM, Thermo Fisher Scientific, Waltham, MA, USA) containing 10\% FBS, $0.002 \%$ Penicillin, $100 \mu \mathrm{g} / \mathrm{mL}$ Streptomycin, $25 \mu \mathrm{g} / \mathrm{mL}$ Amphotericin B, 0.002\% L-Ascorbate and 0.01\% L-glutamine (Sigma Chemical Co, St. Louis, MO), cultured in T75 flasks (TPP Techno Plastic Products AG, Trasadingen, Switzerland) and incubated at $5 \% \mathrm{CO}_{2}, 37^{\circ} \mathrm{C}$, and $95 \%$ humidity.

\section{Biaxial mechanical stress}

Once confluent, passage one cells were exposed to TrypLE express (Invitrogen, Carlsbad, CA) for five minutes, then resuspended and seeded $\left(1 \times 10^{5}\right.$ cells/well $)$ in Collagen Type I-coated BioFlex ${ }^{\circledR}$ plates (Flexcell International, NC, USA). Cells were incubated for an additional $48 \mathrm{~h}$ before strain was applied. Then, nutritional culture media was replaced with $0.5 \%$ FBS media for the remaining five days of culture. Fibroblasts were subjected to continuous mechanical stimulation (2-s strain and 10-s relaxation at a $0.5 \mathrm{~Hz}$ frequency) with a biaxial sinusoidal waveform with three different elongation strains (mechanical 
strain deprivation- $0 \%$, physiologic strain- $4 \%$, or supraphysiological strain-10\%) ${ }^{25}$ for 5 days using the Flexcell FX-4000T strain system (Flexcell International, NC, USA) (Fig. $5.1)$.

Biomarker assays

Media was changed every $24 \mathrm{~h}$ and stored at $-20^{\circ} \mathrm{C}$ until analysis. Media from 24 and 120 hours of culture were assessed for various biomarkers. Proteoglycan (GAG) was assessed using the DMMB assay as previously described. ${ }^{26}$ The concentration of prostaglandin $\mathrm{E}_{2}$ (PGE2) (Cayman Chemical, Ann Arbor, MI, USA), cytokines (IL-6, IL-8, KC and MCP1) (Millipore, Billerica, MA, USA), total matrix metalloproteinase (MMP) activity (SensoLyte 520 generic MMP assay, Anaspec, Inc., Fremont, CA), and MMP production (MMP-1, MMP-2 and MMP-3) (R\&D Systems, Minneapolis, MN, USA), were all assessed using commercially available assays according to the manufacturer's protocol. At the end of the strain protocol (120h), cell viability analysis was performed using the resazurin assay (Sigma Aldrich, Saint Louis, MO).

\section{Statistical Analysis}

For statistical analysis, normal distribution was assessed by Shapiro-Wilk test. Because data were not normally distributed, non-parametric tests were used. Differences between male and female dogs were assessed with Mann-Whitney Test at 24 and 120 hours (R Core Team (2019), R version 3.6.2 Vienna, Austria). Comparisons among strains and tissue types were performed using Kruskal-Wallis test and Dunn's test posthoc. Results were reported as median \pm interquartile range (IQR). Significance levels were set at $\mathrm{p}<0.05$. 


\section{Results}

Concentration of PGE2 was significantly increased in all female tendon fibroblasts when mechanical strain was applied but decreased when subjected to mechanical strain deprivation at $24 \mathrm{~h}$ in QT and PT fibroblasts. Additionally, at 120h of supraphysiological strain, production of PGE2 significantly increased in female QT and PT fibroblasts. Similarly, mechanical strain deprivation elicited an increased production of PGE in male PT and HT fibroblasts at 120h (Fig. 5.2).

Cytokine concentration in the culture media tended to significantly increase in female QT and PT fibroblasts at $4 \%$ strain and $10 \%$ strain groups at $24 \mathrm{~h}$. Also, female HT fibroblasts exhibited significantly more production of IL-8 than males at $24 \mathrm{~h}$ of no strain and $10 \%$ strain. Additionally, only female QT stretched at supraphysiological strain produced significantly higher levels of IL-8 at 120h compared to male QT fibroblasts (Fig. 5.3). The production of the chemokine $\mathrm{KC}$ was consistently increased in female QT fibroblasts compared to male fibroblasts at all strains and timepoints. Moreover, female PT also showed an increased KC production compared to male fibroblasts at all strain at $24 \mathrm{~h}$. Interestingly, female and male $\mathrm{HT}$ fibroblastic production of $\mathrm{KC}$ was not significantly different between males and females in response to strain with the exception of mechanical strain deprivation at $24 \mathrm{~h}$ showing increased levels of $\mathrm{KC}$ in female $\mathrm{HT}$ compared to male fibroblasts. Moreover, persistent increase in KC after $120 \mathrm{~h}$ was only observed in female QT fibroblasts at all strain levels (Fig. 5.4).

MCP-1 production significantly increased in female QT fibroblasts compared to males at all strain levels at $24 \mathrm{~h}$ of culture. Moreover, female PT fibroblasts exhibited a significant increase in MCP-1 production at 4\% strain and 10\% strain at 24h. Furthermore, female 
HT fibroblasts showed significantly increased production of MCP-1 at mechanical strain deprivation at 24h. Additionally, only female QT fibroblasts exhibited a sustained increase in MCP-1 production after $120 \mathrm{~h}$ of culture when fibroblasts were loaded at $4 \%$ and $10 \%$ strain. Moreover, HT and PT did not show significant differences between male and females at $120 \mathrm{~h}$ of culture (Fig. 5.5).

When evaluating production of extracellular matrix related markers, we found that GAG concentration increased in the culture media of female QT and PT fibroblasts at all strain levels at 24h. Female HT fibroblasts only increased the production of GAG when subjected to supraphysiological strain at $24 \mathrm{~h}$. Moreover, only female QT fibroblasts stretched at $10 \%$ strain maintained increased GAG production compared to males after 120h of culture (Fig. 5.6).

MMP activity (MMPACT) was found higher in male QT fibroblasts in no strain and 10\% strain groups at $24 \mathrm{~h}$ compared to females. Male PT fibroblasts showed significantly higher levels of MMPACT compared to females in the $10 \%$ strain group at $24 \mathrm{~h}$. Moreover, male HT fibroblasts showed a significantly higher production of MMPACT at mechanical strain deprivation but decreased at physiological strain relative to females at 24h. Interestingly, male QT fibroblasts maintained an increase in MMPACT at all strains, after 120h of culture. Additionally, male PT and HT fibroblasts showed a significantly higher level of MMPACT compared to females at abnormal loads, no strain and 10\% strain groups at $120 \mathrm{~h}$ of culture (Fig. 5.7).

MMP-2 production increased in male QT fibroblasts compared to females at supraphysiological strain at 24h. Additionally, at 120h, male QT fibroblasts showed a significant increase in MMP-2 production compared to females at all strain levels. 
Interestingly, while male QT fibroblasts increased MMP-2 levels with respect to females at all strains, PT and HT did not exhibited significant differences between male and females at any timepoint (Fig. 5.8).

\section{Strain differences in male dogs:}

Metabolic activity was significantly higher in male QT fibroblasts at 10\% strain compared to $4 \%$ strain. However, male PT and HT fibroblasts did not exhibit significant differences between strains (Fig. 5.9).

The production of the inflammatory biomarker PGE2 was significantly increased in male QT and HT fibroblasts at no strain compared to $4 \%$ strain at 24h. However, all male graft fibroblasts did not show differences in PGE-2 production in response to strain at 120h (Fig. 5.10). Additionally, cytokine and chemokine production were not significantly affected by strain in male fibroblasts (Fig, 5.11-5.13).

The extracellular matrix related biomarker GAG did not exhibit significant changes with respect to strain level in any of the fibroblast types at $24 \mathrm{~h}$. However, significantly lower GAG content was seen in male QT fibroblasts at supraphysiological strain levels compared to mechanical strain deprivation at 120h of culture. PT and HT fibroblasts obtained from male dogs did not show significant differences in GAG content in response to strain at 120h (Fig. 5.14). Moreover, neither MMPACT nor MMP-2 production were affected by strain level in any of the tendon fibroblasts at any timepoint (Fig. 5.15-5.16).

When comparing differences among male fibroblastic tissue sources, fibroblasts did not differ among each other in any of the biomarkers evaluated at any strain or timepoint (5.175.24). However, there was a trend towards decreased production of MMP-2 arising from PT fibroblasts at 120h of culture (Fig. 5.24). 


\section{Discussion:}

While it is generally accepted that there are sex differences in ACL injury risk, the effect of sex on outcomes after surgery is less certain. Although the hormonal effects of estrogen on native ACL properties have been widely investigated, information on effect of hormone on ACL tendon grafts is lacking. ${ }^{27}$ Previous studies have indicated that women's tendons tend to have a lower structural quality, less dry mass, express higher level of type III collagen, and have decreased tendon stiffness and resistance to deformation under loading. ${ }^{28}$ Additionally, estrogen has an inhibiting effect on tendon and ligament collagen synthesis. ${ }^{17 ;} 29$ In this study, we demonstrated that tendon graft fibroblasts exhibited different biologic responses as a function of sex, tissue source, and level of mechanical stress, independent of hormonal stimulation.

Magnusson et al., (2007) showed that men and women have different adaptability to tendon loading. ${ }^{30}$ Specifically, women have a decreased tendon hypertrophy response to habitual training, decreased tendon collagen synthesis rate following exercise, a diminished rate of tendon collagen synthesis due to higher levels of estradiol stimulation, and lower mechanical properties. ${ }^{30}$ Moreover, authors also showed that collagen fascicles from patellar tendons obtained from men showed greater ultimate stress than those from women. Circi et al., (2009) showed that the inflammatory response is greater in the presence of endogenous estrogen. ${ }^{31}$ Our results indicate that female fibroblasts may also have an innate higher inflammatory response that is independent of estrogen stimulation, at least for PT fibroblasts. In this study female PT fibroblasts produce higher concentrations of PGE2, cytokines (IL-6, IL-8), and chemokines (KC and MCP-1) than males in a strain dependent manner. Female tendon fibroblasts showed decrease metabolic activity when compared to 
male dogs regardless of the strain levels. This dampened cellular metabolism among female graft cells might explain the decrease in mechanical properties of female tendon grafts. Interestingly, tendon fibroblasts from male dogs tended to show a significant increase in MMP activity compared to females. Further, these responses seem to be tendon specific. Male QT fibroblasts seem to be more sensitive to strain as indicated by an increased production of MMP-2 compared to females, while PT and HT fibroblasts did not show significant differences in MMP-2 production.

Another factor that could influence poor outcomes after ACL reconstruction in females is the tendon to bone integration. It has been shown that the withdrawal of estrogen can lead to decreased bone mineral density and osteoporosis. ${ }^{36-38}$ Moreover, male sex hormone dihydrotestosterone (DHT) has been found to stimulate bone formation via increased alkaline phosphatase (AP) activity and PICP production. Additionally, DHT may enhance anchorage of osteoblastic cells when subjected to cyclic strain through increased expression of fibronectin. ${ }^{39}$ Anchorage of bone cells to the extracellular matrix is a prerequisite for mechanotransduction. Any disruption in the continuity in the bone-tendon junction could lead to impaired healing and higher risk of failure after ACL reconstruction. Our findings support this theory because of the increased MMP activity and MMP-2 production seen in males compared to females, which may be indicative of a greater remodeling process with male tendon grafts. We believe that the increased remodeling activity in male tendons could potentially lead to better bone to tendon integration within the bone tunnel. Further work to evaluate bone turnover markers in vitro would be necessary in order to establish this relationship. 
Previous studies have indicated that inflammatory responses are attenuated in females during pregnancy, and that cell proliferation and inflammatory responses are greater in the presence of endogenous estrogen. ${ }^{31 ; 40 ; 41}$ Our findings suggest that ECM remodeling may be increased in male autografts as indicated by the increased level of MMP activity during culture. Additionally, male graft fibroblasts were more metabolically active than females indicating that application of tension load tend to increase cell density in male tenocytes. Furthermore, female autograft fibroblasts tend to exhibit higher GAG production in high strain conditions, which may be indicative of an injury response. Female autograft fibroblasts also produced higher levels of inflammatory mediators under all strain conditions. This may explain the clinical phenomenon that females tend to report higher pain levels and lower patient-reported outcomes relative to males following ACL surgery. ${ }^{19 ;} 42$ Our collective findings suggest that there are likely sex differences in graft ligamentization after reconstruction which may contribute to differences seen in outcomes after ACL surgery. Moreover, Clatworthy et al., (1999) found that tunnel widening is increased when hamstring autografts are used, which could be the consequence of the release of cytokines that may lead to increased osteolysis. ${ }^{43}$ Hence, HT might have delayed healing and osteointegration in females due their increased cytokine production (IL-8, KC, MCP-1) compared to males at $24 \mathrm{~h}$ when subjected to mechanical strain deprivation.

Some limitations to this work were identified. First, fibroblasts in culture did not produce enough collagen levels to be detected by the hydroxyproline assay. Consequently, interpretation of ECM remodeling and healing need to be based only on the results obtained from MMPs and GAGs, missing an important piece of information for accurate healing analysis. Second, the monolayer-equibiaxial model utilized in this work, may not reflect 
motion in vivo due to the lack of interaction of forces such as shear stress and torsion. Additionally, our experiment only measured biomarker production for five days, therefore, biological processes that happen at a later timepoint were omitted. Hence, the production of biomarkers may be altered, especially ECM remodeling. Consequently, measuring collagen production using more sensitive assays is recommended. Moreover, the use of bioreactors that can apply rotational and shear stress along with tension load to whole tendon explants is suggested in order to replicate in vivo structures. Lastly, later timepoints that allow the assessment of metabolic responses after the initial inflammation period has occurred, may be useful to truly evaluate the effect of mechanical strain on healing responses of tendon grafts.

In summary, the data from this study provides evidence that biological properties of fibroblasts from common tendons used for ACL reconstruction vary significantly between males and females. Furthermore, graft tissue choice and level of mechanical stress may play a significant role in healing of ACL grafts in both, males and females based on the differential production of healing-related molecules such as MMPs and inflammatory mediators in response to tension load in vitro. Understanding how sex and mechanical stress affect the metabolism of the tendon graft may help optimize surgical outcomes by optimizing level of activity and ACL post-operative rehabilitation based on patient sex and ACL graft tissue choice. 


\section{REFERENCES}

1. Mehran N, Moutzouros VB, Bedi A. 2015. A Review of Current Graft Options for Anterior Cruciate Ligament Reconstruction. JBJS reviews 3.

2. Bottoni CR, Smith EL, Shaha J, et al. 2015. Autograft Versus Allograft Anterior Cruciate Ligament Reconstruction: A Prospective, Randomized Clinical Study With a Minimum 10-Year Follow-up. The American journal of sports medicine 43:2501-2509.

3. Dargel J, Gotter M, Mader K, et al. 2007. Biomechanics of the anterior cruciate ligament and implications for surgical reconstruction. Strategies in Trauma and Limb Reconstruction 2:1-12.

4. Menetrey J, Duthon VB, Laumonier T, et al. 2008. "Biological failure" of the anterior cruciate ligament graft. Knee surgery, sports traumatology, arthroscopy : official journal of the ESSKA 16:224-231.

5. Samitier G, Marcano AI, Alentorn-Geli E, et al. 2015. Failure of Anterior Cruciate Ligament Reconstruction. The archives of bone and joint surgery 3:220240.

6. Kiapour AM, Murray MM. 2014. Basic science of anterior cruciate ligament injury and repair. Bone Joint Res 3:20-31.

7. Arendt E, Dick R. 1995. Knee injury patterns among men and women in collegiate basketball and soccer. NCAA data and review of literature. The American journal of sports medicine 23:694-701.

8. Chandrashekar N, Slauterbeck J, Hashemi J. 2005. Sex-Based Differences in the Anthropometric Characteristics of the Anterior Cruciate Ligament and Its 
Relation to Intercondylar Notch Geometry. The American journal of sports medicine 33:1492-1498.

9. Stijak L, Herzog RF, Schai P. 2008. Is there an influence of the tibial slope of the lateral condyle on the ACL lesion? Knee Surgery, Sports Traumatology, Arthroscopy 16:112-117.

10. Lephart SM, Ferris CM, Riemann BL, et al. 2002. Gender differences in strength and lower extremity kinematics during landing. Clinical orthopaedics and related research:162-169.

11. Dai B, Herman D, Liu H, et al. 2012. Prevention of ACL injury, part I: injury characteristics, risk factors, and loading mechanism. Research in sports medicine (Print) 20:180-197.

12. Sigward SM, Powers CM. 2006. The influence of gender on knee kinematics, kinetics and muscle activation patterns during side-step cutting. Clinical Biomechanics 21:41-48.

13. Hewett TE, Myer GD, Ford KR. 2004. Decrease in Neuromuscular Control About the Knee with Maturation in Female Athletes. JBJS 86:1601-1608.

14. Chandrashekar N, Mansouri H, Slauterbeck J, et al. 2006. Sex-based differences in the tensile properties of the human anterior cruciate ligament. Journal of biomechanics 39:2943-2950.

15. Wojtys EM, Huston LJ, Boynton MD, et al. 2002. The Effect of the Menstrual Cycle on Anterior Cruciate Ligament Injuries in Women as Determined by Hormone Levels. 30:182-188. 
16. Yu WD, Panossian V, Hatch JD, et al. 2001. Combined effects of estrogen and progesterone on the anterior cruciate ligament. Clinical orthopaedics and related research:268-281.

17. Liu SH, Al-Shaikh RA, Panossian V, et al. 1997. Estrogen affects the cellular metabolism of the anterior cruciate ligament. A potential explanation for female athletic injury. The American journal of sports medicine 25:704-709.

18. Thompson SM, Salmon LJ, Waller A, et al. 2016. Twenty-Year Outcome of a Longitudinal Prospective Evaluation of Isolated Endoscopic Anterior Cruciate Ligament Reconstruction With Patellar Tendon or Hamstring Autograft. The American journal of sports medicine 44:3083-3094.

19. Tan SH, Lau BP, Khin LW, et al. 2016. The Importance of Patient Sex in the Outcomes of Anterior Cruciate Ligament Reconstructions: A Systematic Review and Meta-analysis. The American journal of sports medicine 44:242-254.

20. Scheffler SU, Unterhauser FN, Weiler A. 2008. Graft remodeling and ligamentization after cruciate ligament reconstruction. Knee surgery, sports traumatology, arthroscopy : official journal of the ESSKA 16:834-842.

21. Claes S, Verdonk P, Forsyth R, et al. 2011. The "ligamentization" process in anterior cruciate ligament reconstruction: what happens to the human graft? A systematic review of the literature. The American journal of sports medicine 39:2476-2483.

22. Falconiero RP, DiStefano VJ, Cook TM. 1998. Revascularization and ligamentization of autogenous anterior cruciate ligament grafts in humans. Arthroscopy : the journal of arthroscopic \& related surgery : official publication 
of the Arthroscopy Association of North America and the International Arthroscopy Association 14:197-205.

23. Sun L, Qu L, Zhu R, et al. 2016. Effects of Mechanical Stretch on Cell Proliferation and Matrix Formation of Mesenchymal Stem Cell and Anterior Cruciate Ligament Fibroblast. Stem cells international 2016:9842075.

24. Lee CY, Liu X, Smith CL, et al. 2004. The combined regulation of estrogen and cyclic tension on fibroblast biosynthesis derived from anterior cruciate ligament. Matrix biology : journal of the International Society for Matrix Biology 23:323329.

25. Wang $\mathrm{T}$, Chen $\mathrm{P}$, Zheng M, et al. 2018. In vitro loading models for tendon mechanobiology. Journal of orthopaedic research : official publication of the Orthopaedic Research Society 36:566-575.

26. Farndale RW, Buttle DJ, Barrett AJ. 1986. Improved quantitation and discrimination of sulphated glycosaminoglycans by use of dimethylmethylene blue. Biochim Biophys Acta 883:173-177.

27. Lee C-Y, Smith CL, Zhang X, et al. 2004. Tensile forces attenuate estrogenstimulated collagen synthesis in the ACL. Biochemical and Biophysical Research Communications 317:1221-1225.

28. Carroll CC, Dickinson JM, Haus JM, et al. 2008. Influence of aging on the in vivo properties of human patellar tendon. Journal of applied physiology (Bethesda, Md : 1985) 105:1907-1915. 
29. Yu WD, Liu SH, Hatch JD, et al. 1999. Effect of estrogen on cellular metabolism of the human anterior cruciate ligament. Clinical orthopaedics and related research:229-238.

30. Magnusson SP, Hansen M, Langberg H, et al. 2007. The adaptability of tendon to loading differs in men and women. International journal of experimental pathology 88:237-240.

31. Circi E, Akpinar S, Balcik C, et al. 2009. Biomechanical and histological comparison of the influence of oestrogen deficient state on tendon healing potential in rats. Int Orthop 33:1461-1466.

32. Hsieh JL, Jou IM, Wu CL, et al. 2018. Estrogen and mechanical loading-related regulation of estrogen receptor- $\beta$ and apoptosis in tendinopathy. PLoS One 13:e0204603.

33. Oshiro W, Lou J, Xing X, et al. 2003. Flexor tendon healing in the rat: a histologic and gene expression study. The Journal of hand surgery 28:814-823.

34. Jones ER, Jones GC, Legerlotz K, et al. 2013. Cyclical strain modulates metalloprotease and matrix gene expression in human tenocytes via activation of TGFbeta. Biochim Biophys Acta 1833:2596-2607.

35. Zhang K, Asai S, Yu B, et al. 2015. IL-1beta irreversibly inhibits tenogenic differentiation and alters metabolism in injured tendon-derived progenitor cells in vitro. Biochem Biophys Res Commun 463:667-672.

36. Turner RT, Riggs BL, Spelsberg TC. 1994. Skeletal Effects of Estrogen*. Endocrine Reviews 15:275-300. 
37. Lindsay R, Hart DM, Aitken JM, et al. 1976. Long-term prevention of postmenopausal osteoporosis by oestrogen. Evidence for an increased bone mass after delayed onset of oestrogen treatment. Lancet 1:1038-1041.

38. Eriksen EF, Colvard DS, Berg NJ, et al. 1988. Evidence of estrogen receptors in normal human osteoblast-like cells. Science 241:84-86.

39. Liegibel UM, Sommer U, Tomakidi P, et al. 2002. Concerted action of androgens and mechanical strain shifts bone metabolism from high turnover into an osteoanabolic mode. The Journal of experimental medicine 196:1387-1392.

40. Goldstein SA, Armstrong TJ, Chaffin DB, et al. 1987. Analysis of cumulative strain in tendons and tendon sheaths. Journal of biomechanics 20:1-6.

41. Hart DA, Reno C. 1999. Pregnancy alters gene expression in normal synovium: influence of age and parity. The Journal of rheumatology 26:1775-1784.

42. Hamrin Senorski E, Svantesson E, Baldari A, et al. 2019. Factors that affect patient reported outcome after anterior cruciate ligament reconstruction-a systematic review of the Scandinavian knee ligament registers. Br J Sports Med $53: 410-417$.

43. Clatworthy MG, Annear P, Bulow JU, et al. 1999. Tunnel widening in anterior cruciate ligament reconstruction: a prospective evaluation of hamstring and patella tendon grafts. Knee surgery, sports traumatology, arthroscopy : official journal of the ESSKA 7:138-145. 
Figures

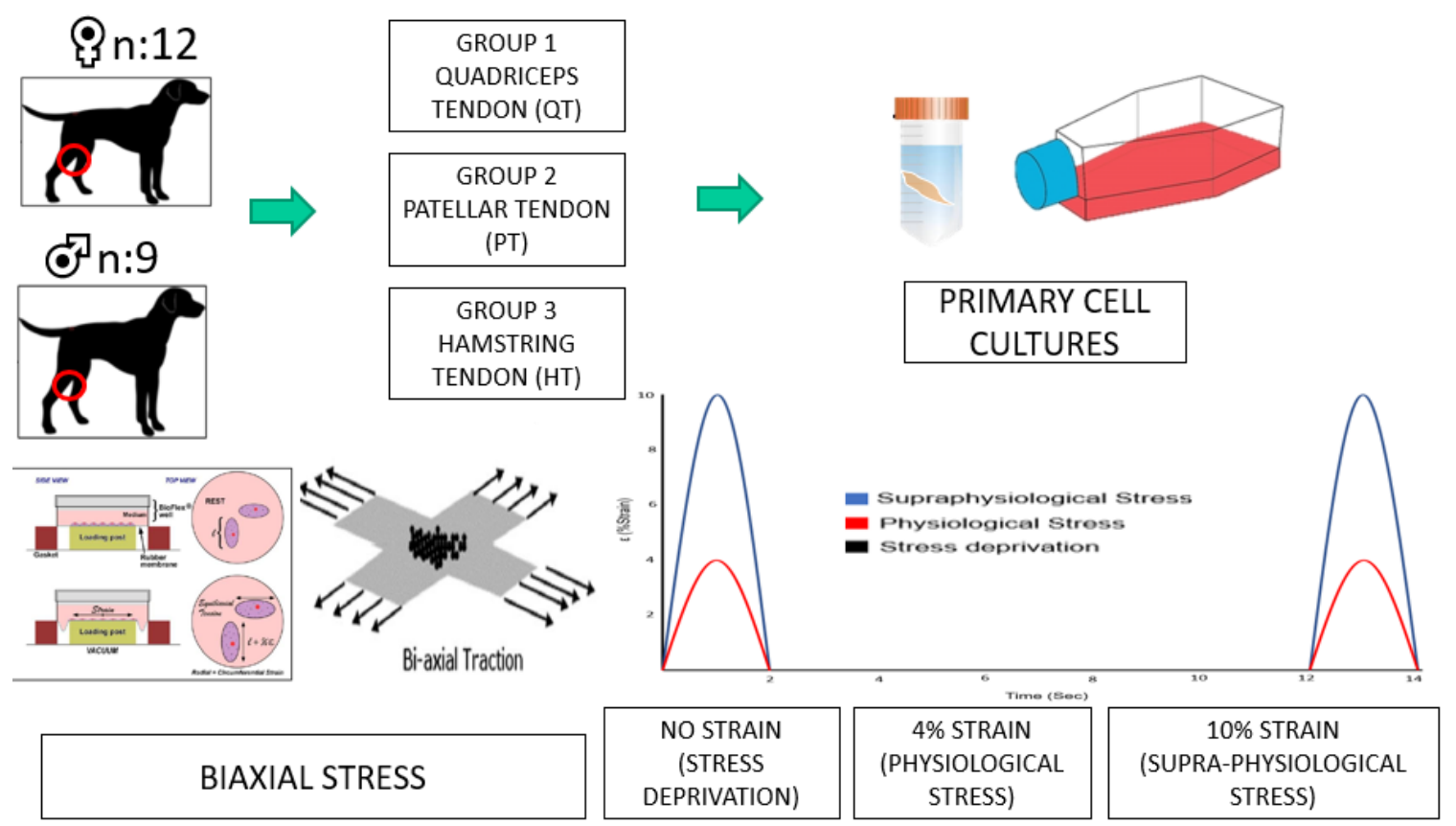

Figure 5.1. Experimental Design 

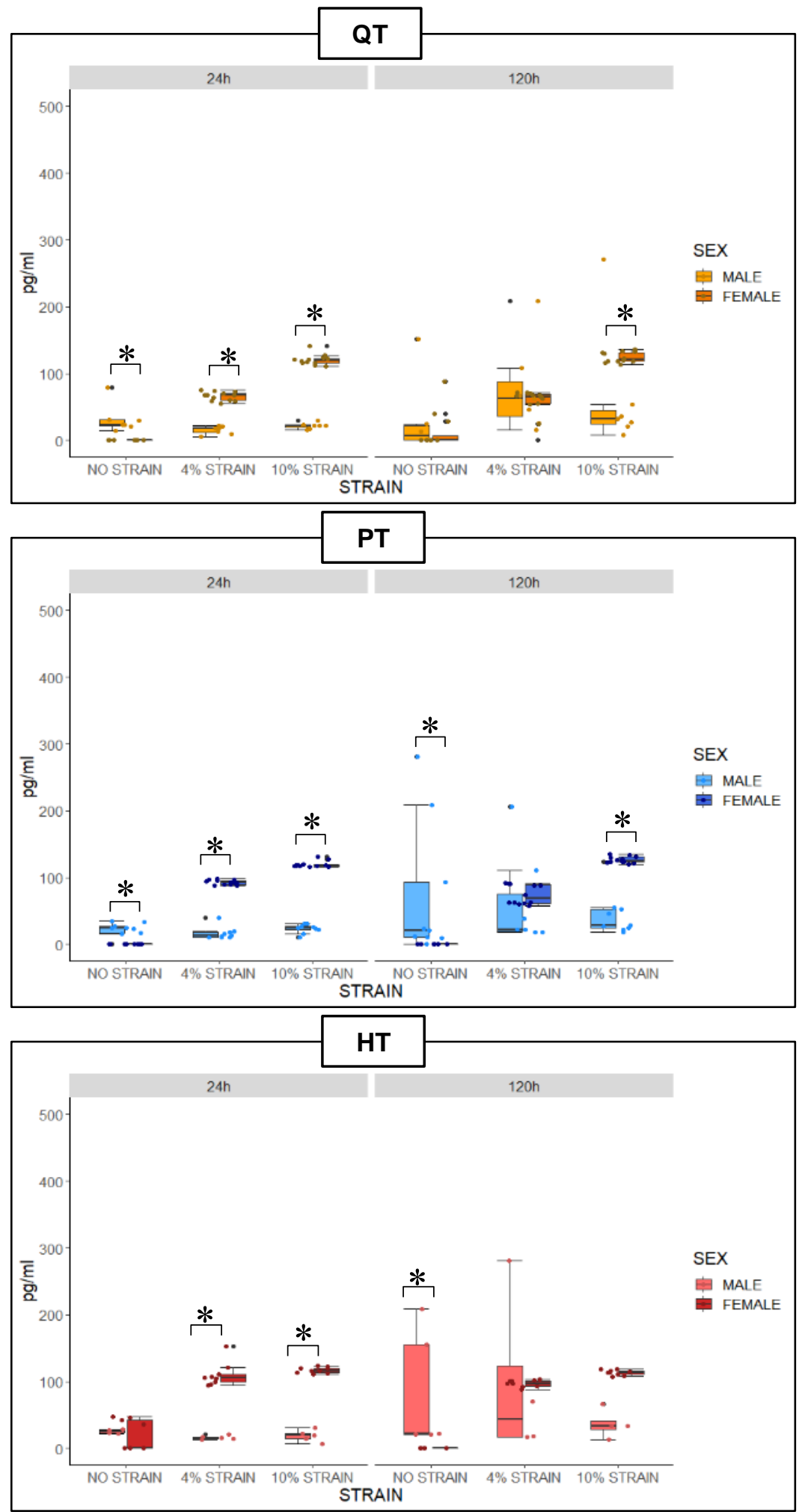

Figure 5.2. Concentration of prostaglandin E2 (PGE2) released to the media. * significant difference between males and females at 24 and 120 hours (Mann-Whitney test). QT: Quadriceps tendon; PT: Patellar tendon, HT: Hamstring tendon 

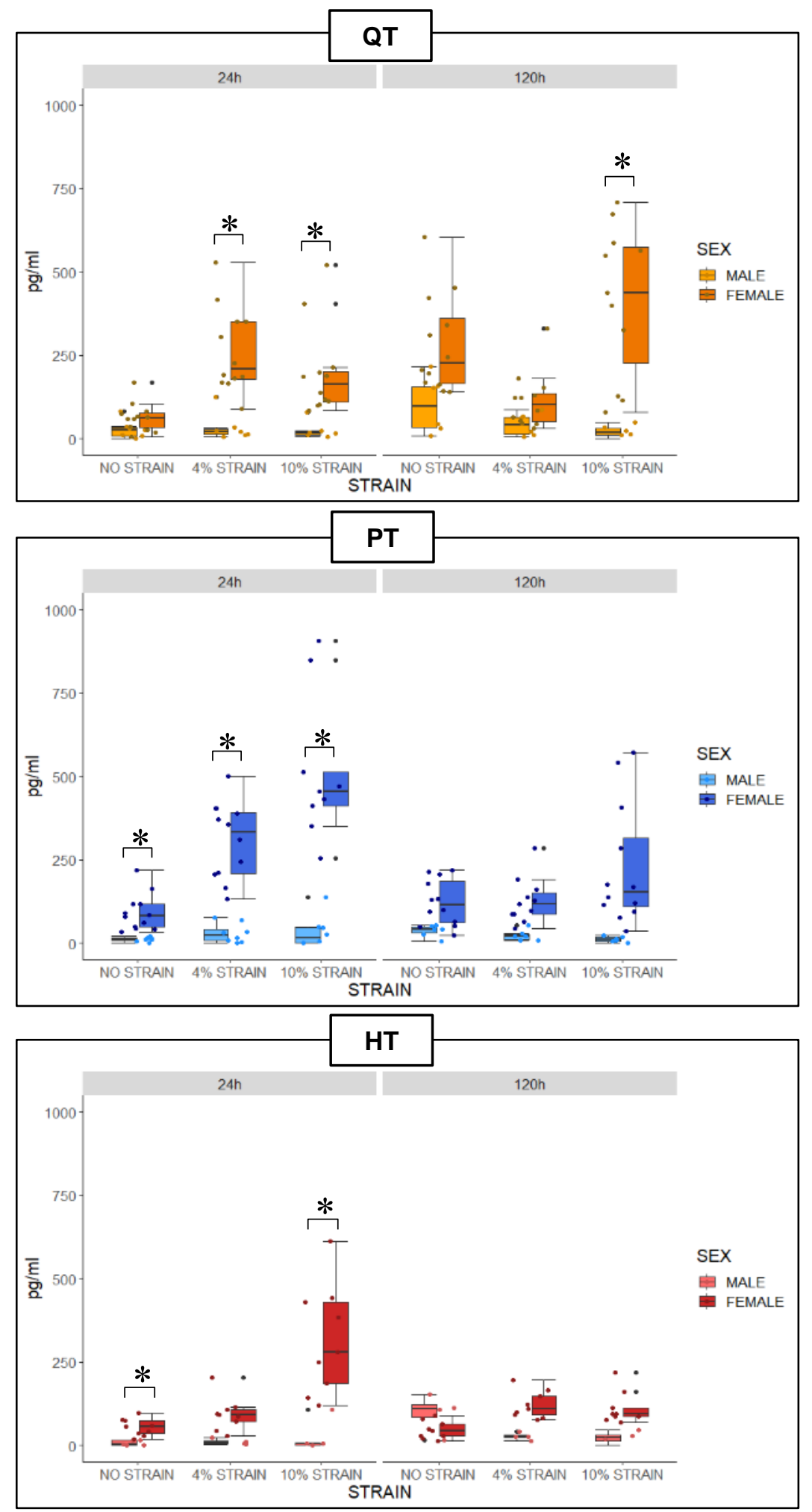

Figure 5.3. Concentration of intereukin- 8 (IL-8) released to the media. * significant difference between males and females at 24 and 120 hours (Mann-Whitney test). QT: Quadriceps tendon; PT: Patellar tendon, HT: Hamstring tendon 

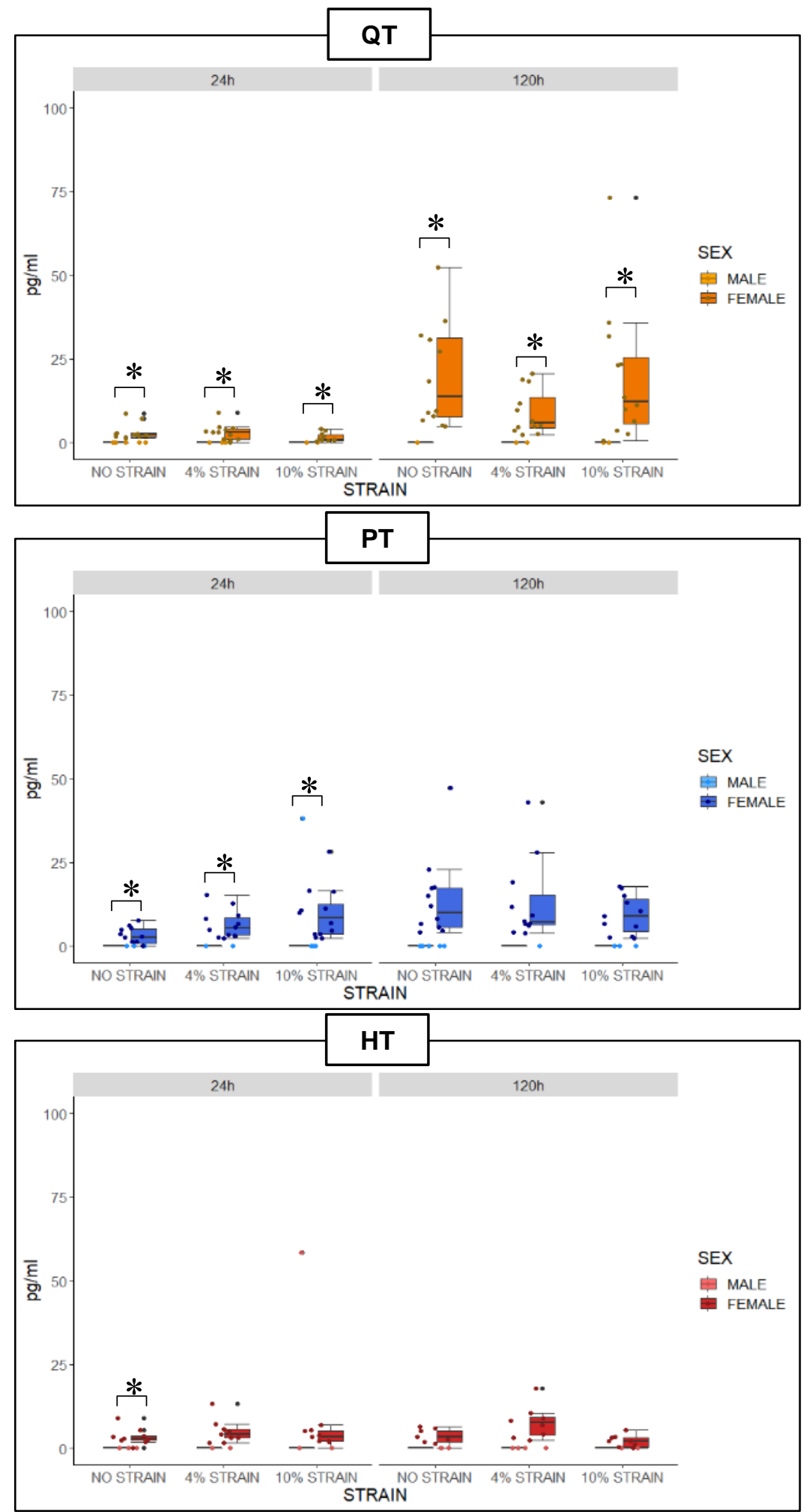

Figure 5.4. Concentration of keratinocyte chemoattractant protein $(\mathrm{KC})$ released to the media. * significant difference between males and females at 24 and 120 hours (Mann-Whitney test). QT: Quadriceps tendon; PT: Patellar tendon, HT: Hamstring tendon 

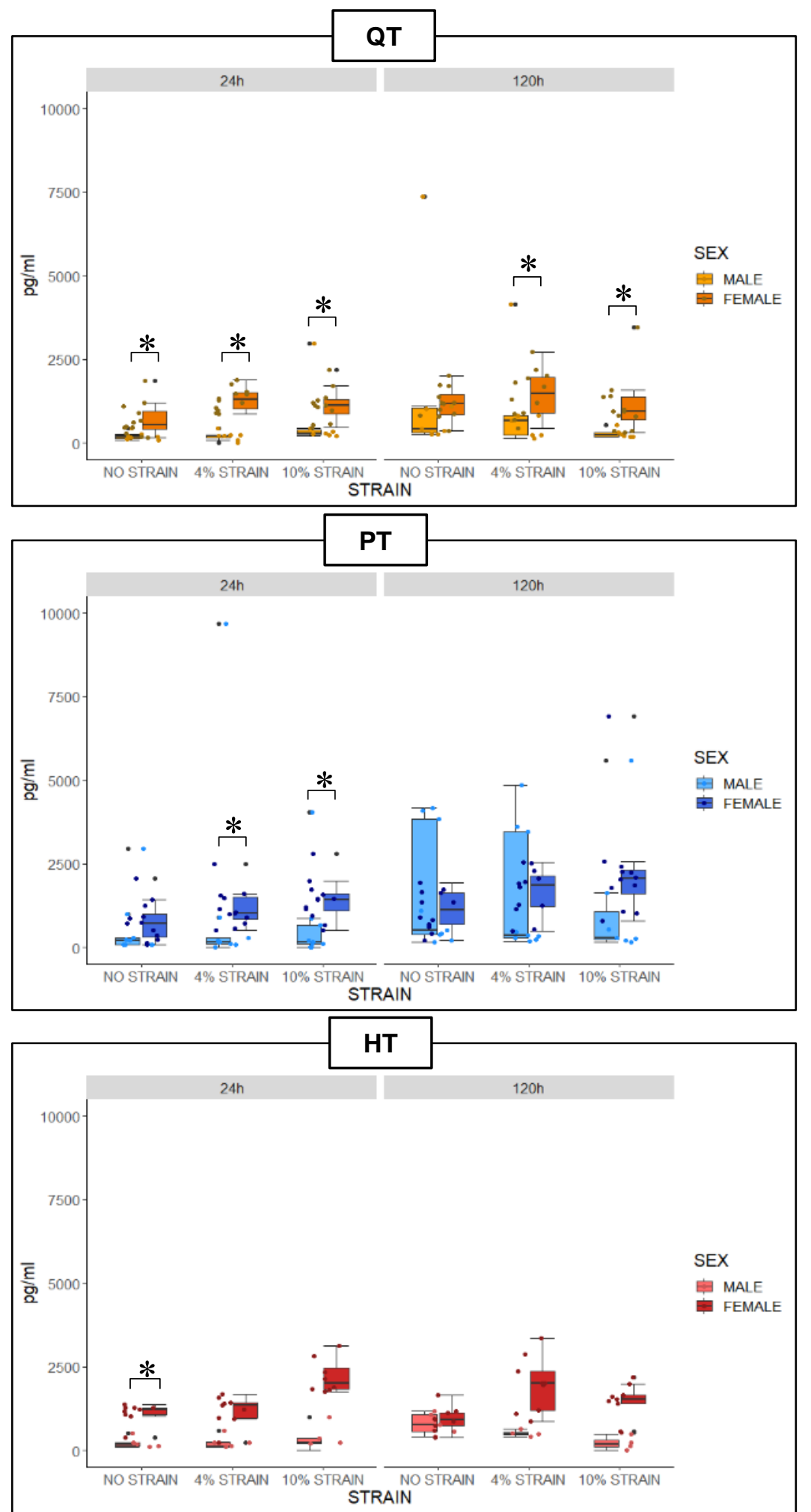

Figure 5.5. Concentration of monocyte chemoattractant protein-1 (MCP-1) released to the media. * significant difference between males and females at 24 and 120 hours (Mann-Whitney test). QT: Quadriceps tendon; PT: Patellar tendon, HT: Hamstring tendon 

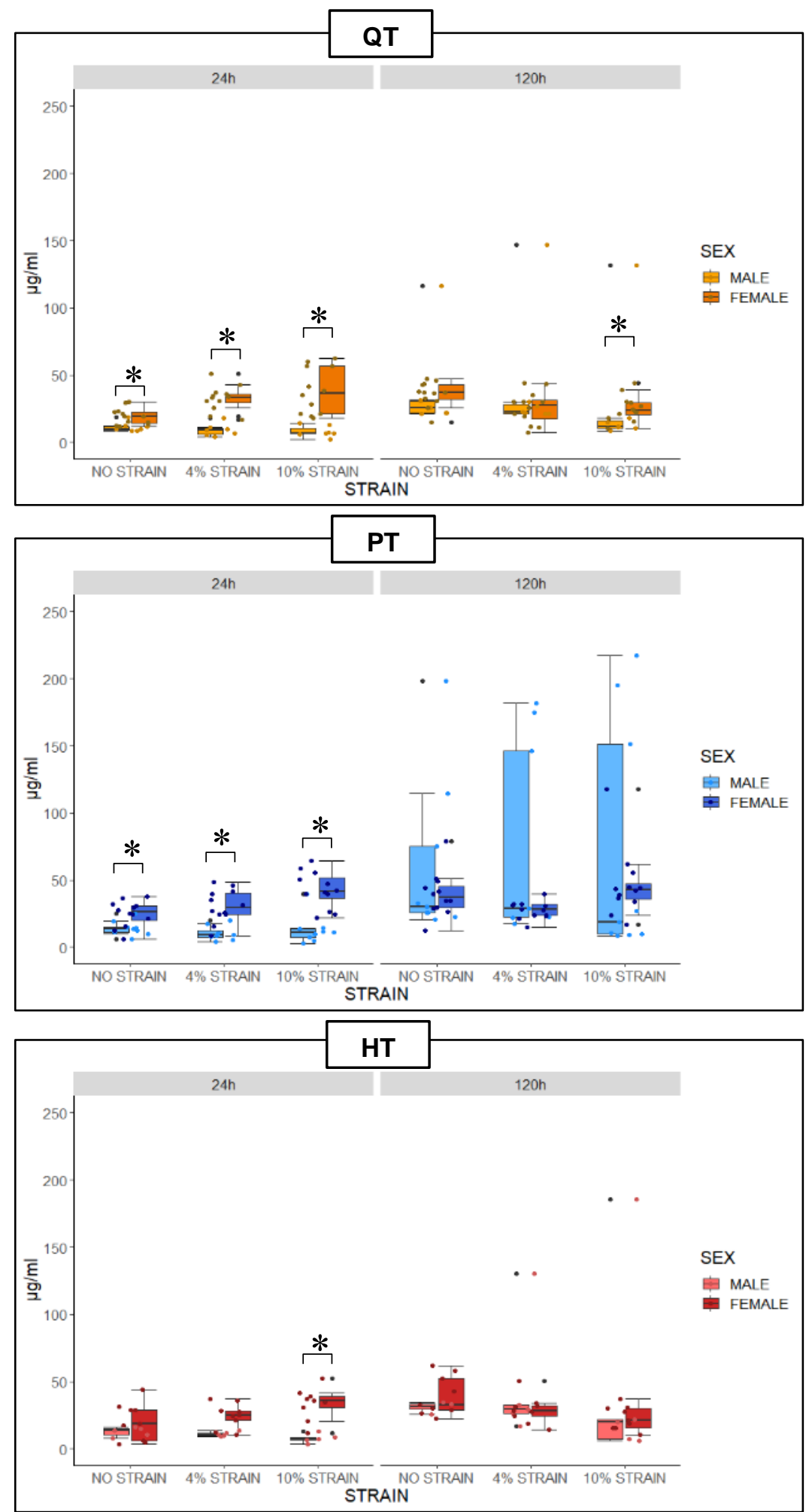

Figure 5.6. Concentration of Glycosaminoglycans (GAG) released to the media. * significant difference between males and females at 24 and 120 hours (Mann-Whitney test). QT: Quadriceps tendon; PT: Patellar tendon, HT: Hamstring tendon 

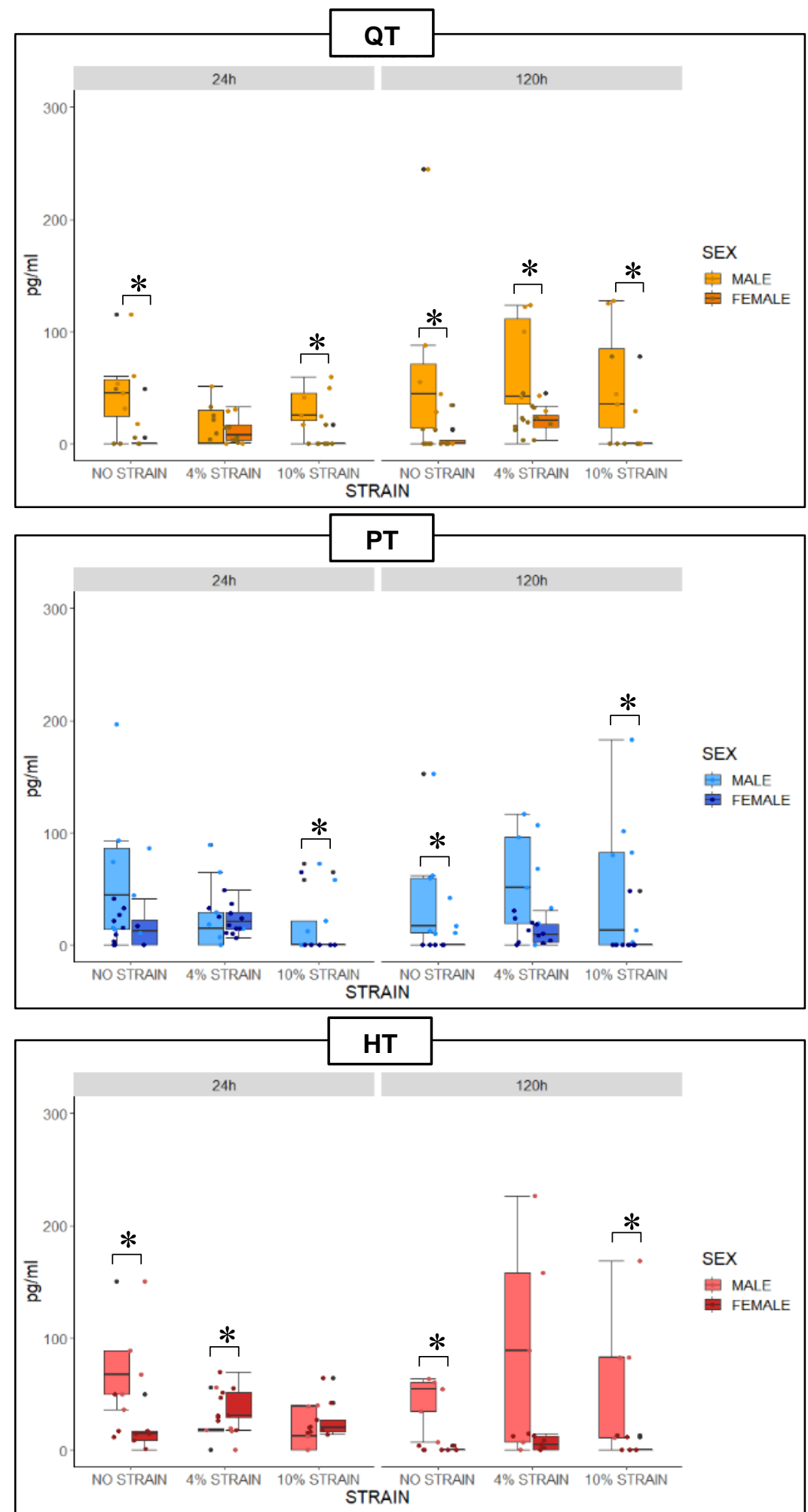

Figure 5.7. Concentration of total MMP activity (MMPACT) released to the media. * significant difference between males and females at 24 and 120 hours (Mann-Whitney test). QT: Quadriceps tendon; PT: Patellar tendon, HT: Hamstring tendon 

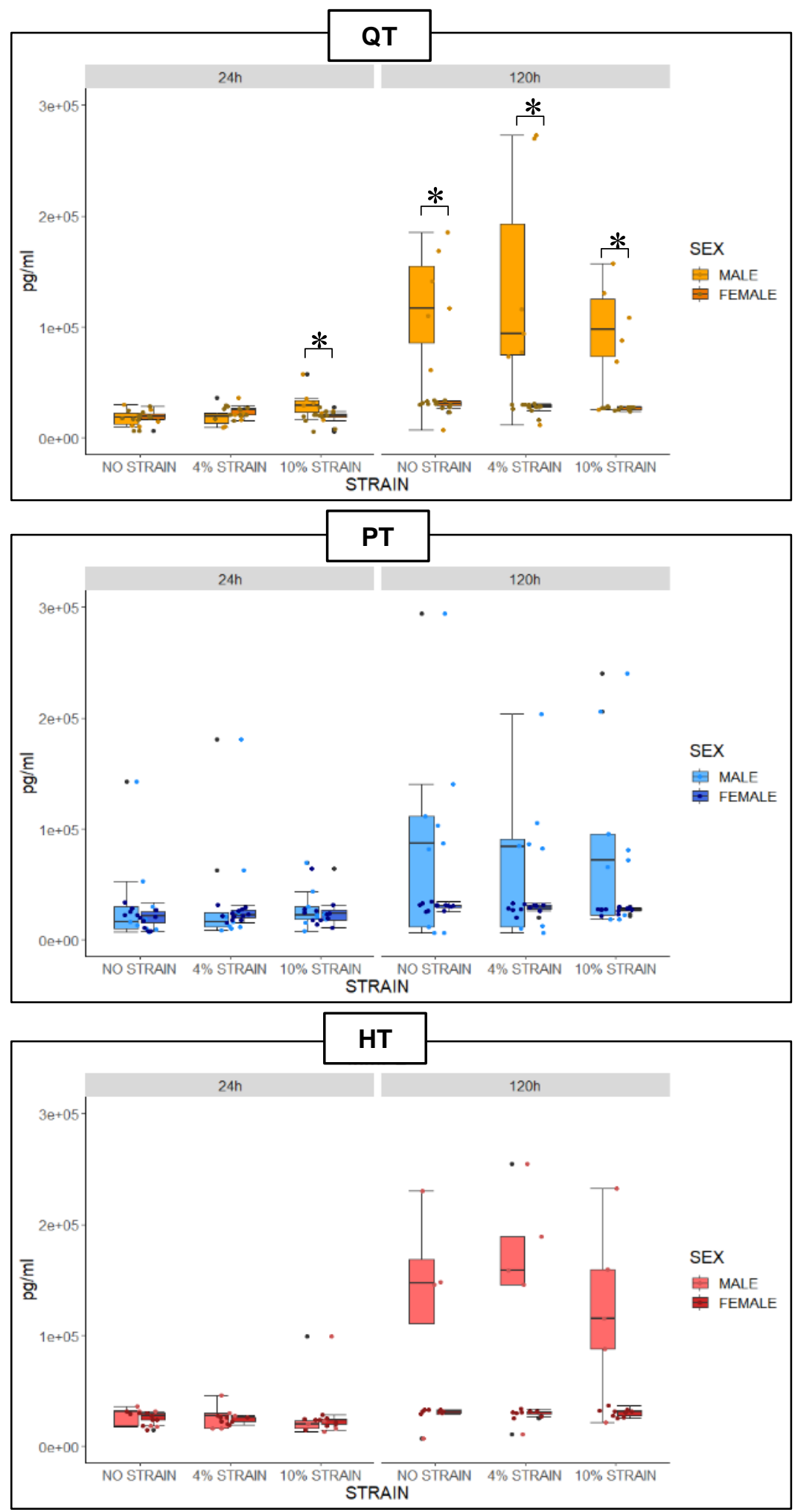

Figure 5.8. Concentration of matrix metalloproteinase 2 (MMP-2) released to the media. * significant difference between males and females at 24 and 120 hours (Mann-Whitney test). QT: Quadriceps tendon; PT: Patellar tendon, HT: Hamstring tendon 

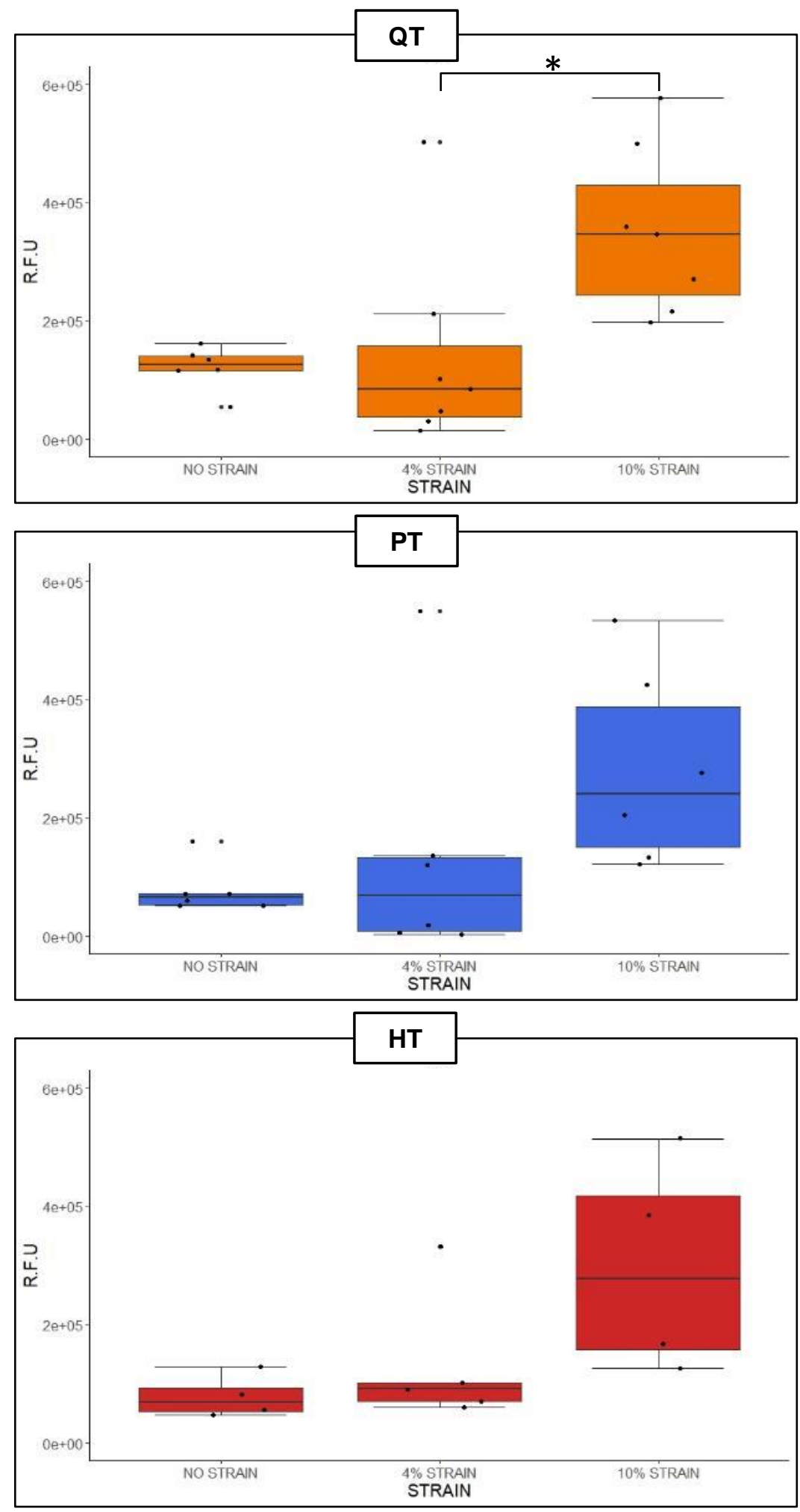

Figure 5.9. Cellular Metabolic Activity in male fibroblasts after 120 hours. * significant difference between tissues. QT: Quadriceps tendon; PT: Patellar tendon, HT: Hamstring tendon: RFU: Resazurin Fluorescent Units 


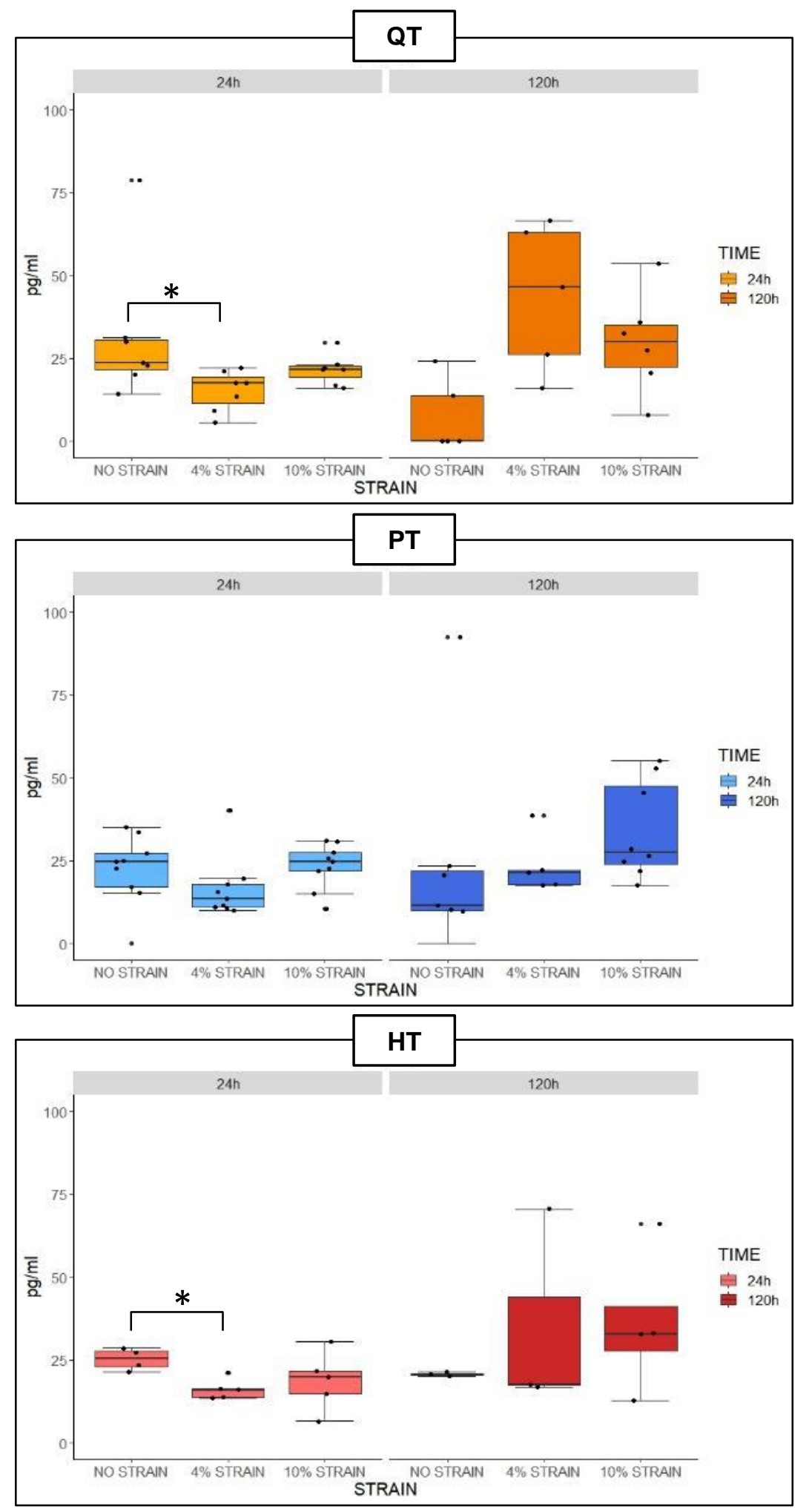

Figure 5.10. Concentration of PGE2 released to the media in males. * significant difference between graft type at 24 and 120 hours (Kruskal-Wallis test). QT: Quadriceps tendon; PT: Patellar tendon, HT: Hamstring tendon 

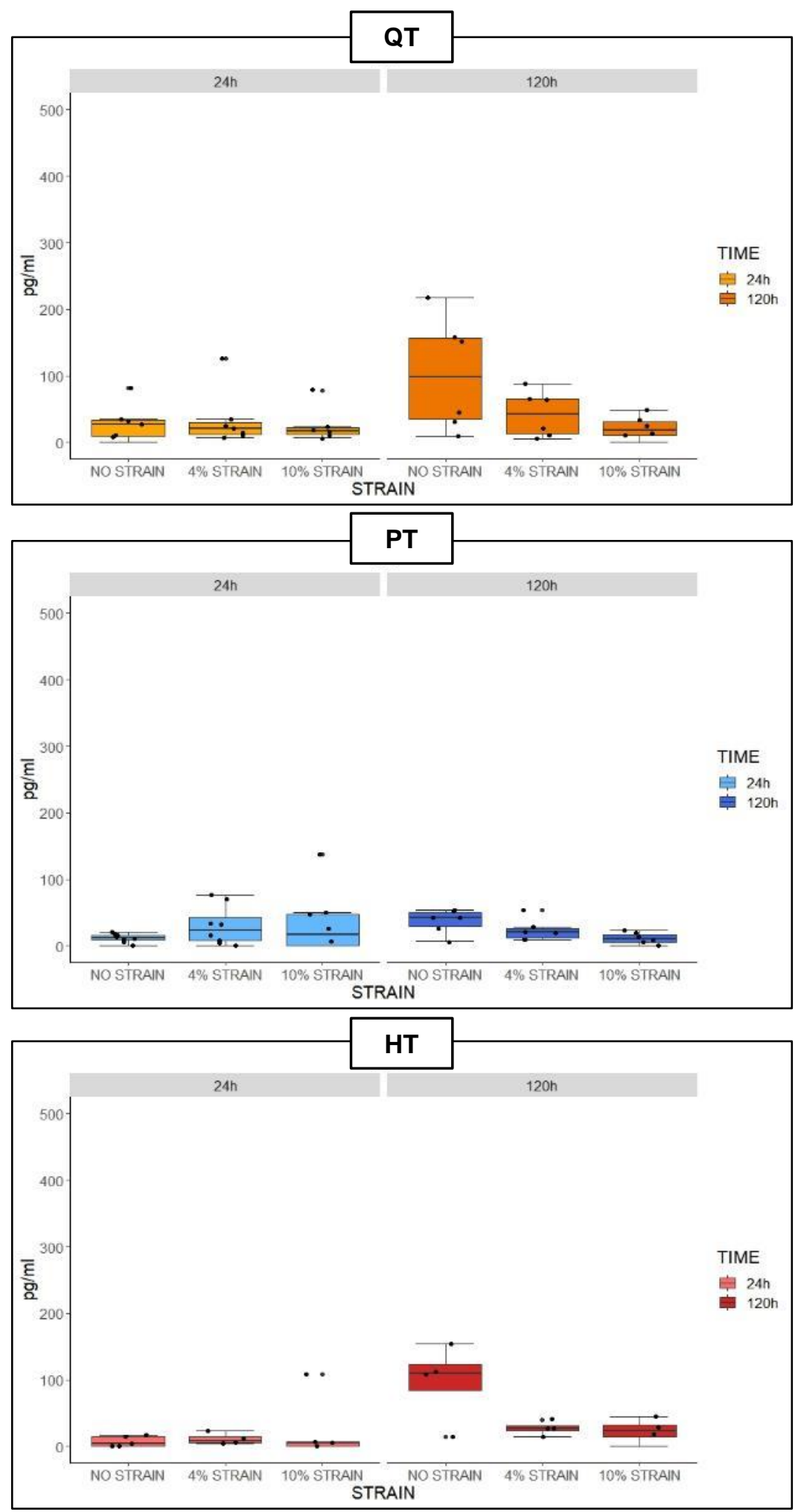

Figure 5.11. Concentration of IL-8 released to the media in males. * significant difference between graft type at 24 and 120 hours (Kruskal-Wallis test). QT: Quadriceps tendon; PT: Patellar tendon, HT: Hamstring tendon 

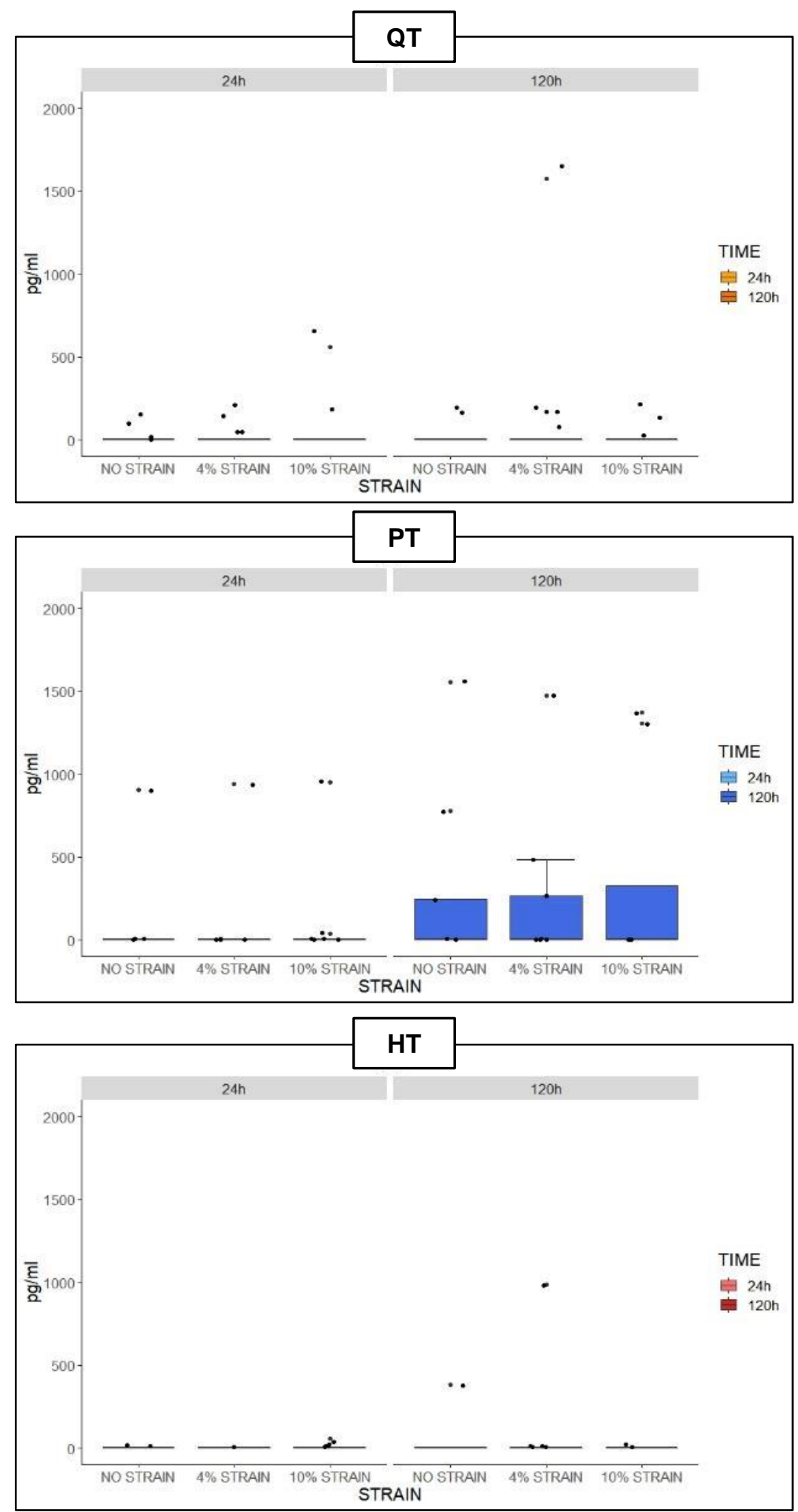

Figure 5.12. Concentration of $\mathrm{KC}$ released to the media in males. * significant difference between graft type at 24 and 120 hours (Kruskal-Wallis test). QT: Quadriceps tendon; PT: Patellar tendon, HT: Hamstring tendon 

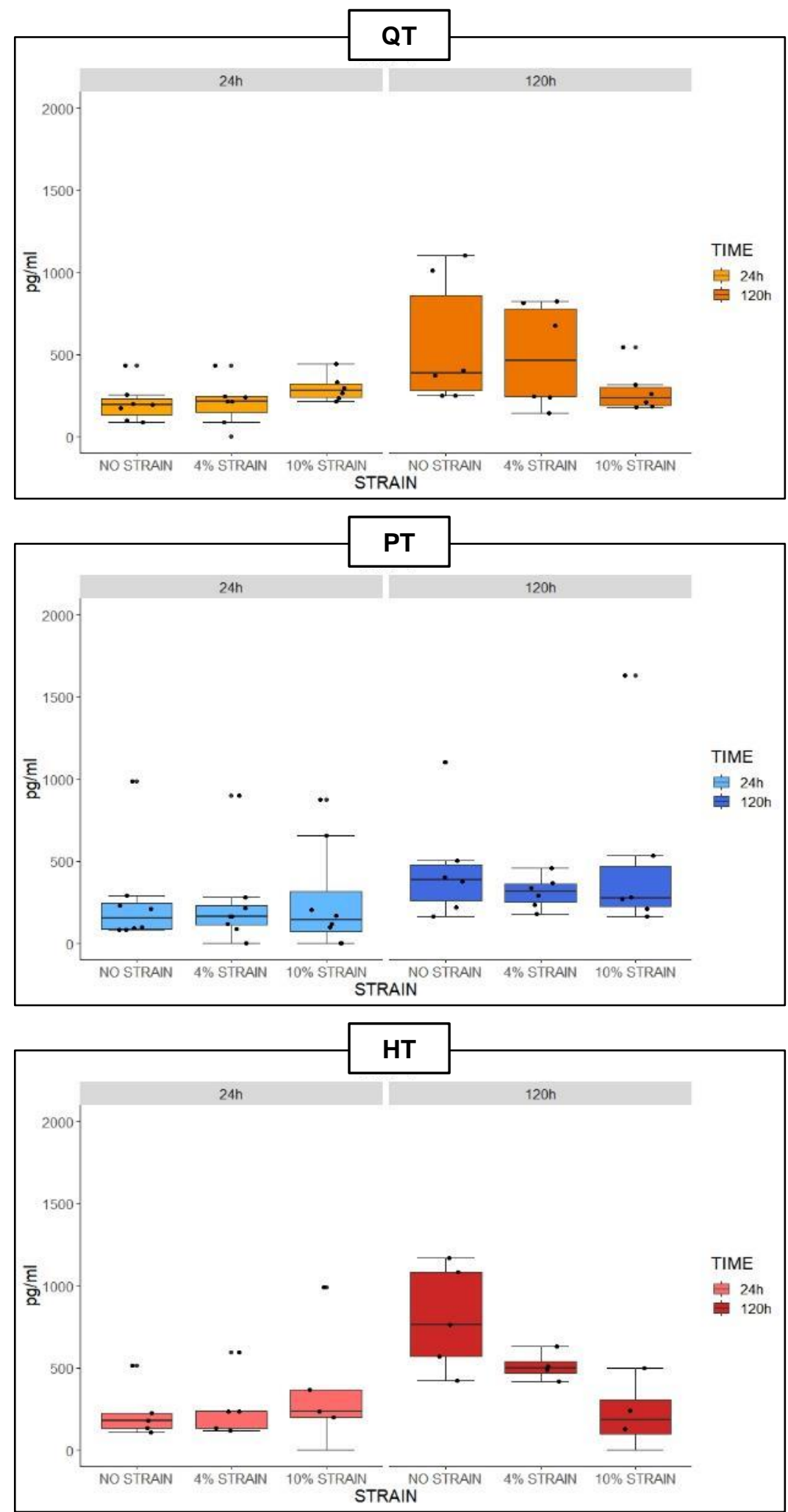

Figure 5.13. Concentration of MCP-1 released to the media in males. * significant difference between graft type at 24 and 120 hours (Kruskal-Wallis test). QT: Quadriceps tendon; PT: Patellar tendon, HT: Hamstring tendon 

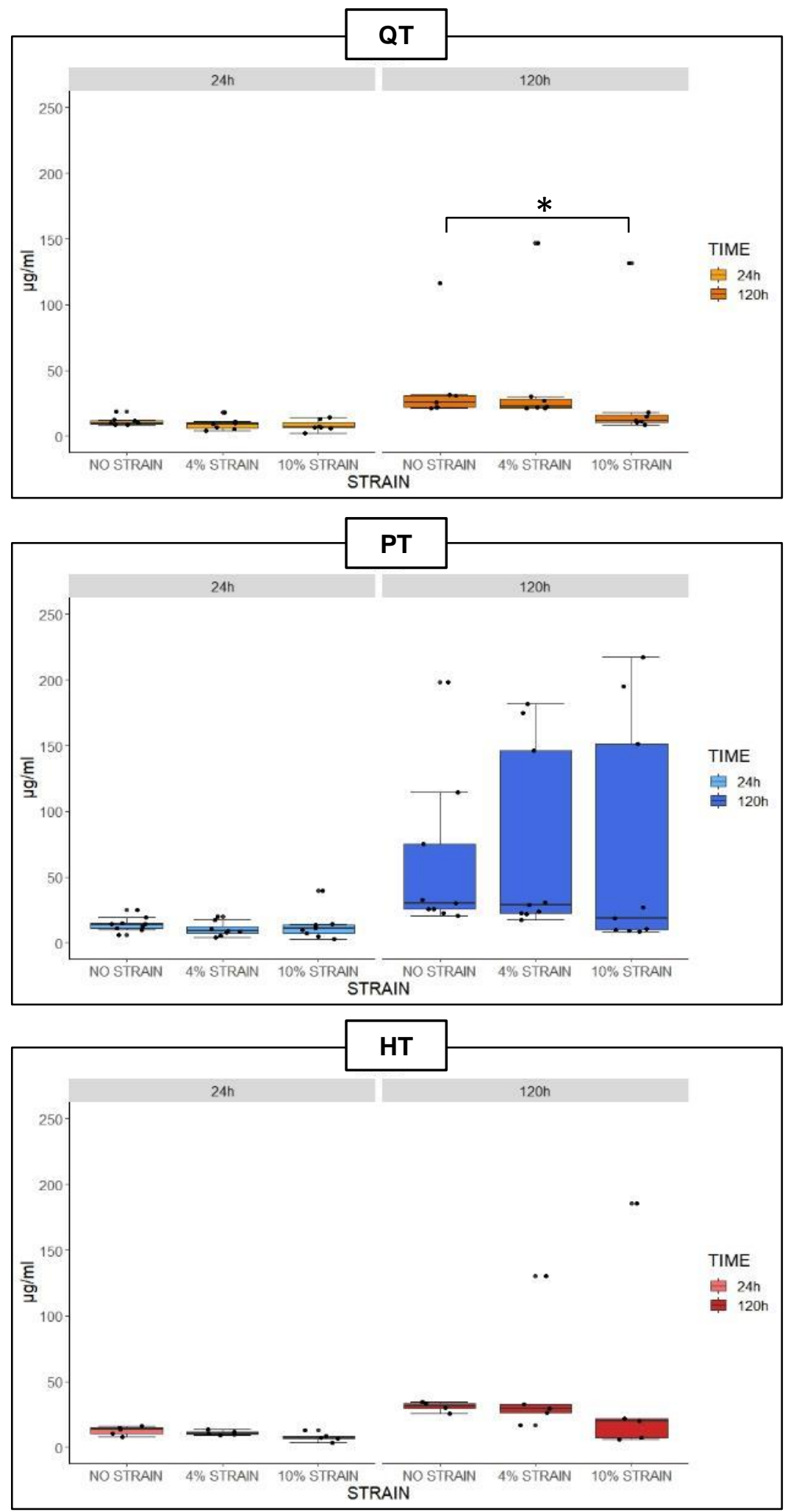

Figure 5.14. Concentration of GAG released to the media in males. * significant difference between graft type at 24 and 120 hours (Kruskal-Wallis test). QT: Quadriceps tendon; PT: Patellar tendon, HT: Hamstring tendon 

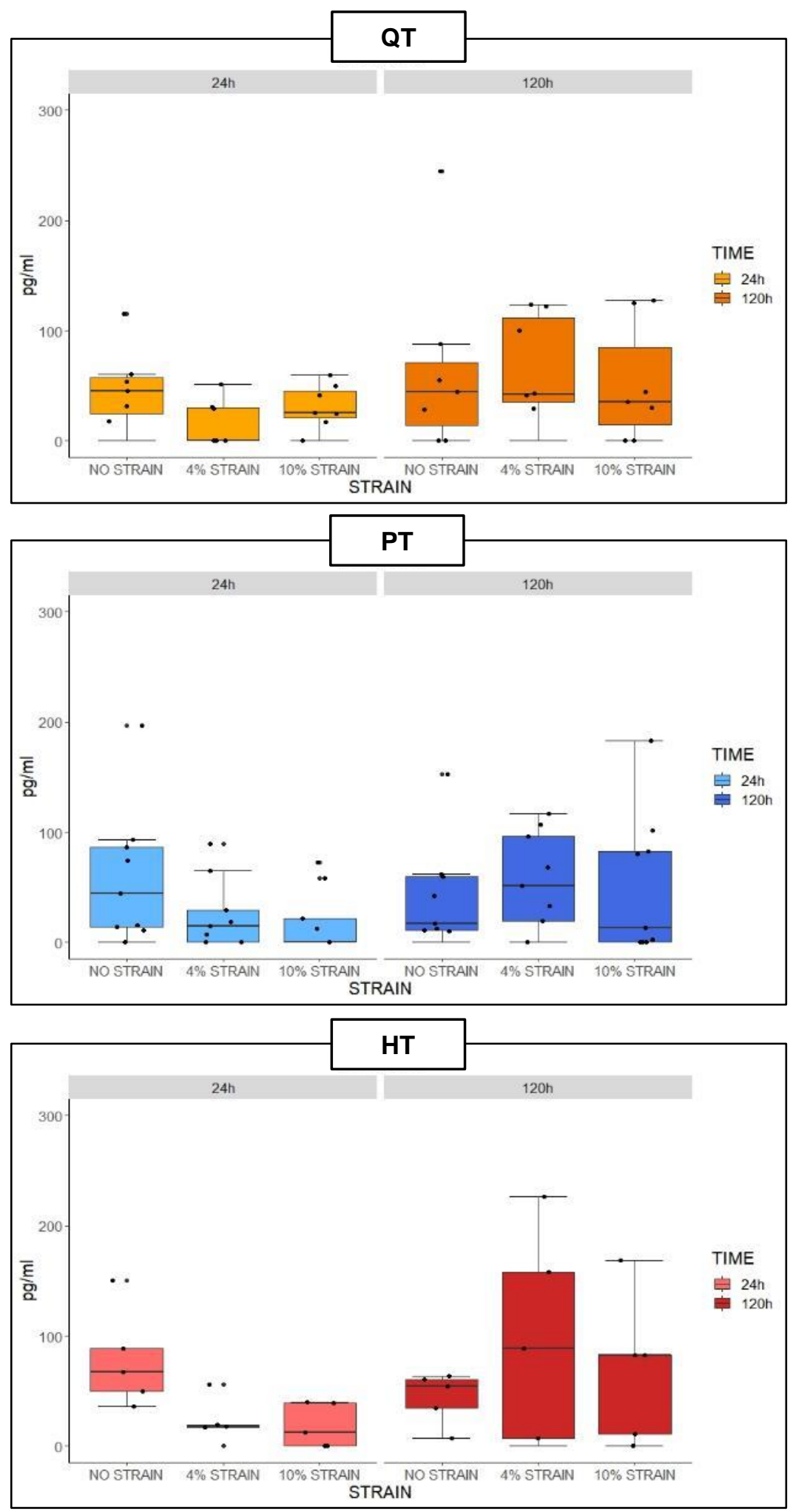

Figure 5.15. Concentration of MMPACT released to the media in males. * significant difference between graft type at 24 and 120 hours (Kruskal-Wallis test). QT: Quadriceps tendon; PT:

Patellar tendon, HT: Hamstring tendon 

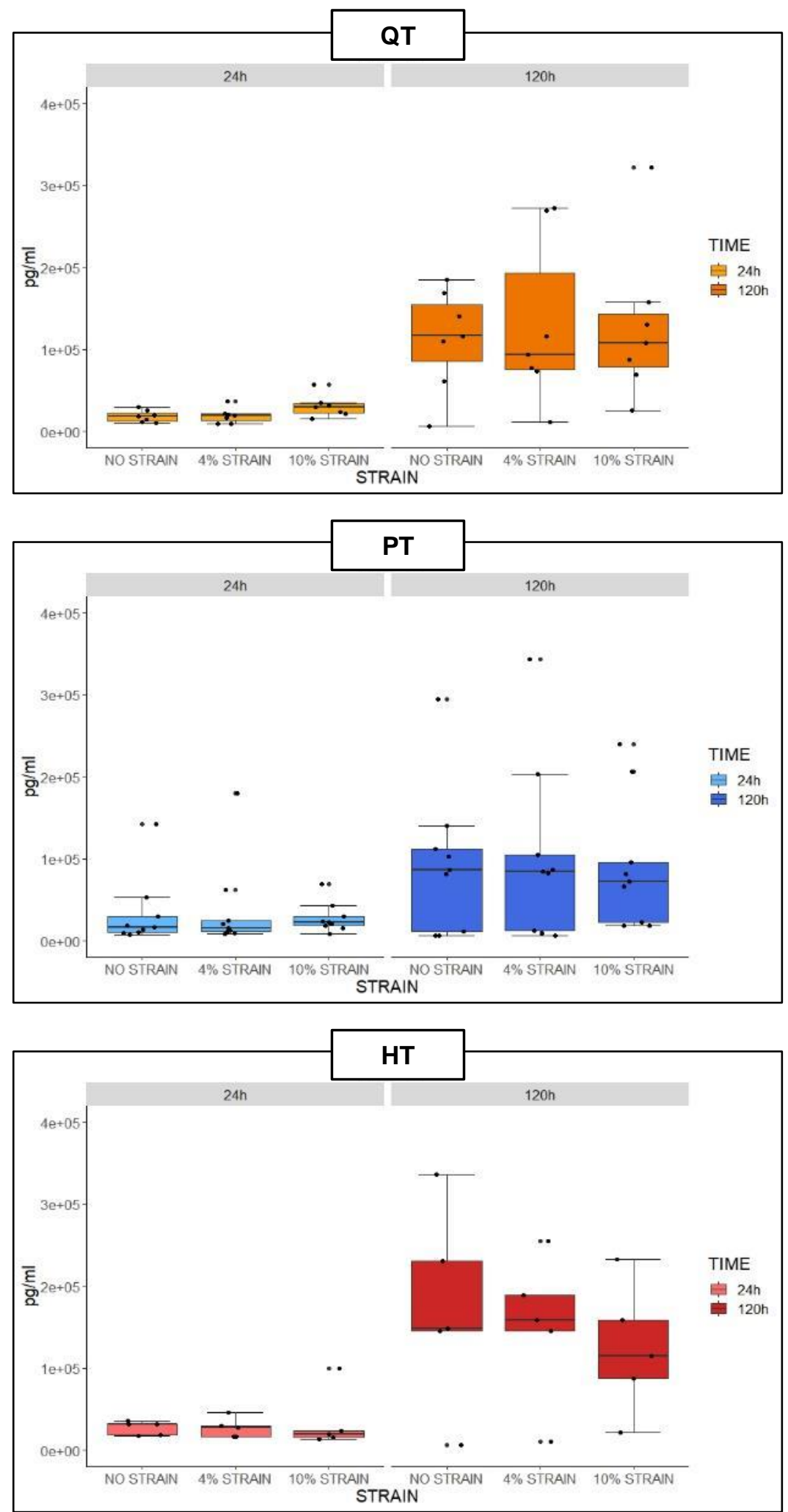

Figure 5.16. Concentration of MMP-2 released to the media in males. * significant difference between graft type at 24 and 120 hours (Kruskal-Wallis test). QT: Quadriceps tendon; PT: Patellar tendon, HT: Hamstring tendon 

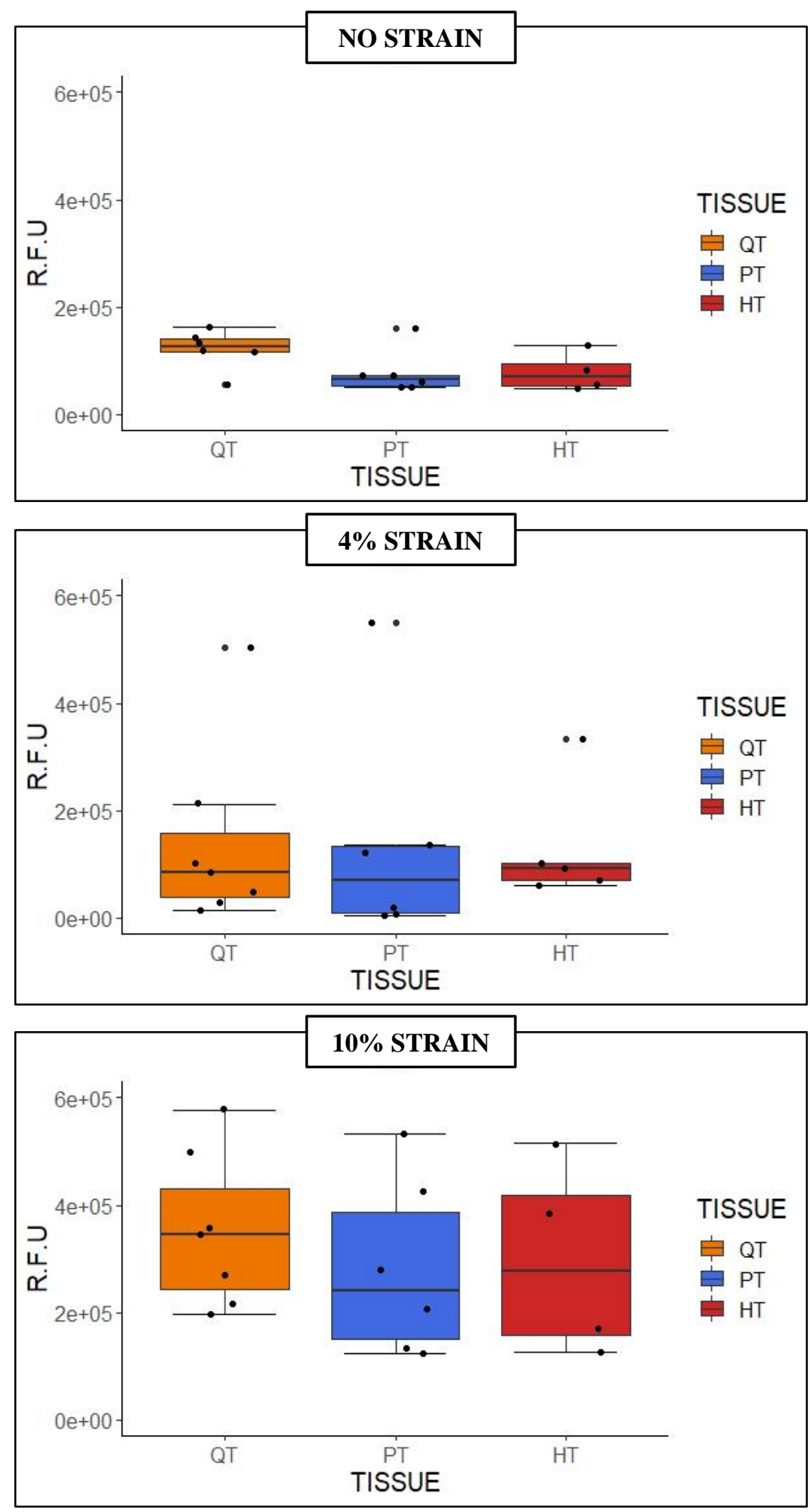

Figure 5.17. Cellular Metabolic Activity in males after 120 hours. * significant difference between strains. QT: Quadriceps tendon; PT: Patellar tendon, HT: Hamstring tendon: RFU: Resazurin Fluorescent Units 

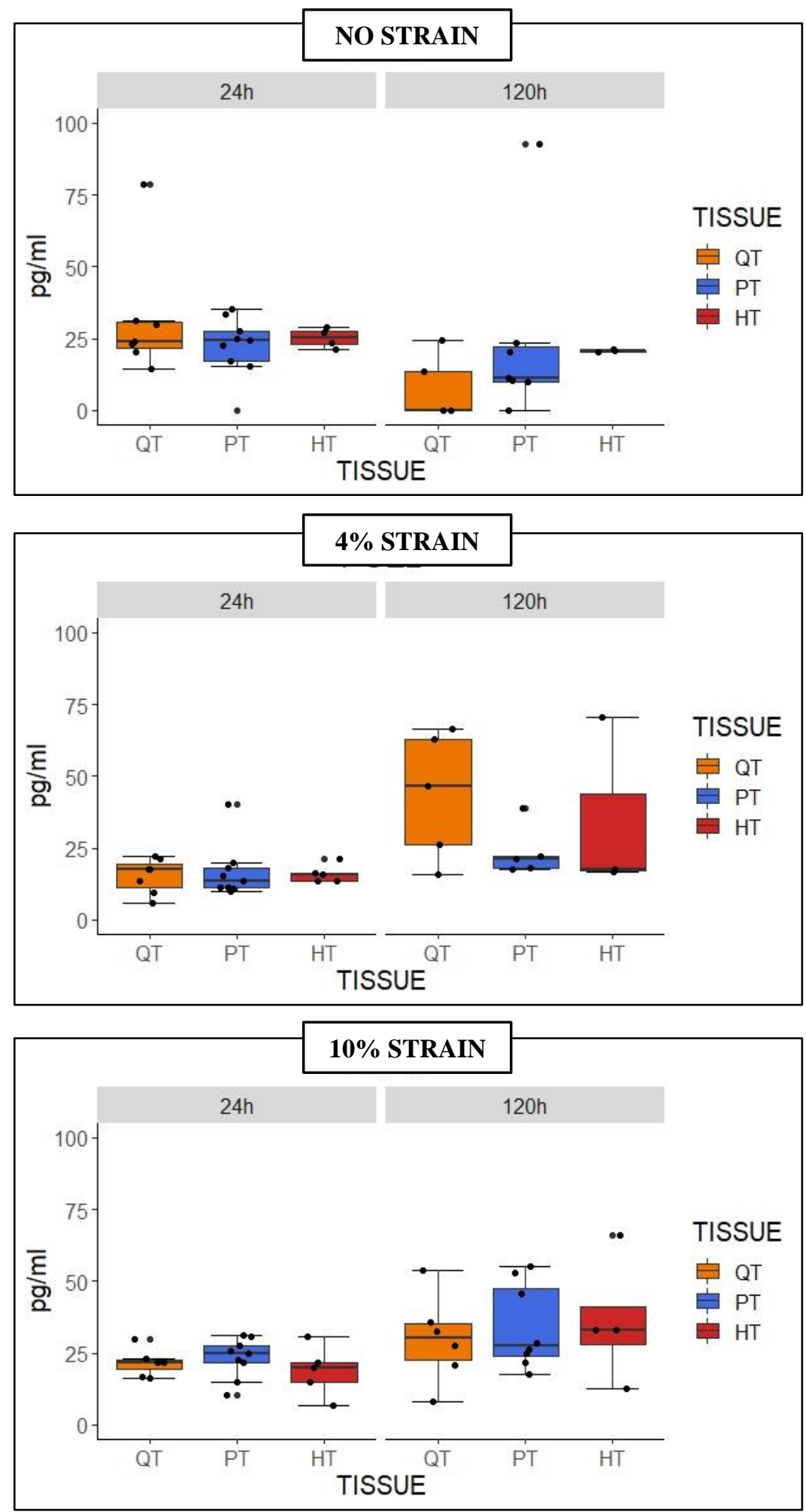

Figure 5.18. Concentration of PGE2 released to the media in males. * significant difference between TISSUES at 24 and 120 hours (Kruskal-Wallis test). QT: Quadriceps tendon; PT: Patellar tendon, HT: Hamstring tendon 

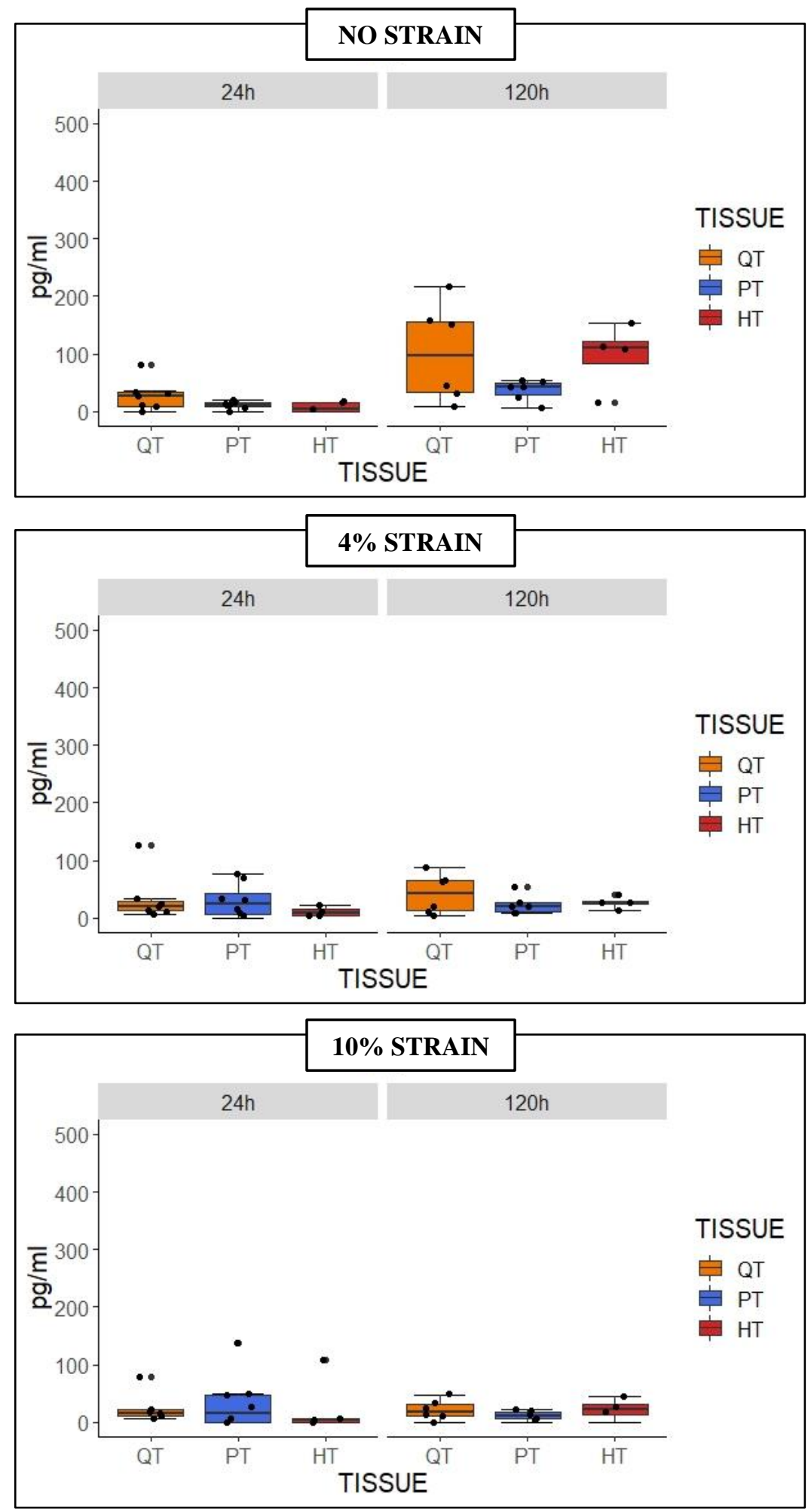

Figure 5.19. Concentration of IL-8 released to the media in males. * significant difference between TISSUES at 24 and 120 hours (Kruskal-Wallis test). QT: Quadriceps tendon; PT: Patellar tendon, HT: Hamstring tendon 

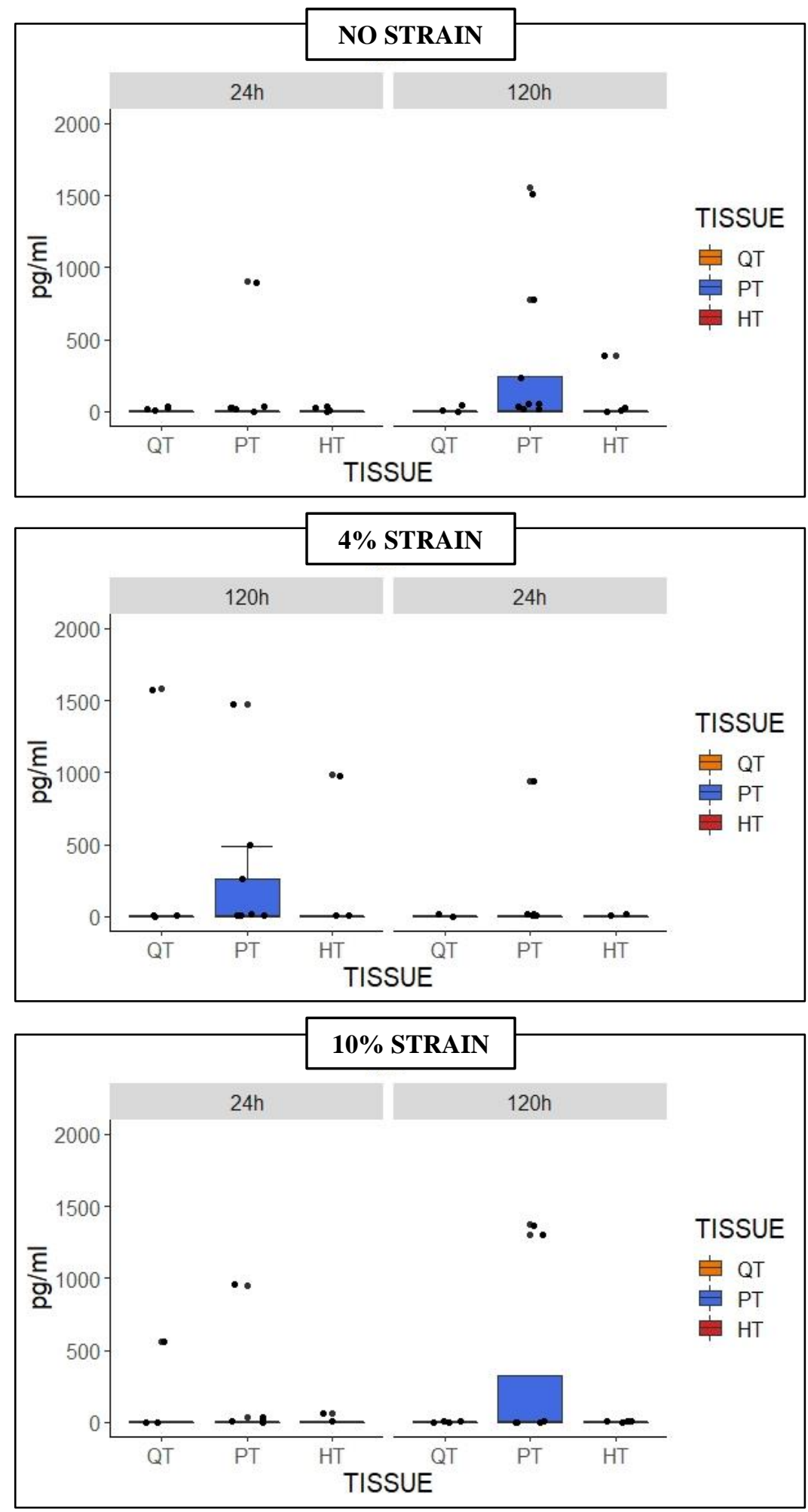

Figure 5.20. Concentration of $\mathrm{KC}$ released to the media in males. * significant difference between TISSUES at 24 and 120 hours (Kruskal-Wallis test). QT: Quadriceps tendon; PT: Patellar tendon, HT: Hamstring tendon 

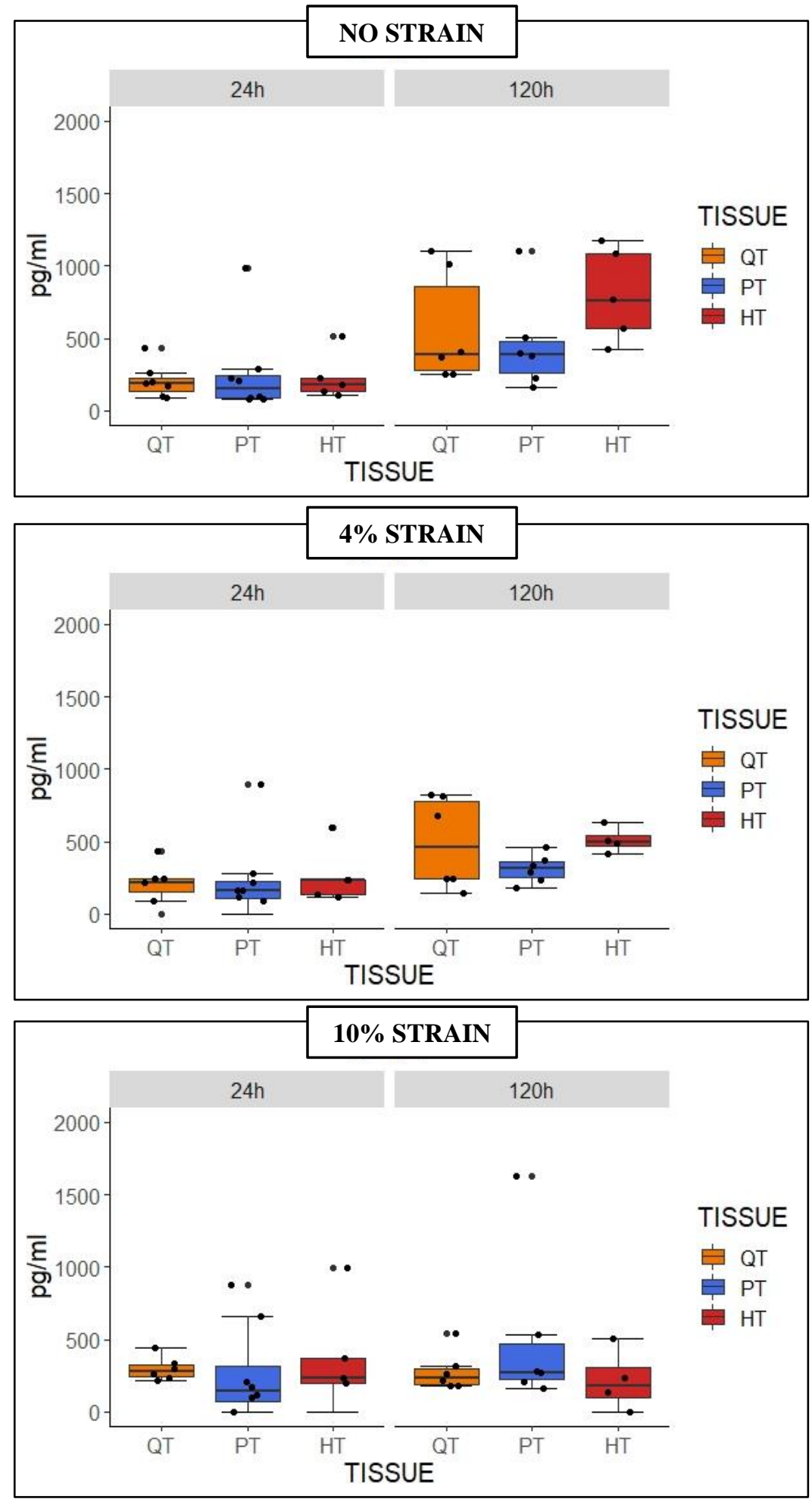

Figure 5.21. Concentration of MCP1 released to the media in males. * significant difference between TISSUES at 24 and 120 hours (Kruskal-Wallis test). QT: Quadriceps tendon; PT: Patellar tendon, HT: Hamstring tendon 

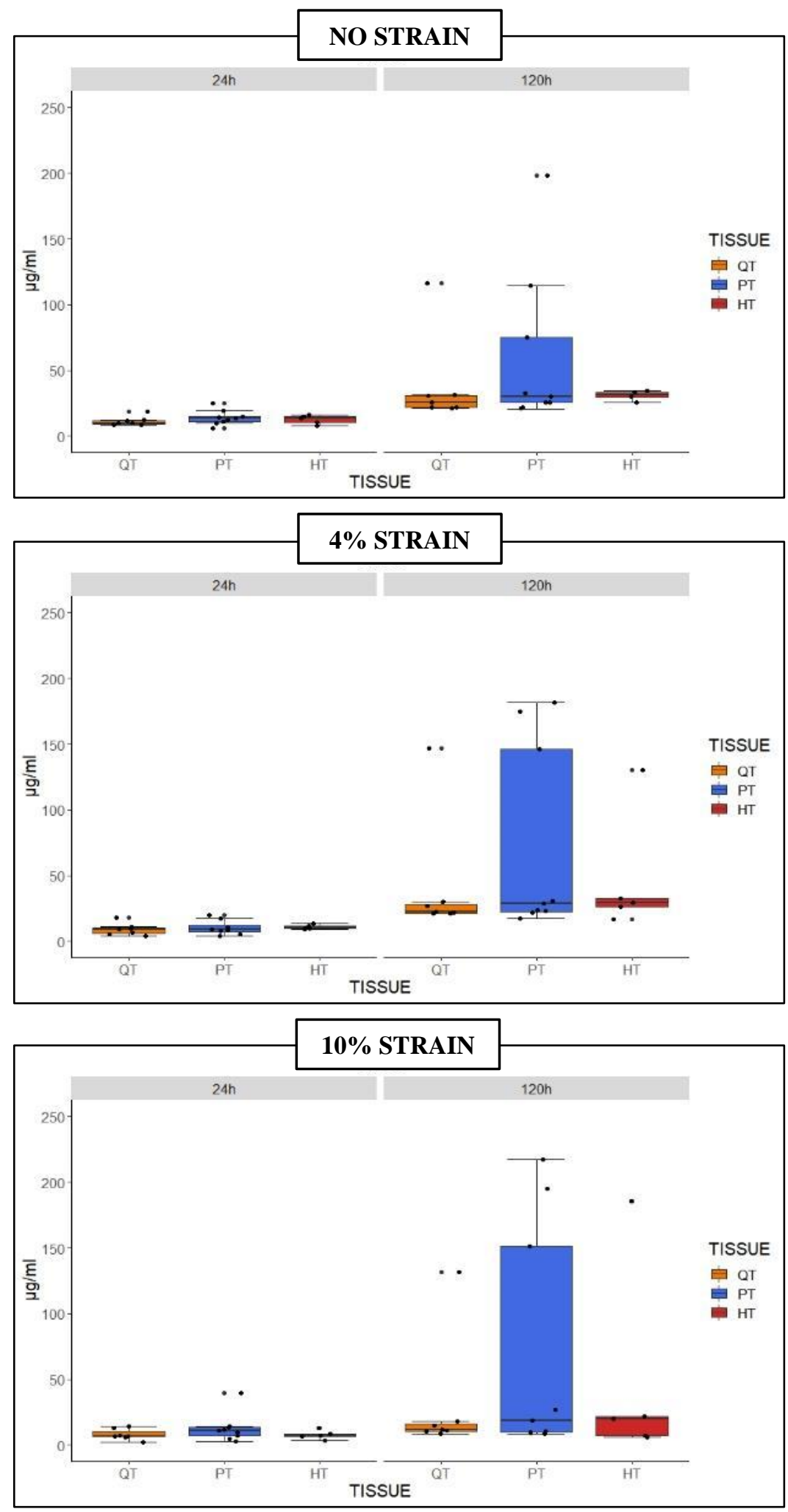

Figure 5.22. Concentration of GAG released to the media in males. * significant difference between TISSUES at 24 and 120 hours (Kruskal-Wallis test). QT: Quadriceps tendon; PT: Patellar tendon, HT: Hamstring tendon 

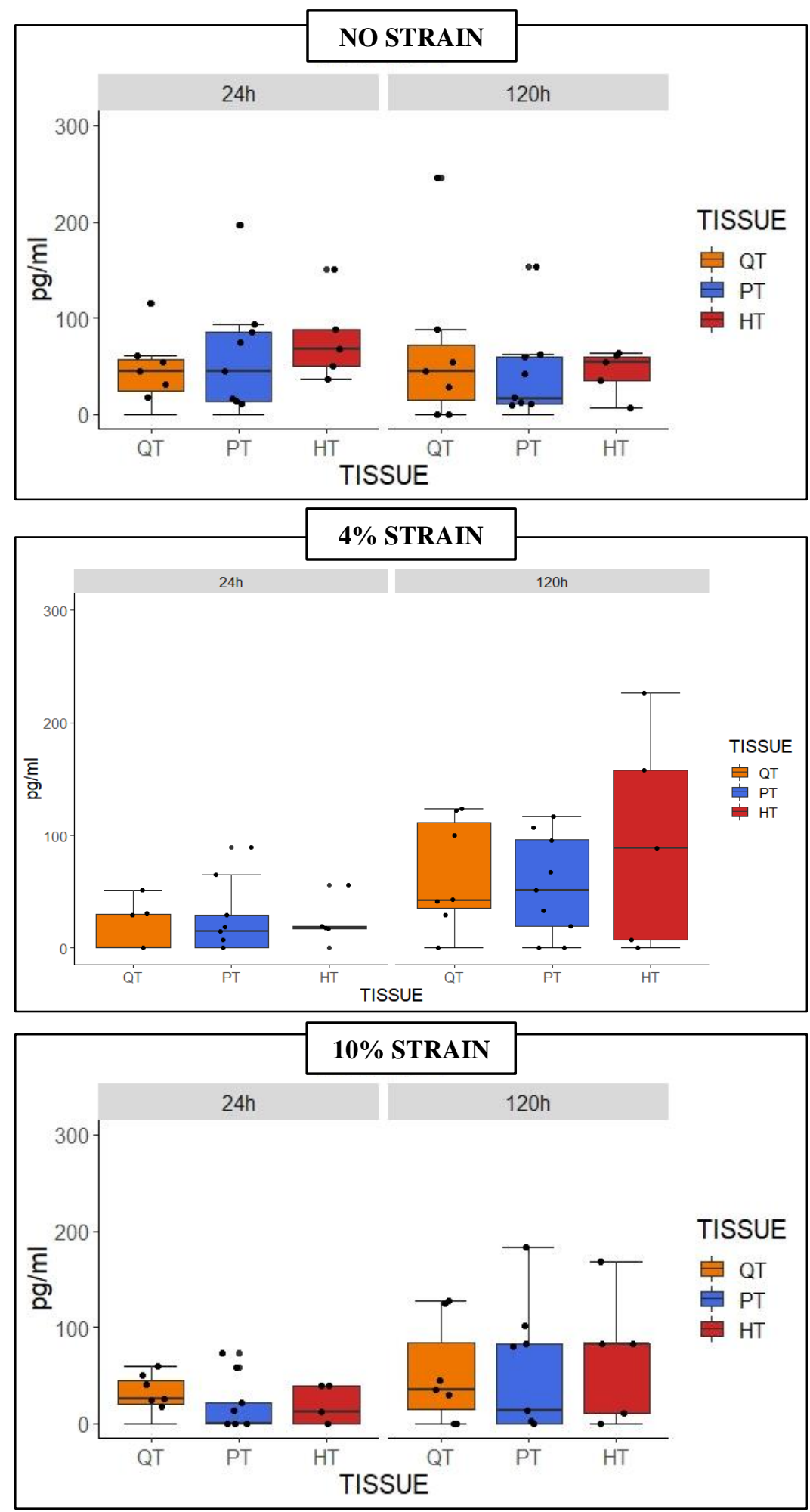

Figure 5.23. Concentration of MMPACT released to the media in males. * significant difference between TISSUES at 24 and 120 hours (Kruskal-Wallis test). QT: Quadriceps tendon; PT:

Patellar tendon, HT: Hamstring tendon 

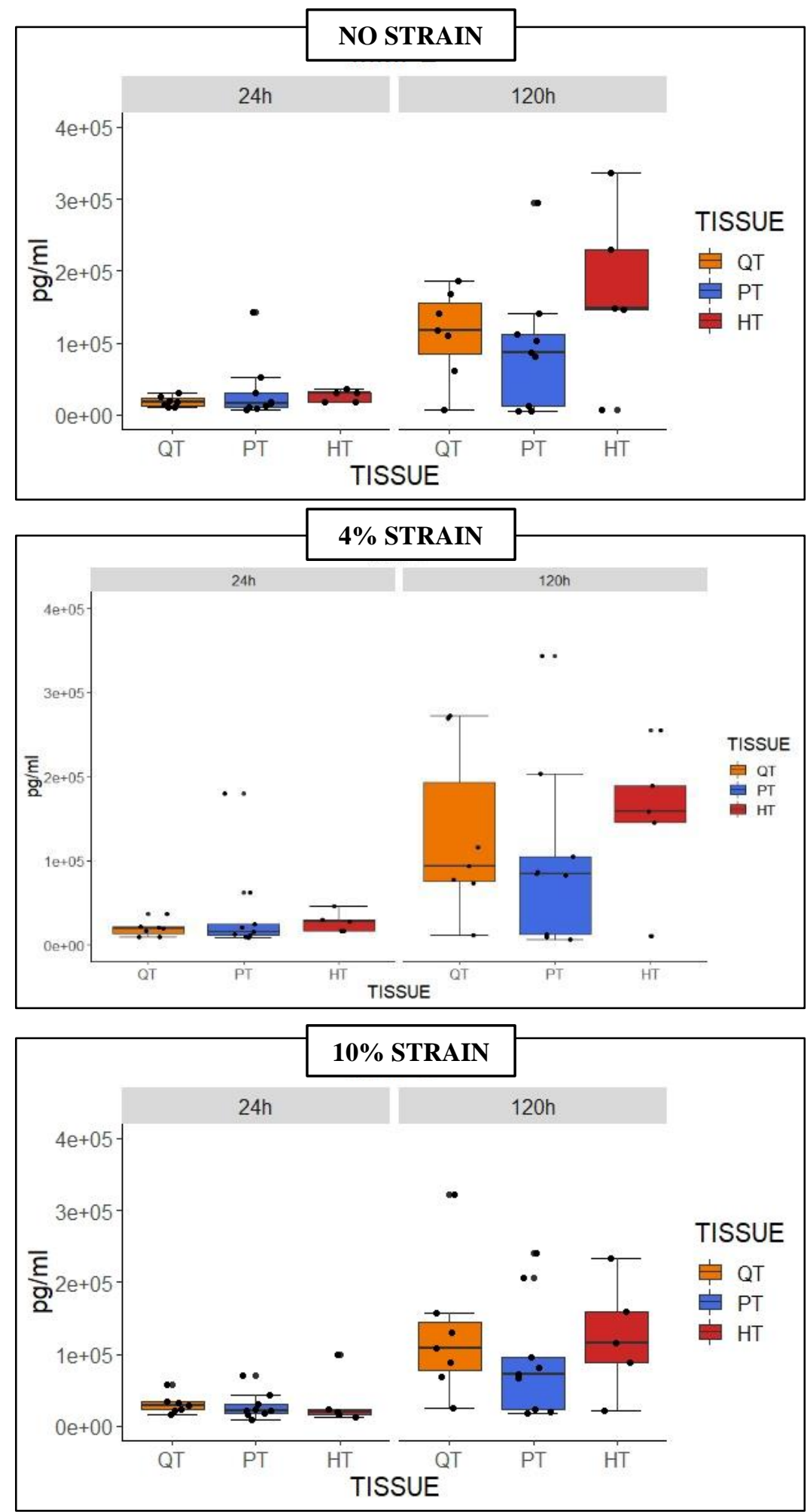

Figure 5.24. Concentration of MMP- 2 released to the media in males. * significant difference between TISSUES at 24 and 120 hours (Kruskal-Wallis test). QT: Quadriceps tendon; PT: Patellar tendon, HT: Hamstring tendon 

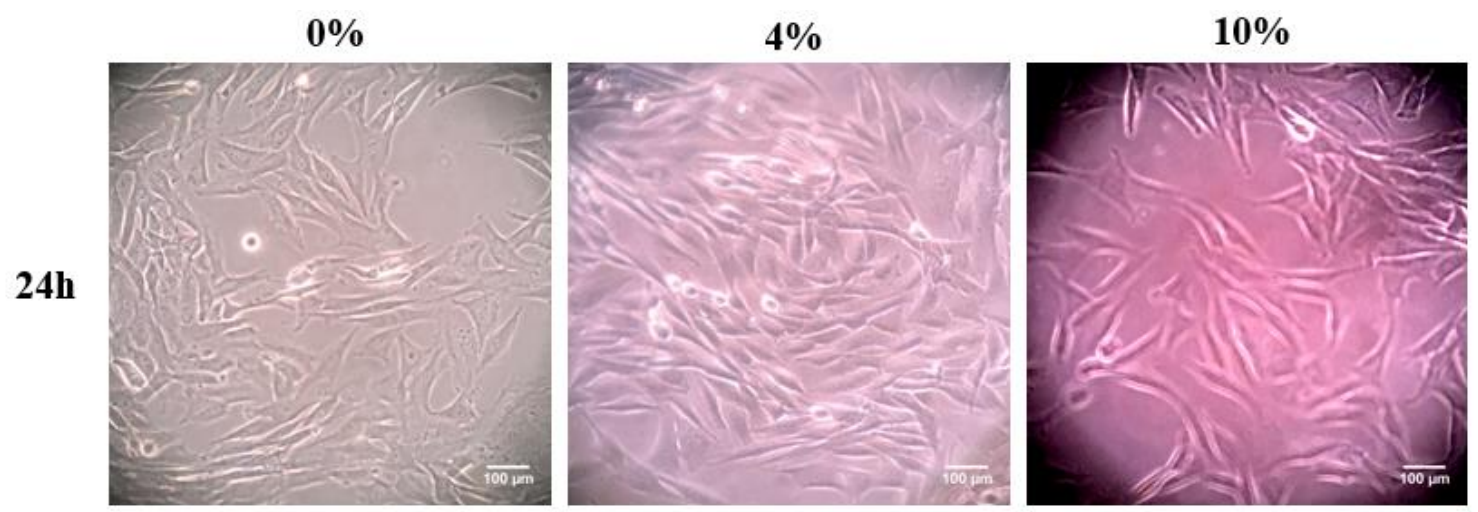

$120 h$
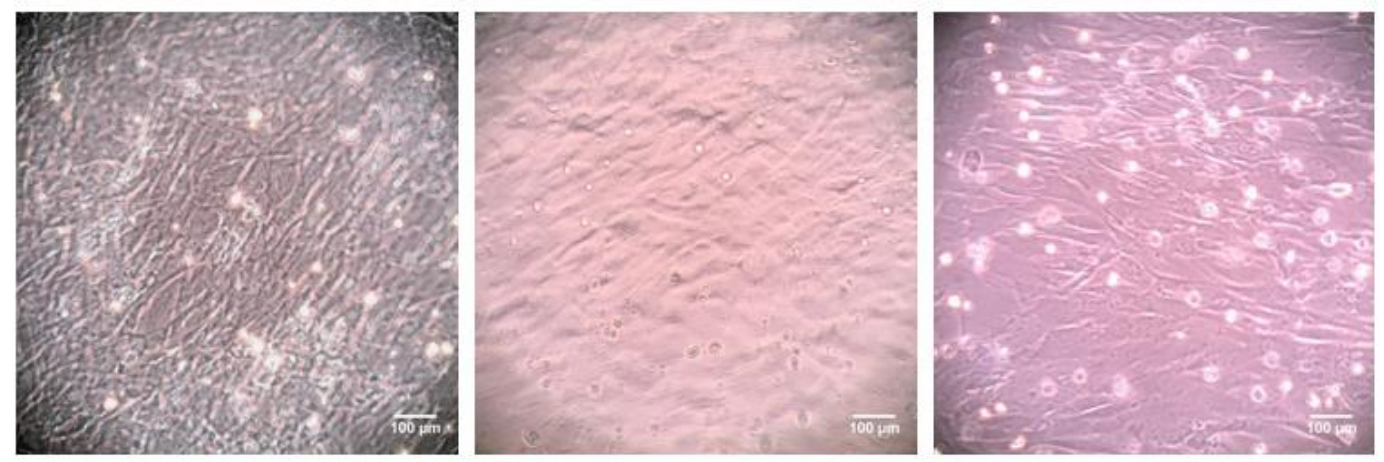

Figure 5.25. Quadriceps tendon (QT) fibroblasts stretched after 24 and 120 hours at three different stain magnitudes.

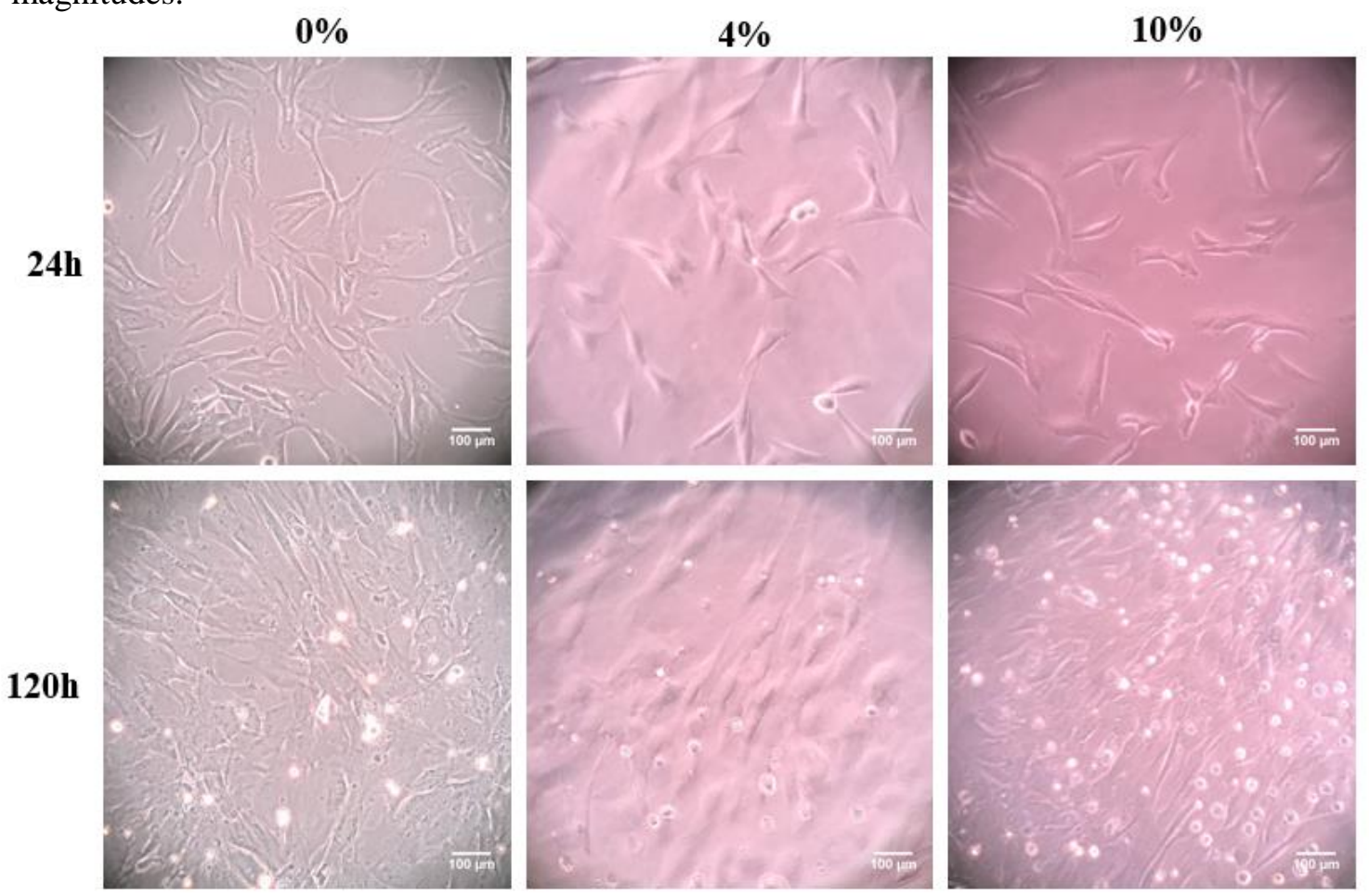

Figure 5.26. Patellar Tendon (PT) fibroblasts stretched after 24 and 120 hours at three different stain magnitudes. 


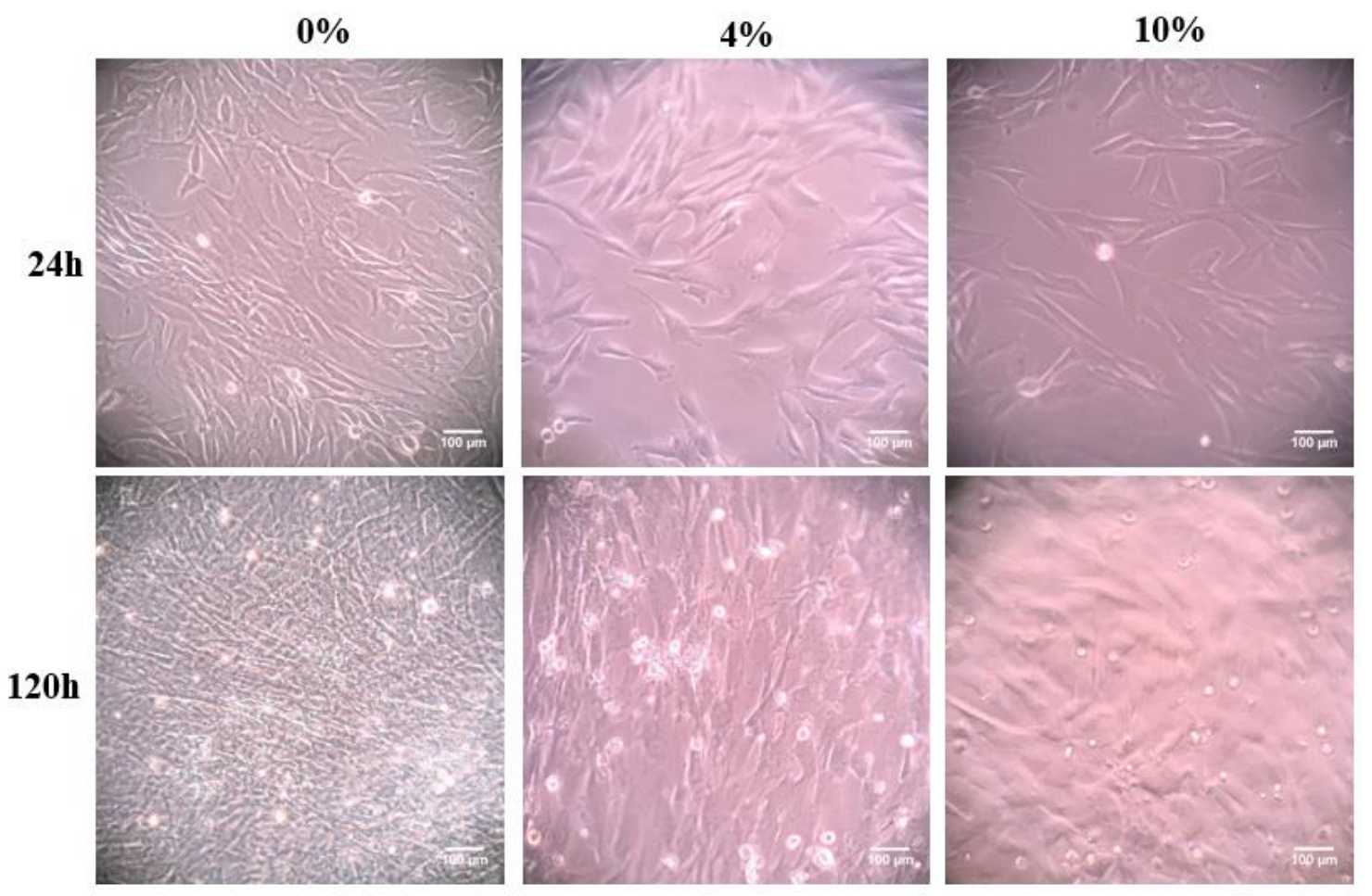

Figure 5.27. Hamstring Tendon (HT) fibroblasts stretched after 24 and 120 hours at three different stain magnitudes. 


\section{CHAPTER 6}

\section{NORMAL CANINE QT, PT, HT CELLS CO-CULTURED WITH SYNOVIOCYTES AND EXPOSED TO MECHANICAL STRAIN}

\section{Introduction}

Anterior cruciate ligament (ACL) injury is one of the most common musculoskeletal injuries, compromising knee stability and function and places individuals at risk for further injuries and post-traumatic osteoarthritis (PTOA). Symptomatic ACL injuries are often surgically treated with reconstruction using a tendon autograft or allograft. Common tendon grafts used in ACL reconstruction include the quadriceps tendon (QT), patellar tendon (PT), and the hamstring tendon (HT). Regardless of the graft used, healing of the ACL graft involves the remodeling of the extracellular matrix (ECM) and ligamentization of the tendon graft. The healing of the ACL graft is influenced by the intraarticular environment, and the ligamentization process requires a biological compositional shift from tendon to ligament-like tissue resulting in changes in its biomechanical properties. ${ }^{1}$ Hence, the evaluation of the metabolic responses in mechanosensitive tissues such as the tendon grafts, constitutes a key part in the understanding of the intraarticular healing responses.

The synovium may play a role in graft ligamentization after ACL reconstruction. While the native ACL is intraarticular in location, it is extrasynovial and is encased by a synovial sheath. The synovial sheath may play an important role in various tendon

pathologies such as carpal tunnel syndrome (CTS). ${ }^{2}$ Early tenosynovial changes seem to correlate with CTS symptoms. Marked ECM remodeling in the tenosynovium is an important characteristic of tendon healing and degradation. The synovial membrane that 
line the intraarticular knee joint is also vital for regulating the joint homeostasis by maintaining or amplifying the inflammatory signals. ${ }^{3}$ Elevated levels of cytokines and growth factors within the knee intraarticular environment are often considered to be the contribution of synovium. ${ }^{4}$ Furthermore, the cross talk between synoviocytes and intraarticular ligaments has been shown to influence the healing response when fibroblasts are subjected to mechanical deformation. ${ }^{5}$ There is, however, no information on the role of the synoviocytes in ACL graft healing when subjected to mechanical strain levels commonly found in perioperative loads.

The biomechanical environment that the tendon graft experiences plays an important role in the graft ligamentization process after ACL reconstruction. ${ }^{6-11}$ Successful intraarticular graft remodeling and intra-tunnel graft incorporation depend on the interplay of many factors. Depending on the mechanical stimulus that the ACL graft experiences, these forces can modulate bone formation and the tendon-to-bone tunnel healing process. ${ }^{12}$ This could be explained by the ability of the tenocytes to remodel the ECM in response to different biomechanical environments. ${ }^{13 ; 14}$

In vitro systems for modeling the ACL and its remodeling usually evaluate cellular responses in a static environment on isolated fibroblasts in monoculture. ${ }^{15-17}$ However, these static monoculture systems does not consider the interplay between various tissues within a complex biologic ecosystem such as the knee joint. Since the intraarticular environment of the knee is constantly subjected to mechanical strain, cellular responses of multiple tissues might be affected by the magnitude of strain that is applied, and in turn, affect one another. Given the importance of the synovium and its central role in modulating the intraarticular knee environment, we aimed to evaluate the effects that synoviocytes 
have on common ACL graft fibroblasts after mechanical stress is applied in a dynamic coculture system. We hypothesized that graft tendon fibroblasts co-cultured with synoviocytes will significantly increase the metabolic activity, and the production of proinflammatory biomarkers and remodeling biomarkers of common graft tendon fibroblast cells under mechanical stress when compared to monocultured graft fibroblasts.

\section{Materials and methods}

Tissue processing and culture

With ACUC approval (ACUC\#9163, 9164), QT, PT, HT and synovial (SYN) (13 PT, 10

HT, 12 QT and 1 SYN) tissues were harvested from skeletally mature dogs (n=13) euthanatized for studies unrelated to the current work and free of any orthopedic disease based on orthopedic examination and radiographs. Tissues were aseptically minced into $0.5-1.0 \mathrm{~cm}^{2}$ pieces and digested overnight in Type 1A Clostridium histolyticum collagenase solution (Sigma-Aldrich, St. Louis, MO, USA) at a concentration of 0.5 $\mathrm{mg} / \mathrm{mL}$. The aliquot was then suspended with nutritional media (Gibco DMEM, Thermo Fisher Scientific, Waltham, MA, USA) containing 10\% FBS, 0.002\% Penicillin, 100 $\mu \mathrm{g} / \mathrm{mL}$ Streptomycin, $25 \mu \mathrm{g} / \mathrm{mL}$ Amphotericin B, 0.002\% L-Ascorbate and 0.01\% Lglutamine (Sigma Chemical Co, St. Louis, MO), cultured in T75 flasks (TPP Techno Plastic Products AG, Trasadingen, Switzerland) and incubated at $5 \% \mathrm{CO}_{2}, 37^{\circ} \mathrm{C}$, and 95\% humidity.

\section{Biaxial mechanical stress}

Once confluent, graft fibroblasts were resuspended and seeded in Collagen Type I-coated BioFlex ${ }^{\circledR}$ plates $\left(1 \times 10^{5}\right.$ cells/well). Polyethylene terephthalate $(\mathrm{PET})$ coated co-culture inserts (Corning $\odot)$ were seeded with SYN $\left(5 \times 10^{4}\right.$ cells/well) and placed in the Bioflex ${ }^{\circledR}$ 
plates using a Flexcell@ transwell holder. Cells were incubated with 10\% FBS media for an additional $48 \mathrm{~h}$ before strain was applied. Then, nutritional culture media was replaced with $0.5 \%$ FBS media for the remaining five days of culture. Fibroblasts were subjected to continuous mechanical stimulation (2-s strain and $10-\mathrm{s}$ relaxation at a $0.5 \mathrm{~Hz}$ frequency) with a biaxial sinusoidal waveform with three different elongation strains chosen to represent three physiologic conditions (mechanical stress deprivation-0\%, physiologic strain-4\%, and supraphysiologic strain-10\%) ${ }^{18}$ for 5 days using the Flexcell FX-4000T strain system (Flexcell International, NC, USA) (Fig. 6.1). Culture media were changed every $24 \mathrm{~h}$ and stored at $-20^{\circ} \mathrm{C}$ until analysis. At the end of the strain protocol (120h), cell viability was assessed using the resazurin assay.

\section{Cell viability assay}

The resazurin dye added to the culture media is a cell permeable redox indicator used to monitor viable cells. Viable cells with active metabolism can reduce resazurin into the highly fluorescent metabolite resorufin. The quantity of resorufin produced is proportional to the number of viable cells in culture. ${ }^{19}$ Briefly, $10 \%$ volume of resazurin (Sigma-Aldrich, Saint Louis, MO) stock solution $(0.1 \mathrm{mg} / \mathrm{mL})$ was added to the media at the end of the culture period $(120 \mathrm{~h})$ and incubated at $37^{\circ} \mathrm{C}$ for two hours. Then, fluorescence excitation and emission were measured at 540/580 nm.

\section{Biomarker assays}

Media from 24 and 120 hours of culture were assessed for various biomarkers.

Proteoglycan (GAG) was assessed using the DMMB assay as previously described. ${ }^{20}$ The concentration of prostaglandin $\mathrm{E}_{2}$ (PGE2) (Cayman Chemical, Ann Arbor, MI, USA), cytokines (IL-6, IL-8, KC and MCP-1) (Millipore, Billerica, MA, USA), total matrix 
metalloproteinase (MMP) activity (SensoLyte 520 generic MMP assay, Anaspec, Inc., Fremont, CA), and MMP production (MMP-1, MMP-2 and MMP-3) (R\&D Systems, Minneapolis, MN, USA), were all assessed using commercially available assays according to the manufacturer's protocol.

\section{Statistical Analysis}

For statistical analysis, normal distribution was assessed by Shapiro-Wilk test. Data were not normally distributed so non-parametric analyses were used. Comparisons between monoculture and co-culture at either 24 or 120 hours of culture were performed using Mann-Whitney U test. Additionally, comparisons among strains and tissue types were performed using Kruskal-Wallis test and Dunn's test posthoc (R Core Team (2019), R version 3.6.2 Vienna, Austria). Results were reported as median \pm interquartile range (IQR). Significance levels were set at $\mathrm{p}<0.05$.

\section{Results}

\section{Differences between monoculture and co-culture}

Co-culture with synoviocytes resulted in significantly higher metabolic activity in QT and PT fibroblasts co-cultured with SYN compared to monocultured fibroblasts regardless of the strain. Similarly, HT fibroblasts exhibited increased metabolic activity at no strain an $10 \%$ strain when cocultured with SYN compared to monoculture (Fig. 6.2).

Inflammatory mediator PGE2, significantly increased at 24h in QT fibroblasts when cocultured with SYN relative to monoculture at no strain. Moreover, at 120h, PGE2 production significantly increased in cocultured QT fibroblasts relative to monoculture regardless of the strain. Similarly, PT fibroblasts cocultured with SYN, increased the production of PGE2 at $24 \mathrm{~h}$ and $120 \mathrm{~h}$ of mechanical strain deprivation compared to 
monoculture. Moreover, HT fibroblasts in cocultured showed an increased production of PGE2 at no strain 120h of culture. Interestingly, physiological strain elicited an increased production of PGE2 in monocultured QT fibroblasts compared to coculture at 24h. Similarly, PT and HT fibroblasts in monoculture, exhibited an increased production of PGE2 at $24 \mathrm{~h}$ and $120 \mathrm{~h}$ when subjected to physiological strain. Additionally, at $120 \mathrm{~h}, \mathrm{PT}$ fibroblasts in monoculture also showed higher production of PGE2 compared to cocultured fibroblasts (Fig. 6.3).

IL-6 production was not significantly different in cocultured when compared to monoculture in any of the tendon fibroblast types evaluated at any timepoint (Fig. 6.4). However, all the other cytokines were significantly increased in coculture.

The production of IL-8 was significantly increased in QT fibroblasts cocultured with SYN at 24h in the supraphysiological strain group. Moreover, at 120h, cocultured QT fibroblasts exhibited a significant increase in IL-8 production relative to monoculture in all strain groups. Similarly, cocultured PT fibroblasts, also exhibited a significantly higher production of IL-8 compared to monoculture at $120 \mathrm{~h}$ in all strain groups. Interestingly, cocultured HT fibroblasts exhibited a significant decrease in IL-8 production relative to monoculture when subjected to $4 \%$ strain at $24 \mathrm{~h}$ and $120 \mathrm{~h}$. Nevertheless, cocultured HT fibroblasts showed a significantly increased production of IL-8 compared to monoculture at 120h (Fig. 6.5).

The production of the chemokine $\mathrm{KC}$ was significantly higher in monocultured QT fibroblasts compared to co-culture in the no strain and 10\% strain groups at 24h. However, at $120 \mathrm{~h}$, cocultured QT fibroblasts exhibited a significantly increased production of KC compared to monoculture regardless of the strain applied. Moreover, physiological strain 
elicited an increased production of $\mathrm{KC}$ in monocultured PT and HT fibroblasts relative to coculture at 24h and 120h. However, at 120h, cocultured PT and HT fibroblasts exhibited significantly higher production of KC compared to monoculture at no strain (HT) and 10\% strain (PT and HT) (Fig. 6.6).

The production of MCP-1 in cocultured QT fibroblasts was significantly higher than monoculture at $24 \mathrm{~h}$ and $120 \mathrm{~h}$ of culture regardless of the strain. Moreover, at $24 \mathrm{~h}$, cocultured PT fibroblasts exhibited increased production of MCP-1 compared to monoculture at no strain and $10 \%$ strain. At 120h, cocultured PT fibroblasts showed significantly higher levels of MCP-1 compared to monoculture in all strain groups. Additionally, cocultured HT fibroblasts exhibited significantly higher levels of MCP-1 compared to monoculture at $24 \mathrm{~h}$ in all strain groups. At $120 \mathrm{~h}$, cocultured HT fibroblasts showed significantly higher production of MCP-1 compared to monoculture in the no strain and 10\% strain groups (Fig. 6.7).

GAG production in co-cultured QT fibroblasts was significantly higher than monoculture in all strain groups at $24 \mathrm{~h}$ of culture. Moreover, at $120 \mathrm{~h}, \mathrm{GAG}$ production was significantly higher in cocultured QT fibroblasts subjected to mechanical strain deprivation compared to monoculture. Similarly, cocultured PT fibroblasts showed significantly higher production of GAG than monoculture in the $10 \%$ strain group at $24 \mathrm{~h}$. However, at $120 \mathrm{~h}$, cocultured PT fibroblasts exhibited significantly lower production of GAG compared to monoculture when subjected to mechanical strain deprivation but significantly increased in cocultured PT fibroblasts at 4\% strain relative to monoculture. Moreover, cocultured HT fibroblasts significantly increased production of GAG at $24 \mathrm{~h}$ in the no strain and $4 \%$ strain 
groups. Similarly, at 120h, cocultured HT fibroblasts showed significantly higher levels of GAG content when subjected to physiological strain compared to monoculture (Fig. 6.8). The level of MMPACT was consistently higher in co-cultured QT fibroblasts compared to monoculture at $24 \mathrm{~h}$ in the no strain and $10 \%$ strain, but significantly lower in the $4 \%$ strain group. Similarly, at $120 \mathrm{~h}$, cocultured QT fibroblasts exhibited significantly decreased levels of MMPACT compared to monoculture in the $4 \%$ strain group. On the other hand, cocultured PT fibroblasts showed a significant increase in MMPACT compared to monoculture in the $4 \%$ strain and $10 \%$ strain groups at $24 \mathrm{~h}$. Also, at $120 \mathrm{~h}, 4 \%$ strain showed a significant increase in MMPACT relative to monocultured PT fibroblasts. Moreover, cocultured HT fibroblasts showed significantly higher levels of MMPACT compared to monoculture in the $10 \%$ strain group at $24 \mathrm{~h}$. MMPACT was also significantly higher at $120 \mathrm{~h}$ of supraphysiological strain in cocultured HT fibroblasts relative to monoculture (Fig. 6.9).

Co-culture conditions typically resulted in significantly decreased MMP-1 production in QT and PT fibroblasts compared to monoculture at all strains at both $24 \mathrm{~h}$ and $120 \mathrm{~h}$. Similarly, cocultured HT fibroblasts produced significantly less MMP-2 in the no strain and $4 \%$ strain groups at $24 \mathrm{~h}$ and in all strain groups at $120 \mathrm{~h}$ (Fig. 6.10). On the other hand, production of MMP-2 was significantly increased in cocultured QT fibroblasts relative to monoculture in all strain groups at $24 \mathrm{~h}$ of culture. However, at $120 \mathrm{~h}$, cocultured QT fibroblasts exhibited significantly less MMP-2 production compared to monoculture in the no strain group. Moreover, at 24h, there were no significant differences between cocultured and monocultured PT and HT fibroblasts. Interestingly, at 120h, MMP-2 production 
significantly increased in monocultured PT fibroblasts compared to coculture but decreased in HT co-culture compared to monoculture at physiological strain (Fig. 6.11). The production of MMP-3 was significantly higher in monocultured QT fibroblasts relative to monoculture in the no strain group at $24 \mathrm{~h}$ and $120 \mathrm{~h}$. Moreover, MMP-3 production was significantly lower in monocultured PT fibroblasts relative to coculture in the no strain and $10 \%$ strain groups at $24 \mathrm{~h}$. No significant differences between cocultured and monocultured HT fibroblasts were seen in any strain group at $24 \mathrm{~h}$ and $120 \mathrm{~h}$ (Fig. 6.12).

Differences between strain loads within fibroblast cell type during co-culture When evaluating strain responses in cocultured grafts it was observed that co-cultured QT fibroblasts subjected to mechanical strain deprivation had a significantly increased metabolic activity when compared to physiological and supraphysiological strain groups Moreover, cocultured PT fibroblasts exhibited a significantly decreased metabolic activity supraphysiological strain relative to mechanical strain deprivation and physiological strain (Fig.6.13). Additionally, with mechanical deprivation, co-cultured QT fibroblasts had significantly higher metabolic activity compared to co-cultured PT and HT fibroblasts. 4\% and $10 \%$ strain did not elicit significant differences in metabolic activity in any of the tissues (Fig. 6.21).

While the production of PGE2 in QT fibroblasts co-cultured with synoviocytes was not significantly different among strains, it typically resulted in significant increases in PGE2 from PT fibroblasts at no strain relative to $4 \%$ and $10 \%$ strain groups at $120 \mathrm{~h}$ of culture. Moreover, the production of PGE2 by co-cultured HT fibroblasts was significantly lower at physiological stress compared to mechanical strain deprivation and supraphysiological strain at 24h. Further, at 120h, the production of PGE2 by co-cultured HT fibroblasts at 
physiological stress was significantly lower than the production by co-cultured HT fibroblasts at mechanical strain deprivation and supraphysiological strain (Fig. 6.14).

The production of IL-8 production was consistently low in co-cultured QT fibroblasts subjected to mechanical strain deprivation relative to supraphysiological strain at $24 \mathrm{~h}$ and 120h of culture. Cocultured PT fibroblasts exhibited a significantly decreased production of IL-8 after $120 \mathrm{~h}$ of physiological strain relative to no strain and $10 \%$ strain groups. Moreover, cocultured HT fibroblasts exhibited significantly lower levels of IL-8 at 4\% strain relative to $10 \%$ strain at $24 \mathrm{~h}$ of culture. Additionally, at $120 \mathrm{~h}$, cocultured HT fibroblasts produced significantly lower levels of IL-8 in the physiological strain group compared to the mechanical strain deprivation and supraphysiological strain groups (Fig. $6.15)$.

KC production significantly increased by co-cultured tendon fibroblasts in the supraphysiologic strain groups compared to the physiological strain groups at $120 \mathrm{~h}$ of culture. Cocultured QT fibroblasts showed significantly increased levels of KC after 120h of supraphysiological strain relative to no strain and $4 \%$ strain groups. Moreover, cocultured PT fibroblasts exhibited a significantly higher production of $\mathrm{KC}$ in the $10 \%$ strain group relative to the $4 \%$ strain group at $120 \mathrm{~h}$ of culture. Additionally, co-cultured HT fibroblasts showed significantly lower levels of KC after $24 \mathrm{~h}$ of physiological strain relative to $10 \%$ strain. Similarly, at $120 \mathrm{~h}$, cocultured HT fibroblast showed significantly lower production of $\mathrm{KC}$ in the $4 \%$ strain group relative to no strain and $10 \%$ strain groups (Fig. 6.16).

Further, co-cultured QT fibroblasts significantly lower production of MCP-1 at no strain compared to $4 \%$ and $10 \%$ strain at $24 \mathrm{~h}$. Similarly, at $120 \mathrm{~h}$, cocultured QT fibroblasts at no 
strain produced significantly less MCP-1 than at 10\% strain. Moreover, cocultured PT and HT fibroblasts produced significantly less MCP-1 at 4\% strain compared to no strain and $10 \%$ strain at $24 \mathrm{~h}$ and $120 \mathrm{~h}$ of culture (Fig. 6.17).

The production of GAG in co-cultured QT fibroblasts was significantly lower in the supraphysiological strain group compared to the no strain and $10 \%$ strain groups at $24 \mathrm{~h}$ of culture. Moreover, cocultured PT fibroblasts showed significantly higher levels of GAG after120h of physiological strain relative to mechanical strain deprivation. Additionally, cocultured HT fibroblasts did not show significant changes among strain groups at any time point (Fig. 6.18).

The level of MMPACT was significantly lower in co-cultured QT fibroblasts at physiological strain compared to no strain and $10 \%$ strain at $24 \mathrm{~h}$. Moreover, co-cultured QT fibroblasts showed a significant increase in MMPACT when cells were subjected to mechanical strain deprivation compared to $4 \%$ and $10 \%$ strain at $120 \mathrm{~h}$ of culture. Additionally, co-cultured PT fibroblasts showed exhibited a significant decrease in MMPACT in the no strain group relative to $4 \%$ and $10 \%$ strain at $24 \mathrm{~h}$ of culture. Also, at 120h, cocultured PT fibroblasts showed a significantly higher production of MMPACT at $4 \%$ strain relative to no strain and $10 \%$ strain groups. In cocultured HT fibroblasts, MMPACT was significantly higher at $10 \%$ strain compared to $4 \%$ strain at $24 \mathrm{~h}$ (Fig. 6.19) The production of MMP-2 was significantly lower in cocultured QT fibroblasts subjected to physiological strain compared to no strain and $10 \%$ strain groups at $24 \mathrm{~h}$. Moreover, at 120h, supraphysiological strain elicited a significant increase in MMP2 production compared to no strain and $4 \%$ strain. Additionally, cocultured PT fibroblasts did not show significant differences in the production of MMP-2 at any strain level in any timepoint. 
However, cocultured HT fibroblasts showed significantly increased levels of MMP-2 when subjected to $4 \%$ strain relative to no strain and $10 \%$ strain at $120 \mathrm{~h}$ of culture (Fig. 6.20) Differences between fibroblast types at each strain load during co-culture

PGE2 production was significantly different among tissue type fibroblasts. During mechanical deprivation, co-cultured PT and HT fibroblasts produced significantly higher levels of PGE2 compared to co-cultured QT fibroblasts at 24h of culture. Moreover, 4\% strain elicited a significantly increased production of PGE2 in cocultured QT fibroblasts relative to PT and HT at $120 \mathrm{~h}$. PGE2 production was also significantly higher in cocultured QT fibroblasts relative to cocultured PT fibroblasts when subjected to supraphysiological strain at $24 \mathrm{~h}$ and $120 \mathrm{~h}$ (Fig. 6.22).

Mechanical strain deprivation did not elicit significant differences in IL-8 production among tendon fibroblasts at any timepoint. However, $4 \%$ and $10 \%$ strain elicited a significant increase in IL-8 production in cocultured QT fibroblasts relative to cocultured PT and HT fibroblasts at 120h (Fig. 6.23).

The production of KC was significantly higher in cocultured HT fibroblasts relative to QT after $120 \mathrm{~h}$ of mechanical strain deprivation. Moreover, at 4\% strain, cocultured QT fibroblasts exhibited a significantly higher production of $\mathrm{KC}$ relative to $\mathrm{PT}$ and $\mathrm{HT}$ fibroblasts at $120 \mathrm{~h}$ of culture. Additionally, at $10 \%$ strain, cocultured HT fibroblasts showed a significantly increased production of KC relative to QT fibroblasts at $24 \mathrm{~h}$ (Fig. 6.24).

MCP-1 production was significantly lower in cocultured QT fibroblasts compared to HT fibroblasts at $24 \mathrm{~h}$ of mechanical strain deprivation. Similarly, after $120 \mathrm{~h}$ of mechanical strain deprivation, a significant decrease in MCP-1 production was seen in cocultured QT 
fibroblasts relative to PT and HT cocultured fibroblasts. Moreover, physiological strain elicited a significant increase in MCP-1 production in cocultured QT fibroblasts relative to PT and HT fibroblasts at 24h and 120h, respectively. No significant differences among tissues were seen at $10 \%$ strain in any of the tissue types at any time point (Fig. 6.25). The production of GAG was significantly lower in cocultured PT fibroblasts relative to QT and HT fibroblasts at $120 \mathrm{~h}$ of mechanical deprivation. Also, at $4 \%$ strain, cocultured PT fibroblasts exhibited a significant increase in GAG production relative to cocultured QT fibroblasts at $120 \mathrm{~h}$. There were no significant differences in GAG production among the different tissue fibroblasts at any timepoint (Fig. 6.26).

The level of MMPACT was not significantly different between tissue types at any time point in the mechanical strain deprivation and supraphysiological strain groups. However, during physiological strain, the level of MMPACT in cocultured PT fibroblasts was significantly higher than QT fibroblasts at $24 \mathrm{~h}$ of culture. Also, the production of MMPACT was significantly lower in co-cultured QT fibroblasts compared to PT and HT fibroblasts at $120 \mathrm{~h}$ of culture (Fig. 6.27).

The production of MMP-2 was not significantly different among tissue fibroblast types at mechanical strain deprivation at any time point. However, at $4 \%$ strain, cocultured QT fibroblasts produced significantly more MMP-2 than PT and HT fibroblast at 24h of culture. Moreover, at 120h, $4 \%$ strain elicited a significant increase in MMP-2 production in cocultured HT fibroblasts relative to QT and PT fibroblasts. At supraphysiological strain, the production of MMP-2 by cocultured QT fibroblasts was significantly higher than PT fibroblasts at 24h of culture (Fig. 6.28). 


\section{Discussion}

The synovial membrane that surrounds the cruciate ligaments plays key roles in their nutrition and blood supply. ${ }^{4 ;} 21$ Synoviocytes are also responsible for the production of synovial fluid constituents and maintenance of joint homeostasis by regulating blood/synovial exchange, phagocytic signaling, and cell-cell and cell-matrix interactions. ${ }^{22}$ The synovial membrane is disrupted when ACL is ruptured, which renders the ACL intrasynovial such that the internal epiligament is exposed to breakdown products and proteolytic enzymes that affect healing. ${ }^{23 ; 24}$

Different tissues within the knee joint contribute to the production of metalloproteinases, after ACL injury. Tang et al., (2009) showed that synovium from ACL-injured rats had a significantly higher concentration of MMP-2 when compared to normal non-injured rats. ${ }^{4}$ Synovial tissue had the greatest ability to convert pro-MMP-2 to the activated isoform of MMP-2 and release MMP-2 to synovial fluid. ${ }^{4}$ Further, it has been reported that release of MMP-2 is increased after type B synoviocytes are overstretched in an equi-biaxial bioreactor. $^{4}$ Our results show that MMP-2 production is increased when QT graft fibroblasts are co-cultured with synoviocytes. Moreover, at supraphysiological strain levels (10\%) the production of MMP-2 by co-cultured QT fibroblasts was significantly higher than mono-cultured QT fibroblasts at $24 \mathrm{~h}$ of culture. Interestingly, the production of MMP2 was significantly higher in mono-cultured PT fibroblasts compared to co-cultured PTfibroblasts at physiological strain but not at mechanical strain deprivation or supraphysiological strain, suggesting that the regulatory function exerted by synoviocytes is tissue-dependent and that graft fibroblastic degradative responses are highly dependent on strain magnitude. 
Intraarticular fibroblasts are involved in extracellular matrix (ECM) production including different types of collagen, proteoglycans and hyaluronic acid that are essential components in healing after injury or surgical repair. ${ }^{25-29} \mathrm{ECM}$ turnover is also affected by cross-talk among tissues and differentially regulated by strain magnitude. ${ }^{30 ; 31}$ Our results suggest that ACL tendon grafts in co-culture with synoviocytes were associated with increased production of GAG and decreased MMP activity after five days of supraphysiological strain.

Animal models have shown that cytokine concentrations (IL-1 $\beta$, IL-6 and TNF- $\alpha$ ) increased in the synovial fluid within three days after ACL injury. ${ }^{4}$ Authors suggest that synovial tissue might be the main producer of cytokines which in turn activate MMP production during an attempted healing response. ${ }^{4}$ Similarly, other authors have reported IL-6 overproduction in inflammatory and degenerative conditions such as osteoarthritis and ligament disease. ${ }^{32}$ In the present study, production of IL-6 was not detected. Other studies have reported that measurable IL-6 production from fibroblasts requires stimulation with a pro-inflammatory mediator. ${ }^{33 ;} 34$ Importantly, the inability to detect IL-6 with mechanical insults alone in the present study highlights the limitations of in vitro models in not fully mimicking in vivo conditions. ${ }^{32 ; 33 ; 35 ; 36}$

The roles of cytokines on synovial fibroblasts under mechanical stress in monoculture have been demonstrated previously. ${ }^{37}$ Cytokines and chemokines in the present study were produced at significantly higher levels when graft fibroblasts were co-cultured with synoviocytes. Moreover, non-physiological strain, either mechanical deprivation or supraphysiological strain conditions, induced increased production of cytokines and chemokines (IL-8, KC and MCP-1) in co-cultured fibroblasts compared to those in 
monoculture. Interestingly, physiological stress decreased KC and IL-8 production levels by HT fibroblasts co-cultured with synoviocytes.

Co-cultured PT fibroblasts subjected to mechanical deprivation showed the highest increase in PGE2 relative to physiological strain. This might suggest that the reported increased risk of developing OA after BPTB reconstruction could be related to decreased fibroblastic stimulation and inadequate levels of postoperative activity or physical therapy ${ }^{38-40}$. Moreover, Hirata et al., (2004) showed that synovial fibroblasts subjected to a $20 \%$ strain deformation increased production of both VEGF and PGE2 after 6 hours. $^{2}$ Consequently, authors suggest that PGE2 might be functionally linked to VEGF and may contribute to tenosynovial pathology in carpal tunnel syndrome (CTS) when subjected to supraphysiological stresses. ${ }^{2}$

Xie et al., (2018) showed that PCL fibroblasts co-cultured with synoviocytes and subjected to supraphysiological strain (12\% strain), expressed more MMP-2 than the same cell types in mono-culture and that MMP-2 activity increased with higher synoviocyte density in coculture. ${ }^{41}$ In our experiment, MMP-1 and MMP-3 production was relatively low and not affected by culture condition, cell type, or strain level. Since MMP production responds to both, pro-inflammatory and growth factors stimulation, the low production of these MMPs in this study may relate to the lack of inflammatory stimulation in the model to stimulate the production of these degradative enzymes by the cells during in vitro culture. ${ }^{37 ; 42}$ Huisman et al., (2016), showed that human HT fibroblasts subjected to $10 \%$ strain exhibited higher MMP-2 gene expression compared to low strain (2\%). ${ }^{43}$ Additionally, Jiang et al., (2012) showed that strain deprivation elicited a decreased collagen I synthesis in human PT fibroblasts compared to $4 \%$ and $8 \%$ strain. ${ }^{44}$ Moreover, Demirag et al., (2005) 
demonstrated that blockage of MMPs with $\alpha_{2}$-Macroglobulin in a rabbit model of ACL reconstruction with semitendinosus graft, had higher ultimate load to failure and denser connective tissue around the bone tunnel compared to the control group (no $\alpha_{2-}$ macroglobulin). ${ }^{45}$ In this work, the production of MMP-2 was variable. There was decreased MMP-2 production in cocultured QT fibroblasts compared to monoculture after 120h of mechanical strain deprivation. Additionally, at 4\% strain, while PT fibroblasts in coculture exhibited significantly lower production of MMP-2 than monoculture, cocultured HT fibroblasts showed higher MMP-2 production than in monoculture conditions. These findings demonstrate that MMP-2 production is both, strain and tissue dependent and that activity level should be matched appropriately to the type of graft. ${ }^{46}$

In the present study, physiological load decreased the production of GAG in co-cultured fibroblasts, and increased levels of IL-8 and KC in mono-culture fibroblasts after 24 hours of culture. It is possible that synoviocytes protect graft fibroblasts from initial excessive ECM remodeling. However, graft type appears to play key roles in ECM production shown by the different GAG production profiles of each graft. Moreover, supraphysiological stress (10\%), led to an initial (24h) increase in GAG and MMP-2 production by QT and HT fibroblasts when co-cultured, as well as a late (120h) increases in IL-8 and PGE2 production, showing the differential adaptability of each graft type to load.

This study demonstrates important interactions between ACL graft tenocytes and synoviocytes, and the importance of synoviocytes in regulating inflammatory and metabolic responses when graft fibroblasts are subjected to clinically relevant strain loads. Our findings suggest that there is an increased ECM remodeling during co-culture as indicated by the increased production of GAG from graft fibroblasts, especially at non- 
physiological loads. This could be related to the decreased organizational structure (scar tissue) provoked by mechanical deprivation or microdamage due to overloading fatigue. ${ }^{8}$ Moreover, our results indicate that both graft type and mechanical strain are important factors to consider before selecting therapeutic protocols after ACL reconstruction. These data suggest that current perioperative activity protocols should be adjusted to each specific graft type in order to optimize the ligamentization of the tendon graft and potentially decrease failure rates after surgery. ${ }^{38 ;}$ 47-51 The data from this study indicates that synoviocytes can affect the metabolism of tendon grafts after ACL reconstruction, and that the effects of synoviocytes are tendon cell type and strain magnitude dependent. 


\section{REFERENCES}

1. Scheffler SU, Unterhauser FN, Weiler A. 2008. Graft remodeling and ligamentization after cruciate ligament reconstruction. Knee surgery, sports traumatology, arthroscopy : official journal of the ESSKA 16:834-842.

2. Hirata H, Nagakura T, Tsujii M, et al. 2004. The relationship of VEGF and PGE2 expression to extracellular matrix remodelling of the tenosynovium in the carpal tunnel syndrome. The Journal of Pathology 204:605-612.

3. Iwanaga T, Shikichi M, Kitamura H, et al. 2000. Morphology and functional roles of synoviocytes in the joint. Archives of histology and cytology 63:17-31.

4. Tang Z, Yang L, Wang Y, et al. 2009. Contributions of different intraarticular tissues to the acute phase elevation of synovial fluid MMP-2 following rat ACL rupture. Journal of orthopaedic research : official publication of the Orthopaedic Research Society 27:243-248.

5. Wang C, Xie J, Jiang J, et al. 2015. Differential expressions of the lysyl oxidase family and matrix metalloproteinases-1, 2, 3 in posterior cruciate ligament fibroblasts after being co-cultured with synovial cells. Int Orthop 39:183-191.

6. Dagher E, Hays PL, Kawamura S, et al. 2009. Immobilization modulates macrophage accumulation in tendon-bone healing. Clinical orthopaedics and related research 467:281-287.

7. Bedi A, Kovacevic D, Fox AJ, et al. 2010. Effect of early and delayed mechanical loading on tendon-to-bone healing after anterior cruciate ligament reconstruction. The Journal of bone and joint surgery American volume 92:2387-2401. 
8. Killian ML, Cavinatto L, Galatz LM, et al. 2012. The role of mechanobiology in tendon healing. J Shoulder Elbow Surg 21:228-237.

9. Brophy RH, Kovacevic D, Imhauser CW, et al. 2011. Effect of short-duration low-magnitude cyclic loading versus immobilization on tendon-bone healing after ACL reconstruction in a rat model. The Journal of bone and joint surgery American volume 93:381-393.

10. Ma R, Stasiak M, Deng XH, et al. 2018. A Preclinical Model to Study the Influence of Graft Force on the Healing of the Anterior Cruciate Ligament Graft. The journal of knee surgery.

11. Ma R, Schar M, Chen T, et al. 2018. Effect of Dynamic Changes in Anterior Cruciate Ligament In Situ Graft Force on the Biological Healing Response of the Graft-Tunnel Interface. The American journal of sports medicine 46:915-923.

12. Zong JC, Ma R, Wang H, et al. 2017. The Effect of Graft Pretensioning on Bone Tunnel Diameter and Bone Formation After Anterior Cruciate Ligament Reconstruction in a Rat Model: Evaluation With Micro-Computed Tomography. The American journal of sports medicine 45:1349-1358.

13. Chiquet M, Gelman L, Lutz R, et al. 2009. From mechanotransduction to extracellular matrix gene expression in fibroblasts. Biochim Biophys Acta 1793:911-920.

14. Kim SG, Akaike T, Sasagaw T, et al. 2002. Gene expression of type I and type III collagen by mechanical stretch in anterior cruciate ligament cells. Cell structure and function 27:139-144. 
15. Hannafin JA, Attia EA, Henshaw R, et al. 2006. Effect of cyclic strain and plating matrix on cell proliferation and integrin expression by ligament fibroblasts. Journal of orthopaedic research : official publication of the Orthopaedic Research Society 24:149-158.

16. Henshaw DR, Attia E, Bhargava M, et al. 2006. Canine ACL fibroblast integrin expression and cell alignment in response to cyclic tensile strain in threedimensional collagen gels. Journal of Orthopaedic Research 24:481-490.

17. Breshears LA, Cook JL, Stoker AM, et al. 2010. The effect of uniaxial cyclic tensile load on gene expression in canine cranial cruciate ligamentocytes. Veterinary surgery : VS 39:433-443.

18. Wang T, Chen P, Zheng M, et al. 2018. In vitro loading models for tendon mechanobiology. Journal of orthopaedic research : official publication of the Orthopaedic Research Society 36:566-575.

19. Riss TL, Moravec RA, Niles AL, et al. 2004. Cell Viability Assays. In: Sittampalam GS, Grossman A, Brimacombe K, et al. editors. Assay Guidance Manual. Bethesda (MD): Eli Lilly \& Company and the National Center for Advancing Translational Sciences.

20. Farndale RW, Buttle DJ, Barrett AJ. 1986. Improved quantitation and discrimination of sulphated glycosaminoglycans by use of dimethylmethylene blue. Biochim Biophys Acta 883:173-177.

21. Dandy DJ, Pusey RJ. 1982. The long-term results of unrepaired tears of the posterior cruciate ligament. The Journal of bone and joint surgery British volume 64:92-94. 
22. Bhattaram P, Chandrasekharan U. 2017. The joint synovium: A critical determinant of articular cartilage fate in inflammatory joint diseases. Seminars in cell \& developmental biology 62:86-93.

23. Murray MM, Martin SD, Martin TL, et al. 2000. Histological changes in the human anterior cruciate ligament after rupture. The Journal of bone and joint surgery American volume 82:1387-1397.

24. Andrish J, Holmes R. 1979. Effects of synovial fluid on fibroblasts in tissue culture. Clinical orthopaedics and related research:279-283.

25. Neurath MF, Stofft E. 1992. Structure and function of matrix components in the cruciate ligaments. An immunohistochemical, electron-microscopic, and immunoelectron-microscopic study. Acta anatomica 145:387-394.

26. Nguyen DT, Ramwadhdoebe TH, van der Hart CP, et al. 2014. Intrinsic healing response of the human anterior cruciate ligament: An histological study of reattached ACL remnants. 32:296-301.

27. Alexander RM. 2002. Tendon elasticity and muscle function. Comparative biochemistry and physiology Part A, Molecular \& integrative physiology 133:1001-1011.

28. Bliss S, Rawlinson JJ, Todhunter R. 2013. Tissues of the musculoskeletal system. In: Tobias KM, Johnston SA editors. Veterinary Surgery: Small Animal-EBOOK: 2-Volume Set: Elsevier Health Sciences; pp. 553-564.

29. Yang G, Rothrauff BB, Tuan RS. 2013. Tendon and ligament regeneration and repair: clinical relevance and developmental paradigm. Birth defects research Part C, Embryo today : reviews 99:203-222. 
30. Arnoczky SP, Tian T, Lavagnino M, et al. 2002. Activation of stress-activated protein kinases (SAPK) in tendon cells following cyclic strain: the effects of strain frequency, strain magnitude, and cytosolic calcium. Journal of Orthopaedic Research 20:947-952.

31. Simmons CA, Matlis S, Thornton AJ, et al. 2003. Cyclic strain enhances matrix mineralization by adult human mesenchymal stem cells via the extracellular signal-regulated kinase (ERK1/2) signaling pathway. Journal of biomechanics $36: 1087-1096$.

32. Fujita Y, Hara Y, Nezu Y, et al. 2006. Proinflammatory Cytokine Activities, Matrix Metalloproteinase-3 Activity, and Sulfated Glycosaminoglycan Content in Synovial Fluid of Dogs with Naturally Acquired Cranial Cruciate Ligament Rupture. Veterinary Surgery 35:369-376.

33. Irie K, Uchiyama E, Iwaso H. 2003. Intraarticular inflammatory cytokines in acute anterior cruciate ligament injured knee. Knee 10:93-96.

34. Troyer LD, Stoker A, Ma R, et al. 2019. Metabolic Responses of Ligament and Tendon to Cytokine Stimulation ex vivo. In: ORS editor. 2019 ORS annual meeting. Austin, TX.

35. Venn G, Nietfeld JJ, Duits AJ, et al. 1993. Elevated synovial fluid levels of interleukin-6 and tumor necrosis factor associated with early experimental canine osteoarthritis. Arthritis and rheumatism 36:819-826.

36. Hui AY, McCarty WJ, Masuda K, et al. 2012. A systems biology approach to synovial joint lubrication in health, injury, and disease. Wiley interdisciplinary reviews Systems biology and medicine 4:15-37. 
37. Wang Y, Yang L, Zhang J, et al. 2010. Differential MMP-2 activity induced by mechanical compression and inflammatory factors in human synoviocytes. Molecular \& cellular biomechanics : MCB 7:105-114.

38. Sauerschnig M, Stolberg-Stolberg J, Schmidt C, et al. 2018. Effect of COX-2 inhibition on tendon-to-bone healing and PGE2 concentration after anterior cruciate ligament reconstruction. European journal of medical research 23:1.

39. Li X, Ellman M, Muddasani P, et al. 2009. Prostaglandin E2 and its cognate EP receptors control human adult articular cartilage homeostasis and are linked to the pathophysiology of osteoarthritis. Arthritis and rheumatism 60:513-523.

40. Attur M, Al-Mussawir HE, Patel J, et al. 2008. Prostaglandin E2 exerts catabolic effects in osteoarthritis cartilage: evidence for signaling via the EP4 receptor. $\mathrm{J}$ Immunol 181:5082-5088.

41. Xie J, Wang CL, Yang W, et al. 2018. Modulation of MMP-2 and MMP-9 through connected pathways and growth factors is critical for extracellular matrix balance of intra-articular ligaments. J Tissue Eng Regen Med 12:e550-e565.

42. Sternlicht MD, Werb Z. 2001. How matrix metalloproteinases regulate cell behavior. Annual review of cell and developmental biology 17:463-516.

43. Huisman E, Lu A, Jamil S, et al. 2016. Influence of repetitive mechanical loading on MMP2 activity in tendon fibroblasts. Journal of Orthopaedic Research 34:1991-2000.

44. Jiang C, Shao L, Wang Q, et al. 2012. Repetitive mechanical stretching modulates transforming growth factor-beta induced collagen synthesis and apoptosis in 
human patellar tendon fibroblasts. Biochemistry and cell biology $=$ Biochimie et biologie cellulaire 90:667-674.

45. Demirag B, Sarisozen B, Ozer O, et al. 2005. Enhancement of tendon-bone healing of anterior cruciate ligament grafts by blockage of matrix metalloproteinases. The Journal of bone and joint surgery American volume 87:2401-2410.

46. Escamilla RF, Macleod TD, Wilk KE, et al. 2012. Anterior cruciate ligament strain and tensile forces for weight-bearing and non-weight-bearing exercises: a guide to exercise selection. J Orthop Sports Phys Ther 42:208-220.

47. Schwarting T, Pretzsch S, Debus F, et al. 2015. The Effect of Cyclooxygenase Inhibition on Tendon-Bone Healing in anIn VitroCoculture Model. 2015:1-10.

48. Yang G, Im H-J, Wang JHC. 2005. Repetitive mechanical stretching modulates IL-1 $\beta$ induced COX-2, MMP-1 expression, and PGE2 production in human patellar tendon fibroblasts. Gene 363:166-172.

49. Thomopoulos S, Williams GR, Soslowsky LJ. 2003. Tendon to bone healing: differences in biomechanical, structural, and compositional properties due to a range of activity levels. Journal of biomechanical engineering 125:106-113.

50. Killian ML, Cavinatto L, Galatz LM, et al. 2012. The role of mechanobiology in tendon healing. Journal of shoulder and elbow surgery 21:228-237.

51. Filbay SR, Grindem H. 2019. Evidence-based recommendations for the management of anterior cruciate ligament (ACL) rupture. Best practice \& research Clinical rheumatology 33:33-47. 
Figures

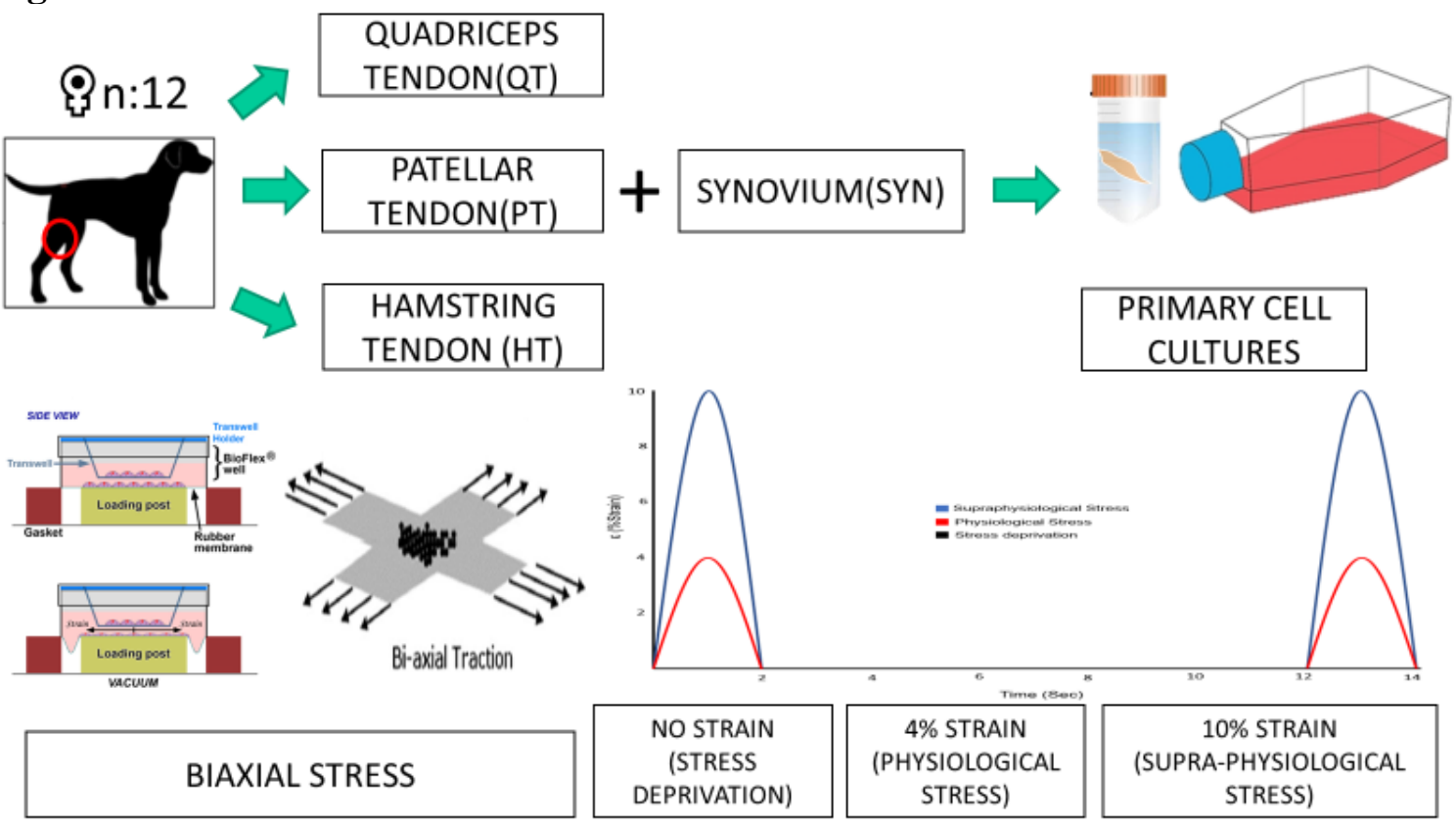

Figure 6.1. Experimental Design. 

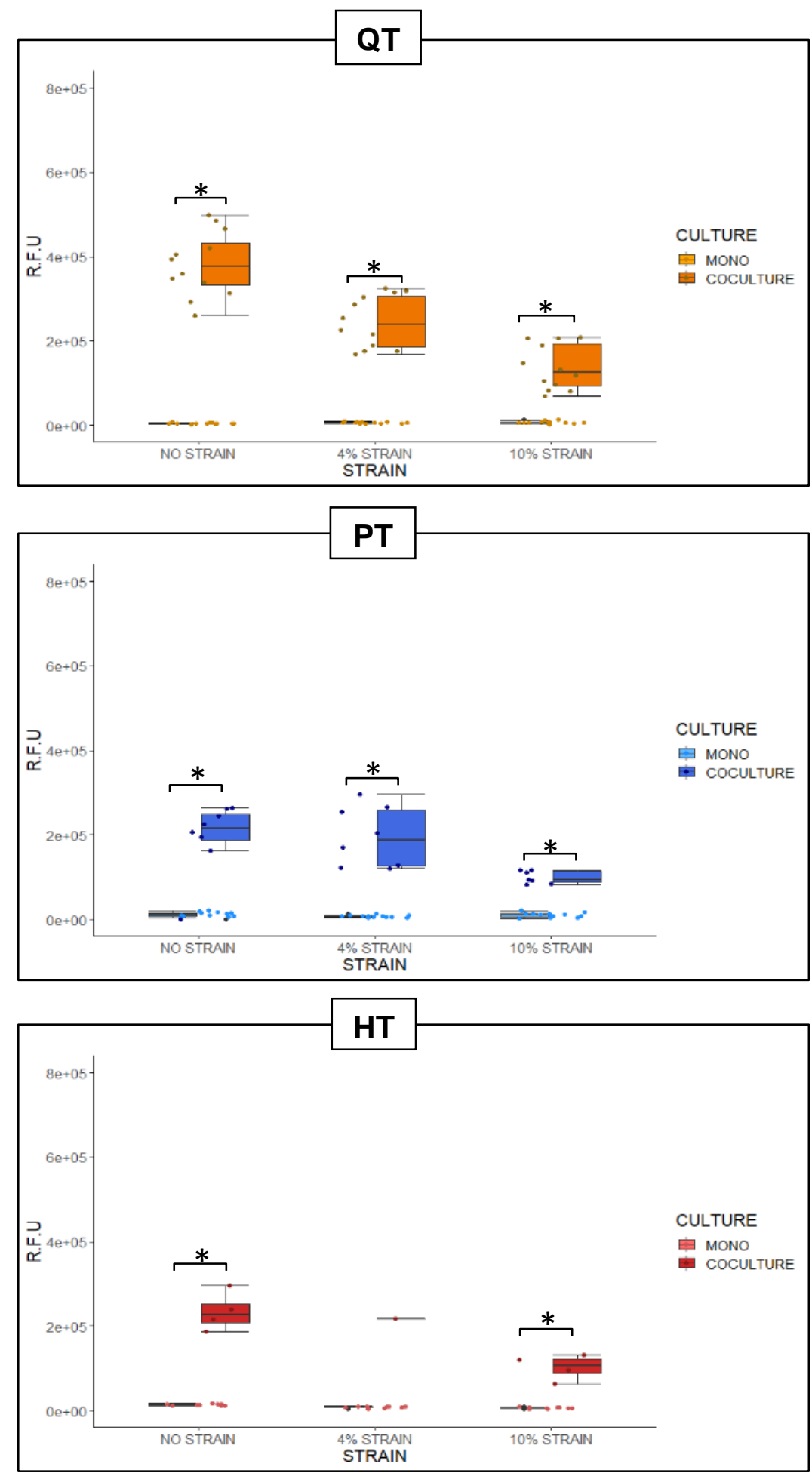

Figure 6.2. Metabolic Activity after $120 \mathrm{~h}$ of culture. * significant difference between monoculture (MONO) and co-culture (Signed Rank test). QT: Quadriceps tendon; PT: Patellar tendon, HT: Hamstring tendon 

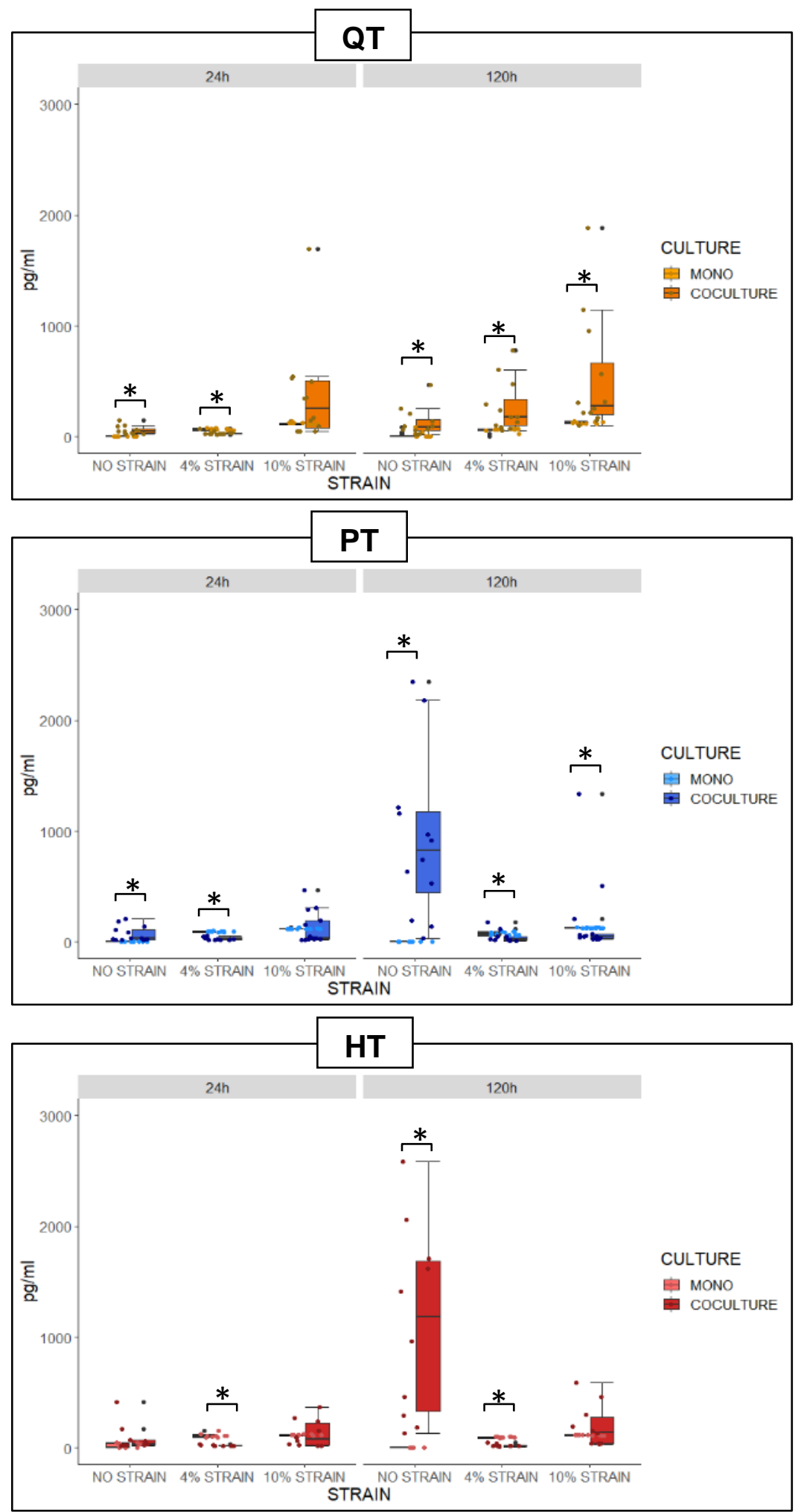

Figure 6.3. Concentration of prostaglandin E2 (PGE2) released to the media. * significant difference between monoculture (MONO) and co-culture at 24 and 120 hours (Signed Rank test); QT: Quadriceps tendon; PT: Patellar tendon, HT: Hamstring tendon 

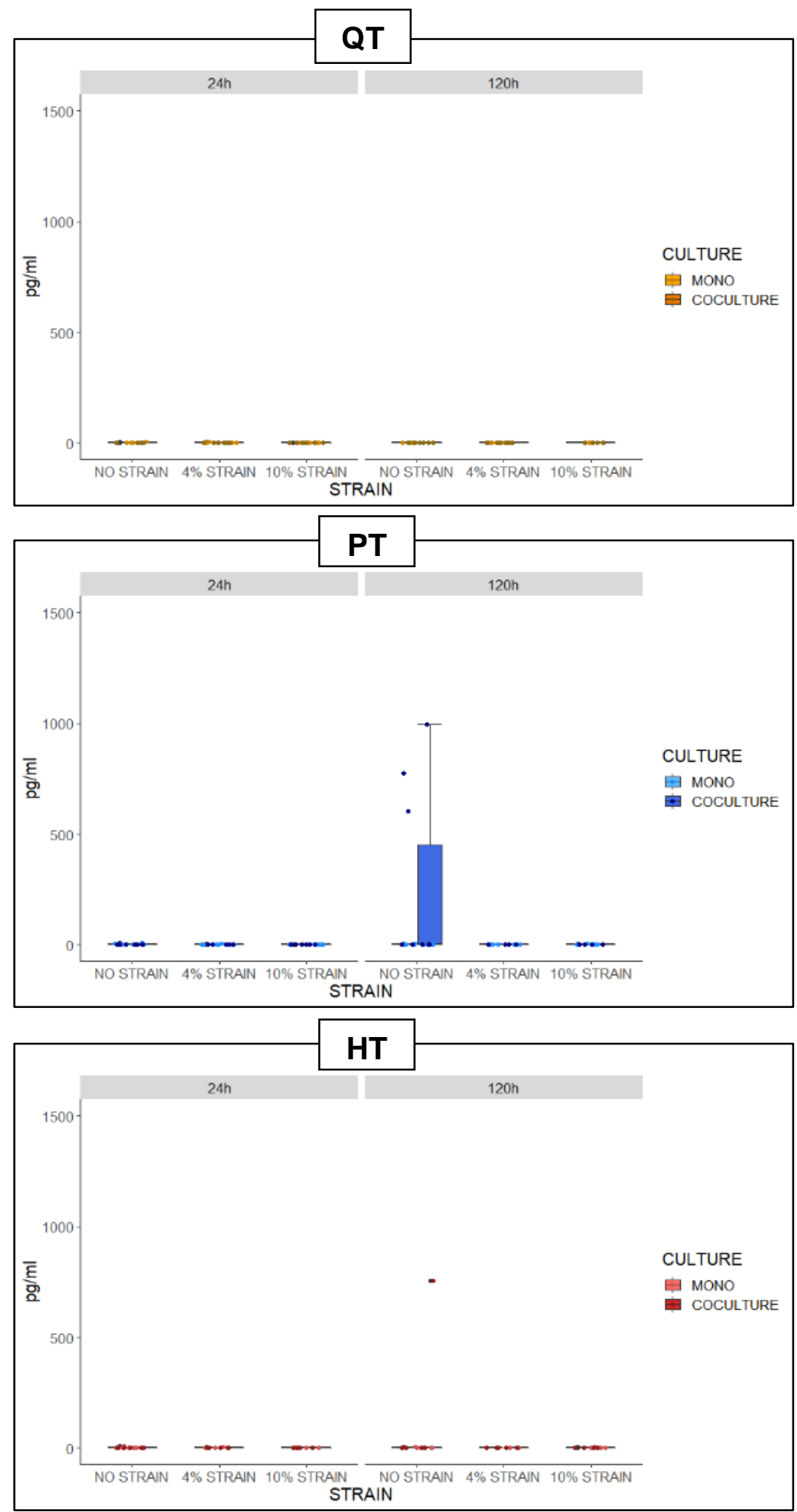

Figure 6.4. Concentration of interleukin-6 (IL-6) released to the media. * significant difference between monoculture (MONO) and co-culture (CO) at 24 and 120 hours (Signed Rank test). QT: Quadriceps tendon; PT: Patellar tendon, HT: Hamstring tendon 

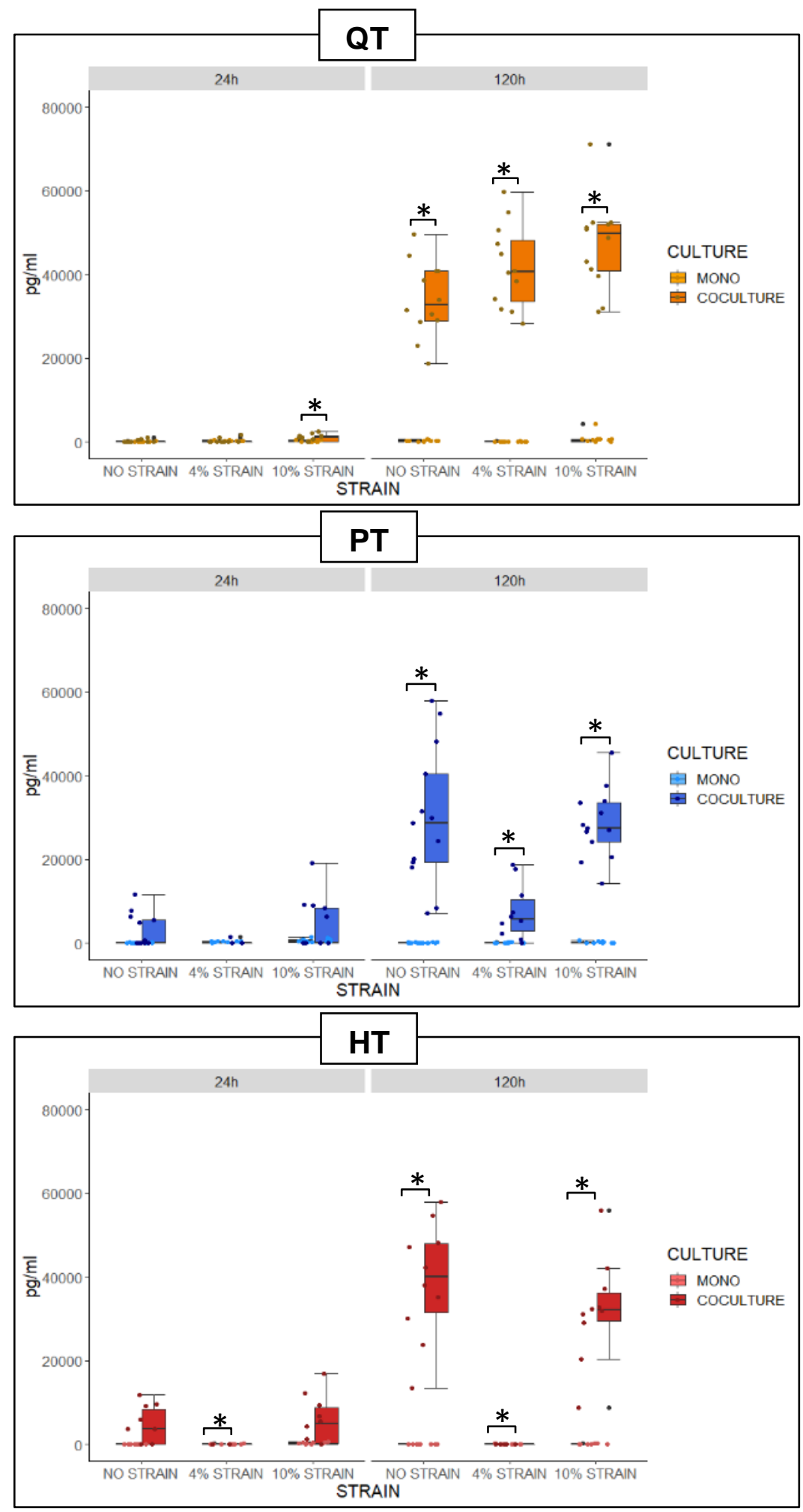

Figure 6.5. Concentration of intereukin-8 (IL-8), released to the media. * significant difference between monoculture (MONO) and co-culture at 24 and 120 hours (Signed Rank test). QT: Quadriceps tendon; PT: Patellar tendon, HT: Hamstring tendon 


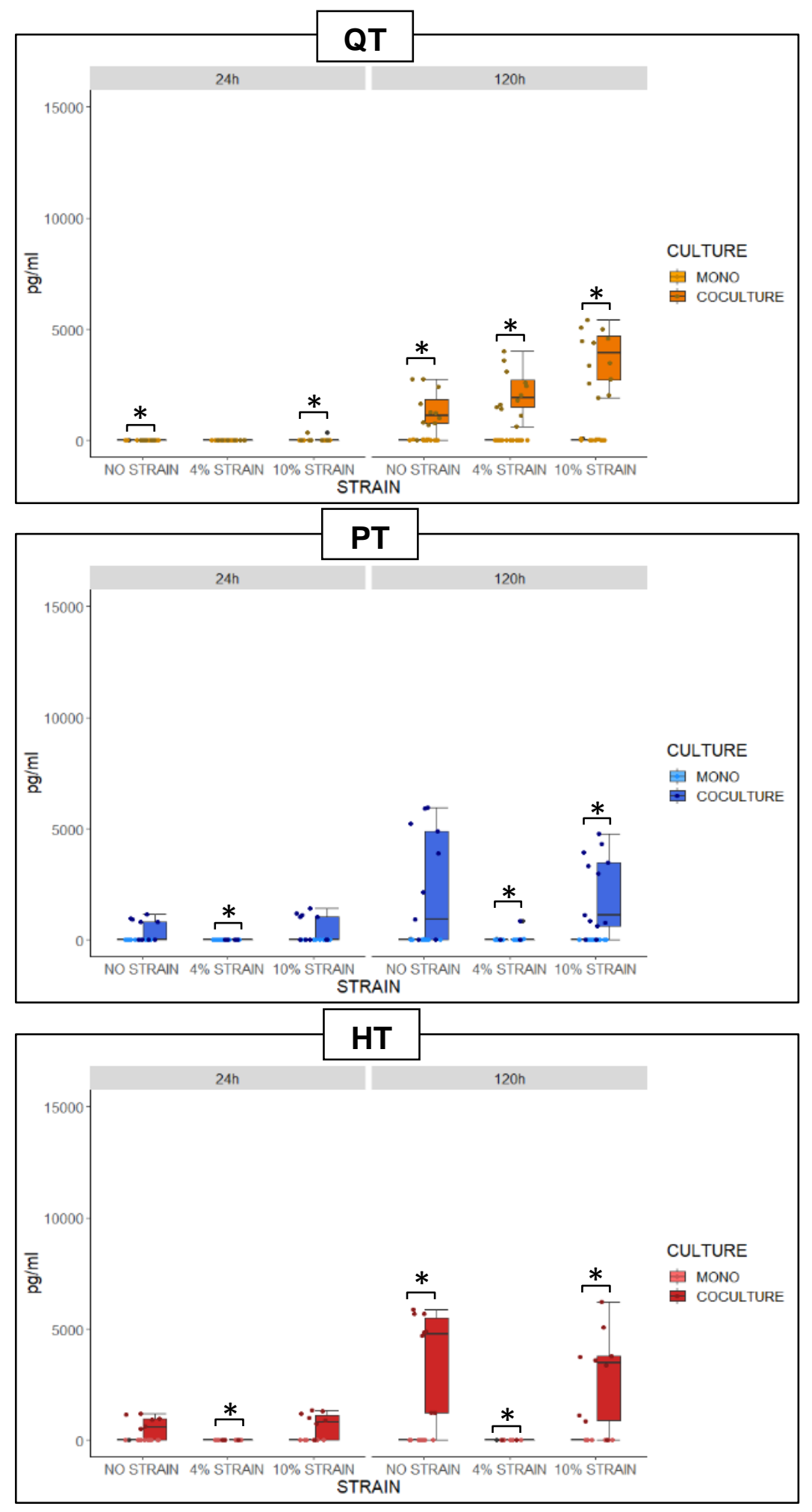

Figure 6.6. Concentration of keratinocyte chemoattractant protein $(\mathrm{KC})$ released to the media. * significant difference between monoculture (MONO) and co-culture at 24 and 120 hours (Signed Rank test). QT: Quadriceps tendon; PT: Patellar tendon, HT: Hamstring tendon 


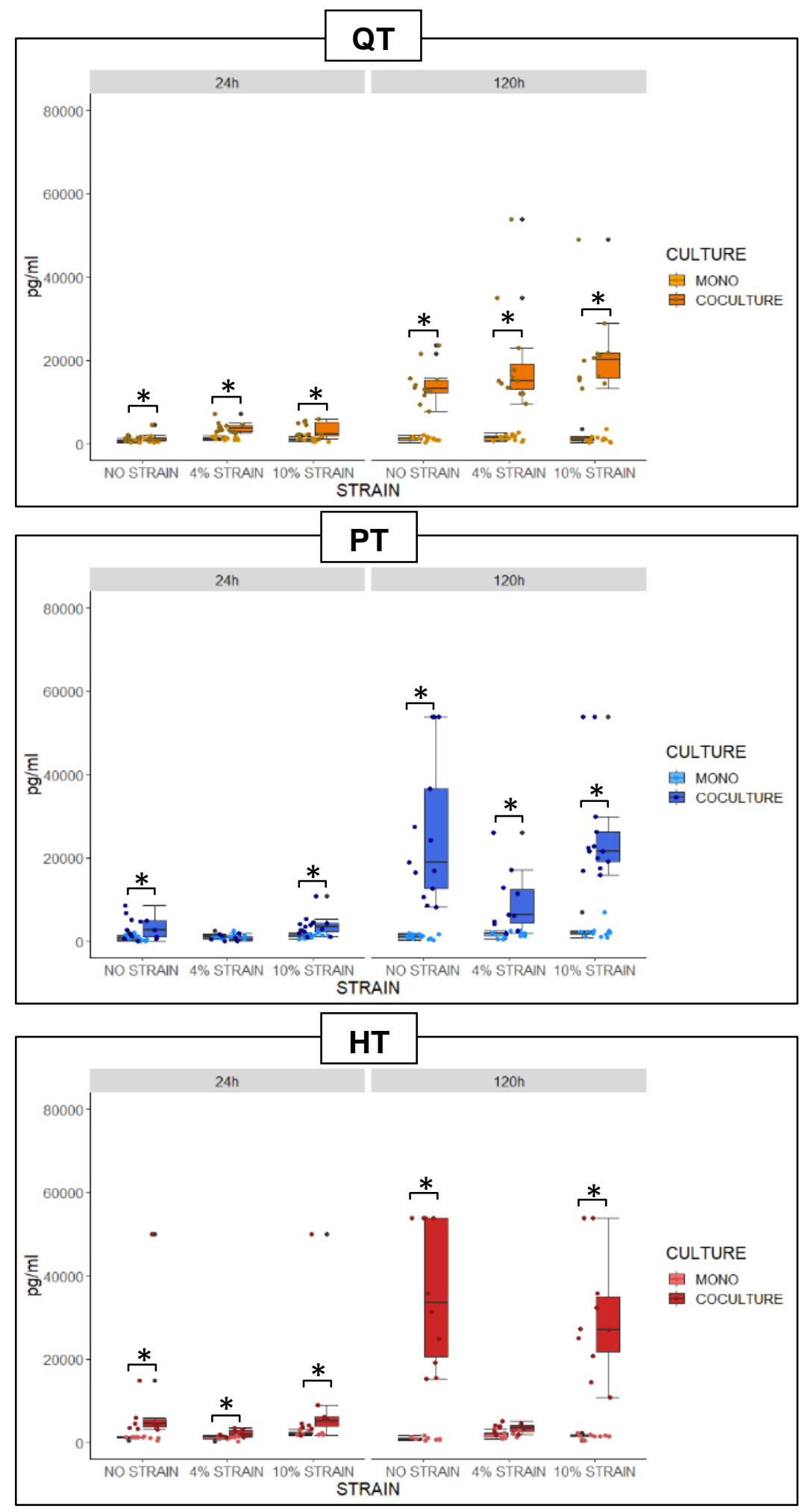

Figure 6.7. Concentration of monocyte chemoattractant protein-1 (MCP-1) released to the media. * significant difference between monoculture (MONO) and co-culture at 24 and 120 hours (Signed Rank test). QT: Quadriceps tendon; PT: Patellar tendon, HT: Hamstring tendon 

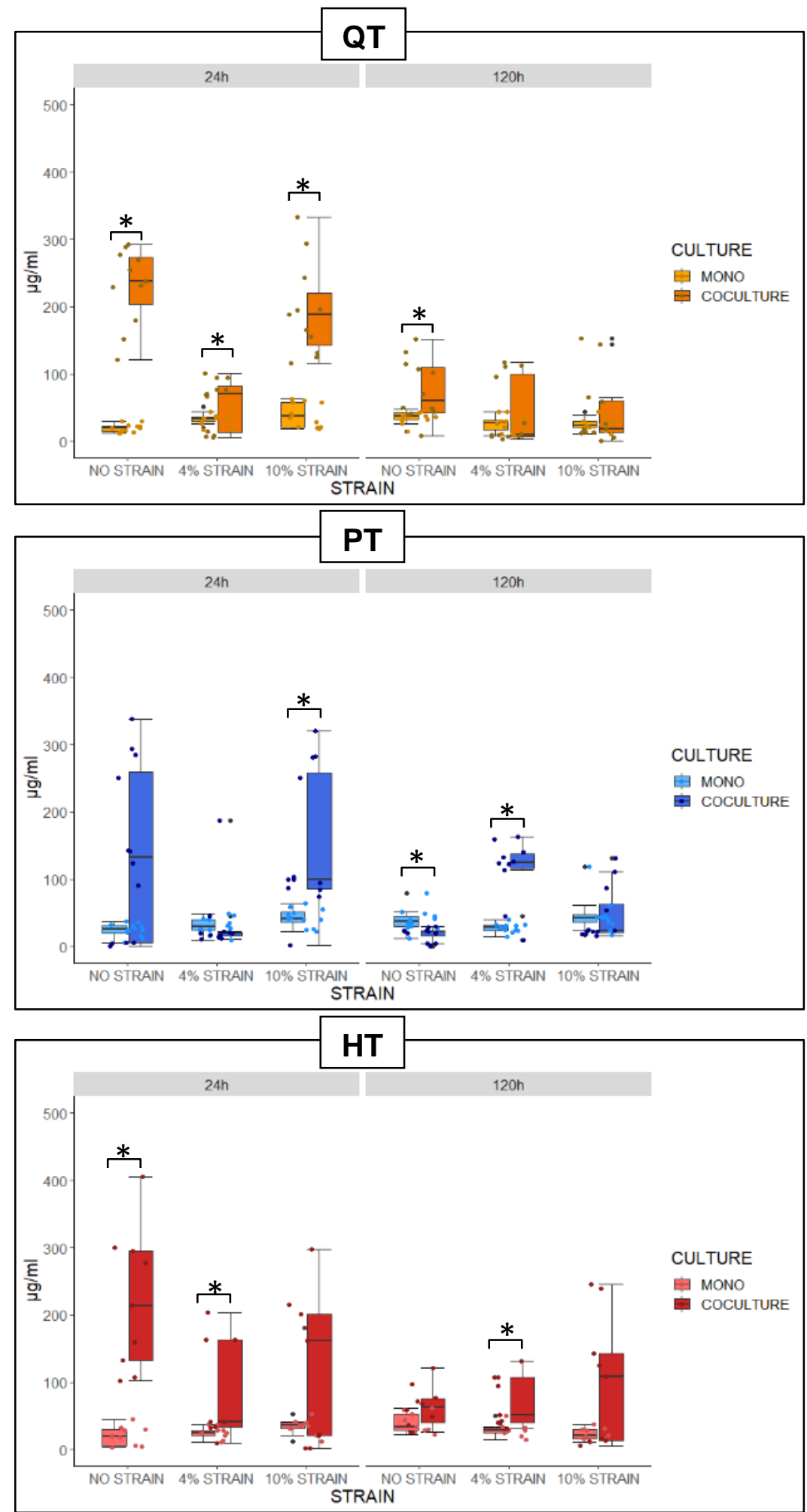

Figure 6.8. Concentration of Glycosaminoglycans (GAG) released to the media. * significant difference between monoculture (MONO) and co-culture at 24 and 120 hours (Signed Rank test). QT: Quadriceps tendon; PT: Patellar tendon, HT: Hamstring tendon 


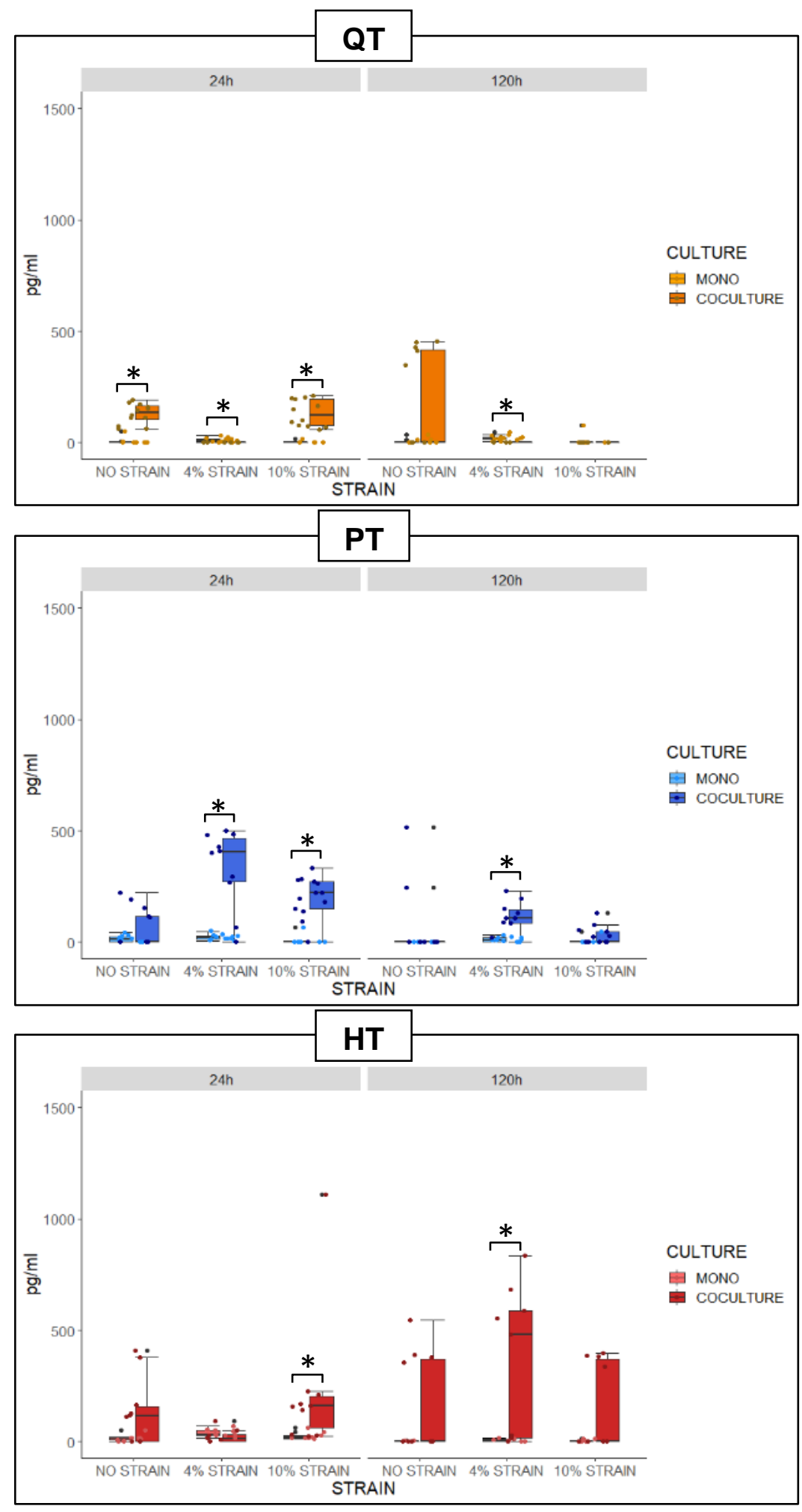

Figure 6.9. Concentration of total MMP activity (MMPACT) released to the media. * significant difference between monoculture (MONO) and co-culture at 24 and 120 hours (Signed Rank test). QT: Quadriceps tendon; PT: Patellar tendon, HT: Hamstring tendon 

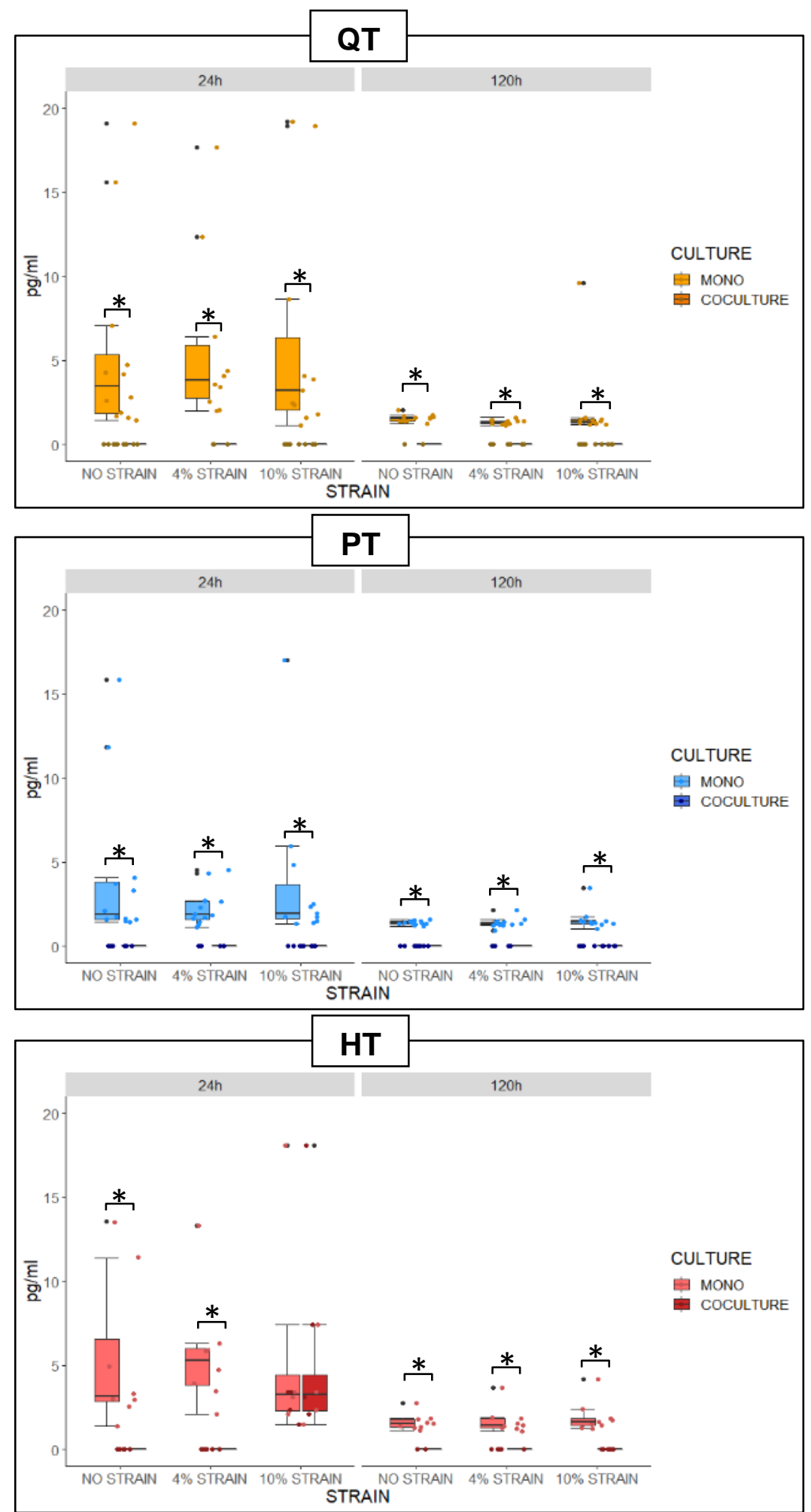

Figure 6.10. Concentration of MMP-1 released to the media. * significant difference between monoculture (MONO) and co-culture at 24 and 120 hours (Signed Rank test). QT: Quadriceps tendon; PT: Patellar tendon, HT: Hamstring tendon 

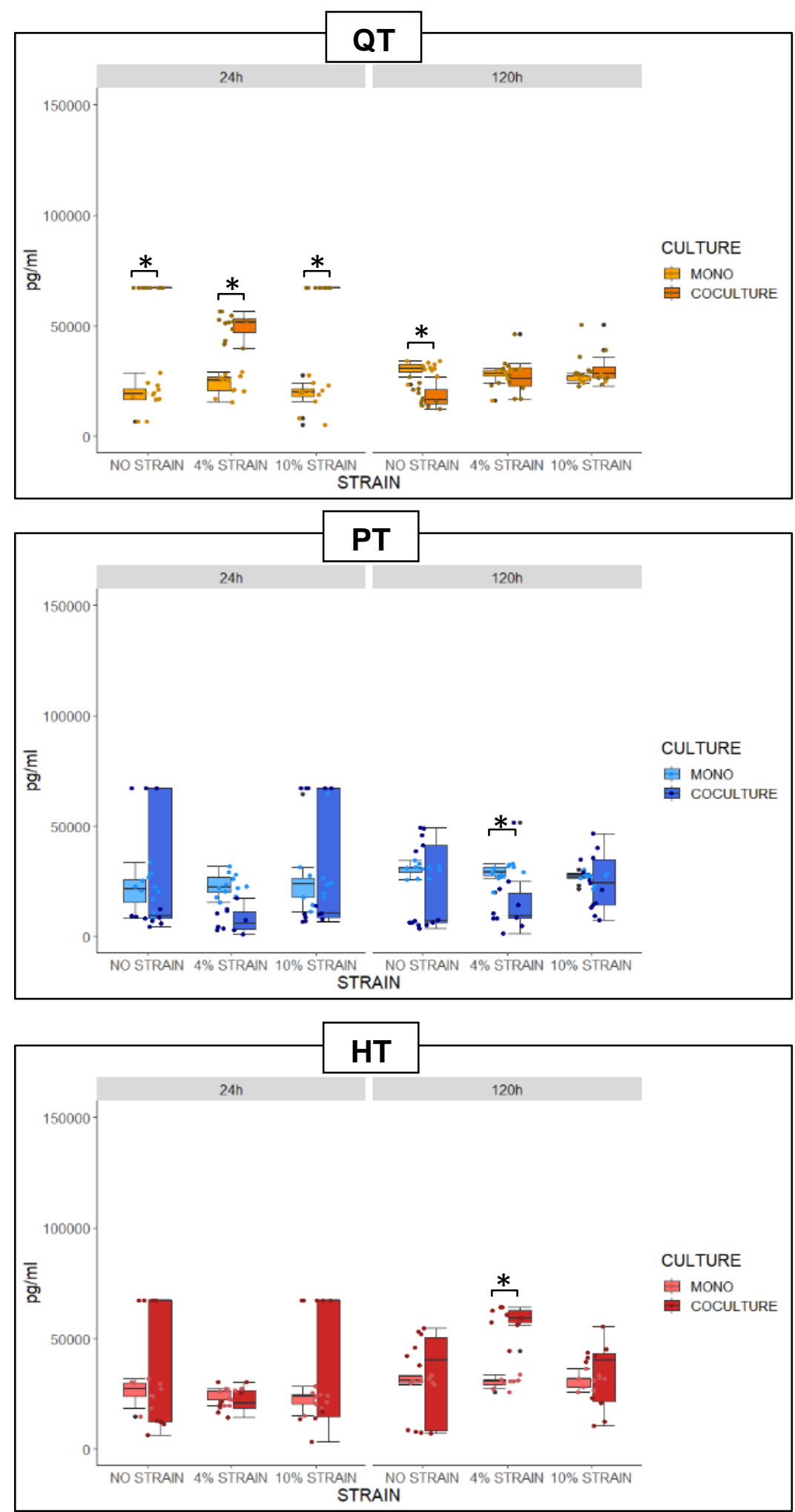

Figure 6.11. Concentration of MMP-2 released to the media. * significant difference between monoculture (MONO) and co-culture at 24 and 120 hours (Signed Rank test). QT: Quadriceps tendon; PT: Patellar tendon, HT: Hamstring tendon 


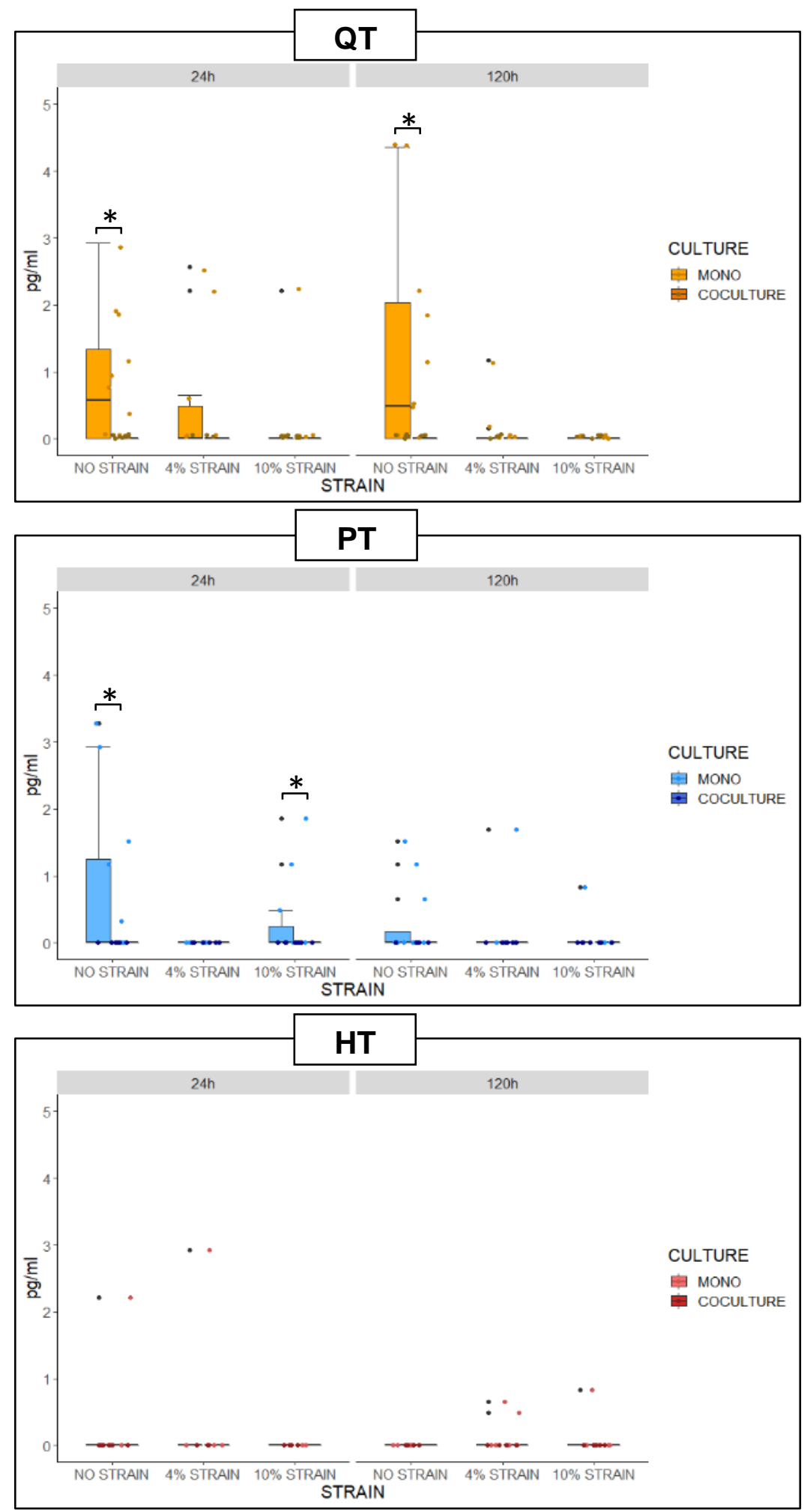

Figure 6.12. Concentration of MMP- 3 released to the media. * significant difference between monoculture (MONO) and co-culture at 24 and 120 hours (Signed Rank test). QT: Quadriceps tendon; PT: Patellar tendon, HT: Hamstring tendon 

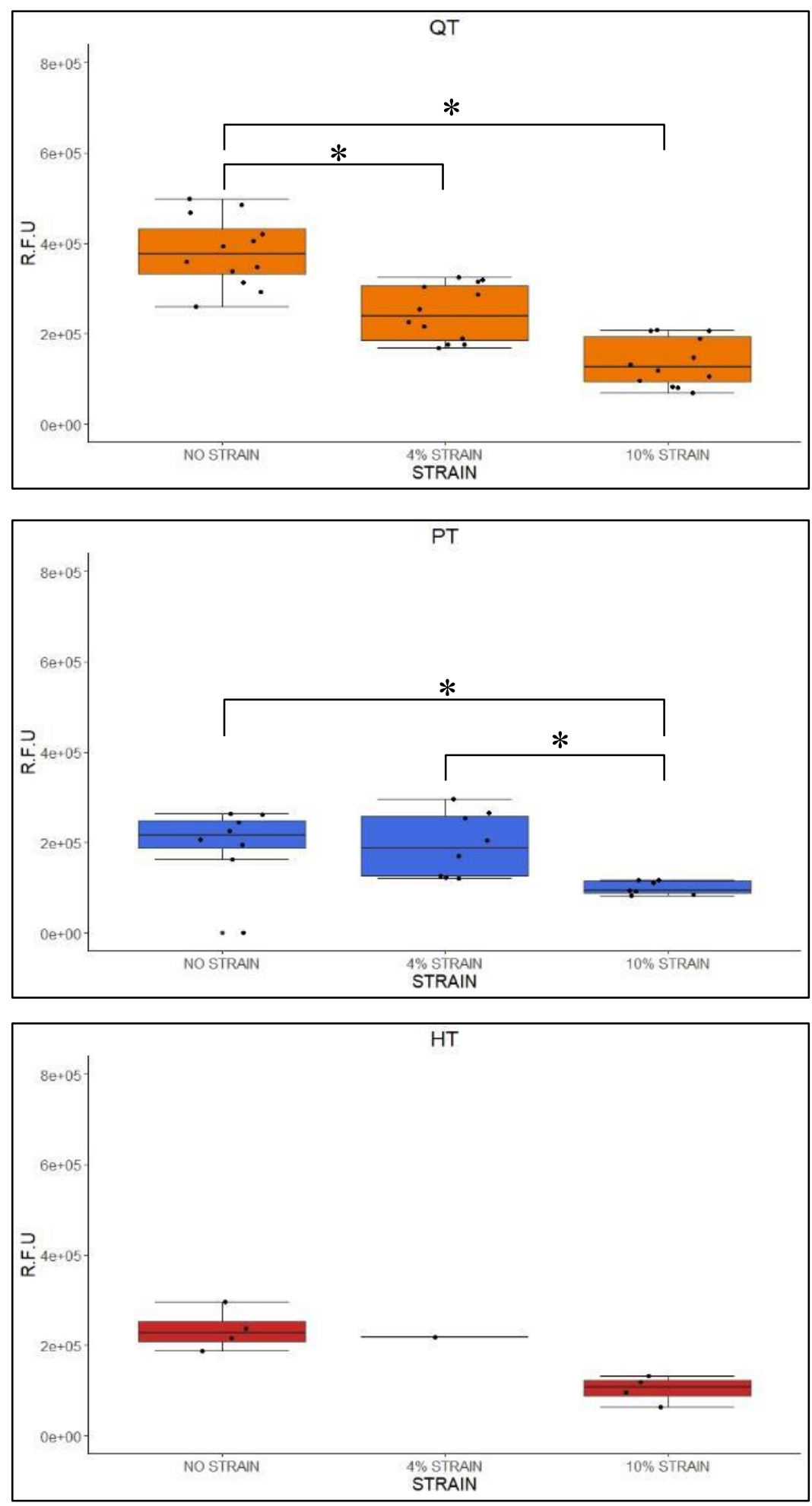

Figure 6.13. Metabolic Activity of graft fibroblasts. * significant difference between STRAINS at 120 hours (Kruskal-Wallis test). QT: Quadriceps tendon; PT: Patellar tendon, HT: Hamstring tendon. 

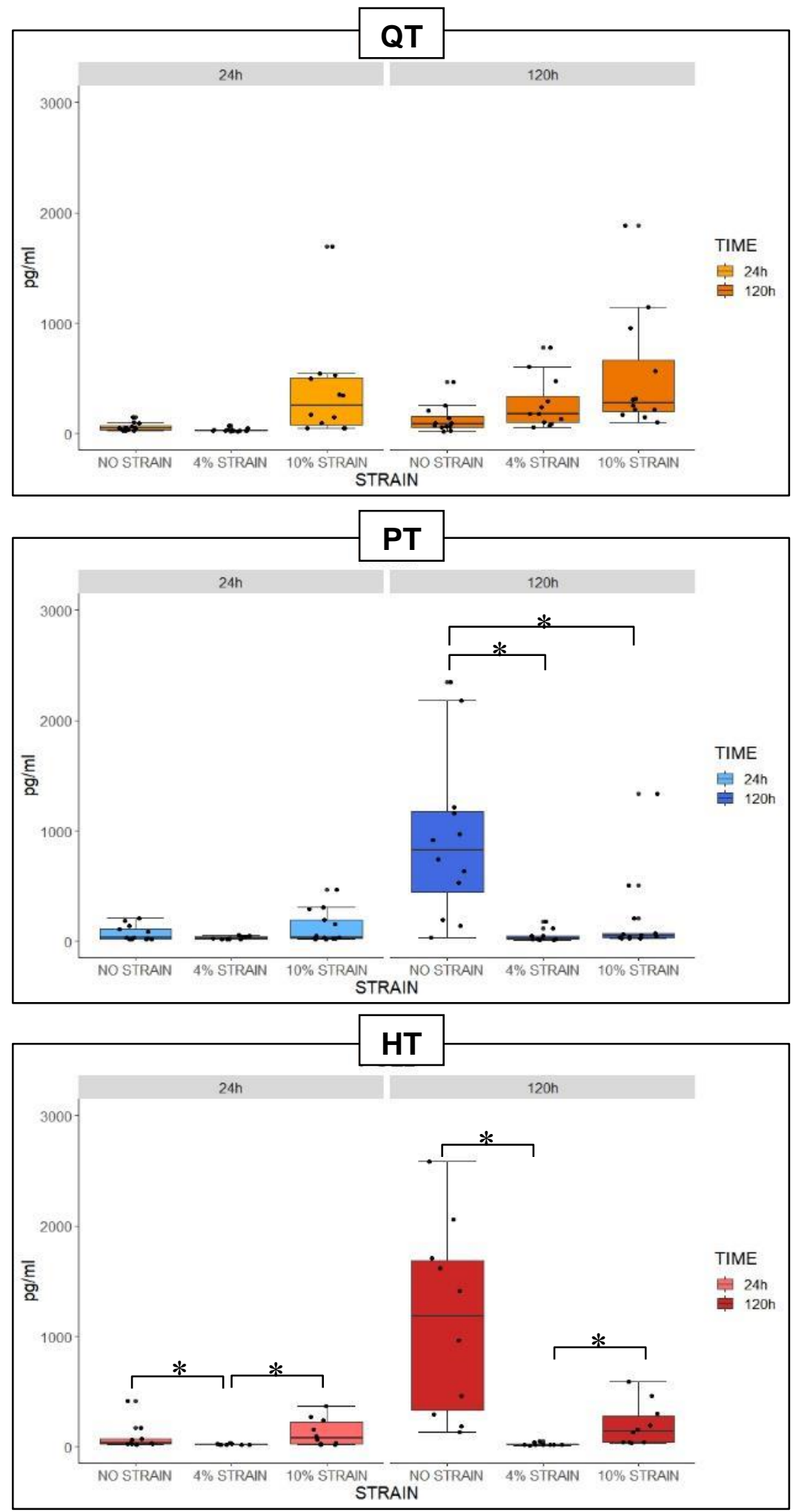

Figure 6.14. Concentration of PGE-2 released to the media when co-cultured with SYN. * significant difference between STRAINS at 24 and 120 hours (Kruskal-Wallis test). QT: Quadriceps tendon; PT: Patellar tendon, HT: Hamstring tendon 

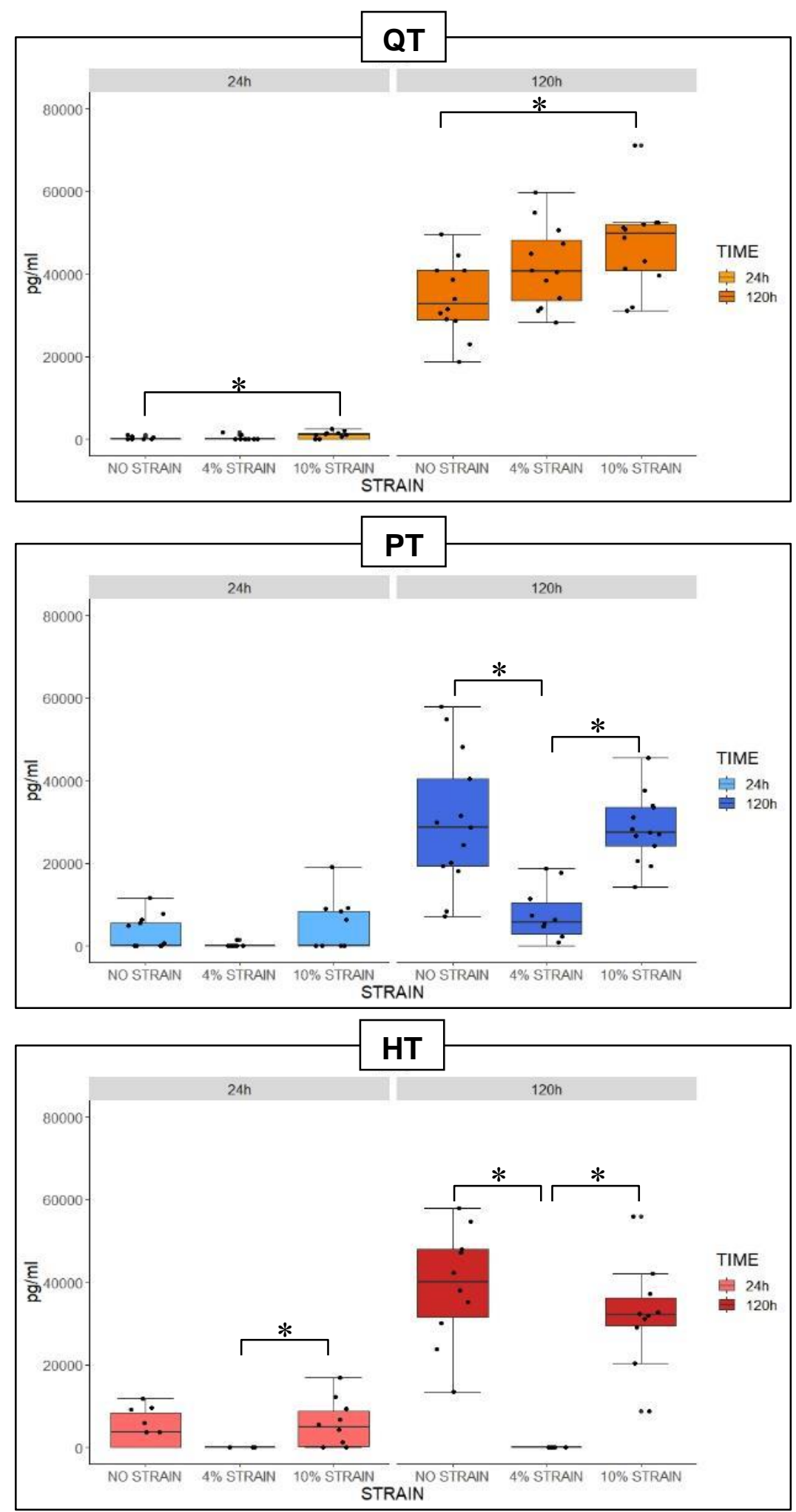

Figure 6.15. Concentration of IL-8 released to the media when co-cultured with SYN. * significant difference between STRAINS at 24 and 120 hours (Kruskal-Wallis test). QT: Quadriceps tendon; PT: Patellar tendon, HT: Hamstring tendon 

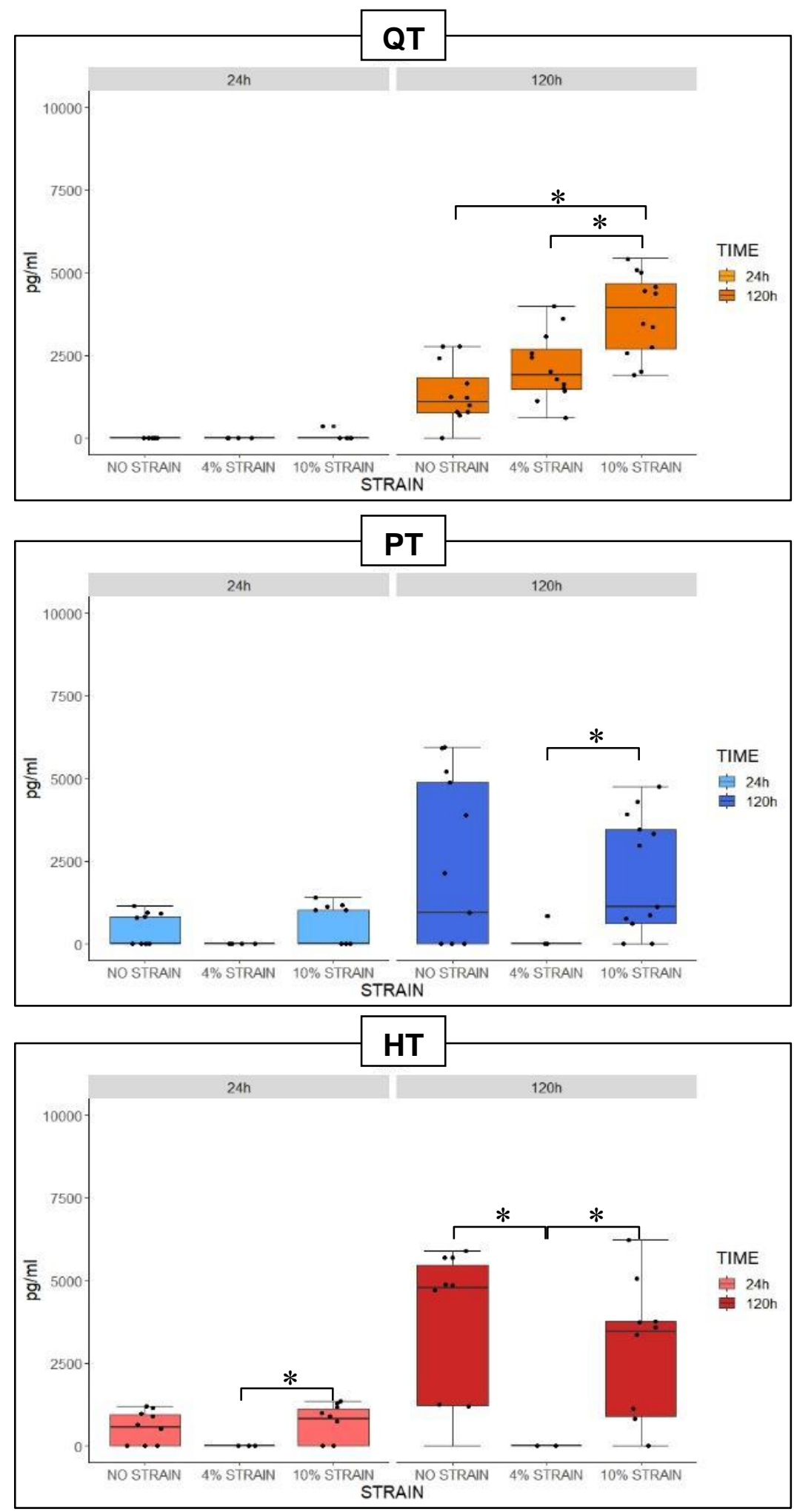

Figure 6.16. Concentration of $\mathrm{KC}$ released to the media when co-cultured with SYN. * significant difference between STRAINS at 24 and 120 hours (Kruskal-Wallis test). QT: Quadriceps tendon; PT: Patellar tendon, HT: Hamstring tendon 

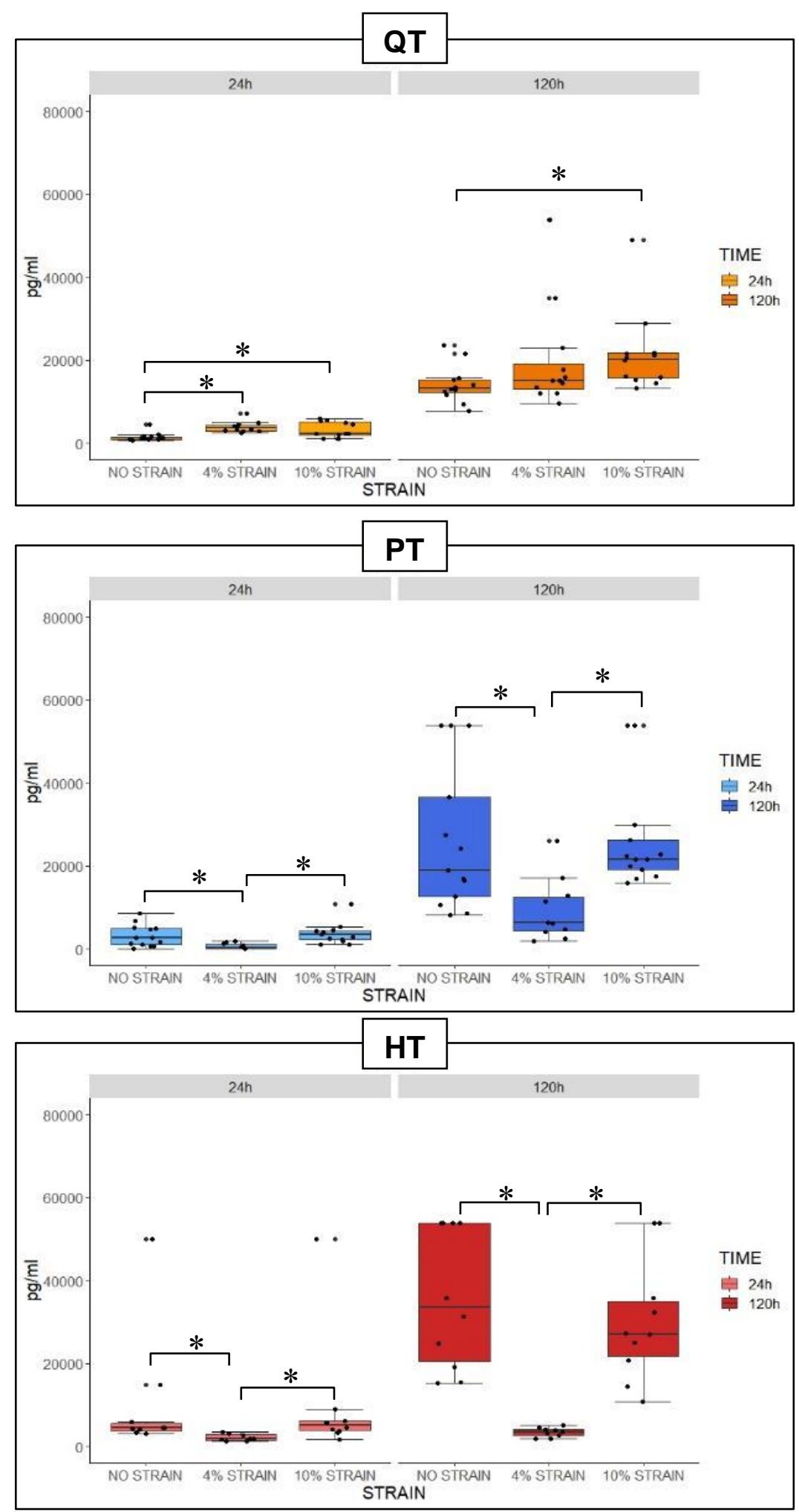

Figure 6.17. Concentration of MCP1 released to the media when co-cultured with SYN. * significant difference between STRAINS at 24 and 120 hours (Kruskal-Wallis test). QT: Quadriceps tendon; PT: Patellar tendon, HT: Hamstring tendon 

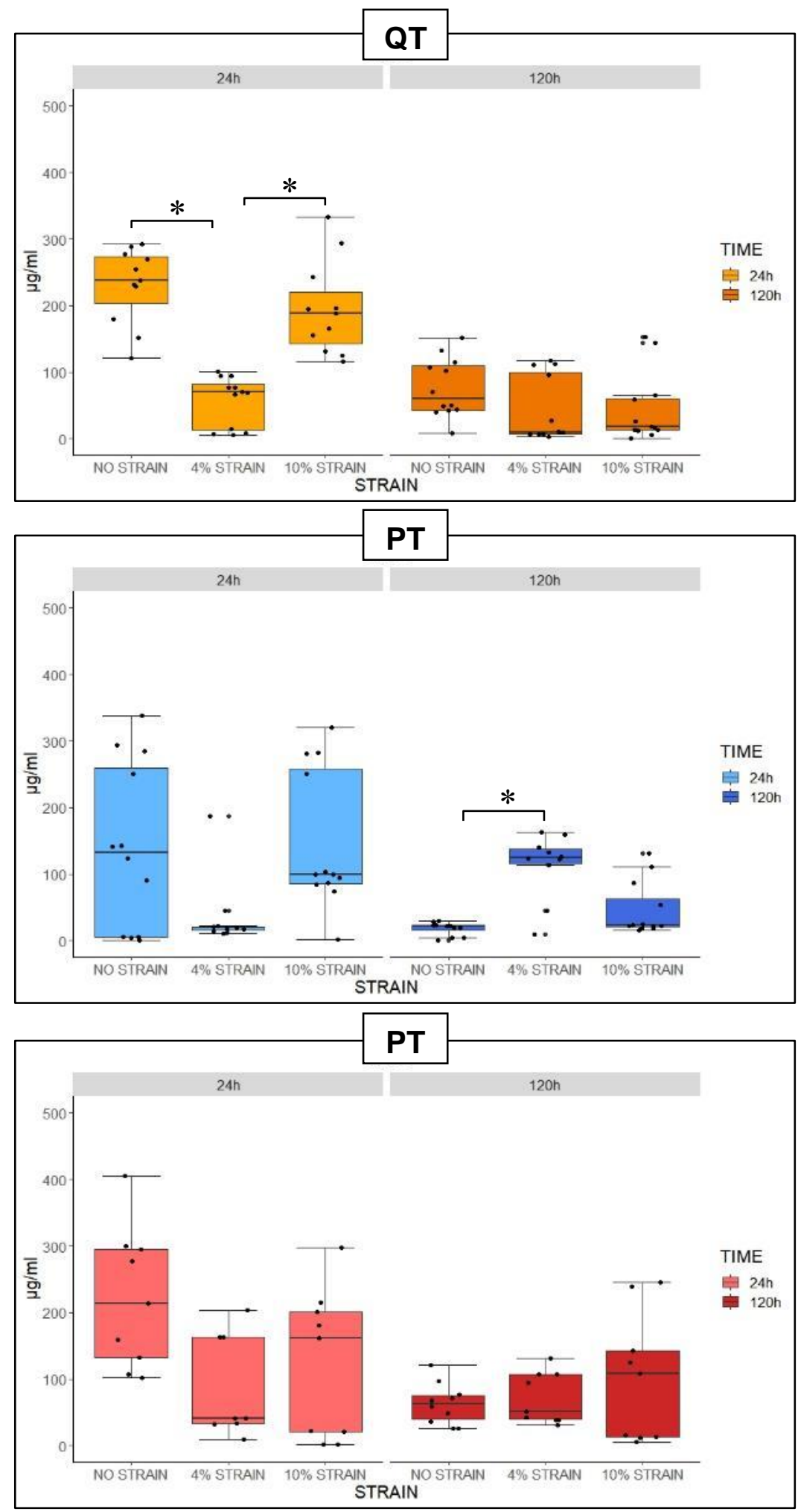

Figure 6.18. Concentration of GAG released to the media when co-cultured with SYN. * significant difference between STRAINS at 24 and 120 hours (Kruskal-Wallis test). QT: Quadriceps tendon; PT: Patellar tendon, HT: Hamstring tendon 

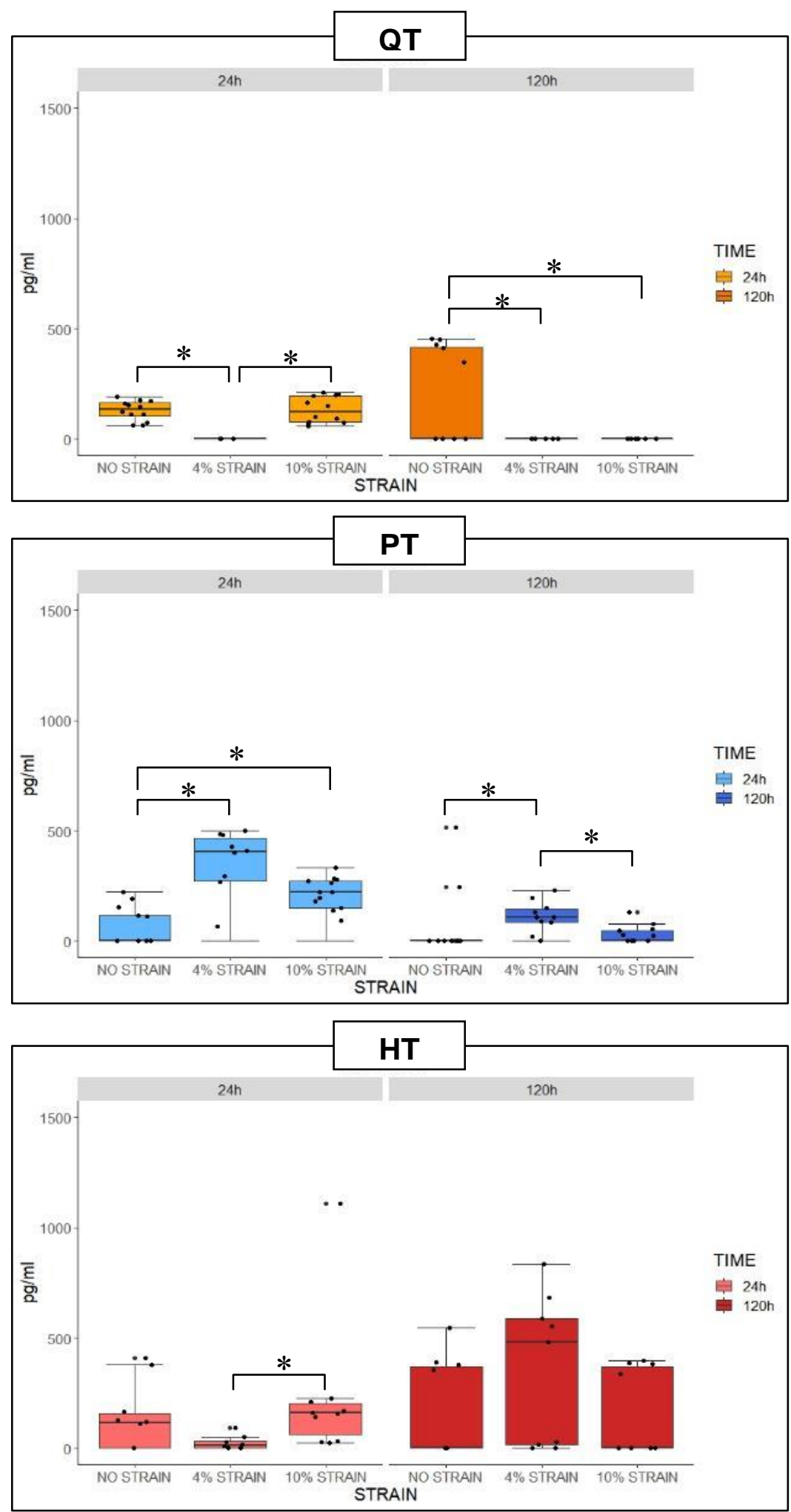

Figure 6.19. Concentration of MMPACT released to the media when co-cultured with SYN. * significant difference between STRAINS at 24 and 120 hours (Kruskal-Wallis test). QT: Quadriceps tendon; PT: Patellar tendon, HT: Hamstring tendon 

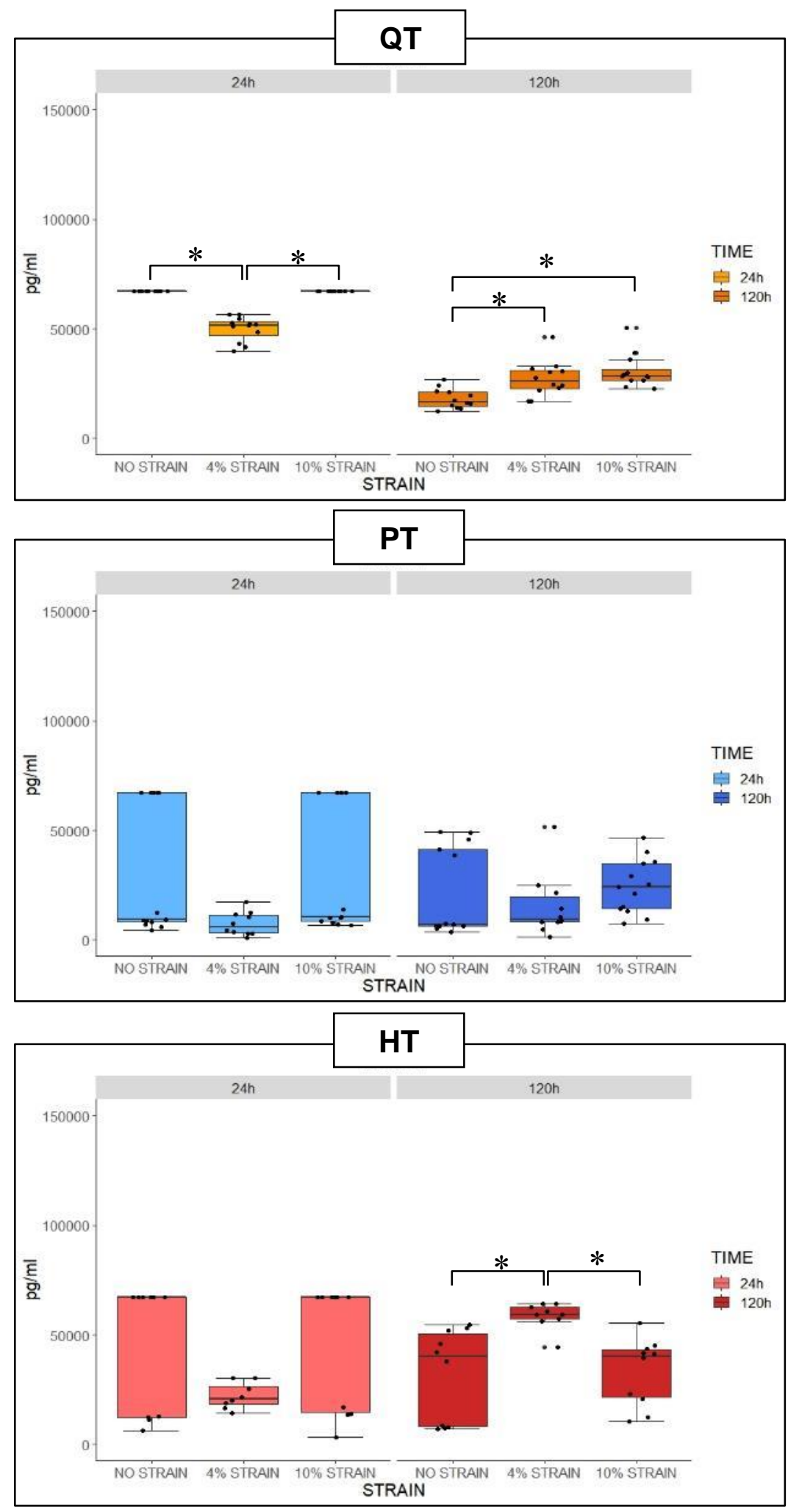

Figure 6.20. Concentration of MMP-2 released to the media when co-cultured with SYN. * significant difference between STRAINS at 24 and 120 hours (Kruskal-Wallis test). QT: Quadriceps tendon; PT: Patellar tendon, HT: Hamstring tendon 

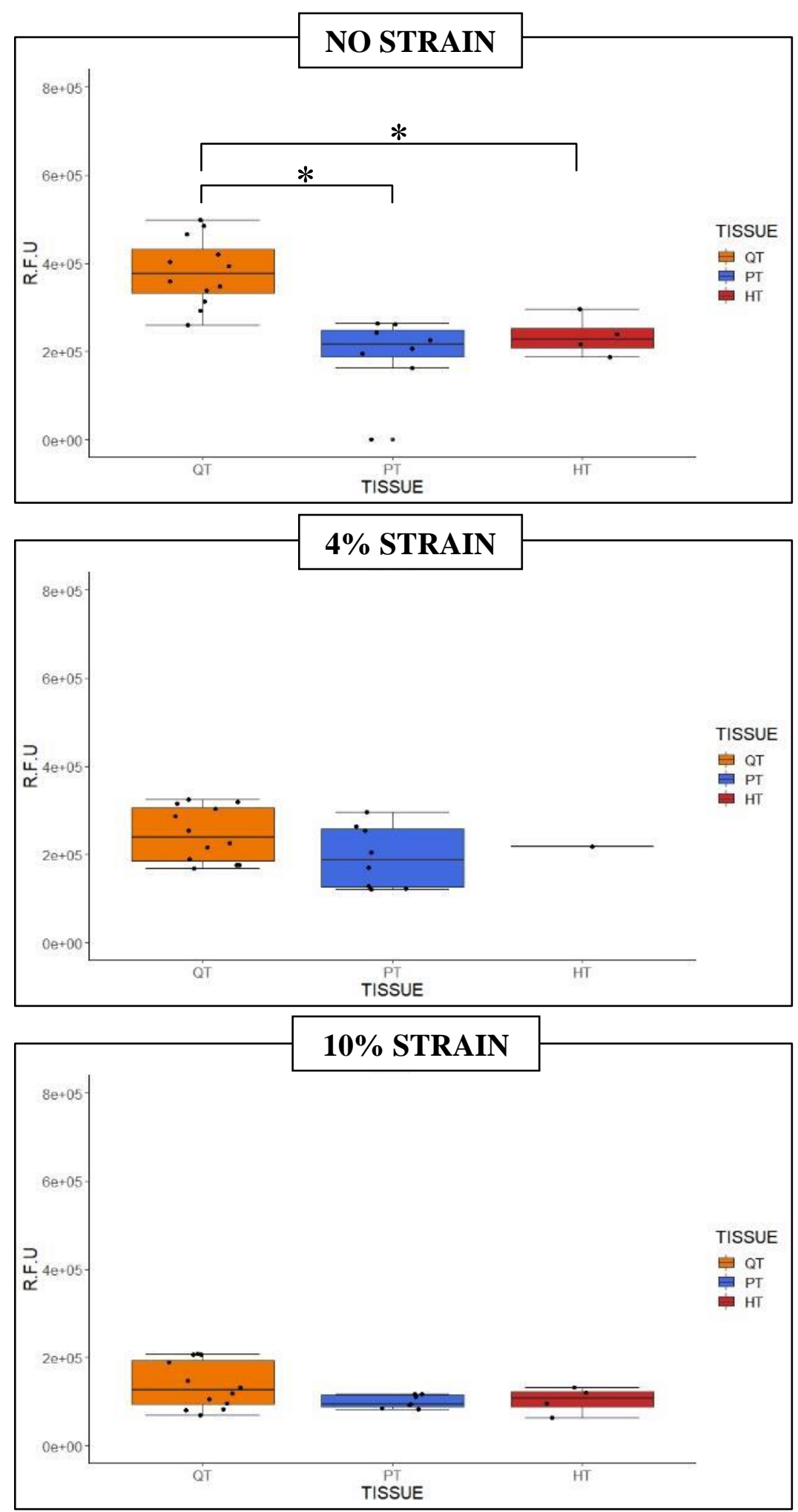

Figure 6.21. Metabolic Activity of graft fibroblasts. * significant difference between TISSUES at 120 hours (Kruskal-Wallis test). QT: Quadriceps tendon; PT: Patellar tendon, HT: Hamstring tendon. 

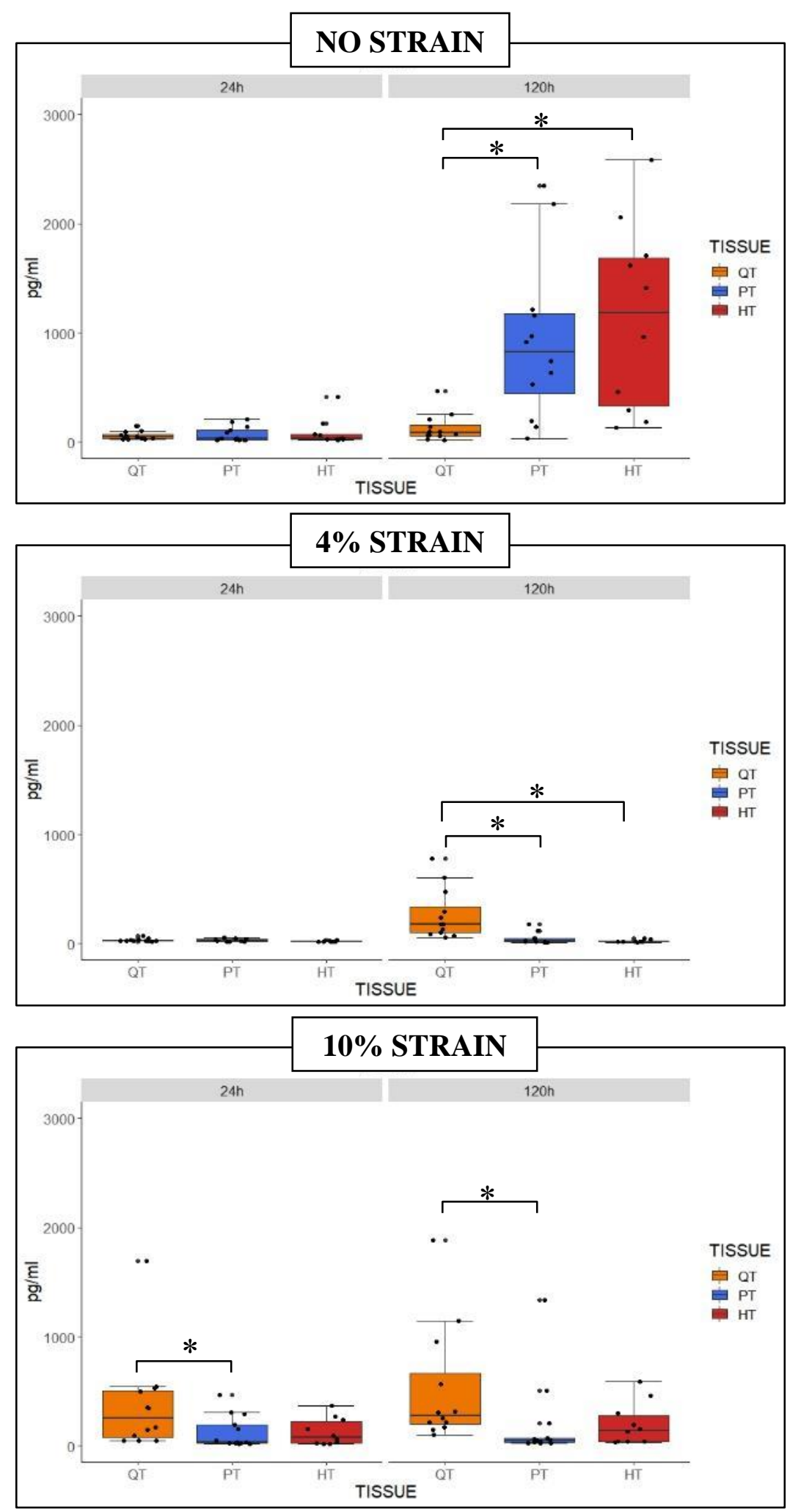

Figure 6.22. Concentration of PGE2 released to the media when co-cultured with SYN. * significant difference between TISSUES at 24 and 120 hours (Kruskal-Wallis test). QT: Quadriceps tendon; PT: Patellar tendon, HT: Hamstring tendon 

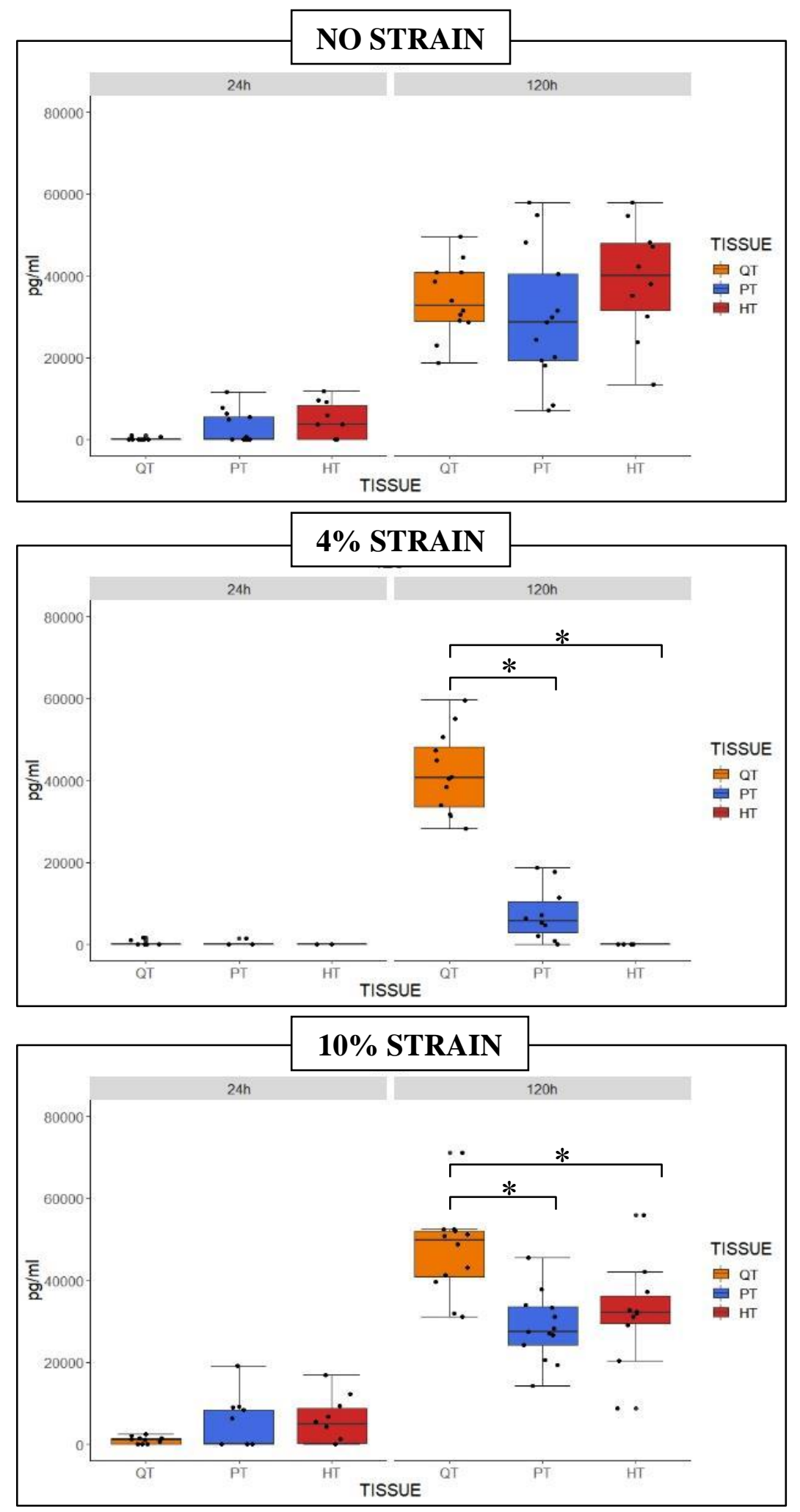

Figure 6.23. Concentration of IL-8 released to the media when co-cultured with SYN. * significant difference between TISSUES at 24 and 120 hours (Kruskal-Wallis test). QT: Quadriceps tendon; PT: Patellar tendon, HT: Hamstring tendon 

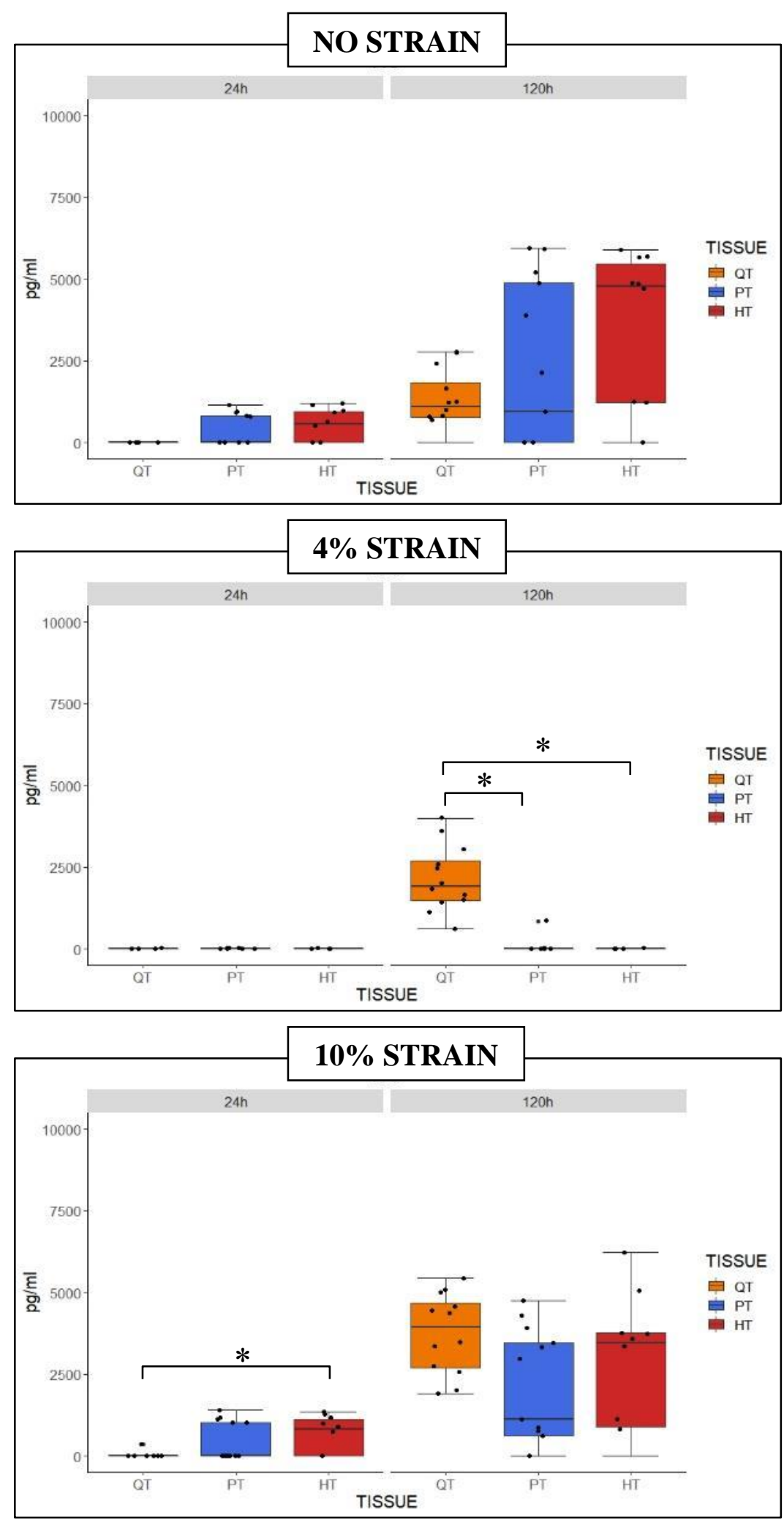

Figure 6.24. Concentration of KC released to the media when co-cultured with SYN. * significant difference between TISSUES at 24 and 120 hours (Kruskal-Wallis test). QT: Quadriceps tendon; PT: Patellar tendon, HT: Hamstring tendon 

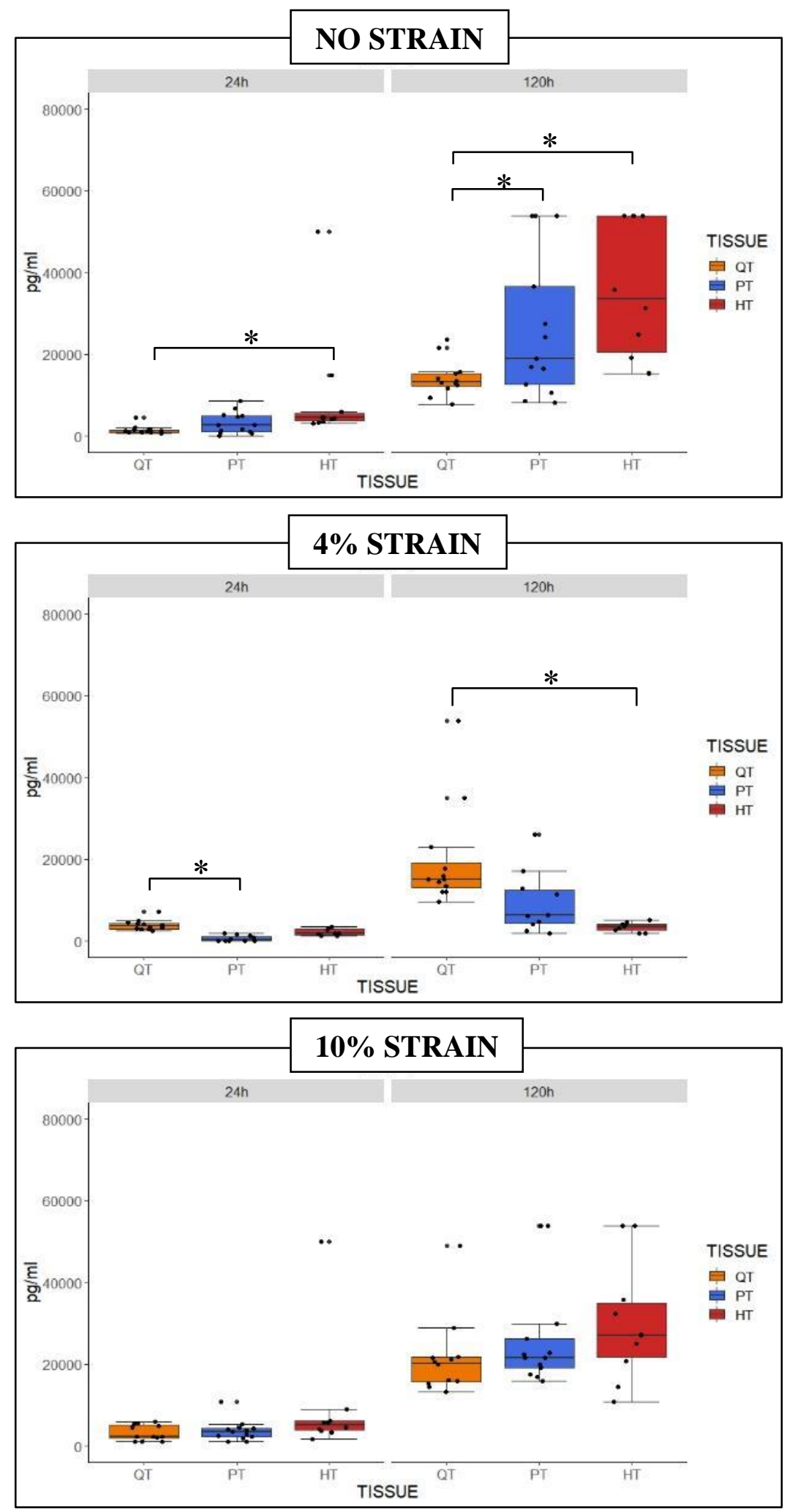

Figure 6.25. Concentration of MCP1 released to the media when co-cultured with SYN. * significant difference between TISSUES at 24 and 120 hours (Kruskal-Wallis test). QT: Quadriceps tendon; PT: Patellar tendon, HT: Hamstring tendon 

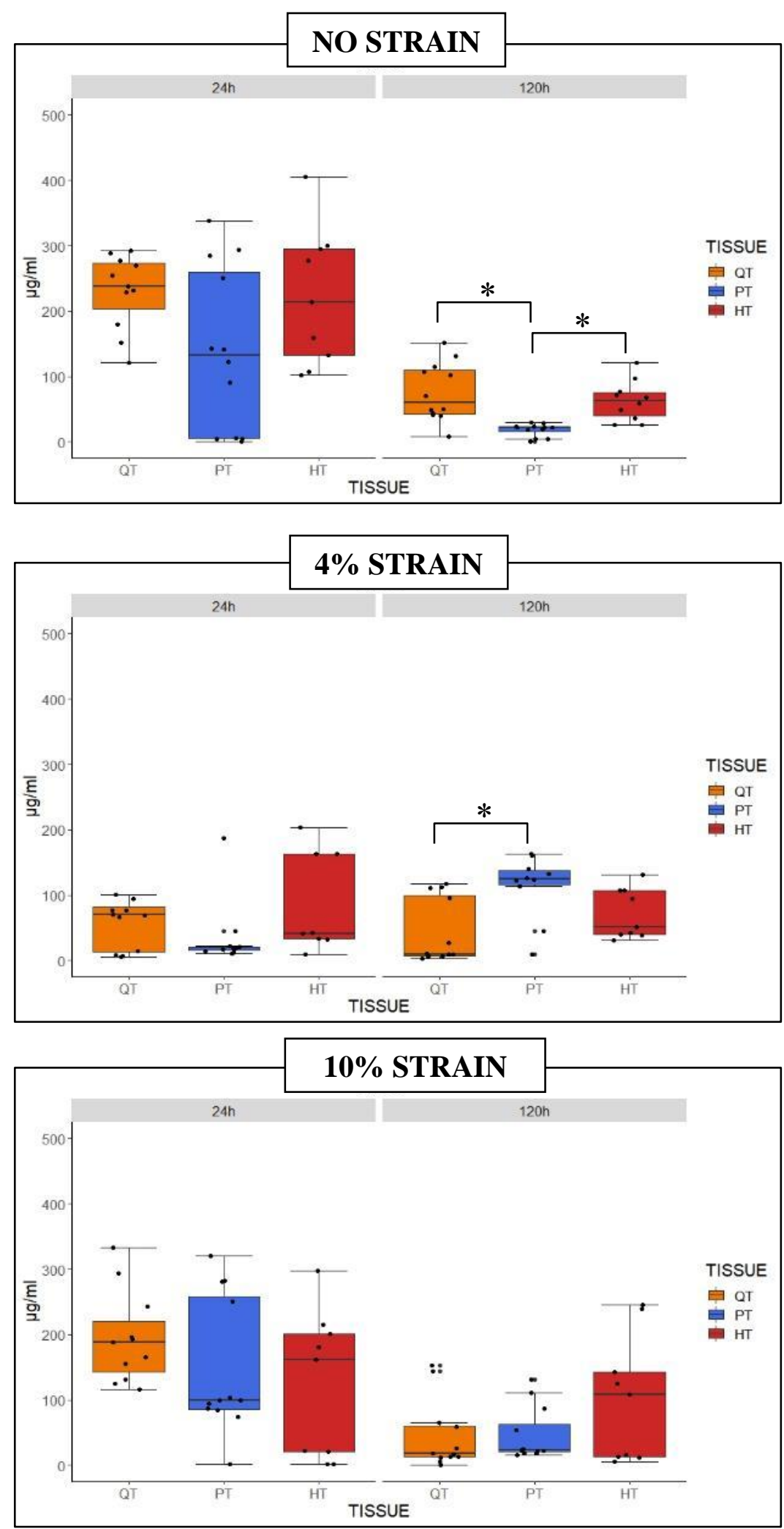

Figure 6.26. Concentration of GAG released to the media when co-cultured with SYN. * significant difference between TISSUES at 24 and 120 hours (Kruskal-Wallis test). QT: Quadriceps tendon; PT: Patellar tendon, HT: Hamstring tendon 

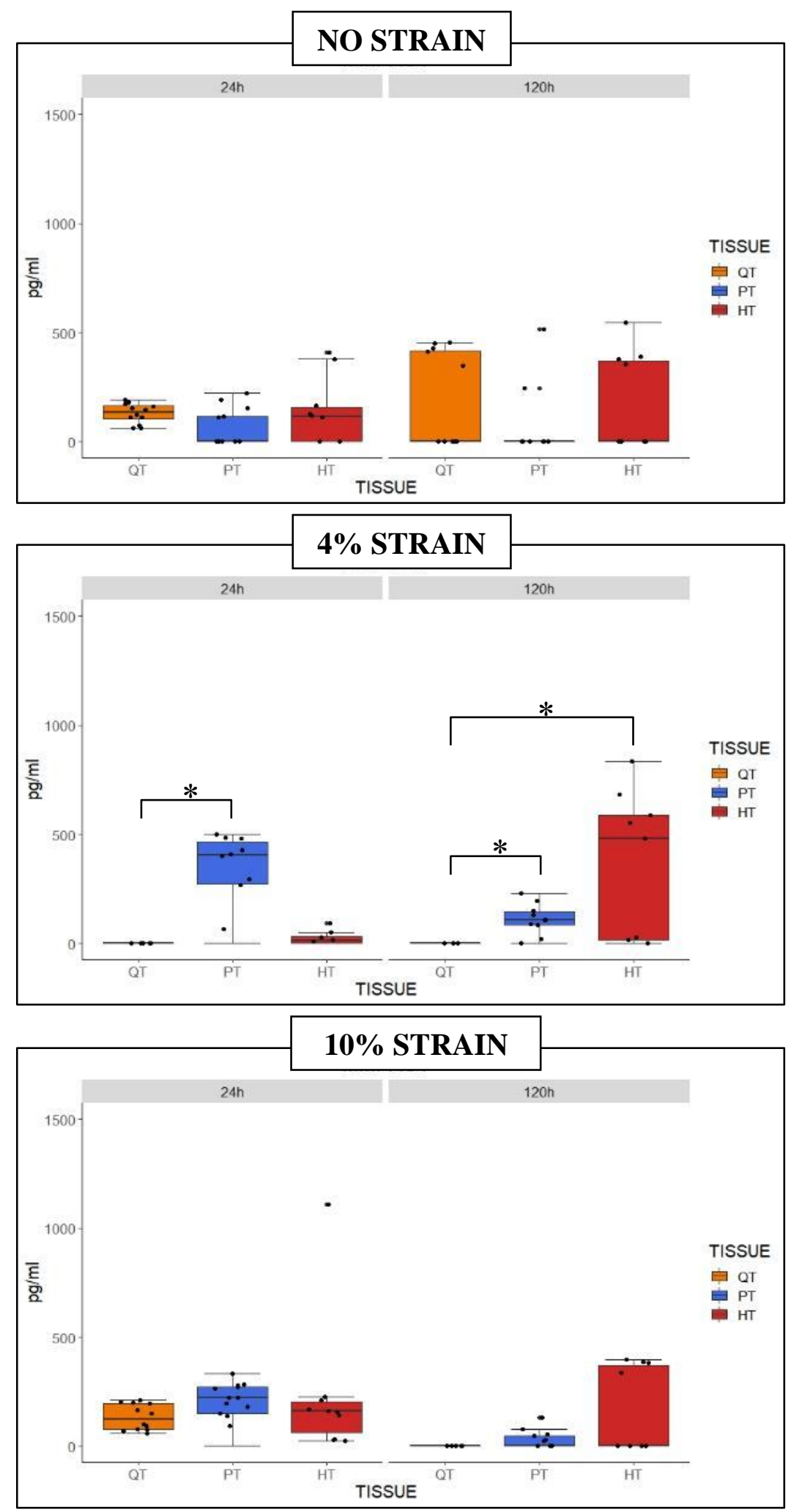

Figure 6.27. Concentration of MMPACT released to the media when co-cultured with SYN. * significant difference between TISSUES at 24 and 120 hours (Kruskal-Wallis test). QT: Quadriceps tendon; PT: Patellar tendon, HT: Hamstring tendon 

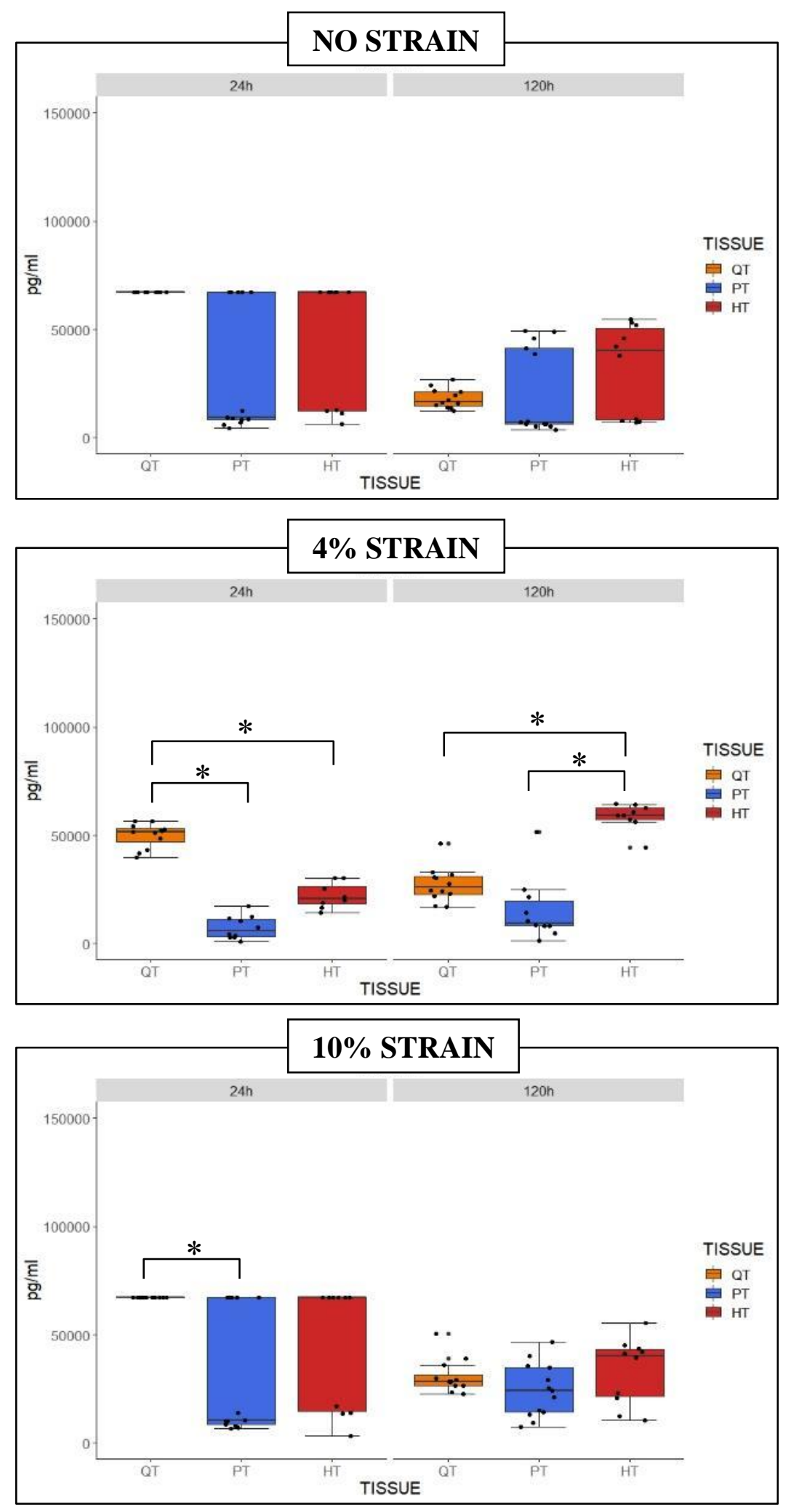

Figure 6.28. Concentration of MMP-2 released to the media when co-cultured with SYN. * significant difference between TISSUES at 24 and 120 hours (Kruskal-Wallis test). QT: Quadriceps tendon; PT: Patellar tendon, HT: Hamstring tendon 


\section{CHAPTER 7}

\section{EFFECTS OF MECHANICAL STRAIN AND SEX ON METABOLIC RESPONSES OF HUMAN ANTERIOR CRUCIATE LIGAMENT AND SYNOVIUM}

\section{Introduction}

There are more than 100,000 anterior cruciate ligament (ACL) injuries in the United States every year. ${ }^{1 ; 2}$ The knee joint is now considered an organ with all of its constituent tissues (i.e. cartilage, meniscus, ligament, synovium) playing important roles during homeostasis and during injury or disease states. These tissue responses are driven by specific molecules released by the varying cell types found within the knee joint.

The synovium is a principal constituent involved in regulating knee joint homeostasis by modulating injury signals through the release of molecules such as cytokines, chemokines and adipokines, and plays important roles in determining downstream effects on articular cartilage. ${ }^{3-6}$ The intraarticular environment may also negatively impact the healing process after ACL tears, preventing primary healing. ${ }^{7-12}$

The individual's sex is known to contribute to risk of ACL injury. ${ }^{13-15}$ Females are noted to have a 2-10 fold increase risk of ACL injury when compared to their male counterparts. ${ }^{13 ; 16 ; 17}$ Possible causes for the female risk in ACL injury include hormonal variations, decreased structural and biochemical quality of female tendons and ligaments and differences in neuromuscular control and specific sex-related anatomical variants. ${ }^{14 ; 18-}$ 22. Additional reasons for increased risk of ACL rupture in females include anatomic factors such as intercondylar notch size, posterior tibial plateau slope and ACL elevation 
angle among others. ${ }^{21 ;} 22$ However, sex differences in metabolic responses of relevant tissues after ACL tears are not fully elucidated.

Characterizing metabolic responses of the various tissues in the knee is important for understanding the injury as was as healing after treatment for ACL injuries. ACL reconstruction using a tendon graft has been the standard-of-care for surgical management of ACL tears in humans. However, there are long-recognized limitations with surgical reconstruction for ACL injuries. Morbidity from ACL reconstructive surgery including risk for graft laxity and clinical failure and inability to consistently mitigate onset of posttraumatic osteoarthritis (PTOA) despite successful reconstruction are notable limitations. ${ }^{23 ;}$ ${ }^{24}$ There is also an ongoing debate if a reconstructed ACL recapitulates all of the properties of a native ACL including its proprioceptive or reflex responses ${ }^{25}$. Therefore, a renewed interest in primary ACL repair has developed.

While attractive, an unreliable healing response has been associated with primary ACL repair. This has been attributed to several factors, including a hostile intra-synovial environment, specific post-inflammatory responses, and intrinsic cell deficiencies. ${ }^{26 ; 27}$ The effects of sex in the injury response and the subsequent healing process of the injured ACL tissue are also unknown. Furthermore, given that ACL cells are likely sensitive to mechanical stimulus, sex-dependent differences in response to external mechanical stimuli need to be more fully characterized. Therefore, understanding the early healing phase characterized by cellular release of molecules that regulate adequate balance between extracellular matrix (ECM) degradation and proliferation, as well as how these processes are impacted by external mechanical stimulus and patient sex, may be crucial to case selection for ACL repair and optimizing rehabilitation after that surgery. 
One method to evaluate cellular interactions involves in vitro models, which also permit evaluating cell responses to mechanical stress. ${ }^{28}$ The Flexcell system has been used widely in both monolayer culture and 3D systems for this purpose. ${ }^{29}$ The bi-axial loading system permits investigators to reliably model ligament-tendon fibroblastic load conditions while allowing for easy manipulation and accessibility. ${ }^{28}$

Since the metabolic responses of the cellular component of knee intraarticular tissues are likely not only interdependent but also susceptible to mechanical strain, we aimed to evaluate the responses of ACL and SYN to three different biaxial mechanical loads that simulate strains associated with perioperative rehabilitation after ACL repair. ${ }^{28 ; 30 ; 31}$ Additionally, we aimed to assess the responses of ACL and SYN after rupture to the conditions described above. We hypothesized that SYN will significantly increase the production of inflammatory and degradative markers compared to ACL fibroblasts in a strain dependent manner.

\section{Materials and Methods:}

\section{Tissue processing and culture}

With IRB approval (IRB\#2009879), explants from injured intraarticular tissues (20 ACL, 19 SYN) that would otherwise be discarded were collected at the time of cruciate ligament reconstruction (Fig.7.1). Explants were aseptically minced into $0.5-1.0 \mathrm{~cm}^{2}$ pieces and digested for two hours in Type 1A Clostridium histolyticum collagenase solution (Sigma-Aldrich, St. Louis, MO, USA) at a concentration of $0.2 \mathrm{mg} / \mathrm{mL}$. The aliquot was then suspended with nutritional media (Gibco DMEM, Thermo Fisher Scientific, Waltham, MA, USA) containing 10\% FBS, 0.002\% Penicillin, $100 \mu \mathrm{g} / \mathrm{mL}$ Streptomycin, $25 \mu \mathrm{g} / \mathrm{mL}$ Amphotericin B, 0.002\% L-Ascorbate and 0.01\% L-glutamine 
(Sigma Chemical Co, St. Louis, MO), cultured in T25 flasks (passage zero) and T75 flasks (passage one) (TPP Techno Plastic Products AG, Trasadingen, Switzerland) and incubated at $5 \% \mathrm{CO}_{2}, 37^{\circ} \mathrm{C}$, and $95 \%$ humidity. Once confluent, passage one cells were stored at $-80^{\circ} \mathrm{C}$ until used for culture.

\section{Biaxial mechanical stress}

Passage one cells were re-expanded in T75 flasks and when confluent, exposed to TrypLE express (Invitrogen, Carlsbad, CA) for five minutes, then resuspended and seeded $\left(1 \times 10^{5}\right.$ cells/well) in Collagen Type I-coated BioFlex ${ }^{\circledR}$ plates (Flexcell International, NC, USA). Cells were incubated for an additional $48 \mathrm{~h}$ in $10 \% \mathrm{FBS}$ nutritional media before strain was applied. Before fibroblasts were subjected to continuous mechanical stimulation media was changed to $2 \% \mathrm{FBS}$ for the duration of the loaded culture (6 days). ${ }^{32}$ Strain loads (2-s strain and 10 -s relaxation at a $0.5 \mathrm{~Hz}$ frequency) with a biaxial sinusoidal waveform with three different elongation strains that replicate 3 physiologic conditions (rest or mechanical stress deprivation-0\%, physiologic strain-4\%, and injury or high strain-10\% $)^{28}$ was applied for 6 days using the Flexcell FX4000T strain system (Flexcell International, NC, USA). Media were changed at day three and day six and stored at $-20^{\circ} \mathrm{C}$ until analysis.

\section{Biomarker assays}

Media was changed every three days and stored at $-20^{\circ} \mathrm{C}$ until analysis. Media from three (3d) and six (6d) days of culture were assessed for various biomarkers. Proteoglycan (GAG) was assessed using the DMMB assay as previously described. ${ }^{33}$ The concentration of prostaglandin E2 (PGE2) (Cayman Chemical, Ann Arbor, MI, USA), cytokines (IL-6, IL-8, KC, MCP-1 and VEGF) (Millipore, Billerica, MA, USA), total 
matrix metalloproteinase (MMP) activity (SensoLyte 520 generic MMP assay, Anaspec, Inc., Fremont, CA), and MMPs and TIMPs production (MMP-1, MMP-2, MMP-3, TIMP 1 and TIMP 2) (R\&D Systems, Minneapolis, MN, USA), were all assessed using commercially available assays according to the manufacturer's protocol. At the end of the strain protocol (6d), cell viability analysis was performed using resazurin assay (Sigma Aldrich, Saint Louis, MO).

\section{Statistical Analysis}

For statistical analysis, normal distribution was assessed by Shapiro-Wilk test. Because data were not normally distributed, non-parametric analyses were used. Comparisons among strains and tissue types were performed using Kruskal-Wallis test and Dunn’s test posthoc. Additionally, comparisons between males and females were performed using Mann-Whitney U test (R Core Team (2019), R version 3.6.2 Vienna, Austria). Results were reported as median \pm interquartile range (IQR). Significance levels were set at $\mathrm{p}<0.05$.

\section{Results}

\section{Differences in ACL and Synovial Fibroblast to Mechanical Strain}

Metabolic activity was not statistically affected by any strain in SYN fibroblasts.

However, the metabolic activity of ACL fibroblasts at supraphysiological strain was significantly lower than ACL fibroblasts at physiological strain (Fig. 7.2).

PGE2 concentration in SYN fibroblasts was significantly increased at supraphysiological strain relative to mechanical strain deprivation at six days of culture. The production of PGE2 by ACL fibroblasts increased significantly with increasing mechanical load. Supraphysiological stress was significantly higher than both, mechanical deprivation and 
physiological stress at three days of culture. Also, at 3d, ACL derived fibroblasts

subjected to $4 \%$ strain produced significantly more PGE2 than no strain. Moreover, at six days, with stress deprivation there was a significant decrease in PGE2 concentration compared to $4 \%$ and $10 \%$ strain in ACL cultures (Fig.7.3)

The production of IL-6 by SYN fibroblasts was significantly higher at physiological stress compared to supraphysiological stress at day 6 of culture. Further, the production of IL-6 by ACL fibroblast at supraphysiological strain levels was significantly lower than at physiological and mechanical deprivation strain levels after six days of culture. While the production of IL-8 was not significantly affected by strain in SYN fibroblasts at any timepoint, the production of IL-8 by ACL fibroblasts at supraphysiological strain levels was significantly lower than at physiological strain levels at day 3 of culture (Fig. 7.4). The production of KC was significantly decreased by ACL fibroblasts subjected to supraphysiological strain compared to physiological strain through 6 days of culture. Moreover, MCP-1 production was significantly decreased by ACL fibroblasts subjected to supraphysiological strain compared to stress deprivation at day 3 of culture. $\mathrm{KC}$ and MCP1 did not show a significant change at any strain and timepoint in SYN fibroblasts (Fig. 7.5).

The production of VEGF was not significantly different among strains in SYN fibroblasts at any timepoint. However, VEGF production was significantly increased by ACL fibroblasts at physiological strain compared to supraphysiological strain at three days of culture. Additionally, MIP-1 $\beta$ production was significantly decreased by SYN fibroblasts at supraphysiological strain levels compared to both stress deprivation and physiological strain level at day 3 of culture. Similarly, ACL fibroblasts produced significantly less 
MP1 $\beta$ at $10 \%$ strain compared to no strain at day 3 of culture. Also, at day 6 , supraphysiological strain elicited a significant decrease in MP1 $\beta$ production compared to mechanical strain deprivation and physiological strain (Fig. 7.6).

Physiologic stress significantly increased the production of GAG by ACL fibroblasts compared to stress deprivation and supraphysiologic strain levels at days 3 and 6 of culture. There were no significant differences between groups for GAG production by SYN fibroblasts (Fig. 7.7). Further there were no significant differences between ACL and SYN fibroblast groups for MMP (Fig.7.8) or TIMP (Fig. 7.9) production at any timepoint.

\section{Differences between ACL and Synovial Fibroblast Response to Mechanical Strain}

Metabolic activity was not significantly different between SYN and ACL fibroblasts at low strains (no strain and $4 \%$ strain). However, at supraphysiological strain levels, the metabolic activity of ACL fibroblasts was significantly lower than SYN fibroblasts (Fig. 7.10).

PGE2 production was significantly lower in ACL compared to SYN fibroblasts when subjected to mechanical strain deprivation at three and six days of culture. Conversely, PGE2 production was significantly increased in ACL compared to SYN fibroblasts when subjected to physiological strain at day three and six of culture. No differences between ACL and SYN fibroblasts were seen at supraphysiological strain at any timepoints (Fig. 7.11).

The production of IL-6 and KC by SYN fibroblasts was significantly higher than ACL fibroblasts at day 3 of culture regardless of the strain applied (Fig. 7.12 and 7.14 respectively). Additionally, the production of IL-8 by SYN fibroblasts at 
supraphysiological strain levels was significantly higher than ACL fibroblasts at day 3 , but not day 6, of culture (Fig. 7.13).

The production of MCP-1 and VEGF by SYN fibroblasts was significantly higher than ACL fibroblasts at supraphysiological strain levels at day three of culture (Fig.7.15 and 7.16). The production of MIP-1 $\beta$ was significantly higher in ACL fibroblasts compared to SYN fibroblasts only when stress deprived at day six of culture. No significant differences in MP1 $\beta$ production were seen between SYN and ACL fibroblasts at $4 \%$ and $10 \%$ strain at any timepoints (Fig. 7.17).

The production of GAG was significantly higher by ACL fibroblasts compared to SYN fibroblasts at stress deprivation and physiological stress levels at day 3 of culture. Moreover, at day 6 of culture, the production of GAG by SYN fibroblasts was significantly higher than ACL fibroblasts during stress deprivation. No significant differences between SYN and ACL fibroblasts were seen at supraphysiological strain at any time point (Fig. 7.18).

The production of MMPs and TIMPS by the ACL and SYN fibroblasts were similar at most strain levels and time points tested. No significant differences were seen in MMP-1, MMP-2, MMP-3 and TIMP-1 between SYN and ACL fibroblasts at any strain level at any timepoint (Fig. 7.19, 7.20, 7.21, 7.22). Only the production of TIMP-2 by ACL fibroblasts was significantly higher than SYN fibroblasts when stress deprived after day 3 of culture (Fig. 7.23).

Males vs Females Differences in ACL and Synovial Fibroblast Response to Mechanical Strain 
There were few significant differences between male and female ACL or SYN fibroblasts regardless of strain or day of culture (Fig. 7.24-7.28). The metabolic activity of male SYN fibroblasts was significantly higher than female SYN fibroblasts at $4 \%$ strain (Fig. 7.24). The production of MIP-1 $\beta$ by male SYN fibroblast was significantly higher than female SYN fibroblasts after six days of supraphysiological stress (Fig.7.27).

\section{Discussion}

This work was able to demonstrate that SYN and ACL fibroblasts modulate the production of inflammatory and metabolic markers in a strain dependent manner.

Moreover, we were able to show that such responses are significantly different between intraarticular tissue fibroblasts such as synovium and ACL. However, it appears that molecular response to load is not significantly different between males and females. The inflammatory marker PGE2, seems to be increased with increasing loads. Interestingly, lower cytokine production, was found at higher strain levels. Moreover, cytokines such as IL-6 and KC in SYN fibroblasts appear to consistently increase in response to raising strains while other inflammatory markers such as IL-8, MCP-1 and VEGF had a more erratic reaction.

This behavior may be explained by the direct reaction of cellular component of the ACL as a response to injury after ACL rupture ${ }^{12 ; 13 ; 3435}$. The synovial lining of the knee joint is also a major player in knee injury responses and the intraarticular environment after an injury ${ }^{5 ; 36}$. Therefore, understanding the time at which degradative and inflammatory molecules are produced by each tissue is important for understanding how healing may occur after surgery. 
Some authors have suggested that ACL reconstruction should be delayed at least three weeks after injury in order to optimize the outcome in reconstruction scenarios. ${ }^{37}$ In contrast, delay in surgery for primary ACL repair may not be advantageous. Magarian et al (2010) demonstrated that a two- or six-weeks delay may decrease functional outcomes after primary ACL repair in a pre-clinical model. ${ }^{38}$ Authors suggest that although late repair may significantly decrease synovitis, the structural composition of the injured ACL and its retracted torn ends make the repair more difficult. This could be due in part from a decrease in ligament composition such as reduced GAG content when the ACL is stress deprived for $120 \mathrm{~h}$.

Clearly, early molecular events after ACL injury are likely important although not fully understood. Moreover, how the biomechanical environment affects the ACL healing capacity is not fully understood. Therefore, we aimed to evaluate how fibroblasts from injured ACL and synovium respond to mechanical stresses and how these individual tissue's biologic response may contribute to the intraarticular knee environment after ACL injury.

Ligaments are mechanosensitive tissues. Due to their multiscale hierarchical structure, loads applied to tissues can be sensed and transduced by fibroblasts into biochemical responses, transforming their extracellular matrix and ultrastructure. ${ }^{39-41}$ Mechanical stress applied to cells also play an important role in inflammation. ${ }^{42-44}$ This work demonstrates that ACL fibroblasts may be more sensitive to extreme mechanical environments, either immobilization or high strain loads, responding with increased inflammatory production. In contrast, physiological strains led to increased release of cytokines and degradative molecules from synovial fibroblasts. This may be related to the 
fact that in this work, synovial fibroblasts were isolated from the ECM and their activity may need to be regulated by other cell types. ${ }^{32 ; 45-48}$

Differential fibroblastic responses to load seen between ACL and SYN may also play a role in ACL injury and repair. ${ }^{3 ; 32 ; 36}$ Since matrix and inflammatory biomarkers exhibit an opposite trend, higher GAG and TIMP-2 and lower cytokine production in ACL compared to SYN fibroblasts, it appears that mechanobiological regulation of healing is tissue dependent and, once again, providing the right amount of biomechanical stress is a key factor for proper healing and decreased injuries.

Sex differences in ACL injury has been the focus of a vast amount of research. This has led to many possible assumptions including anatomical variants, neuromuscular control differences, hormonal levels and variations. ${ }^{21 ; 22 ; 49}$ Recently, rather than the hormonal levels themselves, hormonal variation in females has been suggested as a main factor for increased rates of ACL injury in females. It seems that the cyclical periodic variation in hormone levels, estrogen and prostaglandin play a significant role in ACL injury. ${ }^{50}$ Moreover, conflicting data has been found whether exogenous hormonal therapies are protective or not against ACL injury in female athletes. ${ }^{18 ; 51-53}$ Surprisingly, our current study did not find many biologic differences between male and female ACL and synovial fibroblasts in response to same levels of strain. The absence of differences may suggest that the main risk factors for increased ACL injuries in females do not lie in the cellular ability of injured tissues to withstand load rather than external factors. ${ }^{54}$

It is advantageous that this analysis included human tissues from injured specimens. This helps better reflect the current condition of the tissues present in the ACL injured knee. We also included a reasonable sample size that permitted comparisons between males 
and females. However, inclusion of more males in the study is strongly suggested. Additionally, because we included a wide range of patients, age and ethnicity are expected to play a role in diverse biological responses of intraarticular tissues. Hence, a greater study that allows to control for age and ethnicity seems an interesting option. There were also limitations associated with this experiment. First, this study was only performed for six days. While it is a long in vitro study using stressed cells in culture, it does not reflect typical conditions of a native tissue, disregarding many events in the healing process. ${ }^{10 ; 12 ; 55}$ Second, we did not measure collagen production or degradation. Since many of the markers evaluated directly affect collagen structure, it would have been interesting to analyze how fibroblasts would respond in terms of collagen synthesis/degradation. Lastly, the lack of other molecular techniques such as gene expression or fluorescent imaging prevents us for making a "healing targeted" approach where we could see how biological processes and how they are regulated in terms of inflammation/degradation resolution.

We conclude that inflammatory markers are being significantly produced in response to a variety of tension loads in the cellular environment of the intraarticular tissues. Moreover, SYN appears to contribute to some of the inflammatory markers in response to varying mechanical stresses. It seems that degradative enzymes are not being significantly affected by strain, at least at this point, and that remodeling will take place in future stages of the healing process. Lastly, it appears that metabolic response to stretch is not different between males and females. Future directions include the assessment of a dynamic co-culture using both SYN and ACL fibroblasts to understand the cross talk that 
is taking place in the injured knee. Moreover, knowing the source of molecules involved in injury and healing is important for successfully preventing OA after surgery ${ }^{56}$. 


\section{REFERENCES}

1. Gornitzky AL, Lott A, Yellin JL, et al. 2016. Sport-Specific Yearly Risk and Incidence of Anterior Cruciate Ligament Tears in High School Athletes: A Systematic Review and Meta-analysis. The American journal of sports medicine 44:2716-2723.

2. Moses B, Orchard J, Orchard J. 2012. Systematic review: Annual incidence of ACL injury and surgery in various populations. Research in sports medicine (Print) 20:157-179.

3. Bhattaram P, Chandrasekharan U. 2017. The joint synovium: A critical determinant of articular cartilage fate in inflammatory joint diseases. Seminars in cell \& developmental biology 62:86-93.

4. Guerne PA, Zuraw BL, Vaughan JH, et al. 1989. Synovium as a source of interleukin 6 in vitro. Contribution to local and systemic manifestations of arthritis. The Journal of clinical investigation 83:585-592.

5. Iwanaga T, Shikichi M, Kitamura H, et al. 2000. Morphology and functional roles of synoviocytes in the joint. Archives of histology and cytology 63:17-31.

6. Tang Z, Yang L, Wang Y, et al. 2009. Contributions of different intraarticular tissues to the acute phase elevation of synovial fluid MMP-2 following rat ACL rupture. Journal of orthopaedic research : official publication of the Orthopaedic Research Society 27:243-248.

7. Nguyen DT, Ramwadhdoebe TH, van der Hart CP, et al. 2014. Intrinsic healing response of the human anterior cruciate ligament: An histological study of reattached ACL remnants. 32:296-301. 
8. Georgiev GP, Landzhov B, Kotov G, et al. 2018. Matrix Metalloproteinase-2 and -9 Expression in the Epiligament of the Medial Collateral and Anterior Cruciate Ligament in Human Knees: A Comparative Study. Cureus 10:e3550.

9. Strand T, Molster A, Hordvik M, et al. 2005. Long-term follow-up after primary repair of the anterior cruciate ligament: clinical and radiological evaluation 15-23 years postoperatively. Arch Orthop Trauma Surg 125:217-221.

10. Murray MM, Fleming BC. 2013. Biology of anterior cruciate ligament injury and repair: Kappa delta ann doner vaughn award paper 2013. Journal of orthopaedic research : official publication of the Orthopaedic Research Society 31:1501-1506.

11. Spindler KP, Clark SW, Nanney LB, et al. 1996. Expression of collagen and matrix metalloproteinases in ruptured human anterior cruciate ligament: an in situ hybridization study. Journal of orthopaedic research : official publication of the Orthopaedic Research Society 14:857-861.

12. Murray MM, Martin SD, Martin TL, et al. 2000. Histological changes in the human anterior cruciate ligament after rupture. The Journal of bone and joint surgery American volume 82:1387-1397.

13. Kiapour AM, Murray MM. 2014. Basic science of anterior cruciate ligament injury and repair. Bone Joint Res 3:20-31.

14. Herzberg SD, Motu'Apuaka ML, Lambert W, et al. 2017. The Effect of Menstrual Cycle and Contraceptives on ACL Injuries and Laxity: A Systematic Review and Meta-analysis. Orthopaedic Journal of Sports Medicine $5: 232596711771878$. 
15. Lin CY, Casey E, Herman DC, et al. 2018. Sex Differences in Common Sports Injuries. 10:1073-1082.

16. Arendt E, Dick R. 1995. Knee injury patterns among men and women in collegiate basketball and soccer. NCAA data and review of literature. The American journal of sports medicine 23:694-701.

17. Kaeding CC, Leger-St-Jean B, Magnussen RA. 2017. Epidemiology and Diagnosis of Anterior Cruciate Ligament Injuries. Clin Sports Med 36:1-8.

18. Yu WD, Liu SH, Hatch JD, et al. 1999. Effect of estrogen on cellular metabolism of the human anterior cruciate ligament. Clinical orthopaedics and related research:229-238.

19. Liu SH, Al-Shaikh RA, Panossian V, et al. 1997. Estrogen affects the cellular metabolism of the anterior cruciate ligament. A potential explanation for female athletic injury. The American journal of sports medicine 25:704-709.

20. Carroll CC, Dickinson JM, Haus JM, et al. 2008. Influence of aging on the in vivo properties of human patellar tendon. Journal of applied physiology (Bethesda, Md : 1985) 105:1907-1915.

21. Smith HC, Vacek P, Johnson RJ, et al. 2012. Risk factors for anterior cruciate ligament injury: a review of the literature - part 1: neuromuscular and anatomic risk. Sports Health 4:69-78.

22. Dai B, Herman D, Liu H, et al. 2012. Prevention of ACL injury, part I: injury characteristics, risk factors, and loading mechanism. Research in sports medicine (Print) 20:180-197. 
23. Barenius B, Nordlander M, Ponzer S, et al. 2010. Quality of life and clinical outcome after anterior cruciate ligament reconstruction using patellar tendon graft or quadrupled semitendinosus graft: an 8-year follow-up of a randomized controlled trial. The American journal of sports medicine 38:1533-1541.

24. Murray JR, Lindh AM, Hogan NA, et al. 2012. Does anterior cruciate ligament reconstruction lead to degenerative disease?: Thirteen-year results after bonepatellar tendon-bone autograft. The American journal of sports medicine 40:404413.

25. Nagelli CV, Cook JL, Kuroki K, et al. 2017. Does Anterior Cruciate Ligament Innervation Matter for Joint Function and Development of Osteoarthritis? The journal of knee surgery 30:364-371.

26. Woo SL, Vogrin TM, Abramowitch SD. 2000. Healing and repair of ligament injuries in the knee. The Journal of the American Academy of Orthopaedic Surgeons 8:364-372.

27. Andrish J, Holmes R. 1979. Effects of synovial fluid on fibroblasts in tissue culture. Clinical orthopaedics and related research:279-283.

28. Wang T, Chen P, Zheng M, et al. 2018. In vitro loading models for tendon mechanobiology. Journal of orthopaedic research : official publication of the Orthopaedic Research Society 36:566-575.

29. Tondon A, Haase C, Kaunas R. 2014. Mechanical Stretch Assays in Cell Culture Systems. Handbook of Imaging in Biological Mechanics:313-322. 
30. Escamilla RF, Macleod TD, Wilk KE, et al. 2012. Anterior cruciate ligament strain and tensile forces for weight-bearing and non-weight-bearing exercises: a guide to exercise selection. J Orthop Sports Phys Ther 42:208-220.

31. Lubowitz JH. 2014. Anatomic ACL reconstruction produces greater graft length change during knee range-of-motion than transtibial technique. Knee Surgery, Sports Traumatology, Arthroscopy 22:1190-1195.

32. Xie J, Wang CL, Yang W, et al. 2018. Modulation of MMP-2 and MMP-9 through connected pathways and growth factors is critical for extracellular matrix balance of intra-articular ligaments. J Tissue Eng Regen Med 12:e550-e565.

33. Farndale RW, Buttle DJ, Barrett AJ. 1986. Improved quantitation and discrimination of sulphated glycosaminoglycans by use of dimethylmethylene blue. Biochim Biophys Acta 883:173-177.

34. Yoshida M, Fujii K. 1999. Differences in cellular properties and responses to growth factors between human ACL and MCL cells. Journal of Orthopaedic Science 4:293-298.

35. Bigoni M, Sacerdote P, Turati M, et al. 2013. Acute and late changes in intraarticular cytokine levels following anterior cruciate ligament injury. Journal of orthopaedic research : official publication of the Orthopaedic Research Society $31: 315-321$.

36. Tang Z, Yang L, Wang Y, et al. 2009. Contributions of different intraarticular tissues to the acute phase elevation of synovial fluid MMP-2 following rat ACL rupture. Journal of Orthopaedic Research 27:243-248. 
37. Evans S, Shaginaw J, Bartolozzi A. 2014. Acl reconstruction - it's all about timing. International journal of sports physical therapy 9:268-273.

38. Magarian EM, Fleming BC, Harrison SL, et al. 2010. Delay of 2 or 6 weeks adversely affects the functional outcome of augmented primary repair of the porcine anterior cruciate ligament. The American journal of sports medicine $38: 2528-2534$.

39. Killian ML, Cavinatto L, Galatz LM, et al. 2012. The role of mechanobiology in tendon healing. J Shoulder Elbow Surg 21:228-237.

40. Lavagnino M, Wall ME, Little D, et al. 2015. Tendon mechanobiology: Current knowledge and future research opportunities. Journal of orthopaedic research : official publication of the Orthopaedic Research Society 33:813-822.

41. Wang JHC. 2006. Mechanobiology of tendon. Journal of biomechanics 39:15631582.

42. Archambault JM, Hart DA, Herzog W. 2001. Response of rabbit Achilles tendon to chronic repetitive loading. Connective tissue research 42:13-23.

43. Yang G, Im H-J, Wang JHC. 2005. Repetitive mechanical stretching modulates IL-1 $\beta$ induced COX-2, MMP-1 expression, and PGE2 production in human patellar tendon fibroblasts. Gene 363:166-172.

44. Zhang J, Wang JH. 2010. Production of PGE(2) increases in tendons subjected to repetitive mechanical loading and induces differentiation of tendon stem cells into non-tenocytes. Journal of orthopaedic research : official publication of the Orthopaedic Research Society 28:198-203. 
45. Schett G, Tohidast-Akrad M, Steiner G, et al. 2001. The stressed synovium. Arthritis research \& therapy 3:80.

46. McCarthy N. 2010. Cell signalling: Regulation and crosstalk. Nature reviews Molecular cell biology 11:390.

47. Mullen L, Adams G, Foster J, et al. 2014. A comparative study of matrix metalloproteinase and aggrecanase mediated release of latent cytokines at arthritic joints. Annals of the rheumatic diseases 73:1728-1736.

48. Wang C, Xie J, Jiang J, et al. 2015. Differential expressions of the lysyl oxidase family and matrix metalloproteinases-1, 2, 3 in posterior cruciate ligament fibroblasts after being co-cultured with synovial cells. Int Orthop 39:183-191.

49. Mall NA, Chalmers PN, Moric M, et al. 2014. Incidence and trends of anterior cruciate ligament reconstruction in the United States. The American journal of sports medicine 42:2363-2370.

50. Park SK, Stefanyshyn DJ, Loitz-Ramage B, et al. 2009. Changing hormone levels during the menstrual cycle affect knee laxity and stiffness in healthy female subjects. The American journal of sports medicine 37:588-598.

51. Yu WD, Panossian V, Hatch JD, et al. 2001. Combined effects of estrogen and progesterone on the anterior cruciate ligament. Clinical orthopaedics and related research:268-281.

52. Herzberg SD, Motu'apuaka ML, Lambert W, et al. 2017. The Effect of Menstrual Cycle and Contraceptives on ACL Injuries and Laxity: A Systematic Review and Meta-analysis. Orthop J Sports Med 5:2325967117718781. 
53. Wojtys EM, Huston LJ, Boynton MD, et al. 2002. The Effect of the Menstrual Cycle on Anterior Cruciate Ligament Injuries in Women as Determined by Hormone Levels. The American journal of sports medicine 30:182-188.

54. Hewett TE, Zazulak BT, Myer GD. 2007. Effects of the Menstrual Cycle on Anterior Cruciate Ligament Injury Risk:A Systematic Review. The American journal of sports medicine 35:659-668.

55. Wang JH, Thampatty BP, Lin JS, et al. 2007. Mechanoregulation of gene expression in fibroblasts. Gene 391:1-15.

56. Paschos NK. 2017. Anterior cruciate ligament reconstruction and knee osteoarthritis. World journal of orthopedics 8:212-217. 


\section{Tables}

\begin{tabular}{ccccc}
\multicolumn{2}{c}{ Tissue source (n) } & \multicolumn{2}{c}{ Sex (n) } & Age Range \\
\hline SYN & $(19)$ & Males & $(4)$ & $17-35$ \\
& & Females & $(15)$ & $14-62$ \\
ACL & $(20)$ & Males & $(4)$ & $17-22$ \\
& & Females & $(16)$ & $14-62$ \\
\hline
\end{tabular}

Table 7.1. Patient sex and age distribution by tissue source

Figures:

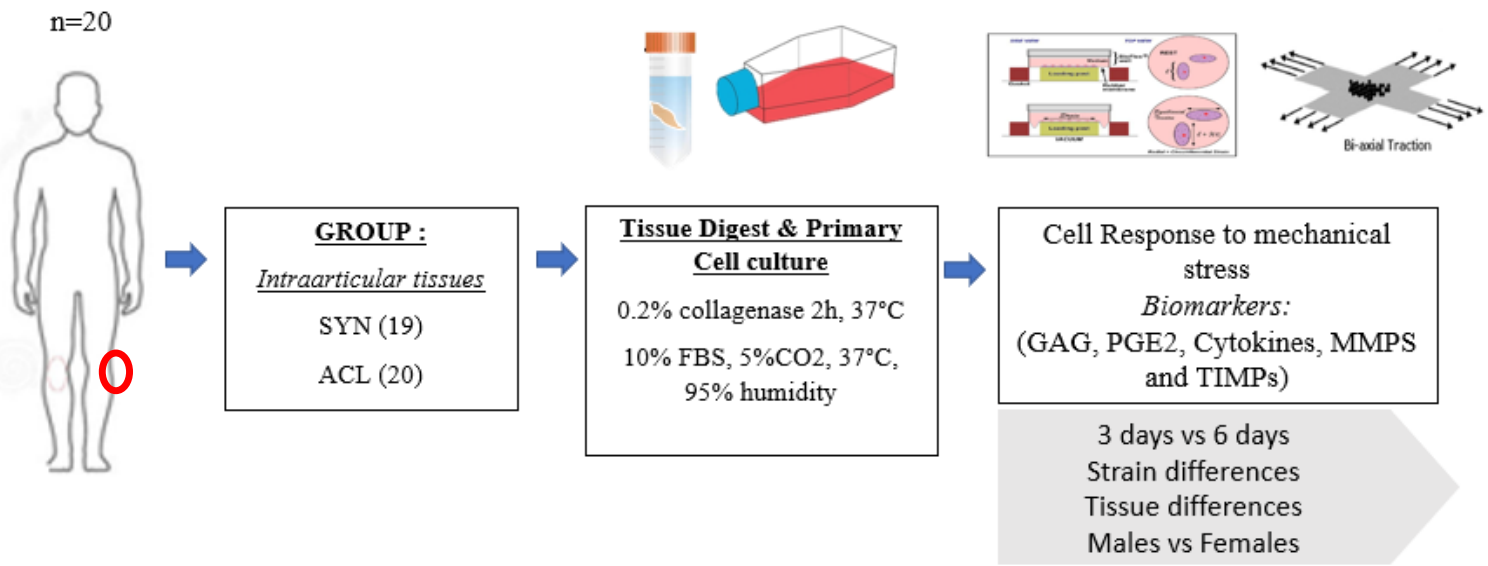

Figure 7.1: Experimental design 

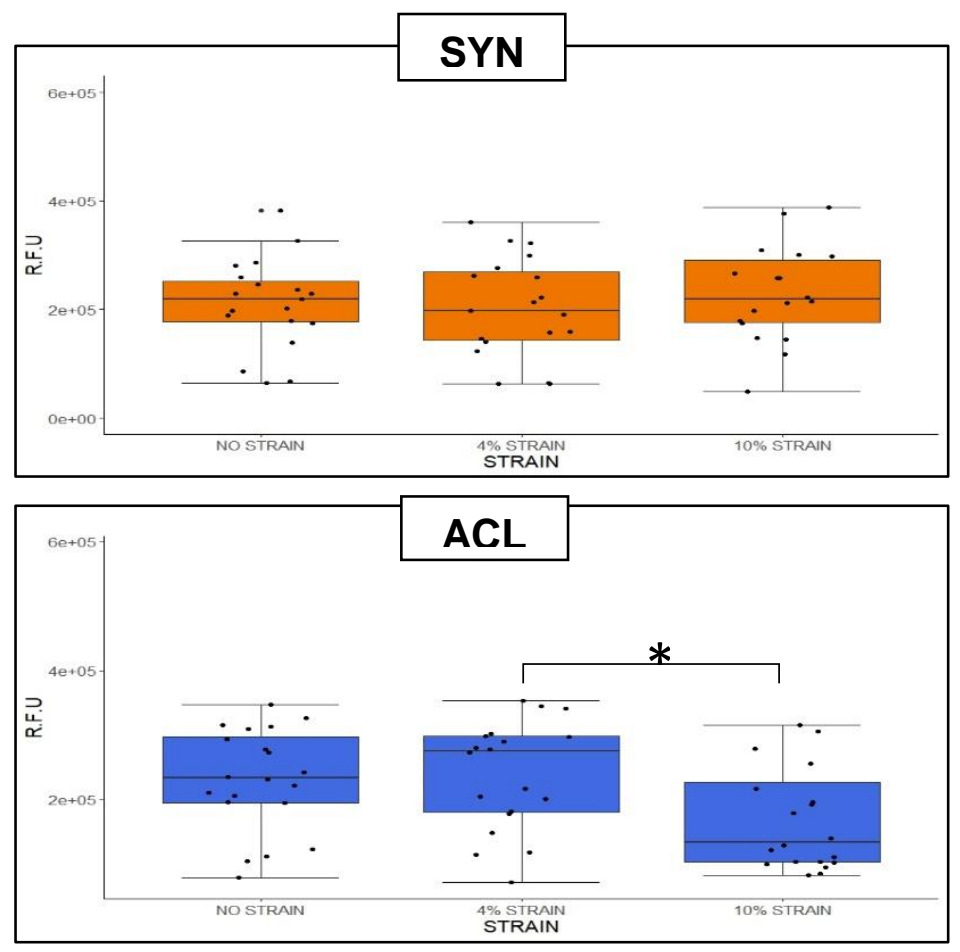

Figure 7.2. Metabolic Activity of intraarticular fibroblasts. * significant difference between STRAINS after 120 hours of culture (Kruskal-Wallis test). SYN: Synovium; ACL: Anterior Cruciate Ligament; R.F.U: Resazurin Fluorescent Units

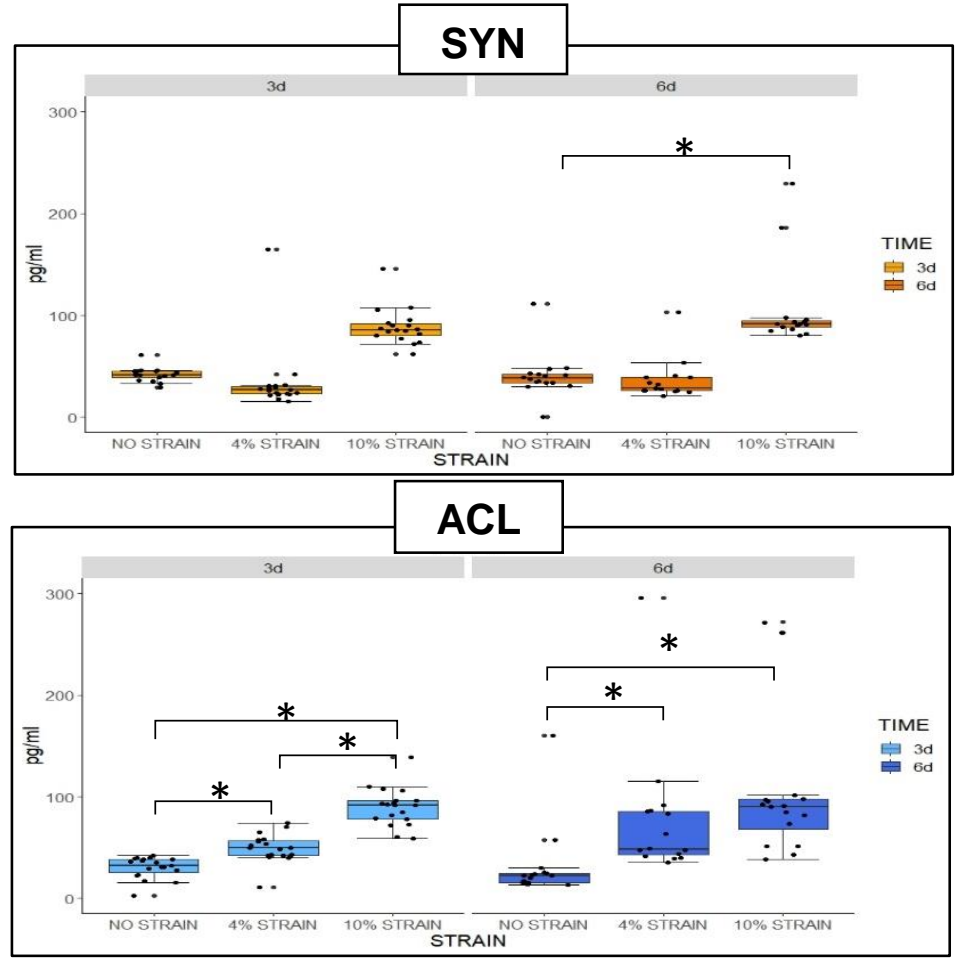

Figure 7.3. Concentration of PGE2 released to the media. * significant difference between STRAINS at 24 and 120 hours (Kruskal-Wallis test). SYN: Synovium; ACL: Anterior Cruciate Ligament 

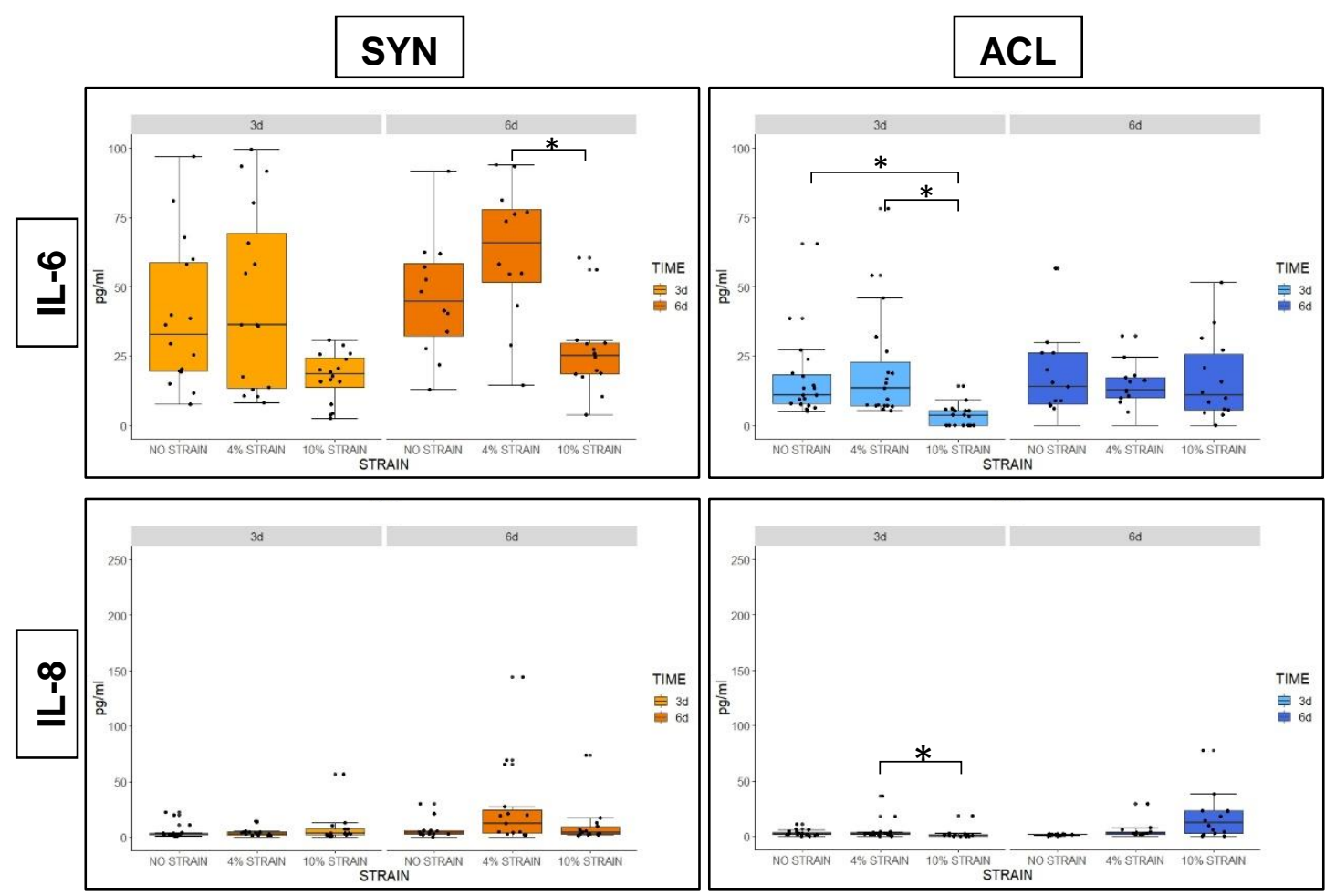

Figure 7.4. Concentration of interleukins 6 (IL-6) and 8 (IL-8) released to the media. * significant difference between STRAINS at 24 and 120 hours (Kruskal-Wallis test). SYN: Synovium; ACL: Anterior Cruciate Ligament

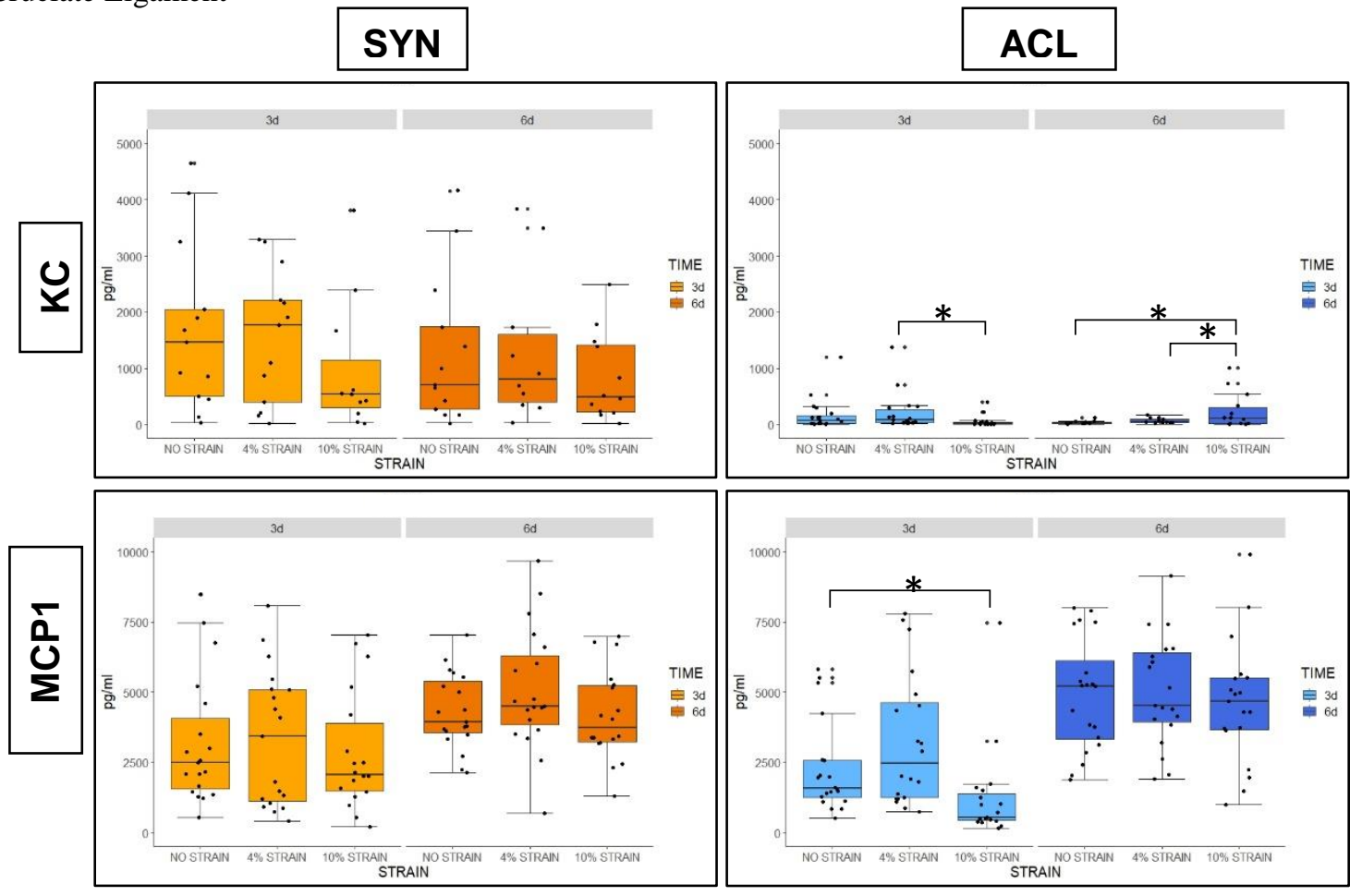

Figure 7.5. Concentration of chemokines (KC and MCP1) released to the media. * significant difference between STRAINS at 24 and 120 hours (Kruskal-Wallis test). SYN: Synovium; ACL: Anterior Cruciate Ligament 

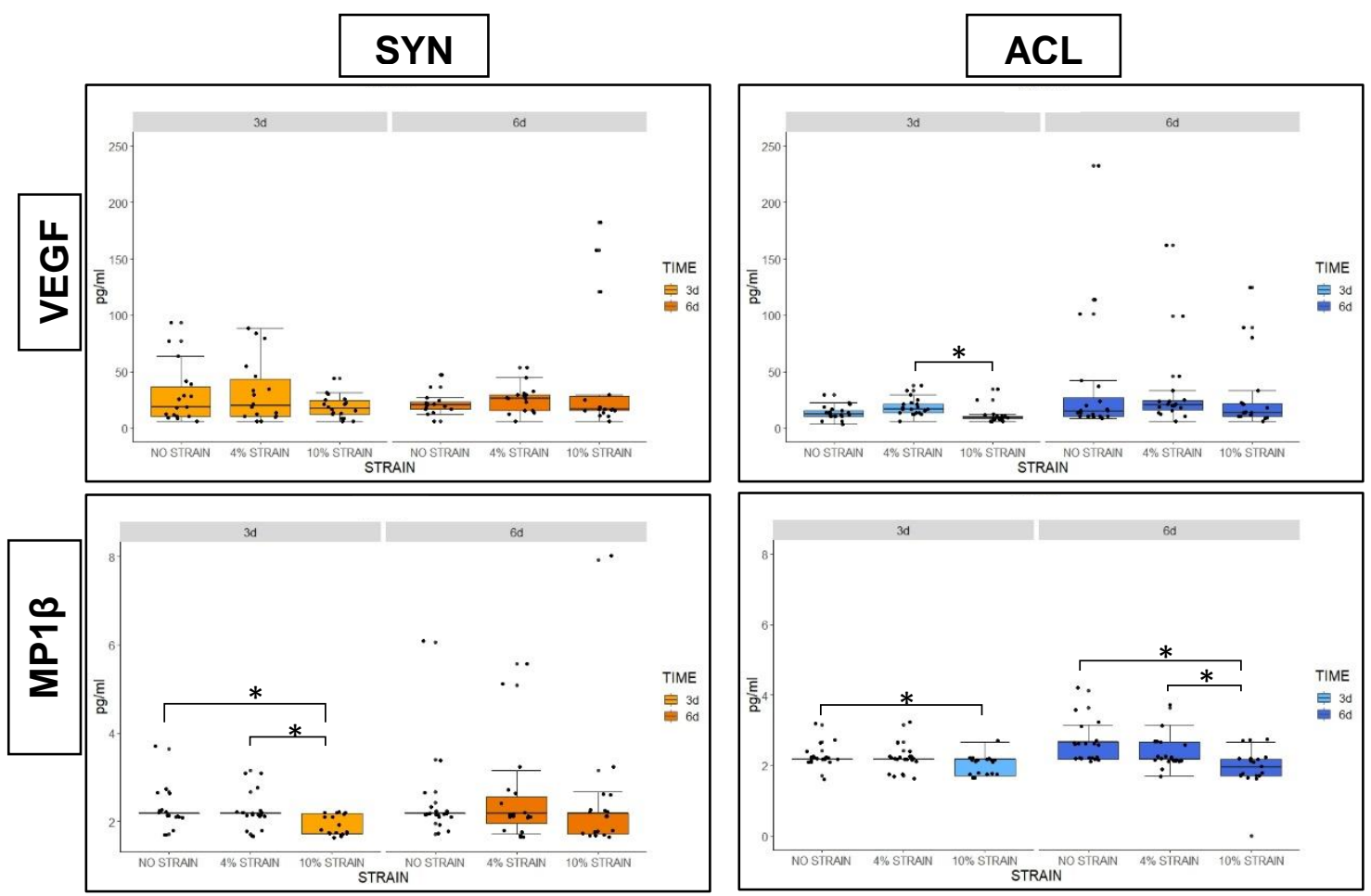

Figure 7.6. Concentration of Vascular Endothelial Growth Factor (VEGF) and macrophage inflammatory protein $1 \beta$ (MP1 $\beta$ ) released to the media. * significant difference between STRAINS at 24 and 120 hours (Kruskal-Wallis test). SYN: Synovium; ACL: Anterior Cruciate Ligament
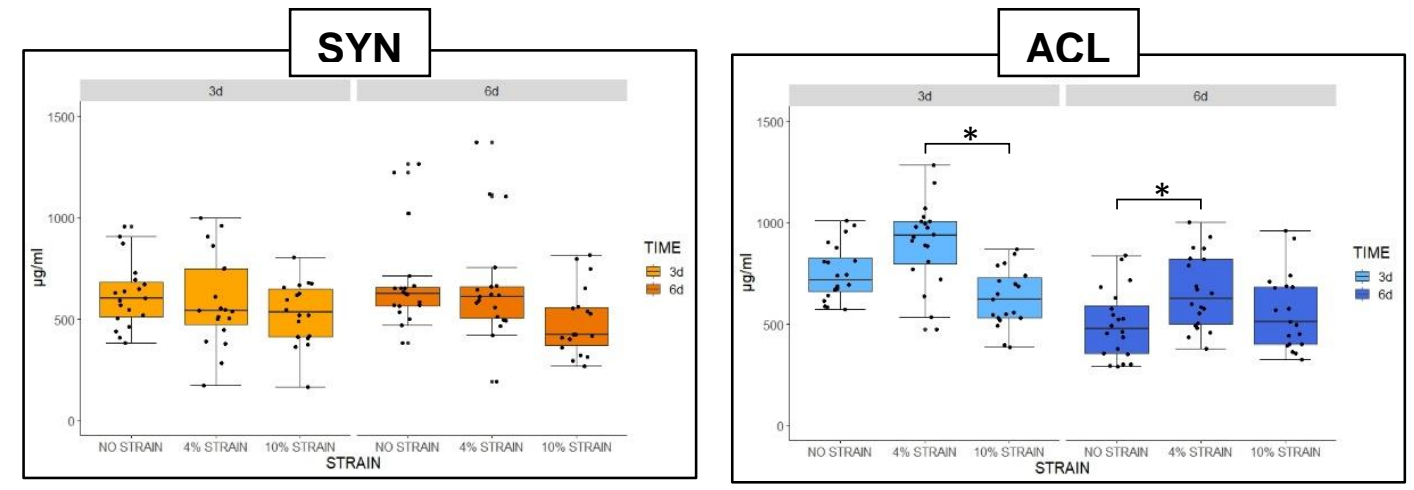

Figure 7.7. Concentration of GAG content released to the media. * significant difference between STRAINS at 24 and 120 hours (Kruskal-Wallis test). SYN: Synovium; ACL: Anterior Cruciate Ligament 

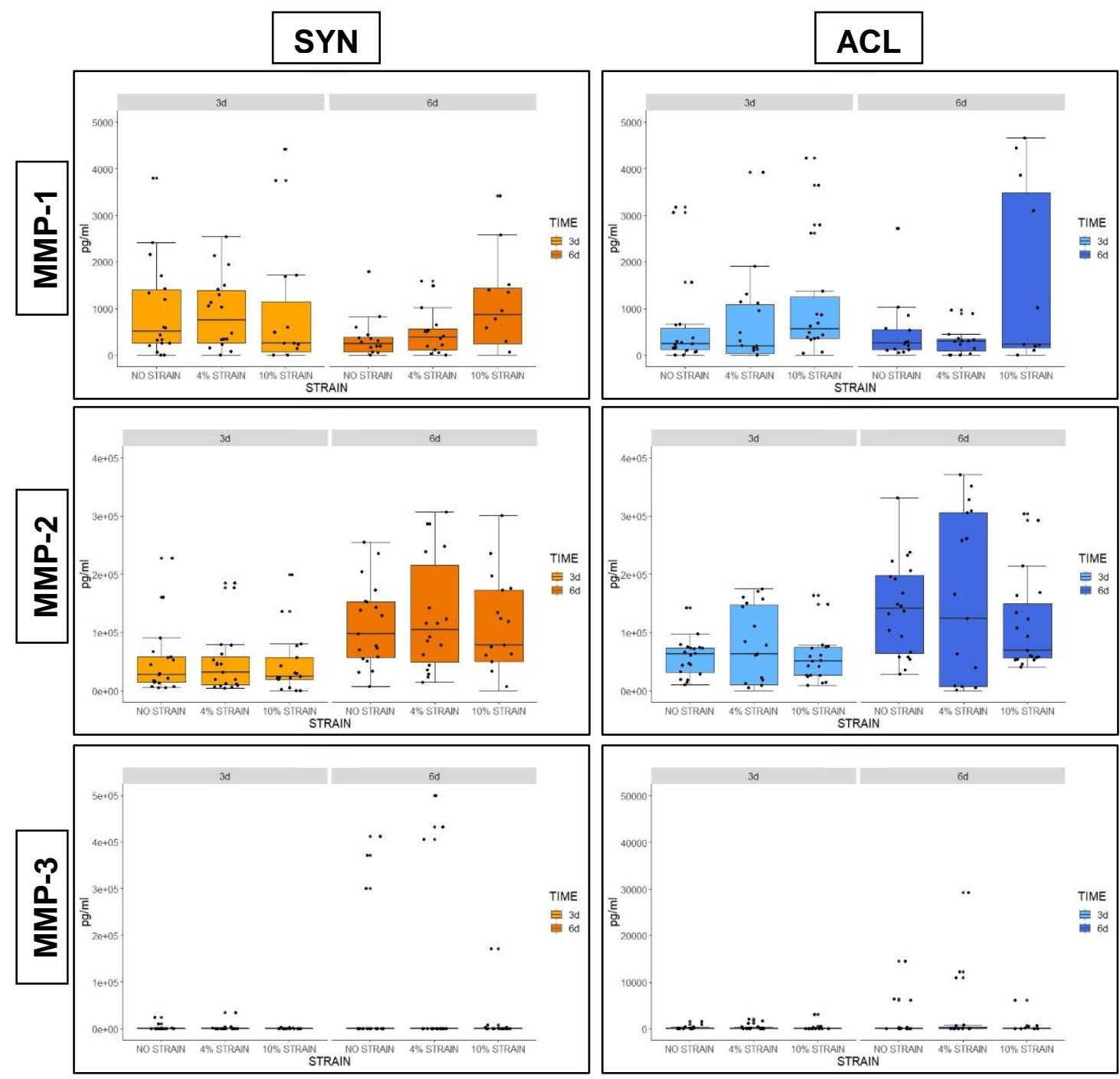

Figure 7.8. Concentration of matrix metalloproteinases (MMP) 1,2 and 3 released to the media. * significant difference between STRAINS at 24 and 120 hours (Kruskal-Wallis test). SYN: Synovium; ACL: Anterior Cruciate Ligament 

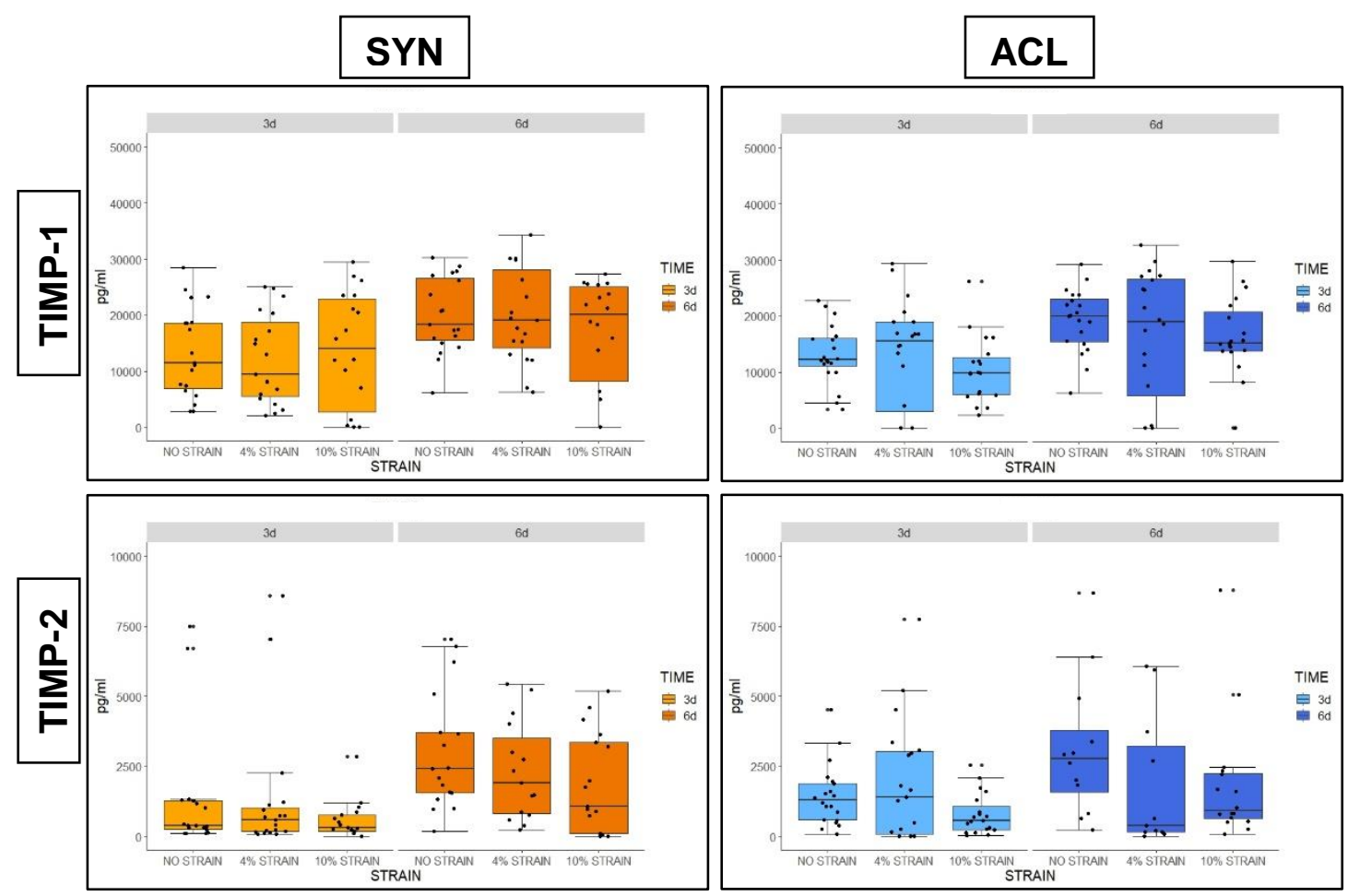

Figure 7.9. Concentration of tissue inhibitor of matrix metalloproteinases (TIMPs) 1 and 2 released to the media. * significant difference between STRAINS at 24 and 120 hours (Kruskal-Wallis test). SYN:

Synovium; ACL: Anterior Cruciate Ligament 

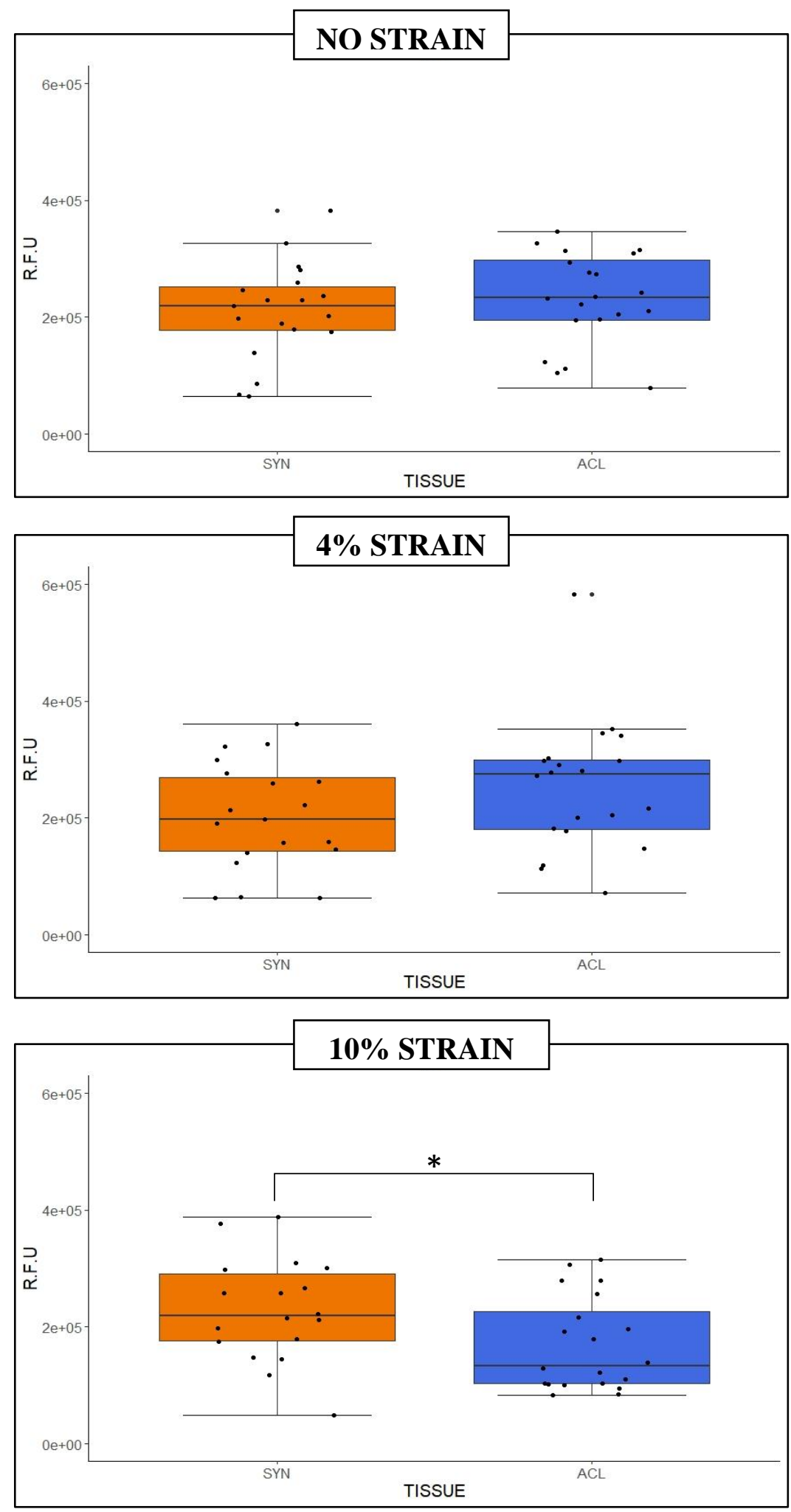

Figure 7.10. Metabolic Activity of fibroblasts after $120 \mathrm{~h}$ of culture. * significant difference between TISSUES. SYN: Synovium; ACL: Anterior Cruciate Ligament; R.F.U: Resazurin Fluorescent Units. 

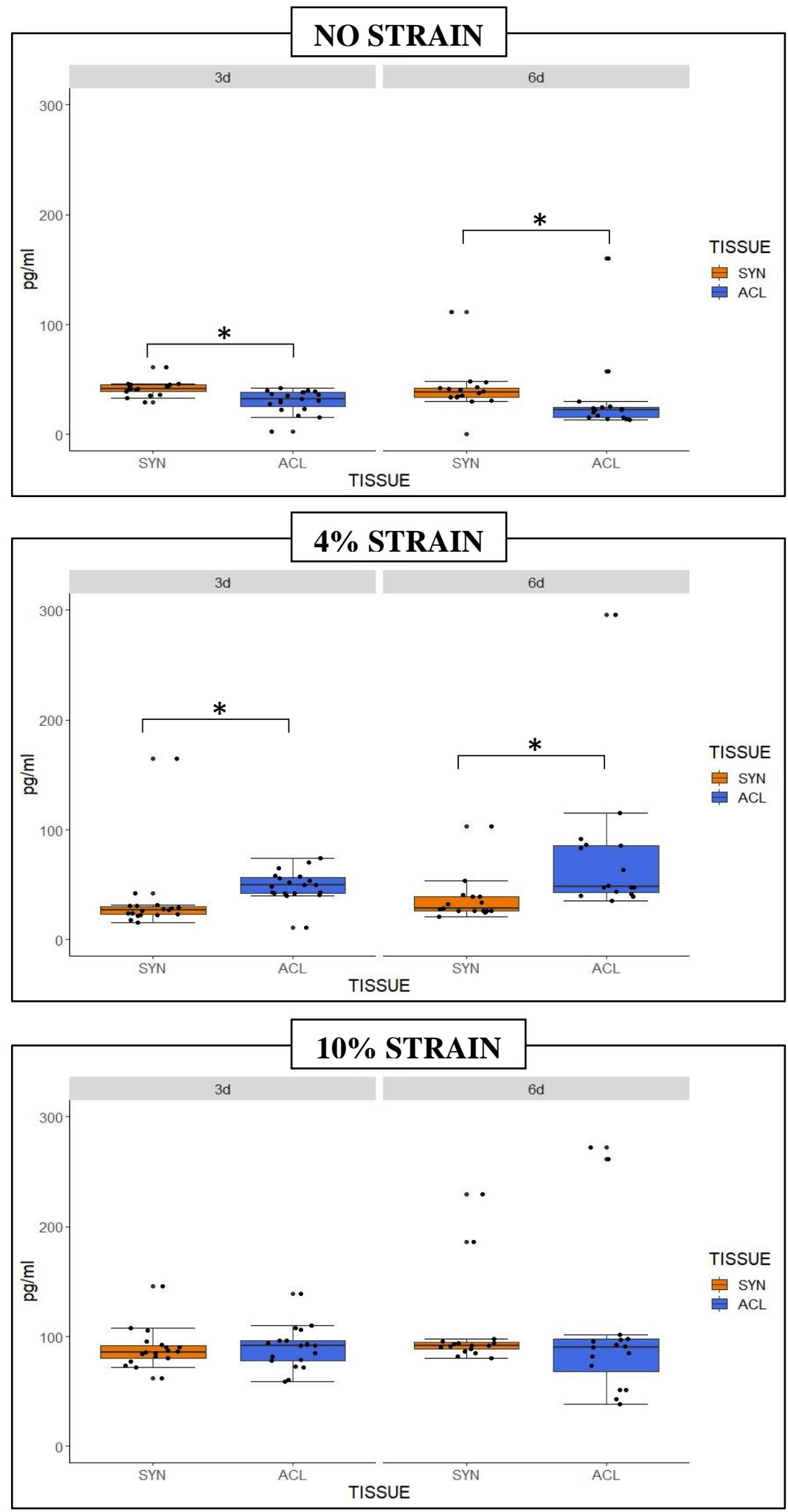

Figure 7.11. Concentration of PGE2 released to the media. * significant difference between TISSUES at 24 and 120 hours (Mann-Whitney test). SYN: Synovium; ACL: Anterior Cruciate Ligament. 

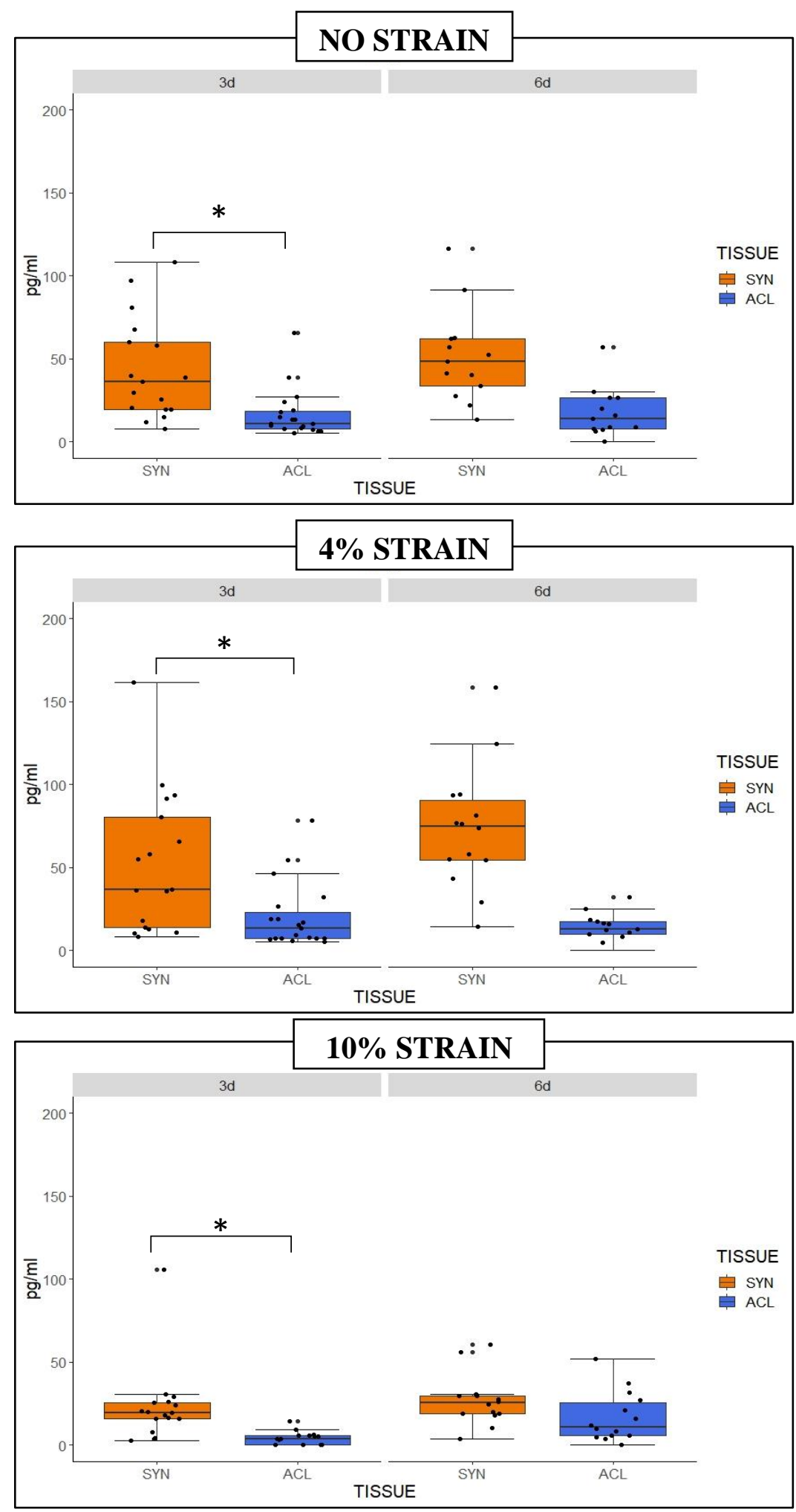

Figure 7.12. Concentration of IL-6 released to the media. * significant difference between TISSUES at 24 and 120 hours (Mann-Whitney test). SYN: Synovium; ACL: Anterior Cruciate Ligament. 

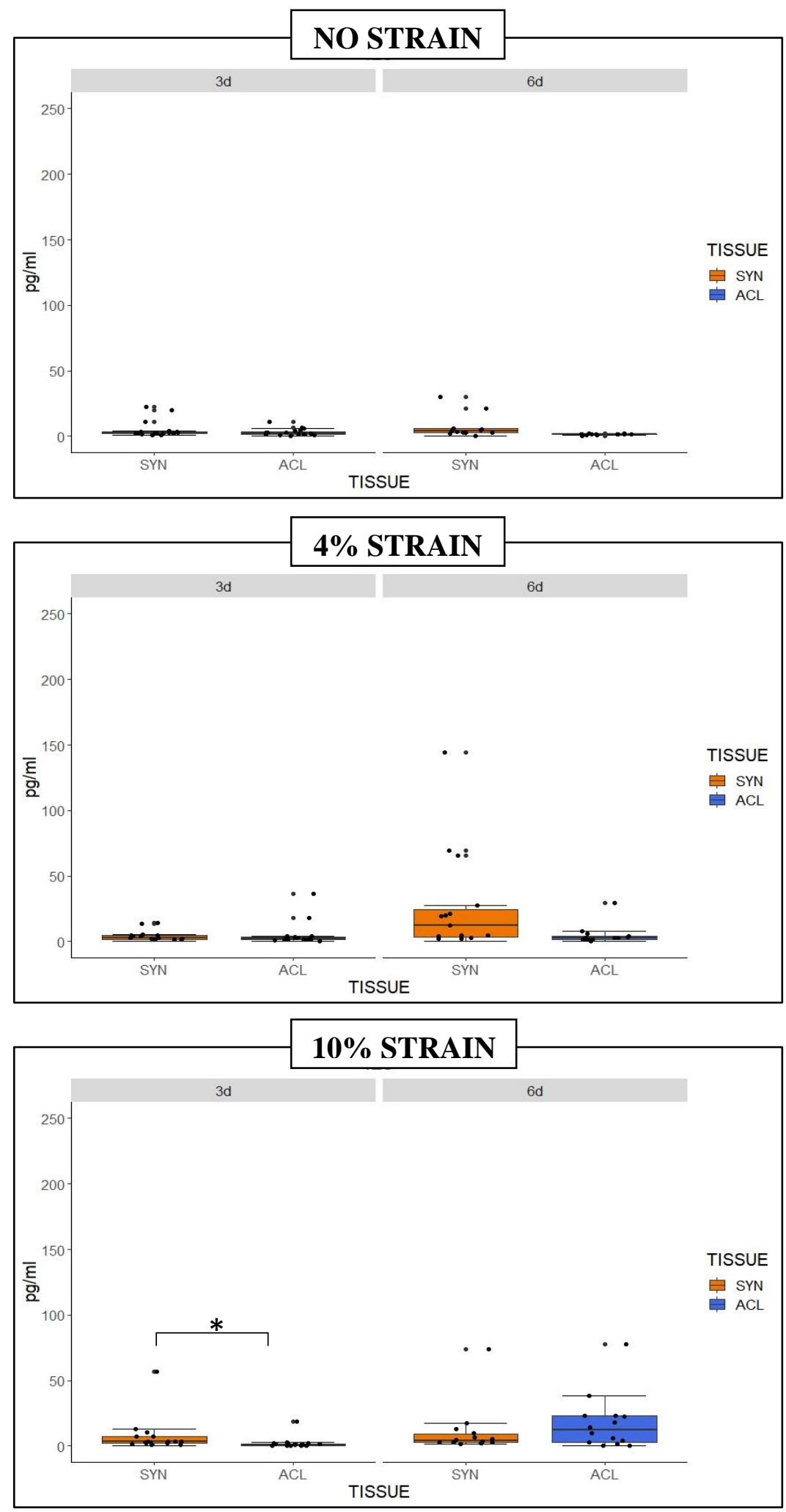

Figure 7.13. Concentration of IL-8 released to the media. * significant difference between TISSUES at 24 and 120 hours (Mann-Whitney test). SYN: Synovium; ACL: Anterior Cruciate Ligament. 

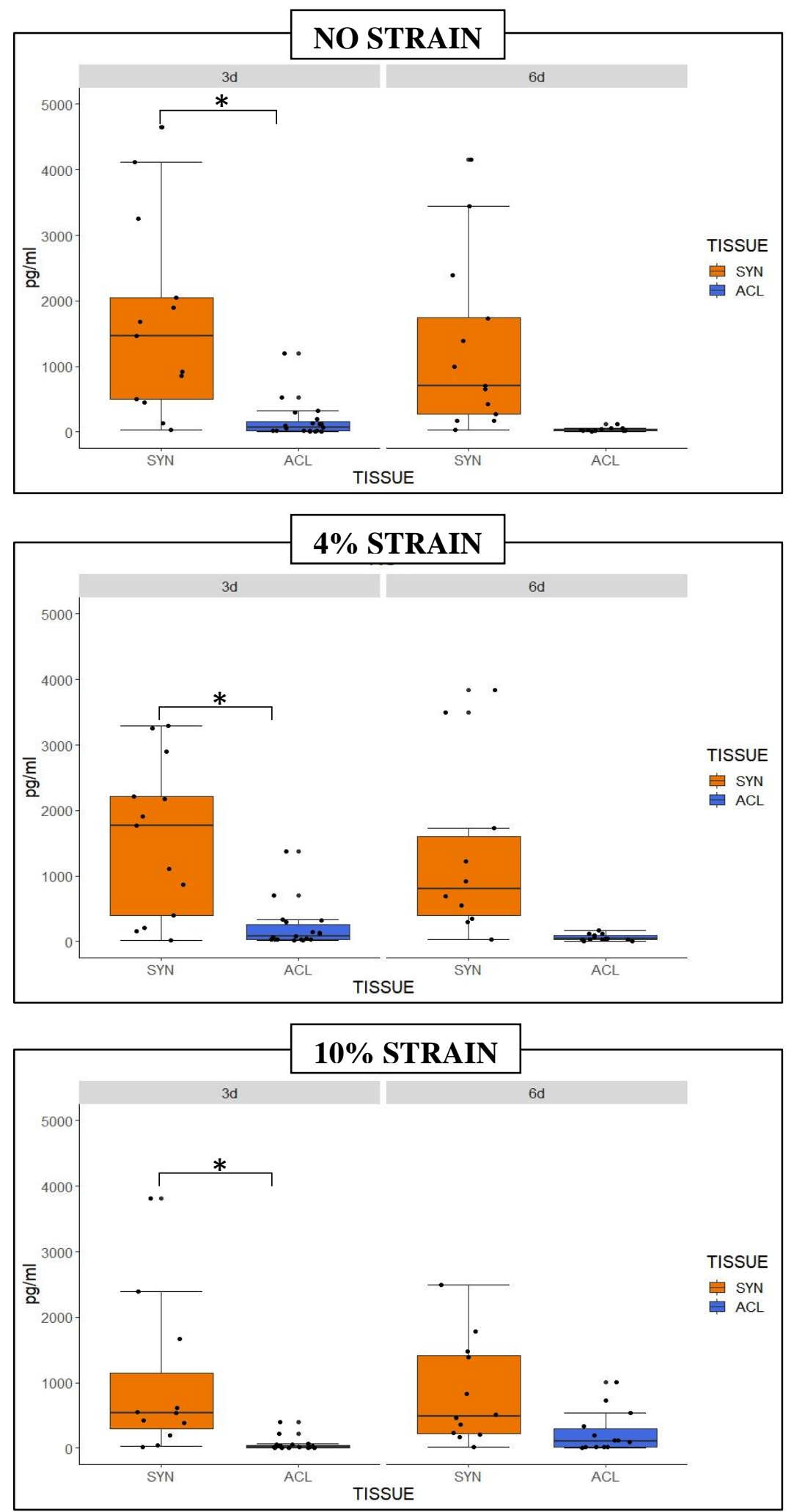

Figure 7.14. Concentration of KC released to the media. * significant difference between TISSUES at 24 and 120 hours (Mann-Whitney test). SYN: Synovium; ACL: Anterior Cruciate Ligament. 

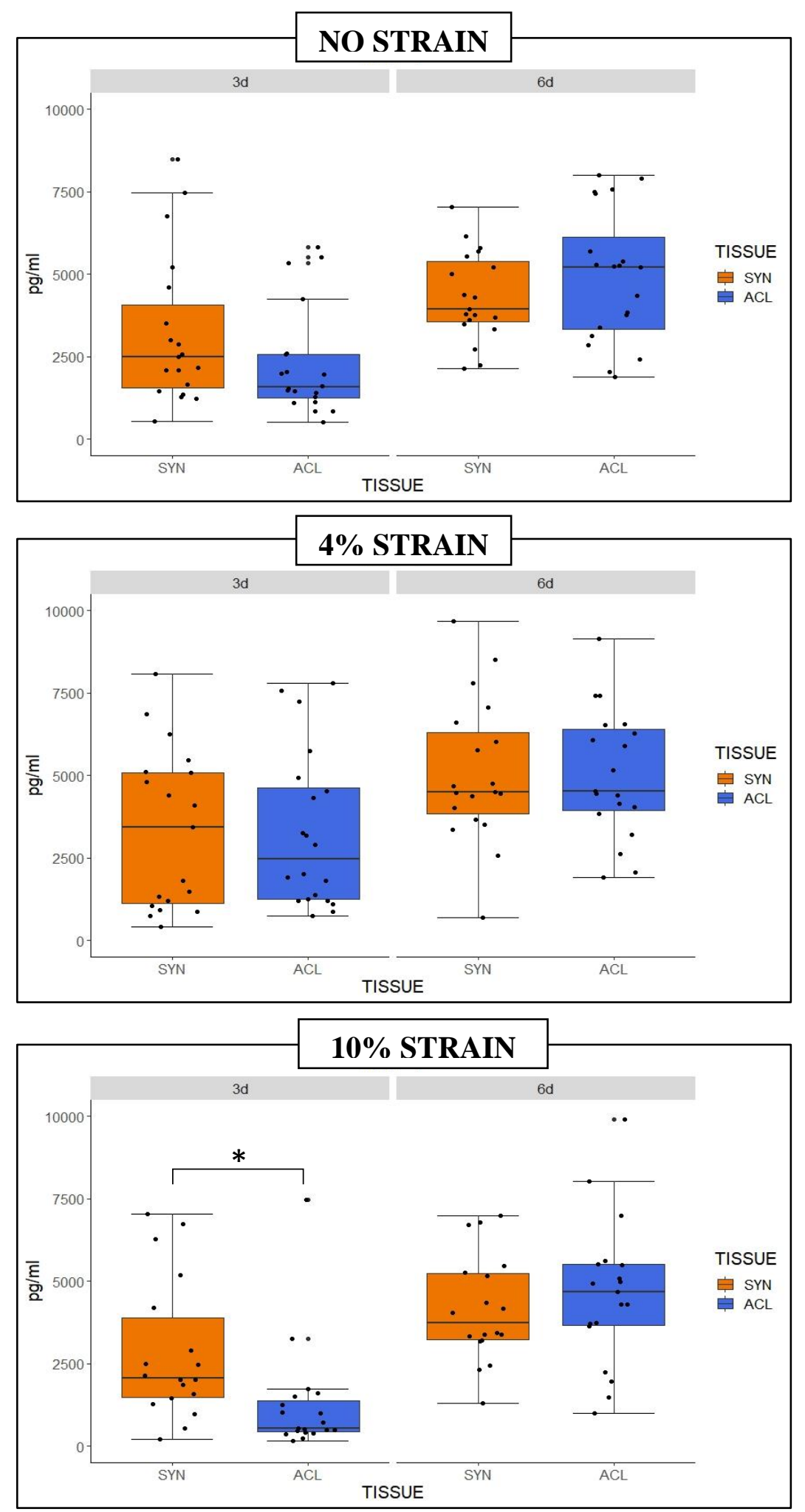

Figure 7.15. Concentration of MCP1 released to the media. * significant difference between TISSUES at 24 and 120 hours (Mann-Whitney test). SYN: Synovium; ACL: Anterior Cruciate Ligament. 

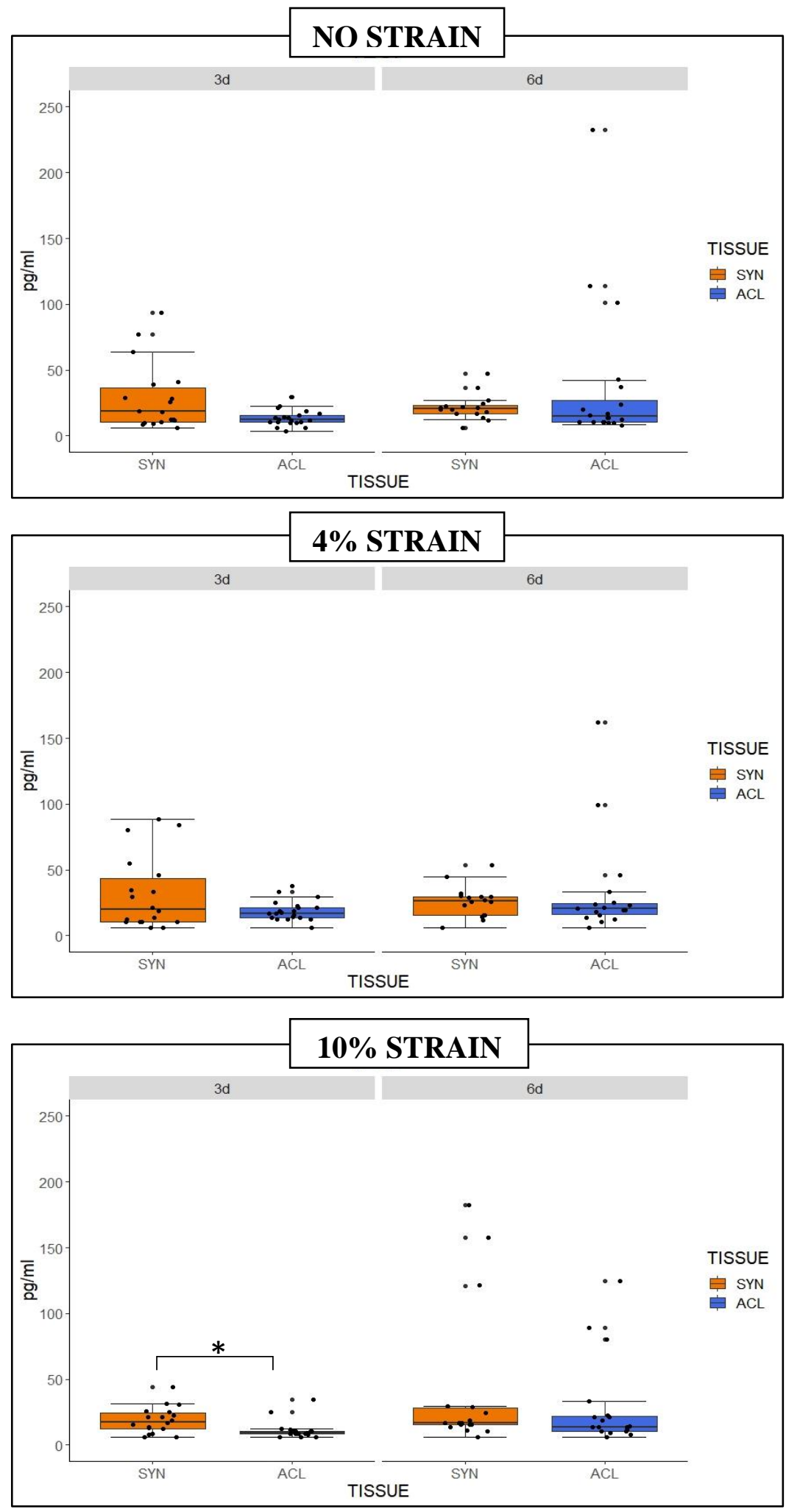

Figure 7.16. Concentration of VEGF released to the media. * significant difference between TISSUES at 24 and 120 hours (Mann-Whitney test). SYN: Synovium; ACL: Anterior Cruciate Ligament. 

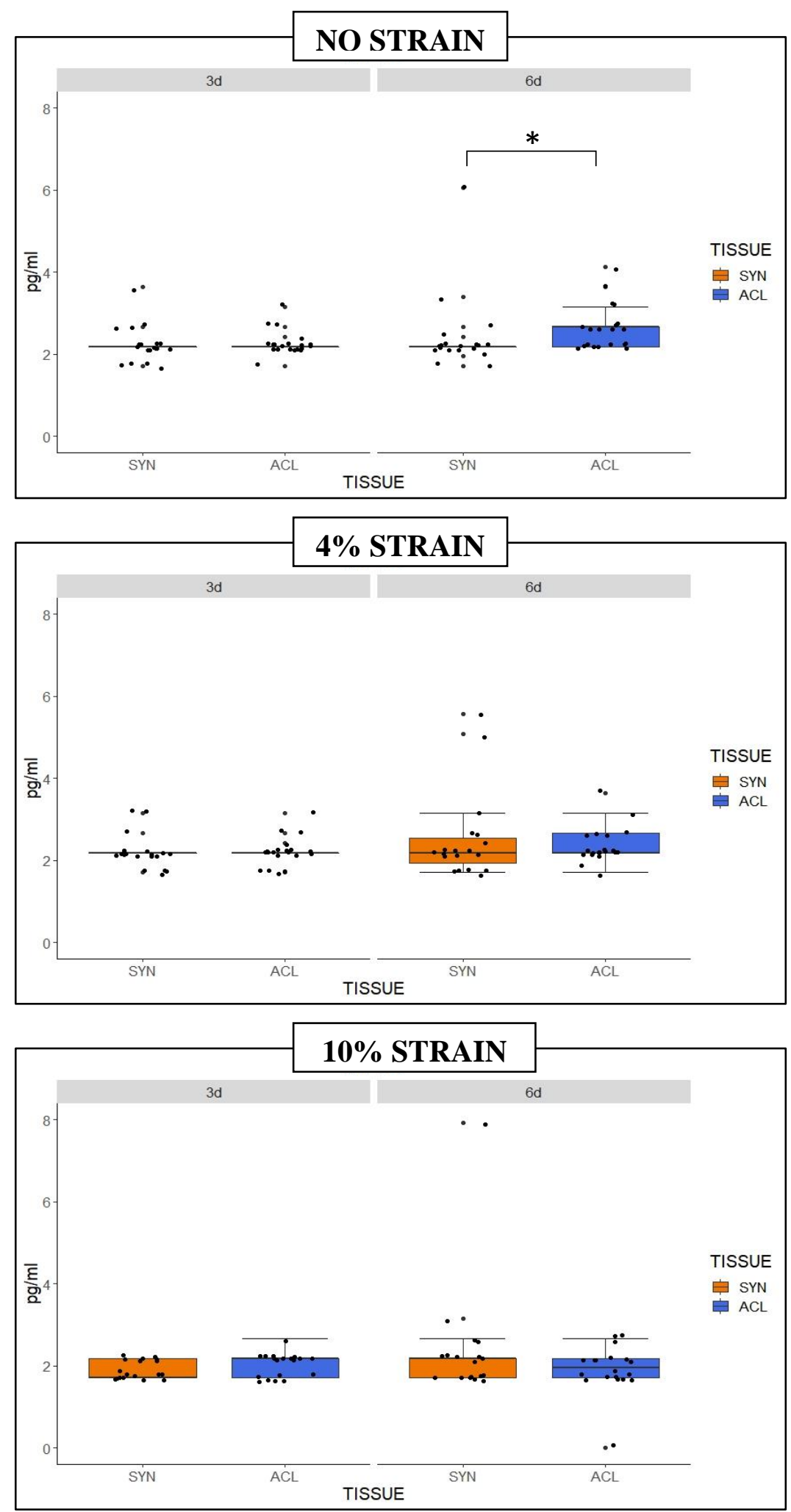

Figure 7.17. Concentration of MP1 $\beta$ released to the media. * significant difference between TISSUES at 24 and 120 hours (Mann-Whitney test). SYN: Synovium; ACL: Anterior Cruciate Ligament. 

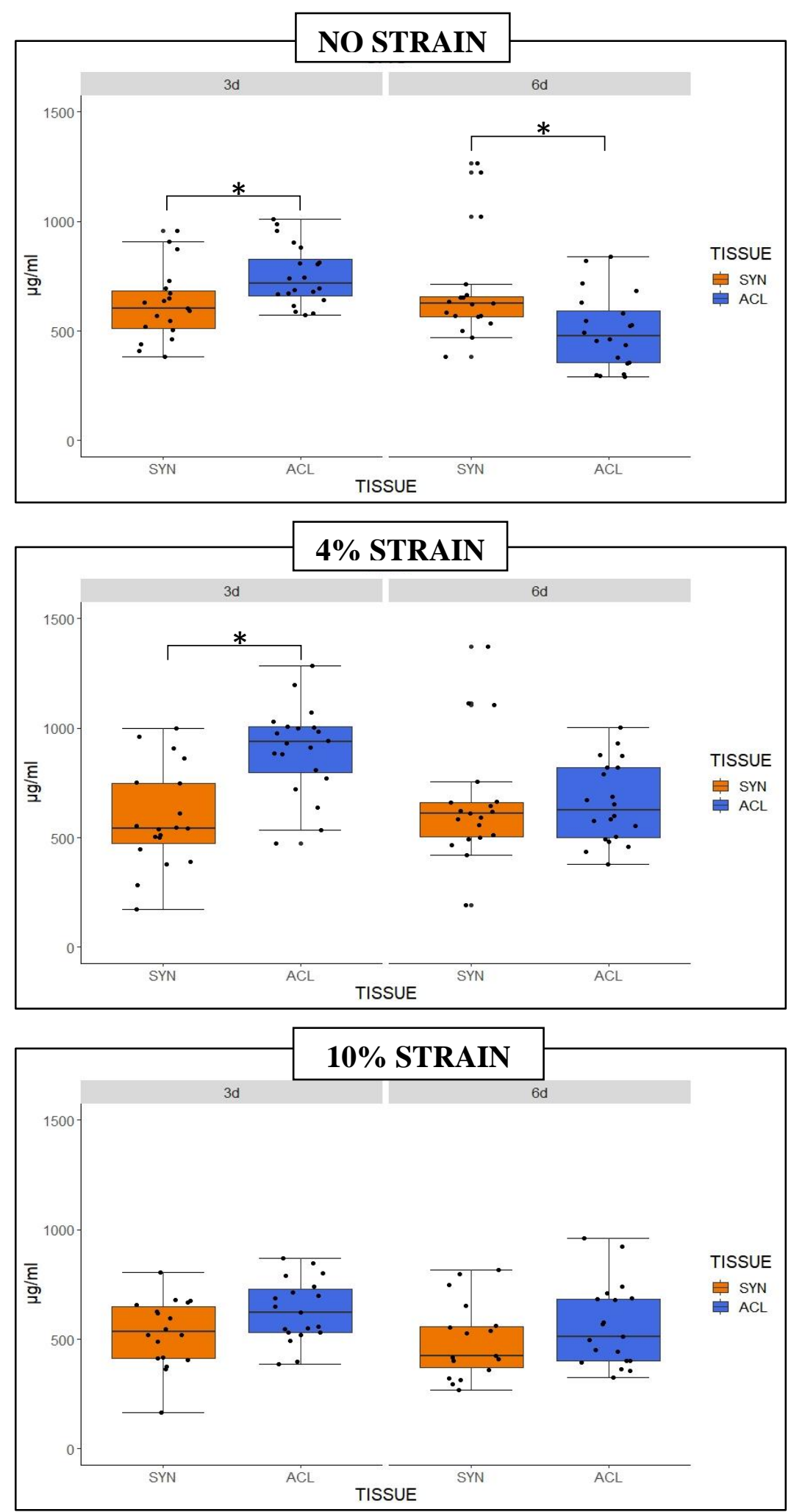

Figure 7.18. Concentration of GAG released to the media. * significant difference between TISSUES at 24 and 120 hours (Mann-Whitney test). SYN: Synovium; ACL: Anterior Cruciate Ligament. 

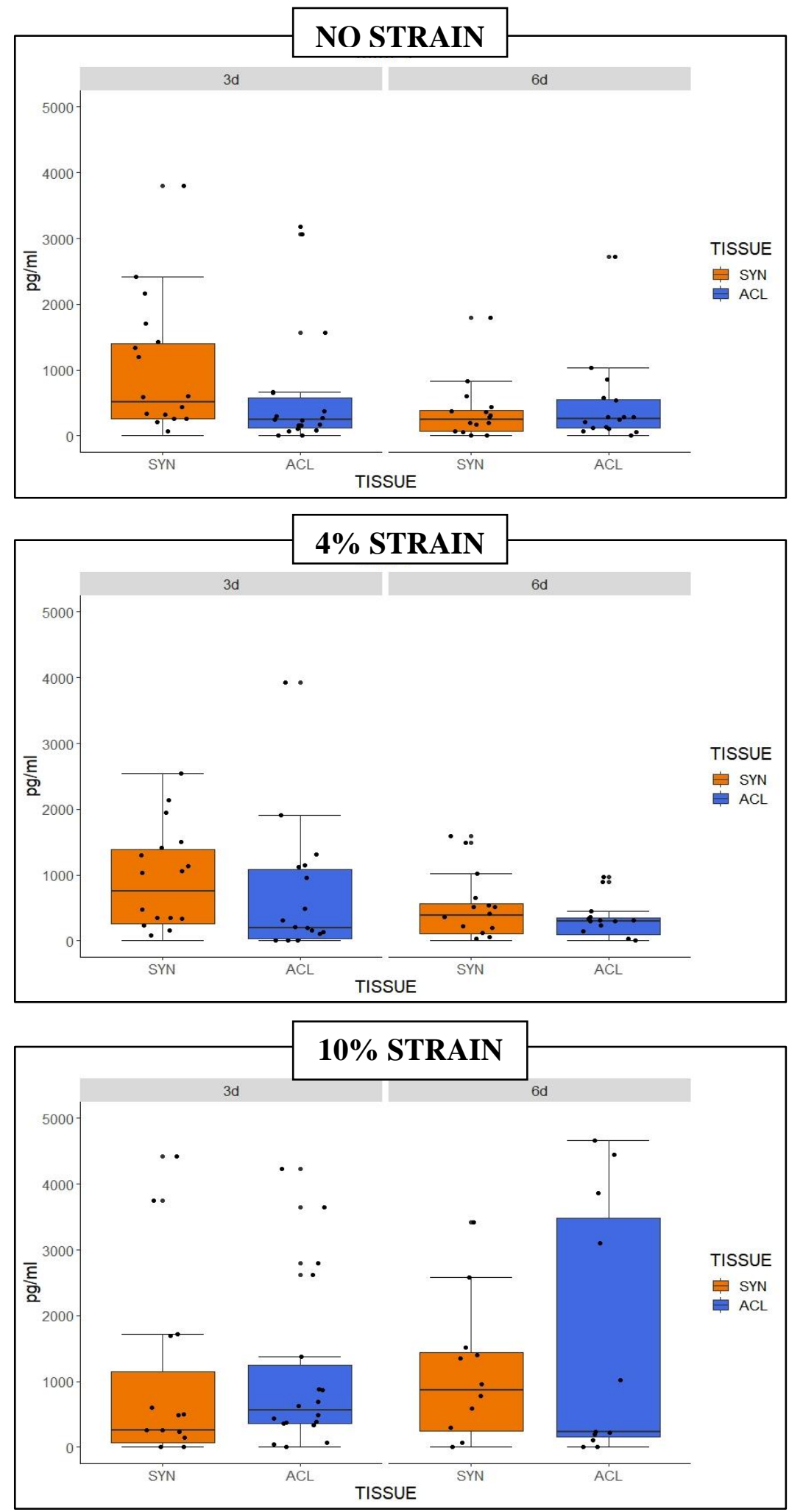

Figure 7.19. Concentration of MMP-1 released to the media. * significant difference between TISSUES at 24 and 120 hours (Mann-Whitney test). SYN: Synovium; ACL: Anterior Cruciate Ligament. 

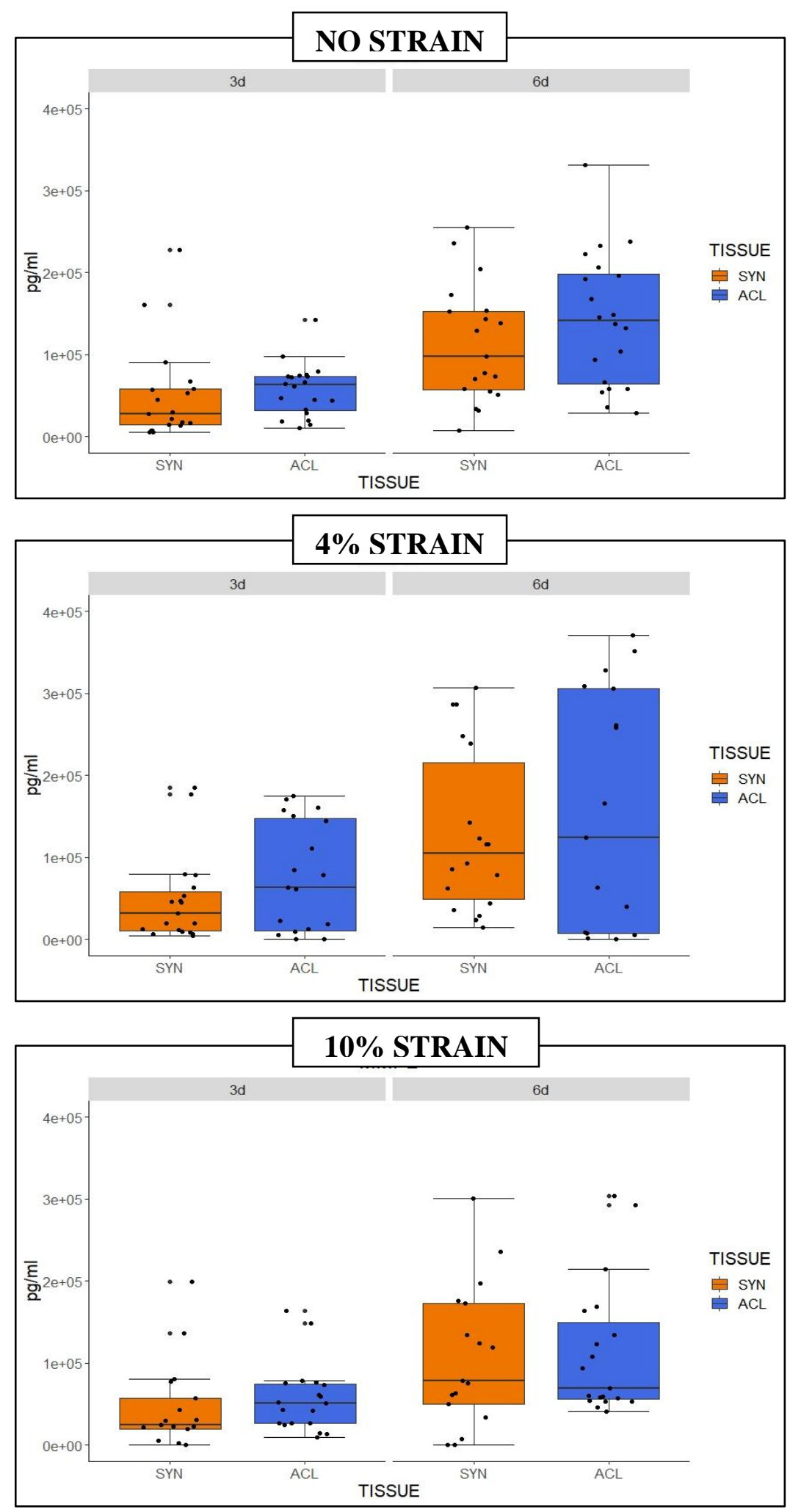

Figure 7.20. Concentration of MMP-2 released to the media. * significant difference between TISSUES at 24 and 120 hours (Mann-Whitney test). SYN: Synovium; ACL: Anterior Cruciate Ligament. 

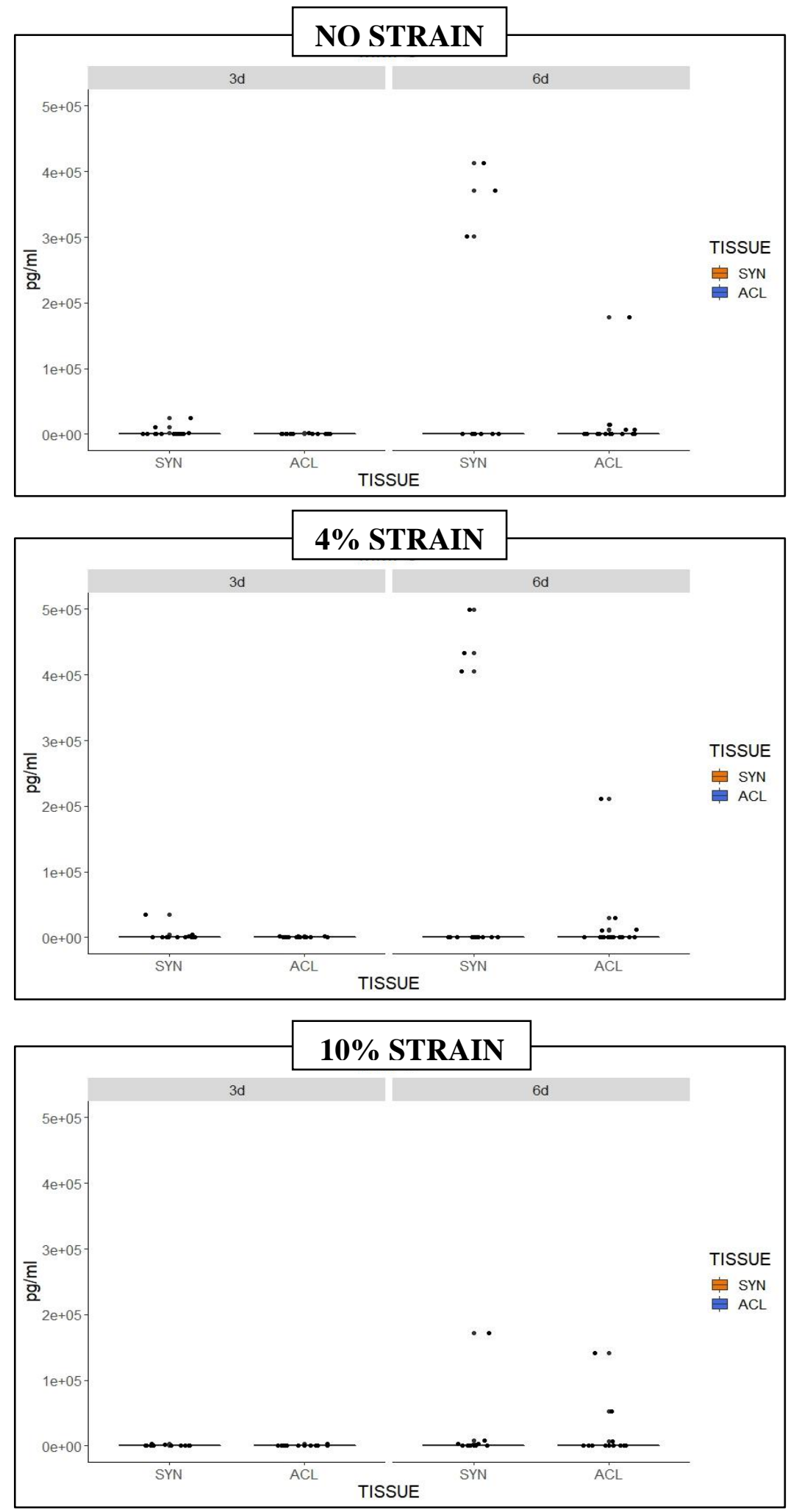

Figure 7.21. Concentration of MMP-3 released to the media. * significant difference between TISSUES at 24 and 120 hours (Mann-Whitney test). SYN: Synovium; ACL: Anterior Cruciate Ligament. 

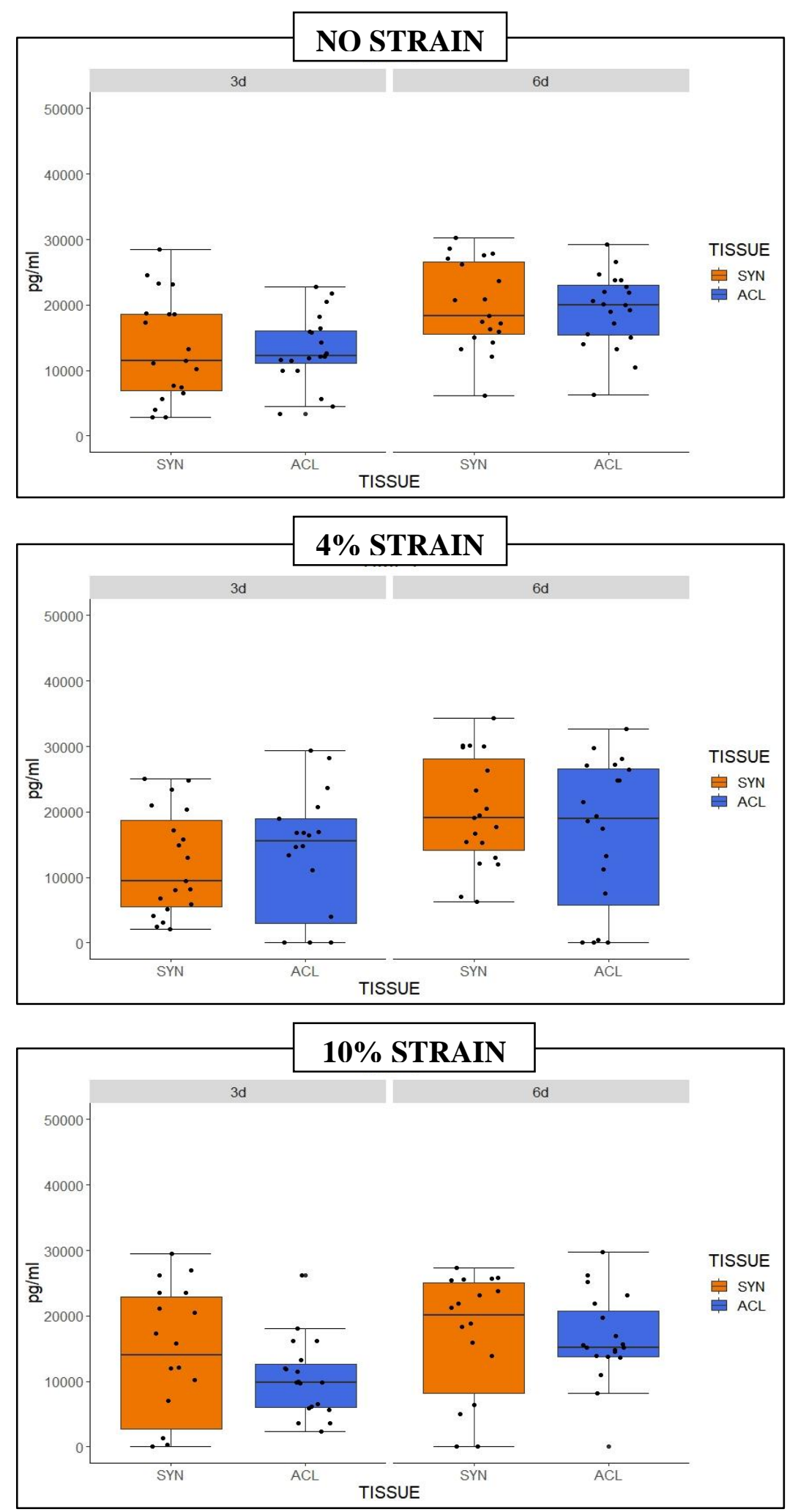

Figure 7.22. Concentration of TIMP-1 released to the media. * significant difference between TISSUES at 24 and 120 hours (Mann-Whitney test). SYN: Synovium; ACL: Anterior Cruciate Ligament. 

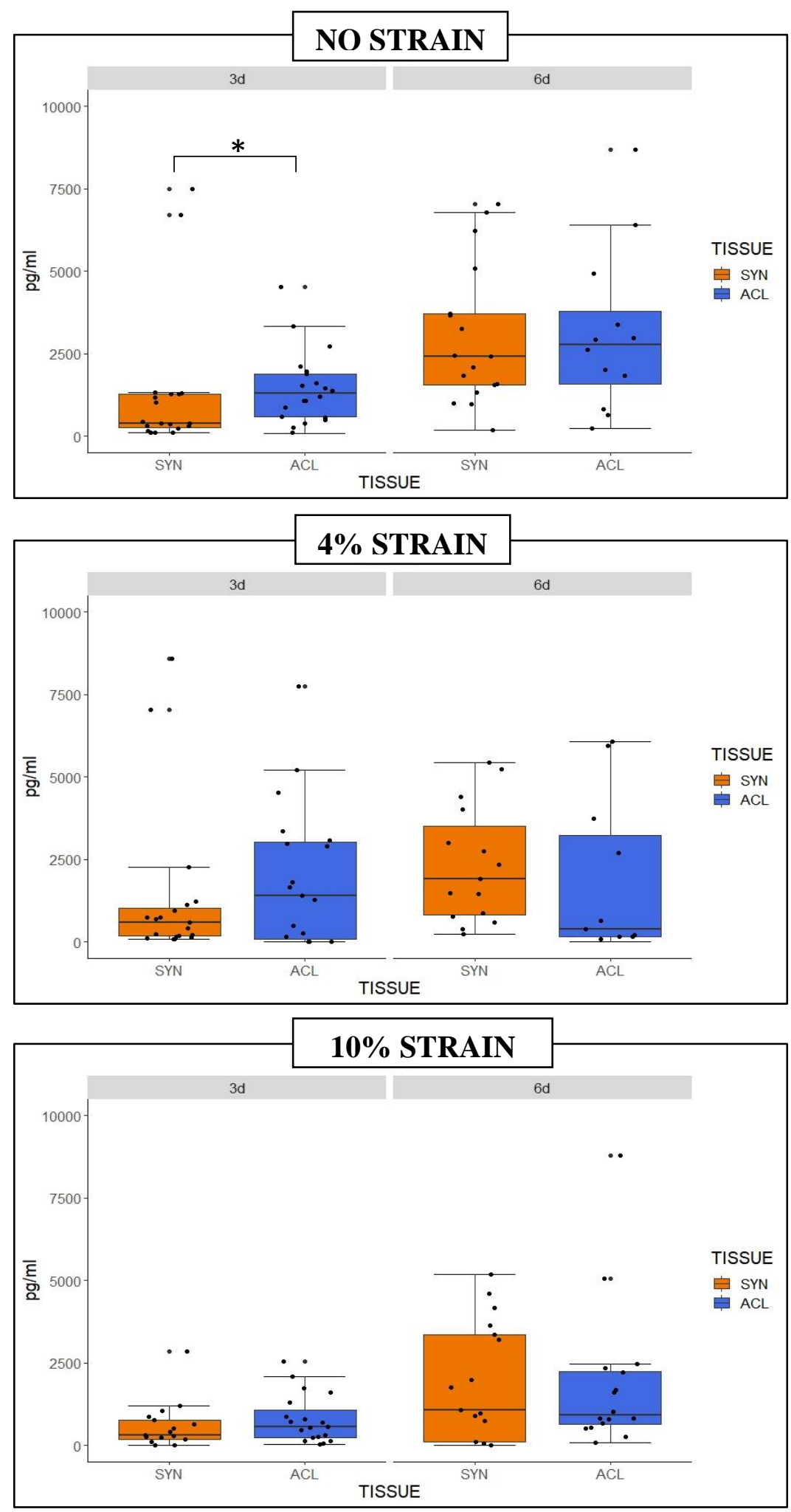

Figure 7.23. Concentration of TIMP-2 released to the media. * significant difference between TISSUES at 24 and 120 hours (Mann-Whitney test). SYN: Synovium; ACL: Anterior Cruciate Ligament. 

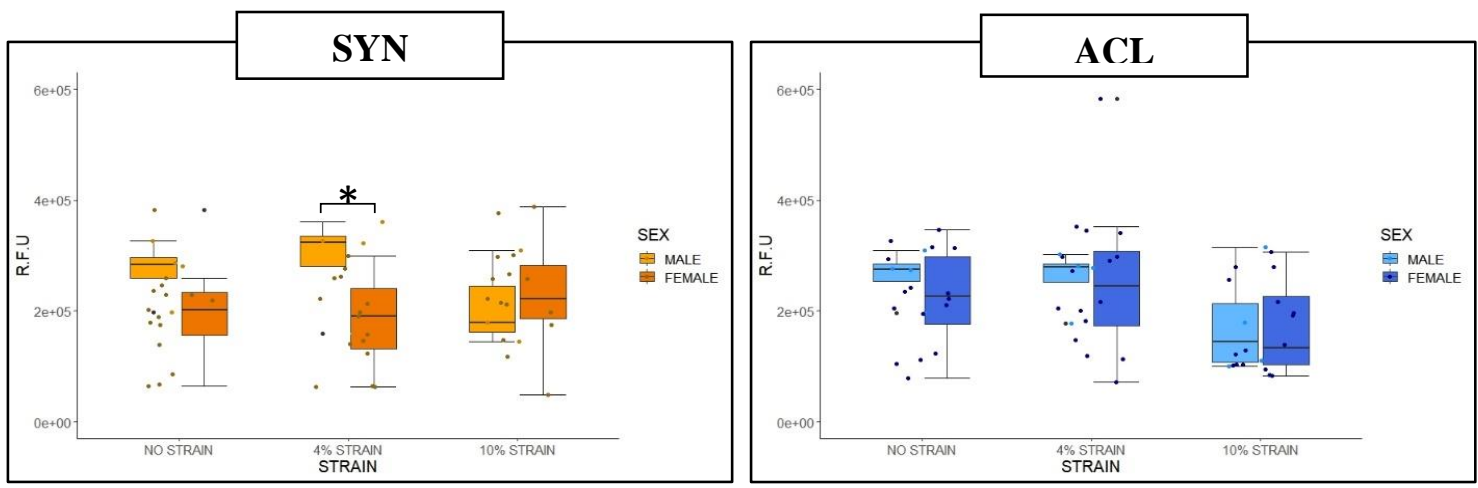

Figure 7.24. Metabolic activity of fibroblasts after $120 \mathrm{~h}$ in culture. * significant difference between males and females (Mann-Whitney test). SYN: Synovium; ACL: Anterior Cruciate Ligament; R.F.U: Resazurin Fluorescent Units.
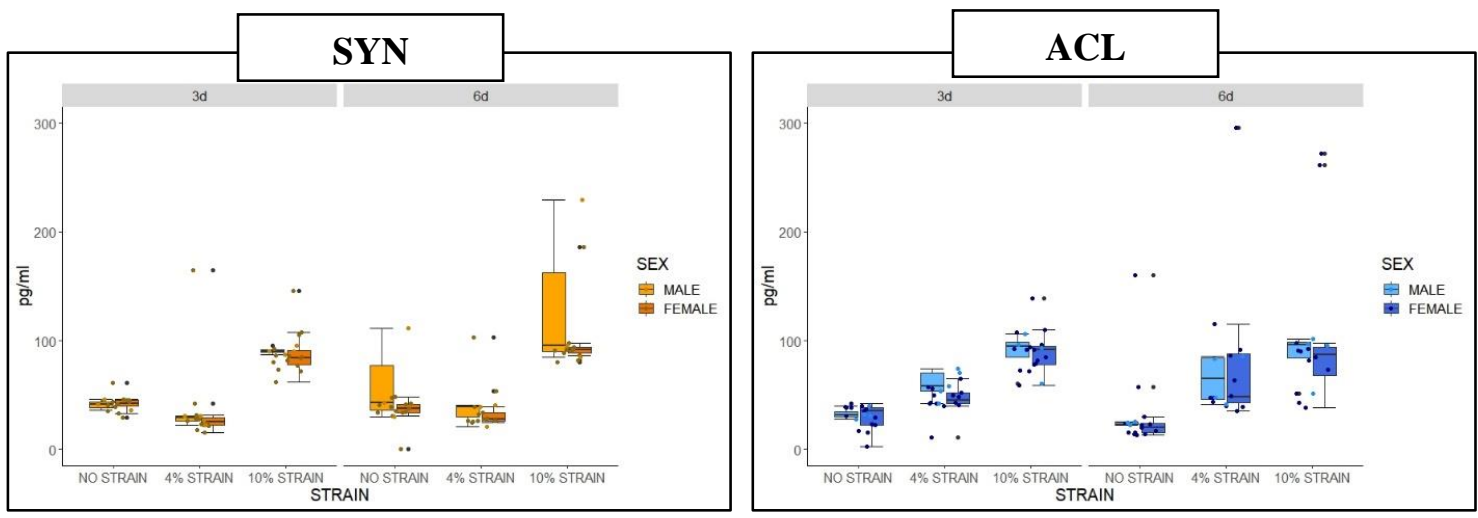

Figure 7.25. Concentration of PGE2 released to the media. * significant difference between males and females at 24 and 120 hours (Mann-Whitney test). SYN: Synovium; ACL: Anterior Cruciate Ligament
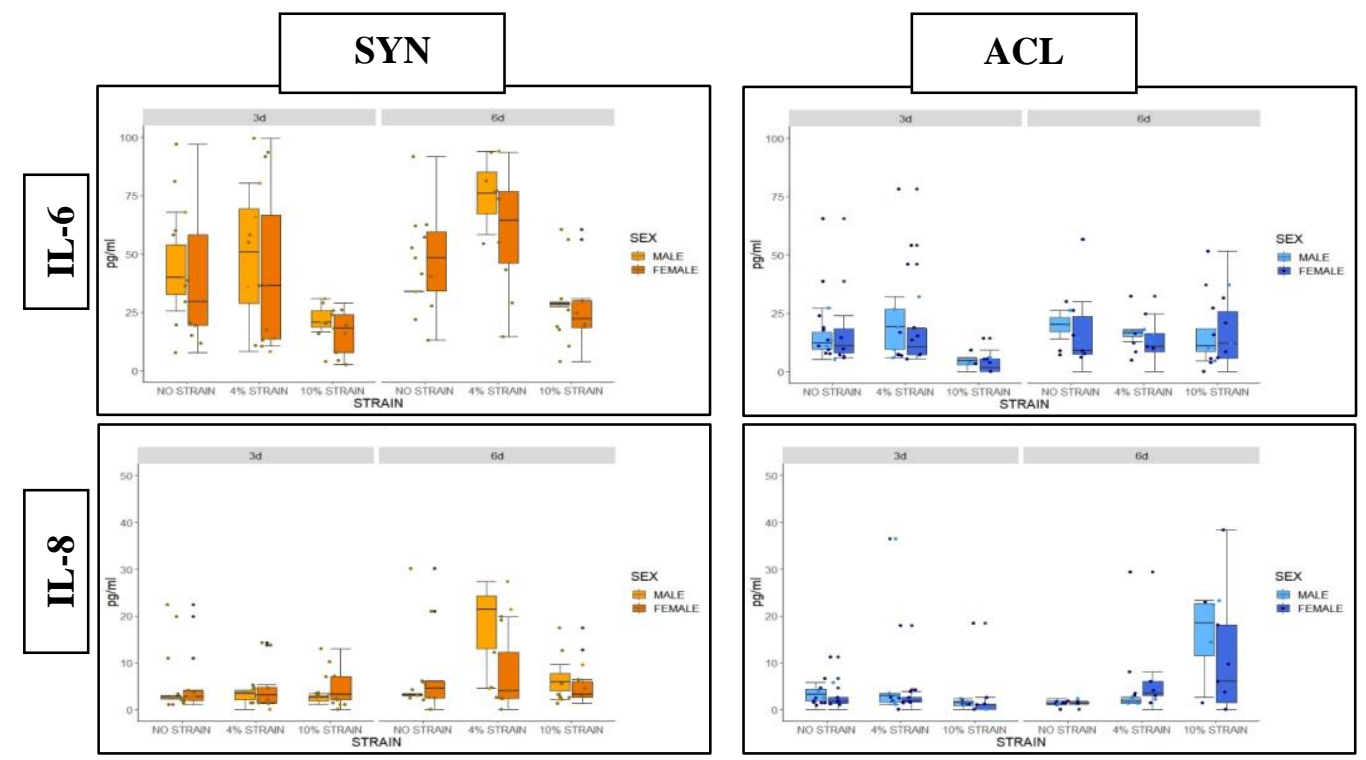

Figure 7.26. Concentration of Interleukins 6 (IL-6) and (IL-8) released to the media. * significant difference between males and females at 24 and 120 hours (Mann-Whitney test). SYN: Synovium; ACL: Anterior Cruciate Ligament 

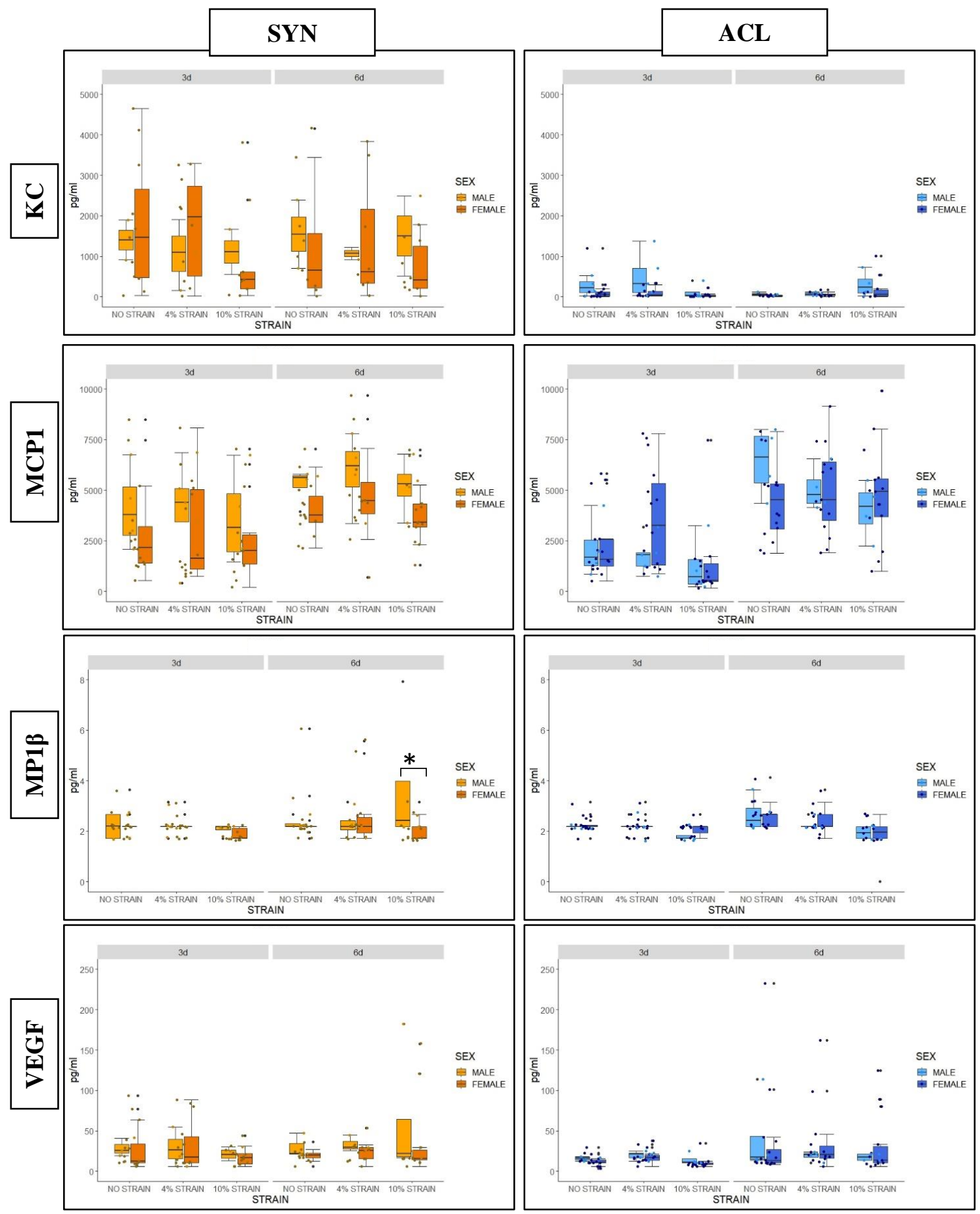

Figure 7.27. Concentration of chemokines (KC, MCP1, MP1B) and Vascular Endothelial Growth Factor (VEGF) released to the media. * significant difference between males and females at 24 and 120 hours (Mann-Whitney test). SYN: Synovium; ACL: Anterior Cruciate Ligament 

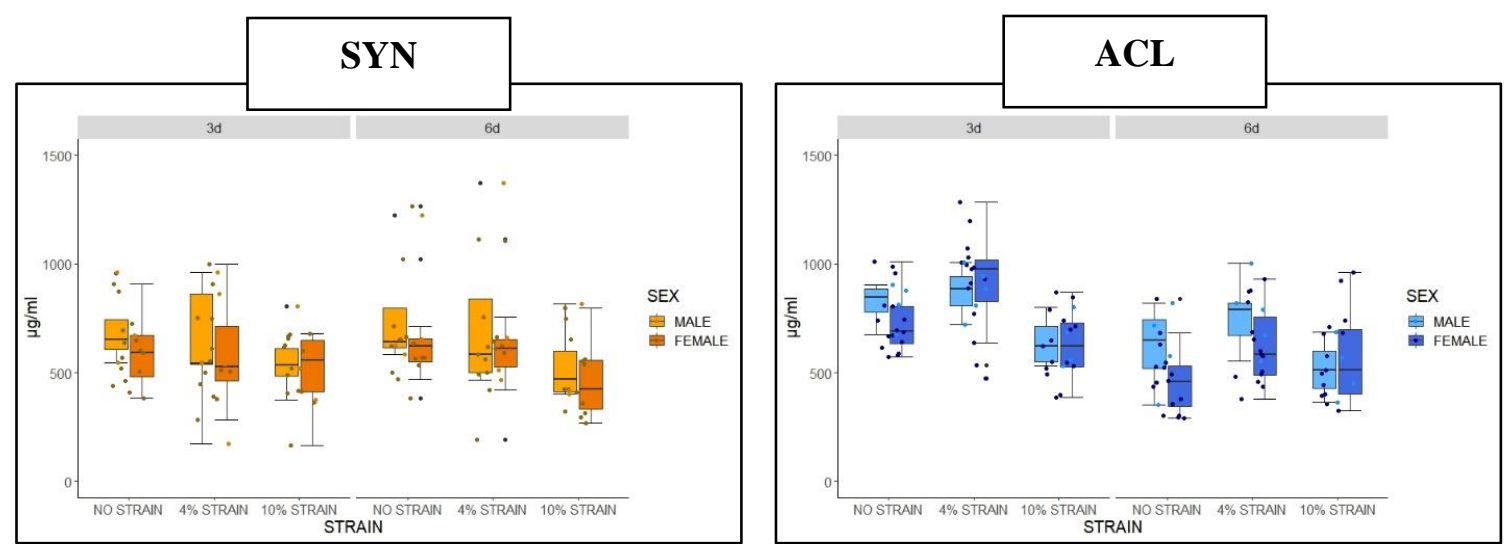

Figure 7.28. Concentration of GAG content released to the media. * significant difference between males and females at 24 and 120 hours (Mann-Whitney test). SYN: Synovium; ACL: Anterior Cruciate Ligament
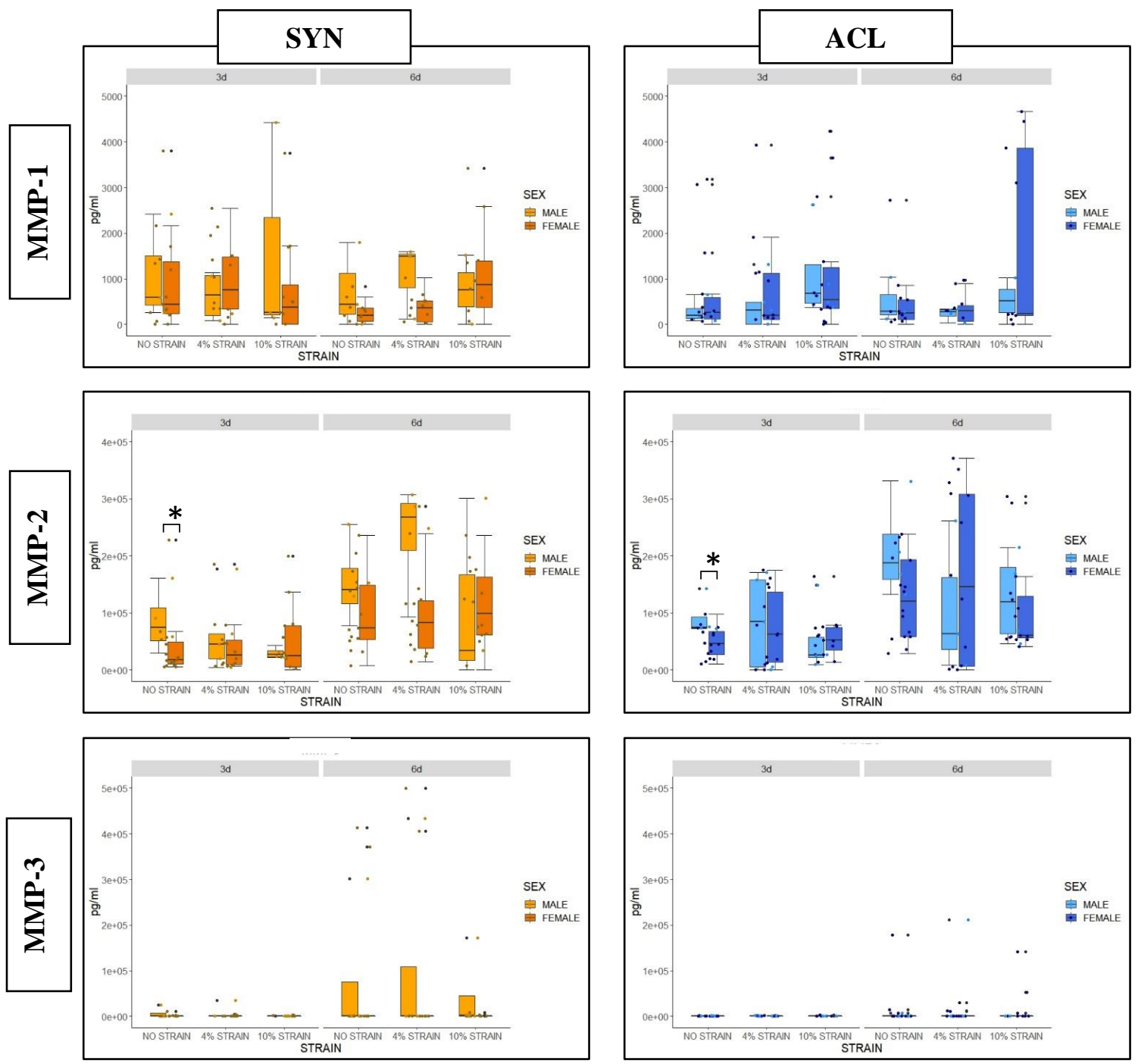

Figure 7.29. Concentration of Matrix Metalloproteinases (MMP-1, 2 and 3) released to the media. * significant difference between males and females at 24 and 120 hours (Mann-Whitney test). SYN: Synovium; ACL: Anterior Cruciate Ligament 

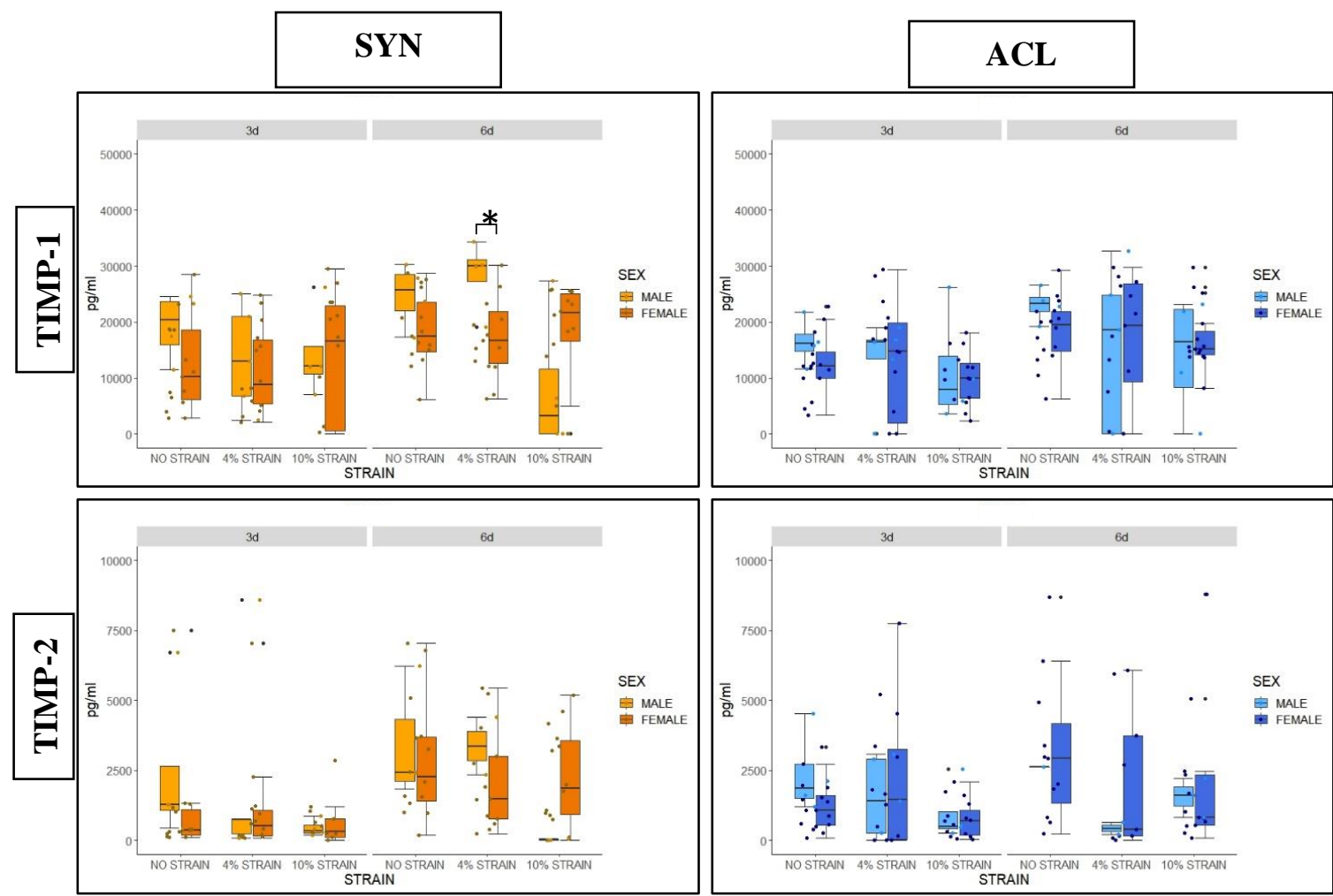

Figure 7.30. Concentration of Tissue Inhibitor of Matrix Metalloproteinases (TIMP-1 and 2) released to the media. * significant difference between males and females at 24 and 120 hours (Mann-Whitney test). SYN: Synovium; ACL: Anterior Cruciate Ligament 

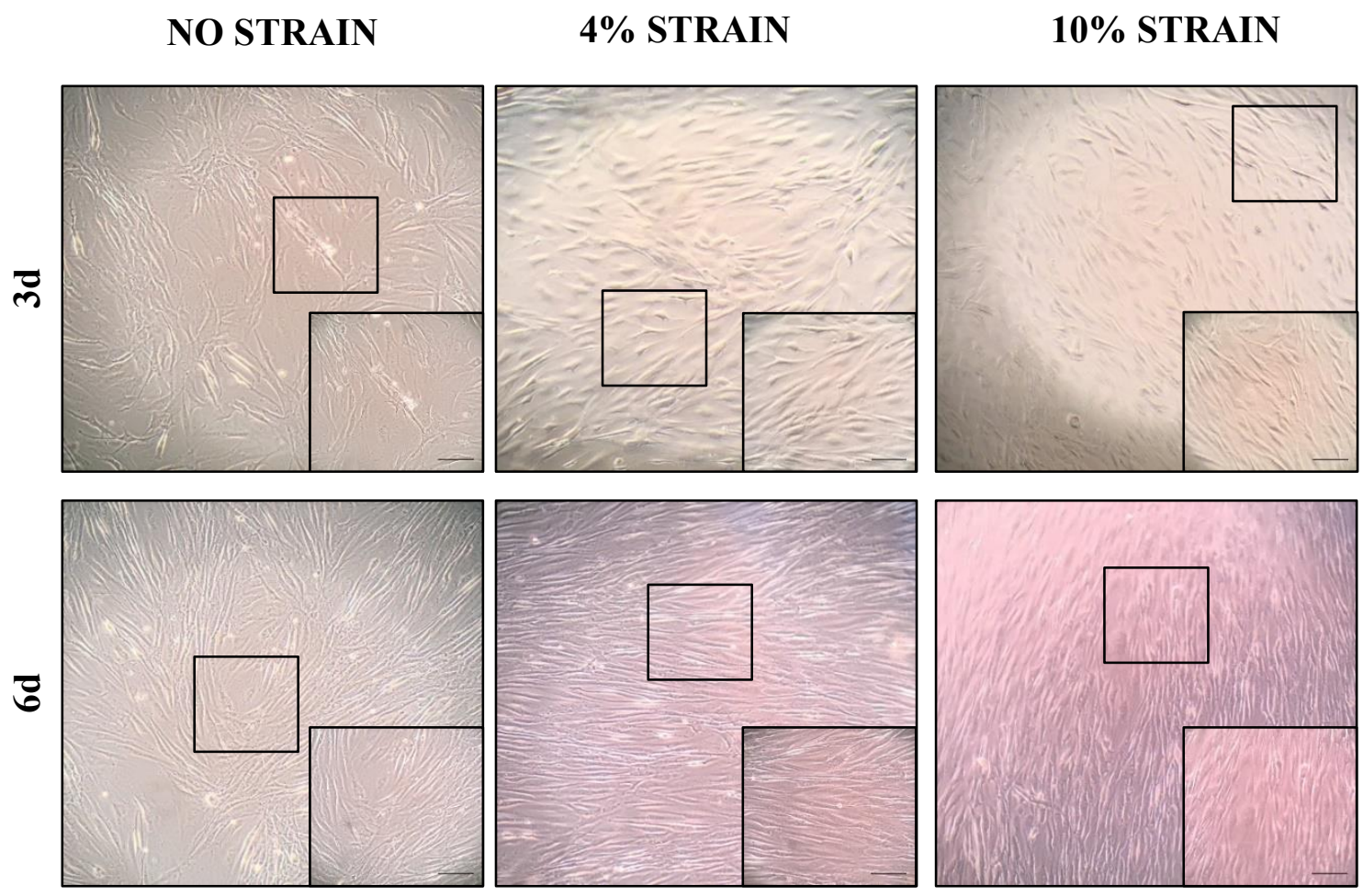

Figure 7.31. Synovium (SYN) fibroblasts stretched after three days and six days at three different stain

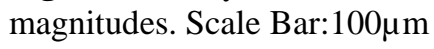

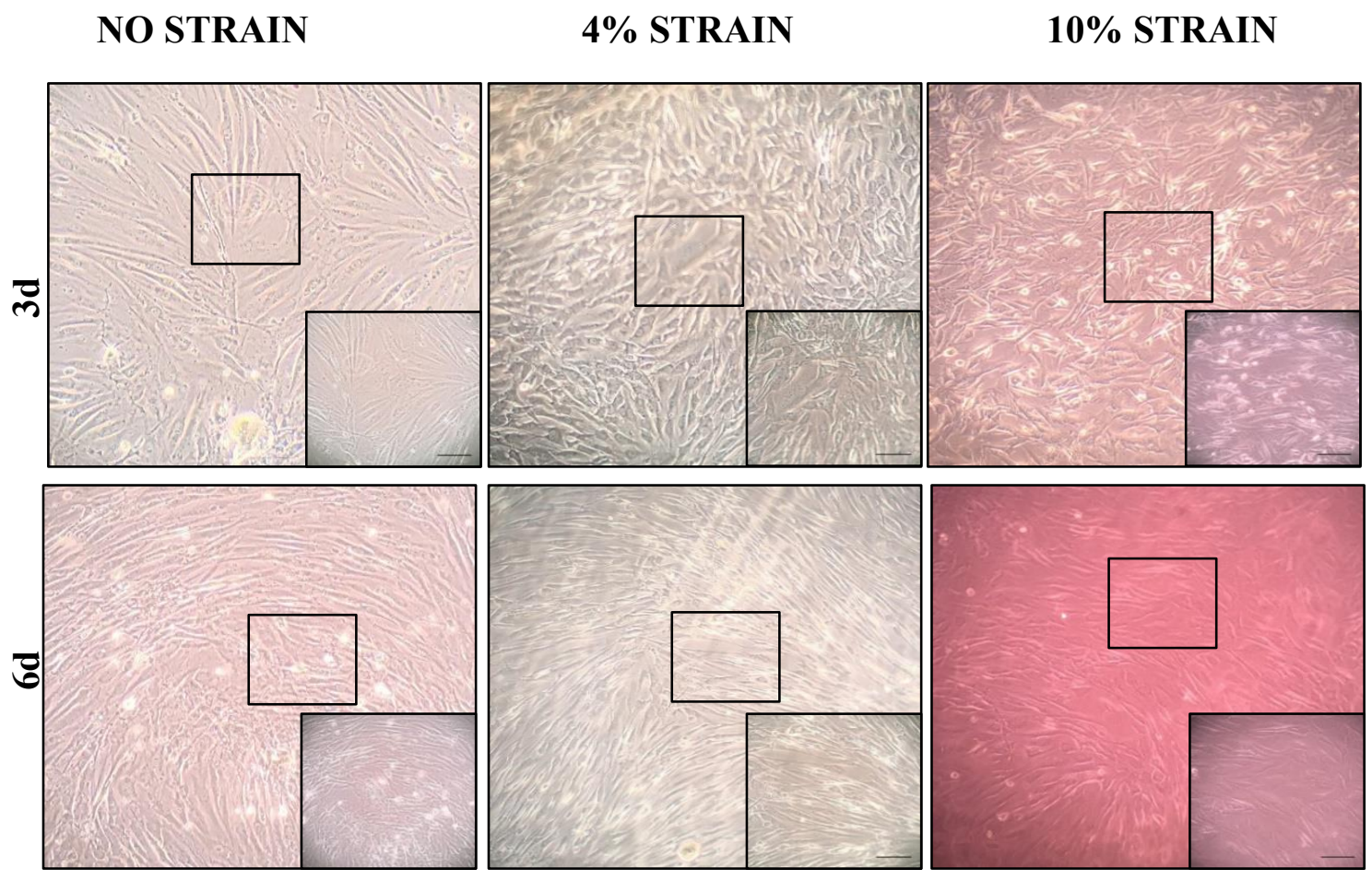

Figure 7.32. Anterior Cruciate Ligament (ACL) fibroblasts stretched after three days and six days at three

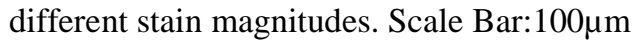




\section{CHAPTER 8}

\section{NORMAL HUMAN TENDON DERIVED FIBROBLASTS EXPOSED TO MECHANICAL STRAIN}

\section{Introduction}

Anterior cruciate ligament (ACL) reconstruction with tendon autografts is the "gold standard" for surgical treatment of ACL injuries. ${ }^{1}$ Common tendon graft choices include patellar tendon (PT), semitendinosus/gracilis tendon (HT), or quadriceps tendon (QT). In recent studies, the choice of which tendon graft used for ACL reconstruction has been associated with different outcomes including variable risk of developing recurrent knee instability or re-injury, osteoarthritis (OA), and harvest site morbidity such as kneeling pain. ${ }^{23 ;}$ As an example, ACL reconstruction with PT have been associated with greater risk of develop post-surgical OA. This may be related to altered patellofemoral kinematics following harvest of the PT tissue. Hamstring tendon graft harvest, in comparison, have associated persistent knee flexion weakness. There are also concerns that hamstring autograft ACL reconstructions may have greater risk of laxity or rerupture after surgery versus PT autograft in certain patient populations. ${ }^{5}$ Furthermore, while QT autografts appear to have better structural qualities, it has not been widely used and more studies are needed. ${ }^{6}$ Consequently, there is a lack of consensus regarding the most adequate biomechanical environment for any specific graft choice.

The healing of the ACL graft after reconstruction is unique since the tissue is subject to mechanical stresses during post-operative rehabilitation that play an important role in proper graft maturation after reconstruction. Dynamic cell culture in vitro models have 
been developed to investigate the effects of mechanical forces on relevant cell populations, which may have particular relevance in the study of ACL graft tissues and tissue specific responses to the mechanical environment. ${ }^{7}$ Specifically, cyclic stretching of cells can affect its extracellular matrix (ECM) synthesis and remodeling ${ }^{8}$, potentially modifying tissue structure, composition, and function. ${ }^{9}$ Abnormal mechanical loading can result in injury, altering tissue homeostasis, and leading to pathological changes and potential tissue ECM failure. It is not known if there is an equivalence in biologic response of common tendon grafts if its cells were exposed to the same level of mechanical stress.

This study was designed to determine if there are differences in metabolic and inflammatory responses to mechanical stress between common tendon fibroblasts used for ACL reconstruction. Additionally, we aimed to evaluate the differences between male and female tendon fibroblasts under these tension loads. We hypothesized that strain and tendon source of ACL-graft fibroblast would significantly influence cellular metabolic responses to mechanical stress. Moreover, we hypothesized that female tendon fibroblasts will exhibit increased inflammatory and altered metabolism in response to load. Furthermore, extracellular matrix (ECM) remodeling driven by cellular responses to load can influence disease, as well as response to treatment. The rationale for this proposal is based on previous reports and preliminary observations on canine fibroblasts, showing that an optimal level of mechanical load facilitates healing while decreasing scar tissue formation. We believe that cellular response correlates with healing status and can determine the amount of ECM produced.

\section{Materials and Methods:}




\section{Tissue processing and culture}

With IRB approval (IRB\#2009879), tendon grafts (9 QT, 7 PT and 6 HT) were harvested from patients (11 males and 11 females, age range 14-43) at the time of anterior cruciate ligament reconstruction (Table 8.1, Fig.8.1). This tissue is typically removed and discarded following the surgical procedure. Tissues were aseptically minced into $0.5-1.0$ $\mathrm{cm}^{2}$ pieces and digested for two hours in Type 1A Clostridium histolyticum collagenase solution (Sigma-Aldrich, St. Louis, MO, USA) at a concentration of $0.2 \mathrm{mg} / \mathrm{mL}$. The aliquot was then suspended with nutritional media (Gibco DMEM, Thermo Fisher Scientific, Waltham, MA, USA) containing 10\% FBS, 0.002\% Penicillin, $100 \mu \mathrm{g} / \mathrm{mL}$ Streptomycin, $25 \mu \mathrm{g} / \mathrm{mL}$ Amphotericin B, 0.002\% L-Ascorbate and 0.01\% L-glutamine (Sigma Chemical Co, St. Louis, MO), cultured in T25 flasks (passage zero) and T75 flasks (passage one) (TPP Techno Plastic Products AG, Trasadingen, Switzerland) and incubated at $5 \% \mathrm{CO}_{2}, 37^{\circ} \mathrm{C}$, and $95 \%$ humidity. Once confluent, passage one cells were stored at $-80^{\circ} \mathrm{C}$ until analysis.

\section{Biaxial mechanical stress}

Passage one cells were re-expanded in T75 flasks in media described above and when confluent, exposed to TrypLE express (Invitrogen, Carlsbad, CA) for five minutes, then seeded $\left(1 \times 10^{5}\right.$ cells/well) on Collagen Type I-coated BioFlex ${ }^{\circledR}$ plates (Flexcell International, NC, USA). Cells were incubated for an additional 48h in 10\% FBS nutritional media described before strain was applied. Before fibroblasts were subjected to continuous mechanical stimulation media was changed to $2 \%$ FBS to keep cells alive for six days (120h). ${ }^{10} \mathrm{~A}$ tension load (2-s strain and 10 -s relaxation at a $0.5 \mathrm{~Hz}$ frequency) with a biaxial sinusoidal waveform with three different elongation strains was applied for 
6 days using the Flexcell FX-4000T strain system (Flexcell International, NC, USA). The strain magnitude were chosen to approximate 3 different strain levels that may be experienced by the graft tissue during the post-operative healing process after ACL reconstruction, immobilization or mechanical stress deprivation-0\%, physiologic strain$4 \%$, and supraphysiologic strain- $10 \%{ }^{7}$ Media was changed at day three and day six and stored at $-20^{\circ} \mathrm{C}$ until analysis.

\section{Biomarker assays}

Media was changed every three days and stored at $-20^{\circ} \mathrm{C}$ until analysis. Media from three (3d) and six (6d) days of culture were assessed for various biomarkers. Proteoglycan (GAG) was assessed using the DMMB assay as previously described. ${ }^{11}$ The concentration of prostaglandin $\mathrm{E}_{2}$ (PGE2) (Cayman Chemical, Ann Arbor, MI, USA), cytokines (IL-6, IL-8, KC, MCP-1 and VEGF) (Millipore, Billerica, MA, USA), total matrix metalloproteinase (MMP) activity (SensoLyte 520 generic MMP assay, Anaspec, Inc., Fremont, CA), and MMPs and TIMPs production (MMP-1, MMP-2, MMP-3, TIMP 1 and TIMP 2) (R\&D Systems, Minneapolis, MN, USA), were all assessed using commercially available assays according to the manufacturer's protocol. At the end of the strain protocol (6d), cell viability analysis was performed using resazurin assay (Sigma Aldrich, Saint Louis, MO).

\section{Statistical Analysis}

For statistical analysis, normal distribution was assessed by Shapiro-Wilk test. Because data were not normally distributed, non-parametric analyses were used. Comparisons among strains and tissue types were performed using Kruskal-Wallis test and Dunn's test posthoc. Additionally, comparisons between males and females were performed using 
Mann-Whitney U test (R Core Team (2019), R version 3.6.2 Vienna, Austria). Results were reported as median \pm interquartile range (IQR). Significance levels will be set at $\mathrm{p}<0.05$.

\section{Results}

\section{Differences in Graft Tendon Fibroblasts to Mechanical Strain}

The metabolic activity was significantly higher in HT fibroblasts exposed to physiological strain compared to supraphysiological strain at day 6 of culture. There were no significant differences in metabolism of QT or PT fibroblasts in response to strain level applied at any timepoint (Fig.8.2).

Differences in inflammatory mediators released to the media were also seen as a function of strain level and tissue source. The production of PGE2 by QT and PT fibroblasts was significantly higher during mechanical strain deprivation compared to physiological strain at day 3 of culture. In contrast, the production of PGE2 by HT fibroblasts was significantly higher at supraphysiological strain levels relative to physiological strain levels at day 6 of culture (Fig. 8.3).

The production of IL-6 (Fig. 8.4) and IL-8 (Fig. 8.5) by PT fibroblasts was significantly lower when subjected to supraphysiological strain levels compared to physiological strain and mechanical strain deprivation at day 3 of culture. Significant differences in IL-6 and IL-8 production were not observed for HT or QT fibroblasts based on strain level at either time point.

Cellular chemoattractant levels also fluctuated depending on tissue type and strain levels. The production of KC by QT fibroblasts was significantly increased when subjected to physiological strains compared to supraphysiological strain at day 3 of culture (Fig. 8.6). 
The production of MCP-1 did not show differences among different strain magnitudes in any of the tissue fibroblasts at any timepoint (Fig. 8.7). The production of MIP-1 $\beta$ was significantly higher in HT fibroblasts subjected to mechanical strain deprivation compared to supraphysiological strain at day 3 of culture (Fig. 8.8). Moreover, VEGF was not statistically different in any tendon fibroblasts at any strain at either $3 \mathrm{~d}$ or $6 \mathrm{~d}$ (Fig. 8.9).

The production of GAG (Fig.8.10) and MMP-1 (Fig. 8.11) by the tendon fibroblasts were not significantly different in response to strain in any of the tendon fibroblasts at any timepoint. However, the production of MMP-2 by HT fibroblasts was significantly lower at supraphysiological strain compared to both, mechanical strain deprivation and physiological strain levels (Fig. 8. 12). The production of MMP-3 by PT fibroblasts was significantly higher during mechanical strain deprivation compared to supraphysiological strain at day 3, but not at day 6 of culture (Fig. 8.13). Moreover, the production of TIMP1 by PT fibroblasts was significantly lower at supraphysiological strain levels compared to mechanical strain deprivation and physiological strain at day 3 of culture (Fig.8.14). Further, the production of TIMP-2 by PT fibroblasts was significantly lower at supraphysiological strain levels compared to mechanical strain deprivation and physiological strain levels at day 3 and day 6 of culture, respectively (Fig. 8.15).

\section{Differences between QT, PT and HT Fibroblast Response to Mechanical Strain}

The level of metabolic activity for HT fibroblasts was significantly higher than QT fibroblasts at mechanical stress deprivation and physiological strain levels, but not at supraphysiological strain. Additionally, the level of metabolic activity for HT fibroblasts 
was significantly higher than PT fibroblasts in the mechanical strain deprivation group (Fig. 8.16).

The production of PGE2 by HT fibroblasts was significantly lower than PT and QT fibroblasts at all strain levels and time points tested (Fig. 8.17). Moreover, the production of IL-6 by HT fibroblasts was significantly higher than PT fibroblasts at supraphysiological strain on day 3 of culture (Fig. 8.18). Additionally, the production of IL-8 by PT fibroblasts was significantly lower than QT and HT fibroblasts at supraphysiological strain levels on day 3 of culture (Fig.8.19). The production of KC by HT fibroblasts was significantly lower than PT and QT fibroblasts at day 6 of culture at all strain levels. Further, the production of $\mathrm{KC}$ by HT fibroblasts was significantly lower than QT fibroblasts at day 3 of culture at physiological and supraphysiological strain levels (Fig.8.20). The production of MIP-1 $\beta$ by HT fibroblasts was significantly lower than PT and QT fibroblasts at all strain levels and timepoints at days 3 and 6 of culture (Fig.8.21). The production of MCP-1 and VEGF were not significantly different between tissue types at all strain levels and days of culture analyzed (Fig.8.22 and 8.23 respectively).

The production of GAG by HT fibroblasts was significantly higher than PT and QT fibroblasts in the mechanical strain deprivation group on day 6 of culture (Fig.8.24). Moreover, the production of MMP-1 by QT fibroblasts was significantly higher than PT and HT fibroblasts at mechanical strain deprivation and physiological strain levels on day 3 of culture (Fig.8.25). The production of MMP-2 was not significantly different between tissue types at all strain levels and days of culture analyzed (Fig.8.26). Additionally, the production of MMP-3 by PT fibroblasts was significantly lower than QT fibroblasts at 
physiological and supraphysiological strain levels on day 3 of culture (Fig.8.27). The production of TIMP-1 by PT fibroblasts was significantly higher than fibroblasts in the mechanical strain deprivation group on day 3 of culture. However, the production of TIMP-1 was not significantly different between tissue types at physiological and supraphysiological strain levels for any of the days of culture analyzed (Fig. 8.28). Additionally, the production of TIMP-2 was not significantly different between tissue types at all strain levels and days of culture analyzed (Fig.8.29).

When evaluating sex differences, we found that the metabolic activity of female QT fibroblasts was significantly higher than male QT fibroblasts at supraphysiological strain (Fig. 8.30). However, there were no other statistically significant differences between male and female fibroblasts at any strain level or timepoint tested in this study (Fig.8.318.43). There were some trends toward a difference between male and female PT fibroblasts for IL-6, MMP-1 and MMP-2 production, but these differences were not statistically different (Fig. 8.32, 8.39 and 8.40 respectively).

\section{Discussion}

It is known that mechanical stimulation plays a key role in normal or altered tendon and ligament development and maturation. ${ }^{12}$ In the present study, we were able to demonstrate that tendon fibroblasts from grafting tissue respond differentially to varying levels of biaxial cyclic strain. Moreover, these differences were unique dependent on fibroblast tissue source. PT fibroblasts were more sensitive to changes in strain levels as demonstrated by more frequent differences within fibroblast from this tendon, especially in response to extremes loads such as mechanical stress deprivation and high strain levels. 
Successful healing of the tendon graft after ACL reconstruction involves the biological processes that occur during graft necrosis, remodeling, maturation, and incorporation. Fibroblasts are dispersed within the tendon extracellular matrix and are the main resident cells of common tendons including patellar, quadriceps, and hamstring tendons. ${ }^{13 ; 14}$ While these cells are integral to production of extracellular matrix, particularly during the response to injury and repair, their role in the ligamentization process after reconstruction is largely not known. There is limited information on metabolism and biosynthesis differences between fibroblasts of various tendon tissues when subjected to mechanical strain.

For this study in vitro strains related to clinical scenarios commonly found in ACL reconstruction and tendon pathology were analyzed. It is known that normal tendon and ligament strain are in the order of 2-4\%. ${ }^{15 ; 16}$ Moreover, strains higher than $8 \%$ are associated with microscopic fiber damage and altered material properties. ${ }^{7 ; 15 ; 16}$ Since the amount of strain applied to the graft after ACL reconstruction in vivo is still not fully understood, current approach is valid to evaluate main responses to strain.

In our study, we demonstrated that fibroblasts from different tendons, not only differed in their production of extracellular matrix, remodeling and inflammatory mediators, but this difference was dependent on level of strain experienced by the cell. Since the cellular density is also vastly different among grafts ${ }^{17}$, a relation between graft fibroblasts and the success of biological graft healing is likely present as these differences may be amplified with differences in density of cells within a given tissue.

Since PGE2 significantly changed within different tendon fibroblasts in response to changing strain levels in our experiment, it appears that PGE2 may be an important 
mechanosensitive marker. The concentration of PGE2 was consistently increased at strain levels outside of physiologic levels. It is possible that extreme strain levels stimulate an inflammatory cascade and that PGE2 overproduction is an early event for tissue repair. ${ }^{18}$ Likewise, the significant increase of interleukins in PT fibroblasts at subnormal strain levels, may indicate that this type of cell requires a minimum of cytokine production, given by physiological strain, and that abnormal strains may facilitate inflammatory signaling in this type of graft.

Decreased MMP-2 concentration correlates with improved mechanical properties of the reconstructed graft. ${ }^{19}$ In this work, MMP-2 production was significantly lower in HT fibroblasts after 3 days of supraphysiological strain compared to mechanical strain deprivation and physiological strain. Similarly, MMP-3 production was significantly lower in PT fibroblasts after 3 days of supraphysiological strain compared to mechanical strain deprivation. Clinically, knee immobilization when using soft tissue grafts for ACL reconstruction intended to restrict motion will supposedly protect the recently operated knee from high strains.$^{20}$ However, our results indicate that mechanical strain deprivation may increase MMP production when using PT and HT grafts with potentially increased risk of graft weakness and failure.

The application of tension load to the reconstructed graft seem to increase tensile strength and to avoid adhesions formation. ${ }^{15}$ However, supraphysiological strain is believed to be detrimental for tendon-to-bone healing ${ }^{12}$. Interestingly, our results showed a consistent decrease in cytokine (IL-6, IL-8) and TIMPs production (TIMP-1 and TIMP-2) in PT fibroblasts at day 3 of culture with supraphysiological strain. These findings might suggest that PT graft could indeed benefit from early mobilization at high strains as 
reported in vivo and that that motion applied to the reconstructed knee may be more beneficial than immobilization. ${ }^{20-22}$

Differences between male and female fibroblasts were also evaluated in this work. Although there were not many sex differences exhibited by tendon fibroblasts in response to strain, there was an increased metabolic activity of female QT fibroblasts compared to males. Since this measure is directly related to the number of cells in culture, it is possible that female QT grafts tend to increase cellularity when high loads are applied. Moreover, considering that cell necrosis of the graft is a main event in early postoperative period, we believe that this may be neutralized by adjusting the proper postoperative physical therapy to each specific graft. ${ }^{19}$

Curiously, the lack of differential responses between male and female fibroblasts to any type of strain may be an example of the simplicity of our in vitro model. Since many of the sex differences in ACL injury are due to neuromuscular control and specific anatomical features, it is not surprising that significant differences between fibroblasts based on sex were not observed during in vitro monolayer culture without these other factors included. ${ }^{23} 24$ Additionally, the low sample size and high variability in this study may be responsible for the trends observed in different biomarkers such as IL-6, MMP-1 and MMP-2, especially in QT and PT fibroblasts. Hence, including a larger sample size may allow for the identification of significant differences in the metabolism of male and female fibroblast metabolism with and without applied strain.

Taken together, results of present study deepen the understanding of the effect of different clinical scenarios where strain applied to the reconstructed ACL graft drives essential biochemical changes. Moreover, this work highlights specific needs in the graft 
healing and ligamentization process such as standardization of physical therapy for each specific graft type. Since many of our results are consistent with literature reports such as increased failure in HT grafts and increased OA in PT grafts, we could potentially use this work to understand biological processes that have an evident gap in knowledge. Importantly, the lack of consensus about the perioperative activity recommended for the patient that undergoes ACL reconstruction should draw the attention of physicianscientists to explore the mechanobiology of tendon grafts to diminish ACL reconstruction failures and revisions.

Since, fibroblasts within the tendon graft respond to mechanical stress by altering the ECM that surrounds them, we believe that modifying the mechanical environment by applying an adequate biaxial stress to the cellular component of the graft, will result in improved biological and structural properties of the tissues after ACL reconstruction. Once again, PT showed decreased production of TIMP-1 and TIMP-2 at high strains. Moreover, an increased production of TIMP-2 at physiological levels of load in PT after 6 days of stretch suggest that controlled motion to the graft is required to regulate excessive remodeling within the knee after ACL reconstruction. ${ }^{25}$ As with any study there are inherent limitations to consider when interpreting the data. This was an in vitro study utilizing monolayer cell culture without the other biological and mechanical factors that are present in vivo. Additionally, since the in vitro system utilized in this work only allows for equi-biaxial strain, in vivo replication of heterobiaxial strain (more tension to the longitudinal axis than the transverse axis) cannot be evaluated. Furthermore, 3D cultures that allow for a longer time period of evaluation will be interesting to see if biomarker production is maintained across time. Finally, the 
samples size for this study was low, and could be responsible of the lack of significant differences seen when analyzing for sex differences. It could be interesting to see if sex plays a role in cellular metabolic responses to strain by increasing sample size.

In conclusion, this study demonstrated that human tendon fibroblasts that are used for ACL reconstruction respond differently to changing mechanical environment. These findings may have applicability given outcomes of ACL reconstruction in certain patient populations seems to be impacted by graft source and selection. This study suggests that early biological differences in fibroblast cellular protein expression between graft source may be one explanation for fundamental differences in clinical outcomes as it relates to graft selection in ACL reconstruction. Therefore, the clinician may need to consider graft type and sex to adjust physical therapy to reduce risk of failure for athletes. In this work we have provided an initial approach to evaluate specific cellular responses within a specific clinical context. Future work should include the evaluation of graft fibroblasts along with other cell types (co-culture) including synovium and cruciate ligamentocytes to replicate the in vivo multi-tissue environment. Ongoing studies in our laboratory are aimed at evaluating the metabolic response of graft fibroblasts co-cultured with various cell types and assess whole-tissue culture systems to better replicate the in vivo environment that the tenocyte experiences. 


\section{REFERENCES}

1. Mahapatra P, Horriat S, Anand BS. 2018. Anterior cruciate ligament repair - past, present and future. Journal of experimental orthopaedics 5.

2. Thompson SM, Salmon LJ, Waller A, et al. 2016. Twenty-Year Outcome of a Longitudinal Prospective Evaluation of Isolated Endoscopic Anterior Cruciate Ligament Reconstruction With Patellar Tendon or Hamstring Autograft. The American journal of sports medicine 44:3083-3094.

3. Fu F. 2011. Anterior cruciate ligament reconstruction with semitendinosus graft provided similar stability and knee function and fewer problems with kneeling compared with the bone-patellar tendon-bone graft. The Journal of bone and joint surgery American volume 93:969.

4. Webster KE, Wittwer JE, O'Brien J, et al. 2005. Gait patterns after anterior cruciate ligament reconstruction are related to graft type. The American journal of sports medicine 33:247-254.

5. Laboute E, James-Belin E, Puig PL, et al. 2018. Graft failure is more frequent after hamstring than patellar tendon autograft. Knee surgery, sports traumatology, arthroscopy : official journal of the ESSKA 26:3537-3546.

6. Mouarbes D, Menetrey J, Marot V, et al. 2019. Anterior Cruciate Ligament Reconstruction: A Systematic Review and Meta-analysis of Outcomes for Quadriceps Tendon Autograft Versus Bone-Patellar Tendon-Bone and Hamstring-Tendon Autografts. The American journal of sports medicine 47:35313540. 
7. Wang T, Chen P, Zheng M, et al. 2018. In vitro loading models for tendon mechanobiology. Journal of orthopaedic research : official publication of the Orthopaedic Research Society 36:566-575.

8. Sarasa-Renedo A, Chiquet M. 2005. Mechanical signals regulating extracellular matrix gene expression in fibroblasts. Scandinavian Journal of Medicine and Science in Sports 15:223-230.

9. Wang JHC, Thampatty BP. 2006. An Introductory Review of Cell Mechanobiology. Biomechanics and Modeling in Mechanobiology 5:1-16.

10. Xie J, Wang CL, Yang W, et al. 2018. Modulation of MMP-2 and MMP-9 through connected pathways and growth factors is critical for extracellular matrix balance of intra-articular ligaments. J Tissue Eng Regen Med 12:e550-e565.

11. Farndale RW, Buttle DJ, Barrett AJ. 1986. Improved quantitation and discrimination of sulphated glycosaminoglycans by use of dimethylmethylene blue. Biochim Biophys Acta 883:173-177.

12. Killian ML, Cavinatto L, Galatz LM, et al. 2012. The role of mechanobiology in tendon healing. J Shoulder Elbow Surg 21:228-237.

13. Bliss S, Rawlinson JJ, Todhunter R. 2013. Tissues of the musculoskeletal system. In: Tobias KM, Johnston SA editors. Veterinary Surgery: Small Animal-EBOOK: 2-Volume Set: Elsevier Health Sciences; pp. 553-564.

14. KJÆR M. 2004. Role of Extracellular Matrix in Adaptation of Tendon and Skeletal Muscle to Mechanical Loading. Physiological reviews 84:649-698.

15. Wang JHC. 2006. Mechanobiology of tendon. Journal of biomechanics 39:15631582. 
16. Hsieh AH, Tsai CM, Ma QJ, et al. 2000. Time-dependent increases in type-III collagen gene expression in medical collateral ligament fibroblasts under cyclic strains. Journal of orthopaedic research : official publication of the Orthopaedic Research Society 18:220-227.

17. Hadjicostas PT, Soucacos PN, Koleganova N, et al. 2008. Comparative and morphological analysis of commonly used autografts for anterior cruciate ligament reconstruction with the native ACL: an electron, microscopic and morphologic study. Knee Surgery, Sports Traumatology, Arthroscopy 16:10991107.

18. Wang JH, Jia F, Yang G, et al. 2003. Cyclic mechanical stretching of human tendon fibroblasts increases the production of prostaglandin E2 and levels of cyclooxygenase expression: a novel in vitro model study. Connective tissue research 44:128-133.

19. Scheffler SU, Unterhauser FN, Weiler A. 2008. Graft remodeling and ligamentization after cruciate ligament reconstruction. Knee surgery, sports traumatology, arthroscopy : official journal of the ESSKA 16:834-842.

20. Escamilla RF, Macleod TD, Wilk KE, et al. 2012. Anterior cruciate ligament strain and tensile forces for weight-bearing and non-weight-bearing exercises: a guide to exercise selection. J Orthop Sports Phys Ther 42:208-220.

21. Shelbourne KD, Gray T. 1997. Anterior cruciate ligament reconstruction with autogenous patellar tendon graft followed by accelerated rehabilitation. A two- to nine-year followup. The American journal of sports medicine 25:786-795. 
22. Benner WR, Shelbourne KD. 2018. The Case for the Bone-Patellar Tendon-Bone Autograft with Anterior Cruciate Ligament Reconstruction. In: Prodromos C editor. The Anterior Cruciate Ligament: Reconstruction and Basic Science EBook: Elsevier Health Sciences.

23. Smith HC, Vacek P, Johnson RJ, et al. 2012. Risk factors for anterior cruciate ligament injury: a review of the literature - part 1: neuromuscular and anatomic risk. Sports Health 4:69-78.

24. Dai B, Herman D, Liu H, et al. 2012. Prevention of ACL injury, part I: injury characteristics, risk factors, and loading mechanism. Research in sports medicine (Print) 20:180-197.

25. Anil U, Jejurikar N, Kenny L, et al. 2019. Changes in Synovial Fluid Biomarker Concentration Before and After ACL Reconstruction. Bulletin of the Hospital for Joint Disease (2013) 77:189-193. 


\section{Tables}

\begin{tabular}{ccccc}
\multicolumn{2}{c}{ Graft source } & Sex & Age Range \\
\hline \multirow{2}{*}{ QT } & 9 & Males & 4 & $17-38$ \\
& & Females & 5 & $14-25$ \\
PT & 7 & Males & 4 & $17-35$ \\
& & Females & 3 & $15-21$ \\
\multirow{2}{*}{ HT } & \multirow{2}{*}{6} & Males & 3 & $18-25$ \\
& & Females & 3 & $17-43$ \\
\hline
\end{tabular}

Table 8.1. Patient sex and age distribution by graft source

\section{Figures}

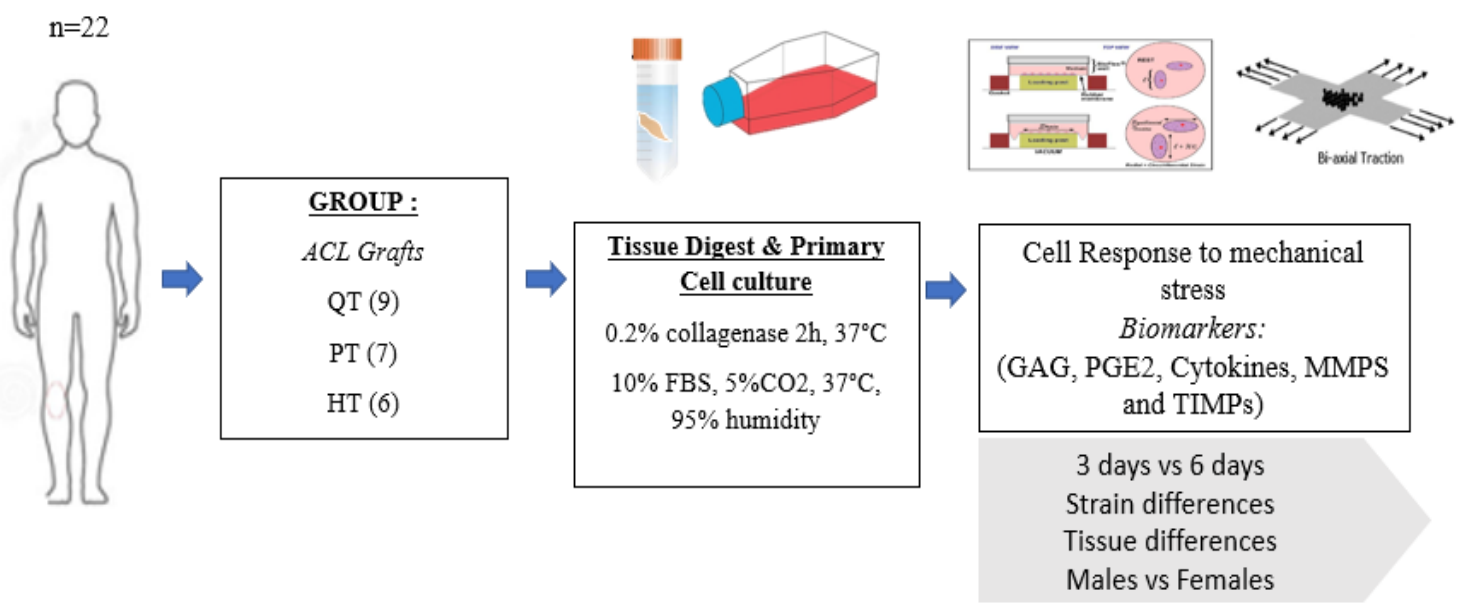

Figure 8.1. Experimental design 

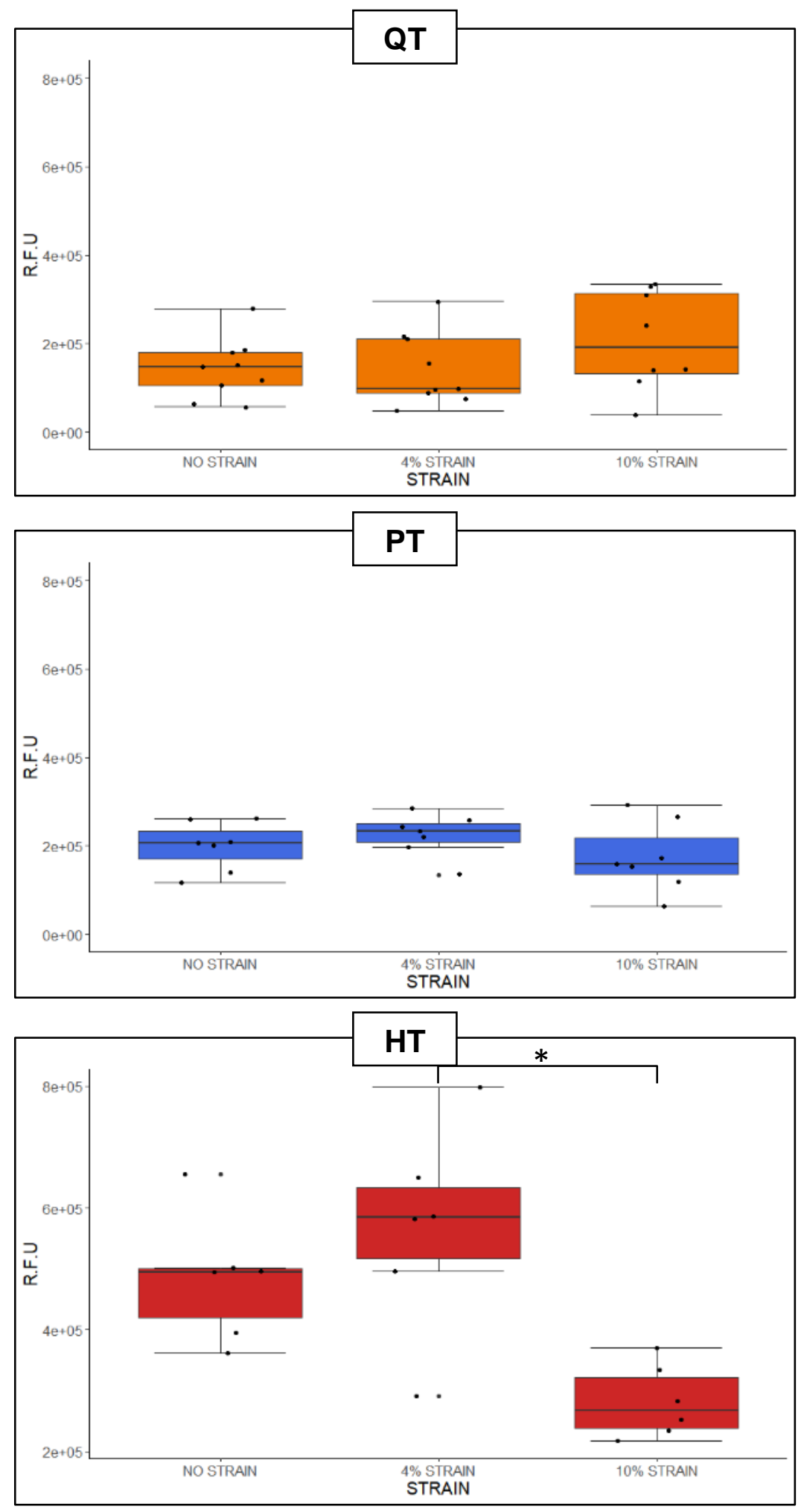

Figure 8.2. Metabolic activity of fibroblasts after six days of culture. ${ }^{*}$ significant difference between STRAINS. QT: Quadriceps tendon; PT: Patellar tendon; HT: Hamstring tendon. R.F.U: Resazurin Fluorescent Units 

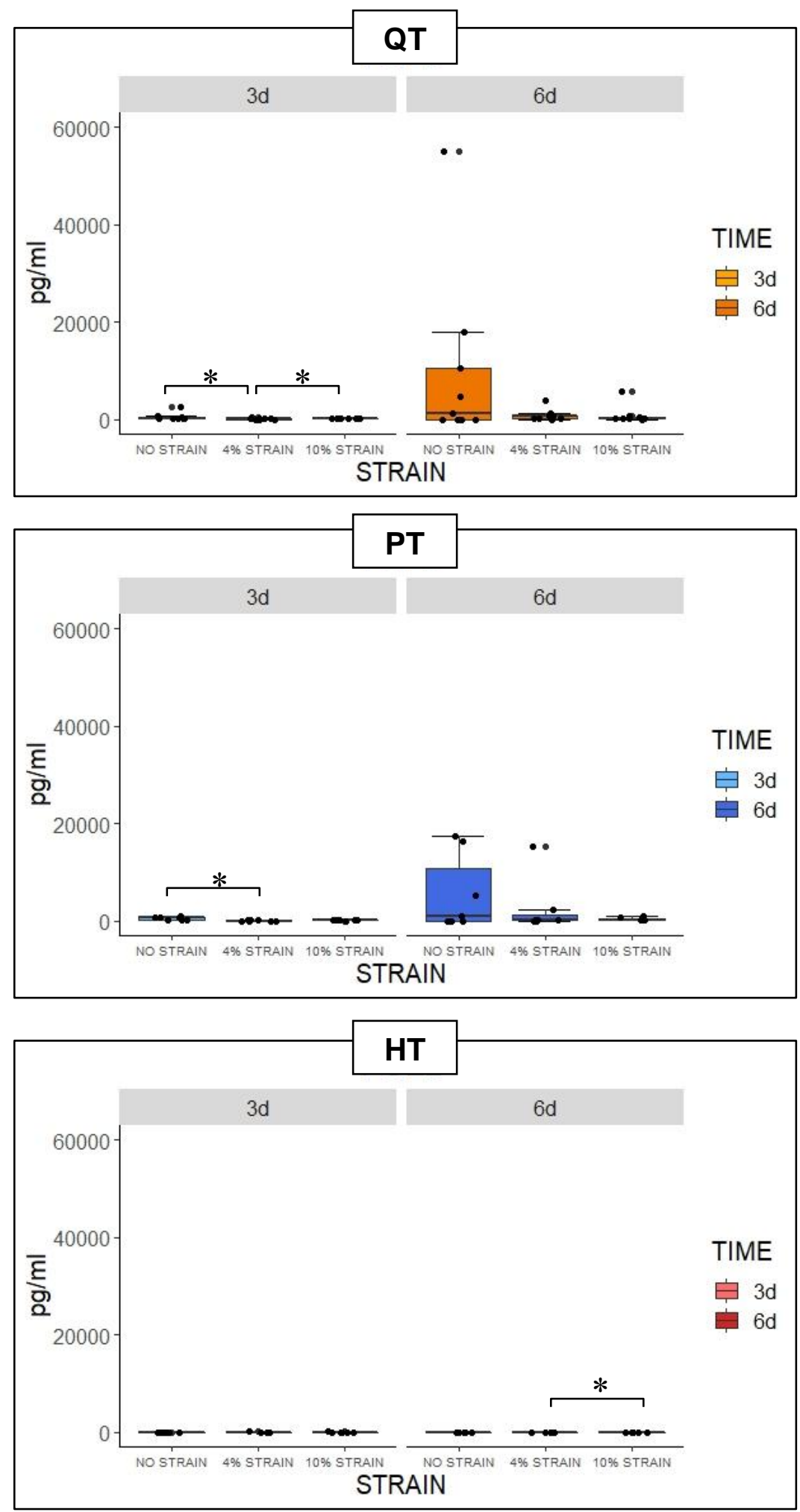

Figure 8.3. Concentration of PGE2 released to the media. * significant difference between STRAINS at three (3d) and six (6d) days of culture. QT: Quadriceps tendon; PT: Patellar tendon; HT: Hamstring tendon. 

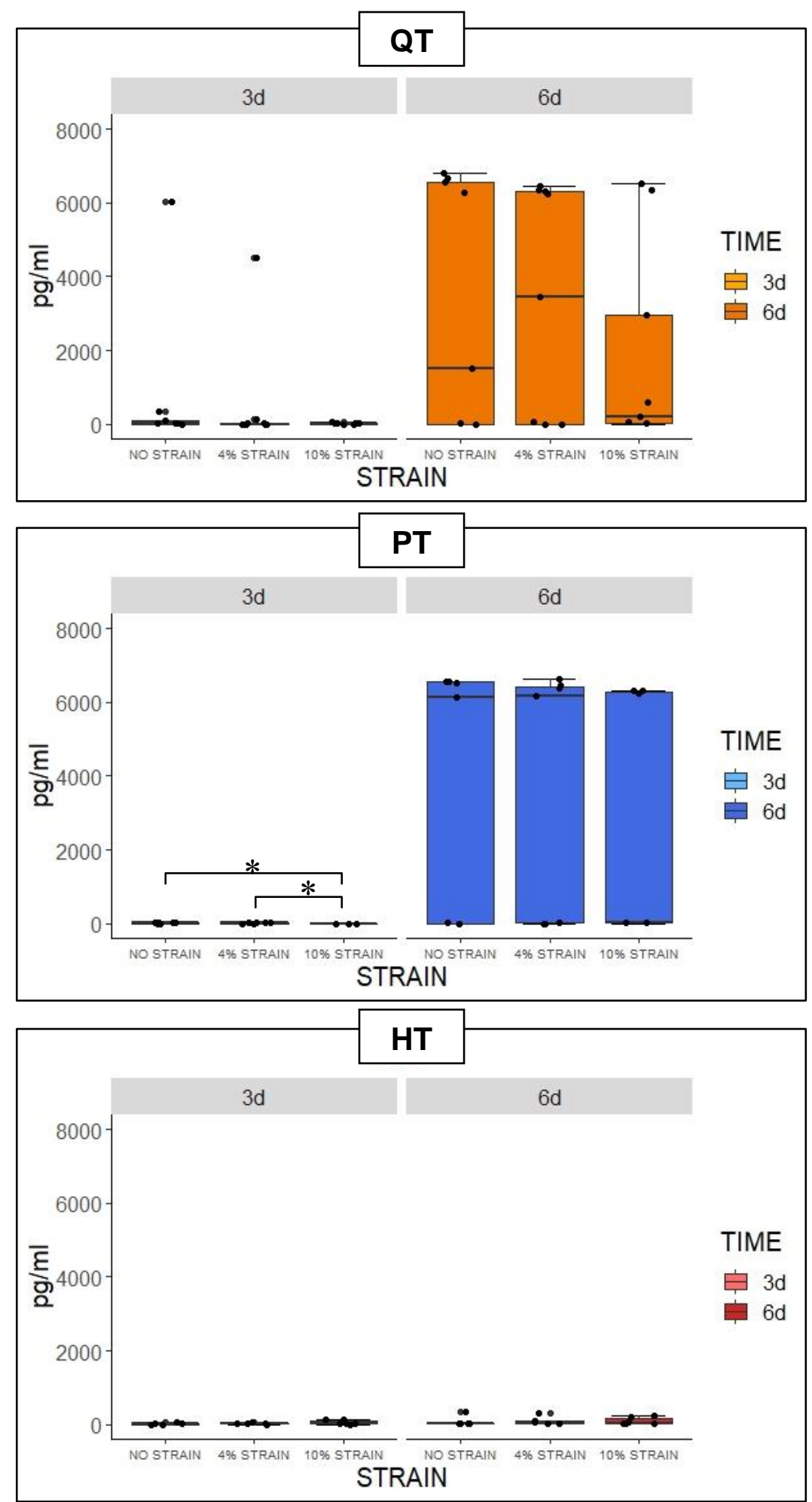

Figure 8.4. Concentration of IL-6 released to the media. * significant difference between STRAINS at three (3d) and six (6d) days of culture. QT: Quadriceps tendon; PT: Patellar tendon; HT: Hamstring tendon. 

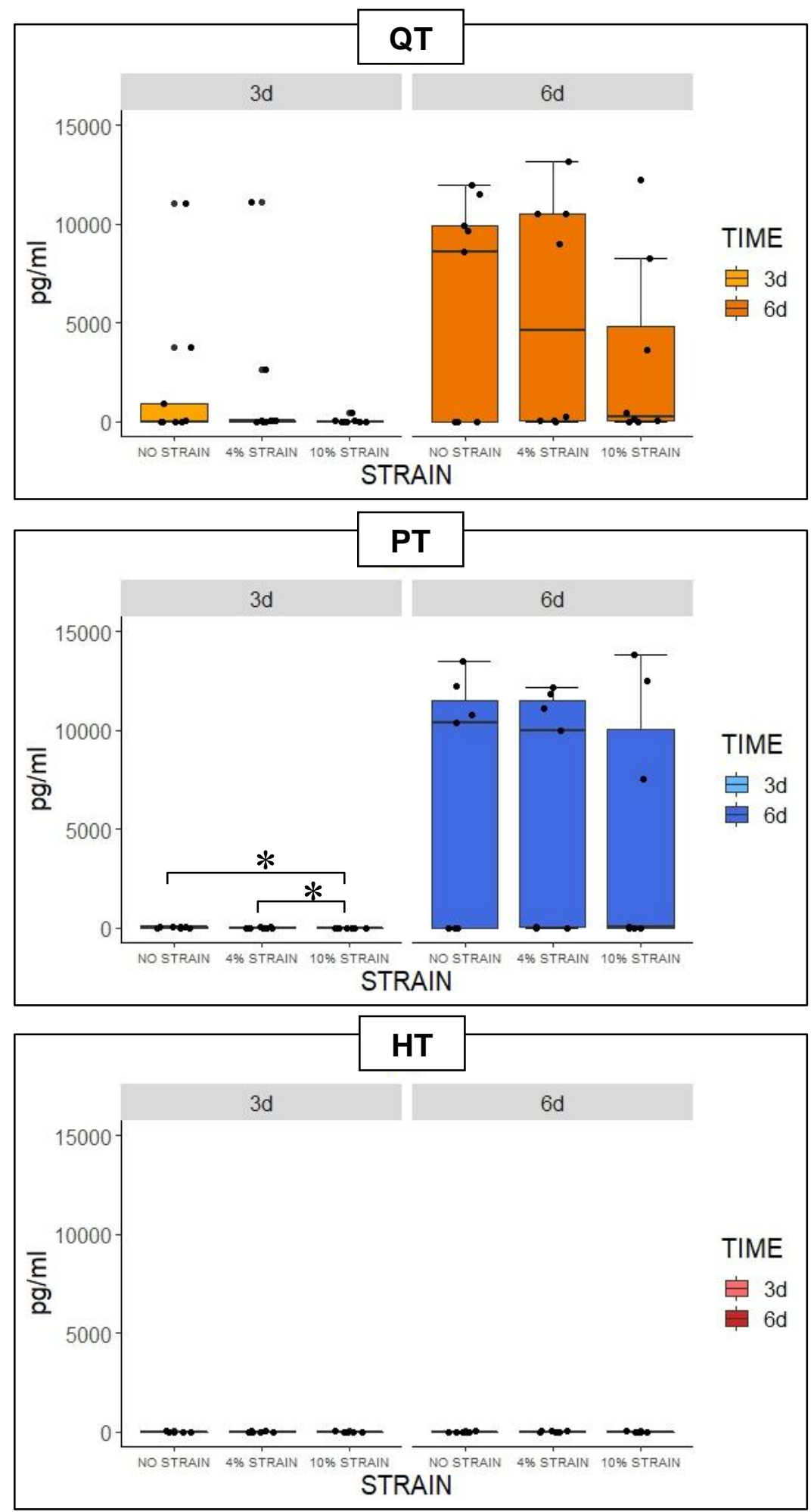

Figure 8.5. Concentration of IL-8 released to the media. * significant difference between STRAINS at three (3d) and six (6d) days of culture. QT: Quadriceps tendon; PT: Patellar tendon; HT: Hamstring tendon. 

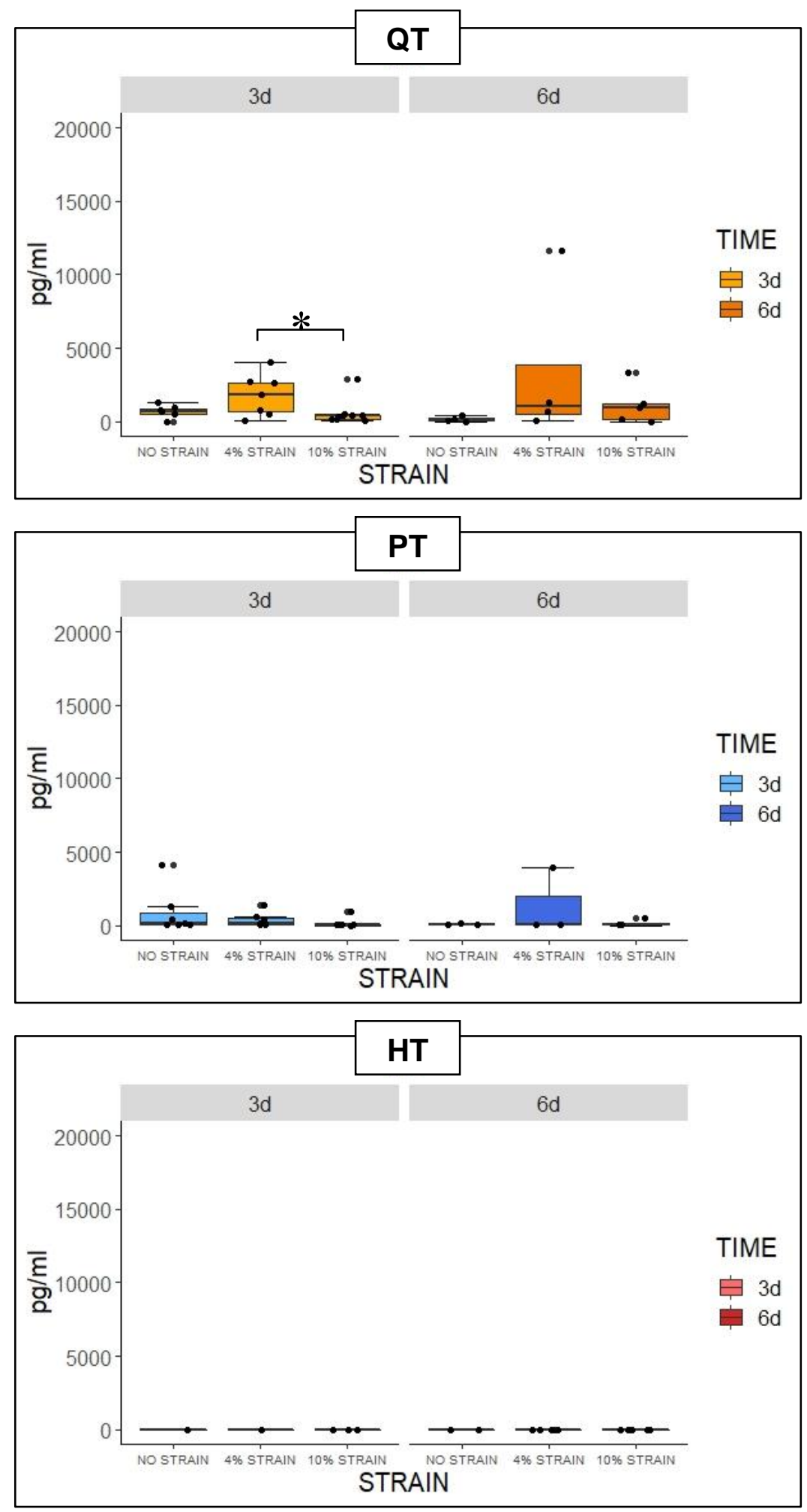

Figure 8.6. Concentration of $\mathrm{KC}$ released to the media. * significant difference between STRAINS at three (3d) and six (6d) days of culture. QT: Quadriceps tendon; PT: Patellar tendon; HT: Hamstring tendon. 

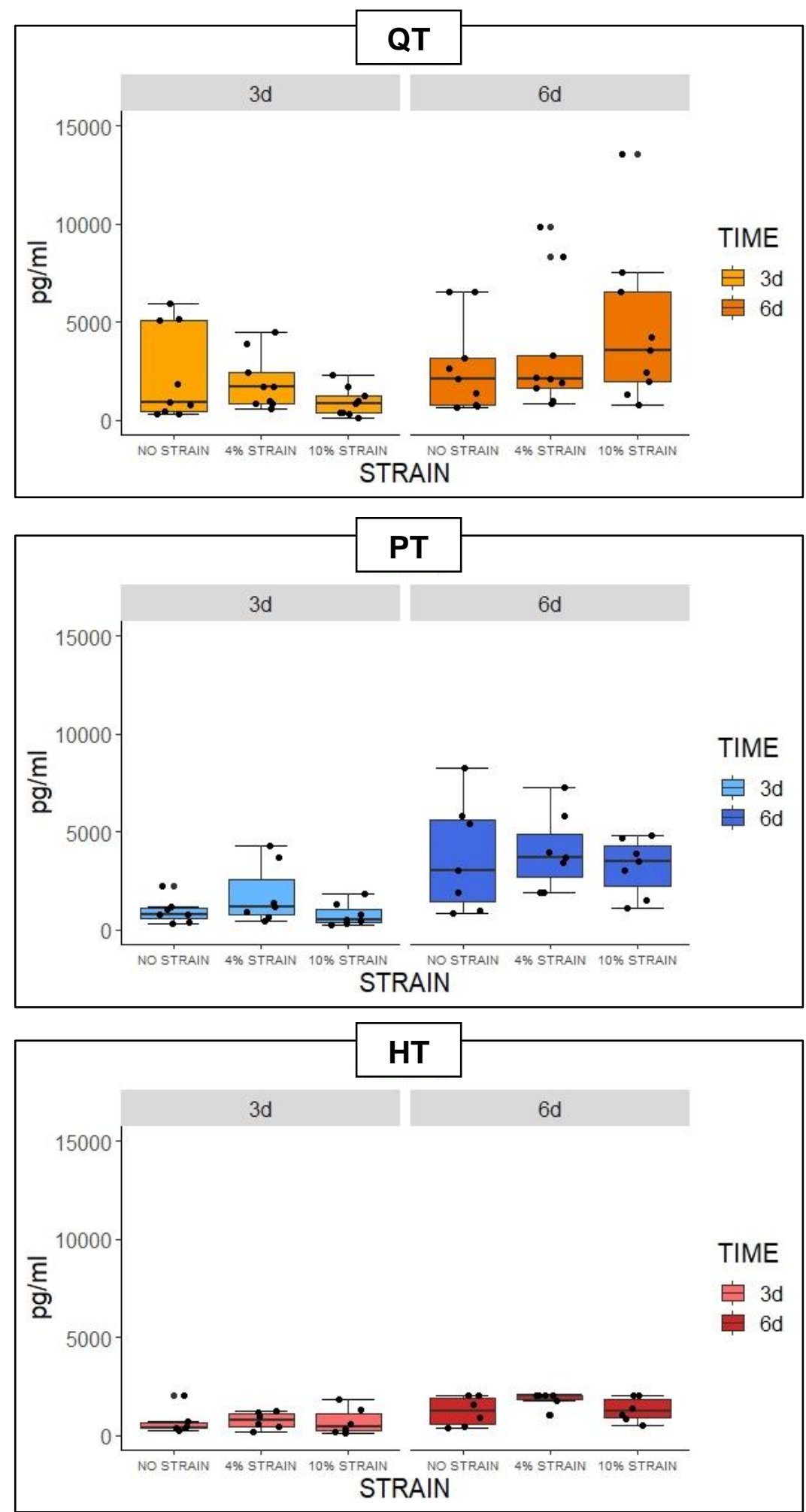

Figure 8.7. Concentration of MCP1 released to the media. * significant difference between STRAINS at three (3d) and six (6d) days of culture. QT: Quadriceps tendon; PT: Patellar tendon; HT: Hamstring tendon. 

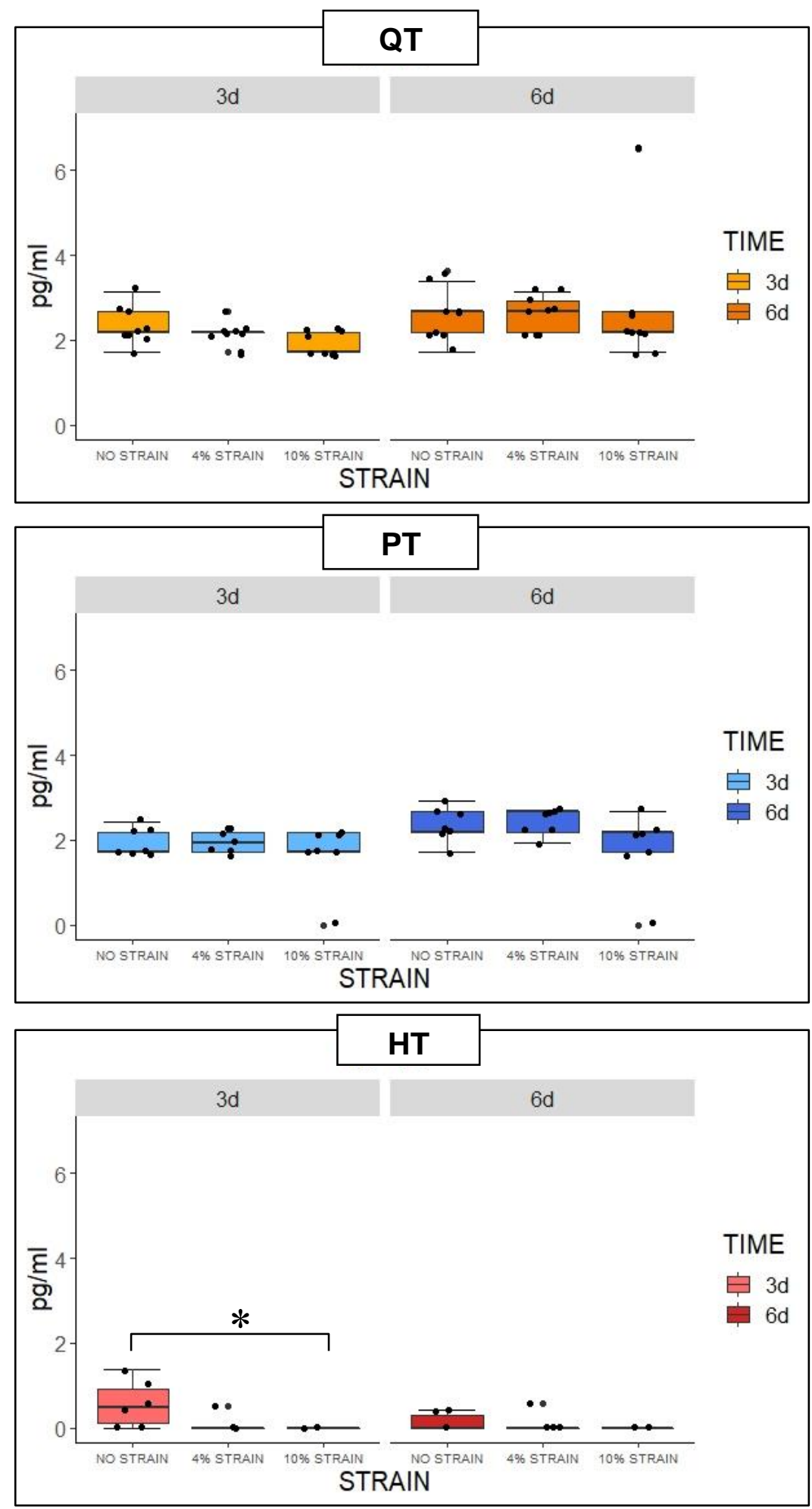

Figure 8.8. Concentration of MP1 $\beta$ released to the media. * significant difference between STRAINS at three (3d) and six (6d) days of culture. QT: Quadriceps tendon; PT: Patellar tendon; HT: Hamstring tendon. 

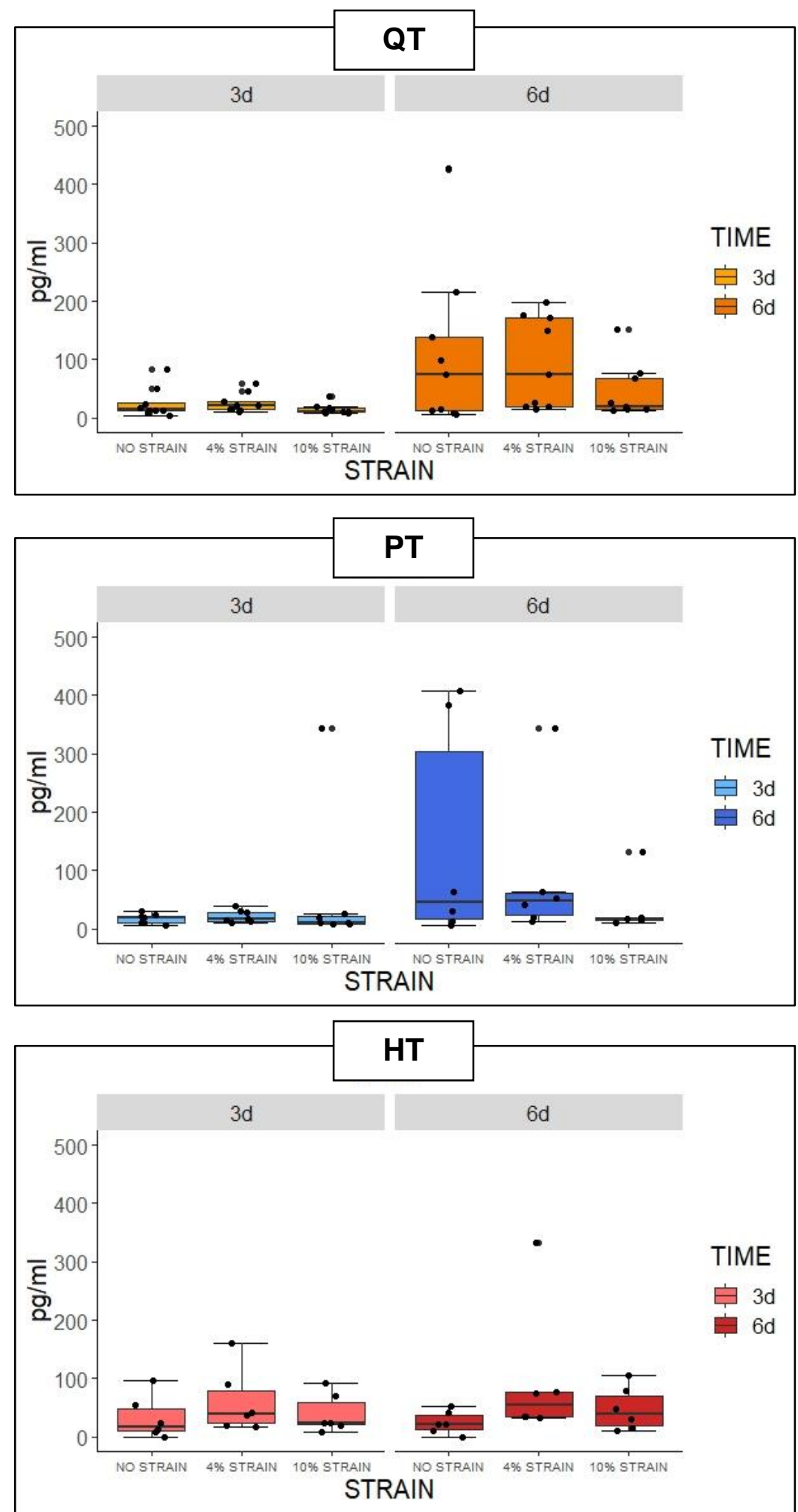

Figure 8.9. Concentration of VEGF released to the media. * significant difference between STRAINS at three (3d) and six (6d) days of culture. QT: Quadriceps tendon; PT: Patellar tendon; HT: Hamstring tendon. 

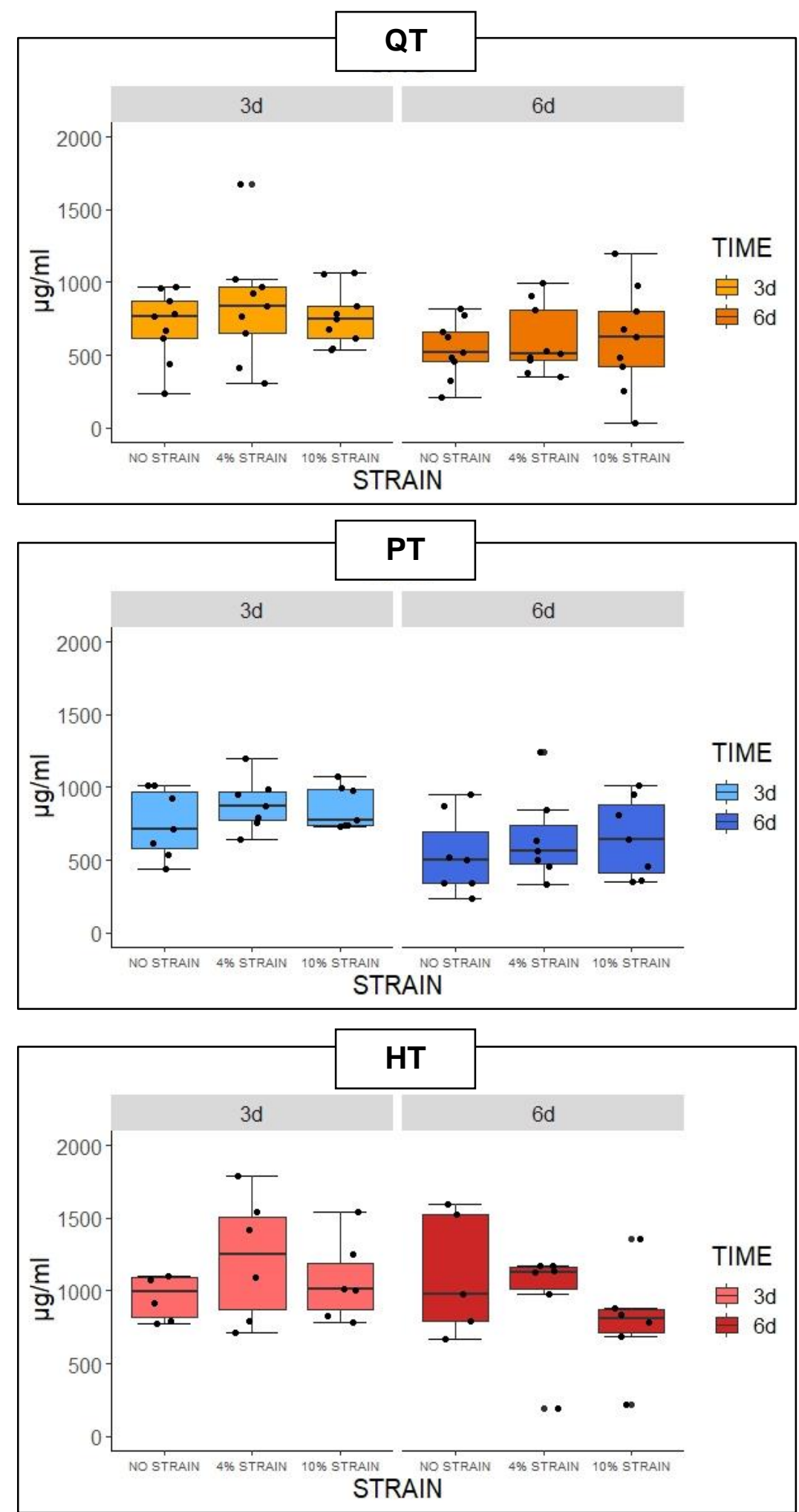

Figure 8.10. Concentration of GAG released to the media. * significant difference between STRAINS at three (3d) and six (6d) days of culture. QT: Quadriceps tendon; PT: Patellar tendon; HT: Hamstring tendon. 

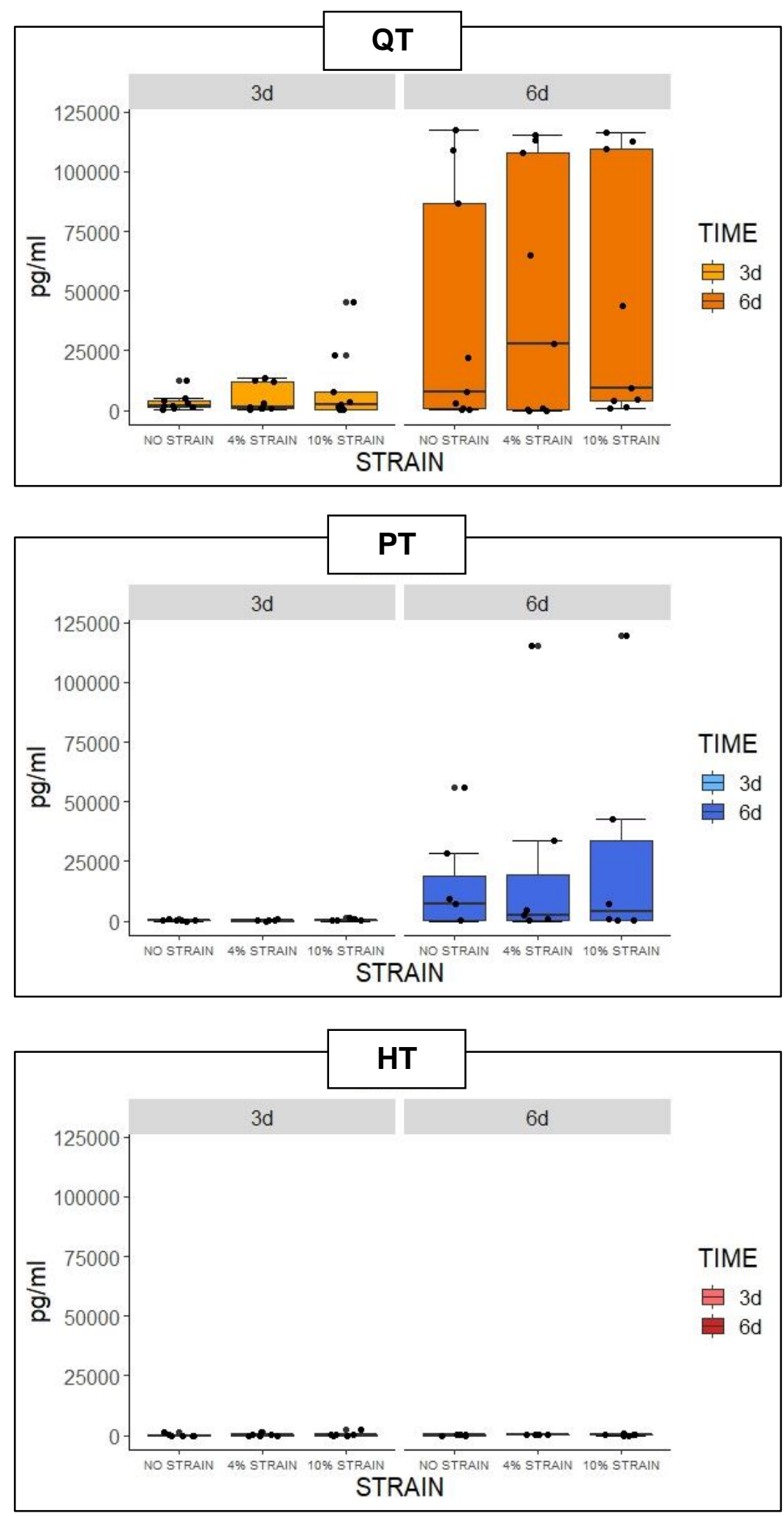

Figure 8.11. Concentration of MMP-1 released to the media. * significant difference between STRAINS at three (3d) and six (6d) days of culture. QT: Quadriceps tendon; PT: Patellar tendon; HT: Hamstring tendon. 

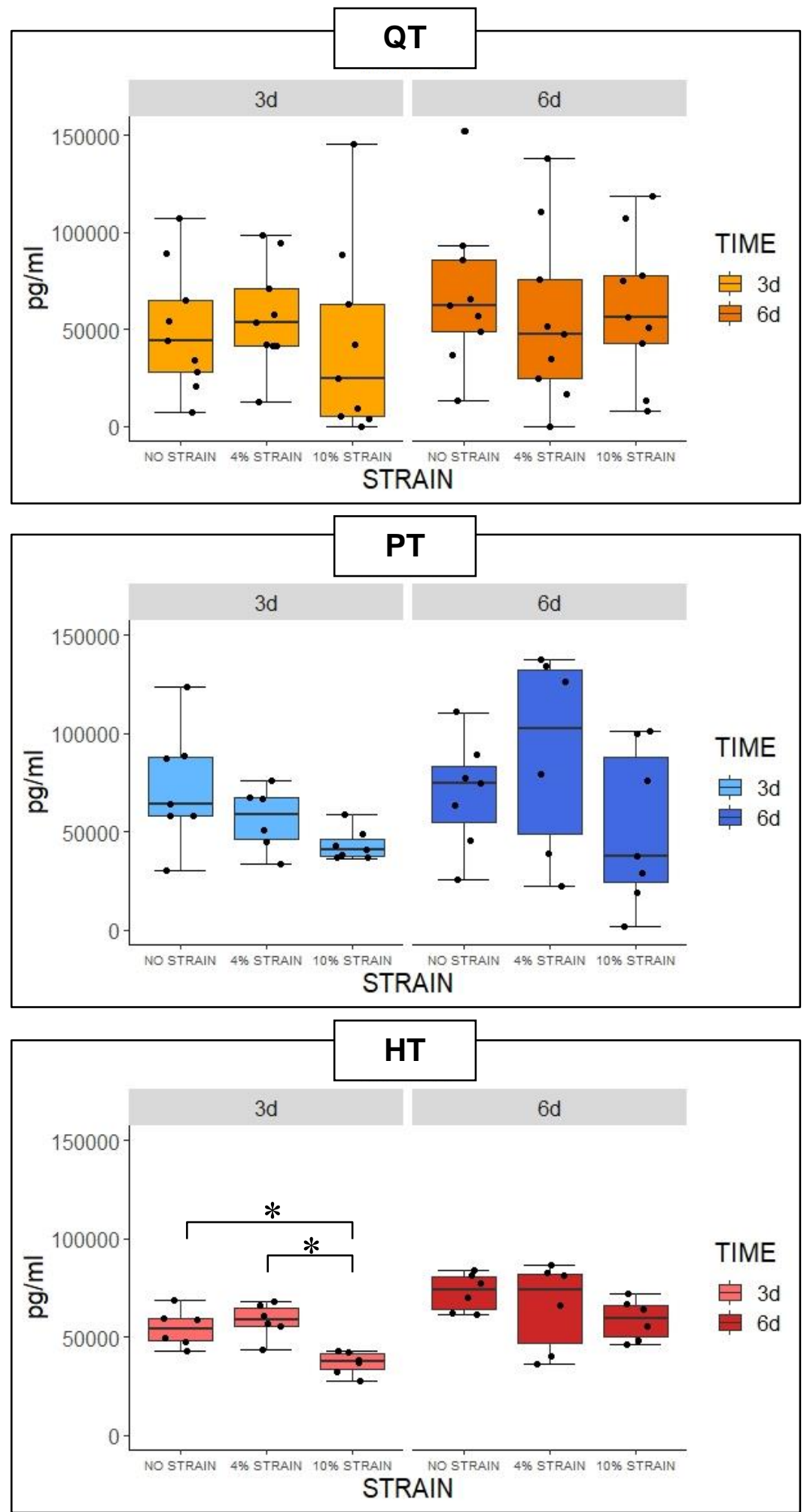

Figure 8.12. Concentration of MMP-2 released to the media. * significant difference between STRAINS at three (3d) and six (6d) days of culture. QT: Quadriceps tendon; PT: Patellar tendon; HT: Hamstring tendon. 

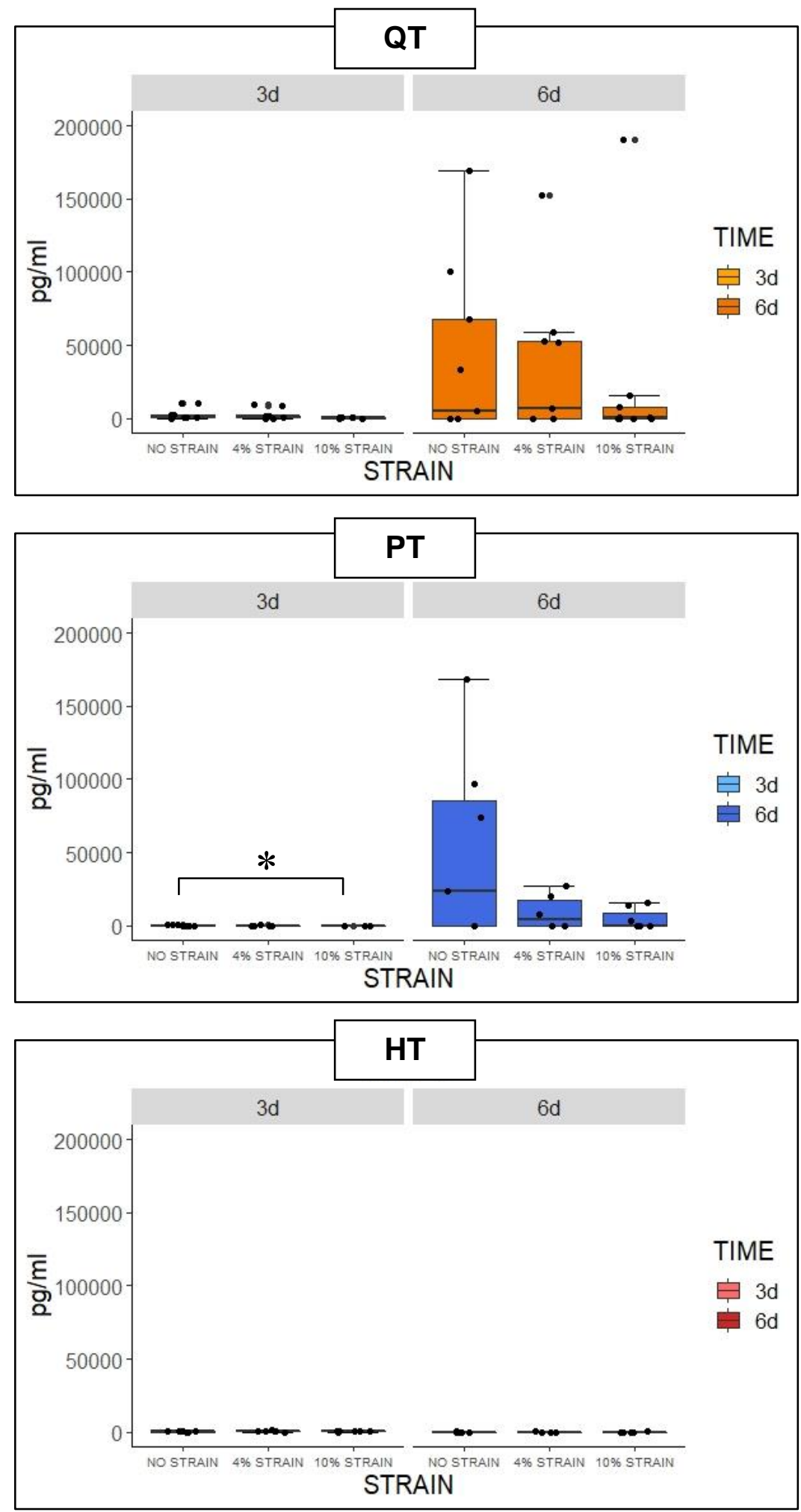

Figure 8.13. Concentration of MMP-3 released to the media. * significant difference between STRAINS at three (3d) and six (6d) days of culture. QT: Quadriceps tendon; PT: Patellar tendon; HT: Hamstring tendon. 

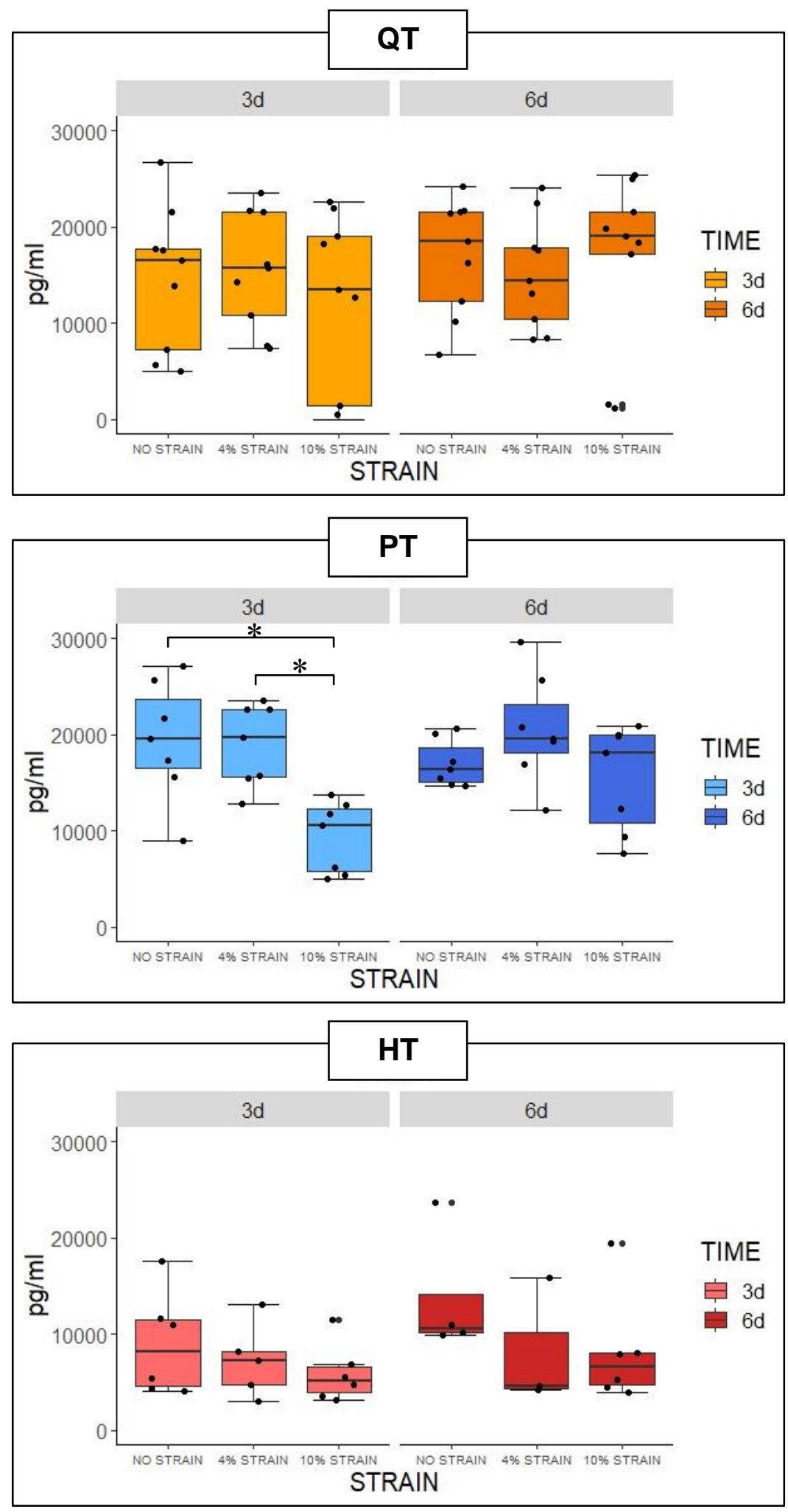

Figure 8.14. Concentration of TIMP-1 released to the media. * significant difference between STRAINS at three (3d) and six (6d) days of culture. QT: Quadriceps tendon; PT: Patellar tendon; HT: Hamstring tendon. 

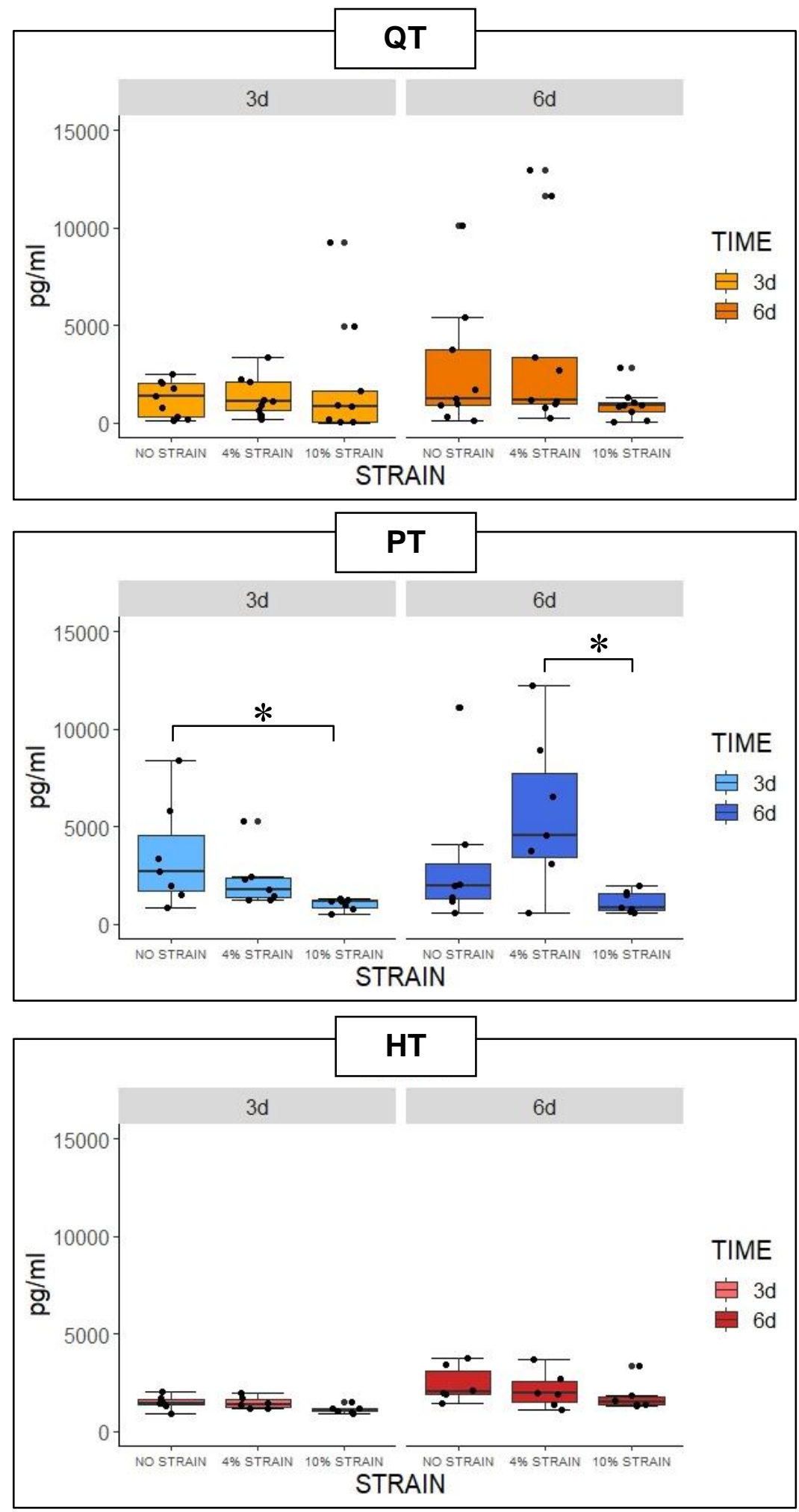

Figure 8.15. Concentration of TIMP-2 released to the media. * significant difference between STRAINS at three (3d) and six (6d) days of culture. QT: Quadriceps tendon; PT: Patellar tendon; HT: Hamstring tendon. 

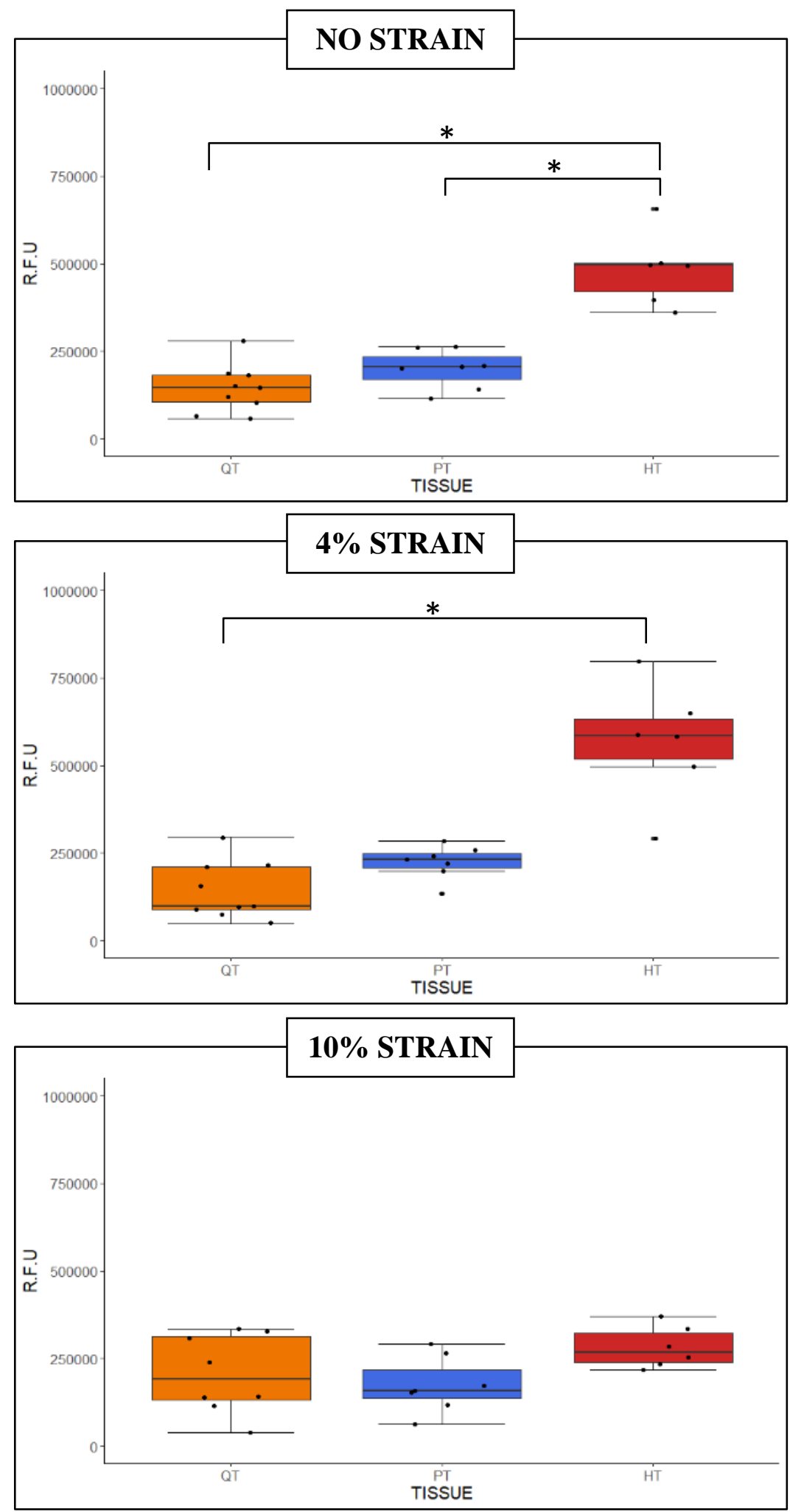

Figure 8.16. Metabolic activity of fibroblasts after six days of culture. * significant difference between TISSUES. QT: Quadriceps tendon; PT: Patellar tendon; HT: Hamstring tendon. R.F.U: Resazurin Fluorescent Units 

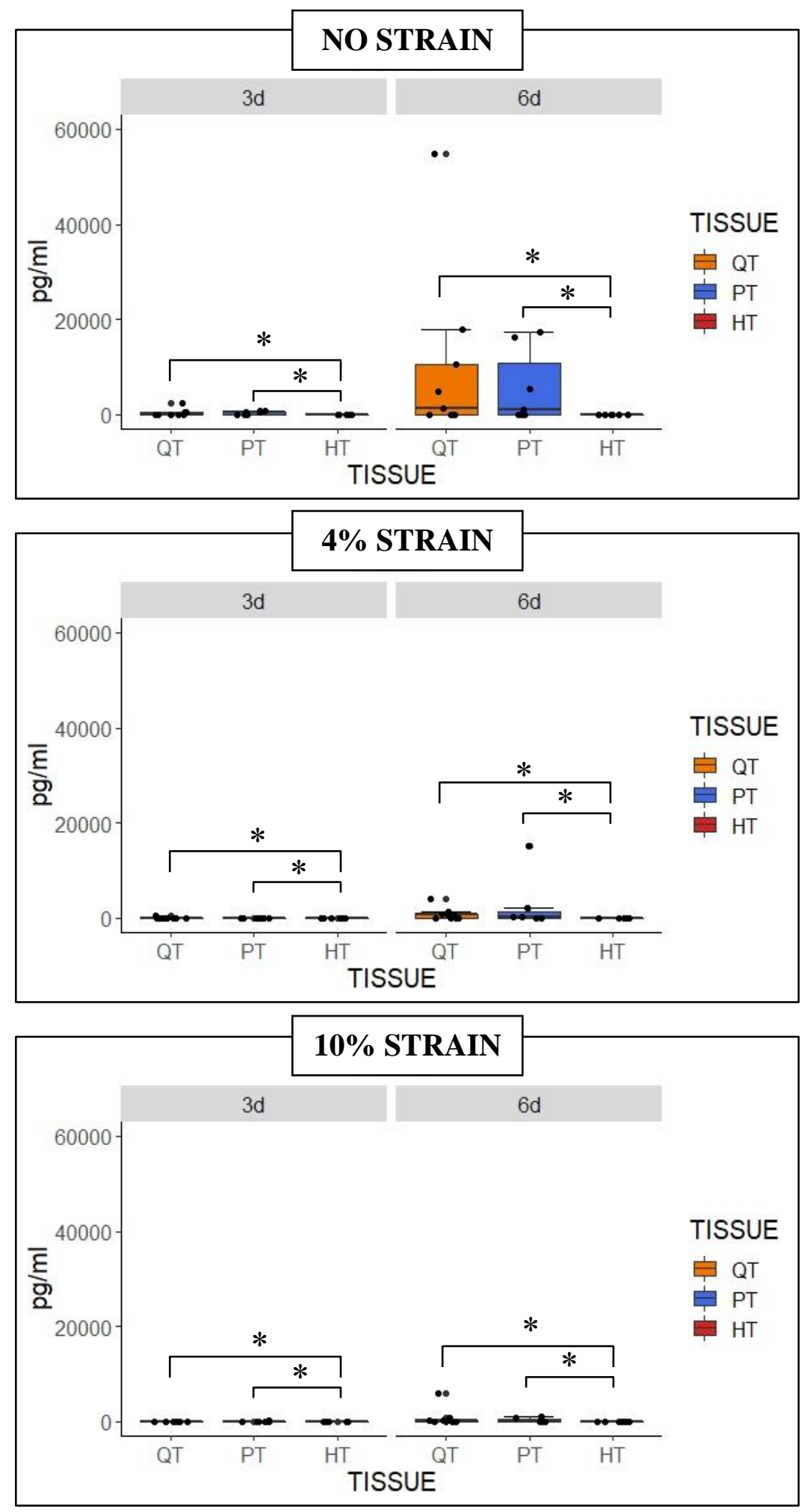

Figure 8.17. Concentration of PGE2 released to the media. * significant difference between TISSUES at three (3d) and six (6d) days of culture. QT: Quadriceps tendon; PT: Patellar tendon; HT: Hamstring tendon. 

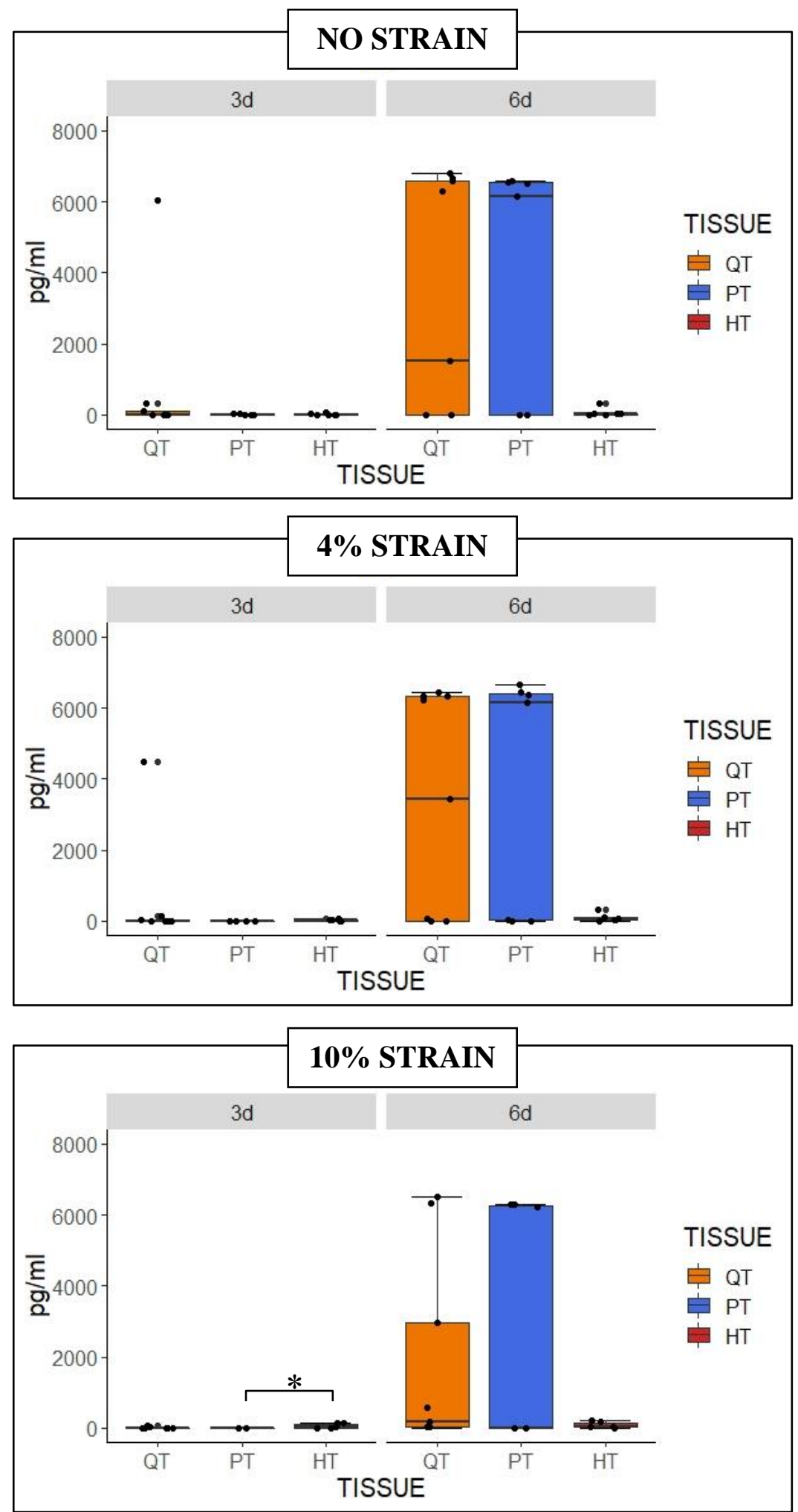

Figure 8.18. Concentration of IL-6 released to the media. * significant difference between TISSUES at three (3d) and six (6d) days of culture. QT: Quadriceps tendon; PT: Patellar tendon; HT: Hamstring tendon. 

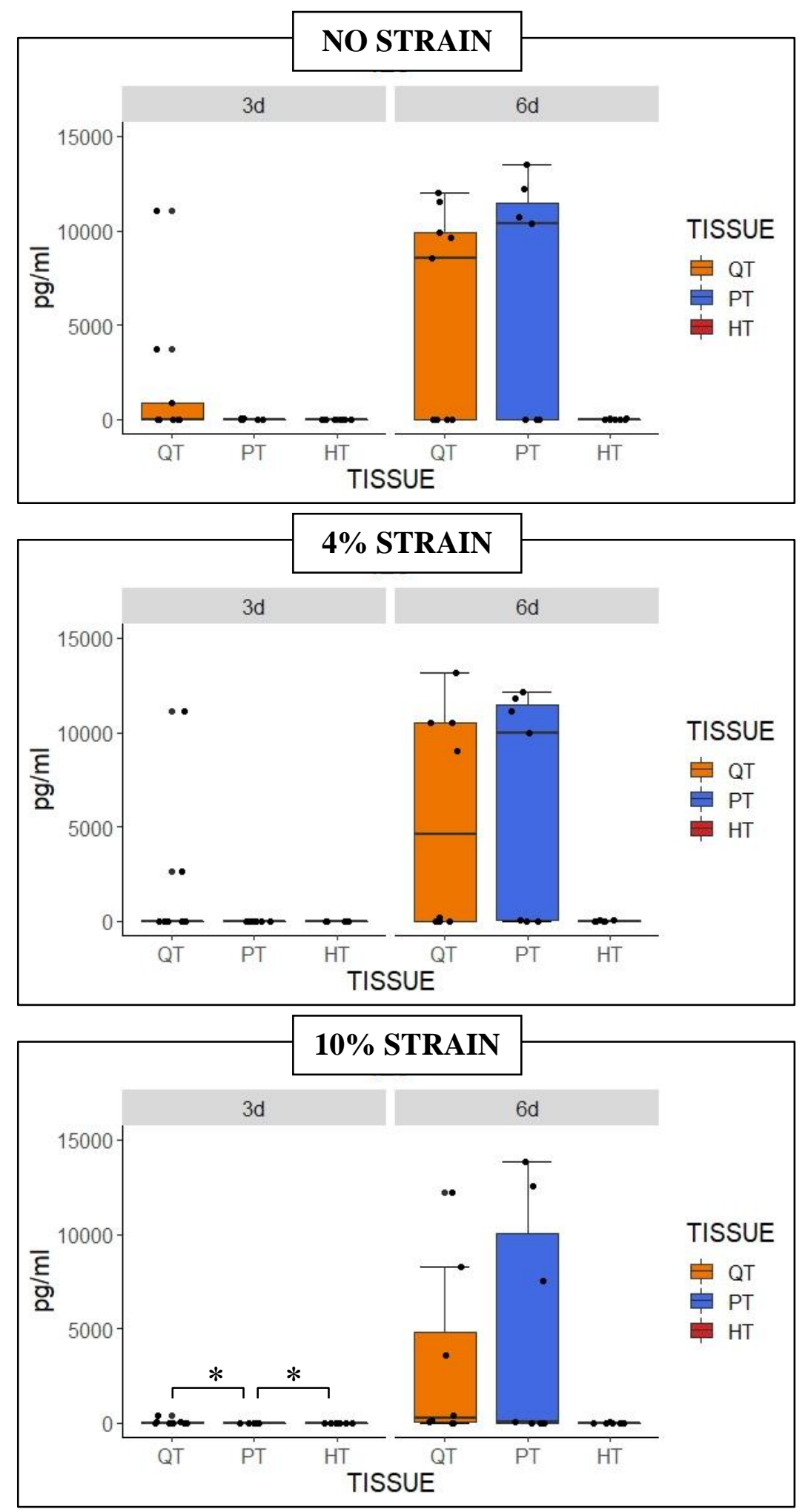

Figure 8.19. Concentration of IL-8 released to the media. * significant difference between TISSUES at three (3d) and six (6d) days of culture. QT: Quadriceps tendon; PT: Patellar tendon; HT: Hamstring tendon. 

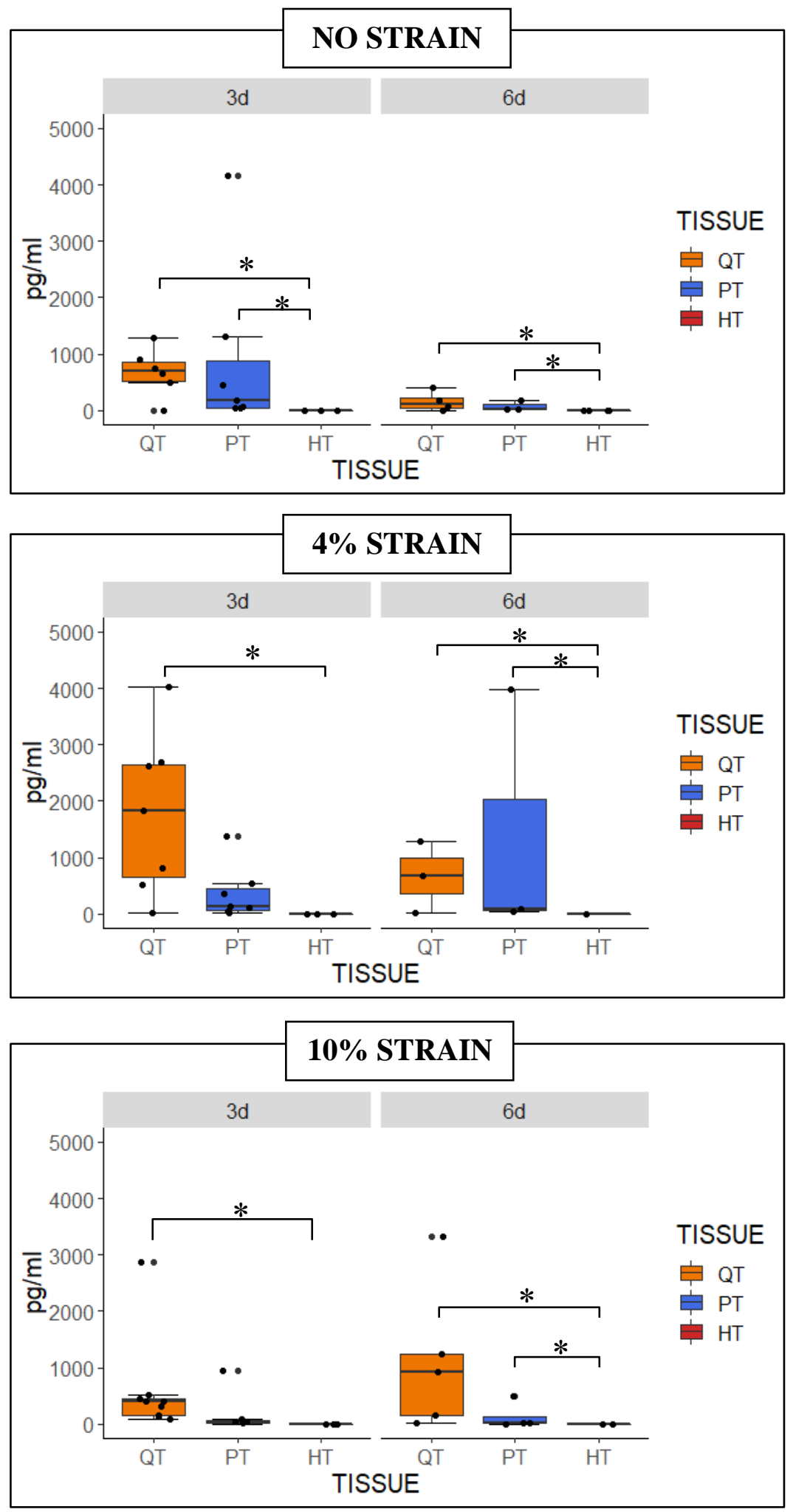

Figure 8.20. Concentration of $\mathrm{KC}$ released to the media. * significant difference between TISSUES at three (3d) and six (6d) days of culture. QT: Quadriceps tendon; PT: Patellar tendon; HT: Hamstring tendon. 

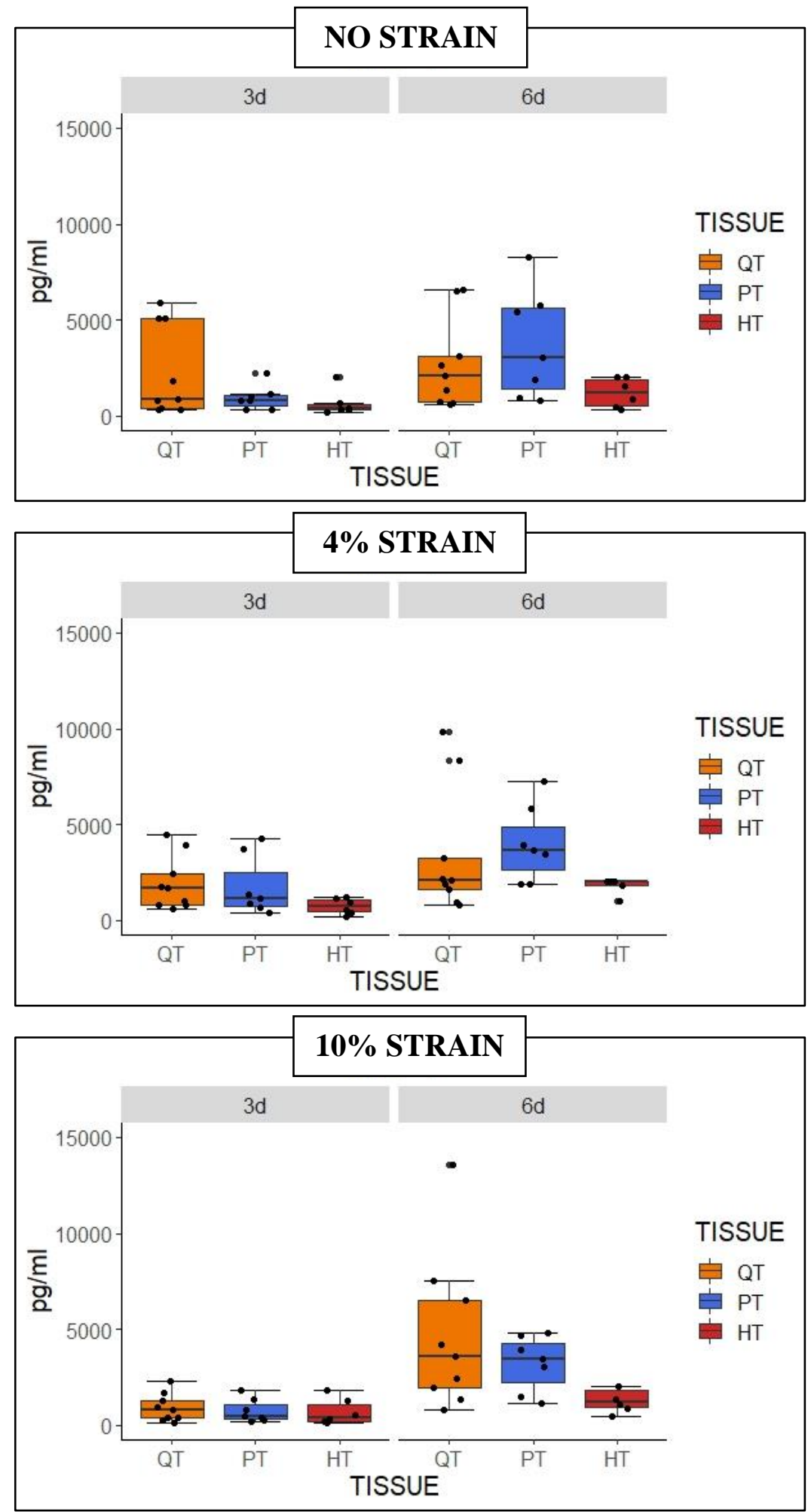

Figure 8.21. Concentration of MCP1 released to the media. * significant difference between TISSUES at three (3d) and six (6d) days of culture. QT: Quadriceps tendon; PT: Patellar tendon; HT: Hamstring tendon. 

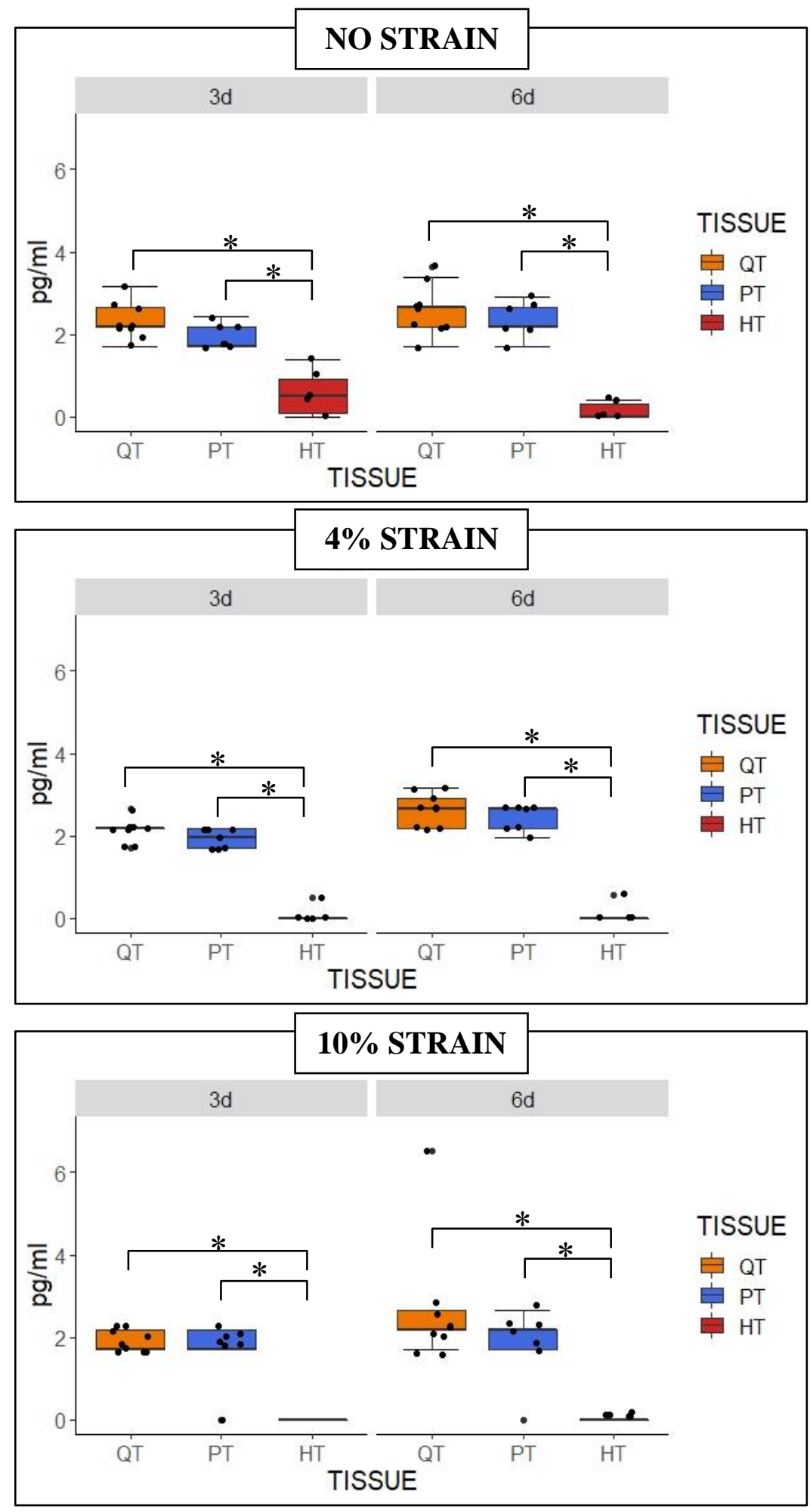

Figure 8.22. Concentration of MP1 $\beta$ released to the media. * significant difference between TISSUES at three (3d) and six (6d) days of culture. QT: Quadriceps tendon; PT: Patellar tendon; HT: Hamstring tendon. 

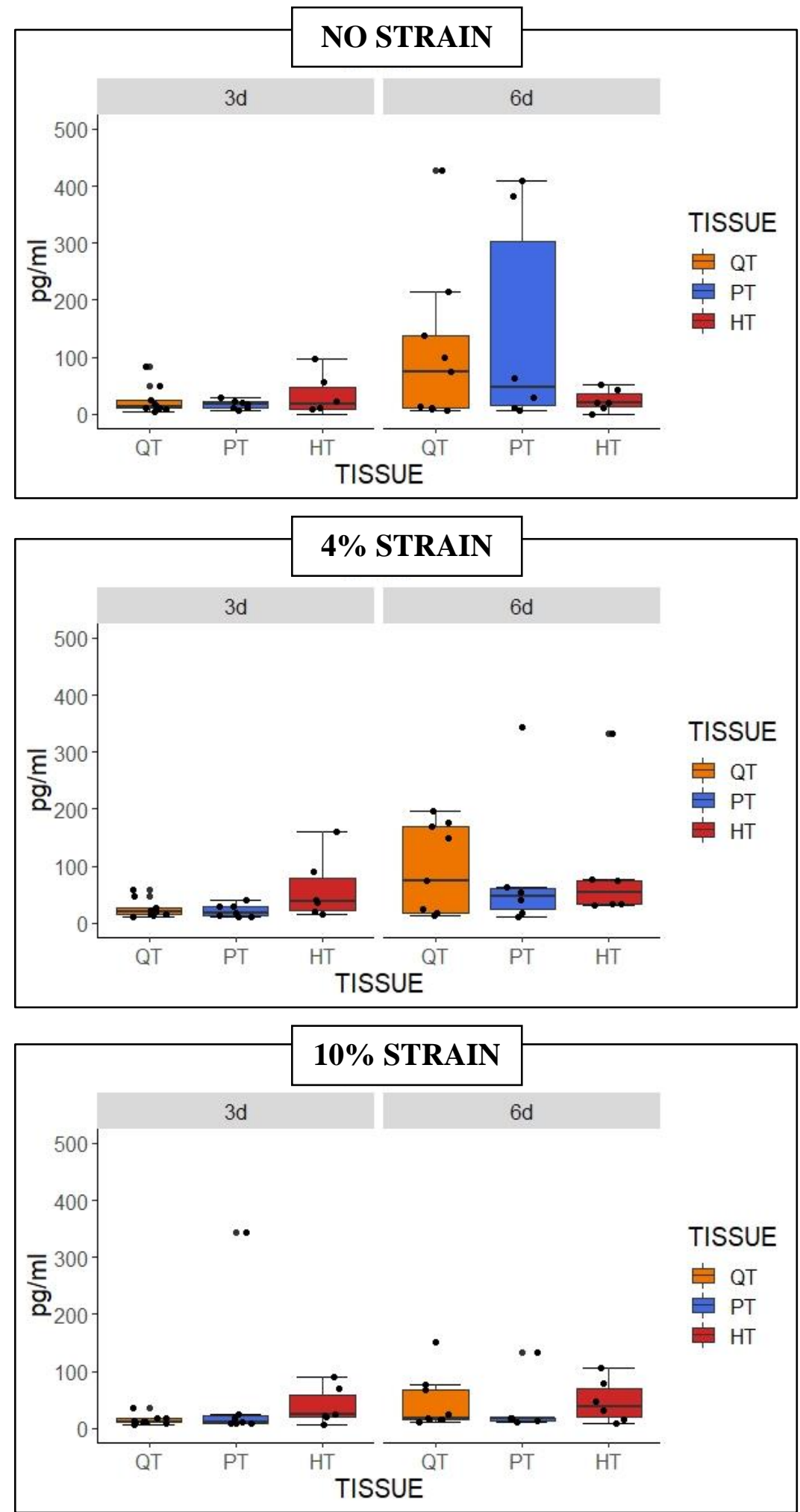

Figure 8.23. Concentration of VEGF released to the media. * significant difference between TISSUES at three (3d) and six (6d) days of culture. QT: Quadriceps tendon; PT: Patellar tendon; HT: Hamstring tendon. 

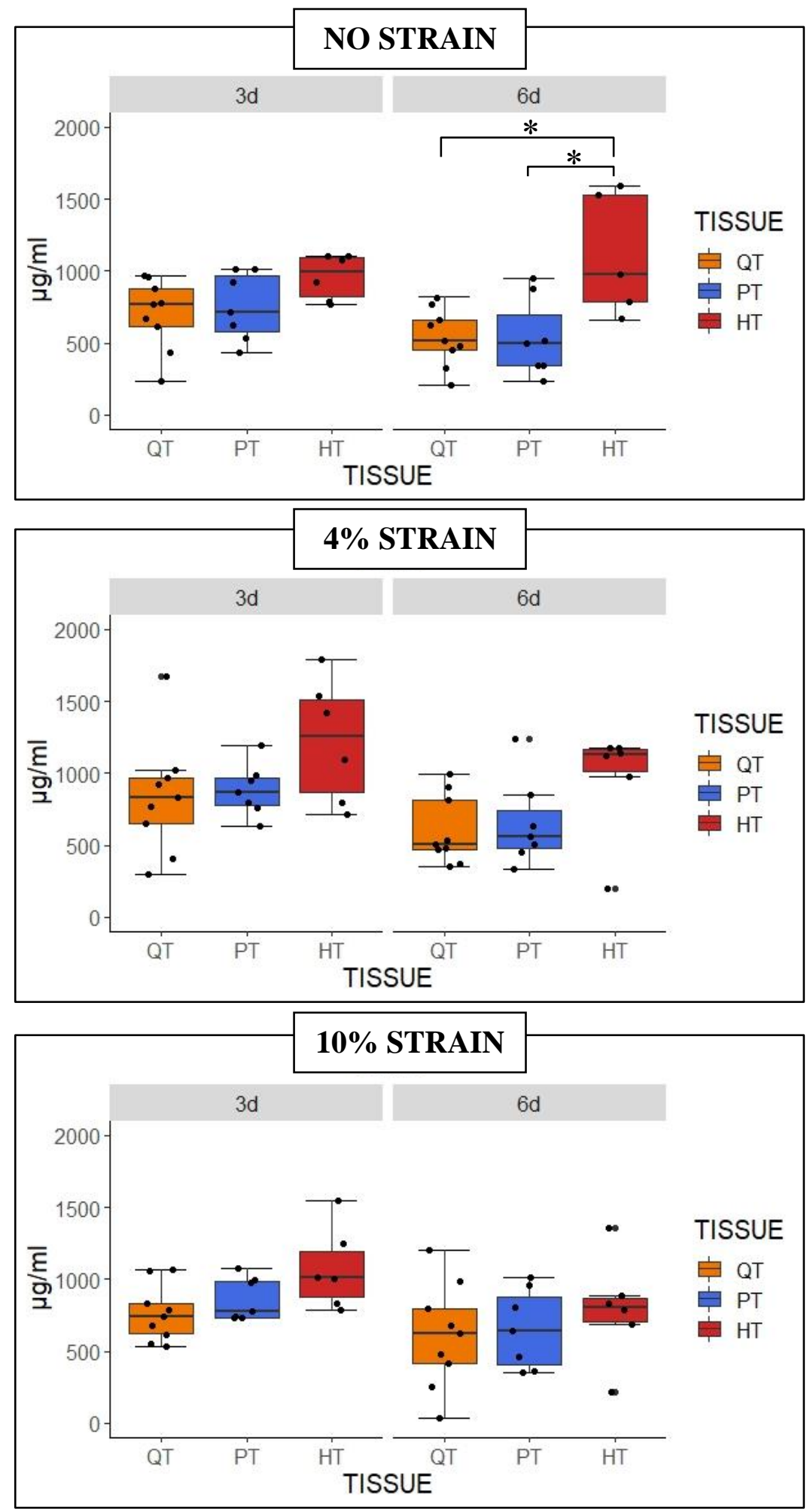

Figure 8.24. Concentration of GAG released to the media. * significant difference between TISSUES at three (3d) and six (6d) days of culture. QT: Quadriceps tendon; PT: Patellar tendon; HT: Hamstring tendon. 

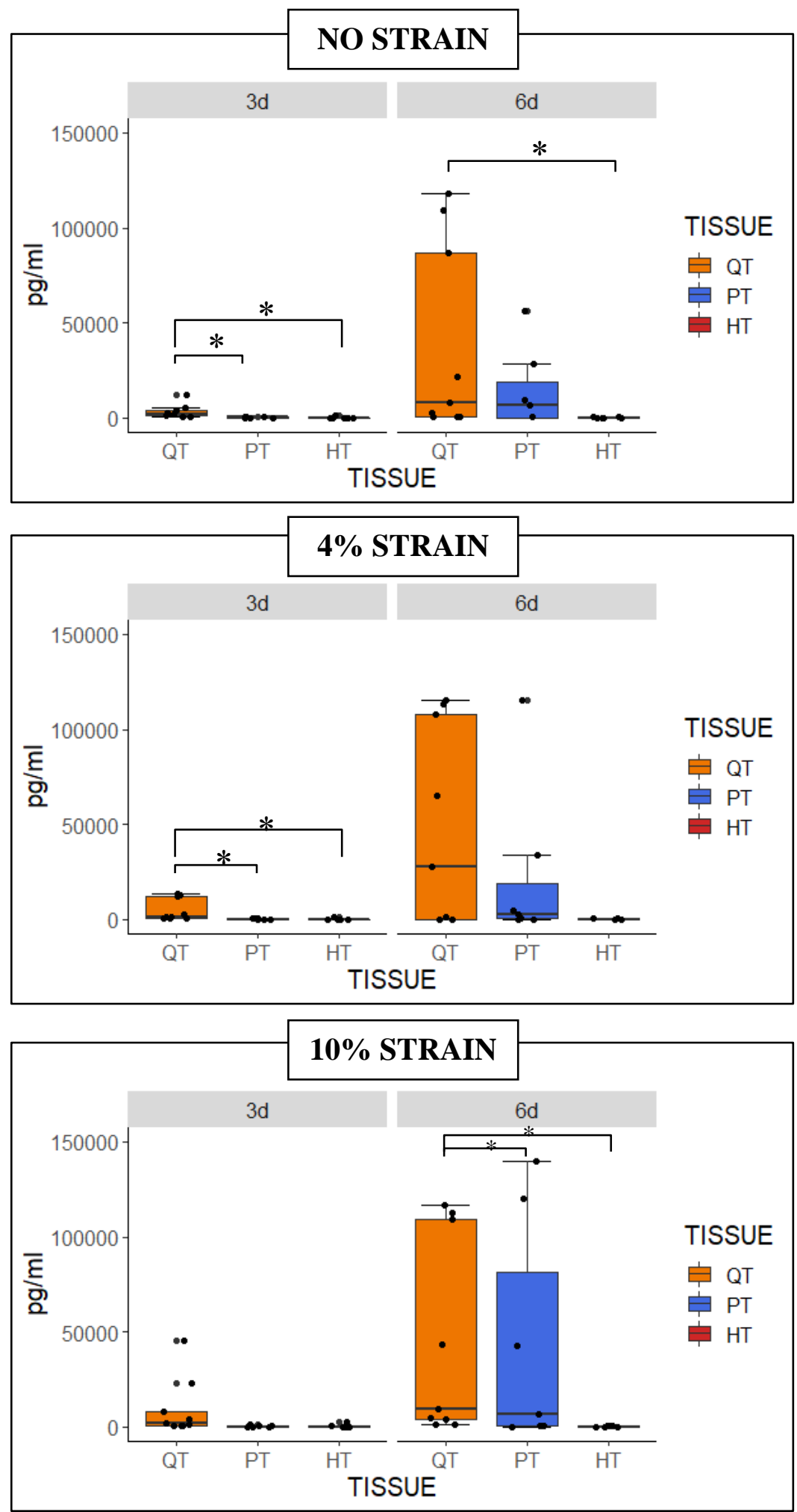

Figure 8.25. Concentration of MMP-1 released to the media. * significant difference between TISSUES at three (3d) and six (6d) days of culture. QT: Quadriceps tendon; PT: Patellar tendon; HT: Hamstring tendon. 

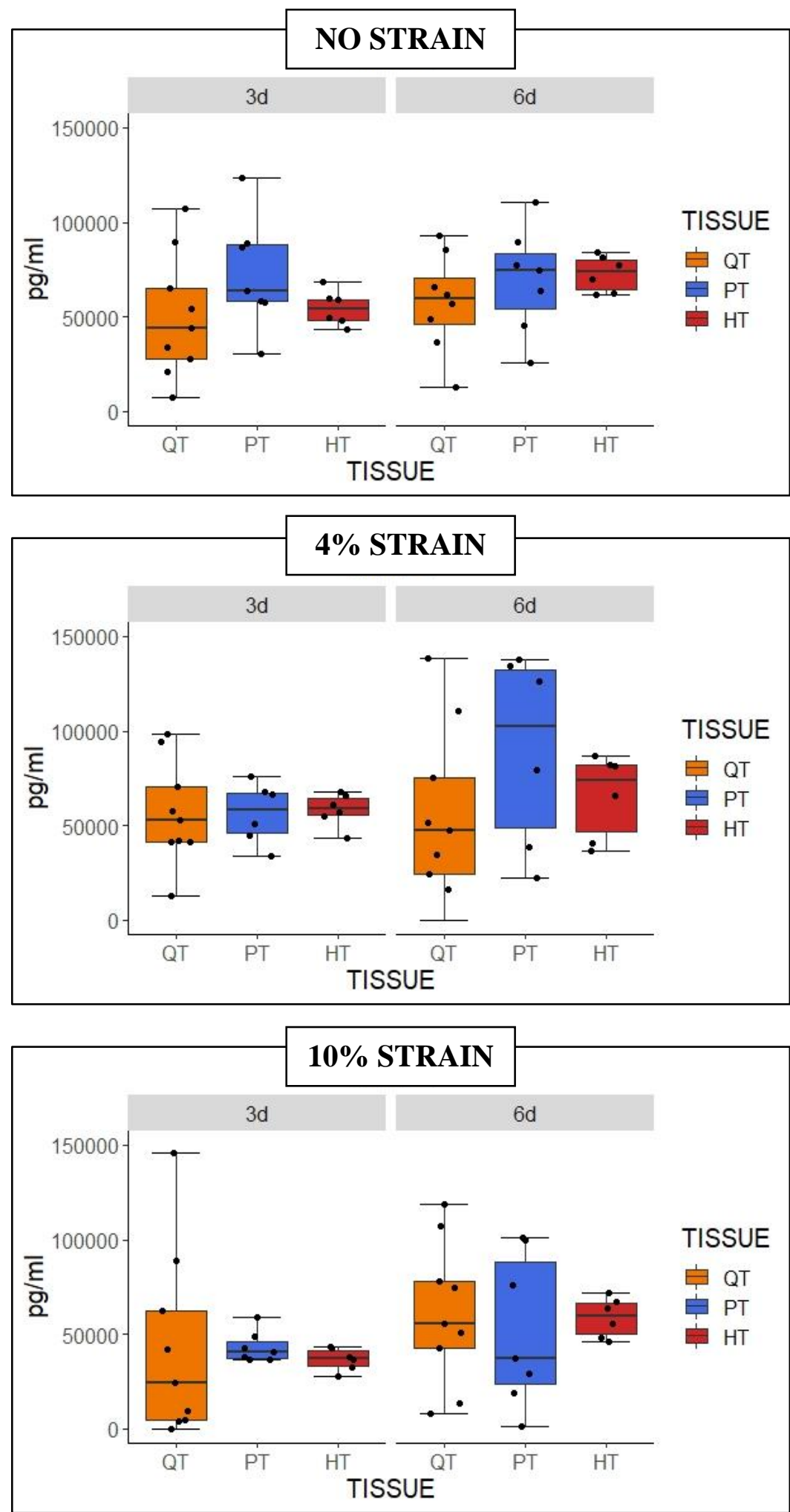

Figure 8.26. Concentration of MMP- 2 released to the media. * significant difference between TISSUES at three (3d) and six (6d) days of culture. QT: Quadriceps tendon; PT: Patellar tendon; HT: Hamstring tendon. 

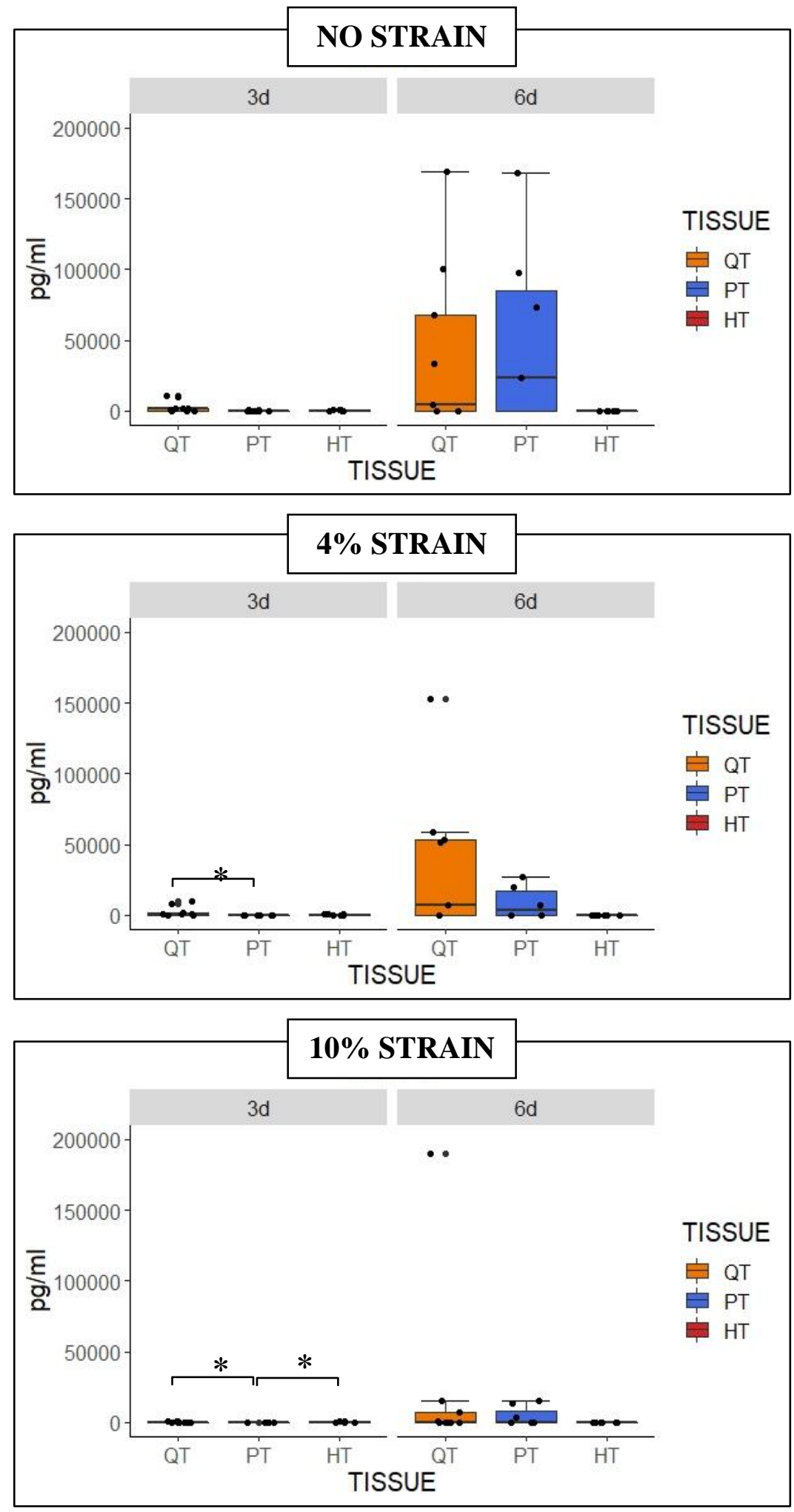

Figure 8.27. Concentration of MMP-3 released to the media. * significant difference between TISSUES at three (3d) and six (6d) days of culture. QT: Quadriceps tendon; PT: Patellar tendon; HT: Hamstring tendon. 

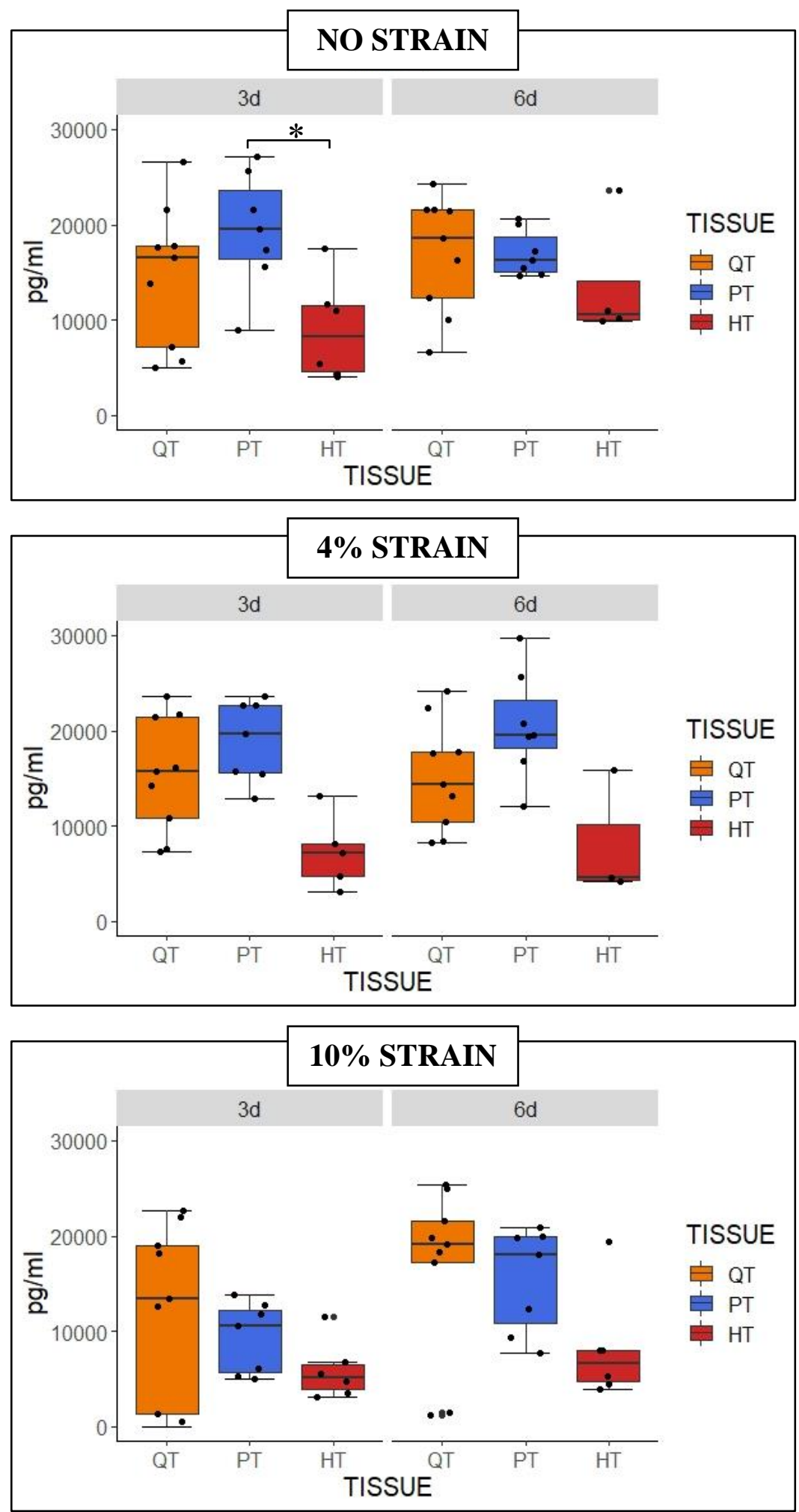

Figure 8.28. Concentration of TIMP-1 released to the media. * significant difference between TISSUES at three (3d) and six (6d) days of culture. QT: Quadriceps tendon; PT: Patellar tendon; HT: Hamstring tendon. 

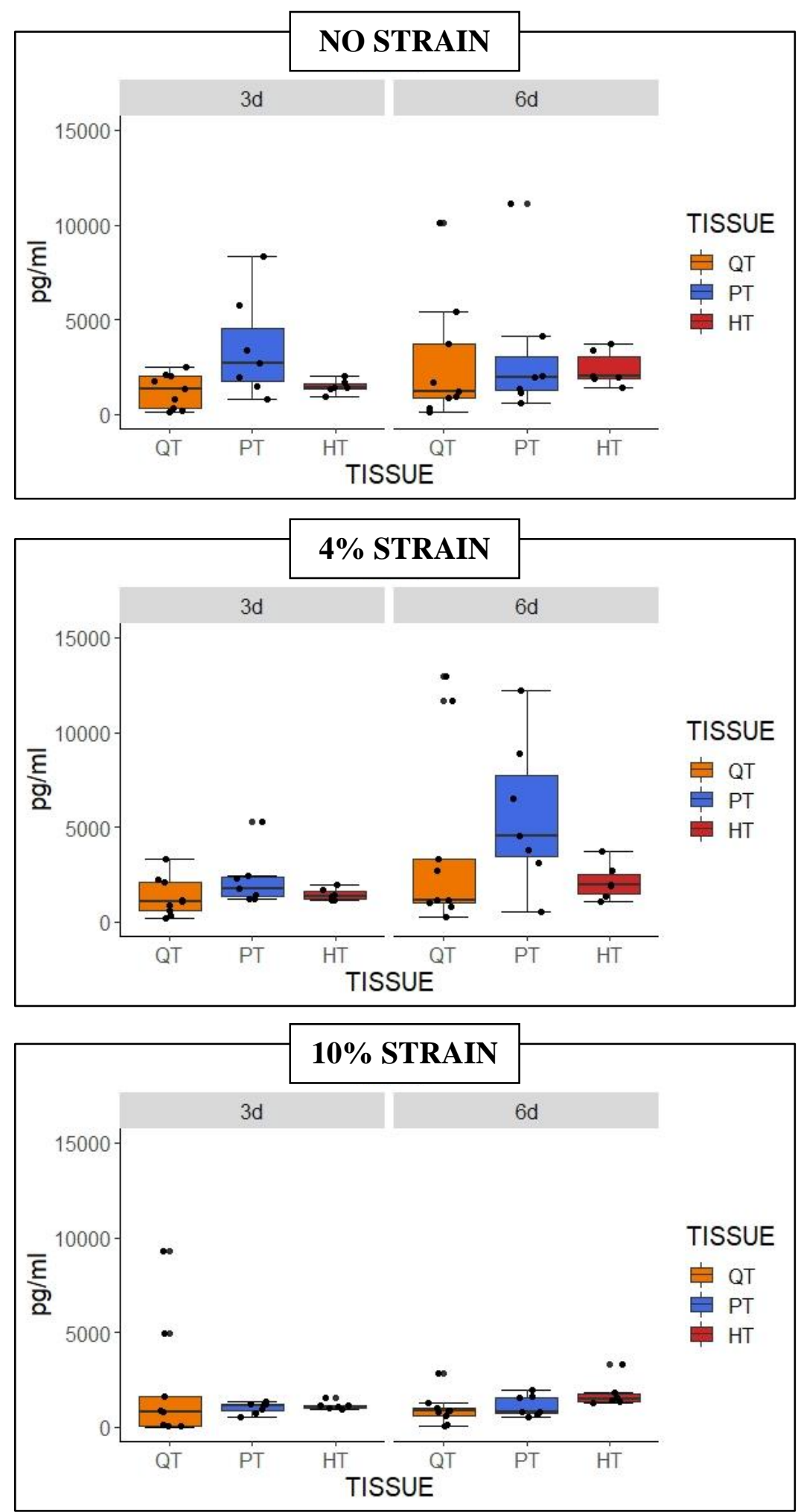

Figure 8.29. Concentration of TIMP- 2 released to the media. * significant difference between TISSUES at three (3d) and six (6d) days of culture. QT: Quadriceps tendon; PT: Patellar tendon; HT: Hamstring tendon. 

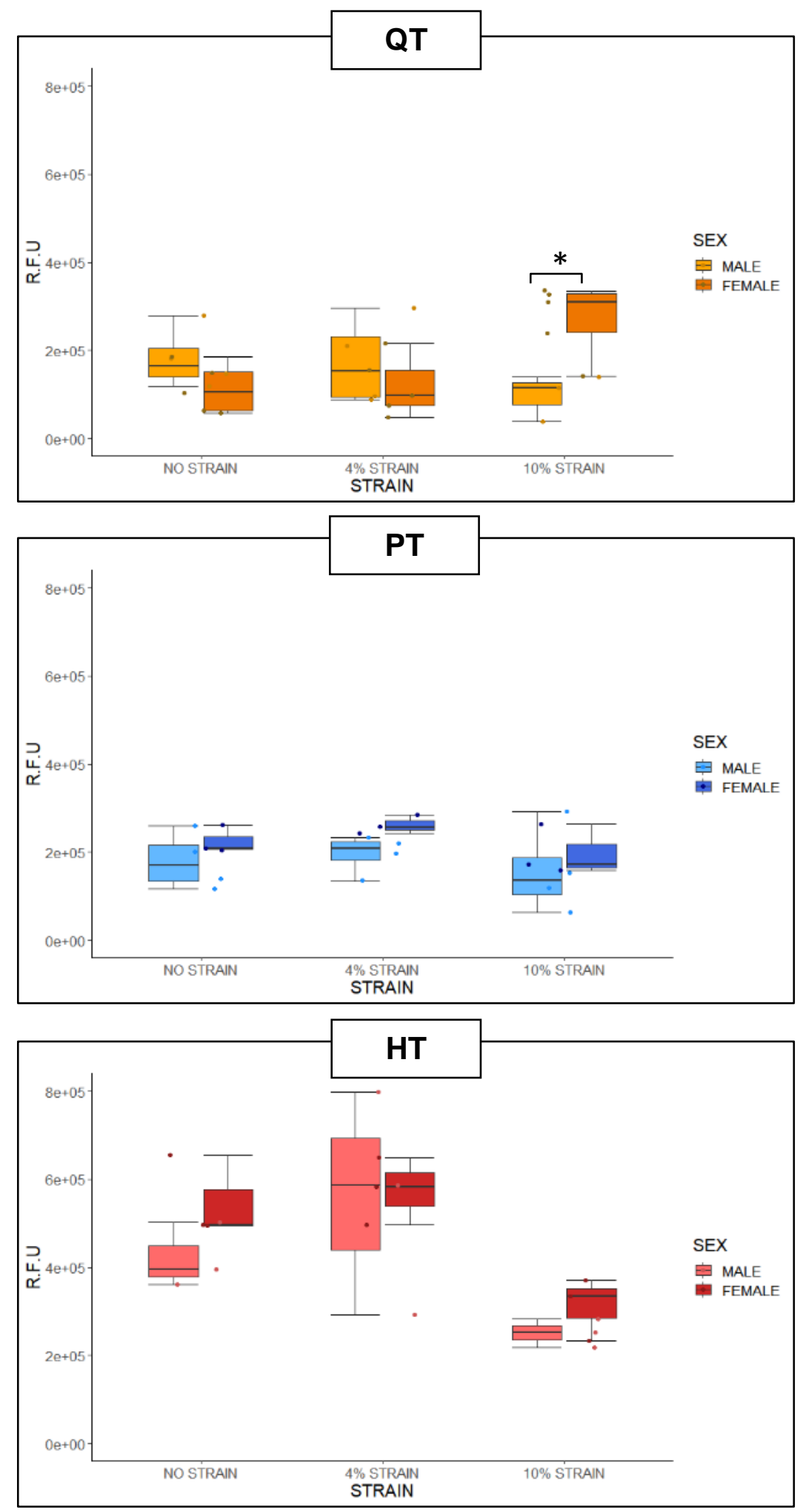

Figure 8.30. Metabolic Activity of fibroblasts after $120 \mathrm{~h}$ of culture. ${ }^{*}$ significant difference between males and females (Signed Rank test). QT: Quadriceps tendon; PT: Patellar tendon, HT: Hamstring tendon 


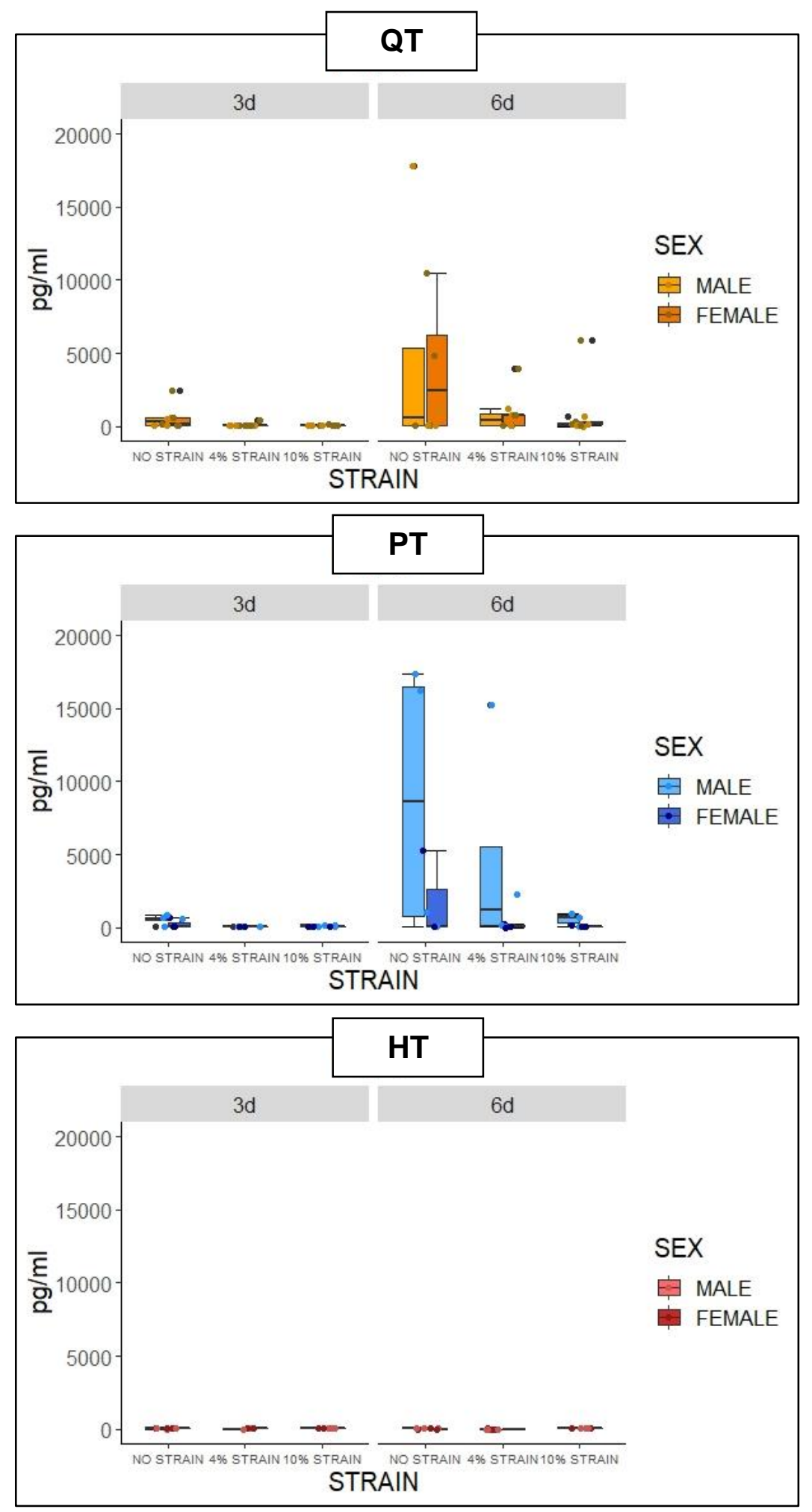

Figure 8.31. Concentration of PGE2 released to the media. * significant difference between males and females at 24 and 120 hours (Signed Rank test). QT: Quadriceps tendon; PT: Patellar tendon, HT: Hamstring tendon 

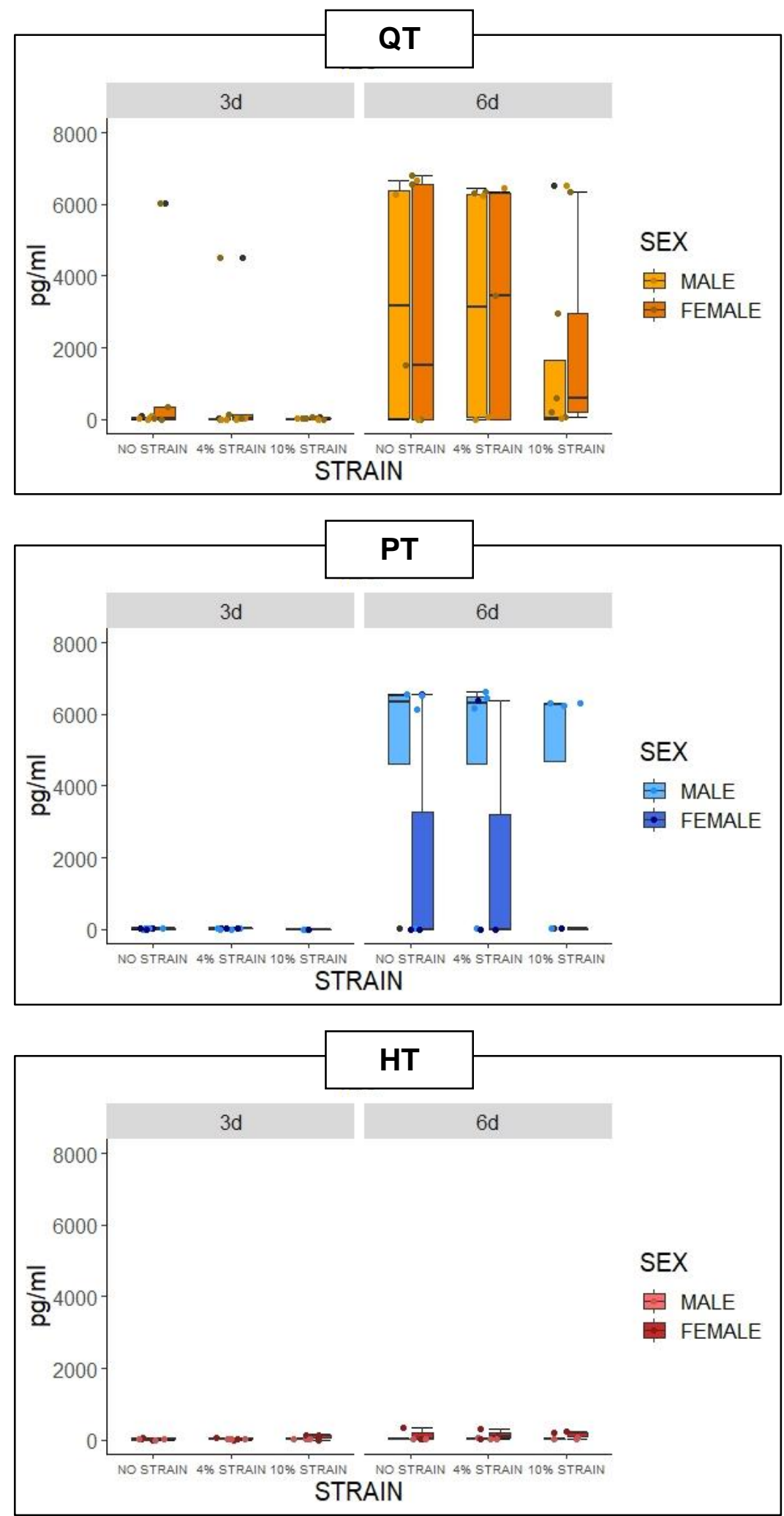

Figure 8.32. Concentration of IL-6 released to the media. * significant difference males and females at 24 and 120 hours (Signed Rank test). QT: Quadriceps tendon; PT: Patellar tendon, HT: Hamstring tendon 

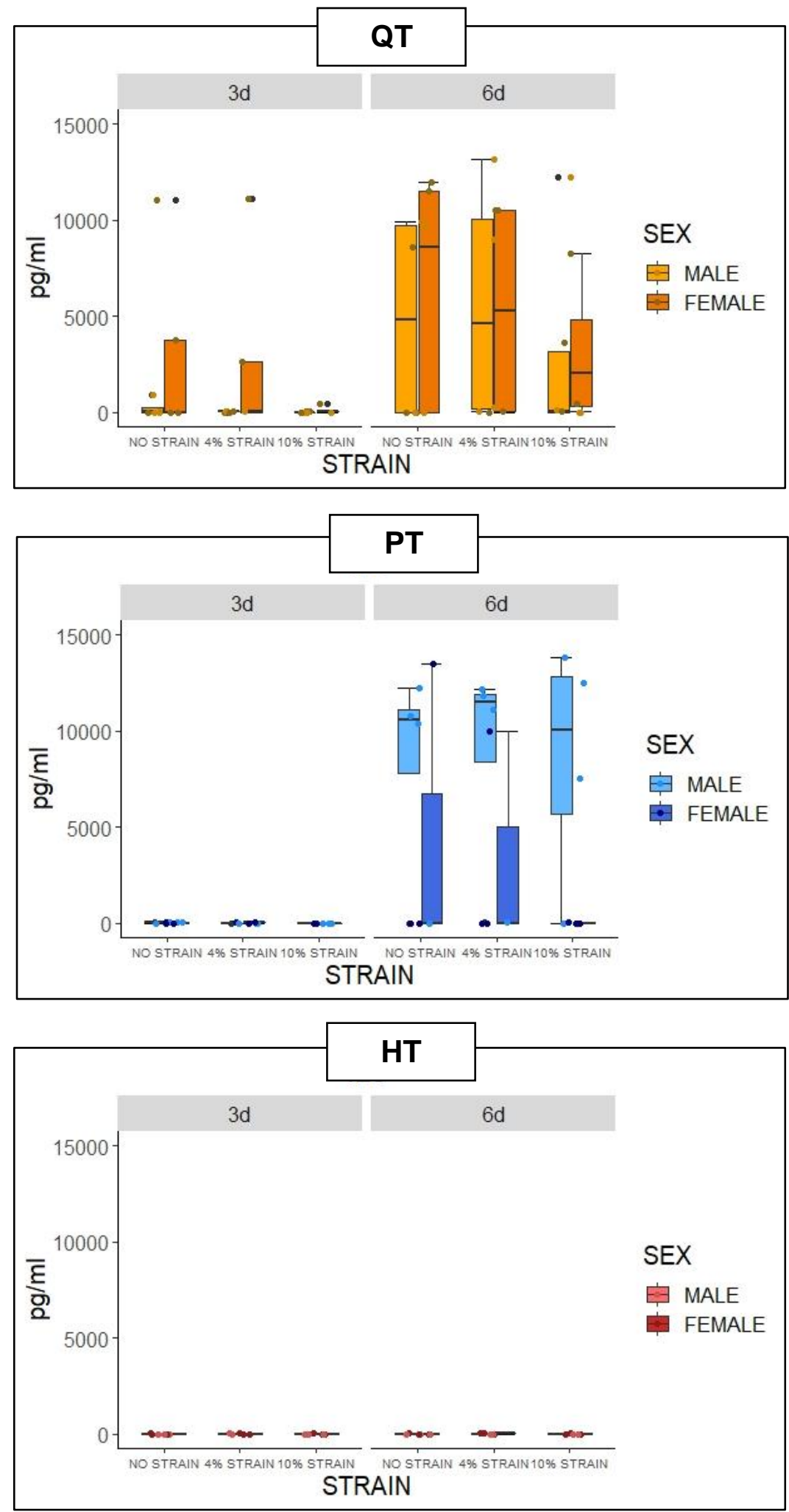

Figure 8.33. Concentration of IL-8 released to the media. * significant difference males and females at 24 and 120 hours (Signed Rank test). QT: Quadriceps tendon; PT: Patellar tendon, HT: Hamstring tendon 

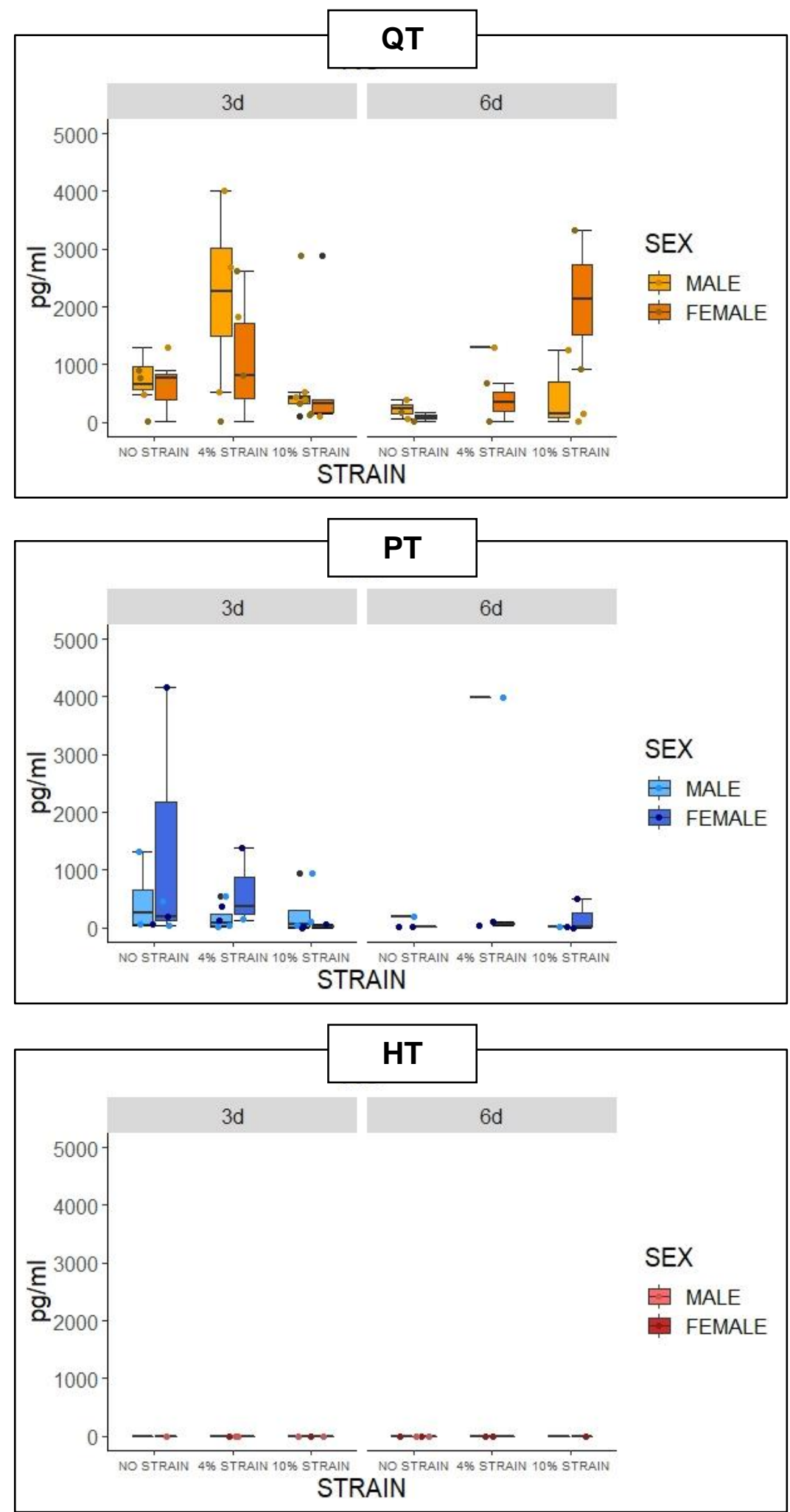

Figure 8.34. Concentration of $\mathrm{KC}$ released to the media. * significant difference between males and females at 24 and 120 hours (Signed Rank test). QT: Quadriceps tendon; PT: Patellar tendon, HT: Hamstring tendon 

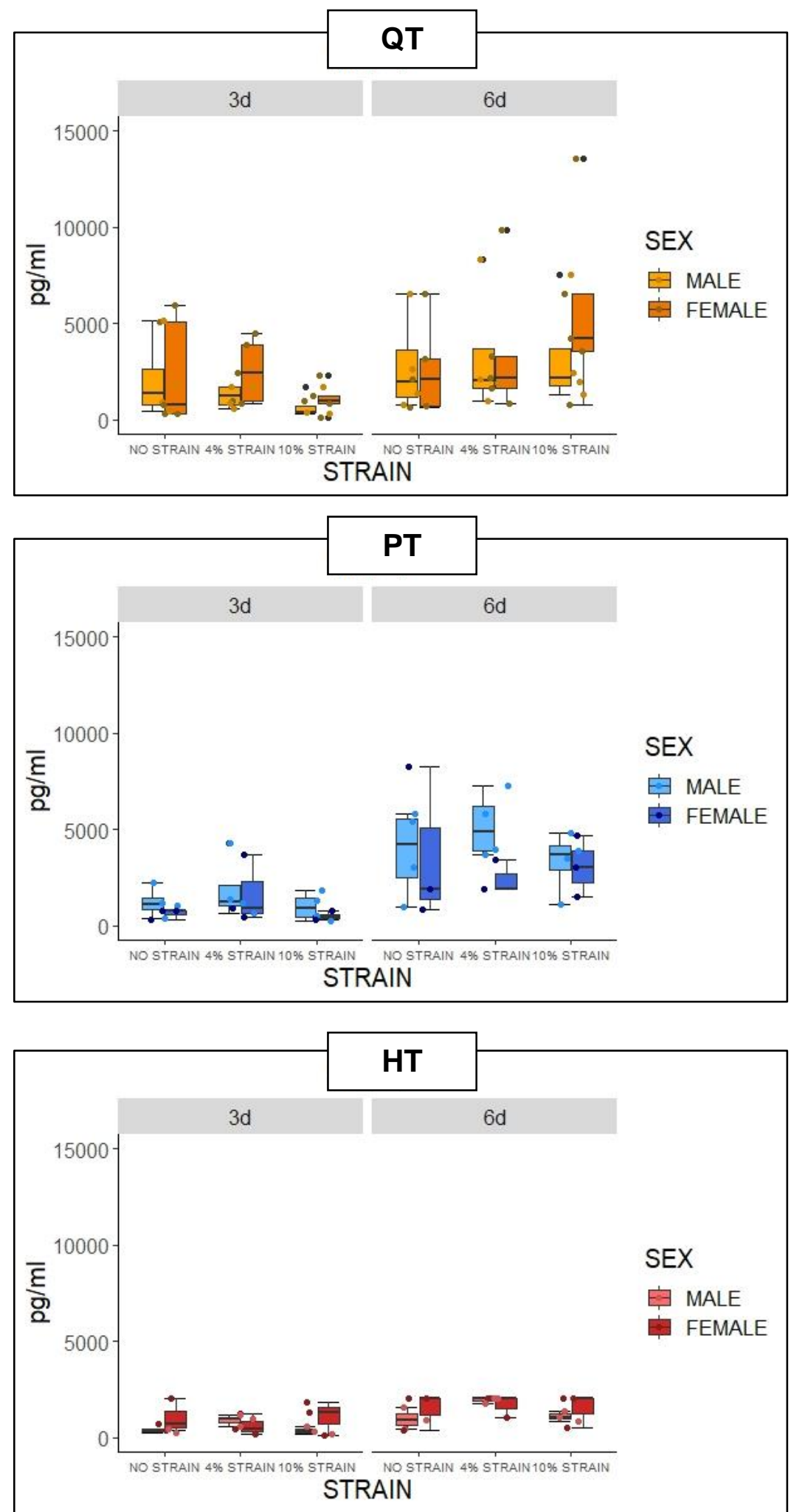

Figure 8.35. Concentration of MCP1 released to the media. * significant difference between males and females at 24 and 120 hours (Signed Rank test). QT: Quadriceps tendon; PT: Patellar tendon, HT: Hamstring tendon 

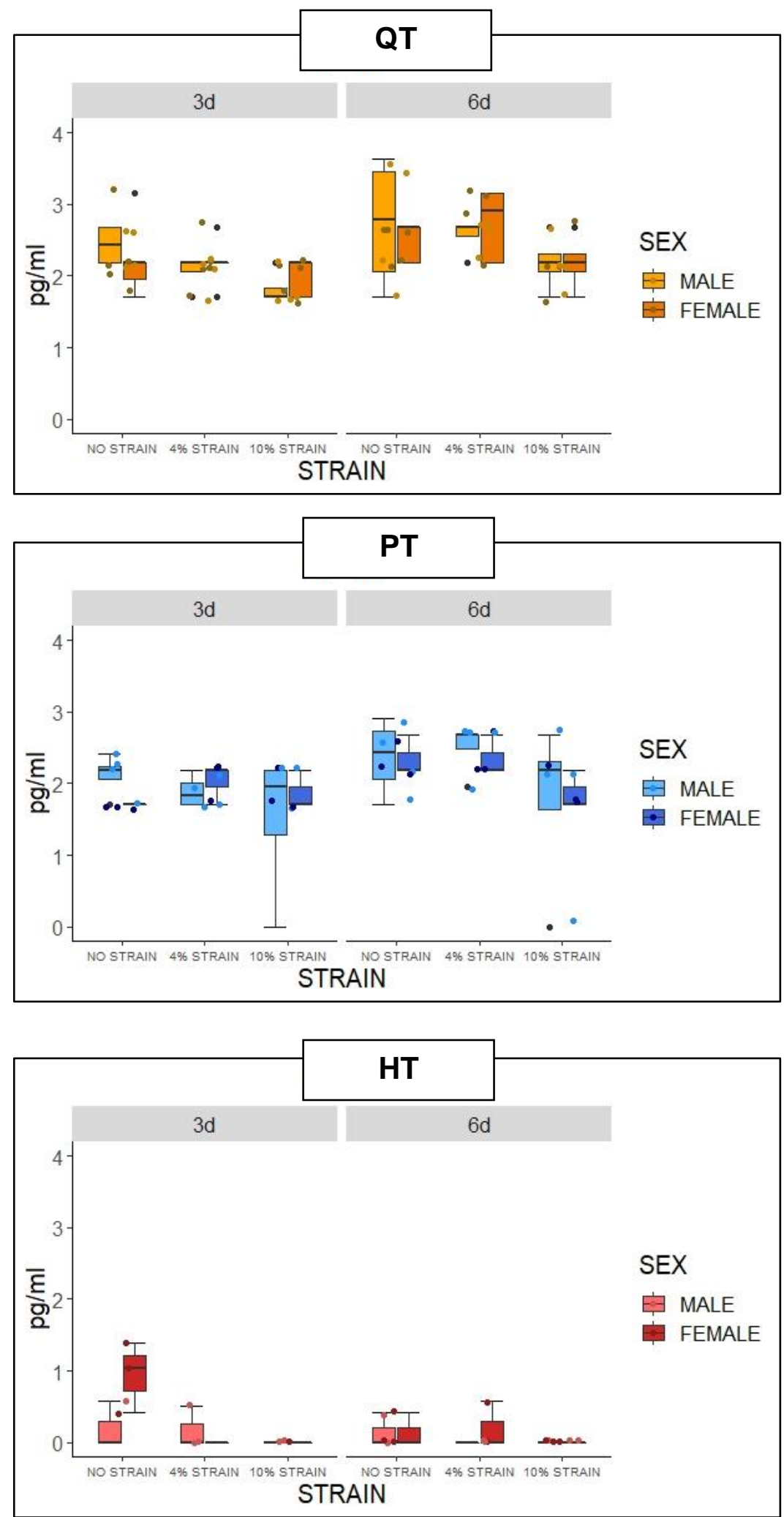

Figure 8.36. Concentration of MP1 $\beta$ released to the media. * significant difference between males and females at 24 and 120 hours (Signed Rank test). QT: Quadriceps tendon; PT: Patellar tendon, HT: Hamstring tendon 

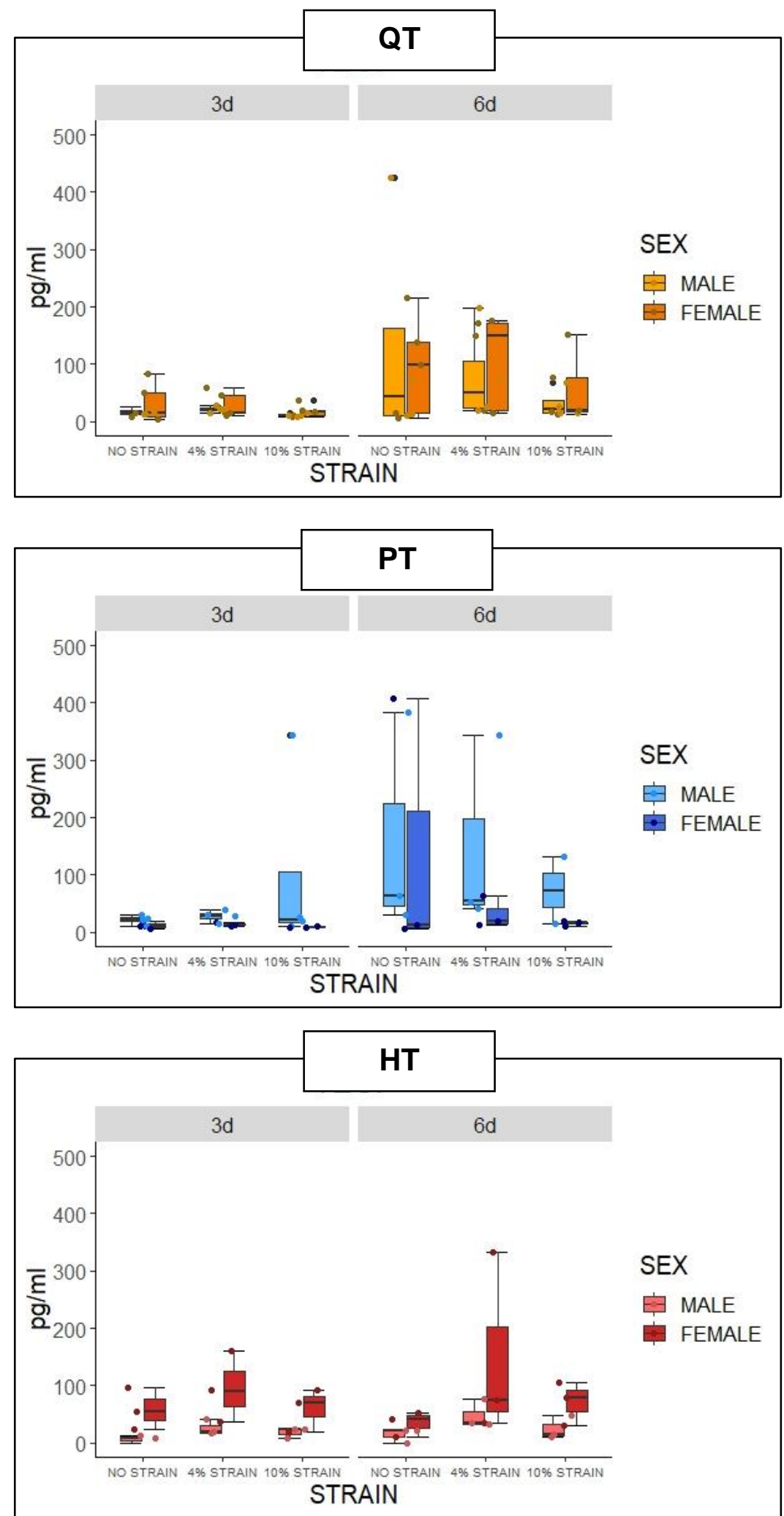

Figure 8.37. Concentration of VEGF released to the media. * significant difference between males and females at 24 and 120 hours (Signed Rank test). QT: Quadriceps tendon; PT: Patellar tendon, HT: Hamstring tendon 

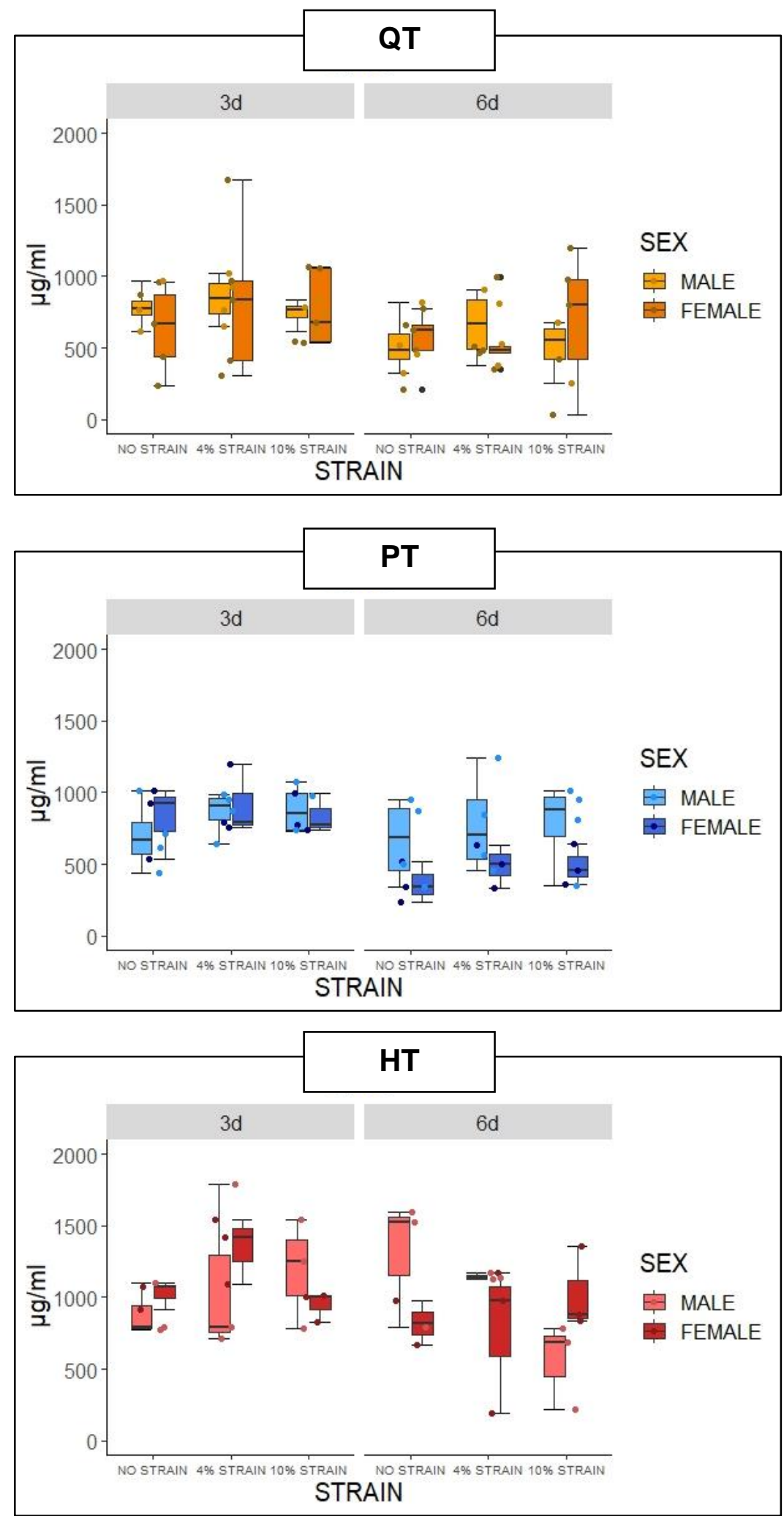

Figure 8.38. Concentration of GAG released to the media. * significant difference between males and females at 24 and 120 hours (Signed Rank test). QT: Quadriceps tendon; PT: Patellar tendon, HT: Hamstring tendon 

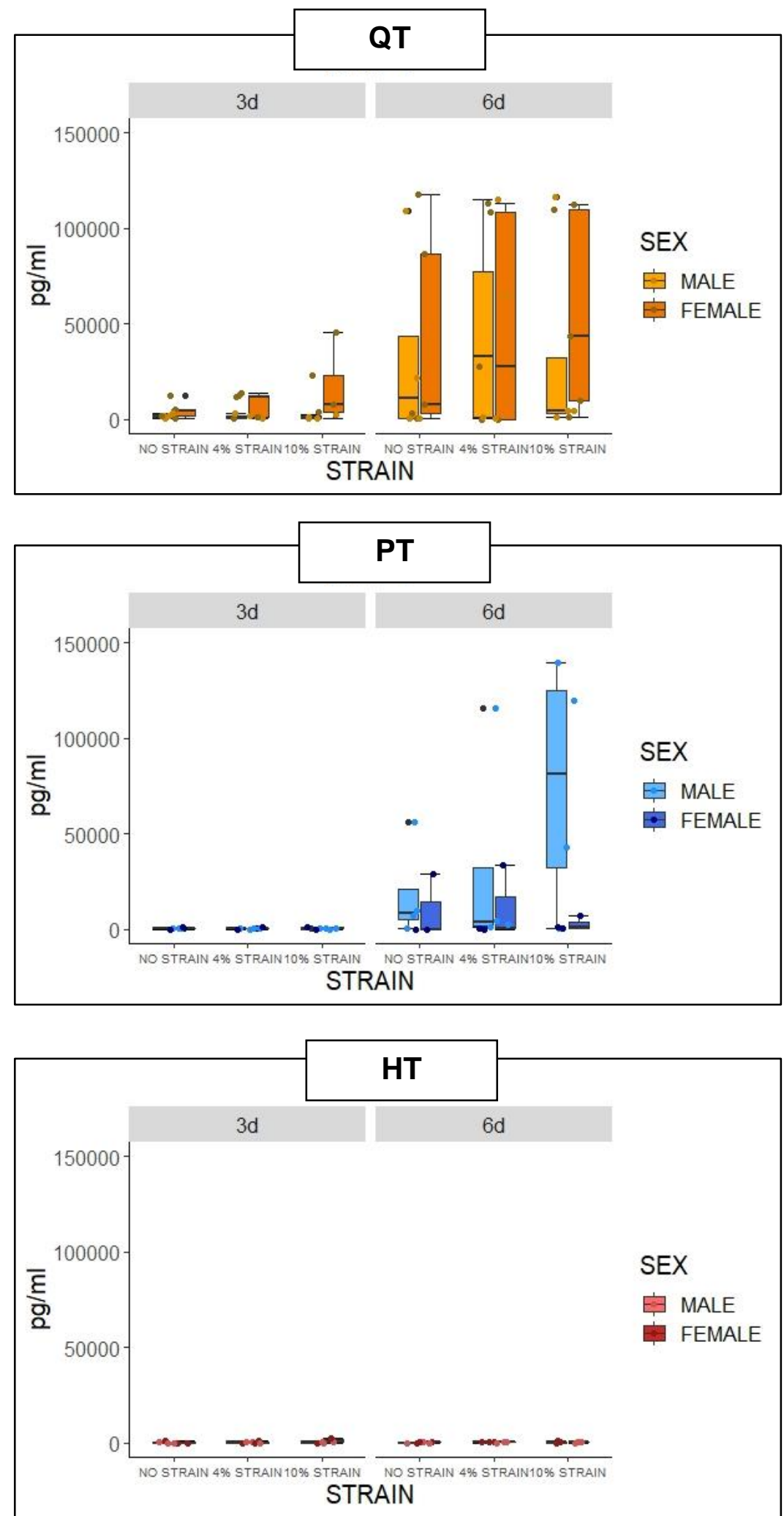

Figure 8.39. Concentration of MMP-1 released to the media. * significant difference males and females at 24 and 120 hours (Signed Rank test). QT: Quadriceps tendon; PT: Patellar tendon, HT: Hamstring tendon 

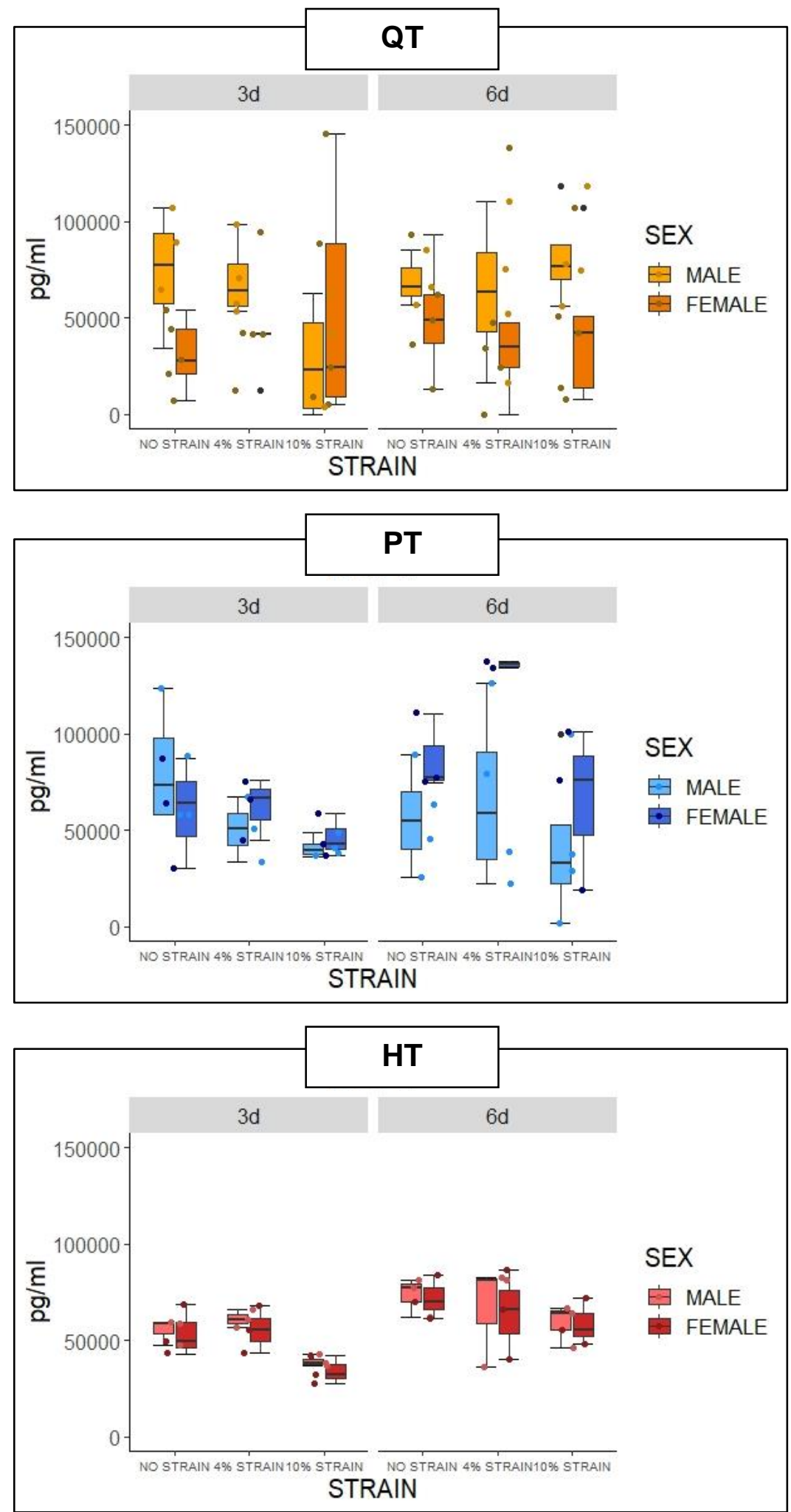

Figure 8.40. Concentration of MMP-2 released to the media. * significant difference between males and females at 24 and 120 hours (Signed Rank test). QT: Quadriceps tendon; PT: Patellar tendon, HT: Hamstring tendon 

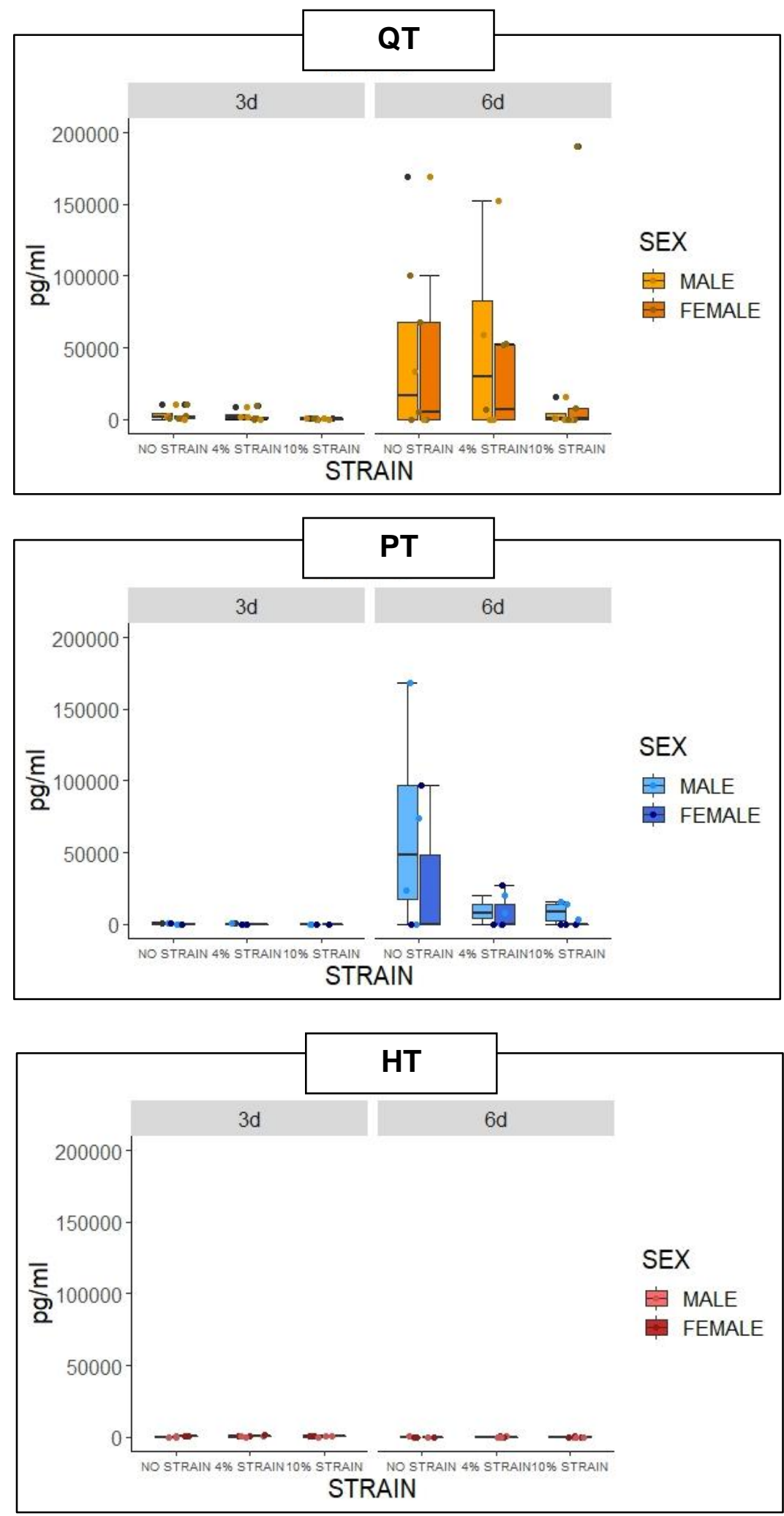

Figure 8.41. Concentration of MMP-3 released to the media. * significant difference males and females at 24 and 120 hours (Signed Rank test). QT: Quadriceps tendon; PT: Patellar tendon, HT: Hamstring tendon 

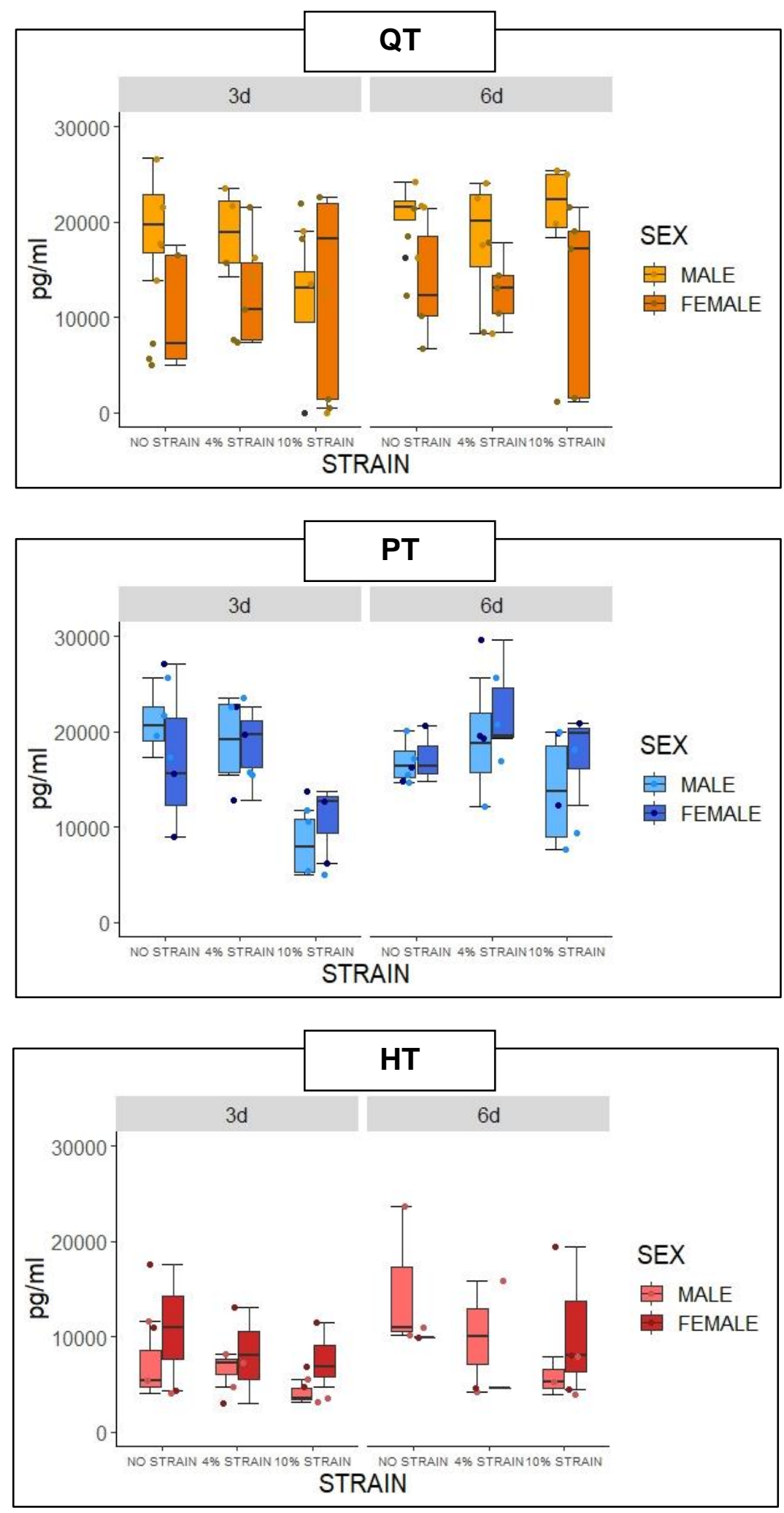

Figure 8.42. Concentration of TIMP-1 released to the media. * significant difference between males and females at 24 and 120 hours (Signed Rank test). QT: Quadriceps tendon; PT: Patellar tendon, HT: Hamstring tendon 

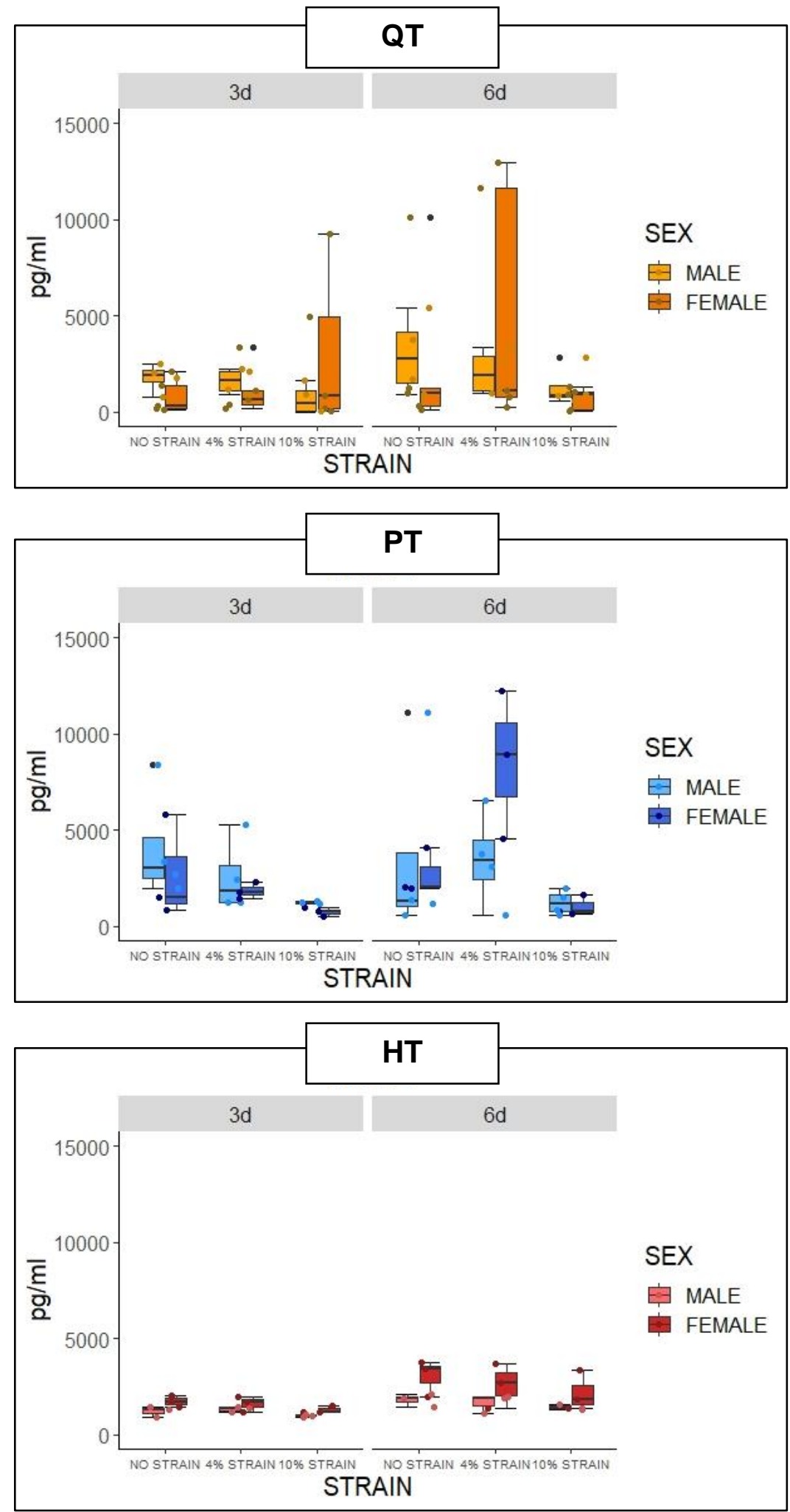

Figure 8.43. Concentration of TIMP-2 released to the media. * significant difference between males and females at 24 and 120 hours (Signed Rank test). QT: Quadriceps tendon; PT: Patellar tendon, HT: Hamstring tendon 


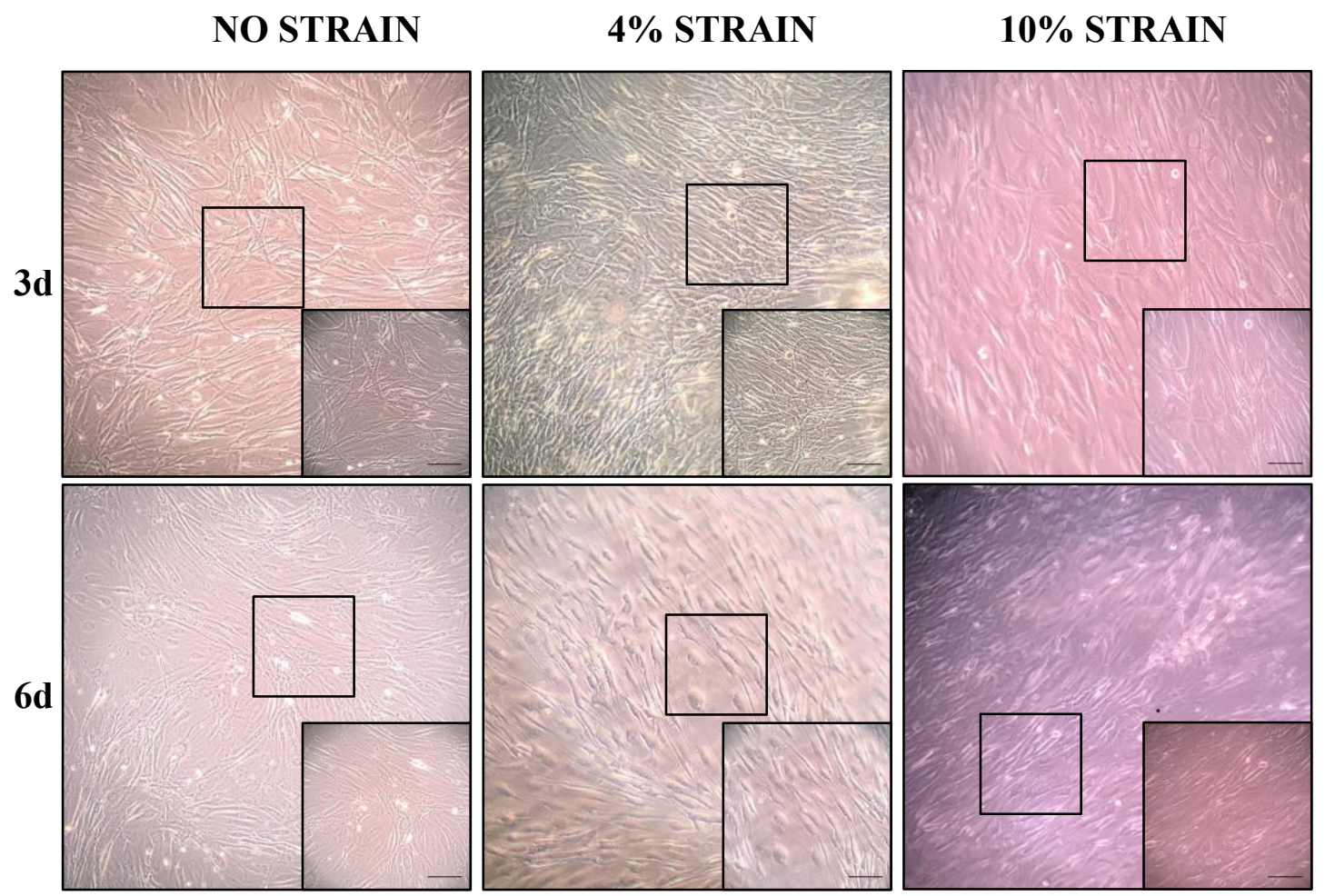

Figure 8.44. Quadriceps Tendon (QT) fibroblasts stretched after three days and six days at three different stain magnitudes. Scale Bar:50 $\mu \mathrm{m}$

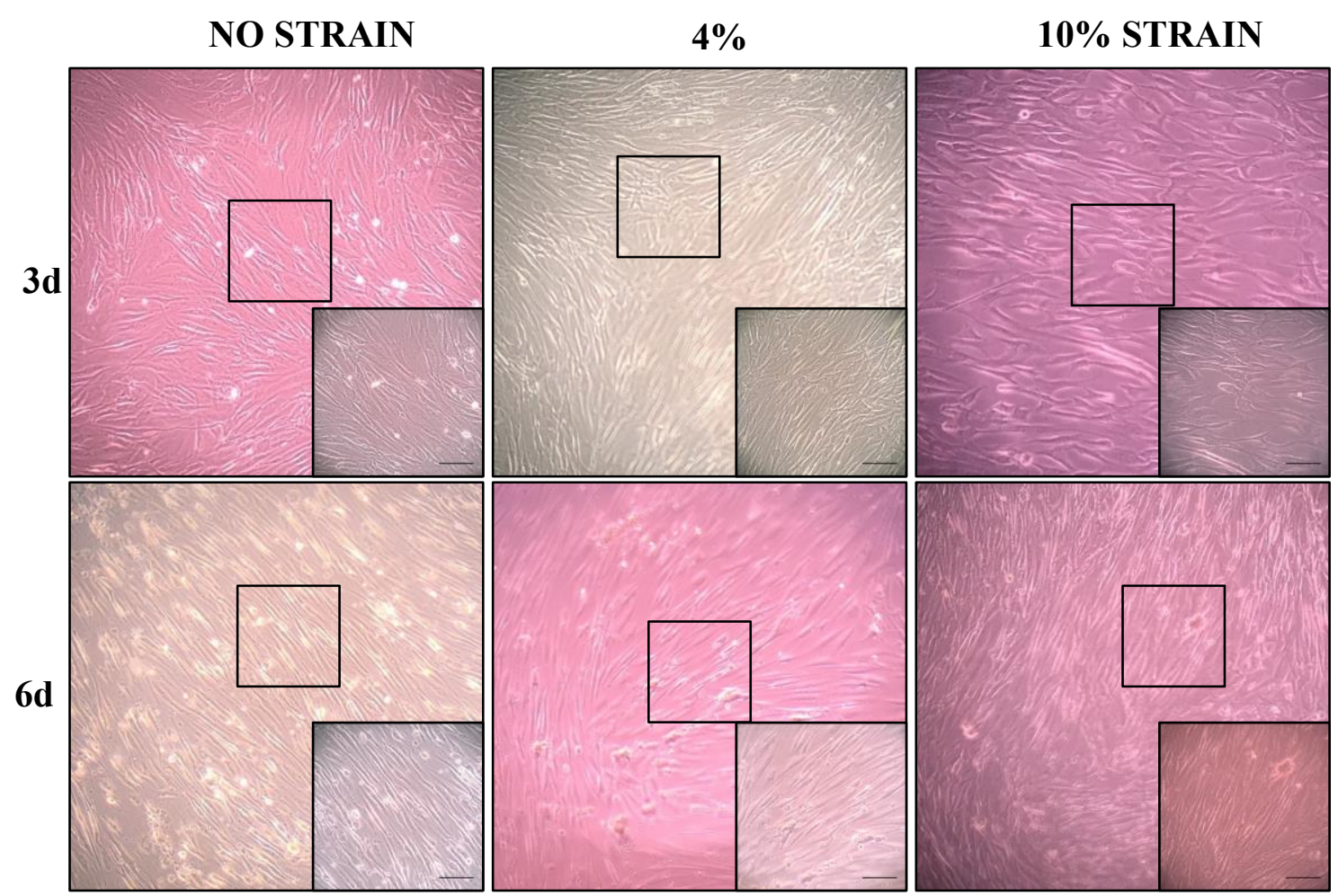

Figure 8.45. Patellar Tendon (PT) fibroblasts stretched after three days and six days at three different stain magnitudes. Scale Bar:50 $\mu \mathrm{m}$ 


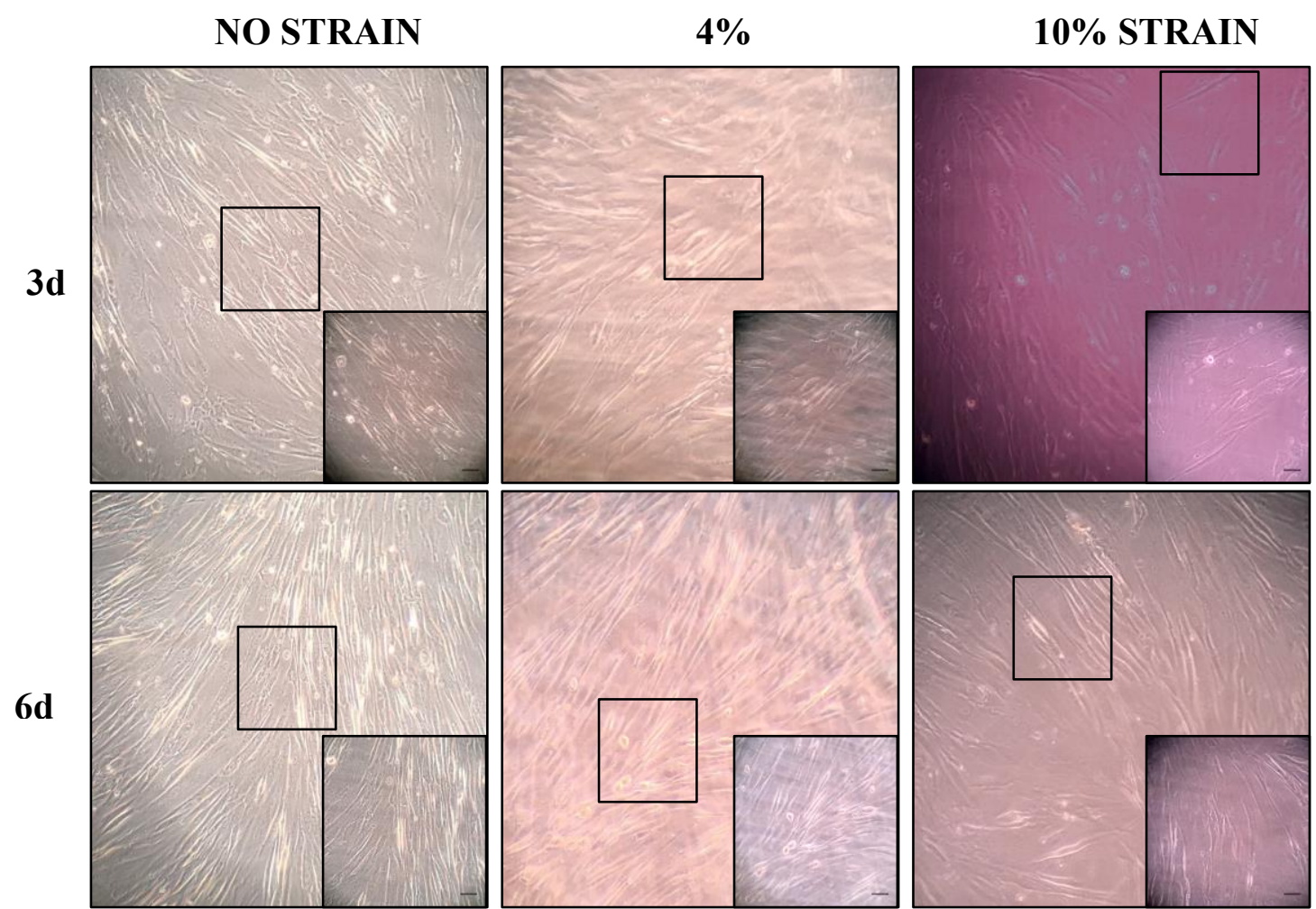

Figure 8.46. Hamstring Tendon (HT) fibroblasts stretched after three days and six days at three different stain magnitudes. Scale Bar:50 $\mu \mathrm{m}$ 


\section{CONCLUSIONS AND FUTURE DIRECTIONS}

This work uses a novel approach to understand cellular biology of the knee intraarticular tissues and tendon grafts. The use of a bioreactor to stress fibroblasts in monolayer, successfully stimulated cells to produce different metabolic biomarkers in response to varying levels of clinically relevant strains. Moreover, these responses are also tissue dependent. We have also demonstrated that fibroblasts obtained from different tissue sources are a prominent supply of metabolic markers in response to cyclic strain. Important relations were noted in response to strain such as the influence of sex and culture conditions. Moreover, our study demonstrated similar patterns in both, dogs and humans, showing that the canine model is suitable for investigating metabolic responses in stressed monolayer tendon and ligament fibroblastic cultures. Subsequent studies will aim to evaluate specific variables such as bone-tendon and cartilage-tendon responses to strain. Further, research is needed to establish if metabolic responses in vitro are also found in vivo. Our lab is currently working on evaluating metabolic markers in synovial fluid and urine from patients undergoing ACL reconstruction. We are interested in evaluating how physical therapy and level of activity will affect ACL graft healing after surgery. Therefore, we recommend a thorough analysis to match the patient and the tendon graft based on sex and level of activity of the individual. 


\section{VITA}

Dr. Sebastian Cardona-Ramirez was born in Manizales, Colombia. He attended Universidad de Caldas where he earned a Bachelor in Veterinary and Animal Sciences degree. He then continued to pursue postgraduate education in his alma mater and enrolled in a Master in Veterinary Sciences degree in 2013 being awarded Cum Laude thesis defense for his work on serum biomarkers of osteoarthritis in dogs with hip dysplasia. He was simultaneously working as a part-time professor in the Universidad del Tolima where he taught different courses including Small Animal Internal Medicine, Semiology and Diagnostic Imaging. He was also working as a veterinary clinician in private practice. In May 2016 he was awarded a Fulbright-COLCIENCIAS scholarship for funding Doctoral studies in the United States. He joined Dr. Cook's team in August 2016 in the Thompson Laboratory for Regenerative Orthopaedics (TLRO) as a full-time graduate student. He has been awarded several research and travel awards including Phi Zeta Grant, John D. Bies travel award and the TLRO Graduate Excellence in Research Award in 2019. He will pursue a postdoctoral fellowship in the TLRO to continue his investigation on In Vitro models for Orthopaedic Research. 